\title{
Michel Foucault et le christianisme
}

Philippe Chevallier

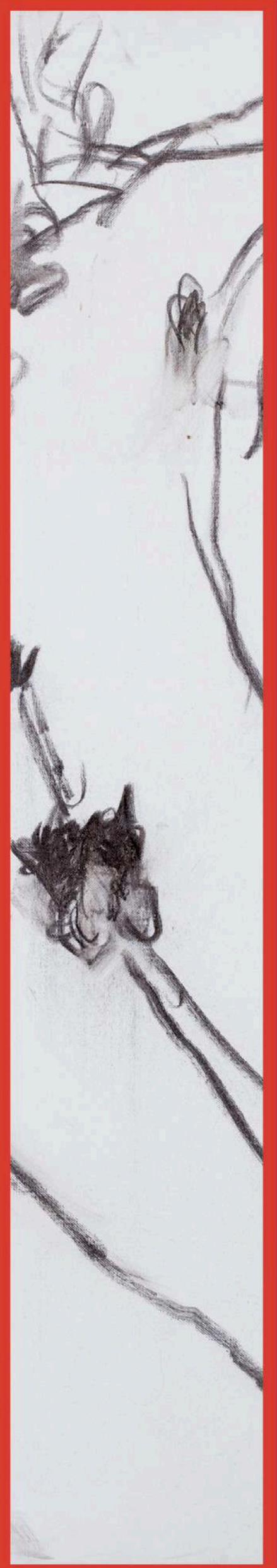




\section{Michel Foucault et le christianisme}

\section{Philippe Chevallier}

DOI : 10.4000/books.enseditions.9714

Éditeur : ENS Éditions

Lieu d'édition : Lyon

Année d'édition : 2011

Date de mise en ligne : 4 juillet 2019

Collection : La croisée des chemins

EAN électronique : 9791036200472

\section{Q OpenEdition \\ Books}

https://books.openedition.org

\section{Édition imprimée}

Date de publication : 1 décembre 2011

EAN (Édition imprimée) : 9782847883251

Nombre de pages : 384

\section{Référence électronique}

CHEVALLIER, Philippe. Michel Foucault et le christianisme. Nouvelle édition [en ligne]. Lyon : ENS

Éditions, 2011 (généré le 28 janvier 2022). Disponible sur Internet : <http://books.openedition.org/ enseditions/9714>. ISBN : 9791036200472 . DOI : https://doi.org/10.4000/books.enseditions. 9714

(c) ENS Éditions, 2011

Conditions d'utilisation

http://www.openedition.org/6540 


\section{RÉSUMÉS}

Des premiers rites baptismaux à la confession moderne, les références au christianisme sont constantes dans l'œuvre de Michel Foucault. Cette constance s'inscrit dans un questionnement philosophique plus large sur notre actualité : comprendre le rapport que nous avons aujourd'hui à nous-mêmes demande de s'interroger sur les actes de vérité que l'Occident a instaurés depuis les premiers siècles chrétiens. Que faut-il dire et manifester de soi pour être transformé dans son être, pardonné, sauvé, jugé ou guéri? Ce livre propose une étude critique de l'ensemble des lectures chrétiennes de Foucault, avec une attention particulière portée au cours Du gouvernement des vivants (1979-1980). Ni chronologique ni thématique, le parcours suivi espère retrouver la logique d'un travail à la fois philosophique et historique : quand et comment le christianisme a-til été constitué par Foucault en objet de recherche, avec quelles pratiques de lecture et quelles conséquences sur l'interprétation? Attentif aux mots plus qu'aux choses, le philosophe repère les glissements sémantiques successifs qui annoncent, entre le $\mathrm{II}^{\mathrm{e}}$ et le $\mathrm{IV}^{\mathrm{e}}$ siècle de notre ère, le passage du monde antique à un univers inédit : celui de la perfection impossible et des fidélités difficiles. Loin de l'image facile d'un christianisme ascétique et intransigeant, Foucault définit l'originalité chrétienne comme la reconnaissance et l'institution paradoxale d'un rapport précaire à la vérité.

\section{PHILIPPE CHEVALLIER}

Docteur en philosophie de l'université Paris-Est, il a récemment publié Être soi, Actualité de Søren Kierkegaard (François Bourin, 2011). Il travaille actuellement à la Bibliothèque nationale de France.

\section{NOTE DE L'ÉDITEUR}

Illustration de couverture : Judit Reigl, Dyonisos crucifié, 1998. ㄷ Adagp, Paris 2011. 
Cet ouvrage est diffusé en accès ouvert dans le cadre du projet OpenEdition Books Select.

Ce programme de financement participatif, coordonné par OpenEdition en partenariat avec Knowledge Unlatched et le consortium Couperin, permet aux bibliothèques de contribuer à la libération de contenus provenant d'éditeurs majeurs dans le domaine des sciences humaines et sociales.

La liste des bibliothèques ayant contribué financièrement à la libération de cet ouvrage se trouve ici :

https://www.openedition.org/22515.

This book is published open access as part of the OpenEdition Books Select project.

This crowdfunding program is coordinated by OpenEdition in partnership with Knowledge Unlatched and the French library consortium Couperin. Thanks to the initiative, libraries can contribute to unlatch content from key publishers in the Humanities and Social Sciences.

Discover all the libraries that helped to make this book available open access: https://www.openedition.org/22515?lang=en.

\section{IAI Sopenedition coupernoren}




\title{
LA CROISÉE DES CHEMINS
}

\author{
Collection dirigée par \\ Pierre-François Moreau et Michel Senellart
}

Recherches, héritages, controverses : telles sont quelques-unes des formes que prend le mouvement des idées. L'histoire de la pensée ne se limite pas à des systèmes grandioses et fermés sur eux-mêmes; elle est constituée également par des discours accumulés, des polémiques, des migrations conceptuelles d'un secteur de la pensée à un autre. La collection «La croisée des chemins » publie des textes consacrés à l'histoire intellectuelle et à ses retentissements actuels : philosophie, théorie politique et juridique, esthétique et enjeux des pratiques scientifiques. Elle s'emploie également à faire connaître la recherche étrangère en ces domaines et à donner à lire les textes fondamentaux qui ont marqué les grands moments de cette histoire. 

LA CROISÉE DES CHEMINS

\section{Michel Foucault et le christianisme}

Philippe Chevallier

ENS ÉDITIONS 


\section{Éléments de catalogage avant publication}

Michel Foucault et le christianisme / Philippe Chevallier - Lyon : ENS Éditions, impr. 2011. - 1 vol. (384 p.) : couv. ill. ; 22 cm. - (La croisée des chemins, ISSN 1765-8128)

Bibliogr. : p. 361-367. Notes bibliogr. Index

ISBN 978-2-84788-325-1 (br.) : 29 EUR

Tous droits de reproduction, de traduction et d'adaptation réservés pour tous pays. Toute représentation ou reproduction intégrale ou partielle faite par quelque procédé que ce soit, sans le consentement de l'éditeur, est illicite et constitue une contrefaçon. Les copies ou reproductions destinées à une utilisation collective sont interdites.

(C) ENS ÉDITIONS 2011

École normale supérieure de Lyon

15 parvis René Descartes

BP 7000

69342 Lyon cedex 07

ISBN 978-2-84788-325-1 
Les aléas de l'existence, pour lesquels je n'étais sans doute pas aussi bien équipé qu' un vrai philosophe, ont failli me faire renoncer plusieurs fois à cette recherche. Si elle touche aujourd'hui à sa fin, c'est grâce aux encouragements constants de Frédéric Gros, mon directeur de thèse, et du père François Marty s.j.

L'équipe de l'ANR corpus 2008-2010 «La bibliothèque foucaldienne » (Philippe Artières, Jean-François Bert, Pascal Michon, Mathieu Potte-Bonneville, Judith Revel, José Ruiz-Funes, Michel Senellart et Jean-Claude Zancarini) a également été une source constante d'inspiration.

Thierry Grillet et Stéphane Duchesne, de la Bibliothèque nationale de France, m'ont apporté leur soutien et leur confiance au moment où j'en avais le plus besoin, tandis que Pascal Gauderon, une fois de plus, était mis à contribution pour relire français, grec et latin le stylo à la main. Il le fit, amigo en El Señor.

Daniel Defert et le père Michel Albaric o.p. m'offrirent leur temps et leurs souvenirs, dans leurs scriptoria respectifs. Gratitude éternelle.

Enfin, ce livre voit le jour grâce à l'accueil généreux de Michel Senellart, ce qui ne fait qu'augmenter une dette contractée depuis bien longtemps déjà.

Ce livre est dédié à Ko-Shu SUN 



\section{MICHEL FOUCAULT, BIBLIOGRAPHIE}

\section{Textes et communications publiés}

Folie et déraison. Histoire de la folie à l'âge classique, Paris, Plon, 1961.

Naissance de la clinique, Paris, PUF (Quadridge), 2007 [1963].

Les mots et les choses, Paris, Gallimard (Tel), 1992 [1966].

L'archéologie du savoir, Paris, Gallimard (Bibliothèque des sciences humaines), 1969.

L'ordre du discours. Leçon inaugurale au Collège de France prononcée le 2 décembre 1970, Paris, Gallimard, 1971.

Histoire de la folie à l'âge classique, Paris, Gallimard (Tel), 1976 [1972].

Surveiller et punir. Naissance de la prison, Paris, Gallimard (Tel), 1993 [1975].

Histoire de la sexualité, t. I : La volonté de savoir, Paris, Gallimard (Tel), 1994 [1976].

Histoire de la sexualité, t. II : L'usage des plaisirs, Paris, Gallimard (Tel), 1997 [1984].

Histoire de la sexualité, t. III : Le souci de soi, Paris, Gallimard (Tel), 1997 [1984].

«Qu'est-ce que la critique?», Bulletin de la Société française de philosophie, t. 84, 1990, p. 35-63.

Dits et écrits, t. I : 1954-1975, D. Defert et F. Ewald éd., Paris, Gallimard (Quarto), $2001[1994]$.

Dits et écrits, t. II : 1976-1988, D. Defert et F. Ewald éd., Paris, Gallimard (Quarto), 2001 [1994].

Il faut défendre la société. Cours au collège de France, 1975-1976, M. Bertani et A. Fontana éd., Paris, Seuil/Gallimard (Hautes études), 1997.

Les anormaux. Cours au Collège de France, 1974-1975, V. Marchetti et A. Salomoni éd., Paris, Seuil/Gallimard (Hautes études), 1999.

L’herméneutique du sujet. Cours au Collège de France, 1981-1982, F. Gros éd., Paris, Seuil/Gallimard (Hautes études), 2001. 
Le pouvoir psychiatrique. Cours au Collège de France, 1973-1974, J. Lagrange éd., Paris, Seuil/Gallimard (Hautes études), 2003.

Sécurité, territoire, population. Cours au Collège de France, 1977-1978, M. Senellart éd., Paris, Seuil/Gallimard (Hautes études), 2004.

Naissance de la biopolitique. Cours au Collège de France, 1978-1979, M. Senellart éd., Paris, Seuil/Gallimard (Hautes études), 2004.

Dialogue (avec Raymond Aron), J.-F. Bert éd., Paris, Nouvelles éditions lignes, 2007. «Introduction à l'Anthropologie », dans E. Kant, Anthropologie du point de vue pragmatique, M. Foucault trad., Paris, Vrin (Bibliothèque des textes philosophiques), 2008, p. 11-79.

Le gouvernement de soi et des autres. Cours au Collège de France, 1982-1983, F. Gros éd., Paris, Seuil/Gallimard (Hautes études), 2008.

Le courage de la vérité. Le gouvernement de soi et des autres II. Cours au Collège de France, 1983-1984, F. Gros éd., Paris, Seuil/Gallimard (Hautes études), 2009.

Leçons sur la volonté de savoir. Cours au Collège de France, 1970-1971, suivi de Le Savoir d'Edipe, D. Defert éd., Paris, Seuil/Gallimard (Hautes études), 2011.

\section{Textes et communications inédits}

Répertoire $A Z$, Répertoire bibliographique, 1975 ?-1978 ? - Manuscrit; $17 \mathrm{~cm}$ [Archives Daniel Defert].

La chair et le corps. Fragment de la $1^{\text {re }}$ version du tome II d'Histoire de la sexualité, 1978. - Manuscrit, 40 pages ; $30 \mathrm{~cm}$ [Archives Daniel Defert].

Du gouvernement des vivants. Notes préparatoires, 1980. - Manuscrit; $30 \mathrm{~cm}$ [Archives Daniel Defert].

Du gouvernement des vivants. Cours donné au Collège de France, 1980. - 12 CD audio [Archives IMEC].

Mal faire, dire vrai. Fonctions de l'aveu. Conférences réalisées dans le cadre de la chaire Francqui, Université catholique de Louvain, 1981. - Tapuscrit, 159 pages ; $30 \mathrm{~cm}$ [Archives IMEC].

Subjectivité et vérité. Cours donné au Collège de France, 1981. - 7 CD audio [Archives IMEC]. 


\section{Études sur Michel Foucault}

Artières Philippe, Bert Jean-François, Gros Frédéric et Revel Judith éd., Foucault, Paris, L'Herne (Cahiers de L'Herne ; 95), 2011.

Bernauer James et Carrette Jeremy éd., Michel Foucault and Theology. The Politics of Religious Experience, Aldershot, Ashgate, 2004.

Chevallier Philippe et Greacen Tim éd., Folie et justice : relire Foucault, Toulouse, Érès, 2009.

Deleuze Gilles, Foucault, Paris, Éditions de Minuit (Critique), 1986.

Gros Frédéric et LÉvy Carlos éd., Foucault et la philosophie antique, Paris, Kimé (Philosophie en cours), 2003.

Guenancia Pierre, «Foucault/Descartes : la question de la subjectivité $\gg$, Archives de philosophie, t. 65, vol. 2, 2002, p. 239-254.

Perrot Michelle éd., L'impossible prison. Recherches sur le système pénitentiaire au $x i x^{e}$ siècle, Paris, Seuil (L'univers historique), 1980.

Senellart Michel, « Paradossi e attualità della soggettivazione cristiana », Dopo Foucault. Genealogie del Postmoderno, E. De Conciliis éd., Milan, Mimesis (Eterotopie), 2007, p. 33-52.

\section{Témoignages sur Michel Foucault}

Brown Peter, «A life of learning », Charles Homer Haskins Lecture, American Council of Learned Societies, Occasional Paper, nº 55, 9 mai 2003.

White Edmund, Mes vies, P. Delamare trad., Paris, Plon, 2006. 



\section{INTRODUCTION}

Les références à l'héritage chrétien sont constantes dans l'œuvre de Michel Foucault. Dès la préface à Folie et déraison (1961), le soleil nietzschéen éclaire d'une lumière noire un monde répressif appelé «Occident $\gg^{1}$. Si l'adjectif chrétien n'apparaît pas dans ce texte, la dramaturgie qu'y déploie Foucault croise la question de «la Chute et de l'Accomplissement $\gg^{2}$ et fait signe constamment vers l'implicite d'une culture. Par la suite, tout au long des cours au Collège de France, les dossiers vont s'accumuler sur le bureau du professeur : confession tridentine, pouvoir pastoral, rites baptismaux, parrêsia des martyrs, etc. L'abondance des références ne doit pas nous surprendre : comprendre ce qui, aujourd'hui, nous constitue sujets de nous-mêmes dans des rapports de savoir et de pouvoir demande de s'interroger sur la spécificité du rapport à soi que l'Occident a pensé et institué depuis ce que les historiens ont coutume d'appeler l'Antiquité tardive3. Une étude systématique des références de Foucault aux textes chrétiens, en particulier ceux des premiers siècles $\left(\mathrm{II}^{\mathrm{e}}-\mathrm{V}^{\mathrm{e}}\right.$ siècle $)$, restait cependant encore à faire. Si la récolte est abondante, les ouvriers ont été peu nombreux, malgré le recueil dirigé par James Bernauer et Jeremy Carrette en $2004^{4}$. Ce peu d'empressement

$1 D E \mathrm{n}^{\circ} 4$, «Préface », I, p. 189-19o. Nous citons le recueil des Dits et écrits de Michel Foucault dans sa deuxième édition : M. Foucault, Dits et écrits, t. I : 1954-1975, t. II : 1976-1988, D. Defert et F. Ewald éd., Paris, Gallimard, 2001. Afin que les détenteurs de la première édition puissent également s'y retrouver, nous faisons suivre l'abréviation $D E$ du numéro et du titre de l'article. Les autres ouvrages publiés sous le nom de Michel Foucault seront cités directement, sans nom d'auteur, en mentionnant le titre dans sa version la plus brève et la plus commune. Les éditions utilisées sont indiquées dans la bibliographie, p. 7 .

$2 D E \mathrm{n}^{\circ} 4$, « Préface », I, p. 193.

3 P. Brown, Genèse de l'Antiquité tardive, A. Rousselle trad., Paris, Gallimard, 1983.

4 J. Bernauer et J. Carrette éd., Michel Foucault and Theology. The Politics of Religious 
de la part des spécialistes de l'œuvre de Foucault pourrait s'expliquer par un problème de méthode, auquel nous nous sommes à notre tour heurté : la possibilité ou non de trouver une approche systématique du christianisme chez le philosophe français.

«Michel Foucault et le christianisme »: l'usage de la conjonction «et » dans le titre de notre étude peut en effet surprendre, tant est rare cette situation d'extériorité entre le philosophe et les objets qu'il manie, soucieux qu'il est toujours de les décomposer et les recomposer à volonté, plutôt que de les considérer comme constitués a priori en leur posant des questions de scoliaste. Ainsi du terme «christianisme », renvoyant naturellement à un événement fondateur et un vaste corps doctrinal qui l'interprète, formant un ensemble d'énoncés deux fois millénaires qui a sans doute sa cohérence propre, mais ne correspond pas au niveau d'analyse où opère Foucault. Ce dernier recueille des événements plus ténus, qui ne se groupent pas en ordre serré sous les vastes ensembles thématiques définis par l'habitude ou l'académie. De quoi «christianisme » peut-il donc être le nom ?

Préférant recourir au nuage de points plutôt qu'au trait traditionnel, trop rigide, de l'historien', Michel Foucault dessine à la surface de l'histoire des «problématisations ». Cette notion de «problématisation », qui apparaît dans les derniers entretiens du philosophe, ne décrit pas une totalité organique de pratiques et de discours mais seulement la constitution d'un problème à un moment donné de l'histoire, à partir d'un ensemble de difficultés posées aux individus par un domaine d'action ou un comportement (la folie, le crime, la sexualité). Le problème définit la forme générale dans laquelle ce comportement sera dès lors réfléchi et analysé, structurant ainsi le champ des solutions possibles ${ }^{6}$. Une «problématisation » n'est pas un esprit commun qui habite des

Experience, Londres, Ashgate, 2004. Ce recueil se propose d'utiliser Foucault pour renouveler les approches traditionnelles des sciences théologiques (théologie pastorale, théologie des religions, etc.), ce qui n'est pas ici notre propos. Pour une discussion critique de ce recueil, voir M. Senellart, « Paradossi e attualità della soggettivazione cristiana », Dopo Foucault, Genealogie del Postmoderno, E. De Conciliis éd., Milan, Mimesis, 2007, p. $33-52$.

5 La distinction est faite par Foucault quand il oppose à la caricature (qui simplifie un visage, le réduit à quelques traits simples en le vidant de son contenu historique), le «portrait-charge » qui remplit au contraire le visage des événements historiques dont il n'est plus que le simple transit : $D E \mathrm{n}^{\circ} 167$, « Les têtes de la politique », II, p. 12.

$6 D E \mathrm{n}^{\circ} 342$, « Polémique, politique et problématisations », II, p. 1417 (voir aussi un autre entretien $\mathrm{n}^{\circ} 350$, « Le souci de la vérité », p. 1488-1489). 
discours, encore moins une idée qui fonde et justifie une règle ou un code moral, mais ce moment où la pensée prend du recul par rapport à une conduite particulière et la réfléchit d'une manière nouvelle. Ce n'est donc pas l'esprit d'une époque mais des «événements de pensée $\gg^{7}$ qui sont ainsi désignés, irréductibles à des conditions historiques, sociales ou économiques, même s'ils n'en sont pas indépendants. L'analyse doit par conséquent opérer en deçà des solutions qui, ensuite, s'imposeront et pourront prendre la forme de «mentalités » ou de «systèmes de représentations $\gg$ permettant à une société d'interpréter les actions et les événements. Mais une «problématisation » n'est pas en elle-même un système clos et contraignant, elle est un travail de la pensée qui distingue et répartit selon un ordre nouveau les éléments qui permettront l'élaboration de règles et l'énoncé de jugements discriminants. Ainsi, au lieu de retrouver sous notre expérience de la sexualité un « esprit chrétien $\gg$ qui l'aurait produite, Foucault montre comment une nouvelle problématisation de la sexualité, qui appartient autant aux derniers stoïciens qu'aux premiers chrétiens, a rendu possible, parmi d'autres solutions, la formulation théorique «chrétienne ».

L'analyse traditionnelle des grands ensembles culturels est ainsi déplacée. Reste qu'il faut tout de même partir des formulations théoriques terminales pour retrouver le problème initial. C'est bien en lisant le projet de réaménagement de la ville de Nantes au XVIII ${ }^{\mathrm{e}}$ siècle que Foucault se renseigne sur le «problème » nouveau qui apparaît à cette époque : celui de la sécurité ${ }^{8}$. C’est bien en lisant Jeremy Bentham que Foucault approche du «problème » disciplinaire qui lui est

$7 D E n^{\circ} 340$, « Préface à l'Histoire de la sexualité », II, p. 1399. Nous croisons ici ce texte avec la définition donnée par Foucault de la problématisation dans deux entretiens : $D E$ $\mathrm{n}^{\circ} 341$, « Politique et éthique : une interview », II et $D E \mathrm{n}^{\circ} 350$, « Le souci de la vérité », II. En effet, le texte «Préface à l'Histoire de la sexualité », s'il n'utilise pas le mot «problématisation », met l'accent sur la spécificité d'une « histoire de la pensée » comme lieu de constitution des formes d'expérience. Or, la problématisation est justement l'élément caractéristique d'une histoire de la pensée $\left(D E n^{\circ} 342\right.$, « Polémique, politique et problématisations », II, p. 1416). « Histoire de la pensée » et « histoire des problématisations » sont donc synonymes.

8 Sécurité, territoire, population, p.19-23. Le nombre d'occurrences du terme «problème» est particulièrement important dans ce passage d'une leçon de 1978; ce qui vérifie que le néologisme «problématisation », forgé dans les derniers entretiens, est bien une relecture cohérente des travaux précédents et non une invention tardive. 
contemporain 9 . Les formulations théoriques, incluant pour l'ère chrétienne les formulations théologiques, ne sont donc pas évacuées. Mais si les textes chrétiens sont à lire de près afin de comprendre le problème que le sujet occidental est devenu pour lui-même, faut-il pour autant conférer à l'objet «christianisme » une unité signifiante pour l'analyse ? Si les solutions données à une difficulté inédite dans le champ des pratiques sont multiples et souvent contradictoires, n'en va-t-il pas de même tout au long de l'histoire chrétienne? Plus trivialement : qu'est-ce qui peut unifier l'ascétisme des Pères du désert et la pastorale augustinienne ? La doctrine de la confession à Port-Royal et la doctrine de la confession chez Medina ou Suárez ?

Telle est la question que nous aborderons dans la première partie de notre recherche : avant de réfléchir à l'interprétation que donne Foucault de ce quelque chose que nous appelons communément « christianisme » (troisième partie), il convient de préciser comment, pourquoi, et à partir de quel moment ce complexe historique aux contours incertains est devenu pour le philosophe français un objet de pensée (première partie). Pour parvenir à ce résultat, le cours Du gouvernement des vivants (1979-1980) sera un lieu de référence, en particulier pour la notion de « régime de vérité » qu'il élabore.

Entre la constitution de l'objet christianisme par Michel Foucault et son travail subséquent d'interprétation, s'est alors ouvert pour nous le domaine encore peu exploré du philosophe au travail : comment lit-il les textes, selon quels présupposés méthodologiques, avec quel art de la traduction ? L'attention scrupuleuse avec laquelle Foucault s'est penché sur les écrits chrétiens des premiers siècles nous a semblé une bonne occasion pour entrer dans les coulisses de sa recherche et de son enseignement (deuxième partie).

Objet - Lectures - Interprétations : ces trois moments qui scandent le travail ici présenté sont bien entendu contemporains dans le travail du philosophe ; s'ils viennent à bouger, ils bougent de concert. Aussi avonsnous volontairement rusé pour éviter les redites, privilégiant certaines parties de l'œuvre pour éclairer chacun des moments. Il nous a semblé par exemple plus fructueux d'aborder la question de la confession tridentine dans la partie «Lectures »-puisque c'est d'abord l'usage des

9 Le Panoptique est une «solution » à un «problème » de ratio du pouvoir : Surveiller et punir, p. 242. 
textes qui fait alors problème - et non dans la partie «Interprétations ». De même, le christianisme évoqué par Foucault dans Folie et déraison et certains articles des années 1960 consacrés à la littérature n’a pas encore à nos yeux la fermeté d'un «Objet » ni la rigueur d'une «Lecture »; mais son «Interprétation » fait sens, si nous la mettons en regard du cours de 1979-1980.

Ne suivant ni la logique chronologique ni la logique conceptuelle, le plan retenu a préféré se plier à la logique d'un travail, pour respecter la spécificité d'une pensée qui ne se déploie pas seulement à partir d'ellemême, mais également au rythme des découvertes provoquées par l'archive. À la croisée d'un questionnement philosophique et d'un matériau historique, la pensée de Foucault suit un mouvement complexe où s'enchevêtrent la constitution d'objets, l'affrontement aux textes et l'esquisse d'interprétations. Parce qu'il n'a pas donné lieu à un livre achevé comme le fut Surveiller et punir pour la prison, le christianisme est l'occasion de déplier les multiples paperolles d'une réflexion qui ne se résume pas à l'énoncé de certaines thèses trop simples sur les corps assujettis : il serait doux d'être grec et dur d'être chrétien. Comme nous essaierons de le montrer, le christianisme pour Foucault n'est pas d'abord la religion de l'obéissance et de l'aveu. 



\section{Le christianisme comme objet historique, une question de méthode}

Le christianisme existe-t-il pour Michel Foucault comme ensemble individualisé de pratiques et de discours? Si tel était le cas, cet ensemble pourrait prendre la forme d'un concept historique, au sens où l'est par exemple «l'esprit du capitalisme » pour Max Weber, c'est-à-dire un « complexe de relations présentes dans la réalité historique, que nous réunissons, en vertu de leur signification culturelle, en un tout conceptuel $\gg^{1}$. Au premier abord, rien n'est moins sûr que Foucault ait besoin d'un tel concept, ni même qu'il puisse lui accorder une place à l'intérieur de son propre projet historiographique. De manière hâtive, on peut noter que ce projet semble préférer la dispersion des faits à leur concentration sous des entités idéales. De manière plus fine, on peut relever que les rares efforts entrepris par Foucault pour individualiser un ensemble de faits taillent un costume encore trop étroit ou mal coupé pour l'individu nommé « christianisme ».

Dans un article important de 1968, réponse du philosophe à une série de remarques de la revue Esprit sur son travail ${ }^{2}$, se trouve par exemple la notion $\mathrm{d}^{\prime}$ «individualisation des discours » qui permet de contourner les unités traditionnelles (le texte, l'œuvre, la science) sans se résoudre à la

1 M. Weber, L'éthique protestante et l'esprit du capitalisme, Paris, Plon, 1964, p. 45. Directement ou indirectement, Foucault dialogue régulièrement avec ce livre de Weber. Sans le nommer, il l'évoque dans La volonté de savoir, p. 162. Par la suite, il est explicitement cité au début de ses conférences à l'université du Vermont en 1982 : $D E n^{\circ}{ }_{363}$, « Les techniques de soi », II, p. 1603 . 
dispersion. Au sein du projet d'archéologie du savoir, Foucault a ainsi tenté d'établir des critères d'unification des formations discursives. Rappelons qu'une formation discursive n'est pas une science constituée, mais une collection d'énoncés entre lesquels une régularité est repérable à travers les objets qu'ils traitent, les concepts qu' ils définissent, les types de validation qu' ils requièrent. Dans sa discussion avec la revue Esprit, Foucault fournit une première liste de critères, revue et corrigée quelques mois plus tard dans sa réponse au Cercle d'épistémologie ${ }^{3}$. Cette liste peut-elle nous aider à constituer le christianisme en individualité historique ? Assurément, non. Ces critères formels concernent d'abord et avant tout les coupures épistémologiques dans le champ du savoir, auxquelles les pratiques concrètes ne se greffent que secondairement. Or, les textes chrétiens étudiés dans les cours au Collège de France, en particulier Du gouvernement des vivants (1979-1980), ne sont pas seulement des espaces où des objets et des concepts apparaissent et se transforment, mais ils sont également le lieu d'un engagement direct du sujet dans ce qu'il dit : profession de foi et acte d'aveu. Il ne s'agit pas seulement pour Foucault d'étudier la régularité interne d'un discours et la place qu'il ménage au sujet de l'énonciation, mais aussi les règles de conduite, les modes d'existence qui permettent à un sujet de dire ce qu'il dit. Les critères énoncés dans le contexte de l'archéologie du savoir ne sont donc pas ici immédiatement pertinents.

Cette remarque souligne la difficulté pour Foucault de constituer le «christianisme » en concept historique, difficulté redoublée par son propre choix de pratiquer une description stratégique du matériau historique. Or, c'est ce modèle stratégique qui domine les recherches de Foucault dans la plus grande partie des années 1970, alors même qu'il commence à visiter quelques lieux chrétiens majeurs, tels la confession tridentine ou le pouvoir pastoral. Dans un premier temps, il nous faut définir ce modèle stratégique, ses conséquences sur l'écriture de l'histoire pratiquée par Foucault, afin de savoir, dans un deuxième temps, si quelque chose comme «le christianisme »- unité historique de pratiques et de discours - peut ou non s'y inscrire, et sous quelles conditions.

3 La première liste contient les critères de «formation », de «transformation » et de « corrélation» (ibid., p.703-704). Les critères de la seconde sont plus nombreux et différents : « référentiel », « écart énonciatif », « réseau théorique » et «champ de possibilités stratégiques $\left(D E n^{\circ} 59\right.$, « Sur l'archéologie des sciences. Réponse au Cercle d'épistémologie », I, p. 739-747). 


\section{CHAPITRE UN \\ Définition du modèle stratégique}

\section{Usage des concepts dans l'historiographie de Michel Foucault}

Si Foucault débat peu avec ses contemporains philosophes, il débat sans cesse avec ses contemporains historiens. Ce débat ne peut être réduit à la critique aisée des histoires totales et globales, qui caricature bien souvent les positions en présence. Nulle historiographie ne peut en effet prétendre éviter le labeur du concept et le risque de la généralité.

En ce qui concerne l'opération historiographique, il convient de distinguer les objets que l'historien se donne au départ comme présupposé théorique minimal (la conscience individuelle, l'idéologie, la mentalité, le savoir/pouvoir, etc.) de la manière dont il organise les faits qu' $i l$ a isolés grâce à ces objets. Cette distinction que nous proposons peut disparaitre chez certains historiens pour qui l'organisation des faits se borne à donner un contenu historique cohérent à un objet préalablement défini : présupposant qu'une classe sociale a une structure psychique qui lui est commune, Lucien Goldmann tente ainsi de définir cette structure dans le cas particulier de la noblesse de robe aux grandes heures du jansénisme $^{1}$. Mais ce travail d'organisation des faits peut également dépasser l'objet de départ, voire dans certains cas l'effacer, pour construire une

1 C'est la vision tragique de l'homme déchiré entre des exigences contradictoires :

L. Goldmann, Le Dieu caché. Étude sur la vision tragique dans les Pensées de Pascal et dans le théâtre de Racine, Paris, Gallimard (Bibliothèque des idées), 1955. Pour la méthodologie appliquée par Lucien Goldmann - dite «structuralisme génétique » - voir L. Goldmann, Sciences humaines et philosophie, suivi de Structuralisme génétique et création littéraire, $2^{\mathrm{e}}$ édition augmentée, Paris, Gonthier, 1966. Foucault et Goldmann se sont croisés 
forme historique à la fois plus générale et plus complexe ( $\ll$ l'esprit du capitalisme moderne » chez Max Weber, «le subjectivisme religieux au XVII ${ }^{\mathrm{e}}$ siècle » pour Leszek Kołakowski, «la rationalité punitive au $\mathrm{XIX}^{\mathrm{e}}$ siècle $\gg$ pour Michel Foucault). Dans ce cas, il faut remarquer qu'un tel effort de conceptualisation est tout autre chose qu'un simple usage de concepts prédéfinis. Max Weber le souligne quand il précise qu'un concept historique comme l'esprit du capitalisme doit être le point d'aboutissement et non le point de départ de l'analyse, qu'il doit donc être « composé graduellement, à partir de ses éléments singuliers qui sont à extraire un à un de la réalité historique $\gg^{2}$. De plus, cette opération seconde ne produit pas nécessairement un nouvel objet absolument circonscrit, mais tout aussi bien, et le plus souvent, un champ ouvert de relations, à la description jamais achevée, aux limites toujours incertaines. Dès 1964, Leszek Kołakowski, dans sa tentative de définition du subjectivisme religieux au XVII ${ }^{\mathrm{e}}$ siècle, affiche sa conviction «que les structures fermées n'existent pas, et donc que la reconstitution de n'importe quelle structure ne peut jamais avoir de forme définitive $\gg^{3}$. La distinction de ces deux moments de l'opération historiographique - la boîte à outils initiale et la conceptualisation progressive - va nous permettre de ne pas définir de manière trop simple l'écart pratiqué par Foucault par rapport aux historiens qui lui sont contemporains ; écart dont l'affirmation semble parfois d'autant plus forte du côté de l'intéressé que la proximité de fond reste grande.

Par rapport aux objets que certaines écoles historiques se donnent a priori, la position de Foucault est claire, et, pour une fois, invariable : refus d'une « histoire des mentalités » et refus d'une « histoire des idéologies $\gg$. Il le dit successivement en 1973 et en 1976 au Collège de France, il le redira au début du cours de $1983^{4}$. Précisons les termes.

et affrontés lors d'un débat à la Société française de philosophie, le 22 février 1969: $D E$ no 69, « Qu'est-ce qu'un auteur? », I, p. 840-849.

2 M. Weber, L'éthique protestante et l'esprit du capitalisme, Paris, Plon, 1964, p. 45, nous soulignons.

3 L. Kołakowski, Chrétiens sans Église. La conscience religieuse et le lien confessionnel au xvie siècle, Paris, Gallimard, 1969, p. 51.

4 Pour la distance prise par rapport à l'histoire des mentalités : Le pouvoir psychiatrique, p.14. Pour la distance par rapport à la critique des idéologies : Il faut défendre la société, p. 30. Dans son cours de 1983, Foucault use d'une autre distinction : il oppose l'histoire des mentalités à l'histoire des représentations (Le gouvernement de soi et des autres, p. 4-5) et divise cette dernière en histoire des idéologies et histoire des « valeurs représentatives des systèmes 
Le terme de mentalité fut emprunté par Lucien Febvre à Lucien Lévy-Bruhl, par l'intermédiaire de Charles Blondel, psychologue auteur

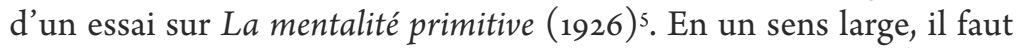
entendre par mentalité les « façons de sentir et de penser $»^{6}$, conscientes ou non, qui habitent une communauté à un moment donné de l'histoire. Dans cette perspective, les comportements et attitudes individuels sont en partie déterminés par des structures mentales collectives qui peuvent aussi être nommées « vision du monde » ou «esprit d'une époque ». L'enquête historique croise alors la psychologie, avec une priorité donnée, selon la sensibilité des historiens, à l'approche individuelle (Lucien Febvre) ou à l'approche collective (Marc Bloch). De manière significative, pour prendre un exemple contemporain de Foucault, Mythe et pensée chez les Grecs de Jean-Pierre Vernant est sous-titré en 1965 Étude de psychologie historique ${ }^{7}$, revendiquant ainsi le croisement des deux disciplines.

Le terme d'idéologie, s'il apparaît dès le XVIII ${ }^{\mathrm{e}}$ siècle, renvoie ici à l'histoire marxiste, en particulier au texte source de Karl Marx et Friedrich Engels : L'idéologie allemande (1845-1846), dans lequel les deux auteurs fustigent la postérité hégélienne représentée par Feuerbach, Bauer et Stirner. Une idéologie est une construction intellectuelle qui a pour fonction de justifier l'activité d'une classe dominante en masquant ses motivations d'abord matérielles : «Les pensées dominantes ne sont pas autre chose que l'expression idéale des rapports matériels dominants, elles sont ces rapports matériels dominants saisis sous forme d'idées $\gg^{8}$. Dissimulant une réalité de luttes sociales, cette projection idéale finit par se prendre pour un pur produit de la pensée. L'idéologie présente ainsi comme universel ce qui ne relève en fait que d'un intérêt particulier. À l'époque de Foucault, Louis Althusser continue de faire un large usage de cette notion

de représentations ». Nous préférons en rester à la première partition binaire (idéologies/ mentalités), d'autant plus qu'à certains endroits Foucault assimile l'histoire des représentations tantôt à l'histoire des idées $\left(D E \mathrm{n}^{\circ} 342\right.$, « Polémique, politique et problématisations », II, p.1416), tantôt à l'histoire des mentalités (Le pouvoir psychiatrique, p. 14).

5 Pour une histoire de l'histoire des mentalités, on peut se référer à : J. Le Goff, « Les mentalités : une histoire ambiguë », Faire de l'histoire, t. III : Nouveaux objets, J. Le Goff et P. Nora éd., Paris, Gallimard, 1974.

6 M. Bloch, La société féodale, Paris, Albin Michel, 1994, t. I, $1^{\mathrm{re}}$ partie, Livre II, chap. II.

7 J.-P. Vernant, Mythe et pensée chez les Grecs. Étude de psychologie historique, Paris, Maspero, 1965.

8 K. Marx et F. Engels, L'idéologie allemande, précédé de Karl Marx, Thèses sur Feuerbach, Paris, Éditions sociales, 1982, p. 111. 
qu'il oppose à la science en tant que, pour l'idéologie, « la fonction pratico-sociale $\gg^{9}$ prime sur la fonction de connaissance.

Qu'ils les révèlent dans leur structure historique collective ou les dénoncent comme instrument de domination, ces deux types d'histoire s'attachent aux productions de l'esprit humain : idées, théories, images, mythes. Avant d'apparaître dans l'archive d'une époque, ces productions ont eu une première existence comme représentations de la conscience. L'historien leur reconnait ainsi un domaine propre originel qui est un esprit tapissé d'images, selon la conception platonicienne de l'image mnémonique (દikóv). Offrant une métaphore heureuse pour penser la prégnance d'une mentalité ou d'une idéologie sur un individu - comme nous y invite Paul Ricœur -, Platon compare l'esprit à un bloc de cire et l'image mnémonique à une empreinte à sa surface ${ }^{10}$. Dans cette perspective, idées, théories, images et mythes n'ont pas d'autre contenu pour l'individu et pas d'autre efficace pour son activité que de marquer d'un sceau sa mémoire. Leurs effets réels, qu'ils soient de conditionnement (mentalité) ou de dissimulation (idéologie), passent nécessairement et exclusivement par cette fonction de représentation qui, sous la métaphore platonicienne, est d'abord une impression. Mais il reste à expliciter le rapport de ce qui vient ainsi s'imprimer dans l'esprit à une situation historique - politique, sociale, économique - donnée. Or, selon Foucault, c'est devant une telle explication que les historiens battent en retraite. Même si, ni pour les historiens des mentalités ni pour les marxistes, le domaine de l'esprit n'est séparé de ses déterminations sociales, le lien qu'il entretient à la réalité matérielle est défini par des termes que le philosophe juge insatisfaisants, à la fois trop simples et imprécis. L'esprit serait le « reflet $\gg, l^{\prime} \ll$ expression $\gg$ ou encore la «traduction $\gg^{11}$ d'une situation concrète, laissant ainsi, jusque dans des

$\ll[\ldots]$ exactement comme lorsqu'en guise de signature nous imprimons la marque de nos anneaux, quand nous plaçons ce bloc de cire sous les sensations et sous les pensées, nous imprimons sur lui ce que nous voulons nous rappeler, qu'il s'agisse de choses que nous avons vues, entendues ou que nous avons reçues dans l'esprit »: Platon, Théétète, 191d, M. Narcy trad., $2^{\mathrm{e}}$ édition corrigée, Paris, Flammarion, 1995, p. 250 . Ce texte est longuement commenté par Paul Ricœur dans son ouvrage sur l'historiographie : P. Ricœur, La mémoire, l'histoire, l'oubli, Paris, Seuil, 200o, p. 8-18. Ricœur s'inscrit lui-même dans cette tradition grecque de l'sikóv pour penser à nouveaux frais une histoire des mentalités, lui préférant la dénomination plus dialectique d'histoire des représentations (p. 292-301). 
visions de l'histoire farouchement opposées à la philosophie idéaliste, une dernière solution de continuité entre la pensée et le réel.

Ces productions mentales, Foucault ne les contourne pas pour retrouver une vérité «brute » des pratiques, il les intègre au contraire à ses recherches. Mais il montre que leur origine n'est pas logée exclusivement dans l'esprit, puisqu'elle naît constamment et de manière diffuse à même les corps. Discours réformistes, philosophies de la peine, utopies architecturales, plans raisonnés : les théories se lisent à toutes les pages de Surveiller et punir, mais elles ne sont pas considérées comme le subtil reflet de mentalités qui habiteraient les confins de la conscience, ni comme des résidus d'idéologies qui cacheraient la réalité. Pour éviter le détour par la représentation, Foucault va utiliser une curieuse notion, empruntée à la philosophie stoïcienne par l'intermédiaire de Gilles Deleuze : «l'incorporel ».

Même s'il se la réapproprie très librement, cette notion lui permet de penser une réalité qui ne soit pas de l'ordre des choses sans recourir pour autant à la médiation d'une conscience individuelle pour expliquer sa genèse. Les «incorporels » ne naissent pas dans la conscience, mais à la surface des corps aux prises avec un pouvoir dont ils sont à la fois l'effet et l'instrument. Dans Surveiller et punir, la délinquance est ainsi qualifiée de « réalité incorporelle $\gg^{12}$. Mais c'est la description de «l'âme moderne », dans laquelle Foucault se refuse à voir une simple vue de l'esprit ou une idéologie, qui offre sans doute le meilleur exemple de production d'un incorporel :

Il ne faudrait pas dire que l'âme est une illusion, ou un effet idéologique. Mais bien qu'elle existe, qu'elle a une réalité, qu'elle est sans cesse produite en permanence, autour, à la surface, à l'intérieur du corps par le fonctionnement d'un pouvoir qui s'exerce sur ceux qu'on punit [...]. Cette âme réelle, et incorporelle, n'est point substance; elle est l'élément où s'articulent les effets d'un certain

qui relient les comportements aux mentalités : ils les «précèdent », les «suivent », les «traduisent $»$, les «prescrivent », les « masquent », les « justifient » (Le gouvernement de soi et des autres, p. 4). Les deux derniers verbes semblent d'ailleurs concerner l'histoire des idéologies plus que celle des mentalités. La même critique se trouvait déjà en 1968 dans la réponse à la revue Esprit : les pratiques politiques ne se « reflètent » ni ne se «traduisent $\gg$ ni ne «s'expriment $\gg$ dans les discours scientifiques $\left(D E n^{\circ} 58\right.$, « Réponse à une question $\gg, \mathrm{I}, \mathrm{p} .718$ ).

12 Surveiller et punir, p. 296 : « [la prison] fabrique [des délinquants] en cet autre sens qu'elle a introduit dans le jeu de la loi et de l'infraction, du juge et de l'infracteur, du condamné et du bourreau, la réalité incorporelle de la délinquance qui les lie les uns aux autres ». 
type de pouvoir et la référence d'un savoir [...]. Sur cette réalité-référence, on a bâti des concepts divers et on a découpé des domaines d'analyse : psyché, subjectivité, personnalité, conscience, etc. ; sur elle on a édifié des techniques et des discours scientifiques; à partir d'elle on a fait valoir les revendications morales de l'humanisme. ${ }^{13}$

Lestée d'une technicité que ne reprend pas Surveiller et punir, cette notion d'incorporel se trouve être au centre de l'essai consacré en 1969 à la philosophie stoïcienne par Gilles Deleuze ${ }^{14}$, lui-même marqué comme Foucault par la lecture du maître livre d'Émile Bréhier sur le sujet. Dans cette étude classique datant de 1907, la «surface » est en effet définie comme le lieu où les événements existent dans l'ordre des $\operatorname{corps}^{15}$. La référence de la part de Foucault est d'autant plus claire qu'il avait longuement commenté l'essai de Gilles Deleuze dans son article «Theatrum philosophicum » publié en 1970 dans la revue Critique ${ }^{16}$. Rappelons brièvement ce dont il est ici question : pour les Stoïciens, ce qui est un «quelque chose » se divise en « corporel» (les corps avec leurs qualités propres et leurs actions mutuelles) et «incorporel » (les effets de ces actions qui n'appartiennent pas au corps mais en sont les attributs, exprimés par des verbes comme «être coupé », «brûler», «mourir »). L'incorporel est un événement causé par le heurt des corps entre eux, sans pouvoir à son tour être cause de quoi que ce soit et sans s'objectiver dans une réalité nouvelle. La causalité modifie mais ne crée rien. Émile Bréhier met alors l'accent sur la double appartenance de l'incorporel au monde et au langage : son surgissement appartient au premier et subsiste dans le second, toujours sous la forme d'un verbe. Par exemple, l'hypocondrie n'est pas cause de la fièvre, mais du fait que «la fièvre arrive $»^{17}$. Si l'on transpose la leçon stoïcienne à Surveiller et punir, l'incorporel qui surgit à la surface des corps punis aurait pour exprimable «le corps a une âme ». Pour Foucault, lecteur de Deleuze et de Bréhier, cette réalité subsistante, qui n'est cependant pas un être physique, émerge à la limite des rapports de pouvoir, comme ce qui arrive aux corps et ce qu'on dit de lui. Ce qui explique que les concepts et les

Ibid., p. 38, nous soulignons.

G. Deleuze, Logique du sens, Paris, Éditions de Minuit, 1969.

É. Bréhier, La théorie des incorporels dans l'ancien stö̈cisme, Paris, Vrin, 1997, p. 13.

$D E n^{\circ} 80$, «Theatrum philosophicum », I, p. 949-951.

É. Bréhier, La théorie des incorporels dans l'ancien stoïcisme, déjà cité, p. 12. 
théories puissent y trouver leur origine, même si «l'âme » n'agit ni ne pâtit, comme le passage de Surveiller et punir que nous venons de citer le vérifie. Foucault fait en effet de l'incorporel une réalité plus fondamentale que les productions de l'esprit humain; il est le pur effet de la prise du pouvoir sur les corps, avant toute reprise réflexive.

En se débarrassant des activités propres à l'esprit humain comme fausses explications que présupposent l'histoire des mentalités et la critique des idéologies, Foucault propose une histoire de ce qui arrive aux corps, incorporels inclus. Après avoir converti les idéologies et les représentations en événements de «surface », Foucault doit organiser les faits qu'il a ainsi isolés. Lorsqu'il aborde dans les années 1970 une analyse des pratiques après celle des discours, il s'agit pour lui de forger de nouvelles notions qui répondent à deux critères : 1) n'avoir de valeur qu' heuristique ; 2) ne pas faire de la conscience et de ses représentations un passage obligatoire, voire un principe explicatif. Ainsi des deux notions centrales de « pouvoir » et de «savoir ».

Faisant suite à une première tentative de morphologie de la « volonté de savoir » dans le cours de 1970-1971, le doublet «pouvoir-savoir » est défini pour la première fois dans le cours Théories et institutions pénales (1971-1972). Ce doublet permet de contourner les partages traditionnels entre des domaines dont la délimitation reste problématique et trompeuse : principalement le partage entre, d'un côté, «la science », et de l'autre, «la société $»$ et $\ll l^{\prime}$ État $\gg^{18}$. Au terme de ce contournement, il n'y a même plus à se soucier de la moindre délimitation, à partir du moment où l'historien modifie la question posée à l'histoire. À la question «quoi ? » (qu'est-ce que la folie, qu'est-ce que la sexualité ?), qui risque toujours de nous faire retrouver une essence cachée derrière la dispersion des phénomènes, Foucault choisit de substituer la question «comment cela se passe? ». Cette interrogation volontairement naïve et empirique est présentée dans un article de 1982 comme un questionnement $\ll$ critique $\gg^{19}$, qui vient nettoyer le champ historique de toute substance ou essence dont les « contenus assignables » (ibid., p. 1051) n'auraient pas été préalablement et fermement établis. Dans ces conditions, les notions de «savoir » et de « pouvoir » utilisées par le 
philosophe doivent être considérées non comme des entités ou des puissances - au fond, se demande même Foucault, peut-être le « pouvoir » n'existe-t-il pas $^{20}$ ? - mais uniquement comme des opérateurs de la recherche, dont le rôle est de réduire les phénomènes à un espace commun, sans valeur ni légitimité a priori, composé des connaissances acceptables à un moment donné de l'histoire (savoir) et des mécanismes qui induisent des discours et des comportements (pouvoir) ${ }^{21}$. Ils ne sont que des «noms » qui demandent à recevoir, pour chaque situation historique donnée, un contenu singulier et déterminé, contre la tentation du recours aux universaux historiques.

La conséquence de ce nominalisme méthodologique, revendiqué par Foucault à la suite de Paul Veyne ${ }^{22}$, est de déplacer les champs de recherche initialement annoncés dans le titre des ouvrages : Histoire de la folie à l'âge classique, Naissance de la clinique, Histoire de la sexualité. Toute histoire est toujours histoire de quelque chose ; mais encore faut-il être conscient du rôle fictif ${ }^{23}$ que joue le génitif. L'inconscience, à en croire Foucault, n'est pas tant le fait des historiens eux-mêmes que des philosophes quand ils se servent de l'histoire que d'autres ont constituée pour eux - reproche formulé en particulier à l'encontre de l'école de Francfort ${ }^{24}$. Répandu dans les études marxistes, cet usage ne s'interroge pas suffisamment sur l'objet visé par la recherche, et le considère préparés par les historiens et accueillis tout faits comme des faits » (《Qu'est-ce que la critique? $\gg$, p. 45 ). 
comme naturellement constitué et circonscrit pour la simple raison qu'il est nommé. Le recours aux termes génériques fait croire à l'existence naturelle de ce qu'ils désignent et recouvre la fragilité, voire la facticité des unités. Or, même l'histoire des sciences n'échappe pas aux ruptures radicales qui viennent mettre en question la continuité des procédures et la cohérence des concepts. La seule exception à la règle serait une science qui pourrait intégrer chacune de ses mutations dans un système formel toujours plus vaste. Mais, nous dit Foucault dans un article de 1968, seules les mathématiques pourraient éventuellement être à la hauteur de cette prétention :

Quand on parle de la psychiatrie, ou de la médecine, de la grammaire, de la biologie, ou de l'économie, de quoi parle-t-on ? Quelles sont ces curieuses unités qu'on croit pouvoir reconnaître au premier coup d'œil, mais dont on serait bien embarrassé de définir les limites ? [... ] Peut-être y a-t-il des discours qui peuvent à chaque instant redéfinir leur propre individualité (par exemple, les mathématiques peuvent réinterpréter en chaque point du temps la totalité de leur histoire) ; mais, dans aucun des cas que j’ai cités, le discours ne peut restituer la totalité de son histoire dans l'unité d'une architecture formelle. ${ }^{25}$

Aucun ensemble de discours ne peut donc prétendre être a priori formellement individualisé. La difficulté est redoublée quand le discours étudié est inséparable dans sa production et sa diffusion de pratiques individuelles ou collectives. Foucault avoue ainsi son embarras pour définir la cohérence d'un domaine complexe comme celui de la clinique - qui n'est pas seulement un savoir et n'est pas seulement une pratique, qui est plus large que le discours médical et n'englobe pourtant pas la totalité du savoir sur le corps humain ${ }^{26}$. Au passage, il est intéressant de noter que cet embarras se fait jour non pas à la publication de Naissance de la clinique en 1963, mais une fois accomplie la description pure des formations discursives, c'est-à-dire après la publication des Mots et les choses en 1966. Comment lier cette description pure avec les événements historiques, les décisions politiques, les règlements administratifs, et former ainsi l'unité historique de la clinique?

《ensemble énonciatif à la fois théorique et pratique, descriptif et institutionnel, analytique et réglementaire, composé aussi bien d'inférences que de décisions, d'affirmations que de décrets $\gg\left(D E n^{\circ} 59, \ll\right.$ Sur l'archéologie des sciences. Réponse au Cercle d'épistémologie », I, p. 750). 
Une telle question, récurrente dans les discussions méthodologiques de la fin des années $1960^{27}$, encore marquées par le modèle de l'histoire des sciences, va en fait complètement disparaître des préoccupations de Foucault dans les années 1970 : le binôme «pouvoir-savoir» fait désormais seul le lien entre des discours et des pratiques hétérogènes. Non seulement les unités traditionnelles sont brisées, mais il n'y a plus à se soucier de leur en substituer de nouvelles, en aval de ce binôme. Le titre que Foucault choisit pour chacun de ses ouvrages n'est plus alors qu'un repérage provisoire, un point d'accroche pour la description d'une connexion singulière de pouvoir et de savoir, dont la fonction et les effets se révéleront toujours plus larges que le sujet initial : le dispositif de sexualité qui se met en place aux XVIII ${ }^{e}$ et XIX ${ }^{e}$ siècles n'est qu'un maillon d'un certain type de pouvoir sur la vie qui engage bien autre chose que la seule sexualité des individus et touche à l'exploitation du travail salarié, les théories de l'hérédité, etc. La rationalité punitive qui s'élabore au XIX est à replacer dans le cadre plus large des pratiques disciplinaires dont les origines remontent aux communautés éducatives du Moyen Âge. La recherche s'étend ainsi par capillarité entre des domaines en apparence différents, non par grandes scansions théoriques.

Il reste alors à définir la manière dont, malgré sa défiance vis-à-vis des idéalités, Foucault organise conceptuellement le matériau historique qu' il rassemble, et définit ainsi certaines formes historiques intelligibles. L'historien ne peut en effet éviter ce passage à un niveau général d'analyse sans lequel l'histoire resterait muette, livrée à l'aléa des pures singularités. Une seule fois, Foucault a tenté de préserver cette fugacité de l'événement : dans «La vie des hommes infâmes » en 1977, présentation d'une anthologie des archives de l'Hôpital général et de la Bastille. Volontairement, la matière première des procès-verbaux y est livrée par l'historien dans le « lyrisme frugal de la citation $»^{28}$, sans être organisée d'une manière systématique ni chargée de commentaires. Délestés de leur interprétation, aucun des événements recueillis ne prétend avoir de signification historique privilégiée ou majeure. Mais si Foucault, dans la première partie de son texte, fait du respect de la singularité un principe

Les lieux majeurs sont les réponses à Esprit $\left(D E \mathrm{n}^{\circ} 58\right.$, « Réponse à une question 》, I, p. 701-723) et au Cercle d'épistémologie ( $D E \mathrm{n}^{\circ} 59$, « Sur l'archéologie des sciences. Réponse au Cercle d'épistémologie $\gg$, I, p. 724-759). 
éditorial, dans la seconde, il replace ces fragments dans le contexte général de la naissance, à la fin du XVII ${ }^{\mathrm{e}}$ siècle, d'un nouveau type de pouvoir : celui qui porte sur l'ordinaire des vies (ibid., p. 243-253). L'instantanéité de l'enregistrement de ces vies et de leurs méfaits, le violent hasard qui leur a fait un jour rencontrer le pouvoir royal, n'effacent pas une histoire longue de plusieurs siècles dont une intelligibilité peut être proposée.

Chez Foucault, le niveau général d'analyse pourrait être décrit comme celui des «formes de rationalités », le philosophe employant cette expression à plusieurs reprises dans les dernières années de sa réflexion ${ }^{29}$. Il faut donner ici au terme de «forme » un sens large, peu connoté philosophiquement, comme le vérifient les expressions variées de « régime de rationalité $»^{30}$, de $\ll$ type de rationalité $\gg^{31}$, ou encore de $\ll$ style pénal $\gg^{32}$. Dans la ligne des analyses de Max Weber, cette pluralité des formes de rationalités ne renvoie pas à l'unité de la Raison ou de la Science, mais est inséparable d'un champ historique où elles prennent naissance et opèrent ${ }^{33}$. Une forme de rationalité n'obéit pas à une finalité univoque instituée par un groupe; elle ne recouvre pas non plus le sens subjectif que les individus confèrent à leurs actions. Elle est le croisement historique de tactiques de pouvoir et de techniques de savoir dont l'appui réciproque produit certains effets sur les individus. Plus précisément, puisque nulle intention n'est à l'œuvre dans ce croisement : c'est parce que deux tactiques ou techniques hétérogènes trouvent soudain à se renforcer mutuellement que le système qu'elles forment peut perdurer

$D E n^{\circ} 278$, « Table ronde du 20 mai 1978 », II, p. 845. L'expression se trouve déjà subrepticement en $D E \mathrm{n}^{\circ}$ 234, « La scène de la philosophie », II, p. 572. On la retrouve régulièrement plus tard : $D E \mathrm{n}^{\circ} 310$, « Espace, savoir et pouvoir », II, p. $1091 ; \mathrm{n}^{\circ} 330$, « Structuralisme et poststructuralisme », II, p.1261 et 1268-1269; no 339 , « Qu'est-ce que les Lumières? », II, p. 1395 . $D E \mathrm{n}^{\circ} 277$, «La poussière et le nuage », II, p. 845 , nous soulignons.

$D E \mathrm{n}^{\circ}$ 291, «"Omnes et singulatim" : vers une critique de la raison politique », II, p. 955, nous soulignons. Surveiller et punir, p.14, nous soulignons.

«De là des variétés considérables de rationalisation dans les divers domaines de la vie et selon les civilisations. Pour en caractériser les différences, du point de vue de l'histoire des civilisations, il est nécessaire de déterminer quels sont les domaines rationalisés et dans quelle direction ils le sont $\gg$ (M. Weber, L'éthique protestante et l'esprit du capitalisme, déjà cité, p. 23-24). En reprochant à Weber de faire de la rationalité un «invariant anthropologique $\left(D E n^{\circ} 278\right.$, « Table ronde du 20 mai $1978 »$, II, p. 846), Foucault ne lui rend pas tout à fait justice. 
et se doter d'un rythme régulier d'entrainement réciproque savoir/pouvoir. Le résultat du croisement peut donc être relativement stable - et former ainsi une technologie unifiée et analysable -, alors que les éléments qui le composent ne le sont pas. Ses effets peuvent être généralisés à un ensemble très divers d'activités, alors que les objectifs initiaux ne sont que locaux. En changeant d'échelle, le pouvoir gagne en régularité tout en perdant son intentionnalité subjective, c'est-à-dire les mobiles explicites qui guidaient encore les tactiques à un niveau local, et que l'historien peut retrouver dans les déclarations d'intentions de groupes ou d'individus ${ }^{34}$.

Sans expliciter la possibilité de ce passage du local à l'étendu, Foucault pose que l'irrégularité de départ n'empêche pas le surgissement d'une régularité anonyme, dans un domaine et à un moment donnés de l'histoire. Il rejoint sur ce point l'école autant italienne que britannique de la microhistoire et son effort pour redonner toute leur place, à l'intérieur de changements globaux d'apparence homogène, à des stratégies locales toujours incertaines. Mais le philosophe français s'en distingue par l'économie qu'il fait des catégories explicatives de cette école, majoritairement subjectives et réflexives : histoire « vécue », conscience des acteurs, choix, compromis ${ }^{35}$. « Micro $»$ ne désigne pas non plus chez Foucault un lieu géographique délimité, comme le village piémontais de Giovanni Levi ${ }^{36}$. Tout «lieu » historique est fluctuant pour le philosophe, même si on peut en donner une délimitation provisoire pour les besoins de la recherche : l'histoire de la « rationalité punitive » au XIX ${ }^{\mathrm{e}}$ siècle tournera ainsi autour $\mathrm{du}$ lieu « prison $»$. Mais ce que révèle la question $\mathrm{du} \ll$ comment cela se

Des stratégies initialement différentes peuvent produire « des effets permanents et solides qu'on pourrait parfaitement comprendre dans leur rationalité même, bien qu'ils ne soient pas conformes à la programmation première $\gg\left(D E \mathrm{n}^{\circ} 278\right.$, « Table ronde du 20 mai $1978 \gg$, II, p. 847). Voir aussi La volonté de savoir, p. 124-125.

Influencée par des historiens britanniques, la microstoria se développa en Italie au début des années 1970. Son représentant le plus connu en France, Giovanni Levi, étudia l'exercice du pouvoir à l'échelle micro-historique d'un village. Il remarqua que les stratégies personnelles et familiales semblaient toujours in fine tendre vers un équilibre social relatif (Le pouvoir au village. Histoire d'un exorciste dans le Piémont du xvie siècle, Paris, Gallimard, 1989, p. 12). Jacques Revel fit une présentation très foucaldienne de ce livre, lors de sa publication en France : «Le pouvoir n'est pas une chose. [...] il s'identifie à un réseau relationnel constamment mobile $\gg$ (ibid., p. xxviII). De son côté, Paul Ricœur en fera une lecture différente, plus réflexive, insistant sur «l'appropriation » personnelle, «l'accord » des acteurs entre eux, visant un « vivre ensemble 》 (P. Ricœur, La mémoire, l'histoire, l'oubli, déjà cité, p. 274-277 et 290). 
passe ? », ce sont à la fois les mutations parfois radicales qu' un champ prédéfini de pratiques et de discours peut connaître, et les interactions de ce champ avec d'autres éléments qui lui semblent étrangers. La prison, avec son encadrement juridique, ses règlements et son architecture, peut croiser dans l'analyse le domaine de la pédagogie, celui de la formation militaire ou encore la philosophie empirique anglo-saxonne.

Foucault risque ainsi des rapprochements audacieux entre des institutions, des discours et des techniques dont les rapports ne sont pas constitutifs mais uniquement stratégiques. Ce dernier adjectif est déterminant et définit le travail généalogique des années 1970. Au-delà des distinctions thématiques souvent accentuées par les commentateurs, il permet de rassembler au niveau de leur méthode un ensemble de travaux aux objets divers, qui s'étend de mai 1973 (conférences à Rio de Janeiro, «La vérité et les formes juridiques ») à avril 1979 (fin du cours Naissance de la biopolitique). Nous approchons de notre objectif: définir le modèle stratégique à l'œuvre dans les travaux des années 1970, afin d'établir les ensembles historiques qu'il permet de penser et ceux qu' il refuse.

\section{La stratégie comme connexion de l'hétérogène}

Au sein de l'œuvre aujourd'hui publiée, le terme de «stratégie » est utilisé pour la première fois par Foucault de manière significative dans les conférences de Rio de Janeiro en $1973^{37}$. Accolé au terme de «lutte $»$, il $y$ décrit une nouvelle manière d'analyser le discours ${ }^{38}$. Cette utilisation croise en fait deux influences distinctes, explicitement nommées par Foucault en 1973 : la philosophie anglo-saxonne des «jeux de langage »

Le terme apparaît néanmoins parmi les têtes de chapitres de l'ouvrage de 1969 (L'archéologie du savoir, II.vI : «La formation des stratégies »), mais avec un sens différent : le terme «stratégie »y désigne les thèmes et les théories qui se déploient à l'intérieur d'une formation discursive donnée comme la médecine ou l'économie.

$D E \mathrm{n}^{\circ} 139$, «La vérité et les formes juridiques », I, p. 1407 et 1499-1501. La mention de la «lutte » permet de rapprocher la conférence de Rio de l'article sur Nietzsche publié en $1971\left(D E n^{\circ} 84\right.$, « Nietzsche, la généalogie, l'histoire », I). Si le terme de «stratégie » n’y apparaît pas, la « lutte » et la « ruse » sont bien utilisées comme matrices d'intelligibilité de l'histoire. Une différence notable demeure néanmoins : Nietzsche, contrairement à Foucault, ne décrit que des stratégies ratées, énonçant «les erreurs, les fautes d'appréciation, les mauvais calculs » (ibid., p. 1009), là où les analyses foucaldiennes décrivent des positivités, des stratégies qui fonctionnent. 
et les luttes verbales des sophistes; la notion de «jeu $\gg$ faisant le lien entre les deux références ${ }^{39}$. Arrêtons-nous sur la première référence. Dans la discussion qui suit la conférence, Foucault cite plus précisément les noms de Ludwig Wittgenstein, John L. Austin, Peter F. Strawson et John R. Searle. Ces références ne sont pas fortuites : L'archéologie $d u$ savoir avait déjà discuté la pertinence d'une analyse de l'énoncé en termes de speech act ${ }^{40}$ et une conférence de 1978 reconnaît à nouveau cette dette vis-à-vis de la pensée analytique ${ }^{41}$. À cinq ans d'écart, ces deux mentions permettent de synthétiser les principes que Foucault a retenus de l'approche anglo-saxonne du langage :

1) mettre entre parenthèses la question de la référence des mots et lui substituer la question de leur usage (use). Retirant à la logique son primat dans l'intelligibilité des propositions, Wittgenstein pose que la signification d'un mot est d'abord son usage, qui renvoie à une « forme de vie $\gg^{42}$ comme pratique sociale historiquement et culturellement déterminée;

2) mettre entre parenthèses la question de l'essence unitaire du langage et lui substituer celle de la pluralité des «jeux de langage» (language games) obéissant chacun à un ensemble singulier de règles. Parler n'est pas accomplir une unique destinée des mots, comme révéler ou dissimuler l'être, mais simplement jouer à de multiples jeux.

La notion de jeu s'affirme au $\$ 23$ des Investigations philosophiques, où Wittgenstein propose une liste non exhaustive de ce que l'on peut faire avec le langage, bien au-delà de la seule représentation des « états de choses $\gg 43$. Foucault peut à juste titre y inscrire les jeux de pouvoir entre deux partenaires. Le discours y sera dès lors analysé comme une succession d'actions et de réactions qui ne cachent ni ne révèlent rien d'autre que les moments stratégiques d'une lutte. tein, Tractatus logico-philosophicus, suivi de Investigations philosophiques, P. Klossowski trad., Paris, Gallimard, 1961, p. 125). 
Cette notion de stratégie court tout au long de Surveiller et punir et de La volonté de savoir; elle constitue par ailleurs le relais méthodologique principal des cours au Collège de France de 1973 à $1979^{44}$. Jamais définitivement abandonnée, elle se retrouve encore dans les derniers entretiens de Foucault ${ }^{45}$. Mais la longévité du concept n'exclut pas les mutations. Relevons deux inflexions notables :

1) son utilisation se libère de plus en plus du modèle de la lutte, comme grille d'intelligibilité des relations de pouvoir, et ne conserve que l'idée de jeu, comme possibilité constante de réaction de la part des partenaires et de transformation de la relation. C'est le cours Il faut défendre la société (1975-1976) qui signe l'adieu à ce modèle de la lutte et de la guerre; ce que vérifient les entretiens qui le suivent de près ${ }^{46}$;

2) le champ d'application de cette notion va peu à peu s'étendre du domaine du discours à tout ce qui peut être utilisé dans une relation de pouvoir pour la relancer, l'intensifier ou la renverser : pratiques, institutions, connaissances, etc., en posant comme principe l'hétérogénéité des éléments utilisés.

Contredisant les analyses dialectiques développées dans le sillage de Marx et de Hegel, la stratégie empêche de faire des antagonismes apparus dans l'histoire - pensons à l'opposition entre le savoir du médecin et le pouvoir du juge au XIX ${ }^{\mathrm{e}}$ siècle - des puissances appelées nécessairement à faire bouger la structure d'ensemble. Même si elle repose sur des forces opposées, une forme de rationalité peut parfaitement et durablement fonctionner, sans jamais muter ou produire de troisième terme homogène. Un complexe de savoir/pouvoir s'accommode très bien de la contradiction.

44 Peu de temps après la mention du terme «stratégie » à Rio de Janeiro, Foucault le réutilise dans le cours au Collège de France de la même année (Le pouvoir psychiatrique, p. 16 et 18). Bien plus tard, dans le cours de 1977-1978, quand s'annonce le virage d'une étude des disciplines vers une étude de la gouvernementalité, Foucault précise que c'est toujours une analyse en termes de «technologie, tactiques, stratégies » qui sera encore pratiquée (Sécurité, territoire, population, p.123, note *), ce que vérifie l'étude de la gouvernementalité libérale (Naissance de la biopolitique, p. 44). Même importance accordée à la stratégie dans la communication à la Société française de philosophie, le 27 mai 1978 : «Qu'est-ce que la critique? $\gg$, p. 52 .

$45 D E \mathrm{n}^{\circ} 356$, « L'éthique du souci de soi comme pratique de la liberté », II, p. 1546-1547; ou encore dans le dernier grand article publié en $1984: D E n^{\circ} 339$, « Qu'est-ce que les Lumières? », II, p. 1395 .

46 Foucault s'interroge à plusieurs reprises sur le sens et la pertinence de la notion marxiste de «lutte »: $D E \mathrm{n}^{\circ} 200$, « Non au sexe roi », II, p. $268 ; D E \mathrm{n}^{\circ} 235$, « Méthodologie pour la connaissance du monde : comment se débarrasser du marxisme », II, p. 606. 
Dans la leçon au Collège de France du 17 janvier 1979, l'analyse «stratégique » est ainsi définie par opposition à une analyse de type dialectique :

$[\ldots]$ la logique dialectique, c'est une logique qui fait jouer des termes contradictoires dans l'élément de l'homogène. Et à cette logique de la dialectique je vous propose de substituer, plutôt, ce que j'appellerai une logique de la stratégie. [... ] La logique de la stratégie, elle a pour fonction d'établir quelles sont les connexions possibles entre des termes disparates et qui restent disparates. La logique de la stratégie, c'est la logique de la connexion de l'hétérogène. ${ }^{47}$

«Connexion »: le vocabulaire est ici volontairement technicien. Dans un sens similaire, il arrive à Foucault de parler d'《échangeur $\gg$ ou de $\ll$ point de jonction ${ }^{48}$. Pour étendre sa domination, une pratique disciplinaire peut ainsi se mettre en rapport avec un domaine qui lui est absolument extérieur, et ce de trois manières différentes, détaillées lors de la table ronde de 1978 autour de Surveiller et punir : 1) transfert de modèles techniques, 2) calcul tactique répondant à une situation particulière, 3) application de schéma théorique ${ }^{49}$. «Transfert », « calcul » ou «application » : autant de gestes circonstanciés mais toujours circonstanciels, qui se contentent d'instrumentaliser ce dont ils se saisissent. L'hétérogénéité est donc maintenue d'un bout à l'autre d'une analyse qui n'a pas pour but de la dépasser mais seulement d'y organiser des connexions rendant compte en quoi le réel a été, non pas nécessaire, mais simplement possible ${ }^{50}$ : comment, par exemple, le croisement d'un discours juridique et d'un discours médical a eu pour effet, au XIX ${ }^{\mathrm{e}}$ siècle, la production d'une nouvelle rationalité punitive. La « connexion »se borne à constater qu'un type de connaissance s'est appuyé sur des pratiques coercitives ou qu'une pratique coercitive a emprunté à un savoir n'ayant a priori rien à voir avec elle un élément qui renforce ses effets propres. Il ne s'agit donc pas de déduire la naissance de la prison des objectifs avoués des politiques ou des juristes de l'époque, mais de réinsérer cette institution dans un ensemble de relations où se croisent des questions aussi bien éducatives, industrielles que démographiques. Le travail de l'historien consiste à « fixer les points d'ancrage d'une stra- 
tégie $\gg^{51}$, en arrimant à la réforme du système pénal les éléments souvent flottants qui l'ont relayée, prolongée, intensifiée, alors même qu'ils appartenaient à des domaines régis par des objectifs différents.

Ces connexions peuvent être multiples et complexes, au point de dessiner un véritable maillage, elles n'obéissent pour autant qu'à un calcul conjoncturel de coût et de rendement. Foucault réduit ici la raison d'être d'une connexion à un « ratio » (ibid., p. 832), c'est-à-dire un rapport chiffré entre deux grandeurs, ouvrant ainsi la voie à une analyse du pouvoir en termes d'économie. Par «économie », notion centrale de Surveiller et punir $^{52}$, il faut entendre un effort raisonné pour distribuer de manière régulière les investissements et les effets du pouvoir. Connecter, c'est calculer $^{53}:$ le pouvoir agit sur des quantités, non sur des qualités (puisque ces dernières sont des incorporels, comme « avoir une âme »). Désignant initialement la gestion « convenable » de la maison, le terme «économie »,

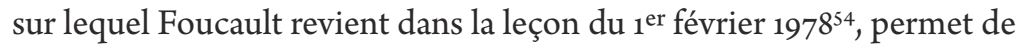
ne pas limiter le ratio du pouvoir à la recherche du meilleur rendement financier - qui ne décrirait alors que le pouvoir disciplinaire moderne, opposé au caractère dispendieux du pouvoir féodal. Il désigne plus largement la gestion d'une multiplicité, une manière de disposer les choses pour en maitriser l'évolution. Cette analyse économique n'est donc pas une ruse pour réintroduire une forme plus subtile de téléologie qui trouverait du côté des mathématiques les principes conjugués de sa rigueur et son anonymat. Elle l'est d'autant moins que toute prévision reste hasardeuse : si les interactions décrites entre domaines hétérogènes sont bien au départ le résultat d'un calcul rationnel de la part de groupes ou d'individus, leurs conséquences ne sont pas maîtrisables sur le long terme, car tout élément connecté peut être par la suite relié à d'autres éléments qui

$D E \mathrm{n}^{\circ} 277$, « La poussière et le nuage $\gg$, II, p. 833 .

Relevons les expressions : «"économie politique" du corps » (Surveiller et punir, p. 33), «économie du pouvoir » (ibid., p. 95 et 106), « économie des illégalismes » (p.103), etc. L'usage du terme ne s'applique pas seulement pour Foucault au pouvoir capitaliste du $\mathrm{XIX}^{\mathrm{e}}$ siècle, contemporain du développement de la société industrielle. Il désigne également et plus largement une manière nouvelle pour l'historien d'analyser le pouvoir.

Dans la réponse à Jacques Léonard, «l'intention réfléchie » à l'œuvre dans une mutation du pouvoir se dit « ratio » et aussi « calcul $\gg\left(D E n^{\circ} 277\right.$, « La poussière et le nuage $\gg$, II, p. 832). Foucault s'efforce toujours de retrouver la raison mathématique, quantifiable, sous la raison qualitative : ainsi du «principe de calcul » caché derrière l'appel humaniste à la modération des peines (Surveiller et punir, p. 108).

Sécurité, territoire, population, p. 98-99, p. 110. 
se trouvent à d'autres niveaux : « [...] de sorte que [explique Foucault en 1978], d'une certaine façon, aucune de ces interactions n'apparaît ou primaire ou absolument totalisante. Chacune peut être replacée dans un jeu qui la déborde $\gg^{55}$. Prévoir l'évolution reviendrait à trouver le ou les points d'équilibre d'un système, à l'image des équations différentielles permettant d'étudier la dynamique des populations. Ce qui est en soi possible; mais encore faudrait-il que le nombre d'équations soit déterminé à l'avance - ce qui n'est pas le cas pour un dispositif de pouvoir. Nous ne sommes ni dans les mathématiques de l'aléatoire ni dans les mathématiques déterministes, mais entre les deux : dans une intrigue historique qui modifie sans cesse son système d'équations. Les prédictions sont donc possibles, mais seulement à court terme.

Avant d'aborder la question de la causalité, concluons sur la description pratiquée par Foucault. S'il fallait trouver une métaphore géométrique pour exprimer les liens ainsi définis, ce serait en premier lieu celle de lignes qui se coupent. On ne sera donc pas étonné que Foucault définisse un événement comme «le point d'intersection entre deux durées, deux vitesses, deux évolutions, deux lignes d'histoire $\gg^{56}$. La diversité des matériaux collectés demande de multiplier les lignes et donc les croisements. Cette multiplication, qui empêche d'obtenir une figure géométrique fermée, conduit l'analyse à suivre une loi de dispersion, sans point d'origine unique, ni même - contrairement aux analyses wébériennes 《d'affinités électives ${ }^{57}$ entre un événement et ses antécédents. Si la dispersion totale est conjurée par l'existence de séries d'événements tracées par les documents eux-mêmes - pensons aux archives de la Bastille pour Foucault, à celles du port de Séville pour $\mathrm{Chaunu}^{58}-$ l'analyse abandonne toute relation de nécessité entre les séries. Le fond de l'histoire serait alors de ne pas en avoir ou d'en avoir de multiples : comme un croisement incessant et hasardeux de séries singulières. Que reste-t-il alors de la vieille notion de causalité?

Le premier type de causalité fermement exclu est celui de « cause finale », notion aristotélicienne que le détour par Nietzsche en 1971 avait déjà suffi

55 《u'est-ce que la critique? », p. 52 .

$56 D E \mathrm{n}^{\circ}$ 234, «La scène de la philosophie », II, p. 581.

57 M. Weber, L'éthique protestante et l'esprit du capitalisme, déjà cité, p. 107.

58 P. et H. Chaunu, Séville et l'Atlantique, Paris, Sevpen, 11 volumes, 1955-1960. Souvent cité par Foucault, ce travail se trouve présenté dans $D E \mathrm{n}^{\circ}{ }_{103}$, « Revenir à l'histoire », I, p. 1144-1145. 
à rejeter ${ }^{59}$. La « connexion de l'hétérogène » est d'abord la description d'un branchement et de ses effets et non une explication qui s'appuierait, pour l'imputation causale, sur la fin en vue de laquelle une chose a lieu. La discussion avec Aristote ne semble cependant pas se prolonger au-delà de la simple remarque selon laquelle il faut substituer à la considération des fins celle des effets - les seconds recoupant rarement les premières dans l'exercice d'un pouvoir ${ }^{60}$. On peut donc émettre l'hypothèse que les réflexions plus poussées de Foucault sur le problème de la causalité, concentrées autour de l'année 1978, ont un autre vis-à-vis que le philosophe macédonien. À plusieurs reprises cette année-là, Foucault revient sur la notion de cause pour préciser combien, loin de la refuser, ses analyses visent au contraire à produire, contre toute détermination univoque et globale, un « réseau causal à la fois complexe et serré ${ }^{61}$, ou encore une 《démultiplication causale $\gg^{62}$. À en rester cependant à ces seules expressions, l'originalité de Foucault ne serait pas ici probante ${ }^{63}$. Ces réflexions ne valent pas tant en fait par ce qu'elles proposent, que par la manière dont elles exposent les modèles qu'elles repoussent. À cette occasion, l'un des vis-à-vis anonymes de Foucault pourrait bien être démasqué. En particulier, dans la communication du 27 mai 1978 à la Société française de philosophie, Foucault présente brièvement une conception ternaire et traditionnelle de la causalité historique qui semble bien recouper la tripartition définie par Raymond Aron dans son Introduction à la philosophie de l'histoire (1938). La possibilité d'un dialogue muet entre les deux

Il ne s'agit pas de « rendre compte d'une émergence par le terme final. Comme si l'œil était apparu, depuis le fond de temps, pour la contemplation $\gg\left(D E \mathrm{n}^{\circ} 84\right.$, « Nietzsche, la généalogie, l'histoire », I, p. 1011). Pour l'exposé des différents types de causes chez Aristote : Aristote, Organon, t. IV : Les seconds analytiques, II, 11, J. Tricot trad., Paris, Vrin, 1938, p. 197-203.

Toujours hétérogènes aux fins initialement cherchées, les effets peuvent être à leur tour repris, organisés, rationalisés, pour de nouvelles fins : $D E \mathrm{n}^{\circ} 346$, « Qu'appelle-t-on punir? », II, p. 1458-1459.

61 «u'est-ce que la critique?», p. 51 .

$62 D E$ no $^{278}$, « Table ronde du 20 mai $1978 \gg$, II, p. 843 .

63 Même Max Weber accepterait cette démultiplication causale. S'il procède à une concentration et à une simplification causale dans L'éthique protestante et l'esprit du capitalisme, il précise aussitôt que cela ne signifie pas que l'éthique protestante soit l'unique cause du développement du capitalisme. Il invite à compléter l'analyse par l'attention au rôle joué par le rationalisme humaniste, l'empirisme philosophique et scientifique, et les progrès techniques. Il invite également à réinscrire l'éthique protestante dans les conditions économiques et sociales qui l'ont rendue possible : M. Weber, L'éthique protestante et l'esprit $d u$ capitalisme, déjà cité, p. 251-252. 
penseurs n'a d'ailleurs, d'un point de vue aussi bien intellectuel que biographique, rien de surprenant - Foucault ayant participé au séminaire de Raymond Aron à la Sorbonne en mars 1967, ainsi qu'à un entretien radiophonique avec lui au mois de mai de la même année ${ }^{64}$.

Reprenons tout d'abord la communication de Foucault : n'a traditionnellement de valeur causale en histoire que 1) ce qui vise « une dernière instance valorisée comme profonde et elle seule, économie pour les uns, démographie pour les autres $\gg ; 2$ ) «ce qui obéit à une pyramidalisation pointant vers la cause ou le foyer causal, l'origine unitaire »; 3 ) 《ce qui établit une certaine inévitabilité ou du moins ce qui approche de la nécessité ${ }^{65}$. Relevons aussitôt que ces trois acceptions de la causalité ne s'épaulent pas forcément mais pourraient également, dans bien des cas, s'opposer : les lois de l'économie ou la constatation en démographie d'une itération ne renvoient pas nécessairement à un unique foyer causal. Mais il y a bien trois lignes de force en histoire qui tentent d'estomper la complexité du réel. Le rapprochement avec l'étude de Raymond Aron permet alors d'éclairer cette présentation serrée, de révéler peut-être ce qui la soustend, au moins de mesurer l'écart ultérieurement pratiqué par Foucault. Il est possible en effet d'entendre ces trois types de causalité comme correspondant respectivement aux trois questions du philosophe, du juge et du savant - tripartition qui permettait à Raymond Aron de déployer en 1938 l'éventail de la pensée causale en histoire ${ }^{66}$. Rappelons les étapes de sa démonstration, à la section III de l'Introduction à la philosophie de l'histoire:

Premier cité, le juge pointe l'unique responsable parmi les antécédents d'un événement : il isole une singularité. «À qui [ou à quoi] la faute? » Notons que pareillement, dans son deuxième point, Foucault insiste sur $l^{\prime} \ll$ origine unitaire $\gg, \ll l a$ cause $\gg^{67}$.

Arrivant en second, le savant cherche au contraire les liens constants de coexistence et de succession : il établit une loi et une régularité. Ceci nous rapprocherait du troisième point de Foucault évoquant l'« inévitabilité » et la « nécessité » (ibid., p. 51).

Aron montre alors que la donnée isolable sans laquelle le fait ne se

64 R. Aron, M. Foucault, Dialogue, J.-F. Bert éd., Paris, Nouvelles éditions lignes, 2007.

65 «u'est-ce que la critique? », p. 50-51.

66 R. Aron, Introduction à la philosophie de l'histoire. Essai sur les limites de l'objectivité historique, édition revue et augmentée, Paris, Gallimard, 1981, p.196-198 et 329-330.

$67 \ll$ Qu'est-ce que la critique ?», p. 50, nous soulignons. 
serait pas produit (consécution unique cherchée par le juge) n’est pas immédiatement conciliable avec une loi générale qui rend compte, dans l'histoire, de réitérations approchant de la nécessité (établie par le savant). Dans ces conditions, seule l'analyse du philosophe peut permettre à l'historien de réconcilier l'analyse du juge et celle du savant. Cette conciliation est une prise de hauteur par rapport à l'histoire, qui reprend les singularités et les généralités dans l'ensemble du déterminisme historique, c'est-à-dire dans une philosophie de l'histoire décidant de ce « qui mène le monde ${ }^{68}$ : Dieu ou l'Idée, l'individu ou les peuples. Nous ne sommes plus alors du côté de causes isolables ni de lois générales, mais du côté d'une structure d'intelligibilité qui permet de lier les unes aux autres. Cette structure peut être rapprochée de ce que Foucault nomme dans son premier point «l'instance profonde $»^{69}$. Même si, dans une histoire matérialiste, cette instance peut prendre la figure de «l'économie » ou de «la démographie », elle n'en reste pas moins une décision philosophique de la part de l'historien : choisir de comprendre l'histoire comme essentiellement mue par les rapports marchands ou la dynamique des populations. La conclusion de Raymond Aron fait de cette décision à la fois la chance et la limite de l'explication historique :

[...] pour organiser événements et régularités en fonction les uns des autres et dans un ordre global, faute de systématisation objective, toute science historique devient philosophique, et c'est pourquoi l'intention du philosophe ne se juxtapose pas aux deux enquêtes analytiques [du juge et du savant], mais les dépasse et cherche à les réconcilier. Du même coup se trouvent précisées les limites de la pensée causale et de l'objectivité de celle-ci. ${ }^{70}$

Or, c'est bien un tel saut de la science historique à la philosophie que cherche à éviter Foucault. L'évitement laisse alors juxtaposées, sans pouvoir être conciliées, les causalités définies respectivement par le juge et le savant. Pour s'en convaincre, il suffit de parcourir le réseau causal de la rationalité disciplinaire, tel qu'il est exposé dans le résumé du cours La société punitive (1972-1973) ${ }^{71}$ et dans Surveiller et punir ${ }^{72}$ : les événements singuliers (la Révolution française, les révoltes populaires)

$69 \ll$ Qu'est-ce que la critique ?», p.50, nous soulignons.

70 R. Aron, Introduction à la philosophie de l'histoire, déjà cité, p. 330.

$71 D E \mathrm{n}^{\circ} 131$, «La société punitive », I, p. 1334-1336. 
y croisent les régularités macroscopiques (poussée démographique, croissance économique), sans structure d'intelligibilité commune. Se limitant à une évaluation des possibilités offertes par la rencontre de ces deux « vitesses » différentes d'histoire, Foucault se refuse à les composer dans un mouvement unique.

Il faut donc écrire l'histoire dans ce seul espace continu des corps et des pratiques, et poser en même temps l'hétérogénéité des éléments qu' ils manipulent. Centrifuge, l'analyse parcourt à partir d'un lieu singulier un domaine historique a priori indéfini ${ }^{73}$, multipliant les connexions entre des intérêts et des stratégies distincts. Par conséquent, l'étude de la rationalité d'une pratique n'a aucun corpus prédéfini ni aucune ère géographique bien délimitée - labilité problématique que relève avec perspicacité la rédaction d'Hérodote, dans un entretien avec Michel Foucault publié en 1976 :

On peut relever ainsi un souci rigoureux de périodisation qui contraste avec le flou, la relative indétermination de vos localisations. [... ] Vous privilégiez, de fait, le facteur temps, au risque de délimitations ou de spatialisations nébuleuses, nomades. Spatialisations incertaines qui contrastent avec le souci de découper des tranches, des périodes, des âges. ${ }^{74}$

Ce principe revendiqué de nomadisme semble bien être le résultat méthodologique auquel est parvenu Foucault à la fin des années 1970, après les travaux sur la pénalité et la sexualité. Ses analyses en étoiles, qui peuvent à volonté multiplier les branchements - étant donné que la description d'une rationalité n'est jamais close -, viennent cependant croiser une analyse centripète qui ne les contredit pas forcément : celle qui s'efforce d'établir, non pas a priori mais au cours de la recherche, des unités relativement stables au sein des pratiques et des discours. Nous les avons repérées au passage sous l'expression «forme de rationalité », mais sans préciser exactement leur degré d'idéalité par rapport à la mobilité du réel. Il nous faut maintenant les accorder aux principes de l'analyse stratégique tels qu'ils viennent d'être décrits.

73 Si le livre sur la prison a un aspect de monographie, « [son] corpus est en un sens indéfini » $\left(D E \mathrm{n}^{\circ}{ }_{156}\right.$, « Entretien sur la prison : le livre et sa méthode », I, p. 1609).

$74 D E n^{\circ} 169$, « Questions à Michel Foucault sur la géographie », II, p. 31. 


\section{Les «formes de rationalité » sont-elles des idéalités?}

Pour articuler ces deux mouvements de l'analyse, description d'un réel éclaté et effort de resserrement par le concept, il serait tentant de rapprocher la pensée de Foucault de celle de Max Weber. Le sociologue allemand évite en effet la contradiction en distinguant fermement l'ontologique du méthodologique, la réalité mouvante des concepts élaborés par le chercheur. Si les sciences de la culture ne peuvent suivre les procédures des sciences de la nature et avancer par voie déductive, il leur reste la voie conceptuelle $\mathrm{du}$ 《 tableau de pensée » (Gedankenbild), aussi utile pour la recherche (il guide l'élaboration des hypothèses) que pour l'exposé des faits (il leur fournit un moyen d'expression univoque). Ce tableau est un produit de l'esprit du chercheur qui « réunit des relations et des événements déterminés de la vie historique en un cosmos non contradictoire de relations pensées $\gg 75$. Il est donc toujours de l'ordre d'un «idéal », d'une «utopie » utile. Foucault ne se ralliet-il pas d'ailleurs à cette prudence méthodologique wébérienne quand il précise, en réponse à des critiques, qu'une situation relativement stable de pouvoir n'est également qu'une « photographie instantanée de luttes multiples $\gg 7^{6}$, justifiant ainsi l'écart avec le réel par la position de l'observateur? Il n'y a pas loin de la photographie au tableau.

Par deux fois cependant, dans la table ronde du 20 mai 1978, l'auteur de Surveiller et punir s'efforce de se distinguer des analyses de Weber et des « wébériens ». Pour parvenir à ses fins, on peut penser qu'il simplifie grandement la pensée du sociologue allemand, en faisant par exemple de l'«idéaltype » une catégorie qui permet « de ressaisir une "essence" (du calvinisme, ou de l'État, ou de l'entreprise capitaliste) $\gg 77$. Synonyme $\mathrm{du} \ll$ tableau de pensée $\gg, l^{\prime} \ll$ idéaltype $\gg$ est bien une construction de l'historien qui décrit une rationalité idéale de pratiques. Pour Weber, celle-ci est et restera toujours étrangère aux éléments de la réalité historique et même aux idées qui ont effectivement gouverné les hommes. $L^{\prime}$ «idéaltype »s'arrache au vécu immédiat des individus pour tenter a posteriori de ressaisir une activité rationnelle finalisée qui échappe à 
leur conscience. Or, comme le précise Weber, les synthèses logiques que cette catégorie permet restent problématiques jusque dans leur délimitation; elles requièrent ainsi une multiplication des concepts utilisés à mesure que l'analyse embrasse une histoire plus vaste et plus complexe. L'«idéaltype » ne présuppose donc à aucun moment l'unité, dans le domaine empirique, de quelque chose comme le «libéralisme», le « socialisme » ou encore le « christianisme », trois exemples justement cités par Weber pour appeler à la prudence ${ }^{78}$. Autrement dit, il s'agit de pratiquer un idéalisme méthodologique, jamais métaphysique.

Le débat est pourtant ouvert et force Foucault à préciser pourquoi les «formes de rationalité » qu'il décrit ne sont pas des «idéaltypes ». Cette question semble d'autant plus importante pour notre sujet que Weber, malgré la prudence ci-dessus mentionnée, n'exclut pas la possibilité de considérer le christianisme comme un «idéaltype », à partir du moment où cette considération n'est qu'une commodité méthodologique qui ne présage nulle unité empirique de l'objet 79 . Se dessinerait alors la possibilité pour Foucault de donner au terme « christianisme » une stricte valeur heuristique, évitant ainsi d'en faire une essence, une mentalité ou une idéologie. Mais Foucault semble également se méfier des facilités heuristiques. Le débat autour des «idéaltypes » s'annonce donc serré.

«Il y a chez vous, dans un sens que vous n'accepteriez sans doute pas, quelque chose comme un "type idéal" $\gg^{80}$. Le rapprochement proposé par un intervenant de la table ronde de 1978 n'est pas gratuit; il vient après les sévères remarques de l'historien Jacques Léonard, reprochant à Surveiller et punir de sous-estimer l'importance « du désordre, du laisser-aller, de la jungle, de la pagaille en somme $\gg^{8_{1}}$. Dans un long compte-rendu du livre publié en 1976, il oppose «la poussière des faits

78 M. Weber, Essais sur la théorie de la science, déjà cité, p. 187-19o.

79 «Tous les exposés qui ont pour thème l'"essence" du christianisme sont des idéaltypes qui n'ont nécessairement et constamment qu'une validité relative et problématique, s'ils revendiquent la qualité d'un exposé historique du donné empirique ; par contre ils ont une très grande valeur heuristique pour l'exposé, si on les utilise simplement comme moyens conceptuels pour comparer et mesurer à eux la réalité » (ibid., p.191-192).

$D E n^{\circ} 278$, « Table ronde du 20 mai $1978 \gg$, II, p. 846.

81 «'historien et le philosophe. À propos de Surveiller et punir », L'impossible prison. Recherches sur le système pénitentiaire au xixe siècle, M. Perrot éd., Paris, Seuil, 1980, p. 12. L'article avait été initialement publié dans les Annales historiques de la Révolution française, dirigées par l'historien Albert Soboul, membre influant du parti communiste. Enseignant à Rennes, Jacques Léonard était spécialiste d'histoire de la médecine. Le dialogue avec 
concrets $»^{82}$ à la thèse, prêtée à Foucault, de la « normalisation massive », rappelant au passage que la plupart des modèles disciplinaires décrits dans le livre n'ont jamais été effectivement mis en place. En premier lieu : le Panoptique de Jeremy Bentham (1748-1832), que les analyses foucaldiennes contribuèrent à remettre sur le devant de la scène après des décennies d'oubli du côté des historiens. Dès le cours sur Le pouvoir psychiatrique en 1973-1974, Foucault avait lui-même relevé le faible nombre de réalisations effectives : principalement Pentonville en Angleterre et la Petite-Roquette en France ${ }^{83}$. C'est en effet en vain, et jusqu'à la ruine personnelle, que Bentham chercha à faire appliquer son système de surveillance en France, en Irlande et en Angleterre. Dans ces conditions, la notion d'《idéaltype » aurait pu offrir une bouée de sauvetage pour sauver la proposition de Foucault de la noyade : un livre comme Surveiller et punir décrirait finalement une rationalité idéale - efficacité parfaite, fonctionnement sans obstacle de la société panoptique - dont il resterait ensuite à mesurer l'écart avec la réalité historique, afin de réajuster le modèle aux connaissances empiriques.

Regardons de près la manière dont Foucault présente la maison de surveillance conçue par le philosophe anglais. Par deux fois, il commente l'ouvrage de Bentham intitulé en français Le Panoptique (Panopticon, or, the Inspection-house, Dublin, 1791), comprenant textes et planches ${ }^{84}$ : dans le cours au Collège de France de 1973-1974 et dans Surveiller et punir. D'un commentaire à l'autre, le philosophe n'aborde pas l'œuvre

Foucault se poursuivit jusque dans les titres de ses livres : J. Léonard, La médecine entre les savoirs et les pouvoirs, Paris, Aubier, 1980.

82 J. Léonard, «L'historien et le philosophe. À propos de Surveiller et punir », déjà cité, p. 13.

83 Le pouvoir psychiatrique, p. 75. Il faut cependant ajouter à cette liste la prison de Millbank, sur la rive droite de la Tamise, qui reprend certains aspects du projet de Bentham. Sur Millbank, voir D. Neil, « Des "corps dociles" ? Le Panoptique de Jeremy Bentham en théorie et en pratique », L'un sans l'autre : racisme et eugénisme dans l'aire anglophone, M. Prum éd., Paris, L'Harmattan, 2005, p. 207-230.

84 Imprimé d'abord à Dublin, puis à Londres, comprenant vingt et une lettres, deux postscriptum et trois planches, cet ouvrage est en partie le fruit d'un séjour de Bentham en Russie, de février 1886 à novembre 1887 , au cours duquel il découvrit un nouveau type de surveillance des ouvriers mis au point par son frère Samuel. Le terme français « Panoptique » apparaît dans le document présenté à l'Assemblée nationale en 1791, qui n'est pas exactement une traduction du Panopticon, mais plutôt une adaptation, œuvre de l'éditeur de Bentham, Étienne Dumont : J. Bentham, Panoptique : mémoire sur un nouveau principe pour construire des maisons d'inspection, et nommément des maisons de force, E. Dumont éd., Paris, 1791. Foucault travaille pour sa part sur une édition anglaise. 
de Bentham de la même manière, et seul le premier commentaire permet effectivement de jeter un pont entre sa pensée et celle de Weber.

Dans la leçon du 28 novembre 1973, Foucault souligne que le Panoptique n'est pas un rêve, même s'il a rarement été réalisé dans la forme architecturale précise que Bentham avait conçue initialement pour une maison de force. Il rappelle que, dans l'esprit de Bentham, le projet devait pouvoir fonctionner aussi bien pour un hôpital, une école, un atelier, etc., qu'il faut donc l'abstraire des objectifs immédiats et concrets (soigner, punir, éduquer) pour le considérer comme le «schéma formel $\gg^{85}$, la « forme générale $\gg(i b i d ., p .81)$ d'un pouvoir. Le nom propre «Panoptique » devient alors, dans la leçon du 28 novembre, un nom commun («panoptisme ») et même un adjectif («panoptique ») pour décrire, non pas la prison rêvée par Bentham, mais un opérateur spatial applicable dans n'importe quelle institution et même hors les murs de toute institution particulière. Cet opérateur vise à optimiser l'exercice d'un pouvoir, le rendre à la fois plus intense, plus constant, plus précis. Le panoptisme est une distribution du regard par une certaine gestion de l'espace qui permet une division de la multiplicité en individus, couplée sur une extraction permanente de connaissances. Foucault laisse alors entendre que cet opérateur résume le pouvoir disciplinaire et décrit effectivement la société :

La trame commune à ce qu'on pourrait appeler le pouvoir exercé sur l'homme en tant que force de travail et le savoir sur l'homme en tant qu'individu, il me semble que c'est le mécanisme panoptique qui la donne. Si bien que le panoptisme pourrait, je crois, apparaître comme une forme générale ; on pourrait parler d'une société disciplinaire ou aussi bien d'une société panoptique. Nous vivons dans le panoptisme généralisé par le fait même que nous vivons à l'intérieur d'un système disciplinaire. (Ibid., p. 80-81)

Il faut accorder à l'orateur la prudence de son cheminement, le conditionnel précédant curieusement l'indicatif. La direction suivie par l'analyse pose cependant deux problèmes majeurs au regard du projet historiographique de Foucault.

1) Alors que Foucault prétendait s'affranchir d'une histoire des représentations, n'y retombe-t-il ici avec éclat? Les planches commentées de Bentham sont finalement la représentation spatiale parfaite de l'idée de la société disciplinaire, qui tend à se réaliser concrètement au XIX ${ }^{e}$ siècle. 
Cette société en genèse aurait alors son expression dans les productions intellectuelles de l'époque, dans ce qui habite l'esprit d'un Jeremy Bentham ou de l'obscur docteur N. H. Julius. Que leurs représentations aient été plus ou moins bien appliquées ne leur retire pas leur fonction de médium entre l'idée et sa réalisation. Dans l'épure de leur dessin, les planches du Panoptique se rapprocheraient alors du schème kantien, comme procédé général permettant de donner à un concept son image. Derrière le Panoptique et sa disposition spatiale particulière, serait à l'œuvre la représentation, dans l'imagination, d'une méthode pour appréhender sensiblement, et d'abord visuellement, le concept de discipline.

2) Alors que le modèle stratégique insiste sur la connexion de tactiques hétérogènes, la notion de panoptisme ne vient-elle pas homogénéiser l'exercice du pouvoir en la réduisant à une unique «forme »? Prisons, écoles, ateliers, hôpitaux ont des objectifs immédiats différents, mais ils appliquent, comme à la chaîne, dans la monotonie d'un pouvoir univoque, la même «forme » que Foucault extrait de Bentham. Tel est bien le motclé du commentaire proposé par le cours de 1973-1974: «forme», «forme disciplinaire générale », «schéma formel $», ~ «$ forme générale ${ }^{86}$.

Le deuxième point nécessite un commentaire prolongé car la notion de «forme » n'a pas ici le sens large d'une certaine modalité d'existence (comme dans «forme de rationalité »), mais le sens précis d'une configuration spatiale, strictement reconnaissable et répétable. Cette possibilité itérative offerte par la forme nous rapproche en fait des structures formelles que l'archéologie du savoir tentait de retrouver sous les pratiques discursives. La méthode archéologique déployée dans les années 1960 écartait de ce fait la question des connexions réelles entre événements, pour lui substituer la mise en lumière d'isomorphismes. Précisons ce terme qui renvoie aux débats auxquels Foucault a été mêlé, autour et au-delà du structuralisme.

Après la publication des Mots et les choses en 1966, certains commentateurs s'étaient interrogés sur l'absence dans le livre de tout ce qui déborde le domaine régulier du discours : situation économique, secousses politiques, mouvements sociaux. À cela, Foucault n'eut de cesse de répondre qu'il en allait seulement d'un choix méthodologique temporaire et non d'un postulat inamovible : il fallait d'abord défaire les 
liens trop simples entre les pratiques discursives et les autres pratiques, pour qu'apparaissent de nouvelles règles autonomes de transformation des discours. Les mots et les choses ne prétendaient pas balayer d'un revers de main la poussière des faits non langagiers, mais les laisser pour un temps en suspens, afin de voir plus précisément comment ils retombaient ensuite sur le discours ${ }^{87}$. De ce point de contact possible entre les énoncés et la vie des sociétés, avant que ne domine dans ses travaux le modèle stratégique, Foucault a proposé deux schémas différents : «l'isomorphisme » et «les conditions d'existence historique d'un énoncé $\gg$.

Dans un entretien de 1966, Foucault use d'une métaphore mathématique pour rendre compte des relations, à une époque donnée, entre pratiques, institutions et théories : entre ces domaines, l'archéologie cherche à définir un «isomorphisme $»^{88}$. Ce terme, déjà utilisé par Foucault dans ses discussions sur le roman ${ }^{89}$, et commun à l'époque à plusieurs sciences humaines, est ici appliqué pour la première fois à son propre travail. Rappelons sa définition dans la théorie des ensembles mathématiques : une bijection est une fonction $\mathrm{f}: \mathrm{x} \rightarrow \mathrm{y}$, telle que pour tout $y$ de l'ensemble d'arrivée il existe un et un seul $x$ dans l'ensemble de départ. Un isomorphisme est alors une bijection telle que les relations algébriques entre les éléments de l'ensemble d'arrivée sont les mêmes qu'entre leurs antécédents respectifs. Plus simplement, disons que la structure algébrique est conservée par la fonction. C'est bien ce dernier point qui nous importe pour comprendre l'usage que Foucault fait de cette métaphore, qu'il versera ensuite à son vocabulaire usuel : même si les contenus entre les champs étudiés n'ont en apparence rien à voir, on peut repérer, à un niveau formel, les mêmes types de relations entre leurs éléments. Ces relations forment ainsi, sous des domaines hétérogènes quant à leurs objets, un invariant commun qui se maintient ou se transforme indépendamment des éléments qu'il contient.

Mais le recours à l'isomorphisme comme modèle pour relier pratiques, institutions et théories n'a en fait, dans les commentaires de Foucault sur son propre travail, que l'occurrence que nous venons de

87 «Mais il n'y a d'intérêt à décrire cette couche autonome des discours que dans la mesure où on peut la mettre en rapport avec d'autres couches, de pratiques, d'institutions, de rapports sociaux, politiques, etc. $\gg\left(D E \mathrm{n}^{\circ} 48\right.$, « Sur les façons d'écrire l'histoire $\left.», \mathrm{I}, \mathrm{p} .618\right)$.

$88 D E \mathrm{n}^{\circ} 34$, « Michel Foucault, Les mots et les choses 》, I, p. 526.

$89 D E n^{\circ} 17$, « Distance, aspect, origine », I, p. 301 et $305 ; D E n^{\circ} 22$, « Débat sur le roman », I, p. $366 ; D E n^{\circ} 23$, «Débat sur la poésie », I, p. 423. 
mentionner. Si le terme est ensuite régulièrement utilisé pour désigner le type de relations que l'on peut établir entre des formations discursives différentes (par exemple entre l'analyse des richesses, l'histoire naturelle et la grammaire générale ${ }^{90}$ ), il ne sera plus employé pour désigner la relation entre des formations discursives et les pratiques extradiscursives qui les accompagnent ou les jouxtent. Il y a là, après 1966, un silence déroutant. Dans ces mêmes années, les allusions de Foucault à l'œuvre de Georges Dumézil croisent également cette notion d'isomorphisme, pour ne cesser ensuite de la corriger, de la déplacer ${ }^{91}$.

À cet abandon, il y a sans doute une première raison : l'isomorphisme dit une similitude formelle, là où Foucault, répondant aux critiques dont il est l'objet, cherche d'abord une articulation historique et concrète. La réponse à la revue Esprit en 1968 éclaire les débats du moment et les efforts de Foucault pour redonner à l'archéologie une portée pratique et politique, contre ceux qui n'y avaient vu qu'une fuite de tout engagement concret. On comprend alors que la métaphore de l'isomorphisme soit soigneusement évitée dans un débat où l'auteur des Mots et les choses doit d'abord répondre de l'accusation de formalisme. Au lieu d'une relation formelle, Foucault définit alors un point d'articulation concret entre pratiques et discours, en reprenant l'exemple de la médecine : entre la pratique politique et le discours médical, le point de rencontre historique

Les trois champs étudiés dans Les mots et les choses fournissent l'exemple le plus clair $\mathrm{d}^{\prime} \ll$ isomorphisme $\gg\left(\right.$ voir $D E \mathrm{n}^{\circ} 72$, « Préface à l'édition anglaise », I, p. 877).

91 Dans un entretien de 1967, Foucault use du terme «isomorphisme » pour décrire le rapport chez Dumézil entre la religion romaine et les légendes scandinaves $\left(D E \mathrm{n}^{\circ} 48\right.$, «Sur les façons d'écrire l'histoire », I, p. 618). Il s'agit bien d'une identité de relations entre des objets appartenant à des ensembles différents. Dans une conférence de 1970, Foucault évoque à nouveau Dumézil et l'isomorphisme, mais il insiste désormais sur le fait que cette identité ne doit pas être considérée comme le but de la recherche et n'est pas non plus l'originalité de Dumézil ( $D E \mathrm{n}^{\circ}$ 103, «Revenir à l'histoire », I, p.1142). Que le modèle de l'isomorphisme soit cependant resté pour Foucault une voie possible d'investigation, les discussions de Rio en 1973 le montrent. Ces discussions ne se contentent pas d'évoquer une fois de plus la similitude de relations établies entre deux mythes, mais apportent un élément nouveau : pour Foucault, Dumézil n'en est pas resté à une comparaison des discours, mais a tout aussi bien comparé un mythe et une pratique judiciaire. Le terme d'isomorphisme n'est pas employé, mais l'idée est la même : Dumézil, explique Foucault, «admet que les mêmes relations puissent intervenir aussi bien dans un discours que dans un rituel religieux ou dans une pratique sociale $\gg\left(D E \mathrm{n}^{\circ} 139, \ll \mathrm{La}\right.$ vérité et les formes juridiques », I, p.1503-1504). Nous sommes alors très près d'un isomorphisme discours/pratiques. 
est à chercher au niveau des $\ll$ conditions d'existence $\gg^{92}$ du discours médical. Les mouvements administratifs ou politiques n'imposent pas de nouvelles «idées » médicales, mais ils offrent de nouveaux objets d'observation, de nouveaux lieux d'intervention, de nouveaux critères de formation et de diffusion de la parole du médecin. Ainsi, les règlements statutaires, la définition des espaces, les modes d'enregistrement et de conservation du discours sont autant de lieux où, à un moment donné de l'histoire, se nouent pratiques politiques et discours scientifiques.

Ce nœud est alors opposé par Foucault à la conception traditionnelle un peu lâche de l'histoire des mentalités, qui voudrait que les pratiques «se reflètent » ou «se traduisent» dans les discours d'une époque (ibid., p. 718). Nous voyons poindre dans cette opposition la deuxième raison qui expliquerait la mise à l'écart du modèle de l'isomorphisme. Si le terme est clair pour le mathématicien, son usage métaphorique reste ambigu et pourrait glisser vers une simple ressemblance morphologique entre les domaines. Reviendrait alors subrepticement ce qui est vigoureusement écarté ici par Foucault : des thèmes « expressionnistes $\gg 93$ qui en resteraient à une vague silhouette commune entre discours et pratiques - silhouette apparaissant comme une ombre sur la toile de l'esprit humain.

La réponse à la revue Esprit arrive ainsi dans un contexte où Foucault perçoit en même temps les limites d'une histoire des mentalités et le danger d'une approche strictement formelle qui permettrait de relier des systèmes de pratiques et de discours. À un lien mental ou idéologique, il propose de substituer un lien pratique. On ne sera donc pas étonné de constater que, tardivement, dans un entretien de 1983, Foucault oppose sans ambages la notion de stratégie à celle d'isomorphisme : « [... j j'essaie d'établir des interconnexions qui ne sont pas des isomorphismes $\gg^{94}$.

Devant ces corrections importantes apportées à son propre travail, la leçon du 28 novembre 1973 ne signe-t-elle pas alors un désaveu gênant ? Le commentaire de Bentham semble effectivement conjuguer une histoire des représentations et une histoire «structurale $\gg$ du pouvoir. La première mettrait l'accent sur le Panoptique comme idéalité pensée par Bentham; la seconde sur le «panoptisme », comme formalisation pro-

$D E \mathrm{n}^{\circ} 58$, « Réponse à une question », I, p. 718 .

$93 D E n^{\circ} 85$, «Entretien avec Michel Foucault », I, p. 1031.

$94 \mathrm{DE} \mathrm{n}^{\circ} 330$, « Structuralisme et poststructuralisme », II, p. 1269. 
duite par l'historien. Qu'il ne soit plus question ici de connexions singulières et concrètes entre des événements mais bien d'abstraction, la question que Foucault se pose à lui-même à la fin de son commentaire le vérifie : «Vous me direz : tout cela, c'est très bien, mais est-ce qu'on peut dire véritablement que les dispositifs disciplinaires ont effectivement recouvert la société tout entière ? $\gg 95$ - signe que la description précédente avait bien mis la question entre parenthèses.

Ouvrons maintenant le chapitre «Le panoptisme » de Surveiller et punir. Dans un premier temps, Foucault nous place clairement du côté de l'idéalité. Mais non pas celle que produirait l'historien pour les besoins de sa recherche, uniquement celle de Jeremy Bentham. La société panoptique n'existe que dans l'esprit du philosophe britannique et dans ses travaux : «Bentham rêve » (ibid., p. 243), Bentham « programme » (p. 243), son schéma «pourra être utilisé » (p. 240), « est destiné $\gg$ (p.240), etc. : tous ces verbes ne décrivent aucune effectivité. Surtout, Foucault ne se pose à aucun moment la question de la réalisation plus ou moins réussie, plus ou moins diffusée, de cette curieuse figure architecturale. Pour la simple raison que l'efficacité du Panoptique ne tient pas à son seul caractère de représentation; c'est là le virage du livre de 1975 par rapport au cours Le pouvoir psychiatrique.

Certes, Foucault continue d'y voir l'une des «images $\gg^{96}$ de la discipline qui se déploie à cette époque dans des institutions très diverses ; son schéma spatial reste la représentation d'un mouvement de fond de la société du XIX ${ }^{\mathrm{e}}$ qui fait se ressembler l'école et la prison (ibid., p. 264); il est la «formule abstraite d'une technologie bien réelle $\gg($ p. 261). En cela, Surveiller et punir reprend en partie les analyses de 1973-1974. Mais il fait en même temps autre chose avec le texte de Bentham. Ce n'est plus seulement comme image parfaite de la société en train de se mettre en place que Foucault insère ce document dans l'histoire des disciplines, ce n'est pas seulement comme forme épurée d'un pouvoir omniprésent que le Panoptique doit être pris au sérieux; le texte vaut également comme événement dans l'histoire du discours, dont le caractère «idéal » a en lui-même des effets de réalité.

Pour ne pas se méprendre, il faut relire le chapitre «Le panoptisme » (III.III) en regard de l'avant-dernier chapitre du livre : «Illégalismes et 
délinquance » (IV.II). Tout se passe comme si la présentation parfois éclatante, presque jubilatoire, du panoptisme par Foucault, joignant sa voix à celle de Bentham et de Julius - au point de se confondre par moments avec celles-ci ${ }^{97}$-, n'était elle-même que la manifestation ironique de la principale ruse de la rationalité punitive.

Soit la fin du chapitre «Le panoptisme » (III.III). Notons le vocabulaire de l'«idéal » et l'emploi du conditionnel :

Le point idéal de la pénalité aujourd'hui serait la discipline indéfinie : un interrogatoire qui n'aurait pas de terme, une enquête qui se prolongerait sans limite dans une observation lumineuse et toujours plus analytique, un jugement qui serait en même temps la constitution d'un dossier jamais clos, la douceur calculée d'une peine qui serait entrelacée à la curiosité acharnée d'un examen, une procédure qui serait à la fois la mesure permanente d'un écart par rapport à une norme inaccessible et le mouvement asymptotique qui contraint à la rejoindre à l'infini. (p. 264)

Soit maintenant l'avant-dernier chapitre « Illégalismes et délinquance $\gg$ (IV.II). Au sujet de la production de la délinquance et de son investissement par l'appareil pénal, Foucault commente :

$[\ldots]$ il faut les prendre pour ce qu'ils sont : non pas des résultats acquis une fois pour toutes mais des tactiques qui se déplacent dans la mesure où elles n'atteignent jamais tout à fait leur but. (p. 333)

Entre les deux, Foucault a montré que la prison avait été un échec complet. Mais un échec dont le constat a presque été contemporain de sa mise en place et n'a cessé dès lors de faire entendre sa voix éplorée : les détenus, a-t-on écrit dès les premières enquêtes réalisées sur la prison, ne sont pas bien corrigés, les techniques sont trop rudimentaires, la criminalité ne baisse pas. Dans cette brèche, s'est alors engouffrée toute une littérature réformiste - « champ actif où ont foisonné les projets, les réaménagements, les expériences, les discours théoriques, les témoignages, les enquêtes » (p. 272-273) - qui n'a fait que relancer une technique inefficace en proposant à nouveau et sans cesse un système impossible. À

97 Le glissement est à la fois très net et imperceptible au lecteur pressé, quand Foucault rapporte à l'imparfait les idées de Julius («Julius lisait») avant de passer brusquement au présent («Notre société n'est pas celle du spectacle mais de la surveillance »), comme si l'interprétation de Julius devenait la description d'une réalité (ibid., p. 252-253). C'est un procédé rhétorique courant chez Foucault de dramatisation d'une idée : il l'exprime au présent pour la rendre présente au lecteur. 
côté de la négativité apparente de cette faillite, Foucault met en lumière la positivité qui l'accompagne : la production active et utile de « délinquants $\gg$. Afin de gérer les nouveaux illégalismes populaires, le système carcéral en isole une certaine catégorie, qu'il produit et reconduit sans cesse par les vertus même de son échec. Se dessine ici la fonction précise qu'occupe dans la stratégie carcérale l'ensemble des projets et des idées de réforme, ou, plus généralement, l'ensemble des idéalités.

Dans cette même direction, la table ronde de 1978 va être l'occasion pour Foucault de développer la notion d'《effet de réel ». Il s'agit de donner aux projets qui entourent une rationalité de pouvoir tout leur poids de réalité, même s'ils n'ont pas été matériellement mis en œuvre : «Ces programmations de conduite, ces régimes de juridiction/véridiction ne sont pas des projets de réalité qui échouent. Ce sont des fragments de réalité qui induisent ces effets de réel $\gg^{98}$. La mise en œuvre concrète de ces projets n'est donc que l'un de ces effets, sur lequel l'historien Jacques Léonard a eu tort de se focaliser exclusivement. Au-delà de la question de savoir si le bâtiment a bien été bâti, étudier ces effets dans leur diversité c'est définir d'abord le rôle stratégique que joue l'idéal dans un dispositif de pouvoir. Foucault montre ainsi, dans le cas précis de la rationalité punitive, que si les prisonniers ont finalement été considérés comme incorrigibles, si les prisons sont rapidement apparues aux yeux des observateurs comme des «chaudrons à sorcières » en comparaison de la «belle mécanique benthamienne (ibid., p. 848), c'est bien parce que l'idéal avait réussi à s'imposer comme une « grille d'appréciation $\gg$ (p. 848), et fonctionnait pleinement à ce titre, imposant la réforme perpétuelle comme réquisit et complice d'un système : il faut sans cesse mieux faire, sans rien modifier des présupposés de départ, car la prison n'est pas encore ce qu'elle devrait être.

L'écart entre l'idéal et la réalité doit être considéré sous un angle tactique, et inscrit à ce titre dans la réalité historique. Il n'a donc pas une fonction méthodologique, conséquence des abstractions et des généralisations historiennes. S'il y a un «idéaltype », il n'est pas construit par l'historien mais se trouve déjà dans cette partie conséquente de l'archive, composée de schémas rationnels d'organisation, de règlements, de programmations, de prescriptions diverses, qui ne disent rien en 
eux-mêmes de leur application réelle, sans pour autant être négligeables. L'analyse ne doit pas se limiter à vérifier leur effectivité, mais d'abord et surtout à étudier leurs «effets de réel » dans une stratégie qui utilise justement l'ineffectivité de l'idéal pour reconduire son exercice.

L'écart avec la méthodologie wébérienne est donc vérifié, mais il ne laisse à Foucault qu'une marge de manœuvre étroite pour décrire de manière synthétique ce qu'il appelle une «forme de rationalité ». Pour éviter le degré d'idéalité assumé par Weber, Foucault ne se risque pas à donner une définition univoque et générale de ce qu'est la rationalité punitive au XIX ${ }^{\mathrm{e}}$ siècle - une telle définition risquant toujours de substituer un point de vue fonctionnel trop simple au point de vue stratégique. Or, la fonction officielle d'une institution est non seulement un idéal à distance de la réalité - que l'historien traditionnel tente indéfiniment de réduire - mais elle provoque elle-même, dans cette distance qu'elle pose, des connexions nouvelles indéfinies et des retournements stratégiques incessants. Nous avons vu que la description en apparence homogène des formes panoptiques à l'œuvre dans la société, proposée dans la troisième partie de Surveiller et punir, était ainsi brutalement déplacée et finalement retournée par les ruses de la quatrième partie. Ne reste-t-il alors que la poussière des faits ?

L'existence d'une forme générale du pouvoir est cependant présupposée par Foucault, pour la raison principale que des stratégies locales ne pourraient perdurer et se côtoyer sans produire à un moment donné des effets stables et réguliers, ou encore une «stratégie d'ensemble». Cette expression apparaît conjointement dans Surveiller et punir (p. 97) et La volonté de savoir (p.132), mais garde un statut incertain. Une telle stratégie ne doit pas être comprise comme la somme des tactiques locales et, surtout, elle ne leur est pas homogène : tel est le principe décisif du «double conditionnement », qui pose une solution de continuité entre les niveaux supérieur et inférieur, entre le local et le général99. La stratégie d'ensemble est hétérogène aux intérêts initiaux de groupes ou

«Des unes [stratégies globales] aux autres [stratégies locales], pas de discontinuité [...]; mais pas non plus d'homogénéité » (La volonté de savoir, p. 132). Étrangement, la définition d'un espace continu et hétérogène nous renvoie à la définition de la durée chez Henri Bergson. Pour ce dernier, l'espace se dit au contraire discontinu et homogène. Il serait intéressant d'éclairer cette inversion. 
d'individus, comme aux conjonctures et aux luttes locales, sur lesquels elle ne cesse cependant de s'appuyer. Elle est une «forme terminale » du pouvoir qui a en même temps sa régularité propre ${ }^{100}$. La tentation est alors grande de se tourner vers une superstructure comme l'État pour rendre compte de cette forme apparemment supérieure, sinon ultime. En 1976, La volonté de savoir n'exclut pas cette possibilité.

L'aversion de Foucault pour les explications en termes de structures politiques est en fait moins évidente qu'il n'y paraît. Si le philosophe refuse de voir dans l'État, dans sa souveraineté et ses lois, l'origine du pouvoir, il laisse entendre que c'est bien de ce côté-là qu'il faut chercher sa forme terminale et solidifiée. La multiplicité des rapports de force - décrite par l'historien dans sa dispersion initiale - a pour effet concret la mise en place progressive d'une stratégie «dont le dessin général ou la cristallisation institutionnelle prennent corps dans les appareils étatiques, dans la formulation de la loi, dans les hégémonies sociales » (p.122). Foucault n'évoque-t-il pas lui-même dans Surveiller et punir «l'étatisation des mécanismes de discipline » (p.248) à travers la mise en place de la police et son quadrillage serré du corps social? Il ne faut pas pour autant se méprendre : l'expression «forme terminale » n'est pas synonyme de « forme totale » puisque les processus locaux gardent leur autonomie. Mais il faut reconnaître que le principe stratégique de la connexion de l'hétérogène, qui fait voler en éclats les unités géographiques et fonctionnelles de l'analyse traditionnelle du pouvoir, n'empêche pas Foucault de dessiner à ses risques et périls des «stratégies d'ensemble » dont la localisation du côté de l'État est affirmée.

Nous aurons l'occasion de retrouver ces problèmes quand sera évoquée la question de la gouvernementalité; mais la présentation générale du modèle stratégique nous semble désormais suffisante pour nous permettre de redevenir «chrétiens », avec Foucault. Il nous faut maintenant étudier comment la question du christianisme va croiser ces analyses stratégiques, pour y être successivement émiettée («Le christianisme éclaté ») et simplifiée («Le christianisme comme "gouvernementalité" $\gg)$, rendant ainsi nécessaire la formation d'un nouveau modèle d'analyse («Le christianisme comme régime de vérité »). 



\section{Le christianisme au risque de l'analyse stratégique}

Étudier le rapport de Foucault à un objet idéal et constitué à l'avance comme le christianisme, quel que soit le type de définition que l'on choisit, semble contredire le projet d'une historiographie qui veut en rester à des analyses locales, découpant dans l'archive des séries de discours ou de pratiques dont les croisements ne sont tout au plus que stratégiques. La discussion avec Weber nous a permis de vérifier qu'un tel objet idéal était refusé, même graduellement construit dans une visée strictement heuristique. Hasard ou clin d'œil de l'histoire, les géographes d'Hérodote citent précisément la « chrétienté » comme exemple par excellence chez Foucault de ces «spatialisations nébuleuses, nomades $\gg^{1}$ dont il use et, pour certains, abuse.

Entre 1974 et 1980, Foucault va pourtant se risquer à penser quelque chose comme le christianisme, à travers une série d'essais et d'erreurs que l'on peut regrouper autour de trois moments principaux : l'étude de dossiers historiques utilisant l'adjectif « chrétien » comme simple indicateur culturel (cours de 1974-1975 : Les anormaux) ; l'étude du christianisme comme «gouvernementalité » (cours de 1977-1978 : Sécurité, territoire, population); l'étude du christianisme comme «régime de vérité $\gg$ (cours de 1979-1980: Du gouvernement des vivants). 


\section{Le christianisme éclaté : 1973-1977}

Avant de nous concentrer sur la leçon du 19 février 1975 consacrée aux procédures de confession, il convient de faire une remarque lexicale. $\mathrm{Si}$ l'on cherche le terme «christianisme » dans les cours de 1973-1974 (Le pouvoir psychiatrique) et 1974-1975 (Les anormaux), on ne l'y trouvera pas, alors même que ces cours abordent des dossiers historiques appartenant à la sphère culturelle chrétienne. Le dernier cours cité n'utilise que le terme de $\ll$ christianisation $»^{2}$ pour décrire, au Xvi $I^{\mathrm{e}}$ siècle, le resserrement du pouvoir ecclésial sur la vie individuelle. Si l'on poursuit l'étude lexicale dans l'ensemble des dits et écrits de Foucault, les résultats sont encore plus nets : à l'exception des articles consacrés à la littérature au début des années 1960 qui feront l'objet d'une analyse ultérieure ${ }^{3}$, le « christianisme » n'existe pas avant 1977. Les seules occurrences significatives ${ }^{4}$ du terme avant cette date désignent uniquement les fausses explications historiques de ce que Foucault nomme dans $\mathrm{La}$ volonté de savoir «l'hypothèse répressive »: l'entité historique «christianisme » serait responsable de la répression et du silence qui entoureraient aujourd'hui notre rapport à la sexualité. La pudibonderie de notre société s'expliquerait ainsi par le «lourd héritage qu'elle aurait reçu du christianisme $\gg^{5}-$ argumentation qui renvoie à une histoire des mentalités ou des idéologies, toutes deux refusées par Foucault.

2 Les anormaux, p.164, 189 et 190. Le terme fait implicitement référence au livre de Jean Delumeau : Le catholicisme entre Luther et Voltaire, Paris, PUF, 1971. L'historien sera élu au Collège de France en 1975 à la chaire d'Histoire des mentalités religieuses dans l'Occident moderne. Suivant les règles de fonctionnement du Collège, Michel Foucault rencontrera Jean Delumeau à l'occasion de sa candidature. Les charges académiques empêcheront néanmoins une poursuite du dialogue entre les deux professeurs, travaillant pourtant sur des sujets étonnamment proches. Elles n'empêcheront pas, par contre, l'estime mutuelle (entretien avec Jean Delumeau, 12 janvier 2007).

3 Le terme «christianisme » se trouve précocement dans les articles sur Flaubert ( $D E$ $\mathrm{n}^{\circ}{ }_{20}$, « (Sans titre) $\gg$, I, p. 335), Klossowski $\left(D E \mathrm{n}^{\circ}{ }_{21}\right.$, « La prose d'Actéon », I, p. 354) et Blanchot $\left(D E \mathrm{n}^{\circ} 38\right.$, « La pensée du dehors $», \mathrm{I}$, p. 549). Voir Troisième partie, «Une expérience d'abord littéraire ».

4 L'adjectif «significatif » désigne pour nous un usage du terme « christianisme » qui ne soit pas une synecdoque ou une indication de contexte, mais fasse référence à l'unité historique d'un concept. N'est pas significative par exemple l'unique mention du terme dans La volonté de savoir, page 154 .

$5 D E \mathrm{n}^{\circ} 181$, «L'Occident et la vérité du sexe », II, p. 103. Dans ce même sens : «c'est le christianisme qui aurait imposé $[\ldots] »\left(D E n^{\circ} 233\right.$, «Sexualité et pouvoir », II, p. 559). 
En 1977 cependant, commence à apparaître un usage positif du terme « christianisme » comme lieu de naissance, en Occident, de la procédure d'aveu. Mais plus qu'une causalité remontant à une essence ou à une origine lointaine - le christianisme des premiers siècles -, il est possible de n'y voir que l'indication purement descriptive d'une ère culturelle, sans principe organisateur particulier, souvent assortie d'une datation qui en réduit immédiatement la portée ( «depuis le Moyen Âge $»^{6}$, «à partir de la Réforme ${ }^{7}$ ). Seul un entretien pour la revue lacanienne Ornicar?, publié en juillet 1977, se risque à remonter le temps et évoquer Tertullien, au tournant des $\mathrm{II}^{\mathrm{e}}$ et $\mathrm{III}^{\mathrm{e}}$ siècles de notre ère, mais sans prolonger ce qui reste de l'ordre d'une simple intuition dans une conversation quelque peu débridée . $^{\text {. }}$

Avant 1978, cette impossibilité de recevoir a priori ou de constituer en concept historique quelque chose comme le «christianisme », dans le cadre d'une analyse stratégique des rapports de pouvoir, peut être vérifiée sur un exemple précis. Si le «christianisme » n'existe pas pour Foucault dans les années où il rédige Surveiller et punir et La volonté de savoir, les références à l'histoire de l'Église en Occident y sont cependant fréquentes. Cette abondance répond d'abord à un simple constat historique : la prégnance des techniques chrétiennes de subjectivation dans la société occidentale, depuis que l'Église s'est constituée en pouvoir rapproché sur les corps et les âmes. L'accès privilégié des clercs à l'intime, en des temps où le pouvoir politique ne prétendait pas encore à une telle intrusion, rend ces détours nécessaires pour une histoire de la subjectivité. Les cours au Collège de France ouvrent ainsi des dossiers historiques limités dans le temps et l'espace, mais déterminants pour l'analyse. Citons les principaux : à titre de préliminaire à la compréhension du système judiciaire au XIX ${ }^{\mathrm{e}}$ siècle, Théorie et institutions pénales (1971-1972) visite le modèle inquisitorial; dans

$6 D E n^{\circ} 233, \ll$ Sexualité et pouvoir », II, p. 527.

$7 D E \mathrm{n}^{\circ}$ 216, « Pouvoir et savoir », II, p. 412. Explicitement ou implicitement, toutes ces occurrences renvoient en fait toujours à la procédure médiévale de l'aveu : «La prise de pouvoir sur l'ordinaire de la vie, le christianisme l'avait, pour une grande part, organisée autour de la confession $\gg\left(D E n^{\circ} 198\right.$, «La vie des hommes infâmes », II, p. 245); « depuis le christianisme, l'Occident n'a cessé de dire : "Pour savoir qui tu es, sache ce qu'il en est de ton sexe” $\gg\left(D E n^{\circ} 200\right.$, «Non au sexe roi », II, p. 257).

8 «L bonhomme fondamental, c'est Tertullien ». Mais Foucault minimise aussitôt son propos devant les objections de Jacques-Alain Miller : « C'est pour rigoler [...]. Je dis ça d'une façon fictive, pour rire, pour faire fable $\gg\left(D E \mathrm{n}^{\circ}{ }_{206}\right.$, « Le jeu de Michel Foucault », II, p. 313, 316). 
Le pouvoir psychiatrique (1973-1974), les communautés religieuses médiévales et leurs méthodes pédagogiques apparaissent à la naissance du pouvoir disciplinaire; les mutations de la confession du XII ${ }^{\mathrm{e}}$ au XVII ${ }^{\mathrm{e}}$ siècle sont l'objet d'une leçon entière dans Les anormaux (1974-1975). Mais en aucun cas ces références ne constituent une étude exhaustive de ce que serait le « christianisme » comme corps de doctrines homogène ou totalité historique faite d'institutions, de discours et de pratiques. Tout d'abord, parce que ces références restent périphériques par rapport aux thèmes généraux du cours (respectivement : constitution de la vérité judiciaire en 1971-1972, naissance de la psychiatrie en 1973-1974, définition de la norme et de l'individu dangereux en 1974-1975). Ensuite, parce qu'elles suivent un unique fil, relativement ténu, tiré sans toujours beaucoup de délicatesse du tissu historique chrétien : celui des mécanismes disciplinaires.

Reportons-nous par exemple à la leçon du 19 février 1975 consacrée à la confession chrétienne des péchés. Cette brève étude s'inscrit dans le projet plus large d'une généalogie de la notion d'《anormalité », notion qui a pris toute son extension quand la psychiatrie a brutalement investi le champ de la sexualité dans les années 1845-1850. Cet investissement a été rendu possible par une sexualité devenue, « en Occident ${ }^{9}$, objet de discours, par l'intermédiaire de la procédure de l'aveu contraint et obligatoire. Mais après avoir jeté un filet assez lâche au-dessus de l'histoire - l'aveu « en Occident »-, Foucault resserre aussitôt les mailles et insiste sur le fait :

1) que l'aveu n'appartenait pas au rituel de pénitence des premiers siècles chrétiens, où l'acte relevait d'abord d'une libre décision : seul celui qui considérait avoir gravement fauté allait lui-même trouver l'évêque ;

2) que la pénitence tarifée, qui apparaît au $\mathrm{VI}^{\mathrm{e}}$ siècle et demande de connaître avec précision la faute afin de pouvoir lui appliquer le juste tarif, est le résultat du croisement d'une pratique religieuse avec un modèle strictement laïque, judiciaire, non latin : la pénalité germanique ;

3) que cette tarification précise ne confère pas encore à l'aveu, comme acte du sujet, une valeur et une efficace propres. Ces derniers ne seront reconnus qu'au Moyen Âge (ibid., p. 157-158).

Ces remarques, qui reprennent certains résultats du cours Théories et institutions pénales (1971-1972), brisent les belles continuités dont le 
concept massif d'Occident chrétien aurait pu être le garant. La procédure de l'aveu, que la psychiatrie du XIX ${ }^{\mathrm{e}}$ siècle intègre dans son dispositif de savoir-pouvoir, n'est pas une vieille histoire. C'est dans un mouvement qui va du XIII ${ }^{e}$ siècle (concile du Latran IV) à la Renaissance qu'une certaine pratique pénitentielle, au départ limitée à certaines catégories de personnes et à certains événements, croisant elle-même des éléments extérieurs au christianisme latin, va se voir institutionnalisée par l'Église et étendue à tous les fidèles. Alors seulement l'aveu prendra son efficace singulière, comme verbalisation totalisante et exhaustive de soi, sous une forme qui pourrait être résumée par cette triple obligation : il faut avouer, il faut tout avouer, il faut tout avouer au prêtre.

Qu'est-ce alors que l'aveu pour Foucault? Non pas un vaste mouvement homogène de culpabilisation des consciences, mais une procédure discursive précise, à la mise en œuvre incertaine et aux controverses incessantes, étudiée uniquement à travers les manuels des confesseurs $\mathrm{du} \mathrm{XVII}^{\mathrm{e}}$ et du XVIII ${ }^{\mathrm{e}}$ siècle. Ces incertitudes de l'histoire sont importantes car elles ramènent avec elles les questions dont nous avons débattu autour du Panoptique. Dès 1975, Foucault prend acte de l'écart entre une procédure idéale, lisible comme telle dans l'archive, et une pratique réelle : le dispositif décrit dans les manuels est-il «représentatif $\gg^{10}$ des confessions populaires telles qu'elles se répandent après le concile du Latran? Certainement pas, répond le cours du 19 février 1975. Mais ce dispositif n'est pas non plus une « utopie », une « rêverie », un simple «édifice théorique ( (ibid., p. 178). La proximité lexicale avec le débat sur le Panoptique est ici frappante. Nous retrouvons en fait la même attention aux connexions historiques concrètes, visant toujours la plus grande proximité physique entre les éléments mis en relation. Si les manuels des confesseurs ne sont pas représentatifs des pratiques pastorales - qui demeurent populaires, massives, expéditives -, il faut les étudier du côté de leur diffusion et de leur réception; répondre donc aux questions : quels en ont été les lecteurs, par quelles institutions ont-ils transité ? Or, il se trouve que ces manuels ont d'abord et surtout été appliqués dans les séminaires; séminaires qui ont ensuite fourni le modèle principal des grands établissements d'enseignement secondaire (oratoriens, jésuites ${ }^{11}$ ), permettant ainsi une mise en œuvre 
et une diffusion des techniques de confession auprès de la majorité des élites, futurs éducateurs et gouvernants. Pas de vaste filet, donc, jeté par Foucault sur la conscience occidentale, ni de mentalité soudainement perçue par l'historien comme massivement «avouante ». Ne reste que le simple tracé de la diffusion d'une procédure, qui n'exclut ni le hasard ni les chemins de traverse.

En accordant une place majeure aux élites, Foucault pourrait rejoindre ici les études de Jean Delumeau sur la prolifération, du XIII ${ }^{\mathrm{e}}$ au XVIII ${ }^{\mathrm{e}}$ siècle, des discours du péché et de la peur jusque dans la littérature laïque ${ }^{12}$. La leçon mentionne d'ailleurs les similitudes entre les manuels religieux et les traités des passions de l'époque ${ }^{13}$. Ce n'est cependant pas cette littérature qui fait relais pour Foucault, mais seulement les institutions qu'administrent les élites : école, armée, ateliers. Pour Jean Delumeau au contraire, il y a une correspondance mentale entre l'élite et le peuple, entre celui qui prononce le discours et celui qui l'écoute. Dans son analyse, cette correspondance est assurée par la fluidité que permet l'étude d'un sentiment universel : la peur. L'inquiétude du poète Eustache Deschamps (1346-1406) vient ainsi coïncider avec les aspirations collectives de ceux qui vivent la Peste et la guerre de Cent Ans au quotidien $^{14}$. À rebours de cette histoire qui se réclame explicitement des mentalités, Foucault évoque au contraire une chaîne concrète et localisée de réception.

formation du clergé est décidée lors de la $5^{\mathrm{e}}$ session du concile de Trente, soit en 1546; mais la délimitation de leur rôle reste encore imprécise. La société française de l'Oratoire est fondée à Paris par Bérulle en 1611 et joue effectivement un grand rôle dans la direction couplée de séminaires et de collèges. Par contre, les premiers collèges jésuites, dans leur forme initiale, qui est celle de maisons de formation pour les seuls étudiants jésuites, remontent aux années 1539-1541. À partir de 1546, ces collèges vont commencer à accueillir des étudiants non jésuites, jusqu’à prendre leur autonomie complète par rapport à leur rôle premier. Quoi qu'il en soit de cette évolution, leur organisation s'inspire non pas des séminaires qui leur sont postérieurs, mais des collèges de l'université de Paris, qui reproduisaient eux-mêmes certains modèles pédagogiques des Frères de la vie commune (d'ailleurs mentionnés par Foucault dans Le pouvoir psychiatrique, p. 68-70). Sur cette question, voir J. O'Malley, Les premiers jésuites, 1540-1565, E. Boné trad., Paris, Desclée de Brouwer, 1999, p. 288-300 et 309-323.

12 J. Delumeau, La peur en Occident ( $x v^{e}-x v i n{ }^{e}$ siècles), Paris, Fayard, 1978 ; Le péché et la peur. La culpabilisation en Occident, $x_{11}{ }^{e}-x v u^{e}{ }^{e}$ siècles, Paris, Fayard, 1983.

13 Les anormaux, p. 179.

14 J. Delumeau, Le péché et la peur, déjà cité, p.129-138 («Âge de pleur, d'angoisse et de tourment $\gg$ ) et p. 626-627 (pour la conclusion d'ensemble). 
Dans le même sens, mais à un niveau maintenant diachronique, ce qui est récupéré par la médecine du XIX ${ }^{\mathrm{e}}$ siècle du côté de l'aveu est uniquement une technique, et non une certaine représentation du corps, encore moins une idéologie répressive de l'homme charnel et peccable. Du cours Le pouvoir psychiatrique en 1973-1974 à La volonté de savoir en 1976, Foucault n'a de cesse d'affirmer et d'accentuer les différences historiques entre les discours et les pratiques, malgré les ressemblances apparentes : l'âme moderne de la psychiatrie n'est pas l'âme définie par la théologie et la pastorale chrétiennes (même si elle leur emprunte l'instant décisif de l'aveu ${ }^{15}$, le pouvoir disciplinaire du capitalisme naissant n'est pas la discipline monastique (même s'il en reprend le dispositif cellulaire et l'attention au détail $)^{16}$, le discours médical sur la masturbation est tout autre chose que le discours chrétien sur la chair (même s'il est aussi scrutateur $)^{17}$. «Visible continuité [insiste La volonté de savoir], mais qui n'empêche pas une transformation capitale $\gg^{18}$. Pour la simple raison que les discours qui apparaissent respectivement aux XVIII ${ }^{\mathrm{e}}$ et XIX ${ }^{\mathrm{e}}$ siècles ont des rôles stratégiques différents dans des connexions de savoir-pouvoir inédites. Passer de la moralisation de la chair à la somatisation de la sexualité, c'est engager une autre technologie, c'est-à-dire une autre manière pour le pouvoir d'avoir prise sur les corps. De la chair chrétienne à la psychopathologie sexuelle se déploient ainsi de nouveaux objets (l'organisme et ses maladies et non le désir et ses voluptés), de nouvelles cibles (le sexe de l'enfant, le corps de la femme), de nouveaux relais du pouvoir (l'institution médicale, la famille), de nouvelles organisations de l'espace (l'univers domestique) et de nouveaux objectifs (le gouvernement d'une population). Les anciens discours ne communiquent donc pas immédiatement avec les nouveaux par le biais d'une « mentalité » ou d'une « idéologie » qui traverseraient les siècles, mais ils doivent d'abord repasser par les transformations concrètes qui déplacent non pas d'abord les idées dans la conscience des individus, mais les points d'ancrage et les cibles des stratégies. Lors de l'entretien de 1977 déjà cité, consécutif à la publication de La volonté de savoir, Foucault a soin de préciser :

15 Le pouvoir psychiatrique, p. 54 : c'est « une âme fort différente ».

16 Surveiller et punir, p. 162.

17 Les anormaux, p. 219 : c'est « quelque chose qui est tout à fait différent de ce qu'on pourrait appeler le discours chrétien de la chair ».

18 La volonté de savoir, p. 155 . 
Je ne cherche pas à construire, avec cette notion d'aveu, un cadre qui me permettrait de tout réduire au même, des confesseurs à Freud. Au contraire, comme dans Les Mots et les Choses, il s'agit de mieux faire apparaître les différences. ${ }^{19}$

Concluons alors que la recherche menée sur l'aveu, non seulement ne prétend pas confondre les confesseurs et les psychiatres, mais ne prétend pas remonter en deçà des premiers, c'est-à-dire en deçà du Moyen Âge. Dans La volonté de savoir, le christianisme médiéval marque clairement un point de butée, la limite historique de l'enquête ${ }^{20}$. Si l'aveu contraint et généralisé n'est pas le produit univoque d'un « esprit chrétien », il ne sert à rien en effet de visiter les confins de l'Occident pour en retrouver le noyau essentiel. Ce détour par les premiers siècles non seulement ne s'impose pas, mais ne semble pas avoir sa place dans le projet initial, annoncé dans le cours de 1974-1975, d'une « histoire de l'aveu de la sexualité $\gg^{21}$.

Au sein du premier programme d'Histoire de la sexualité - dont l'édition est annoncée en décembre 1976 avec la publication de La volonté de savoir - le deuxième volume intitulé La chair et le corps devait initialement porter sur les procédures discursives chrétiennes du $\mathrm{X}^{\mathrm{e}}$ au $\mathrm{XVIII}^{\mathrm{e}}$ siècle $^{22}$. Si la chronologie de Daniel Defert dans les Dits et écrits note que Foucault « écrit sur les Pères de l'Église ${ }^{23}$ en août 1977 à Vendeuvre, la teneur de ce deuxième volume ne semble pas pour autant remise en cause quand Foucault en rédige une première version en janvier 1978. Daniel Defert précise au sujet de cette version inédite, dont nous aurons l'occasion de commenter un fragment : « Il s'agit alors d'une généalogie de la concupiscence à travers la pratique de la confession dans le christianisme occidental et de la direction de conscience, telle qu'elle se développe à partir du concile de Trente $»^{24}$. Aux dires de la chronologie, ce n'est qu'en janvier 1979 qu'un nouveau projet voit le jour, incluant cette fois un détour par les premiers siècles chrétiens projet qui deviendra finalement le quatrième volume inédit de l'Histoire de la sexualité, intitulé Les aveux de la chair.

$D E \mathrm{n}^{\circ} 206, \ll$ Le jeu de Michel Foucault », II, p. 318 .

Voir la section 4 《périodisation » du chapitre IV (La volonté de savoir, p. 152-173).

Les anormaux, p. 158.

$D E \mathrm{n}^{\circ} 206, \ll$ Le jeu de Michel Foucault $\gg$, II, p. 319 .

DE I, p. 71.

DE I, p. 73 . 
Ce détour par les Pères ne répond pas à un goût de l'encyclopédisme. Revenir aux premiers siècles, c'est se donner enfin une chance de penser quelque chose comme le «christianisme » comme totalité historique. Cet horizon élargi est rendu possible par l'apparition, à partir de 1978, de nouveaux objets qui opèrent un changement d'échelle. Ce passage à un niveau supérieur d'analyse ne doit cependant pas être considéré comme une remise en cause des précédents travaux. Il recouvre en fait un désir déjà ancien chez Foucault, dont les premières traces se trouveraient dans un entretien de 1966. À cette époque, la méthode archéologique appliquée par Foucault aux discours scientifiques avait eu pour premier résultat d'éclater l'histoire en couches distinctes d'événements ayant chacune leur périodisation propre. Foucault s'était alors demandé si le rapport entre les couches successives était nécessairement de simple juxtaposition et si l'historien pouvait se contenter de décrire des séries autonomes d'événements. La réponse de Foucault était alors ouverte et prudente. Lisons-la attentivement :

[... ] dans ce domaine où l'on ne fait qu'aborder les principes possibles, il est encore bien tôt pour poser le problème des déterminations réciproques de ces couches. Il n'est pas du tout impossible qu'on puisse trouver des formes de déterminations telles que tous les niveaux s'accordent à marcher ensemble d'un pas de régiment sur le pont du devenir historique. ${ }^{25}$

Reconnaissons le caractère quelque peu ironique de la finale, prévenant sans doute l'illusion d'une nouvelle totalisation de l'histoire par un formalisme redoublé (une archéologie de l'archéologie). Mais cette réponse montre en même temps que l'éclatement n'a jamais été le dernier mot dont pouvait simplement se satisfaire l'historien. La première leçon du cours de 1976 Il faut défendre la société ne redoute pas à ce sujet l'autocritique et exprime ce même besoin de synthétiser les résultats acquis mais épars pour échapper au piétinement indéfini de l'analyse ${ }^{26}$.

$25 D E n^{\circ} 48$, « Sur les façons d'écrire l'histoire », I, p. 616.

26 « Tout cela piétine, ça n'avance pas; tout ça se répète et n'est pas lié. Au fond, cela ne cesse de dire la même chose et, pourtant, peut-être, cela ne dit rien; cela s'entrecroise dans un embrouillamini peu déchiffrable, qui ne s'organise guère; bref, comme on dit, ça n'aboutit pas » (Il faut défendre la société, p.5). 


\section{Le christianisme comme «gouvernementalité » : 1978}

Un nouveau regard sur les institutions politiques : Église et État

C'est en 1978 au Collège de France, à l'occasion du virage thématique opéré par la notion de «gouvernement», que Foucault effectue une première tentative pour rassembler sous le concept de christianisme une réalité historique à la fois large et relativement homogène. Avec beaucoup de réserves et de précautions, le terme apparaît dans la leçon du 15 février 1978 :

[... la véritable histoire du pastorat, comme foyer d'un type spécifique de pouvoir sur les hommes, l'histoire du pastorat comme modèle, comme matrice de procédures de gouvernement des hommes, cette histoire du pastorat dans le monde occidental ne commence guère qu'avec le christianisme. Et sans doute ce mot «christianisme »- là, je me réfère à ce qu'a dit souvent Paul Veyne -, le terme «christianisme » n'est pas exact, il recouvre en vérité toute une série de réalités différentes. ${ }^{27}$

Parmi ces réalités différentes, Foucault choisit d'en retenir une seule qui deviendra, dans les leçons suivantes de l'année 1978, la référence historique précise de ce qu'il entend par «christianisme » : la constitution de la religion chrétienne en Église, dans un mouvement qu'une première évaluation situe entre les $\mathrm{II}^{\mathrm{e}}-\mathrm{III}^{\mathrm{e}}$ siècles et le XVIII ${ }^{\mathrm{e}}$ siècle. L'aversion bien connue de Foucault pour les institutions, à partir du moment où leur définition juridico-politique est prise comme schéma explicatif du pouvoir, rend presque provocante une telle décision. Une institution est d'abord une organisation qui se donne une certaine fin, et dont le fonctionnement durable et régulier est explicitement codifié par des lois, des règlements ou une constitution. Or, cet édifice politique majeur dans l'histoire de l'Occident qu'a été l'Église n'est justement pas abordé en 1978 par le biais des définitions juridiques ou théologiques : le cours ne cite ni le droit canon ni les décisions conciliaires. De l'Église, Foucault fait d'abord une certaine technique de «gouvernement des hommes dans leur vie

Sécurité, territoire, population, p. 151. Pour la référence à Paul Veyne, l'édition du cours nous renvoie principalement à son article « La famille et l'amour sous le Haut-Empire romain », Annales. Économies, Sociétés, Civilisations, t. 33, vol. 1, 1978, p. 35-63. 
quotidienne $\gg^{28}$. Le terme de «gouvernement $\gg$ peut être ici défini comme l'art d'aménager, pour des individus, le champ de possibilités de leurs actions, afin que chaque individu participe de lui-même et pour luimême à un certain résultat collectif. Cet art, qui n'est pas essentiellement de contrainte ou de violence, suppose en effet que l'individu reste tout au long du processus sujet de sa conduite ${ }^{29}$. Étymologiquement, comme le rappelle Foucault, gouverner ( $\kappa v \beta \varepsilon \rho v \tilde{a} v$ en grec, gubernare en latin) signifie d'abord diriger un bateau ou un char, tenir un gouvernail ${ }^{30}$. Dans le christianisme, il s'agira ainsi, par un ensemble de techniques d'aveu, d'examen et de direction de conscience, de mener l'ensemble du peuple en général, et chaque individu en particulier, à faire son salut.

D'un point de vue méthodologique, la notion de «gouvernement » permet ainsi de décrire ce que Foucault avait nommé en 1975 et 1976 une «stratégie d'ensemble», en redonnant une place centrale aux institutions qui ont pour objectif la gestion d'une communauté tout entière, sans pour autant réduire leur fonctionnement à l'application d'un modèle juridique. D'un point de vue historique, cette notion permet de jeter un pont entre le modèle du pastorat chrétien et l'État moderne. Mais avant de parcourir cette histoire ecclésiale, il convient de préciser les mutations que vont connaître, dans le cours Sécurité, territoire, population, les concepts massifs d'《État $\gg$, de $\ll$ loi $»$, de « droit $\gg$.

Étudier le fonctionnement d'une institution sous l'angle singulier de la « gouvernementalité », c'est considérer qu'elle n'a pas d'abord prise sur des sujets dont un système juridique codifie les droits et les devoirs fondamentaux, mais sur des hommes concrets en relation avec un environnement. Cet environnement étant appelé à se modifier sans cesse, l'État doit être étudié du côté de sa capacité à se déplacer et à s'adapter aux circonstances, et non là où son pouvoir s'exerce dans l'unité infrangible de la loi, du pouvoir, du sujet. Le cours de 1976 Il faut défendre la société avait fortement mis en question les théories de l'État regroupées sous le

Sécurité, territoire, population, p. 151.

Telle est la définition de la « relation de pouvoir $\gg$ chez Foucault, distinguée de la « relation de violence $\gg: D E n^{\circ} 306$, « Le sujet et le pouvoir $\gg$, II, p. 1055-1056.

Comme le mentionne Foucault dans le cours de 1978, la métaphore du navire est un lieu commun des traités sur le gouvernement du XVI ${ }^{\mathrm{e}}$ au XVIII ${ }^{\mathrm{e}}$ siècle (Sécurité, territoire, population, p. 100). Elle vient de l'Antiquité grecque : elle est dans Edipe roi de Sophocle, chez Platon, Aristote, Cicéron (ibid., p. 117, note 25). 
dénominateur commun de «théorie de la souveraineté $\gg^{31}$. Chez les penseurs politiques comme Hobbes et Rousseau, il s'agit en effet de constituer le droit de la puissance publique à partir des droits sacrés ou naturels des sujets. L'individu est originellement détenteur de droits - ontologiquement premiers par rapport à tout système juridique - qu'il va céder, déléguer, aliéner, pour fonder l'autorité légitime qui, en retour, veillera sur lui. C'est un « cycle du sujet au sujet» (ibid., p. 37), du sujet naturel au sujet politique. Que l'État puisse être étudié sous un autre angle que celui du pouvoir exercé sur des sujets politiques, l'analyse des disciplines en avait déjà apporté la preuve : certains passages de Surveiller et punir décrivaient par exemple - non sans une ambiguité déjà relevée - la lente montée des micro-pouvoirs vers le macro-pouvoir. Ainsi de l'« étatisation des mécanismes de discipline » (p.248) ou de la « généralisation des disciplines qui atteint aux dimensions de l'État » (p.250). Ce mouvement n'était pas un simple recouvrement des techniques de normalisation par la loi, mais un choc de stratégies différentes, rejoignant et débordant à la fois l'exercice de la souveraineté. Pour cette raison, ce processus semblait relégué par Foucault du côté sombre d'un État aux ramifications souterraines et proliférantes, dans un exercice à la fois permanent et insaisissable, dont le philosophe posait la spécificité sans se donner vraiment les moyens de l'analyser. Nous avions relevé à ce sujet la difficulté de définir, hors du modèle juridique, une telle « stratégie d'ensemble ».

La notion de «gouvernementalité » résout la difficulté en réintégrant les tactiques locales et contingentes dans l'exercice régulier de l'État. Prenons l'exemple de la police, développé par Foucault dans les leçons du 29 mars et du 5 avril 1978. Celle-ci naît en France non pas dans une théorie ou dans une loi, mais d'abord dans une pratique administrative. Surveiller et punir avait une première fois étudié sa mise en place centralisée au XVIII ${ }^{\mathrm{e}}$ siècle, mais avec un point de vue différent : le livre insistait sur le constant débordement de l'appareil judiciaire par une police aux pouvoirs indéfinis (p. 248-249). Pour prendre ses distances avec le modèle du droit, Foucault se devait de distinguer le mouvement d'étatisation de la discipline d'un simple encadrement des disciplines par la loi. Sécurité, territoire, population corrige ce caractère extralégal et montre au contraire comment la notion de gouvernementalité peut 
faire la suture entre la gestion du corps social et le domaine juridique. La police a bien la loi pour instrument, mais une loi prise dans son fonctionnement mobile, méticuleux et sans cesse ajustable : tel est bien le domaine du règlement, de l'ordonnance, de la consigne ${ }^{32}$.

Précisons ces termes «loi » et « règlement » pour éviter les oppositions trop simples. Au sein du dispositif juridique français issu de la Révolution, il convient en effet de distinguer :

1) les principes supraconstitutionnels ou droits naturels. Énoncés par la déclaration du 26 août 1789 , ils fixent les « droits naturels inaliénables et sacrés de l'homme »;

2) la constitution, postérieure de fait [1791] et de droit aux principes énoncés en 1 ), soumise à ratification par le peuple ou son équivalent. Celle-ci a pour rôle premier de définir le rôle de la puissance publique : à la fois l'étendue de son pouvoir et sa limite ;

3) les lois, soumises au parlement, contrôlables par le niveau 2);

4) les règlements, édictés par le gouvernement.

Quand Foucault parle de «loi $»$ et de «droit $\gg$ comme modèle repoussoir de l'analyse du pouvoir, il englobe les trois premiers niveaux pour les penser comme un tout ${ }^{33}$. Ce qui explique qu'il dédaigne jusqu'en 1979 toute autre approche du droit, comme celle qui contournerait son fondement idéal pour l'ancrer d'abord dans une réalité historique concrète faite de luttes politiques et économiques non-violentes ${ }^{34}$. Foucault en reste à un droit pensé comme marquage constitutionnel de l'étendue de la puissance publique, appuyé en amont sur les droits inaliénables de sujets contractants (niveau 1), et décliné en aval jusqu'aux

Sécurité, territoire, population, p. 348. Sur la «plasticité » de l'action policière, orientée vers la constitution d'un droit non pas inexistant mais «souple», signalons l'étude importante menée dans le sillage de Foucault sur la police française : P. Napoli, Naissance de la police moderne. Pouvoir, normes, société, Paris, La Découverte, 2003.

À une seule reprise, dans le cours du 14 janvier 1976, Foucault emploie le terme «droit » en un sens élargi à sa mise en œuvre concrète, jusque dans le moindre règlement institutionnel. Ce sens élargi déborde alors clairement les rapports de souveraineté : « quand je dis le droit, je ne pense pas simplement à la loi, mais à l'ensemble des appareils, institutions, règlements, qui appliquent le droit » (Il faut défendre la société, p. 24).

À l'exception de la pensée de Sieyès, que croise Foucault en 1976. Selon Foucault, Sieyès retrouve sous les conditions juridico-formelles de la nation des conditions historicofonctionnelles : une loi requise non pour garantir des droits idéaux, mais pour permettre des travaux (agriculture, artisanat, industrie) et assurer des fonctions préexistantes (armée, justice, Église, administration). Il faut défendre la société, p. 195-200. 
différents codes juridiques (niveau 3). Ce sont d'ailleurs bien les codes napoléoniens qui sont mentionnés dans le cours du 14 janvier 1976 comme produits directs de la théorie de la souveraineté.

Le quatrième niveau - celui des règlements - reste cependant disponible pour l'exercice d'une rationalité gouvernementale qui n'échappe pas pour autant complètement à l'État de droit, sans s'y réduire simplement. Cette rationalité n'est pas la face sombre et insaisissable de l'État, mais seulement son adaptateur. Si l'on voulait risquer un parallèle avec les analyses déjà évoquées de la microstoria, on pourrait dire qu'un rôle similaire à celui de la police et des règlements chez Foucault est joué chez Giovanni Levi par les notables, comme médiation entre la mobilité des conflits locaux et la tendance centralisatrice de l'État moderne ${ }^{35}$.

C'est dans le manuel de Guillaume de La Perrière Le miroir politique, contenant diverses manières de gouverner (1555), commandé à l'auteur par la ville de Toulouse ${ }^{36}$, que Foucault trouve la trace de cette manière nouvelle de considérer le rôle du monarque et de l'État : il ne s'agit pas seulement de définir ce que peuvent faire ou ne pas faire les hommes à l'intérieur de l'État, mais de gérer le rapport qu'ils entretiennent à leurs richesses, à leur espace vital, à leur milieu, et jusqu'à leurs rapports entre eux. Cette intervention - qui est de régulation plus que de coercition doit obéir à une fin particulière qui n'est pas d'abord l'obéissance à la loi et la soumission au souverain, mais une optimisation des ressources naturelles et humaines ${ }^{37}$. Elle requiert pour cela une connaissance et une surveillance des phénomènes concernés, que le développement aux XVI ${ }^{\mathrm{e}}$ et $\mathrm{XVII}^{\mathrm{e}}$ siècles de l'appareil administratif et de la science statistique a traduites à sa manière : est ainsi née la science nouvelle de l'« économie politique $\gg$ sur laquelle, dans un temps seulement second, va se greffer une théorie de la souveraineté et du contrat social. L'État ne doit donc pas être considéré comme une unité définie a priori, qui fonctionnerait rigoureusement à partir de son fondement théorique, mais au contraire

G. Levi, Le pouvoir au village. Histoire d'un exorciste dans le Piémont du xvıe siècle, M. Aymard trad., précédé de L'histoire au ras du sol par Jacques Revel, Paris, Gallimard, 1989, p. 11-12, note 1.

36 Quand Foucault prononce son cours en 1978, on ne sait pas grand-chose de ce roturier de formation juridique, historiographe de la ville de Toulouse. Il a fait depuis l'objet d'une thèse de doctorat: G. Cazals, Guillaume de La Perrière (1499-1554). Un humaniste à l'étude du politique, thèse de doctorat d'histoire du droit, université des sciences sociales, Toulouse-I, 2003. Sécurité, territoire, population, p. 100. 
comme ce qui n'existe qu'à partir d'une technique générale de gouvernement. Cette technique nécessite, pour parvenir à ses fins, de redéfinir et de déplacer sans cesse les limites effectives de l'État.

Des anciens mécanismes disciplinaires à la nouvelle gouvernementalité, un changement important d'échelle est opéré, mais la cartographie initiale reste la même. Le premier domaine était analysé en termes de micro-pouvoirs localisés autour d'institutions bien circonscrites et aux objectifs limités (hôpitaux, prisons) ; le second domaine cherche à définir la rationalité générale à l'œuvre dans les interventions de l'État, la manière dont il calcule sa puissance et augmente son efficacité. Cette notion de gouvernementalité n'est pas pour autant réservée aux superstructures et permet au contraire de faire la suture entre les différents niveaux d'analyse. Comme le précise Foucault dans le cours Naissance de la biopolitique (1978-1979), le gouvernement localisé des fous ou des malades peut être étudié avec les mêmes points de vue que le gouvernement d'une population ${ }^{38}$. Alors que $L a$ volonté de savoir posait, à travers le principe du «double conditionnement », une hétérogénéité sans discontinuité entre le local et le général, l'historien se donne désormais la possibilité d'aller d'un niveau à l'autre, considérant l'exercice du pouvoir étatique comme une simple $\ll$ manière de faire $\gg^{39}$, toujours à la recherche de sa juste puissance par la prise en compte d'une certaine naturalité des phénomènes à réguler. Notons que La Perrière refusait déjà à sa manière le principe d'hétérogénéité posé par $L a$ volonté de savoir : alors que, dans sa méthodologie, le livre de 1976 précisait que le père de famille ne représentait pas l'État, et que le second n'était pas la projection du premier ${ }^{40}$, La Perrière remarquait en 1555 que gouverner se disait aussi bien de l'État que de la maison, et que le deuxième type de gouvernement pouvait par conséquent être dit immanent au premier ${ }^{41}$. Si l'économie politique a eu par la suite toujours plus tendance à se détacher de l'économie domestique, ce n'est qu'en déployant dans une scientificité qui lui est propre, de manière plus affinée et plus constante, les réquisits de La Perrière : une possibilité d'ajuster des choses à la fin qui leur convient. 
Sécurité, territoire, population propose ainsi une interface entre la société et l'organisation politique : «Ce qu'il y a d'important pour notre modernité, c'est-à-dire pour notre actualité, ce n'est donc pas l'étatisation de la société, c'est ce que j'appellerais plutôt la "gouvernementalisation" de l'État » (ibid., p.112). Dans le même geste méthodologique, il va s'agir pour Foucault de «gouvernementaliser » l'Église, d'en décrire la technologie générale de pouvoir dans sa forme à la fois souple (ce qui, dans les pratiques, peut être négocié ou déplacé) et relationnelle (ce qui engage une réponse autonome de la part des sujets). C'est donc une technologie irréductible aux déclarations conciliaires ou au droit canon. Bien que la confession obligatoire n'ait été instituée qu'au concile du Latran IV en 1215, l'analyse doit remonter aux premiers siècles chrétiens pour retrouver, dans la manière dont un pouvoir s'est pensé comme rapport du pasteur à son troupeau, les prémices d'une rationalité. Si Foucault ne remonte pas en deçà du $\mathrm{II}^{\mathrm{e}}$ ou du $\mathrm{III}^{\mathrm{e}}$ siècle, ni ne déborde une certaine ère culturelle, ce n'est nullement pour préserver quelque chose comme «l'essence du christianisme $\gg$, mais pour respecter ce point de vue technologique appliqué au pouvoir : la technologie chrétienne du pastorat se distingue radicalement de la manière dont le monde hébraïque, égyptien et assyrien d'un côté, et le monde grec de l'autre, ont défini la tâche de gouverner. Reprenons les étapes de la démonstration proposée par Foucault.

L'analyse part des trois opérateurs que tout gouvernement met en œuvre dans son exercice : le salut, la loi, la vérité ${ }^{42}$. Du Dieu berger et législateur des Hébreux à la Cité grecque ou au magistrat chez Platon, toutes les formes de gouvernement œuvrent pour un but recherché (soigner, éduquer, guider), édictent une loi générale pour y parvenir et définissent des discours de vérité pour évaluer les étapes et les échecs. L'originalité de ce que Foucault nomme alors le «pastorat chrétien » va être d'exercer le pouvoir en instaurant sous les définitions exhaustives et générales du salut, de la loi et de la vérité, des rapports inédits qui multiplient les points de contrôle et de surveillance, tout en y ménageant des espaces de liberté pour les sujets. Il nous faut même aller plus loin : c'est parce que les sujets ont tout au long du processus des marges d'initiative et d'incertitude que le pouvoir peut pénétrer le détail, le quotidien, l'infiniment petit de leur vie.

Reprenons de manière transversale l'exposé du 22 février 1978, en 
tentant de synthétiser non pas la finalité de ce pouvoir (obéissance pure, destruction de l'ego) mais son dispositif technique. Il s'agit ce faisant de dégager les points nodaux de cette manière de faire chrétienne :

1) le pouvoir pastoral décompose l'objet sur lequel il s'exerce : la responsabilité du pasteur par rapport à ses brebis n'est pas seulement numérique (chacun) mais analytique et qualitative (chaque acte). Le champ de contrôle et de surveillance est donc par définition indéfini;

2) les outils de contrôle (direction de conscience, examen) ne s'appliquent pas à une matière inerte : ils ont des effets à la fois d'individualisation (l'âme est isolée comme une entité qui a sa vie secrète) et de subjectivation (production d'un discours de l'âme sur elle-même);

3) l'agent du pouvoir est personnellement engagé dans la relation, et la qualité de son engagement détermine en partie le résultat : les imperfections du pasteur, sa propre obéissance, son sacrifice pour le troupeau interviennent dans l'économie finale des mérites et démérites. Il y a correspondance et circularité entre le salut du berger et celui de son troupeau;

4) dans l'exercice de son pouvoir, l'agent ne connaît pas de résultat univoque, ni pour lui ni pour son troupeau ; il calcule et corrige simplement, « sans certitude terminale ${ }^{43}$, une trajectoire.

Dans cette économie subtile, sont déjà engagés les deux sens du mot «sujet», tels que Foucault les définira de manière insécable dans un article de 1982: « sujet soumis à l'autre par le contrôle et la dépendance, et sujet attaché à sa propre identité par la conscience ou la connaissance de soi $\gg 44$. Dans l'édition du cours de 1977-1978 un extrait du manuscrit préparatoire indique déjà en note la polysémie du terme : « $[\ldots]$ l'individualisation de l'homme occidental pendant le long millénaire du pastorat chrétien s'est opérée au prix de la subjectivité [... . Il faut devenir sujet pour devenir individu (tous les sens du mot "sujet") $\gg 45$. À cette polysémie répond d'ailleurs immédiatement celle induite par la notion de « conduite » : terme volontairement ambigu en ce qu'il désigne à la fois l'acte de conduire quelqu' un et la manière de se conduire (ibid., p. 196-197).

Ibid., p. 176.

$D E \mathrm{n}^{\circ} 306$, « Le sujet et le pouvoir $\gg$, II, p. 1046.

Sécurité, territoire, population, p. 236, note *, nous soulignons. Notons dans ce passage que le pastorat dure seulement un «long millénaire» pour Foucault, alors qu'il a été thématisé dès les premiers siècles par les Pères de l'Église. La durée de la mise en œuvre bien concrète d'une technologie prime ici sur toute autre considération de l'ordre d'une histoire des idées. 
Malheureusement, le résultat obtenu au terme du cours de 1977-1978 n'est pas à la hauteur des espérances, comme si le nouvel objet finement élaboré avait été trop vite appliqué sur une matière historique nouvelle. Il semble que Foucault n'ait justement pas encore donné à la forme réfléchie du verbe « conduire » toute sa portée. Pour cette raison, la description de la gouvernementalité pastorale finit par épouser de manière répétitive un schéma binaire qui oppose sans cesse, jusqu'à l'épuisement du commentaire, l'autonomie à l'hétéronomie. À partir de deux exemples précis utilisés par Foucault, il nous faut évaluer la pertinence au plan historique de cette opposition monotone. Mais auparavant, une hypothèse peut être formulée pour expliquer une telle lecture univoque, qui taille brutalement dans le vif de l'histoire.

\section{Pastorat chrétien et marxisme : un miroir déformant?}

Il semblerait que Foucault ait en fait à l'esprit un autre phénomène historique qui informe ou déforme son approche du pastorat chrétien. Il ne faut pas croire en effet que ce détour pastoral ne relève que de la curiosité érudite, Foucault ayant toujours eu soin de rappeler qu'il interrogeait le passé à partir du présent. Or, en cette année 1978, ses réflexions sur l'Église ne sont pas séparables d'une réflexion, plus sourde, périphérique, sur le marxisme. Il y a en fait une troublante coïncidence qui se fait jour à l'occasion d'un entretien cette même année avec le philosophe japonais Takaaki Yoshimoto. Foucault y décrit d'abord le marxisme de manière un peu lâche comme une «somme de mécanismes et de dynamiques de pouvoir ${ }^{46}$. Prenant alors le risque de sortir de la seule description, il fait de cet ensemble l'articulation précise entre une «science », une « prophétie » et un «parti » (ibid., p. 600-601). Il définit ainsi à sa manière un concept historique, soigneusement dialectisé. En dépit de leur autonomie apparente, les trois domaines forment en effet une unité indissoluble de pouvoir : la distinction méthodologique entre 1) le marxisme comme somme de propositions prises pour la vérité (science), 2) le marxisme comme discours sur l'avenir de l'humanité (prophétie), et 3) le marxisme comme mouvement incarné dans une organisation politique (parti), ne doit pas recouvrir le fait que ces trois 
aspects sont liés intrinsèquement à l'ensemble des rapports de pouvoir que constitue le marxisme. La science marxiste ne rencontre pas le parti communiste de l'extérieur, mais elle est en elle-même « une dynamique aux effets coercitifs, à propos d'une certaine vérité $\gg$ (p.600).

Or, cette tripartition proposée pour étudier le marxisme est chronologiquement encadrée par deux exposés similaires concernant le christianisme. Comme nous avons déjà eu l'occasion de le relever, trois mois avant le voyage en Asie, dans son cours au Collège de France, Foucault définit effectivement la forme générale du pastorat chrétien comme une manière inédite de se rapporter au «salut $\gg$, à la $\ll$ loi $\gg$ et à la « vérité » 47 . Au surlendemain de son retour du Japon, à l'occasion d'une séance de la Société française de philosophie, Foucault propose une nouvelle tripartition qui concerne uniquement cette fois le rapport à la vérité dans le pouvoir pastoral. Dans le christianisme, ce rapport est bien triple : vérité comme dogme, vérité comme connaissance particulière des individus, vérité comme ensemble de techniques pour produire cette connaissance ${ }^{48}$.

La proximité des trois gestes se lit dans les dates : la leçon est donnée le 22 février 1978 ; l'entretien avec Yoshimoto a lieu le 25 avril 1978; le 27 mai 1978, Foucault prononce sa communication devant la Société française de philosophie. L'homologie est encore plus frappante si l'on note que Foucault insiste sur le caractère unique dans l'histoire occidentale d'un parti qui se pense nécessairement comme État (le parti communiste), de la même manière que le cours de cette année-là avait souligné le caractère unique dans l'histoire des civilisations d'une religion qui se pense comme Église ${ }^{49}$. Il faut enfin noter le curieux rapprochement dans la leçon du ${ }_{1}{ }^{\text {er }}$ mars 1978 entre les dissidents du bloc soviétique et la « révolte des conduites $\gg$ contre le pouvoir ecclésial aux XIII ${ }^{\mathrm{e}}$ et XVI ${ }^{\mathrm{e}}$ siècles $^{50}$.

Derrière ce rapprochement, il est possible de voir se profiler l'œuvre de Leszek Kołakowski - et non pas celle de Norman Cohn qui inspira

Sécurité, territoire, population, p. 170.

48 «Qu'est-ce que la critique?», p. 37 .

49 Pour le lien intrinsèque entre parti communiste et État: $D E \mathrm{n}^{\circ} 235$, « Méthodologie pour la connaissance du monde : comment se débarrasser du marxisme $\gg$, II, p. 600-601; pour le caractère unique d'une religion qui se pense comme Église : Sécurité, territoire, population, p. 151. Sécurité, territoire, population, p. 204. 
pourtant cette même leçon de $1978^{51}$. Alors qu'il est déjà en rupture avec le parti communiste de son pays, le philosophe et historien polonais publie à Varsovie en 1965 un ouvrage colossal sur le christianisme anticonfessionnel des $\mathrm{XVI}^{\mathrm{e}}$ et $\mathrm{XVII}{ }^{\mathrm{e}}$ siècles, opposé au centralisme romain puis à toute forme d'organisation sociale. Sa traduction française, Chrétiens sans Église. La conscience religieuse et le lien confessionnel au xvin siècle, paraît chez Gallimard en 1969 et marque profondément de jeunes historiens comme Michel de Certeau ${ }^{52}$. Si aucun fait biographique ni bibliographique (Foucault ne cite jamais le nom de Kołakowski) ne vient étayer le rapprochement des deux penseurs, les indices de proximité intellectuelle sont nombreux. Chrétiens sans Église prononce en effet un certain nombre d'adieux, qui auraient pu tout aussi bien s'entendre dans la bouche de Foucault.

Contre toute histoire globale, Kołakowski distingue d'abord les niveaux d'intelligibilité historique sans présumer d'une synthèse possible $^{53}$. Contre une certaine historiographie marxiste, il pose ensuite l'ir-

C'est bien à l'historien britannique Norman Cohn, et non à Kołakowski - qui n'emploie pas le terme -, que Foucault emprunte l'application du terme « dissident » à ces révoltés religieux du Moyen Âge. Dans sa grande étude de 1957, Norman Cohn parle en effet à leur égard de religious dissidents : N. Cohn, The Pursuit of the Millennium. Revolutionary Millenarians and Mystical Anarchists of the Middle Ages, chap. II : « The tradition of religious

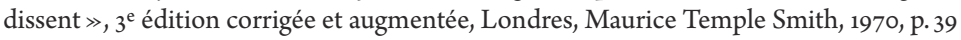
et suiv.; traduction française : Les fanatiques de l'Apocalypse. Courants millénaristes révolutionnaires $d u x l^{e}$ au $x v l^{e}$ siècle. Avec une postface sur le $x x^{e}$ siècle, S. Clémendot, M. Fuchs et P. Rosenberg trad., Paris, Julliard, 1962. Dans son cours de 1977-1978, Foucault discute l'usage du terme « dissidence » pour décrire les révoltes religieuses médiévales et renonce finalement à l'employer (Sécurité, territoire, population, p. 204-205). Si la référence à Cohn est donc bien attestée, le rapprochement avec les dissidents de l'Est ne peut cependant pas venir de l'historien britannique ; d'abord pour des raisons chronologiques évidentes (Cohn écrit avant que l'on ne parle de dissidents pour le bloc de l'Est), ensuite parce que la thèse de The Pursuit of the Millenium est exactement inverse : pour Cohn, les mouvements de révoltes ascétiques et mystiques du Moyen Âge annoncent non pas les dissidences politiques du $\mathrm{xx}^{\mathrm{e}}$ siècle, mais au contraire les mouvements totalitaires que furent le nazisme et le communisme. Lisant Cohn, Foucault a cependant pu y percevoir l'importance de ces mouvements pour comprendre les révolutions du $\mathrm{XX}^{\mathrm{e}}$ siècle. Ernst Bloch n'avait pas dit autre chose en s'intéressant, à la suite de Friedrich Engels, à la figure de l'opposant à Luther, Thomas Münzer : E. Bloch, Thomas Münzer, théologien de la révolution, M. de Gandillac trad., Paris, Julliard, 1964.

52 Michel de Certeau en fit un compte rendu cependant nuancé, voire critique : « La mort de l'histoire globale : L. Kołakowski », Politique aujourd'hui, février 1970, repris dans M. de Certeau, L'absent de l'histoire, Paris, Mame, 1973, p. 109-114.

Étudiant la figure pittoresque de Labadie, Kołakowski distingue trois unités produites par 
réductibilité des phénomènes religieux aux luttes sociales et politiques. Sur ce point précis, il s'oppose à l'historien français Lucien Goldmann - adversaire par ailleurs de Foucault - qui expliquait le jansénisme par la situation de la noblesse de robe sous Louis XIV ${ }^{54}$. Considérant l'expérience et le discours religieux comme un « fait social ${ }^{55}$, devant à ce titre être étudié dans la stricte perspective d'une histoire matérialiste, Kołakowski cherche en même temps à en dégager la structure interne originale. Cette recherche qui se réclame de l'analyse structurale de Roland Barthes doit en fait autant à la sociologie de Max Weber et à la phénoménologie, tout en conservant le vocabulaire marxiste omniprésent de l'idéologie ${ }^{56}$. On ne peut que souligner tout au long du livre le foisonnement déroutant et parfois contradictoire des outils conceptuels et méthodologiques utilisés.

Les résonances personnelles du livre sont évidentes et seront accentuées lors de sa réception française : la traduction paraît en 1969, peu de temps après la révocation de Kołakowski de l'université de Varsovie où il était titulaire de la chaire d'Histoire de la philosophie. À la suite d'un discours officiel volontairement provocateur, l'historien fut accusé d'avoir une lecture révisionniste de Marx. Suite à cette révocation, le philosophe polonais voit son œuvre interdite en Pologne et séjourne quelque temps à Paris. Devenu enseignant aux États-Unis puis à Oxford, il n'oublie pas pour autant son pays d'origine et, comme Foucault, s'engagera dans les années 1980 en faveur du mouvement Solidarność.

De la conscience religieuse en butte avec l'Église à la conscience politique en butte avec le Parti, il n'y a qu'un pas, que firent aisément les lecteurs polonais de l'œuvre en 1965, comme les lecteurs français

le travail historique : unité de la personnalité, unité du phénomène historique, unité de la structure théologique. Or, insiste Kołakowski, ces unités ne sont pas synthétisables; elles pourraient même décrire trois réalités complètement différentes : L. Kołakowski, Chrétiens sans Église. La conscience religieuse et le lien confessionnel au xvit siècle, A. Posner trad., Paris, Gallimard, 1969, p. 782-783.

54 L. Goldmann, Le Dieu caché. Étude sur la vision tragique dans les Pensées de Pascal et dans le théatre de Racine, Paris, Gallimard, 1955. Pour la critique du livre de Goldmann par Kołakowski : L. Kołakowski, Chrétiens sans Église, déjà cité, p. 352.

55 L. Kołakowski, Chrétiens sans Église, déjà cité, p. 31.

56 Si le nom de Max Weber n'apparaît qu'une fois, on notera la mention du « modèleidéal $\gg$ (ibid., p. 23). Roland Barthes a droit quant à lui à une longue citation (p. 51-52). Toujours marqué par l'historiographie marxiste, Kołakowski note en même temps que l'orthodoxie religieuse est «l'idéologie » de la « caste des prêtres » (p. 70). 
en $1969^{57}$. L'étude du christianisme anti-confessionnel des siècles passés rejoint la propre dissidence politique de l'historien polonais, sans l'empêcher pour autant de se livrer à une peinture étonnement sombre, voire féroce, de ces illuminés, schismatiques, irréguliers - « outsiders » comme il aime les nommer - de l'après-Réforme. La férocité a sans doute sa part de désillusion, sinon d'amertume, du côté d'un penseur qui constate non seulement la répression de ces mouvements par l'autorité légitime, mais surtout leur commune et inéluctable autodestruction. Par la logique même propre à leurs idées, ces mouvements étaient en effet voués à l'échec : la protestation religieuse anti-confessionnelle se nie elle-même en refusant de s'inscrire dans un corps social.

Dans son introduction, Kołakowski souligne que cette révolte du subjectivisme religieux est une conséquence conjointe de l'ex opere operato catholique et de la justification par la foi protestante, ce qui explique qu'elle ait atteint les rangs catholiques comme les rangs luthériens et calvinistes, n'épargnant aucune communauté instituée. Dans un cas, l'insistance sur le culte formel, dans l'autre, l'accent mis sur le Credo, ont fait d'une simple donnée extérieure, reconnaissable par une communauté, un critère suffisant d'authenticité. En réaction à cette suffisance, qui accorde une place excessive au sacrement ou à la profession de foi, se sont alors développés des projets de religion mettant l'accent sur l'individu dans son développement moral (Dirk R. Camphuysen) ou spirituel (Jean-Joseph Surin, Madame Guyon). La mystique est la pointe extrême de cette figure impossible du subjectivisme religieux, à l'image d'un Jean de la Croix aux prises avec la difficulté de tenir ensemble l'intériorisation de la vie religieuse et le maintien du culte extérieur ${ }^{58}$. Le divorce entre la mystique et l'institution, entre l'intérieur et l'extérieur, atteint son paroxysme dans les écrits de Miguel de Molinos et de Madame Guyon, dont Kołakowski dresse des portraits peu charitables. La négation théocentrique de la nature, la résorption des distinctions du monde fini dans

Lors de sa publication en Pologne, les étudiants de l'université de Varsovie lisaient et discutaient le livre en ce sens (entretien avec Piotr Witt, le 8 mars 2007). Journaliste polonais, Piotr Witt suivait à cette époque les cours de Kołakowski à Varsovie. Il vit aujourd'hui à Paris. Du côté francophone, ce rapprochement entre la dissidence religieuse et la dissidence politique fut systématiquement fait par les commentateurs du livre dans les années 1969-1970 : voir les comptes-rendus publiés dans la Revue philosophique de Louvain, les Archives des Sciences sociales des Religions et Politique aujourd'hui. 
l'infini, le primat de la grâce sur la nature et de l'expérience sur la raison, l'extinction de la volonté propre et la passivité parfaite : tout ceci dessine « le caractère organique du conflit entre la conscience mystique et l'Église $\gg$ (ibid., p. 482).

Si Foucault n'emploie pas le vocabulaire de la conscience, une telle affirmation se trouve cependant très proche de sa propre lecture de la mystique dans la leçon du $1^{\mathrm{er}}$ mars 1978. Plus généralement, de Kołakowski à Foucault, même si les périodes et les exemples privilégiés sont différents - et dénotent également chez Foucault la fréquentation des travaux de Pierre Chaunu ${ }^{59}$ - les structures de résistance au pouvoir pastoral se recoupent. Ce rapprochement est facilité par l'effort commun aux deux penseurs - absent en revanche chez Chaunu - pour ramener des mouvements historiques divers, non seulement à une « formule généralisatrice » qui permet de les classer, mais également à des « mécanismes historiques types ${ }^{60}$ qui en généralisent les conséquences historiques : anti-confessionnelles pour l'un, anti-pastorales pour l'autre. Nous sommes au-dessus du simple relevé des faits, au niveau de la construction d'un modèle d'intelligibilité historique. Du livre de Kołakowski en 1965 au cours de Foucault en 1978, l'égalitarisme communautaire, la communication immédiate avec Dieu (la mystique), la revendication d'une lecture individuelle des Écritures sont ainsi tour à tour évoqués comme autant de conduites religieuses se révélant essentiellement incompatibles avec l'institution ecclésiale. Isolées comme telles chez Foucault, ces structures de révolte sont disséminées mais omniprésentes dans l'ouvrage de Kołakowski ${ }^{61}$, qui privilégie pour sa part les portraits individuels.

Cathares, Bégards, Béguines, Amis de Dieu, Vaudois, devotio moderna, Wyclif, Jean Hus, Taborites : le dossier historique, qui va chez Foucault du XIII ${ }^{\mathrm{e}}$ au Xv $\mathrm{Xv}^{\mathrm{e}}$ siècle, est certainement en partie de première main (du fait des citations par exemple de Jean Hus), mais il a été accompagné, voire guidé, par le livre de Pierre Chaunu qui parcourt un à un, et dans le même ordre, tous ces mouvements : P. Chaunu, Le temps des réformes. Histoire religieuse et système de civilisation. La crise de la chrétienté. L'éclatement, 1250-1550, Paris, Fayard, 1975. Cette référence à Chaunu se retrouve dans un petit carnet bibliographique tenu par Foucault dans ces années-là, et consacré au christianisme des XIII - XVIII ${ }^{\mathrm{e}}$ siècles : Répertoire AZ, Répertoire bibliographique, 1975 ?-1978 ? - Manuscrit ; 17 cm [Archives Daniel Defert].

60 L. Kołakowski, Chrétiens sans Église, déjà cité, p. 11.

61 Sur l'égalitarisme communautaire chez Kołakowski, voir les cas de Dirk R. Camphuysen (p.105), de Simons (p.158), des collégiants (p.175), etc. Sur la mystique, voir la deuxième moitié du livre de Kołakowski, qui lui est entièrement consacrée (p. 349 et suiv.). Sur l'instruction directe de chacun par l'Écriture, voir les cas d'Arminius (p. 80), des remonstrants (p.85), de Gelenus (p. 185), etc. Illustrés par des exemples médiévaux, ces 
S'il nous semble aller de soi que Foucault n'a pu en aucune façon se passer de la lecture de ce livre, il est plus risqué de déceler une influence directe. Il n'est cependant pas impossible que le « cas » Kołakowski, joignant la vie et l'œuvre d'un penseur iconoclaste, ait éveillé en lui ce rapprochement étrange entre dissidents religieux et dissidents politiques, et, par voie de fait, entre Église et parti communiste. Ce sont en tout cas les mêmes oppositions systématiques entre l'individuel et le collectif, l'expérience immédiate et les médiations institutionnelles, qui se retrouvent chez les deux penseurs, et ont déjà suscité des relectures critiques du maître ouvrage de Kołakowski ${ }^{62}$. De la même manière chez Foucault, nous allons voir qu'une position au départ dialectique se résorbe finalement en une partition simple, qu'il nous faudra discuter sur un plan historique.

\section{Évaluation critique de l'histoire pastorale}

La discussion va se jouer autour d'un néologisme forgé par le cours de 1977-1978: « contre-conduites». Pour les distinguer des «butées externes $\gg^{63}$ que le pouvoir ecclésial rencontre de manière massive et frontale (sorcellerie, hérésies, pouvoir politique), Foucault fait des contre-conduites des butées internes, « dans le champ même du pastorat » (ibid., p. 198), qu'il se propose de rassembler autour des cinq thèmes suivants : ascétisme, vie communautaire, mystique, rapport à l'Écriture, croyances eschatologiques. Ces révoltes peuvent être qualifiées d'internes, car elles ne sont pas des refus massifs de tout gouvernement, mais des oppositions ciblées et adaptées à l'adversaire. Elles ne rejettent du gouvernement que certaines de ses modalités concrètes d'application. Comme le dira Foucault devant la Société française de philosophie quelques mois plus tard, la question posée par ces révoltes est d'abord: «comment ne pas être gouverné comme cela, par cela, au nom de ces

trois opérateurs de révolte sont isolés par Foucault dans Sécurité, territoire, population, pages 214, 216 et 217 .

62 Dès 1975, Pierre Chaunu notait le côté « abrupte » de l'analyse de Kolakowski, tout en louant sa perspicacité : P. Chaunu, Le temps des réformes, déjà cité, p.171. Des études plus récentes nuancent grandement les portraits dressés par l'historien polonais. Pour Jean-Joseph Surin ou Labadie, voir M. de Certeau, La fable mystique, Paris, Gallimard, 1982. Pour Madame Guyon et Fénelon, voir J. Le Brun, Le pur amour de Platon à Lacan, Paris, Seuil, 2002.

63 Sécurité, territoire, population, p. 197. 
principes-ci, en vue de tels objectifs et par le moyen de tels procédés $\gg^{64}$. Les oppositions historiques au gouvernement des conduites ne sont donc pas le produit d'une anarchie généralisée encore moins d'une rétivité originelle de la liberté humaine. Non seulement ces contestations sont corrélatives de tout art de gouverner, mais elles en sont même la «ligne de développement » (ibid.). Cela signifie qu'elles sont toujours récupérables par cela même auquel elles s'opposent ; les Églises protestantes comme la Contre-réforme catholique n'auront ainsi de cesse de réinscrire en leur sein ce que leurs propres dissidents leur opposaient. Foucault pose ici le principe de non-extériorité et de réversibilité de la révolte, dont il fera ultérieurement un important point de méthode ${ }^{65}$.

Mais à mesure que l'analyse avance, la leçon $\mathrm{du} 1^{\mathrm{er}}$ mars 1978 va en fait externaliser les cinq tactiques citées, en les opposant de manière systématique au pastorat mis en œuvre par l'institution ecclésiale. Avec pour résultat principal de vider méthodiquement le christianisme institutionnel de tout ce qui, dans les pratiques individuelles, semble échapper à l'emprise d'un pouvoir extérieur. Toute initiative individuelle, qu'elle soit d'ordre ascétique, mystique ou érudite, se voit définie comme a-gouvernementale et donc anti-ecclésiale. Ce qui mène Foucault à une affirmation finale bien audacieuse par son extension :

$[\ldots]$ le christianisme, dans son organisation pastorale réelle, n'est pas une religion ascétique, ce n'est pas une religion de la communauté, ce n'est pas une religion de la mystique, ce n'est pas une religion de l'Écriture et, bien sûr, ce n'est pas une religion de l'eschatologie. ${ }^{66}$

Conclusion rigoureusement opposée à celle de Leszek Kołakowski, qui préférait quant à lui voir dans les conflits de la communauté, de la mystique ou de l'Écriture, la contradiction même du christianisme, comme tentative idéologique désespérée de faire tenir ensemble la logique de la Loi et la logique de la Grâce, celle d'un monde fini et d'un Dieu infini. Idéologie impossible pour l'historien polonais, le christianisme devient

64 «u'est-ce que la critique? », p. 38, l'éditeur souligne.

$65 D E \mathrm{n}^{\circ} 306$, « Le sujet et le pouvoir », II, p. 1054-1057.

66 Sécurité, territoire, population, p. 218. La fatigue et la fin du cours peuvent expliquer ces raccourcis. La leçon est, à d'autres endroits, plus nuancée : « cet ascétisme est, je crois, un élément, on ne peut pas dire littéralement étranger au christianisme, mais à coup sûr étranger à la structure de pouvoir pastoral autour duquel s'organisait, s'était organisé le christianisme $\gg($ p. 211). 
possible et efficace pour le philosophe français comme gouvernementalité. Mais au prix élevé de la mise à l'écart de toutes ses tensions internes, transformées en un simple jeu d'opposition conduites/contre-conduites. Pour le montrer, reprenons deux des cinq dossiers historiques ouverts par Foucault pour classifier les contre-conduites : l'ascèse et l'eschatologie. Ces deux dossiers sont par ailleurs les plus originaux par rapport à l'analyse de Kołakowski, qui ne les mentionnait qu'occasionnellement.

\section{L'ascèse chrétienne est-elle athlétique?}

S'il est difficile de commenter des remarques dont Foucault demande expressément à son auditeur d'excuser la généralité, on peut se reporter à certaines références d'apparence mineure, qui ont cependant l'avantage de la précision. Écoutons celle-ci :

Troisièmement, l'ascétisme est également une forme de défi, ou plutôt c'est une forme de défi intérieur si l'on peut dire, c'est aussi le défi à l'autre. Et alors là, les histoires qui donnent des descriptions de la vie des ascètes, des anachorètes orientaux, égyptiens ou syriens, sont remplies de ces histoires où d'ascète à ascète, d'anachorète à anachorète, on apprend que l'un fait un exercice d'une extrême difficulté, à quoi l'autre va répondre en faisant un exercice d'une encore plus grande difficulté : jeûner pendant un mois, jeûner pendant un an, jeûner pendant sept ans, jeûner pendant quatorze ans. ${ }^{67}$

L'édition du cours précise que ces citations ne se trouvent pas dans le recueil de paroles des Pères du désert : les Apophthegmata Patrum (ibid., p. 228, note 47). L'absence de toute référence exacte est effectivement embarrassante car elle ne permet pas de juger si une telle compétition entre moines est recommandée - auquel cas on ne voit pas en quoi elle constituerait une contre-conduite - ou au contraire critiquée. Il nous faut donc revisiter les lieux possibles de recherche dans le massif montagneux de la patristique, et apporter quelques précisions sur les éditions dont pouvait disposer Foucault.

Ce texte des Apophthegmata Patrum, dont l'origine remonte au $\mathrm{V}^{\mathrm{e}}$ siècle, a été reproduit par l'abbé Migne au XIX ${ }^{\mathrm{e}}$ siècle dans le volume 65 de sa Patrologie grecque. Cette version, que Migne emprunta à une édition de 1677, était un bon candidat pour retrouver la citation, d'autant plus que l'édition du célèbre abbé semble avoir été utilisée par Foucault 
dans ses toutes premières recherches patristiques ${ }^{68}$. Il faut cependant remarquer que Foucault ne fait pas mention directement des Apophthegmata Patrum dans ses leçons, mais de l'Histoire lausiaque ${ }^{69}$, dont les éditions sont multiples, $y$ compris chez Migne, qui en distille sans beaucoup de recul critique des versions grecque et latine à divers endroits de sa Patrologie. Or, l'Histoire lausiaque, œuvre du $\mathrm{v}^{\mathrm{e}}$ siècle rédigée en grec par l'évêque Palladius, offre plusieurs descriptions des agissements du moine Macaire d'Alexandrie qui semblent bien correspondre à l'allusion du cours. Ces passages étaient déjà chez Migne, mais dans des ensembles n'ayant pas toujours l'Histoire lausiaque pour titre ${ }^{70}$. Ce détail laisse à penser que Foucault s'est plutôt reporté à l'édition critique de l'Histoire lausiaque par Lucot, datant de 1912, dont nous citons ici la traduction :

$S$ 'il a entendu parler de quelque chose quelque part, [Macaire] l'a certainement réalisé en perfection. En effet, ayant appris de quelques-uns que les Tabennésiotes mangent pendant tout le carême de ce qui n’a pas été soumis au feu, il décida de ne pas manger pendant sept ans de ce qui passe par le feu, et à l'exception de plantes potagères crues, si parfois il s'en trouva, et de légumes à cosses trempés [sic], il ne goûta à rien. Ayant donc réalisé en perfection cette vertu, il apprit encore au sujet d'un autre qu'il mange une livre de pain; et ayant rompu son biscuit et l'ayant versé dans des saites en poterie, il résolut de manger autant que sa main retirerait.71

Macaire corse ainsi ses exercices ascétiques dans une surenchère dont il semblait coutumier et qui lui valut, de la part de Dom Butler, le surnom de $\ll$ recordman du désert $\gg^{72}$. Dans le texte de Palladius, ces prouesses semblent cependant tout à l'honneur de l'《 athlète ${ }^{73}$

68 Entretien avec Daniel Defert, le 6 décembre 2006.

69 Sécurité, territoire, population, p. 179.

70 L'Histoire lausiaque a eu une histoire éditoriale compliquée, que Migne s'est bien gardé de démêler. Le passage qui nous intéresse plus particulièrement se trouve dans sa Patrologie latine, mais dans un livre intitulé Héraclidis Paradisius (Patrologia Latina, t. 74, J.-P. Migne éd., p. 270).

71 Palladius, Histoire lausiaque (Vies d'ascètes et de Pères du désert), A. Lucot éd. et trad., Paris, Librairie A. Picard et Fils, 1912, p. 119.

72 Cité dans J. Bremond, Les Pères du désert, $2^{\mathrm{e}}$ édition, Paris, J. Gabalda (Les Moralistes chrétiens), 1927, vol.1, p. Xxxvir. Dom Cuthbert Butler est l'auteur de la première édition critique de l'Histoire lausiaque (Cambridge, 1898-1904), qui servit de référence à l'édition française de Lucot en 1912.

73 D'emprunt stoïcien, le terme d'《athlète » est constant dans le vocabulaire des Pères et se trouve déjà chez saint Paul. Dans le seul Préambule de l'Histoire lausiaque, on trouve quatre mentions de l'«athlète » (Palladius, Histoire lausiaque, déjà cité, p. 5, 7 et 9) : «athlète invincible », «nobles athlètes », «athlète du Christ» (deux occurrences). Cette dernière expression sera plus loin appliquée à Macaire (ibid., p.112). 
spirituel et ne suscitent nulle mise en garde. Cette volonté de dépasser ce que fait autrui en matière d'ascèse peut d'ailleurs avoir chez Macaire un sens non pas de simple compétition, mais de pédagogie : afin que les jeunes prétendants à l'exploit ascétique ne se prennent pas pour des héros, il est bon qu'un ancien les dépasse. Un autre extrait de l'Histoire lausiaque le vérifie : cette fois-ci incognito, Macaire revient à la charge chez les Tabennésiotes dont la «magnifique règle de vie » est objet de louanges. Ne reculant devant rien pour leur donner une leçon de modestie, il entame un jeûne de quarante jours qui les laisse pantois et furieux. Reconnu par l'higoumène, celui-ci lui déclare :

Allons, beau vieillard! Tu es Macaire et tu t'es caché de moi. [... ] Je t'ai de la reconnaissance pour avoir fait sentir ta poigne à mes petits enfants, afin qu'ils n'aient pas des pensées de superbe à propos de leurs ascèses. (Ibid., p. 131)

Cette émulation collective se retrouve en fait dans bien d'autres passages de la tradition des Pères, et, en premier lieu, dans un livre qui constitue le porche d'entrée de cette littérature : la Vie d'Antoine. Ce sont même les premiers mots que l'évêque Athanase adresse à ses lecteurs, qui auraient d'abord été, selon les historiens, des moines d'Occident :

C'est un bon combat ( $a \check{\mu} \mu \lambda \lambda)$ que vous avez engagé avec les moines d'Égypte, en vous proposant de les égaler ou même de les dépasser par votre ascèse pour parvenir à la vertu. ${ }^{74}$

Le terme ă $\mu \lambda \lambda a$ signifie « combat » ou encore « rivalité ». Or, ce livre, rédigé peu après la mort d'Antoine en 356, est ultérieurement présenté comme une «règle de vie monastique » par Grégoire de Nazianze, qui en loue grandement son auteur. Dans ses Confessions, Augustin lui confère la même autorité et parle de deux jeunes gens que sa lecture a immédiatement convertis ${ }^{75}$. L'influence de la Vie d'Antoine sera telle qu'elle peut être considérée comme le modèle de la littérature hagiographique chrétienne, appelé à se répandre bien au-delà des cercles monastiques. Athanase invite d'ailleurs à faire lire son livre à tout le monde, sans restriction, même aux païens ${ }^{7}$. Louis Bouyer a pu voir dans la première phrase que nous citons

74 Athanase d'Alexandrie, Vie d'Antoine, 1, 1, G. J. M. Bartelink trad., Paris, Cerf (Sources chrétiennes; 400), 1994.

75 Augustin, Confessions, 8, 15, E. Tréhorel et G. Bouissou trad., Euvres de saint Augustin, t.XIV, Paris, Desclée de Brouwer, 1962.

76 Athanase d'Alexandrie, Vie d'Antoine, 94, 1, déjà cité. 
la trace d'un «premier monachisme, encore inorganisé », confondant ascèse et $\ll$ compétition sportive ${ }^{77}$, mais ce rapprochement n'est pas étayé. Les excès particuliers dont le désert de Syrie a été le théâtre ${ }^{78}$ ont effectivement provoqué une régulation doctrinale et une limitation de l'érémitisme, mais rien ne permet de les relier au texte d'Athanase, originaire d'Égypte et haute figure du patriarcat d'Alexandrie. Le lien entre

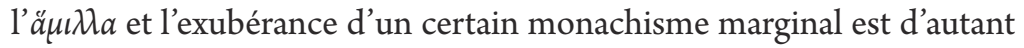
plus difficile à établir que les premiers destinataires de la Vie d'Antoine, comme nous l'avons signalé, seraient des moines d'Occident - peut-être du côté de Trèves où résida Athanase - qui connaissaient une vie religieuse sous les deux formes (érémitisme et cénobitisme) déjà bien organisée.

Surtout, la mention de l'á $\mu \iota \lambda \alpha a$ semble bien être une recommandation traditionnelle, et certainement pas la manifestation d'une dissidence. Pour appuyer cela, il est intéressant de noter que l'Histoire lausiaque place ce terme à son fronton, puisqu'il apparaît dès le prologue du livre. Louant Lausus, Palladius explique que ce dernier «a excité notre paresseux esprit à la contemplation de ce qui est mieux, pour porter à l'imitation et à la rivalité ( $\left.a^{\prime} \mu \iota \lambda \alpha\right)$ avec les vertus de l'ascèse de nos saints et immortels pères spirituels $\gg 79$.

Cette importance accordée au combat engagé entre moines doit en fait être lue à l'horizon de deux thèmes majeurs du monachisme ancien : la nécessité pour le moine de dépasser sans cesse la loi commune, et l'importance de l'exemple d'autrui dans la vie ascétique. Le premier thème ouvre un chemin indéfini de perfection, le second définit l'un des moteurs de cette perfection.

Prenons le premier thème. Homme de gouvernement s'il en est, témoin principal du vaste effort institutionnel pour réguler la vie monastique, Cassien insiste à plusieurs reprises sur le fait que le moine, en tant qu' homme juste et parfait, n'est pas esclave de la loi et ne peut donc jamais s'en contenter. En cela, il se distingue de l'homme ordinaire : ce dernier, vivant dans le monde, occupé par les plaisirs et les affaires, peut limiter sa conduite à l'observance de ce qui est prescrit et au refus de ce qui est des Pères, Paris, Aubier, 1960, p. 373-374.

78 Pour une étude contemporaine sur les excès (réclusion, stylitisme, pratiques spectaculaires) du monachisme syrien, voir P. Escolan, Monachisme et Église : le monachisme syrien $d u v^{e}$ au $v^{e} \iota^{e}$ siècle, Paris, Beauchesne, 1999. Palladius, Histoire lausiaque, déjà cité, p. 5. 
défendu. Pour sa part, l'homme juste doit au contraire chercher à toujours faire mieux que le commandement, afin d'atteindre la perfection évangélique. Ainsi, dans la conférence XXI, Jean Cassien écrit-il : « [...] l'homme juste et parfait n'est point lié par la loi du carême, et ne peut se contenter du joug d'une règle aussi modeste $\gg^{80}$. Or, cette majoration singulière de la loi, qui échappe à toute généralité, est bien l'exercice de la volonté, dans l'espace libéré par la sortie d'un joug à la fois trop réglé et trop modeste :

$[\ldots]$ les justes, pour qui n'est point faite la Loi, prouvent qu' ils ne sont pas sous la Loi en ceci, que, non satisfaits d'accomplir la justice de la Loi, ils s'efforcent de la dépasser. Leur dévotion est supérieure au commandement; et, mettant le comble (major) à l'observance du précepte, ils ajoutent une part volontaire (volontaria) à ce qui est dû strictement. ${ }^{81}$

Or, dans l'engagement de cette part volontaire, l'exemple d'autrui va jouer un rôle prépondérant. Quand Antoine commence sa vie solitaire, Athanase nous explique qu'il part d'abord s'instruire auprès des « hommes pleins de zèle » pour les égaler, voire les dépasser, dans leur ascétisme :

Il admirait l'un pour ses austérités, l'autre pour ses jeûnes et son repos sur la terre nue. $[\ldots]$ Fort de tout cela, il revenait à l'endroit où lui-même se livrait à l'ascèse, puis rassemblant en lui-même ce qu' il avait vu chez chacun, il s'efforçait de montrer en lui toutes ces vertus. De ceux de son âge, il n'était jaloux que sur un seul point : ne pas leur paraître inférieur dans le mieux. ${ }^{82}$

Rappelons ici qu'Athanase a par ailleurs soin de donner une image extrêmement orthodoxe d'Antoine, dont il souligne le respect pour les évêques et les prêtres (ibid., 67, 1) et l'opposition aux hérésies $(69,1)$.

Cette possibilité de dépassement incessant, qui ouvre la voie à l'émulation entre ascètes, Foucault la réfère à la $\ll$ joute de l'athlète $\gg^{8_{3}}$ qui se distinguerait radicalement de l'état de passivité et de renoncement à l'ego cherché par le gouvernement pastoral. Telle est la distinction faite dans la leçon du 22 février 1978 entre l'á $\pi \dot{a} \theta \varepsilon ı a$ grecque, qui serait exclusivement

Jean Cassien, Conférences, t. III, XXI, 29, E. Pichery trad., Paris, Cerf (Sources chrétiennes; 64), 1971.

81 Ibid., XXI, 3. Ce thème se retrouve dans le récit de la mise en place de la première règle des Tabennésiotes, dictée par un ange à Pacôme : « Quant aux parfaits, ils n'ont pas besoin de réglementation $\gg$ (Palladius, Histoire lausiaque, déjà cité, p. 219).

82 Athanase d'Alexandrie, Vie d'Antoine, 4, 1, déjà cité.

83 Sécurité, territoire, population, p. 210. 
du côté de la maîtrise de soi, et l'ảंá $\theta \varepsilon$ cla chrétienne, qui serait du côté de l'abandon de la volonté propre (ibid., p. 181-182). De Palladius et de Cassien Foucault fait justement les témoins principaux de cette pensée chrétienne de l'abnégation de soi et de la dépendance absolue (p.179). Mais l'examen des exemples auxquels semble se référer Foucault ne permet pas d'exclure aussi facilement la joute ascétique de la vie réglée du moine, telle qu'elle commence à se codifier aux IV $\mathrm{V}^{\mathrm{e}}$ et $\mathrm{V}^{\mathrm{e}}$ siècles. Rien ne permet même de conclure avec certitude qu'elle aurait fait problème. La seule limite posée à l'áuı $\lambda a$ - et, sur ce point, Foucault a raison d'accorder une place centrale au renoncement à la volonté propre - est le risque de verser dans la « vaine gloire », thème constant chez Palladius. Mais ce risque ne suffit pas à exclure le recours à l'émulation. L'Histoire lausiaque n'hésite pas à en présenter les fruits, tout en appelant à jeûner raisonnablement pour se garder de toute vanité ascétique, «car l'action de boire du vin avec raison est chose meilleure que de boire de l'eau avec orgueil $(\tau \dot{v} \varphi o v) \gg^{84}$.

\section{Gouvernementalité et apocalyptique}

Nous voudrions visiter un deuxième champ que la leçon du $1^{\text {er }}$ mars 1978 exclut radicalement du christianisme entendu comme technique de gouvernement : la croyance eschatologique. Cette croyance est référée au moine calabrais Joachim de Flore (1135 ?-1202), dont le découpage de l'histoire du monde en trois temps distincts (temps du Père, temps du Fils, temps de l'Esprit) se retrouvera au XIX ${ }^{\mathrm{e}}$ siècle chez Hegel et Auguste Comte, et sera encore louée au $\mathrm{XX}^{\mathrm{e}}$ siècle par Ernst Bloch dans sa période marxiste. Écoutons Foucault :

Après tout, l'autre manière de disqualifier le rôle du pasteur, c'est d'affirmer que les temps sont accomplis ou qu'ils sont en train de s'accomplir, que Dieu va revenir ou est en train de revenir pour rassembler son troupeau. Il sera le vrai berger. Par conséquent, puisqu'il est le vrai berger venant pour rassembler son troupeau, il peut donner leur congé aux pasteurs. ${ }^{85}$

Foucault mentionne alors «la ligne qui dérive plus ou moins directement de Joachim de Flore » (ibid., p. 217-218). Ce dernier exemple que Foucault donne des contre-conduites pastorales est également celui

84 Palladius, Histoire lausiaque, déjà cité, p. 27. Même mise en garde contre la compétition qui n'est pas habitée par la pureté d'intention (ibid., p. 153).

85 Sécurité, territoire, population, p. 217. 
qu'il présente, faute de temps, de la manière la plus brève et la plus générale. Une discussion néanmoins s'impose, à partir des synthèses sur Joachim et sa postérité que proposèrent Jean Delumeau (1995) et Philippe Lécrivain $(2001)^{86}$.

La référence à l'eschatologie joachimiste est à la fois plus évidente et plus problématique. Plus évidente, car son potentiel de contestation se vérifie dans la longue liste des mouvements millénaristes en opposition souvent violente au pouvoir ecclésial. Plus problématique, car elle s' inscrit dans une tradition qui irrigua non seulement la théologie mais aussi la vie chrétienne depuis les premiers siècles, tendue vers un avenir collectif dont les contours ne sont pas complètement impensables ni incertains.

Rappelons que l'adjectif «millénariste » désigne la croyance en la venue d'un règne de mille ans de bonheur qui précédera la fin des temps, en référence au chapitre 20 du livre de l'Apocalypse. S'il s'inscrit bien dans cette perspective eschatologique par son annonce d'un troisième et dernier temps de l'histoire, Joachim n'utilise cependant pas le mot «millenium ». À partir d'un jeu subtil de concordances entre les deux testaments, Joachim propose une interprétation des textes bibliques qui place soudain l'actualité de l'Église à l'horizon d'un nouveau seuil, situé en l'an 1260. Parcourons alors l'histoire contrastée de la postérité proche du moine calabrais.

1) Un groupe de disciples de Joachim, réunis autour de son secrétaire Luc devenu évêque de Cosenza, rassemble des écrits apocryphes (vers 1235-1246) et s'en prend de plus en plus ouvertement à la politique et à l'administration dans l'Église. Ce mouvement est cependant relativement toléré par les autorités, du fait en particulier du bon accueil que lui font les ordres mendiants récemment fondés (franciscains, dominicains). Le troisième âge annonçant le temps venu des viri spirituales, ces ordres nouveaux se reconnaissent aisément comme la réalisation des prophéties de Joachim. En 1237, la bulle papale de canonisation de Dominique évoque sans difficulté cette lecture de l'histoire et son enra-

86 J. Delumeau, Une histoire du Paradis, t. II : Mille ans de bonheur, Paris, Fayard, 1995. P. Lécrivain, «Les millénarismes du christianisme antique et médiéval », Histoire raisonnée de la philosophie morale et politique, A. Caillé, C. Lazzeri et M. Senellart éd., Paris, La Découverte, 2001, p.151-161. Nous citons ces deux études postérieures à Foucault pour leur clarté et leur concision, mais notons que de grandes études sur Joachim et le joachimisme étaient déjà disponibles en 1978, en particulier : H. de Lubac, Exégèse médiévale. Les quatre sens de l'Écriture, t. II, 2 volumes, Paris, Aubier, 1961 et 1964. 
cinement biblique. Chez les mineurs, une tradition joachimiste durable s'installe, qui a toutes les faveurs de la papauté. Leur fondateur François est alors considéré comme l'ange du sixième sceau de l'Apocalypse. Les idées qui circulent à cette époque sur les trois âges de l'histoire ne sont cependant pas le fait du seul Joachim; elles se trouvent également chez les disciples d'Amaury de Bène.

2) Le franciscain Gérard de Borgo San Domino publie en 1254 un Liber introductorius in Evangelium aeternum, où il annonce que la doctrine de Joachim a surpassé celle du Christ, qui sera définitivement caduque en l'an 1260. Conséquence importante de l'annonce de ce nouvel Évangile : un nouveau sacerdoce surpassera l'actuel, et, à compter de ce moment, nul représentant patenté de l'ancienne Église ne pourra prétendre conduire les hommes à l'esprit de la vie éternelle. Le livre est condamné par le pape Alexandre IV et son auteur est incarcéré. Mais les écrits de Joachim ne sont pas visés par ces sanctions.

3) L'année tant attendue de 1260 voit naître des mouvements populaires extrémistes, parfois violents : les flagellants déferlent sur l'Italie ; Gérard Ségarelli et son successeur Dolcino s'opposent ouvertement à l'Église et leurs adeptes seront réprimés par la force. La situation reste cependant contrastée, les répressions localisées, et le joachimisme continuera d'influencer la pensée de certains évêques comme Jean de Tolède.

Il faut en effet se rappeler que Joachim fut ami des papes de son temps et ne vit jamais sa doctrine mise en cause par les autorités. La définition de la croyance eschatologique comme contre-conduite ne peut donc concerner que certains aspects limités de l' histoire que nous avons retracée. La pensée de Gérard de Borgo San Domino est bien une remise en cause du pouvoir pastoral de l'Église, et un mouvement comme celui des flagellants, justement cité dans la leçon du Collège de France ${ }^{87}$, est l'expression la plus radicale et la plus violente de cette contestation. Estce suffisant pour conclure que le christianisme «n'est pas une religion de l'eschatologie $\gg$ (ibid., p. 218) ?

La venue d'un temps d'accomplissement du temps - c'est-à-dire non seulement une fin de l'histoire mais une fin dans l'histoire - n'est pas une invention de Joachim ; il puise dans les textes bibliques, et, en premier lieu, la grande apocalypse du livre d'Isaïe (chapitres 24-27). Or, ce texte n'est pas 
isolé. Il existait en fait dans le judaïsme hellénistique, puis dans ce que Jean Daniélou a défini comme le «judéo-christianisme $\gg$ des $\mathrm{I}^{\mathrm{er}}$ et $\mathrm{II}^{\mathrm{e}}$ siècles de notre ère, une importante littérature qui non seulement annonçait la fin des temps, mais en donnait de riches descriptions, aux couleurs souvent paradisiaques ${ }^{88}$. La conception juive d'un règne messianique précédant l'ultime jugement fut simplement transposée dans la littérature chrétienne en règne du Christ - l'Apocalypse de Jean reprenant sans doute sur ce point l'Apocalypse syriaque de Baruch, rédigé sensiblement à la même

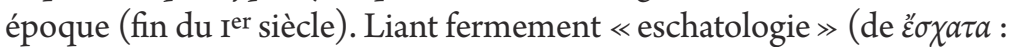

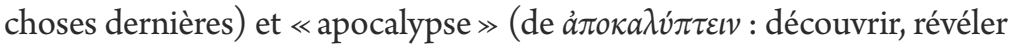
des secrets), cette littérature se retrouve dans le canon des Écritures ${ }^{89}$, mais également dans les apocryphes des deux Testaments ${ }^{\circ \circ}$.

Précisons que ce que Jean Daniélou isole en 1958 sous le terme de «judéo-christianisme » n'a rien à voir avec le raccourci historique simpliste de la «morale judéo-chrétienne », fustigé à raison par Foucault au début de la leçon du $1^{\mathrm{er}}$ mars $1978^{91}$. La catégorie est appliquée par l'historien jésuite à la théologie chrétienne antérieure aux apologistes, qui s'est pensée dans un cadre conceptuel emprunté au monde juif. Cette pensée judéo-chrétienne qui s'étend jusqu'au milieu du $\mathrm{II}^{\mathrm{e}}$ siècle dépasse les seules questions de l'observance de la Loi juive chez les communautés chrétiennes primitives et de la réception du message de Jésus chez certains juifs marginaux pour s'ouvrir à une audacieuse vision de l'histoire. Si certains spécialistes jugèrent que Daniélou avait dès lors trop ignoré les influences hellénistiques ou iraniennes sur cette première théologie, son étude a permis de renouveler l'interprétation d'une littérature auparavant considérée comme sibylline et disparate.

Dans la pensée chrétienne, la place de l'apocalyptique fut régulièrement sujette à controverse. On se rappelle à ce sujet que les écrits johan-

88 J. Daniélou, Histoire des doctrines chrétiennes avant Nicée, t. I : Théologie du Judéo-Christianisme, M.-O. Boulnois éd., chap. XI : «Le millénarisme », $2^{\mathrm{e}}$ édition, Paris, Desclée/Cerf, 1991, p.379-404.

89 Pour l'Ancien Testament, il faut se référer à la littérature prophétique : Am. 9 ; Is. 24-25; Éz. 36-40. Pour le Nouveau Testament : Apoc. 20 ; I Thess. 4 et II Thess. 1 ; I Cor. 15.

90 L'ascension d'Isaïe est un exemple d'apocalypses chrétiennes non canoniques. Rappelons que «canonique » désigne une décision doctrinale largement postérieure à ces textes, qui ne renseigne que bien peu sur l'étendue de leur réception et leur sphère d'influence à l'époque où ils furent écrits. Cette sphère d'influence put, dans certains cas, être très grande. Sécurité, territoire, population, p. 195. 
niques n'entrèrent pas facilement dans le canon final du Nouveau Testament. Certains Pères latins comme Augustin et Jérôme condamnèrent le caractère trop matériel du règne décrit, les jouissances sensibles promises, et préférèrent ne garder que la perspective du jugement dernier. Pour l'évêque d'Hippone, dans le chapitre xx de La Cité de Dieu, le millénaire était déjà commencé, mais en esprit, à travers la vie des saints. Plusieurs Pères de l'Église cependant, et non des moindres, furent ouvertement millénaristes : Justin, Irénée de Lyon, pour les Grecs, Tertullien et Lactance, pour les Latins. Depuis les années 1960, la théologie catholique et protestante a redécouvert l'apocalyptique comme une dimension constitutive non seulement du dogme, mais aussi de la liturgie. Du dogme, parce que la vérité se donne d'abord comme une vision et non comme un discours articulég ${ }^{2}$; de la liturgie, parce que les hymnes de la communauté célèbrent la venue d'un Royaume annoncé comme une «terre nouvelle ». C'est donc la pratique même des fidèles qui est concernée par un accomplissement des temps qui n'est pas seulement spirituel, comme le vivaient au quotidien les communautés de tradition johannique où fut rédigée l'Apocalypse. Cette prégnance du millénarisme a eu par ailleurs des effets concrets sur les pratiques missionnaires chrétiennes : Christophe Colomb et Tommaso Campanella citèrent Joachim comme référence ; les franciscains qui évangélisèrent le Mexique au Xvi ${ }^{\mathrm{e}}$ siècle $^{93}$ et les Pères pèlerins qui débarquèrent en Nouvelle Angleterre en 1620 étaient tous portés par cette même espérance millénariste d'un règne terrestre du Christ. Mais l'exemple le plus probant est certainement celui des réductions jésuites du Paraguay, puisque Foucault en avait fait l'un des modèles des dispositifs disciplinaires dans sa leçon du 28 novembre $1973^{94}$ : dans ce cas précis, croyance eschatologique et pouvoir disciplinaire, loin de s'exclure, se sont mutuellement renforcés.

En soulignant la prégnance du millénarisme sur le christianisme, son inscription jusque dans le canon des Écritures, il ne s'agit pas d'en rester à une histoire des doctrines - que rejetait de toute façon Foucault - mais

92 A. Paul, «De l'apocalyptique à la théologie », Recherches de science religieuse, t. 80, 1992, p. $182-183$.

93 R. de Roux Lopez, « Nouveau Monde, Nouvelle Église », Recherches de science religieuse, t. 8o, 1992, p. 533-550.

94 Le pouvoir psychiatrique, p.70-71. 
de comprendre le rôle déterminant qu'il a joué dans les gestes rituels et les actes militants d'une communauté.

Certes, tous ces contre-exemples pourraient être à leur tour contrés par l'invocation de la réversibilité des luttes. Foucault ne cesse-t-il pas de répéter que ces « éléments-frontière » comme l'ascèse ou l'eschatologie sont « sans cesse repris par l'Église elle-même ${ }^{95}$ et que leur apparition est quasi contemporaine du gouvernement auquel elles s'opposent? Il reste cependant à préciser ce qui permet à l'historien, au sein de cette réversibilité incessante, d'isoler un modèle de contre-conduite relativement stable comme le fait Foucault. Quel critère peut décider qu'une conduite est $\ll$ essentiellement $\gg^{96}$ anti-pastorale et seulement tactiquement récupérée par le pastorat ? Foucault ne peut s'appuyer ici sur un critère d'orthodoxie doctrinale pour deux raisons évidentes : premièrement, ce serait sortir du champ d'étude défini par le thème de la gouvernementalité ; deuxièmement, les exemples historiques contrediraient immédiatement l'hétérodoxie supposée de l'ascèse ou de l'eschatologie. Le seul critère qui reste alors à Foucault est celui de l'autonomie des pratiques. S'il n'est pas affirmé comme tel, ce partage autonomie/hétéronomie opère tout au long de la leçon du $1^{\mathrm{er}}$ mars 1978. La gouvernementalité pastorale, si riche dans sa définition première, est finalement réduite à l'exercice d'un pouvoir qui vient de l'extérieur mettre la main sur les conduites individuelles. L'explication de la rétivité première de l'ascèse au gouvernement est à ce sujet particulièrement éclairante : l'ascèse se refuse au pouvoir car elle est tout d'abord un « exercice de soi sur soi » (p. 209) qui ne passe pas par autrui; ensuite car elle est une figure de l'excès, du «trop qui assure précisément son inaccessibilité pour un pouvoir extérieur $\gg$ (p.211, nous soulignons). Étrange dichotomie qui rejette de part et d'autre de la coupure opérée par l'historien le gouvernement extérieur et le soi intérieur; le second pouvant, par ses tactiques réflexives et ses secrètes intensités, radicalement échapper au premier.

Que Foucault lui-même n'ait pas été satisfait du résultat, il suffit d'écouter le cours de 1979-1980 pour s'en convaincre. Du gouvernement des vivants

96 Nous empruntons volontairement ce terme à Foucault. L'«essence » d'une conduite appartient bien au vocabulaire du cours, qui indique par exemple qu'il y a dans l'obéissance quelque chose d'《essentiellement anti-ascétique » (ibid., p. 209). 
traite du même corpus, développe les mêmes thèmes, lit les mêmes textes et raconte les mêmes histoires, à une différence près : l'exercice de soi sur soi n'est plus un élément frontière de la gouvernementalité chrétienne mais l'élément central. Répétition du cours de 1978, le cours de 1980 l'est sans doute, mais au sens kierkegaardien de reprise à un niveau supérieur. Cette réintégration de l'ascèse, au sens le plus large du terme, va permettre de constituer l'objet christianisme non plus exclusivement comme gouvernement extérieur du soi, mais d'abord comme gouvernement de soi ou encore : « régime de vérité ».

Avant d'aborder cette notion, il convient de remarquer que Foucault corrigea discrètement par deux fois la présentation faite des contreconduites en 1978. Dans une conférence tout d'abord à l'université de Stanford en 1979, le philosophe revient sur la spécificité des luttes autour du pastorat, mais son exposé ne conserve des cinq modèles de résistance définis en 1978 (ascétisme, communauté, mystique, Écriture, eschatologie) que le modèle de la communauté, suivi d'une discrète allusion à la mystique et au millénarisme : il ne s'agit plus tant alors de «luttes antipastorales $\gg$ que de luttes pour une autre forme de pastorat ${ }^{97}$. Mais c'est la toute dernière leçon au Collège de France, le 28 mars 1984, qui porte le coup de grâce au découpage de 1978 : Foucault y définit la mystique commel'un des deux pôles de l'expérience chrétienne (pôle « parrèsiastique »), à la fois aux côtés et contre celui de l'ascétisme (pôle de l'obéissance craintive) ${ }^{98}$. Nous aurons l'occasion d'y revenir dans la troisième et dernière partie de notre recherche.

\section{Le christianisme comme régime de vérité : 1980}

Origine et mutation de la notion de «régime de vérité »

Arrêtons-nous sur cette expression qui constitue le porche d'entrée des recherches de Foucault sur l'Antiquité chrétienne. Si le syntagme semble immédiatement foucaldien à nos oreilles, par son association paradoxale de ce qui s'impose de soi (la vérité) avec un dispositif coercitif (un

97 Ces luttes sont marquées par un « désir » de pastorat : $D E$ no 291, « "Omnes et singulatim" : vers une critique de la raison politique », II, p. 968.

98 Le courage de la vérité, p. 307-308. 
régime), il est relativement rare dans la bouche de Foucault. Apparue subrepticement dans Surveiller et punir'99, l'expression n'a que deux occurrences dans les cours au Collège de France : la leçon du 18 février $1976^{100}$ et celle du 10 janvier $1979^{101}$. Entre ces deux dates, Foucault reprend plusieurs fois le syntagme dans un entretien de juin 1976 qui connut deux publications distinctes - la plus brève étant intitulée « La fonction politique de l'intellectuel $\gg^{102}$. Cet entretien illustre sans ambiguiité l'usage à la fois rare mais homogène de l'expression « régime de vérité » avant 1980.

Après avoir préalablement détaillé les cinq éléments qui composent un régime de vérité - un certain type de 1) discours, 2) mécanismes de distinction, 3) sanctions, 4) techniques de production, 5) statuts des sujets $^{103}$-, Foucault ressaisit tous ces éléments dans la forme d'une circularité entre pouvoir et vérité :

$[\ldots]$ la « vérité » est liée circulairement à des systèmes de pouvoir qui la produisent et la soutiennent, et à des effets de pouvoir qu'elle induit et qui la reconduisent. «Régime » de la vérité. (Ibid., p. 114, repris p. 160)

Si l'expression est nouvelle, l'hypothèse de cette circularité ne l'est pas : elle avait été formulée dans le cours Théories et institutions pénales (1971-1972 $)^{104}$ et n'est finalement qu'une autre manière de parler de ce que Foucault désigne en d'autres endroits par «forme de rationalité ». Un régime de vérité est donc défini comme l'entraînement réciproque d'un système de production de la vérité et des effets de pouvoir induits par cette vérité. C'est avec cette même signification que l'expression apparait dans les cours Il faut défendre la société et Naissance de la biopolitique.

Le terme « régime » n'est pas choisi au hasard et vient explicitement du champ politique. Par deux fois, Foucault opère le rapprochement :

99 Surveiller et punir, p.3o.

100 Il faut défendre la société, p. 145. L'expression y reçoit une définition claire : « un certain pouvoir de partage vérité/erreur $\gg$.

101 Naissance de la biopolitique, p. 20 : «Quand je dis régime de vérité, je ne veux pas dire que la politique ou l'art de gouverner, si vous voulez, accède enfin à cette époque-là à la rationalité. [...] Je veux dire que ce moment que j'essaie d'indiquer actuellement, que ce moment est marqué par l'articulation sur une série de pratiques d'un certain type de discours qui, d'une part, le constitue comme un ensemble lié par un lien intelligible et, d'autre part, légifère sur ces pratiques en termes de vrai ou faux. »

$102 \mathrm{DE} \mathrm{n}^{\circ} 184$, «La fonction politique de l'intellectuel », II : c'est la version brève, publiée en France, d'un entretien paru en Italie : $D E \mathrm{n}^{\circ} 192$, « Entretien avec Michel Foucault », II. $103 D E \mathrm{n}^{\circ}{ }_{184}$, « La fonction politique de l'intellectuel », II, p. 112 $104 D E \mathrm{n}^{\circ} 115$, « Théories et institutions pénales », I, p. 1257-1261. 
Chaque société a son régime de vérité, $\mathrm{sa}$ «politique générale » de la vérité $[\ldots] .{ }^{105}$

[... ce qui est en question, c'est ce qui régit les énoncés et la manière dont ils se régissent les uns les autres pour constituer un ensemble de propositions acceptables scientifiquement $[\ldots]$. Problème en somme de régime, de politique de l'énoncé scientifique. ${ }^{106}$

Il faut entendre ici par politique, avec Foucault, non pas d'abord des institutions, mais une stratégie globale qui essaie de coordonner et de finaliser les rapports de force présents dans une société donnée $e^{107}$. La connotation politique du terme de « régime » est d'autant plus intéressante que nous la retrouvons au tout début de la leçon du 6 février 1980 (cours Du gouvernement des vivants), où « régime de vérité » est employé par analogie avec $\ll$ régime politique $\gg^{108}$. Cependant, la suite du cours va aussitôt faire un grand écart par rapport à l'usage que nous venons de repérer dans l'entretien publié quatre ans auparavant.

Par rapport à l'entretien de 1976, la leçon du 6 février 1980 donne immédiatement une définition beaucoup plus restreinte de l'expression, resserrée autour de l'activité requise de la part du sujet dans la production de la vérité : «Un régime de vérité, c’est ce qui détermine les obligations des individus quant aux procédures de manifestation du vrai $\gg^{109}$. Cette restriction a pour conséquence un double partage qui va modifier en profondeur les analyses menées depuis le début des cours au Collège de France. Ce double partage n'est cependant pas un coup de force méthodologique effectué le 6 février, il est préparé par une succession de décrochages repérables dans des articles et entretiens précédant le cours.

Si l'on reprend les cinq éléments isolés en 1976 comme constitutifs d'un régime de vérité, on se rend compte que Foucault ne distingue pas

$105 D E \mathrm{n}^{\circ} 184$, « La fonction politique de l'intellectuel », II, p.112. Notons que cette expression «politique de la vérité » apparait également dans la communication à la Société française de philosophie, le 27 mai 1978 : «Qu'est-ce que la critique ?», p. 39.

$106 D E \mathrm{n}^{\circ} 192$, «Entretien avec Michel Foucault », II, p. 143, l'auteur souligne.

107 Nous empruntons cette définition à un entretien publié en $1977: D E n^{\circ} 197$, « Les rapports de pouvoir passent à l'intérieur des corps », II, p. 233.

108 L'enregistrement sonore du cours $D$ u gouvernement des vivants, encore inédit, est déposé à l'IMEC, où nous l'avons écouté à l'hiver 2006. Il est aujourd'hui en cours d'édition grâce au travail de Michel Senellart. Nous citons notre propre retranscription, secondée par celle de Michel Senellart, que nous remercions ici chaleureusement.

109 Cours Du gouvernement des vivants, leçon du 6 février 1980. 
le jeu des énoncés entre eux - qui définit une proposition comme étant vraie selon des règles indépendantes des sujets - du rapport des sujets à ces énoncés. Il emporte dans une même dynamique de pouvoir les procédures formelles de validation des énoncés et la manière dont ces énoncés convoquent, engagent, contraignent des sujets. Il dénie donc aux propositions scientifiques une logique propre de vérité, distincte de l'organisation des rapports de force dans une société donnée. Il évite cette distinction d'autant plus facilement que l'analyse ne prend pas en compte les différences entre les types de discours vrais. Le choix méthodologique est discutable mais clair : depuis le cours Théories et institutions pénales (1971-1972), Foucault se refuse à isoler des pratiques sociales un domaine «pur » qui serait celui de la science, et fonctionnerait comme enchaînement systématique de propositions selon des règles strictement formelles. Le fonctionnement effectif, dans une société donnée, de la normativité scientifique ne correspond pas à un type idéal qui se serait d'abord incarné dans les mathématiques ${ }^{10}$; il est toujours une forme historique de pouvoir-savoir. Les conférences de Rio de Janeiro en 1973 vont encore plus loin dans ce sens, en faisant de la pratique judiciaire la racine commune de tous les types de vérités à l'œuvre dans les sociétés occidentales - vérité scientifique comprise ${ }^{111}$.

Le cours de 1979-1980 opère alors un renversement net de perspective, qui est en fait un retour aux analyses des années 1960 : les règles formelles des énoncés scientifiques, qui assurent la systématicité de leurs propositions, sont à nouveau considérées comme hors de tout « régime ${ }^{112}$. À l'affirmation massive de 1976 que la vérité n'est jamais hors pouvoir, fait place un premier partage entre la normativité interne

$110 D E n^{\circ} 85$, «Entretien avec Michel Foucault », I, p. 1036.

111 L'assimilation est sans ambiguité : «Je présenterai quelques esquisses de cette histoire à partir des pratiques judiciaires d'où sont nés les modèles de vérité qui circulent encore dans notre société, qui s'y imposent encore et qui valent non seulement dans le domaine de la politique, dans le domaine du comportement quotidien, mais jusque dans l'ordre de la science $\gg\left(D E \mathrm{n}^{\circ} 139\right.$, « La vérité et les formes juridiques », I, p. 1421).

Rappelons que l'analyse archéologique avait su distinguer la «science $\gg \mathrm{du}$ 《savoir $\gg$, ce que ne faisait plus, par exemple, le résumé du cours de 1971-1972 ( $D E \mathrm{n}^{\circ} 115$, « Théories et institutions pénales », I, p. 1257-1258). Dans une communication de 1968 ( $D E$ n no 59, «Sur l'archéologie des sciences. Réponse au Cercle d'épistémologie », I), Foucault avait pourtant clairement reconnu la légitimité d'une analyse de la science dans son organisation interne et ses normes formelles, détachée du savoir comme lieu et loi historiques de son émergence. Il notait seulement les limites d'une telle épistémologie, qui reste une abstraction et ne 
du discours scientifique et les obligations que les sujets ont à son égard. Pour reprendre les termes mêmes de Foucault, il s'agit, en science, de distinguer ce qui permet de dire «c'est vrai » de ce qui oblige à dire «donc, je m'incline $»^{113}$. Ce «donc , à l'intérieur duquel un sujet s'engage, n'est pas une conséquence de la vérité de la proposition; il est « historique et culturel $\gg$. Est ici distingué de manière très subtile l'acte d'intellection de l'acte d'acquiescement qui engage une réponse de la part du sujet. «Dans ce "tu dois" de la vérité, il y a quelque chose qui ne relève pas de la vérité elle-même dans sa structure $\gg$ (ibid., nous soulignons). Mais dire cela, c'est en même temps rendre à la cohérence interne d'un discours scientifique son autonomie et son efficacité propre, irréductible aux mécanismes sociaux de circulation et de régulation des discours. En particulier, la définition statutaire des sujets de l'énonciation n'entre plus en jeu dans la reconnaissance de la vérité en tant que telle. L'exemple choisi par Foucault est celui de la logique : «si [le logicien] dit "c'est vrai", ce n'est pas parce qu'il est logicien que la proposition est vraie $[\ldots]$. Pour que la proposition soit vraie, il faut et il suffit qu'il y ait des règles de cette logique, des règles de construction, de syntaxe, et que cette logique opère $\gg$ (ibid.). Une telle affirmation semble dénouer d'un seul coup le nœud de pouvoir-savoir auxquels les auditeurs des cours au Collège de France avaient été habitués depuis Théorie et institutions pénales (1971-1972).

Est-ce à dire alors que le régime de la vérité scientifique, comme « effet du vrai $\gg^{114}$ sur le sujet, serait réduit à une pratique politique ajoutée de l'extérieur, incluant les règlements institutionnels et les statuts académiques? Non plus, et c'est là que Foucault va opérer un deuxième partage décisif : si la « vérité » se distingue désormais de son « régime», un « régime de vérité » doit également être distingué de tout « régime politique $\gg$, entendu comme gestion finalisée des rapports de force dans une société. Le maintien du vocable « régime » pourrait d'ailleurs sembler

peut en aucun cas rendre compte de l'existence historique d'une science. Dans un entretien de début 1984, Foucault précisera à nouveau que la mise en lumière du rôle joué par le pouvoir dans la constitution d'un savoir «n'entame aucunement la validité scientifique ou l'efficacité thérapeutique [par exemple] de la psychiatrie. [... ] de toute façon, on ne peut aucunement dire que les jeux de vérité ne sont rien d'autre que des jeux de pouvoir » ( $D E$ $\mathrm{n}^{\circ} 356$, « L'éthique du souci de soi comme pratique de la liberté », II, p. 1543-1544).

113 Leçon du 6 février 1980.

114 Leçon du 6 février 1980. 
ambigu si l'on ne se rappelle, avec le cours de 1977-1978, que le regimen des Pères latins a en fait traduit l'oikovouía des Grecs ${ }^{115}$.

Dans les éléments constitutifs d'un régime de vérité apparaissait pourtant en 1976 le «statut de ceux qui ont la charge de dire ce qui fonctionne comme vrai $\gg^{116}$. Si la définition du statut des sujets disparaît des procédures internes de validation d'une proposition, rendues à leur autonomie, on serait en droit de la retrouver au moins dans l'obligation du sujet de souscrire à cette vérité. Le complémentaire de la proposition « ce n’est pas parce qu'il est logicien que c'est vrai » pourrait alors être, tout naturellement : « par contre, c'est parce qu' il est logicien qu'il s'incline ». Si tel était le cas, la leçon de 1980 prolongerait au moins en partie les affirmations de 1976, en liant l'acte de soumission à une « politique de la vérité », incluant le statut des sujets de connaissance dans une institution scientifique. Ce lien serait d'autant plus évident à établir que Foucault a pris soin de situer l'exemple de la logique dans le cadre d'une conversation concrète entre deux logiciens. Qui ne serait d'accord pour reconnaître, à l'œuvre dans cette conversation, un jeu d'influences et d'autorité qui permettrait d'expliquer la soumission d'un logicien à ce que dit l'un de ses condisciples?

Mais un régime de vérité, dans la définition radicalement nouvelle proposée par le cours de 1979-1980, n’est pas la politique moins la vérité, il n'est en fait ni l'une ni l'autre. Si la soumission au vrai ne vient pas d'une discipline scientifique formellement définie (la logique), elle ne vient pas non plus d'un statut académique historiquement déterminé (le logicien). Si «l'effet du vrai » n'a pas sa raison d'être du côté de règles formelles qui ne dépendent pas du sujet, il n'est pas non plus à chercher du côté de la dépendance institutionnelle ou politique des sujets. Le «statut » n'explique pas la spécificité des effets d'un régime de vérité, et Foucault l'écarte catégoriquement pour la deuxième fois : $\ll[\ldots]$ il pourrait ne pas être logicien de profession et il s'inclinerait de même $\gg^{117}$. Tel est le second partage décisif opéré par Foucault.

Sur l'espace libéré par la mise à l'écart successive de la vérité et de la politique, va alors se dessiner un mouvement singulier. En fait, le seul facteur déterminant du côté du sujet est non pas ce qu'il est - défini par une institution - mais ce qu'il fait. Et ce qu'il fait de la sorte se situe à

115 Sécurité, territoire, population, p. 196.

$116 D E \mathrm{n}^{\circ} 184$, « La fonction politique de l'intellectuel », II, p. 112.

117 Leçon du 6 février 1980. 
l'intérieur d'un mouvement désigné par l'emploi d'un verbe pronominal : se constituer, même si l'initiative peut très bien en revenir à autrui. En effet, si le logicien s'incline :

[... ] c'est parce qu'il fait de la logique, c'est-à-dire parce qu'il s'est constitué luimême - ou qu'il a été invité à se constituer - comme opérateur dans un certain type de pratiques, ou comme partenaire dans un certain type de jeu. Et il se trouve que ce jeu de la logique est tel que le vrai sera considéré comme ayant en lui-même, et sans autre condition, valeur contraignante. ${ }^{118}$

Un régime de vérité est donc l'activité par laquelle un sujet se constitue comme devant établir un certain rapport à une vérité donnée. Pour bien saisir la nouveauté de cette définition, il faut remarquer la substitution de notions entre l'entretien de 1976 et la leçon de 1980 : la place que tenait le statut semble désormais occupée par le terme qualification. En prenant l'exemple du cogito ergo sum après celui de l'inférence logique, Foucault note en effet que le régime de vérité cartésien requiert que «le sujet qui raisonne soit qualifié d'une certaine manière ${ }^{19}$, c'est-à-dire comme n'étant pas fou. Dans un entretien de 1983, Foucault note pareillement chez Descartes un « rapport de soi à soi le qualifiant comme pouvant être sujet de connaissance vraie $\gg^{120}$. La qualification n'a plus ici le sens qu'elle avait encore dans L'ordre du discours en 1971 : des règles ou un rituel codifiant du dehors l'accès des sujets à la parole ${ }^{121}$. Elle désigne bien autre chose qu'un simple statut, si l'on entend par statut une marque extérieure apposée par une institution sur certains individus afin de leur garantir, dans des conditions limitées, un certain nombre de prérogatives. Dans les régimes de vérité tels que les définit l'entretien de 1976, la prérogative attachée au statut est de définir le type de discours fonctionnant comme vrai. Elle ne concerne donc qu'un

118 Ibid., nous soulignons. Cette réflexion permettrait d'éclairer l'expérience menée par Stella Baruk sur l'enseignement des mathématiques en primaire : sur 97 élèves à qui il était demandé de calculer l'âge du capitaine d'un bateau transportant 26 moutons et 10 chèvres, 76 ont donné une réponse (S. Baruk, L'âge du capitaine. De l'erreur en mathématiques, Paris, Seuil, 1985). La raison n'en est pas que les élèves se seraient mépris sur les données du problème, mais qu'ils n'ont pas intégré le régime de vérité qui leur enjoint d'user de leur intelligence de manière méthodique et autonome, indépendamment de l'autorité de l'enseignant et de la puissance magique de l'objet culturel appelé «mathématiques ».

119 Leçon du 6 février 1980, nous soulignons.

$120 \mathrm{DE} \mathrm{n}^{\circ} 344$, «À propos de la généalogie de l'éthique : un aperçu du travail en cours », II, p. 1449, nous soulignons.

121 L'ordre du discours, p. 41. 
petit nombre de sujets, fonctionnant automatiquement comme relais institutionnels. Le terme est en effet mis en série avec des «instances », «mécanismes », «techniques », procédures » qui régissent de l'extérieur l'énoncé du vrai et dont les applications sont immédiates ${ }^{122}$. Dans la leçon de 1980, la « qualification » est au contraire une auto-constitution de soi par un processus qui s'inscrit dans le temps.

Il faut cependant noter qu' il arrive à Foucault, dans un contexte similaire, de parler de cette auto-constitution qualifiante en termes de «statut $\gg$. Le jeu des notions est donc ici subtil et l'exemple de la folie nous offre une intrication intéressante des termes. Depuis la publication de Folie et déraison, Foucault était en effet revenu par deux fois sur la constitution du sujet raisonnable face à la folie, croisant les termes « qualification $\gg$ et $\ll$ statut $\gg$ comme résultats de cette constitution. Il s'agit de la réponse à Jacques Derrida publiée en 1972, « Mon corps, ce papier, ce feu $\gg^{123}$, et du long entretien enregistré à la fin de l'année 1978 pour un journal italien, Il Contributo ${ }^{124}$. Il faut donc être précis sur la distinction opérée. Regardons les textes.

Dans la réponse à Jacques Derrida, publiée initialement au Japon avant de devenir la postface à la réédition de Folie et déraison ${ }^{125}$, Foucault opère un partage qu'il attribue officiellement à sa méthode archéologique, alors qu'il révèle bien autre chose. Attaqué par son cadet sur son interprétation de Descartes, l'historien de la folie distingue la «pure démonstration », qui n'implique pas le sujet du discours, de la «méditation » qui modifie au long de ses événements discursifs le sujet énonçant :

Dans la méditation, le sujet est sans cesse altéré par son propre mouvement; son discours suscite des effets à l'intérieur desquels il est pris; il l'expose à des risques, le fait passer par des épreuves ou des tentations, produit en lui des états, et lui confère un statut ou une qualification, dont il n'était point détenteur au moment initial. ${ }^{126}$

Dans l'entretien pour Il Contributo, cette distinction démonstration/méditation est transposée en distinction connaissance/savoir. Si la connaissance maintient « la fixité du sujet qui enquête », le savoir est, en

$122 D E \mathrm{n}^{\circ} 184$, « La fonction politique de l'intellectuel », II, p. 112.

$123 D E \mathrm{n}^{\circ} 102$, « Mon corps, ce papier, ce feu », I, p. 1113-1136.

$124 D E \mathrm{n}^{\circ}$ 281, «Entretien avec Michel Foucault », II, p. 860-914.

125 Histoire de la folie à l'âge classique, Paris, Gallimard, 1972.

$126 D E \mathrm{n}^{\circ} 102$, «Mon corps, ce papier, ce feu », I, p. 1125, nous soulignons. 
amont, « un processus par lequel le sujet subit une modification $[. .$.$] lors$ du travail qu' il effectue pour connaître $\gg^{127}$. C'est en tant que résultat de ce processus de savoir, que le caractère raisonnable du sujet connaissant peut être dès lors défini comme un «statut fixe et déterminé » (ibid., p. 876).

Nous pouvons vérifier que le sens extérieur et institutionnel conféré au terme «statut » par l'entretien de 1976 est dans les deux cas absent. L'accent est mis sur le résultat d'un engagement du sujet dans son propre savoir qui le transforme lui-même. Le sujet doit se constituer comme n'étant pas fou, mouvement qui n'est pas d'abord effectué par un partage social, mais par un acte réflexif, c'est-à-dire un rapport à soi. Un tel rapport n'est pas applicable par décret, mais il s'établit dans une durée, à l'échelle de la vie individuelle comme de l'histoire générale. C'est «peu à peu» et « euxmêmes », précise Foucault en 1980, que «les hommes s'enserrent [... dans et par la manifestation du vrai $\gg^{128}$. Pour reprendre l'exemple de la folie, on pourrait dire que le sujet occidental se qualifie à partir de l'âge classique comme raisonnable devant l'objet folie (qui se constitue corrélativement dans ce même mouvement), tandis que certains sujets seulement reçoivent, sous certaines conditions, le statut de médecin pour parler de cet objet. Cela ne veut pas dire pour autant que les deux notions soient exclusives l'une de l'autre; nous allons aussitôt voir pourquoi.

Il faut en effet préciser la manière dont les trois domaines ainsi différenciés - « vérité », « régime de vérité » et « régime politique »- peuvent et doivent être articulés. Pour plus de clarté, nous pourrions rebaptiser ces domaines « conditions structurelles », « conditions subjectives » et « conditions juridico-politiques » de la vérité. Dans le cours de 1979-1980, le «savoir » fait le lien entre les deux dernières conditions. Par rapport à l'entretien pour Il Contributo, le cours décale ainsi légèrement son champ d'application. Distingué en 1978 de la «connaissance », le «savoir» semblait alors exactement recouvrir la définition d'un régime de vérité comme engagement et transformation du sujet. En 1980, le «savoir » ne désigne plus ce régime particulier, mais son articulation sur des institutions, des lois, des codes sociaux : «Le régime de savoir, c'est le point où s'articulent un régime politique d'obligation et de contrainte et ce régime d'obligation et de contrainte particulier qu'est la vérité $\gg^{129}$.

$127 D E \mathrm{n}^{\circ} 281$, « Entretien avec Michel Foucault », II, p. 876 .

128 Leçon du 6 février 1980.

129 Leçon du 6 février 1980. 
Si un régime de vérité s'articule avec un régime politique dans un «savoir », il s'articule en même temps avec une systématicité des discours dans une «méditation ». Dans la réponse à Jacques Derrida de 1972, la méditation cartésienne est exactement décrite comme ce point de rencontre entre :

$[\ldots]$ un ensemble de propositions formant système $[\ldots]$ et un ensemble de modifications formant exercice, que chaque lecteur doit effectuer, par lesquels chaque lecteur doit être affecté, s'il veut être à son tour le sujet énonçant, pour son propre compte, cette vérité. ${ }^{130}$

La deuxième articulation trouve ici son modèle. Les Méditations métaphysiques de Descartes sont ainsi un «entrecroisement de la trame démonstrative et la trame ascétique » (ibid., p.1126). D'un côté, il y a un discours qui a ses règles propres d'enchaînement des énoncés, et de l'autre, des «états » (p.1125) subjectifs produits par ces énoncés, qui rendent possibles en retour de nouveaux énoncés et de nouvelles chaînes de déduction. Dans son article, Foucault retrace les différentes étapes de la qualification du sujet comme «sujet raisonnable» et «sujet doutant » en croisant sans cesse les deux mouvements : du sujet méditant, en son actualité, vers la pensée ; et de la pensée, en ses déductions pures, vers le sujet méditant. Le sujet est donc essentiellement mobile, passant par une succession de transformations dont la description par Foucault nous indique que la totalité de l'être du sujet est ici engagée et affectée. Les effets de ce que le cours de 1979-1980 nomme la « force du vrai $»^{131}$ trouvent ainsi dès 1972 une description adéquate : ils sont d'ordre cognitif (《lumière », «obscurité »), moral (《pureté »), «impureté $»)$ ou encore psychologique ( « sérénité », « contrainte des passions $\gg, ~\langle\text { détachement } \gg)^{132}$. Notons que cette lecture très fine des Méditations contraste quelque peu avec la leçon du 6 janvier 1982, dans L'herméneutique du sujet, où le moment cartésien ne sera plus que le divorce consommé entre vérité et ascèse, science et spiritualité ${ }^{133}$.

$130 D E \mathrm{n}^{\circ} 102$, « Mon corps, ce papier, ce feu », I, p. 1126 ; l'auteur souligne.

131 Leçon du 6 février 1980.

$132 D E \mathrm{n}^{\circ} 102$, « Mon corps, ce papier, ce feu », I, p. 1125.

133 L'herméneutique du sujet, p.19-20. La leçon provoque l'agacement des cartésiens : voir P. Guenancia, «Foucault/Descartes : la question de la subjectivité », Archives de philosophie, t. 65, vol. 2, 2002, p. 239-254. L’aspect ascétique des Méditations reviendra cependant dans les entretiens d'avril 1983 avec Hubert Dreyfus et Paul Rabinow. Mais Foucault réaménage curieusement le texte de l'entretien, de la version anglaise traduite par Gilles 
Tentons de préciser la nature des articulations ainsi nommées « savoir » et «méditation ». La première obéit clairement au modèle stratégique - elle opère en effet dans l'hétérogénéité de pratiques historiques : pratiques d'un sujet et pratiques collectives. La seconde est plus délicate à qualifier étant donné que certains passages des Méditations « peuvent se déchiffrer, de manière exhaustive, comme enchaînement systématique de propositions $\gg^{134}$, tandis que d'autres forment des « chiasmes » entre ascèse et déduction. De tels chiasmes revêtent-ils dès lors un caractère nécessaire? Autrement formulé : les états du sujet peuvent-ils être logiquement déduits des résultats de la cogitation? La progression méthodique des Méditations pourrait exclure radicalement le modèle stratégique - étranger qu'il est à toute forme de nécessité - et nous forcer à répondre par l'affirmative. Cependant, un retour tardif au texte de Descartes dans le cours au Collège de France du 3 mars 1982 est sur ce point décisif. Foucault y réinscrit la méditation cartésienne dans la tradition de la spiritualité et la définit comme un «jeu effectué par la pensée sur le sujet lui-même $\gg^{135}$. La métaphore du jeu n'est pas fortuite. Sans hésiter, Foucault reprend ici, pour décrire les rapports de la pensée au sujet, une notion appartenant depuis les conférences de Rio à l'analyse stratégique ${ }^{136}$. On est donc en droit de tenir le caractère imprévisible et contingent des effets d'un tel jeu sur les partenaires.

Le passage méthodologique de deux termes à trois n'est pas un changement purement quantitatif. Foucault a beau protester a posteriori qu'il n'avait jamais confondu pouvoir et savoir ${ }^{137}$, une expression comme celle

Barbedette $\left(D E \mathrm{n}^{\circ} 326\right.$, « À propos de la généalogie de l'éthique : un aperçu du travail en cours », II) à la version française publiée par ses soins $\left(D E n^{\circ} 344\right.$, « À propos de la généalogie de l'éthique : un aperçu du travail en cours $\gg$, II). Initialement, la réponse à la question sur l'âge classique commençait par la mention des Méditations comme «pratiques de soi $\gg\left(D E n^{\circ} 326\right.$, p. 1229), inscrites dans une vieille tradition ascétique, puis notait l'inflexion vers l'accès immédiat à la vérité par l'évidence. La version française fait l'inverse et mentionne Descartes d'abord comme rupture ( $D E \mathrm{n}^{\circ} 344$, p. 1449), ajoutant ensuite une phrase à l'intérieur du paragraphe pour rappeler la démarche des Méditations comme « rapport de soi à soi ». L’accent est mis désormais sur la coupure cartésienne.

$134 D E \mathrm{n}^{\circ}{ }_{102}$, « Mon corps, ce papier, ce feu $\gg$, I, p. 1126.

135 L'herméneutique du sujet, p. 340, nous soulignons.

$136 D E n^{\circ} 139$, « La vérité et les formes juridiques », I, p. 1407.

$137 D E \mathrm{n}^{\circ} 330$, «Structuralisme et poststructuralisme », II, p. 1273-1274; $D E \mathrm{n}^{\circ} 350$, « Le souci de la vérité $\gg$, II, p. 1495 . 
rencontrée en 1976 de «politique de la vérité » ne faisait pas exagérément droit à l'autonomie des domaines. Le régime de vérité, tel que le cours $D u$ gouvernement des vivants le définit, devient au contraire une notion beaucoup plus souple, qui permet de ne pas retomber dans l'opposition binaire science/politique, sans dissoudre pour autant le savoir dans le pouvoir.

Entre les deux domaines préalablement intriqués, vient ainsi se loger à partir de 1980 un ensemble d'effets autonomes qui composent la « force du vrai » sur le sujet. Chaque régime a ainsi sa «force» singulière : si certains régimes supposent un engagement et une transformation totale du sujet, d'autres assignent au contraire à ce sujet une place que l'on pourrait qualifier de « fixe ». Cet adjectif spatial apparait conjointement dans la réponse à Derrida et dans l'entretien d'Il Contributo, et permet à Foucault d'éviter le flou épistémologique de l'adjectif $\ll$ neutre $\gg^{138}$. Dire que le sujet est «fixe »- dans le cadre par exemple de l'acte de connaissance scientifique - ne veut donc pas dire que la vérité se passe de régime, mais que son régime propre est de faire entrer les sujets dans un jeu où ils se disposent à être contraints par la vérité elle-même.

$\mathrm{Au}$ lieu de répéter inlassablement que «la vérité n'est pas horspouvoir $\gg{ }^{139}$, Foucault développe ainsi une analyse différenciée qui déploie les régimes de vérité sur une ligne allant de la vérité s'efforçant de ne tenir que d'elle-même ( «auto-indexation du vrai ${ }^{140}$ ) à la vérité ne tenant que par ce qui lui est extérieur (vérité dogmatique, non vérifiable). Le premier régime de vérité, dont la logique constitue le paradigme, a cette propriété particulière de régner pour s'annuler lui-même; le second désigne au contraire un régime hypertrophié, qui n'est plus que contrainte.

Où placer alors sur cette ligne les régimes religieux de vérité ? En prenant l'exemple du régime chrétien de la vérité, Foucault semble accrocher ceux-ci, sinon à l'extrême opposé du régime scientifique, au moins à bonne distance. Ils en sont « fort éloignés », dit-il en 1980 (ibid.). Mais cela ne signifie pas que la vérité chrétienne n’a pas des procédures de régulation interne de son discours indépendantes des sujets, ou, plus précisément, qui maintiennent la fixité du sujet dans l'opération de connaissance. Il n'est donc pas exclu - pensons à la scolastique médié-

$138 D E \mathrm{n}^{\circ} 102$, « Mon corps, ce papier, ce feu », I, p. $1125 ; D E \mathrm{n}^{\circ}$ 281, « Entretien avec Michel

Foucault $\gg$, II, p. 876 .

$139 D E \mathrm{n}^{\circ} 184$, « La fonction politique de l'intellectuel », II, p. 112.

140 Leçon du 6 février 1980. 
vale - que la théologie comme discours de connaissance puisse accéder à une relative autonomie. C'est même l'un des thèmes de la leçon du 6 janvier 1982 au Collège de France, qui mentionne la longue lutte d'une certaine pensée théologique pour se dégager de l'exigence spirituelle ${ }^{141}$. Mais avant le $v^{e}$ siècle, Foucault insiste d'abord sur le lien qui semble systématiquement s'établir entre le contenu de la foi et une certaine attitude du sujet : l'acte de foi «ne consiste pas simplement à affirmer ces choses pour vraies en soi-même et pour soi-même, mais $[\ldots]$ doit aussi donner des gages, des preuves, des authentifications extérieures selon un certain nombre de règles de conduite ou des obligations rituelles $\gg^{142}$. Foucault va même plus loin et assimile simplement et directement la vérité chrétienne à son régime de vérité. Si le régime logique tend à n'être que sa vérité, la vérité chrétienne tend à n'être que son régime : «Le christianisme, c'est la religion de la confession, à la charnière de la foi et de l'aveu $\gg^{143}$.

Postérité de la notion de «régimes de vérité »:

vers le domaine de l'éthique?

Le vocabulaire utilisé par Foucault pour décrire un régime de vérité - « exercice de soi sur soi », « élaboration de soi par soi », «transformation de soi par soi $\gg^{144}$ - nous permet de le relier directement à ce qui sera désigné quelques années plus tard par le terme d'《éthique ». On objectera cependant que le champ de l'éthique se distingue certes du savoir, mais également et surtout du pouvoir. Or, ce dernier est irréductible à un régime juridico-politique. En effet, Foucault n'a eu de cesse de le répéter, les lois et institutions politiques ne sont tout au plus que des relais d'un pouvoir essentiellement polymorphe et réversible, qui se joue d'abord au niveau des relations physiques entre groupes et individus. Ce décrochage entre le pouvoir et le domaine juridico-politique laisserait entendre que la tripartition savoir/pouvoir/éthique n’a

141 L'herméneutique du sujet, p. 28.

142 Leçon du 6 février 1980.

143 Leçon du 30 janvier 1980, nous soulignons. Ce que Foucault reprend de manière encore plus abrupte dans une conférence donnée à New York quelques mois après le cours parisien : « Le christianisme, comme chacun sait, est une confession $\gg\left(D E \mathrm{n}^{\circ} 295\right.$, « Sexualité et solitude », II, p. 990, nous soulignons).

144 Leçon du 13 février 1980. 
pas encore trouvé en 1980 sa forme achevée, se contentant d'une formulation encore maladroite : vérité / régime juridico-politique / régime de vérité. Il est effectivement habituel de situer l'apparition de la distinction pouvoir /éthique dans les travaux de Foucault portant sur l'Antiquité grecque, aurore d'un rapport à soi où apparaîtrait dans sa pureté éclatante l'autonomie d'une subjectivité encore déliée. Au contraire, la notion de «régime de vérité », pensée ici en contexte chrétien, serait déjà une modalité du pouvoir, qui forcerait par là même Foucault à resserrer ce qui s'en distingue dans le champ étroit du juridico-politique.

Tout ceci ne survit pas à une brève étude des textes. Revenons un moment sur cette tripartition - sans doute trop vite interprétée comme une doxa - du savoir, du pouvoir et de l'éthique. Quand on regarde de près les explications qu'en donne Foucault lui-même entre 1980 et 1984, on se rend compte qu'elles sont pour le moins fluctuantes, manifestant d'un texte à l'autre de nombreux décalages, voire même des permutations. Il faut attendre le printemps 1983 pour que le « pouvoir » soit nommément isolé comme un champ d'étude parmi d'autres. Ce découpage tardif peut être interprété comme une longue réticence à user d'un terme aussi général pour décrire l'un des trois axes. C'est dans trois textes de 1983, quasiment contemporains, rédigés ou relus sur une même période de quelques mois $^{145}$, que le pouvoir est isolé de la sorte, par distinction du savoir et de l'éthique, avant de revenir dans le dernier cours au Collège de France et dans l'ultime entretien accordé par le philosophe ${ }^{146}$. Avant cette date, Foucault insiste au contraire à plusieurs reprises sur le fait que le nouvel axe d'étude apparu dans les recherches sur l'Antiquité - baptisé alors «techniques de soi », « rapport à soi », «subjectivité » - est une modalité parmi d'autres d'exercice du pouvoir entendu en son sens le plus large de gouvernement des conduites. En particulier, le résumé du cours de 19801981, Subjectivité et vérité, situe les techniques de soi à la croisée de la subjectivité et de la gouvernementalité147. Le pouvoir n'est donc pas à côté du soi.

$145 D E n^{\circ} 326$ (et 344), II : «À propos de la généalogie de l'éthique : un aperçu du travail en cours », p. $1212 ; D E n^{\circ} 338$, «Usage des plaisirs et techniques de soi », II, p. $1359 ; D E$ $\mathrm{n}^{\circ} 339$, « Qu'est-ce que les Lumières? », II, p. 1395 .

$146 \mathrm{DE} \mathrm{n}^{\circ} 354$, «Le retour de la morale », II, p.1516. Rappelons que cet entretien a été mené alors que Foucault était déjà dans un grand état d'épuisement. Il ne le relut pas. L'écrivain américain Edmund White, qui connut Foucault, fit un sévère compte-rendu de cette ultime captation dans son autobiographie : Mes vies, P. Delamare trad., Paris, Plon, 2006, p. 228.

$147 D E \mathrm{n}^{\circ}$ 304, «Subjectivité et vérité », II, p. 1033. 
Dans ces conditions, si l'axe du « rapport à soi » est finalement distingué à partir de 1983 de celui du « pouvoir », il semble qu'il faille alors entendre « pouvoir » en un sens plus restreint que celui qu' il avait auparavant. La restriction s'avère d'autant plus nécessaire que ce « rapport à soi $\gg$ ne perd à aucun moment sa dimension de contrainte, inséparable qu'il est du rapport à autrui et à une communauté ; c'est même le thème du livre abandonné en 1983 sur Le gouvernement de soi et des autres $^{148}$. Jusque dans les explications les plus tardives de Foucault, le « rapport à soi » renvoie à des formes historiques et culturelles précises, par lesquelles les individus « peuvent et doivent se reconnaître comme sujets $\gg^{149}$, n'abandonnant jamais complètement une forme extérieure d'obligation. Ce n'est en aucun cas la notion d'autonomie qui définit alors «l'éthique » mais celle de réflexivité. Et c'est uniquement au sein de cette réflexivité que l'analyse peut évaluer, dans des contextes historiques différents, le plus et le moins d'autonomie. Le résumé du cours Subjectivité et vérité (1980-1981) nous fournit à ce sujet une indication précieuse : au lieu d'un partage qualitatif entre autonomie et dépendance, Foucault propose une gradation progressive de la dépendance à l'intérieur du rapport à soi. Ainsi, la connaissance de soi est dite « possible, souhaitable ou même indispensable $\gg^{150}$; l'expérience de soi se fait quant à elle à travers des schémas « définis, valorisés, recommandés, imposés » (ibid., p. 1032) ; enfin, les techniques de soi sont « proposées ou prescrites $\gg($ p. 1032). Du « possible » à l'《indispensable », $\mathrm{du}$ «proposé » au « prescrit », cette quantification de l'autonomie est cohérente avec la définition de l’attitude critique donnée lors de la communication de 1978 devant la Société française de philosophie : non pas une résistance absolue ou une liberté originairement rétive, mais un perpétuel effort de modération, en quête de la « juste mesure ».

Je ne veux pas dire par là que, à la gouvernementalisation, se serait opposée dans une sorte de face à face l'affirmation contraire, «nous ne voulons pas être gouvernés, et nous ne voulons pas être gouvernés $d u$ tout ». [...] En face, et comme contre partie, ou plutôt comme partenaire et adversaire à la fois des

148 Livre proposé au Seuil à la fin 1982 (voir $D E$ I, p. 85). Un entretien de janvier 1984 reprend cette idée : «la gouvernementalité implique le rapport de soi à soi » $\left(D E \mathrm{n}^{\circ} 356\right.$ : «L'éthique du souci de soi comme pratique de la liberté », II, p.1547). Sur ce même thème, voir aussi pages 1533-1534.

$149 D E n^{\circ} 338$, « Usages des plaisirs et techniques de soi », II, p. 1359, nous soulignons.

$150 D E n^{\circ} 304$, « Subjectivité et vérité », II, p. 1032. 
arts de gouverner, comme manière de s'en méfier, de les limiter, de leur trouver une juste mesure $[. .$.$] il y aurait eu quelque chose en Europe [. .$.$] que j'appel-$ lerais tout simplement l'art de n'être pas gouverné ou encore l'art de n'être pas gouverné comme ça et à ce prix. Et je proposerais donc, comme toute première définition de la critique, cette caractérisation générale : l'art de n'être pas tellement gouverné. ${ }^{151}$

Ce texte, qui aborde la question de la «juste mesure $\gg$ dans les arts de gouverner, est important. Pour éviter de l'entendre en un sens trivial, il convient de l'inscrire dans une tradition philosophique qui lui donne toute sa force. Plutôt que de le rapprocher de la métaphore par trop souple $\mathrm{du} \ll \mathrm{Pli} \gg$ deleuzien ${ }^{152}$, il serait plus fructueux de se reporter à l'exercice de la sensibilité chez Aristote, qui révèle avec le texte de Foucault des analogies troublantes. Pour sentir le chaud ou le froid, il ne faut pas l'être en acte - le semblable n'a pas la sensation du semblable - mais il faut l'être en puissance, afin de pouvoir mesurer une différence. Toute sensation conserve nécessairement avec elle la potentialité du contraire, de telle manière qu'il ne saurait y avoir de saturation de la sensibilité, par excès de la chose sentie ${ }^{153}$. À distance des extrêmes, le «toucher » est défini par le philosophe macédonien comme un «juste milieu » ou, plus précisément, un «moyen terme $\gg(\mu \dot{\varepsilon} \sigma o v)^{154}$ entre des couples de contraire (chaud/froid; dur/mou; sec/humide). Le régime des sens offre alors à Aristote cette proposition étonnante : «le moyen terme en

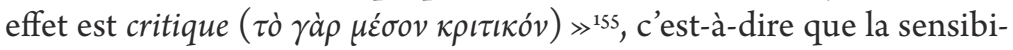
lité s'apparente à un jugement, qui permet un juste toucher. Remis dans sa corporéité, le rapport à soi est exactement chez Foucault l'exercice du moyen terme, entre ces extrêmes potentiels que sont l'autonomie et la domination. On ne sera pas étonné de retrouver la notion de $\mu \varepsilon \dot{\varepsilon} \sigma o v$

151 «u'est-ce que la critique? », p. 37-38, c'est l'éditeur du texte qui souligne. Dans la discussion qui suit la communication, Foucault précise à nouveau que la critique ne renvoie pas à un « anarchisme fondamental, qui serait comme la liberté originaire rétive absolument et en son fond à toute gouvernementalisation » (p. 59).

Deleuze utilise cette métaphore pour décrire l'axe de la subjectivité dans le « dernier »

Foucault : G. Deleuze, Foucault, Paris, Éditions de Minuit, 1986, p. 107 et suiv.

153 Aristote, De l'âme, 423b 12-424a 17.

$154 \mathrm{Ibid}$., 424a 6. D’autres commentateurs préfèrent traduire $\mu \varepsilon \dot{\varepsilon} \sigma o v$ par «médiété ». Voir par exemple P. Pellegrin, Le vocabulaire d'Aristote, Paris, Ellipse, 2001, p. 59.

155 Aristote, De l'âme, 424a 6. Edmond Barbotin traduit pour sa part : « La moyenne ( $\mu \varepsilon \dot{\sigma o v}$ ) est capable de juger » (Aristote, De l'âme, Livre II, 1, E. Barbotin trad., Paris, Les Belles Lettres, 2002, p. 35). 
comme définition chez Aristote de la vertu ${ }^{156}$ - la vertu étant cette disposition qui accomplit toutes les facultés de l'âme, sensibilité comprise.

Une telle considération nous invite à restreindre l'axe du pouvoir, à partir du moment où Foucault choisit de le distinguer de celui du rapport à soi et de l'éthique. La mise en synopse des textes présentant, entre 1980 et 1984, la tripartition indiquée plus haut permet de vérifier cette hypothèse : l'usage du terme «pouvoir» à partir de 1983 apparaît chronologiquement encadré par des expressions telles que «normativité des comportements $\gg 157$, « pratiques de contrôle ${ }^{158}$ et surtout, « politique $\gg$ et $\ll$ structure politique $\gg 159$. Or, c'est bien ce point qui nous intéresse en premier lieu : il arrive à Foucault de resserrer son usage du terme « pouvoir », au point de le faire coïncider avec celui de « politique $»$. Bien entendu, il ne faut pas entendre ici par «structure politique » l'exercice unilatéral d'une légitimité et d'une unité première (l'État), mais bien un jeu stratégique entre des institutions, des individus et des lois.

Concluons ce parcours qui a permis de définir la notion centrale du cours $D u$ gouvernement des vivants. Dans l'expression « régime de vérité », le premier terme semble en apparence tirer vers le pouvoir, tandis que le second voudrait rejoindre le savoir. Foucault va au contraire insister en 1980 sur la stricte autonomie de cet espace où un sujet se modifie dans son rapport à la vérité. Nous avons établi qu'un tel espace était absent des précédents usages, d'ailleurs relativement rares, de l'expression « régime de vérité » chez Foucault. Si le cours de 1979-1980 est sur ce point d'une radicale nouveauté, il est cependant possible de retrouver des mentions précoces de cette dimension ascétique de la subjectivité à l'occasion de la controverse avec Jacques Derrida et d'un important entretien de 1978. Si le terme « régime de vérité $»$ n'apparaît dans aucun de ces deux textes,

156 Aristote, Éthique à Nicomaque, II, 6, 110723.

157 Le 5 janvier 1983 au Collège de France, Foucault explique comment son analyse s'est déplacée d'une «analyse de la norme » à celle des « exercices du pouvoir » puis aux «procédures $[\ldots]$ de gouvernementalité » (Le gouvernement de soi et des autres, p. 6). C'est dans la leçon suivante qu'il utilise la tripartition : axe de la formation des savoirs / axe de la normativité des comportements / axe de constitution du mode d'être du sujet (ibid., p. 41).

$158 D E \mathrm{n}^{\circ} 356$, «L'éthique du souci de soi comme pratique de la liberté », II, p. 1528, où Foucault parle également de «pratiques coercitives ».

$159 \mathrm{DE} \mathrm{n}^{\circ} 342$, « Polémique, politique et problématisations », II, p. 1414. 
s'y dessine cependant une première esquisse de cet espace autonome que recouvrira par la suite le champ des «techniques de soi $\gg$.

Cette nouveauté thématique semble néanmoins se faire jour sur fond d'une continuité méthodologique par rapport aux études des années 1970 : les articulations nouvelles entre les domaines, que nous avons respectivement nommées méditation (articulant les conditions structurelles et subjectives de la vérité) et savoir (articulant les conditions subjectives et juridico-politiques de la vérité), vérifient la cohérence de la notion de « régime de vérité » avec le grand principe de l'analyse stratégique : la rencontre subreptice de champs d'activité hétérogènes. À ce niveau épistémologique, il semble alors possible d'inscrire le cours $D$ u gouvernement des vivants (1979-1980) dans la suite du cours Sécurité, territoire, population (1977-1978), et plus généralement dans la continuité d'une historiographie qui considère l'histoire comme un champ de relations stratégiques entre des sujets, des savoirs et des pratiques instituées. L'ajout d'un nouvel espace de recherche - celui de la subjectivité - ne semble pas en effet modifier le type de connexion établi entre les espaces. Il nous faut cependant le vérifier, au contact du cours lui-même et de ses exemples historiques.

Une double réduction de l'objet «christianisme»

L'analyse proposée du christianisme comme « régime de vérité » n'est pas aussi ténue, encore moins réductrice, qu' il y paraît. Elle pose simplement, à un niveau méthodologique, qu'entre la vérité, comme discours établissant ses principes internes de régulation indépendamment des sujets, et la politique, comme pratique instituée limitant de l'extérieur ce que les sujets peuvent dire ou faire, ily a place pour une analyse autonome de la manière dont la subjectivité est déterminée par la vérité. Cela ne signifie pas que la vérité et la politique, c'est-à-dire ici la théologie et l'organisation ecclésiale, sont exclues des recherches de Foucault sur le christianisme, bien au contraire : non seulement il y a bien articulation entre les domaines, mais il est possible d'utiliser certains outils laissés en d'autres endroits de l'œuvre pour esquisser ce que pourrait être une histoire « politique » et une histoire $\ll$ dogmatique $\gg$ du christianisme selon Foucault ${ }^{160}$. Pour 
autant, ces trois histoires possibles ne sont pas dans la continuité d'une même idéologie ou d'une même mentalité : elles se croisent uniquement dans l'hétérogénéité historique de pratiques et dans les jeux de la pensée avec la subjectivité. En ce sens, le cours de 1979-1980, s'il modifie en profondeur la définition de chaque domaine respectif, ne semble pas remettre en question les principes de l'analyse stratégique que nous avons exposés dans la première partie de notre travail.

Dans ses leçons de 1980 sur les premiers siècles chrétiens, Foucault se livre à une double opération sur l'objet traditionnellement défini comme étant le «christianisme » : il le réduit quant à l'espace et le fragmente quant au temps. Il obtient ainsi non pas un grand cercle fermé, mais des lignes brisées. Relevons les deux réductions successives pratiquées dans la leçon du 30 janvier :

1) le christianisme sera étudié non pas comme un système de dogmescroyances mais comme un régime de vérité, c'est-à-dire un ensemble organisé d'actes dans lesquels des sujets s'engagent personnellement;

2) à l'intérieur de ce régime de vérité, les actes d'aveu seront privilégiés, voire radicalement séparés des actes de foi.

Cette double réduction a une conséquence immédiate sur les corpus et les périodes étudiés par Foucault : non pas tout le christianisme, mais une certaine lignée d'actes de vérité apparue à la fin du $\mathrm{II}^{\mathrm{e}}$ siècle, l'injonction à manifester la vérité de soi. Or, cette injonction, rappelle à plusieurs reprises Foucault, l'Église primitive l'ignorait complètement. Elle n'est donc pas inscrite dans ce qui serait la vérité originelle du christianisme, contenue dans ses premiers écrits ou ses premières expériences communautaires. Pas d'aveu dans la Didachè - ce texte majeur de la fin du I $^{\text {er }}$ siècle ; pas d'examen de soi chez un auteur comme Clément d'Alexandrie (né vers 150); pas de verbalisation des fautes dans les premières préparations baptismales. Par contre, à compter des $\mathrm{II}^{\mathrm{e}}$-III ${ }^{\mathrm{e}}$ siècles, commence pour le christianisme « une histoire qui est profondément nouvelle ${ }^{161}$.

Que veut donc dire encore ici « christianisme » ? Il faudrait répondre que ce n'est justement pas le problème de Foucault. Celui-ci serait plutôt : comment faire l'histoire des actes d'aveu dans leur proximité et leur distance avec, d'une part, les actes de foi, et d'autre part, les ensembles discursifs et institutionnels qui précèdent tout acte du sujet (les dogmes, 
l'épiscope). Il s'agit donc, pour reprendre un néologisme souvent utilisé par Foucault, d'《événementialiser ${ }^{162}$ un régime de vérité, c'est-àdire de rendre intelligible son surgissement dans l'histoire, à partir d'un réseau complexe d'éléments hétérogènes.

Remarquons cependant aussitôt que l'hétérogénéité ne vaut pas pour le rapport entre les actes de foi et les actes d'aveu, dont la continuelle intrication forme au contraire, au moins à partir du $\mathrm{IV}^{\mathrm{e}}$ siècle, l'ensemble homogène de ce que Foucault nomme le « régime général de vérité ${ }^{163}$ chrétien. Si les actes de foi et les actes d'aveu ont des $\ll$ morphologies bien différentes $\gg$ (ibid.), ils se suivent de près comme deux moments successifs d'une même manifestation, s'appuyant toujours l'un sur l'autre. Dire ce que l'on est et dire que l'on croit au dogme; ou encore : dire que l'on est pécheur et dire que l'on croit en un Dieu sauveur, sont les deux pôles de l'expérience chrétienne, deux modalités distinctes d'un même devoir de confession, qui suppose à chaque fois un engagement total de la part du sujet ${ }^{164}$. En particulier, les actes de foi ne sont pas de simples proclamations de vérités extérieures mais bien des « acceptations-engagements $»$, des « adhésionsfidélités » de la part du catéchumène ou du baptisé165. Pas de parole vraie sur Dieu, donc, qui ne s'accompagne d'une parole vraie sur soi. Quelques mois après la fin du cours de 1980, ce lien est à nouveau fermement affirmé dans une conférence donnée aux côtés de Richard Sennett à l'Institute for the Humanities de New York. On retrouve bien les deux séries d'actes, formant une unité qui, pour être apparue dans l'histoire, n'en est pas moins jugée indissoluble, non pas d'un point de vue doctrinal extérieur, mais pour le sujet que Foucault nomme « chrétien $\gg^{166}$. Notons pour le contexte de ce passage, que cette unité décrite pour un sujet permet ensuite à Foucault de distinguer les techniques chrétiennes des techniques bouddhistes :

Ces deux ensembles d'obligations - celles qui concernent la foi, le livre, le dogme, et celles qui concernent le soi, l'âme et le cœur - sont liés. Un chrétien

$162 D E \mathrm{n}^{\circ} 278$, « Table ronde du 20 mai 1978 », II, p. 842 ; « Qu'est-ce que la critique? », p. 47-48.

163 Leçon du 6 février 1980.

164 Leçon du 20 février 1980.

165 Leçon du 6 février 1980.

$166 \mathrm{Si}$ Foucault commence par parler en termes généraux - «le christianisme », «le bouddhisme »-, il préfère ensuite utiliser les termes «le bouddhiste » et « un chrétien », pour décrire les actes posés : $D E n^{\circ} 295$, «Sexualité et solitude », II, p. 991. C'est donc bien le sujet et sa pratique qui font l'unité de ces actes. 
a besoin de la lumière de la foi s'il veut sonder qui il est. Et inversement, on ne peut concevoir qu'il ait accès à la vérité sans que son âme soit purifiée. (Ibid., p. 990-991)

La même circularité entre connaissance de soi et connaissance de la vérité déposée dans les Écritures sera reprise dans la leçon du 17 février 1982 : la connaissance chrétienne de soi est une purification en vue d'accueillir la Parole de Dieu, et en retour, la Parole reçue guide la connaissance de $\operatorname{soi}^{167}$. Ces deux connaissances désignent bien à chaque fois un effort du sujet, qui ne se contente pas de le doter de savoirs ou d'aptitudes (pédagogie) mais qui modifie son mode d'être (psychagogie ${ }^{168}$ ).

Par contre, les croisements de ces deux régimes de vérité avec, d'une part, l'économie dogmatique chrétienne et, d'autre part, l'institution ecclésiale sont bien des événements qui n'obéissent à aucune nécessité intrinsèque, dictée par ce que serait le «christianisme » comme totalité historique. Deux cas de figure sont repérables dans le cours de 19791980 : tantôt l'institution ecclésiale et le dogme reprennent et intensifient les pratiques de soi ; tantôt les pratiques de soi se distinguent au contraire du dogme et des obligations canoniques.

Prenons pour commencer deux exemples de distinction. Apparue dans le monde chrétien au IV ${ }^{\mathrm{e}}$ siècle, une pratique de soi comme la direction de conscience n'a été institutionnalisée que très localement durant un demi-millénaire : dans la vie monastique, exclusivement. Foucault va même plus loin en soulignant l'indépendance originelle de la pratique de direction par rapport à cette forme constitutive du pouvoir ecclésial que Sécurité, territoire, population avait définie comme pouvoir pastoral : «Le thème du pouvoir pastoral n'implique pas une technique de direction,

167 L'herméneutique du sujet, p. 245.

168 Ibid., p. 389-391. L'étymologie indique bien le sens visé : en grec, $\psi v \chi a \gamma \omega \gamma i ́ a$ vient de $\psi v \chi \eta ́$ (âme) et á $\gamma \omega \gamma o ́ s$ (qui conduit). Comme Michel Senellart l'a indiqué dans un article important («La pratique de la direction de conscience», Foucault et la philosophie antique, F. Gros et C. Lévy éd., Paris, Kimé, 2003, p. 153-154), Foucault emprunte ce terme à Paul Rabbow (Seelenführung, Methodik der Exerzitien in der Antike, Münich, KöselVerlag, 1954), par l'intermédiaire d'Ilsetraut Hadot (Seneca und die grieschich-römische Tradition der Seelenleitung, Berlin, Walter de Gruyter \& Co., 1969). Rabbow n'est cependant pas l'inventeur de «Psychagogik». D'abord parce que lui-même reconnaît dans son livre l'avoir emprunté au psychanalyste Georg Wanke et au psychologue Adolf Zeddies. Ensuite, parce que le mot existait déjà dans la littérature grecque, mais avec un sens différent : il désignait une cérémonie religieuse d'invocation des âmes des morts. 
même si plus tard c'est sous le signe du pastorat qu'elle sera placée $\gg^{169}$. Autre exemple de distinction : au sein d'un rituel canonique, une pratique de soi peut se distinguer et devancer l'acquisition des vérités de la foi. Ainsi, dans la préparation au baptême, le mouvement de la pénitence après Tertullien se décroche de l'enseignement des dogmes. La «structure d'épreuve » (discipline pénitentielle) prend ainsi son autonomie par rapport à la «structure d'enseignement » (catéchèse) $)^{170}$.

Soit maintenant deux exemples de reprise et d'intensification. À partir des années 170-180, dans un contexte où le christianisme connait à la fois une diffusion géographique importante, des persécutions politiques et des querelles dogmatiques, on assiste à la reprise ecclésiale et théologique de pratiques de soi qui relevaient auparavant des seuls usages ou traditions communautaires et non d'une procédure codifiée : ainsi de l'institutionnalisation du catéchuménat et du développement de la théologie du baptême ${ }^{171}$. Autre exemple d'une telle reprise : au millénaire suivant, ce sont bien les controverses autour de la définition des vérités de foi et de l'autorité ecclésiale qui provoquent une intensification contrôlée des actes d'aveu, face à l'hérésie cathare puis à la Réforme ${ }^{172}$.

Le « christianisme » n'est donc pas d'abord une idée qui produirait des effets dans l'histoire; il est un croisement singulier de séries autonomes : doctrines, politiques, pratiques de soi. Il ne faut cependant pas considérer ces croisements comme de brusques et soudains retournements. Tout au long du cours de 1979-1980, Foucault insiste au contraire sur la lenteur et surtout la difficulté des transformations décrites - difficulté non seulement externe (pour cause d'hérésies et de persécutions), mais également interne, du fait de la prédominance dans les premiers siècles chrétiens d'un rapport entre le sujet et la vérité encore pensé sur le mode grec de l'illumination définitive ${ }^{173}$. L'apparition de la pénitence, comme dimension permanente de la vie du chrétien, n'est donc pas un basculement de l'histoire mais plutôt une succession de décrochages qui se produisent sur près d'un millénaire. On peut ainsi retrouver dans le cours deux séries de décrochages, qui font appel chaque fois à des

169 Leçon du 19 mars 1980 , nous soulignons.

170 Leçon du 13 février 1980.

171 Leçon du 20 février 1980.

172 Leçon du 30 janvier 1980.

173 Leçons du 13 février 1980. 
éléments singuliers et contingents : le problème pastoral des relaps et le problème doctrinal de la gnose à la fin du $\mathrm{II}^{\mathrm{e}}$ siècle ${ }^{174}$; l'institutionnalisation de la règle monastique et l'introduction du droit germanique à partir du viI ${ }^{\mathrm{e}}$ siècle $^{175}$.

Insister sur ces décrochages serait cependant trompeur au vu de la place marginale qu'ils occupent dans le mouvement d'ensemble des leçons sur le christianisme ancien. Si le modèle stratégique est bien opérationnel en 1980, il semble néanmoins passer au second plan de la description. C'est ce renversement de la topographie habituelle des cours aux Collège de France que nous voudrions maintenant décrire avant de conclure sur l'étude du christianisme comme régime de vérité. Tournant important dans les recherches de Foucault, le cours Du gouvernement des vivants l'est non seulement en ce qu'il fait apparaître un nouveau domaine d'investigation (les régimes de vérité), mais également en ce qu'il modifie les manières de procéder; plus précisément : le rapport aux sources documentaires et la manière de les utiliser dans la description des transformations historiques.

Simple relevé de quelques pratiques de lecture qui se font jour en 1980, les paragraphes suivants annoncent et espèrent justifier à l'avance le long détour de notre deuxième partie, qui reviendra dans le détail sur l'ensemble de ces modifications ${ }^{176}$.

Du cours Sécurité, territoire, population au cours Du gouvernement des vivants, la différence dans le choix des corpus est patente, alors même que les leçons ont en commun un certain nombre de thèmes et d'auteurs, en particulier autour du monachisme ancien. Si le nomadisme dans la description semble encore la loi du cours de 1977-1978 - qui remonte du XIX ${ }^{\mathrm{e}}$ au XIII ${ }^{\mathrm{e}}$ siècle et traverse aussi bien l'épidémie de la variole que le Prince de Machiavel et la mystique -, le cours de 1979-1980 progresse différemment. S'il repasse par des auteurs déjà mentionnés dans les leçons du 15 et 22 février 1978 (comme Cyprien ou Cassien), c'est désormais à l'intérieur d'un corpus historiquement et thématiquement homogène : les textes patristiques. La seule originalité du choix de Foucault est de privilégier à l'intérieur de ce corpus les témoignages

174 Leçon du 27 février 1980.

175 Leçon du 5 mars 1980.

176 Voir Deuxième partie, «Conséquences sur l'usage des textes : traduire les Pères ». 
concernant des pratiques (célébrations liturgiques, rituels du catéchuménat, de la pénitence) sur les traités de théologie (les grandes œuvres dogmatiques d'Origène, des frères cappadociens Basile et Grégoire, et surtout d'Augustin, sont complètement absentes).

Aux rapprochements éclectiques de Sécurité, territoire, population succède donc la lecture systématique et presque chronologique d'un ensemble discursif qui s'étend de la fin du i ${ }^{\text {er }}$ siècle (la Didachè) au début $\mathrm{du}^{\mathrm{e}}$ (les Conférences de Cassien), et dont les contours académiques et confessionnels ne sont pas bousculés. Comme nous aurons l'occasion de le montrer, les œuvres choisies sont parmi les plus commentées des études patristiques et Foucault les lit majoritairement dans les grandes éditions classiques françaises : celle de l'abbé de Genoude ( $\mathrm{XIX}^{\mathrm{e}}$ siècle), celle de Hemmer et Lejay intitulée « Textes et documents pour l'étude historique du christianisme » (début du $\mathrm{Xx}^{\mathrm{e}}$ siècle), et enfin, la collection plus récente des «Sources chrétiennes », fondée en 1943 par les pères Henri de Lubac et Jean Daniélou. En tout, ce sont plus de vingt traités patristiques qui sont utilisés sur sept heures d'enseignement consacrées aux Pères ${ }^{177}$.

S'en tenant strictement à ce corpus prédéfini, le cours ne s'aventure pas hors des sentiers battus : pas de textes marginaux ou d'origine sectaire, même si la gnose est régulièrement évoquée. Les textes retenus par Foucault sont largement reçus par les premières Églises chrétiennes, et certains ont même manqué de peu leur intégration dans le canon des Écritures (ainsi de la Didachè et de l'Épitre de Barnabé). Si le De pudicitia montaniste de Tertullien apparait dans la leçon du 5 mars, c'est d'abord comme témoignage, en creux, de la pratique orthodoxe de la pénitence à son époque. Quant au schisme d'Hippolyte - sans conséquence sur le texte commenté (La tradition apostolique, dans la leçon du 20 février) - il n'est pas même évoqué. Remarquons enfin l'absence de textes appartenant à des domaines extra-religieux : textes juridiques, politiques ou scientifiques.

Mais la différence la plus notable n'est pas dans cet apparent retour à une frugalité érudite ou à une sobriété toute académique. La présence

$177 D$ gouvernement des vivants comprend également cinq leçons consacrées à l'Antiquité gréco-romaine : leçons du 9 janvier au 30 janvier (commentaire d'Édipe roi) et leçon du 12 mars 1980 (sur la direction de conscience gréco-romaine). Pour le décompte des traités patristiques, nous ne gardons que les textes explicitement cités et commentés par Foucault, et non les références plus lointaines (par exemple : une mention sporadique d'Origène, le De anima de Tertullien, ou encore l'unique citation de saint Paul), qui dépendent le plus souvent de sources secondaires. 
des sources textuelles dans le cours n'est plus seulement documentaire : c'est le cours en lui-même qui devient acte de lecture. Foucault intègre pour la première fois dans ses leçons le commentaire de texte. Citant longuement un passage des Pères, discutant un point de traduction, repérant l'occurrence des termes et leurs usages, pratiquant l'étymologie (exomologèse, confession) ${ }^{178}$, le professeur se livre ni plus ni moins à une exégèse des textes patristiques. Des mêmes auteurs, les leçons des 15 et 22 février 1978 n'extrayaient au contraire que des citations brèves, uniquement illustratives, jamais discutées dans leur littéralité. Même la longue présentation d'un passage du Politique de Platon (260e-311c) la même année proposait simplement de «reprendre schématiquement [son] déroulement $\gg^{179}$, sans citer une seule fois le texte, sinon en le paraphrasant. Sans doute parce que l'essentiel ne se jouait pas alors dans les mots mais à leur périphérie : dans les connexions historiques réelles que les textes se contentaient d'indiquer. Désormais au contraire, l'analyse remet les connexions stratégiques à la périphérie et revient à la lettre.

Le second indice de l'effacement du modèle stratégique serait le caractère extrêmement laconique des références historiques et, en particulier, de l'analyse des causes précises des transformations étudiées. Sans développer, encore moins articuler ces causes entre elles, Foucault se contente par moments d'en dresser des listes, comme autant de compilations rébarbatives pour lui-même et l'auditoire. Ainsi de la leçon du 20 février 1980: «Raisons de cette institutionnalisation de l'ordre des catéchumènes ? Je me contente de les signaler $\gg^{180}$. Suit alors la mention désordonnée des persécutions, hérésies, dialogue avec le paganisme, afflux des postulants, etc. Ailleurs encore, les causes deviennent très générales : «Pourquoi dans le monachisme? Deux mots simplement, avec tout ce que ça peut avoir d'aléatoire ${ }^{181}$. Ces deux mots seront,

178 Il est intéressant de noter que les résultats de l'étymologie étaient auparavant historicisés par Foucault comme un objet parmi d'autres de l'analyse : ainsi des remarques dans le cours de 1975-1976 sur les étymologies très politiques du terme «franc » chez l'un des successeurs de Boulainvilliers (Il faut défendre la société, p. 131-132). Voir également les remarques du cours de 1977-1978 sur l'étymologie de vónos chez les pythagoriciens (Sécurité, territoire, population, p.140).

179 Sécurité, territoire, population, p. 144.

180 Leçon du 20 février 1980. La leçon du 5 mars 1980 donne également quelques raisons historiques du développement des actes d'aveu.

181 Leçon du 19 mars 1980. 
sans aucune précision de contexte, l'affrontement du christianisme avec la gnose et le rapport salut/perfection. L'inscription des textes étudiés dans leur tissu historique est si mince - parfois réduite à une simple notice biographique ${ }^{182}$ - qu'elle disparaît même complètement du résumé du cours publié dans l'Annuaire du Collège de France ${ }^{183}$. Or, nous avions vu que non seulement l'analyse stratégique n'évacuait pas la causalité, mais qu'elle la démultipliait. Parmi les causes de l'extension au XIX ${ }^{\mathrm{e}}$ siècle des dispositifs de surveillance, la montée des besoins sécuritaires et économiques tenait ainsi, dans le résumé du cours La société punitive (1972-1973), une place conséquente et bien distincte du reste de l'analyse ${ }^{184}$. À l'opposé, le cours de 1979-1980 semble bien peu se soucier des connexions historiques concrètes entre les pratiques, les discours et les institutions, offrant une lecture en apparence cursive d'un corpus prédéfini de textes. L'homogénéité et la clôture du corpus choisi permettent ici l'analyse autonome d'un régime historique d'actes, dans son rythme propre de transformations. L'étude de l'Antiquité gréco-romaine de 1981 à 1984 reproduira exactement ce modèle, dans la forme lente du commentaire de texte.

Mais cette autonomie nouvelle, qui nous semble avoir été facilitée par la confrontation de Foucault à un corpus déjà unifié d'un point de vue doctrinal et éditorial, modifie la définition du projet d'ensemble. L'historicité des régimes de vérité, rendue ainsi à sa spécificité, maintenue à distance des événements politiques ou sociaux qu'elle ne rejoint que subrepticement, ouvre la possibilité d'écrire une histoire singulière et irréductible des pratiques réfléchies - ce que Foucault appellera plus tard, tout simplement, une « histoire de la pensée ». Dès le cours de 1979-1980, le professeur évoque d'ailleurs la possibilité d'analyser la pensée sans recourir aux idéologies ${ }^{185}$, c'est-à-dire sans supposer que l'activité réflexive, sujette à des illusions telles que les idées politiques ou religieuses, ferait écran à l'exercice réel du pouvoir, aux pratiques de gouvernement.

C'est dans quelques-uns des derniers textes publiés de son vivant que Foucault réinvestit avec une conviction inattendue l'intitulé de sa chaire au Collège de France, « Histoire des systèmes de pensée », qui

182 C'est le cas pour Cassien (ibid.).

$183 D E n^{\circ} 289$, « Du gouvernement des vivants », II, p. 944-948.

$184 D E n^{\circ} 131$, «La société punitive », I, p. 1334-1336.

185 Leçon du 30 janvier 1980. 
opérait déjà un glissement par rapport à l'intitulé de celle de son prédécesseur Jean Hyppolite : « Histoire de la pensée philosophique ». Alors que l'effort de contournement du domaine des représentations, c'està-dire le refus de l'empreinte mnémonique platonicienne, avait éclipsé une décennie durant toute référence à la pensée, Foucault remet celle-ci au cœur de son travail. La définition la plus claire qu'il donne de cette faculté soudain redécouverte se trouve dans la première rédaction - sans doute autour de mars 1983 - de la préface au deuxième volume de l'Histoire de la sexualité :

Par «pensée », j'entends ce qui instaure, dans diverses formes possibles, le jeu du vrai et du faux et qui, par conséquent, constitue l'être humain comme sujet de connaissance; ce qui fonde l'acceptation ou le refus de la règle et constitue l'être humain comme sujet social et juridique; ce qui instaure le rapport avec soi-même et avec les autres, et constitue l'être humain comme sujet éthique. ${ }^{186}$

Si l'on veut élever cette définition à un plus grand degré de généralité, en la délestant de la tripartition particulière savoir/pouvoir/éthique, elle pourrait être reformulée comme suit : la pensée ne désigne pas seulement ce qui, associé à la perception, présente un objet à un sujet selon certaines conditions, mais aussi ce qui légitime (《instaure », «fonde ») chez le sujet l'accès à cet objet, moyennant un certain nombre d'opérations qu'il doit préalablement faire sur lui-même. Cette conception de la pensée joint ainsi au «mode d'objectivation $»$, clause classique de la philosophie de la connaissance simplement historicisée par Foucault, un «mode de subjectivation $\gg$ inédit ${ }^{187}$. Si le mode d'objectivation ouvre un espace de perception et d'intellection bien connu de la tradition critique, le mode de subjectivation constitue un apport original : il fait de l'accès à un objet une question qui ne relève pas seulement de déterminations formelles ou collectives, mais également de l'habitus du sujet, comme activité libre

$186 D E \mathrm{n}^{\circ}$ 340, «Préface à l'Histoire de la sexualité », II, p. 1398. La mention de l'histoire de la pensée est également le porche d'entrée de l'article «Foucault », que l'intéressé rédigea lui-même pour le Dictionnaire des philosophes. Foucault y propose de nommer son travail philosophique une «Histoire critique de la pensée $»\left(D E \mathrm{n}^{\circ} 345\right.$, « Foucault », II, p. 1450).

$187 D E \mathrm{n}^{\circ} 345$, « Foucault », II, p. 1451. Le mode d'objectivation, comme condition de possibilité de la saisie et de la connaissance d'un objet, est en effet chez Foucault un a priori kantien simplement historicisé. Pour qu'un objet apparaisse, il y a des « conditions d'émergence », mais elles ne valent que «pour un temps, une aire et des individus donnés $\gg$ (ibid., p. 1451). D'où l'expression d'《a priori historique » (Les mots et les choses, p. 13). Cette dernière expression est conservée dans le grand article de $1984\left(D E \mathrm{n}^{\mathrm{o}} 345\right.$, «Foucault», II, p. 1451), mais elle englobe désormais aussi les modes de subjectivation. 
et régulière. C'est en commentant le De paenitentia de Tertullien dans la leçon du 5 mars 1980 que Foucault s'arrête sur cette notion d'habitus, qu'il choisit de traduire par $\ll$ manière de vivre $\gg^{188}$. Il ne faut pas seulement être pour faire tel ou tel type d'expérience, mais il faut être exercé. Les exercices spirituels des philosophes de l'Antiquité ne sont qu'un cas particulier d'une vérité générale de l'existence, comme nous le rappelle Montaigne dans ses Essais : le raisonnement et l'instruction n'éclairent l'âme au moment de l'action que si elle s'est préalablement exercée dans des situations concrètes ${ }^{189}$. La pensée chez Foucault établit ainsi des conditions d'expérience qui ne sont pas formelles, mais pragmatiques.

La pensée n'est donc pas ce que le pouvoir contamine de l'extérieur par le biais des idéologies, afin de donner libre cours - à côté et indépendamment - à un gouvernement des corps plus efficace, incitant par exemple le peuple à se soucier de son âme pour le détourner de sa misère sociale. Mais c'est dans la pensée elle-même qu'est engagé un certain gouvernement de soi et des autres. Or, précise aussitôt Foucault en 1983, ce domaine n'est en aucune manière le produit des « déterminations concrètes de l'existence sociale $\gg^{190}$, il leur est irréductible, même s'il n'en est pas indépendant. Il y a une histoire propre aux exercices réfléchis du sujet sur lui-même. Ce que vérifie bien la notion de « régime de vérité » telle que nous venons de la présenter.

188 Leçon du 5 mars 1980 : «C'est une manière de vivre, c'est une manière d'être, c'est une manière de se nourrir. D'ailleurs, le texte [De paenitentia] le dit bien un peu plus haut, c'est une "conversatio", c'est une manière d'exister et d'avoir rapport aux autres, à soi, etc. » $\mathrm{Ce}$ que redit à sa manière l'article pour le Dictionnaire des philosophes : «La question est de déterminer ce que doit être le sujet, à quelle condition il est soumis, quel statut il doit avoir, quelle position il doit occuper dans le réel ou dans l'imaginaire, pour devenir sujet légitime de tel ou tel type de connaissance $\gg\left(D E \mathrm{n}^{\circ} 345\right.$, « Foucault », II, p. 1451).

189 Montaigne, Les essais en français moderne, Livre II, chap. vI : «Sur l'exercice », A. Lanly éd., Paris, Gallimard, 2009, p. 457 : «Il est difficile que le raisonnement et l'instruction, encore que nous ajoutions foi à leurs leçons, soient assez puissants pour nous acheminer jusqu'à l'action si, en outre, nous n'exerçons et ne formons pas notre âme par expérience à l'allure à laquelle nous voulons la régler; autrement, quand elle sera au moment de l'action, elle se trouvera sans aucun doute embarrassée. » Rappelons l'invitation de Foucault au sujet de Montaigne : «Je pense qu'il faudrait relire Montaigne dans cette perspective-là, comme une tentative de reconstituer une esthétique et une éthique du soi » (L'herméneutique du sujet, p. 240).

$190 \mathrm{DE} \mathrm{n}^{\circ} 340$, « Préface à l'Histoire de la sexualité», II, p. 1399. 


\section{CHAPITRE TROIS \\ Foucault et I'historiographie de l'Antiquité tardive}

Si le terme «christianisme » est bien retenu par Foucault en 1980, il doit être entendu pour le moment comme une synecdoque. Àce stade de notre étude, si l'on s'en tient à une définition stricte des régimes de vérité, Foucault n'étudie qu' un champ de pratiques très limité (les actes d'aveu apparus au tournant du $\mathrm{III}^{\mathrm{e}}$ siècle), au prix de réductions franches. Le cours L'herméneutique du sujet (1981-1982) reconnaîtra d'ailleurs la synecdoque en intervertissant le terme de « christianisme » avec celui de « modèle "ascétique-monastique" $\gg^{1}$. En aucun cas, les rapports que ces pratiques entretiennent avec les institutions et les dogmes chrétiens ne se déduisent d'une vérité qui les engloberait - vérité déposée dès le commencement dans la personnalité d'un fondateur (Jésus-Christ) ou un texte source (les premières écritures chrétiennes). La coupure du $\mathrm{III}^{\mathrm{e}}$ siècle dans les régimes de vérité barre ainsi la route à une histoire totale du christianisme, une histoire qui voudrait que tout événement dont elle serait positivement dépositaire remonte à une origine en partie insaisissable, toujours reculée, approchée seulement par des critères théologiques ou spirituels.

Tel était pourtant le principe de la gigantesque Nouvelle histoire de l'Église des historiens Roger Aubert, David Knowles et Ludovicus J. Rogier $^{2}$, achevée quelques années avant le cours de Foucault sur les Pères de l'Église. Dans son introduction générale à ce projet, l'historien Roger Aubert notait qu'il ne pouvait y avoir d'histoire de l'Église sans, « qu'on

$1 \quad$ L'herméneutique du sujet, p. 244.

2 Nouvelle histoire de l'Église, R. Aubert, D. Knowles, L. J. Rogier éd., Paris, Seuil, 1963-1975, 5 volumes. Ces trois historiens réputés étaient professeurs respectivement à Louvain, Cambridge et Nimègue. 
le veuille ou non, certaines options théologiques $\gg^{3}$. Les choix d'Aubert et de ses collaborateurs étaient clairement affirmés sur ce point : selon eux, et dans une ligne qui portait au même moment le concile Vatican II, l'Église n'était pas d'abord une institution hiérarchique, mais le « Peuple de Dieu » confessant Jésus de Nazareth. Il y avait donc bien une décision théologique préalable, avec des conséquences méthodologiques immédiates pour la recherche : leur histoire de l'Église était nouvelle en ce qu'elle intégrait la vie des simples laïcs à côté de celle des papes et des évêques, et faisait droit à la vie quotidienne et spirituelle des croyants, à côté de l'histoire doctrinale.

À cette école de pensée, rompue à la critique historique la plus franche, mais présupposant l'unité essentielle de l'objet «christianisme $\gg$ qui transcenderait les choix individuels et les cultures, il faut associer le nom d'Henri Irénée Marrou (1904-1977), proche de Raymond Aron, collaborateur de la collection «Sources chrétiennes » et corédacteur avec Jean Daniélou du premier volume de cette Nouvelle histoire de l'Église 4 . Professeur à la Sorbonne, où il fut titulaire de 1945 à 1975 de la chaire d'Histoire du christianisme, Marrou avait parfaitement intégré, et même défendu en terrain français, le renouveau de la critique historique venu d'Allemagne. Mais il renâclait à entrer dans une perspective historiciste radicale. Ses carnets en témoignent, en particulier les entrées intitulées par ses soins Ethica ingredientibus dans la liasse XI (1943-1951) :

Oui - tout est là : l'histoire positiviste par une application enfantine du principe de causalité a substitué à l'œuvre proprement historique qui est la compréhension des essences la recherche non de[s] causes, mais des sources, analogies[,] antécédents et $y$ a dissous l'objet. ${ }^{5}$

Longtemps, son séminaire d'« Histoire ancienne du christianisme » à la Sorbonne fédéra les énergies de jeunes chercheurs, parmi lesquels Michel Spanneut, dont Foucault lut la thèse sur le stoïcisme des premiers Pères ${ }^{6}$.

3 Nouvelle histoire de l'Église, t. I : Des origines à Grégoire le Grand, Paris, Seuil, 1963, p. 8.

4 La contribution de Marrou remise dans son contexte intellectuel est résumée dans P. Riché, Henri Irénée Marrou, historien engagé, Paris, Cerf, 2003, p.142-146. Cette biographie offre par ailleurs un intéressant panorama des études patristiques en Sorbonne dans les années 1950-1960.

5 H. I. Marrou, Carnets posthumes, F. Marrou-Flamant éd., Cerf, 2006, p. 409, l'auteur souligne.

6 M. Spanneut, Le stoïcisme des Pères de l'Église. De Clément de Rome à Clément d'Alexandrie, 
Malgré sa fréquentation livresque de ces travaux classiques, Foucault s'est tenu à bonne distance de leurs présupposés essentialistes. L'écart avec ces grands aînés ne se limite pourtant pas au refus de toute option théologique. Ce sont également les questions posées aux textes qui marquent la distance. Pour les principaux contributeurs de la recherche patristique en France dans les années 1960 - et ici, aux côtés d'Henri Irénée Marrou, il faut citer Pierre Courcelle et son cadet Pierre Hadot ${ }^{7}$-, l'accent mis sur l'unité et l'originalité du christianisme n'était nullement en contradiction avec la valorisation de sa profonde imprégnation de culture hellénistique. Ce bain philosophique des Pères était même célébré comme témoignage d'un certain humanisme chrétien. Dès lors, la généalogie des influences païennes était non seulement un objet majeur de la recherche, mais elle était devenue la principale grille de lecture des premiers siècles - la philologie opérant les soudures nécessaires et la mise en synopse des textes donnant à la pensée sa forme un peu raide. Ainsi des articles de Pierre Courcelle sur le néoplatonisme d'Ambroise ${ }^{8}$ ou le stoïcisme d'Augustin ${ }^{9}$; ainsi de la grande étude de Michel Spanneut, préfacée par Marrou, divisant l'aube de la pensée chrétienne entre une première phase stoïcienne (jusqu'en 230) et une seconde phase néoplatonicienne ${ }^{10}$. Qu'a lu tel Père

Paris, Seuil, 1957. Lecture attestée dans l'édition critique du cours de 1981-1982, à partir des références indiquées dans le manuscrit (L’herméneutique du sujet, p. 257, note 16).

7 Spécialiste d'Ambroise et d'Augustin, Pierre Courcelle (1912-1980) fut l'un des maîtres de Pierre Hadot, qui lui succéda au Collège de France en 1983. Voir P. Hadot, Éloge de la philosophie antique. Leçon inaugurale de la chaire d'Histoire de la pensée hellénistique et romaine faite au Collège de France, le vendredi 18 février 1983, Paris, Allia, 1998, p.10: «J'ai parlé d'une étroite liaison entre grec et latin, philologie et philosophie, hellénisme et christianisme. Je crois que cette formule correspond exactement à l'inspiration de l'enseignement de Pierre Courcelle. »

8 P. Courcelle, «Litiges sur la lecture du "Libri Platonicum” par saint Augustin », Augustiniana, t. 4, 1954, p. 225-239 (où est débattue la question de savoir si Augustin a retrouvé la foi avant ou après la lecture des platoniciens); « Nouveaux aspects du platonisme chez saint Ambroise », Revue des études latines, t. 34, 1956, p. 220-239; «De Platon à saint Ambroise par Apulée $\gg$, Revue de philologie, t. 35, 1961, p.15-28.

9 P. Courcelle, «L'immanence dans les Confessions augustiniennes », Hommages à Jean Bayet, M. Renard et R. Schilling éd., Bruxelles-Berchem, Latomus revue d'études latines, 1964, p. 161-171. La conclusion de cet article sur l'origine de la conception augustinienne de la présence de Dieu au cœur de l'homme montre bien que c'est d'abord une question d'influence qui est en jeu : «Augustin me semble donc avoir connu la doctrine de l'immanence à travers la diatribe stoïcienne, mais l'avoir repensée successivement à la lumière de la pensée néoplatonicienne et chrétienne » (p.171). 
et l'a-t-il lu de première ou de seconde main : voilà ce dont il était essentiellement question dans les débats.

À l'opposé, si Foucault mentionne à plusieurs reprises les sources philosophiques païennes des penseurs chrétiens (ainsi du stoïcisme de Tertullien et son écart avec la tradition néoplatonicienne ${ }^{11}$ ), il ne fait à aucun moment d'un débat avec la culture grecque ou romaine la raison d'une transformation dans la manière de réfléchir les pratiques. Est ainsi délaissé le legs païen comme détour nécessaire pour départager entre eux les penseurs chrétiens et approcher de leur singularité propre - souvent réduite chez les chercheurs des années 1960 à n'être qu'une certaine manière de lire Plotin ou de redécouvrir Virgile.

Si Foucault n'a témoigné que peu d'accointances vis-à-vis de cette école française, il s'est rapidement senti en syntonie avec les recherches du professeur Peter Brown. Ce dernier arrive comme enseignant à l'université de Berkeley en 1978 et publie la même année The Making of Late Antiquity ${ }^{12}$ - deuxième ouvrage majeur pour cet historien d'origine irlandaise, de dix ans le cadet de Foucault, qui compte déjà à son actif une biographie remarquée de saint Augustin ${ }^{13}$. Comme Foucault, Brown décrit une transformation historique sans la réduire aux déterminismes politiques, militaires ou économiques, trop souvent convoqués dans une causalité réduite à la linéarité. Contrairement aux thèses traditionnellement acceptées par Eric R. Dodds $s^{14}$, autre historien irlandais, et le père André-Jean Festugière ${ }^{15}$, Brown n'explique pas la nouvelle relation aux

le père Daniélou contesta cette division trop simple, mais uniquement pour complexifier le jeu des influences : J. Daniélou,Histoire des doctrines chrétiennes avant Nicée, t. II : Message évangélique et culture hellénistique aux $\imath^{\mathrm{e}}$ et $\imath^{\mathrm{e}}$ siècles, Paris, Desclée/Cerf, 1990, p.117.

11 Leçon du 13 février 1980.

12 P. Brown, The Making of Late Antiquity, Londres-Cambridge, Harvard University Press, 1978. Traduction française : Genèse de l'Antiquité tardive, A. Rousselle trad., Paris, Gallimard, 1983.

13 P. Brown, Augustine of Hippo. A Biography, Londres, Faber, 1967. Traduction française : La vie de saint Augustin, J.-H. Marrou trad., $2^{\mathrm{e}}$ édition augmentée, Paris, Seuil (Points histoire), 2001.

14 E. R. Dodds, Pagan and Christian in an Age of Anxiety. Some Aspects of Religious Experience from Marcus Aurelius to Constantine, Cambridge, Cambridge University Press, 1990 [1965]. Cette étude de psychologie historique se réfère à William James et Carl G. Jung.

15 Religieux dominicain, grand spécialiste du néoplatonisme et des écrits hermétiques, André-Jean Festugière était un ami de Foucault, avec qui il entretint une longue correspondance. Foucault cite ses Études de philosophie grecque (Paris, Vrin, 1971) et un autre article dans son cours L'herméneutique du sujet, pages 164 et 198. 
dieux dans l'Antiquité tardive par le développement des grandes cités urbaines, l'accumulation des défaites militaires ou la montée d'un sentiment général d'insécurité. Les mutations de la religiosité ont leurs règles propres qui ne répondent pas aux angoisses de la subjectivité face à un monde en déliquescence. Comme Foucault également, Brown écarte toute considération sur l'essence du christianisme, pour insister sur la césure du $\mathrm{III}^{\mathrm{e}}$ siècle dans la vie religieuse et sociale du bassin méditerranéen - césure qui ne passe pas entre la religion païenne et la religion chrétienne, mais entre un «style » de religiosité et un autre.

Cette notion de «style», qui apparaissait dès l'ouverture de la grande biographie d'Augustin ${ }^{16}$, domine le dernier chapitre du livre publié par Peter Brown en 1978 - chapitre consacré à l'anachorèse d'Antoine et de Pacôme. Son apparition furtive dans Du gouvernement des vivants, en 1980, dans un contexte où elle semblerait incongrue sans le lien à Brown (Foucault parle du changement de «style » de la préparation au baptême ${ }^{17}$ ), vérifie que le philosophe français avait bien lu The Making of Late Antiquity à cette époque, sans doute sur la suggestion de Paul Veyne. Reconnaissons cependant que la notion était encore mal définie dans la première édition de l'ouvrage de Brown (Londres et Cambridge, 1978), l'historien irlandais ne la thématisant avec précision qu'ultérieurement : dans l'introduction rédigée pour la traduction française du livre (Paris, 1983 ${ }^{18}$. Préfaçant cette même traduction, Paul Veyne insistera de concert sur cette notion de «style $\gg$, autour de laquelle il rassemblera les noms de Peter Brown, Michel Foucault et le sien propre - sans présomption particulière de paternité ni effort pour vérifier si, de Foucault à Brown, l'usage du terme est strictement homogène (ibid., p. XIX-XXII). Or, après examen, tel n'est pas le cas.

Dans les dits de Foucault, l'expression «style de vie » fait son apparition lors d'un entretien d'octobre 1981 et revient à deux reprises dans le cours de 1982, en lien avec le syntagme «forme de vie » (forma vitae $)^{19}$. Chez les deux penseurs, le «style » renvoie bien à ce qui peut être vu

« Nous verrons Augustin se mesurer tour à tour à des problèmes posés par des milieux nouveaux; son style de vie connaîtra à la longue une transformation inconsciente » (P. Brown, La vie de Saint Augustin, déjà cité, p. 11, nous soulignons).

17 Leçon du 20 février 1980.

18 P. Brown, Genèse de l'Antiquité tardive, déjà cité, p.15-16.

$19 D E \mathrm{n}^{\circ} 313$, «Le triomphe social du plaisir sexuel : une conversation avec Michel Foucault », II, p. 1128 ; L'herméneutique du sujet, p. 406 et 411. 
de soi par autrui. Mais chez Foucault, ce qui est vu est l'expression d'un rapport à soi cherchant pour lui-même un certain résultat, sans volonté d'influer sur la vie sociale; le style est donc une catégorie de la subjectivité, appréhendée comme telle par le philosophe. Chez Brown, ce qui est vu est l'expression d'un rapport au monde dont on peut évaluer l'efficacité et les divers effets, par exemple en termes de pouvoir. C'est donc la manifestation sociale de ce rapport, ou encore la stratégie au sein des rapports intracommunautaires, qui importent en premier lieu à l'historien. La foi dans les miracles ouvre ainsi la possibilité pour le croyant de choisir celui qu'il reconnaîtra comme détenteur de pouvoirs surnaturels $^{20}$. Au croisement des croyances et des pratiques sociales, la notion de «style » chez Brown décrit leur fécondation mutuelle pour produire des tactiques individuelles nouvelles, un nouveau champ de possibilités d'action au sein d'une communauté.

Si l'historien et le philosophe se rencontrent souvent au-dessus des mêmes textes, leurs approches diffèrent donc, sans pour autant se contredire. Ainsi de leur interprétation respective de La clef des songes d'Artémidore, texte $\mathrm{du} \mathrm{II}^{\mathrm{e}}$ siècle de notre ère : Brown voit dans les images oniriques un témoignage de la situation sociale des élites et d'un certain exercice du pouvoir à l'âge antonin. L'interprétation des rêves donnée par le penseur païen dénote une certaine conception de la vie répandue à cette époque autour du bassin méditerranéen ${ }^{21}$. Pour Foucault, si l'onirocritique d'Artémidore trouve place dans son analyse, c'est d'abord comme technique d'existence pour gérer sa vie et se préparer à des événements futurs ${ }^{22}$. Le philosophe français s'attarde sur les procédures de déchiffrement mises en œuvre plus que sur les codes sociaux ou les traits de mœurs que ces pro-

P. Brown, Genèse de l'Antiquité tardive, déjà cité, p. 51. Un autre exemple en serait la description du saint homme d'Égypte : «il ne suffisait pas au pouvoir surnaturel d'exister, il fallait qu'on le voie exister, assigné avec certitude, de façon observable et permanente, à des êtres humains déterminés et non à d'autres. Cela, l'ascète pouvait l'offrir. [...] un style de vie totalement à part, clairement tracé depuis son premier départ de la communauté établie jusqu'au désert, et obstinément maintenue durant des dizaines d'années, était censé lui avoir acquis les pouvoirs qu'il prétendait posséder. Le saint homme devait accomplir des œuvres et être vu à l'œuvre » (p.181-182).

21 P. Brown, Genèse de l'Antiquité tardive, déjà cité, p. 84-85 et 88-89.

22 Le souci de soi, chap. I : « Rêver ses plaisirs 》. Artémidore avait été pour la première fois évoqué par Foucault dans une conférence de $1980\left(D E n^{\circ} 295\right.$, « Sexualité et solitude », II, p. 993). 
cédures attesteraient ${ }^{23}$. Surtout, le texte d'Artémidore retient l'attention de Foucault en ce qu' il superpose le sujet rêvant d'un acte et le sujet de l'acte ${ }^{24}$ : c'est en tant que l'interprétation engage le sujet interprétant que Foucault lit La clef des songes. Pour reprendre une distinction du cours Du gouvernement des vivants : avec l'historien irlandais, nous sommes clairement du côté des « relations d'objectivité », tandis qu'avec le philosophe français, nous sommes du côté des « actes de subjectivité $\gg^{25}$.

Le compagnonnage intellectuel n'en fut pas moins important entre les deux hommes, qui semblent n'avoir cependant véritablement échangé, aux dires de Brown lui-même, qu'à la fin de l'année $1980^{26}$. Laissons au malicieux professeur les derniers mots sur cette relation qui eut, elle aussi, ses historiens impromptus :

J'ai lu le brouillon d'un article qui avançait avec assurance que mon propre travail sur l'Antiquité tardive devait beaucoup à des séminaires avec Mary Douglas en Angleterre et avec Michel Foucault à Berkeley. Cette image proprette de la transmission correcte du savoir, à travers de brillants séminaires et de grands centres d'enseignement était aussi anachronique que ces charmantes lettres apocryphes de l'Antiquité tardive dans lesquelles saint Paul écrit au philosophe Sénèque et où Socrate offre ses services à son collègue Plotin. [... ] Pour ce qui est de Michel Foucault, deux heures d'une discussion enjouée sur la relation entre la notion de concupiscence chez Augustin et la notion de combat spirituel chez Jean Cassien, dans la tanière de l'ours, à Berkeley, vers la fin 1980, ont posé les bases d'une solide amitié. Celle-ci nous poussa à nous rencontrer ultérieurement, à la cafétéria de la bibliothèque Bancroft et à l'hôtel français de Shattuck - conversations toujours intenses mais largement improvisées, trop tôt interrompues par sa mort prématurée. ${ }^{27}$

Participant d'un renouvellement de l'historiographie des premiers siècles, le cours Du gouvernement des vivants constitue un tournant dans le parcours intellectuel de Michel Foucault. Tout d'abord, parce qu'il

Même s'il les mentionne brièvement : Le souci de soi, p. 48 .

Le souci de soi, p. 39.

Leçon du 13 février 1980.

Ce qui est corroboré par la mention, lors du séminaire de novembre 1980 à l'Institute for the Humanities, d'une conversation avec Brown (DE nº 295, « Sexualité et solitude », II, p. 991).

P. Brown, «A Life of Learning », Charles Homer Haskins Lecture, American Council of Learned Societies, Occasional Paper, $n^{\circ} 55,9$ mai 2003 (notre traduction). Brown rendra par deux fois hommage à Foucault dans sa somme The Body and Society (New York, Columbia University Press, 1988), traduction française : Le renoncement à la chair. Virginité, célibat et continence dans le christianisme primitif, P.-E. Dauzat et C. Jacob trad., Paris, Gallimard, 1995, p. 18 et p. 503 , note 1. 
établit un lien entre l'accès à la vérité et certains actes du sujet, accomplis sous seing privé - c'est-à-dire non pas secrets ou intérieurs, mais authentifiés par la seule présence de témoins. Ensuite, parce qu'il déploie un nouveau rapport aux textes, dans la forme de la lecture commentaire. Resserrées autour des actes de pénitence et d'aveu, les leçons chrétiennes s'inscrivent ainsi à la croisée d'une analyse stratégique, héritée des travaux sur la psychiatrie et la pénalité, et d'une histoire de la pensée, qui se fait jour dans les derniers écrits du philosophe. La première histoire se déploie dans l'extériorité de pratiques, de savoirs et d'institutions n'ayant entre eux aucun lien de nécessité. La seconde trace à l'intérieur d'un sujet les lignes de partage de ses champs d'expériences possibles. La description initiale de réalités historiques radicalement hétérogènes est alors temporisée par l'attention nouvelle à l'unité formée par des actes qui sont reconnus indissociables pour un sujet : actes de foi / actes d'aveu. Fort de ces précisions concernant l'objet de la recherche, nous pouvons maintenant étudier de près les pratiques de lecture de Foucault au contact des textes chrétiens. 


\section{Une lecture singulière des Pères}

La notion de « régime de vérité », dans le sens précis que lui donnent la quatrième et la cinquième leçon du cours de $1980^{1}$, restreint le champ de la recherche et guide les choix bibliographiques. Nous l'avons déjà constaté : au sein du vaste corpus patristique, les commentaires des rituels (baptême, seconde pénitence) sont privilégiés par rapport aux traités dogmatiques. C'est donc la codification et la répétition de certains gestes et paroles qui intéressent Foucault et non la manière dont la Bible ou le dogme ont été théoriquement établis comme vrai. Ces gestes et ces paroles manifestent une vérité, ils ne la fondent pas rationnellement. Dans la première leçon du cours de 1980, l'expression « rituel de manifestation de la vérité $\gg^{2}$, appliquée aux textes préchrétiens de Sophocle et Dion Cassius ${ }^{3}$, annonce d'ailleurs celle de «régimes de vérité $»$. Elle

1 Leçons du 30 janvier et, surtout, du 6 février 1980.

2 Leçon du 9 janvier 1980, nous soulignons. Foucault emploie « rituel $\gg$ en un sens bien différent de celui qu'il avait dans la leçon inaugurale au Collège de France en 1970, où il n'est qu'une forme de « restriction » imposée à la prise de parole (L'ordre du discours, p. 40-41).

3 Il s'agit de l'historien grec, né vers 155 après J.-C. Il rédigea à partir de 229 une gigantesque histoire romaine en quatre-vingts livres, des temps primitifs à Alexandre Sévère. L'extrait cité par Foucault (Histoire romaine, LXXIV, 3 ) concerne l'empereur Septime Sévère. Il se trouvait initialement inséré dans un livre perdu de Dion Cassius consacré aux prodiges et aux songes qui annoncèrent l'avènement de l'empereur. Ce texte, jugé souvent bien futile par les commentateurs, est l'occasion pour Foucault de relever cet « excès » de vérité à côté de l'exercice du pouvoir. Il est savoureux que le cours où il va être question du christianisme s'ouvre ainsi sur un empereur qui, après avoir favorisé les chrétiens, les persécuta. Pour des raisons par ailleurs strictement politiques et non religieuses. 
désigne bien « un ensemble de procédés verbaux et non verbaux $\gg^{4}$ par lesquels, dans l'exercice du pouvoir, une vérité est manifestée, à distance des habituelles démonstrations de puissance et de force. La mesure de cette distance est précisément la grande avancée du cours : contre ses précédentes hypothèses, Foucault reconnaît qu'une telle manifestation n'est pas nécessairement instrumentalisée par le pouvoir, mais peut constituer « un supplément $\aleph^{5}$ par rapport aux connaissances requises par le pouvoir pour fonctionner efficacement. Elle nécessite donc une méthode nouvelle d'approche, qui conserve cependant certains principes méthodologiques des précédents travaux : la notion de « rituel $\gg$ permet en effet de ne pas confiner cette manifestation dans la seule conscience des individus et de ne pas réduire la vérité au seul résultat de l'activité rationnelle de connaissance. Les « procédés logico-expérimentaux $\gg^{6}$, précise Foucault, ne sont qu'un rituel de vérité parmi d'autres.

Demandons-nous alors si la décision d'étudier la vérité comme «régime » ou « rituel », au-delà des conséquences évidentes sur les choix bibliographiques, n'induirait pas également une manière particulière de lire et d'interpréter les textes. Autrement dit, cette décision vient-elle se greffer de l'extérieur sur des lectures et des commentaires de textes qui, en eux-mêmes, ne différeraient pas de ceux de n'importe quel philologue ou historien classique? $\mathrm{Ou}$, au contraire, l'art de lire et de traduire les Pères chez Foucault ne serait-il pas guidé, déplacé et constamment plié par cette notion singulière de « régime de vérité »? Une citation de 1978, qui relève au passage une lacune importante dans les travaux de l'école de Francfort, nous invite à privilégier la deuxième hypothèse :

Le rapport avec l'histoire est un élément qui m’a déçu chez les représentants de l'école de Francfort. Il m’a semblé qu' ils faisaient peu d'histoire au sens plein, qu'ils se référaient à des recherches effectuées par d'autres [...]. Je n'entends pas affirmer que chacun doit construire l'histoire qui lui convient, mais il est un fait que je ne me suis jamais pleinement satisfait des travaux des historiens. Même si je me suis référé à de nombreuses études historiques et si je m'en suis servi, j'ai toujours tenu à conduire moi-même les analyses historiques dans les domaines auxquels je m'intéressais. ${ }^{7}$

$7 D E n^{\circ}$ 281, «Entretien avec Michel Foucault », II, p. 894-895. 
Dans cet entretien accordé à la fin 1978, Foucault pose une corrélation entre le domaine de la recherche historique et sa méthode, le premier déterminant la seconde qui ne cesse ainsi de se renouveler ${ }^{8}$. Cette corrélation empêche de considérer l'historiographie comme une discipline neutre, produisant des résultats ayant en eux-mêmes leur valeur explicative, utilisables en l'état par les philosophes. Pour notre étude présente, cette remarque concernant l'époque moderne et l'école de Francfort pourrait aisément être transposée à l'histoire antique et ses spécialistes : si le philosophe se doit d'aller lui-même extraire la matière première dont il a besoin pour ses recherches, c'est bien que sa lecture des Pères ne sera pas la même que celle d'un Pierre Courcelle ou d'un Henri Irénée Marrou. Il reste alors à vérifier avec précision que Foucault s'est bien appliqué à lui-même ce code de bonne conduite.

Mais avant cela, tentons de définir le type des lectures pratiquées par Foucault sur les textes chrétiens avant l'année 1980, en prenant comme exemple le cours de 1974-1975: Les anormaux. Nous pourrons alors mesurer le même écart que dans la première partie de notre recherche, comme si l'usage de certains objets historiques, fabriqués par l'historien, se reportait directement sur la manière de lire les textes.

8 Ce nécessaire renouvellement est noté dès le début de l'entretien de 1978 : « Chacun de mes livres est une manière de découper un objet et de forger une méthode d'analyse » $\left(D E n^{\circ} 281\right.$, «Entretien avec Michel Foucault », II, p. 861). Dans la leçon du 30 janvier 1980, Foucault note le déplacement incessant de son travail théorique : celui-ci n'est pas un système, mais le dessin intelligible de cette succession de déplacements, « la trace des mouvements par lesquels je ne suis plus à la place où j'étais tout à l'heure ». 



\section{CHAPITRE QUATRE Le christianisme dans le texte}

\section{Limites de la première histoire de l'aveu (1974-1978)}

Spécificité de la forme religieuse de l'aveu

Tentons de définir les présupposés de lecture du premier projet d'histoire de l'aveu, tel qu'il s'énonce dans le cours au Collège de France de 1974-1975, Les anormaux. Si le cours ne contient pas d'exposé méthodologique sur la question, nous allons voir que le rapport aux textes y est tout à fait singulier. Précisons tout d'abord un terme que nous risquons d'employer souvent : nous entendons ici par texte, les signes graphiques (graphèmes) déposés sur un support matériel, formant un ensemble que l'on peut à la fois prononcer dans une langue naturelle, éditer sur un autre support et traduire dans une autre langue.

Ce qui intéresse Foucault dans la confession chrétienne est la constitution des individus en sujets de leur existence, par un examen constant de soi qui fait passer les mouvements intérieurs au crible d'observations systématiques, pour les exprimer ensuite dans la forme de l'aveu. Pourquoi doit-on, en Occident, passer par cette objectivation de soi, dans laquelle le sujet énonçant est également le sujet de l'énoncé ? Au sein du vaste domaine des $\ll$ véridictions $\gg^{1}-$ néologisme forgé par Foucault en

1 Dans les conférences à l'université catholique de Louvain en 1981, Foucault se propose de faire une «histoire politique des véridictions ». Nous utilisons la transcription des conférences réalisée par l'université de Louvain, en dépit de sa qualité médiocre (coquilles constantes sur les noms propres, transcription proche de la prise de notes) : Cours Mal faire, dire vrai. Fonctions de l'aveu. Conférences réalisées dans le cadre de la 
référence à un wahr-sagen attribué à Nietzsche ${ }^{2}$-, il faut préciser le rapport tout à fait original qui s'établit, dans l'aveu, entre le sujet et la vérité. Le sujet ne s'y contente pas de constater simplement une vérité qui le concerne, mais il s'engage dans cette parole de vérité et se lie à elle. Dans les conférences inédites de Louvain en 1981 Mal faire, dire vrai, Foucault relève en effet que l'aveu, dans son usage par exemple psychiatrique ou judiciaire, n'apprend communément rien de nouveau, ne dévoile aucune vérité cachée :

L'aveu ne fait pas avancer d'un pouce la connaissance. Ce qui sépare l'aveu d'une déclaration, ce n'est pas ce qui sépare l'inconnu du connu, le visible de l'invisible. Mais ce qu'on pourrait appeler un certain coût d'énonciation. ${ }^{3}$

Étrange magie, étrange pouvoir de ce que Foucault n'hésite pas à appeler un «speech act ${ }^{4}$, qui vaut non pas tant par ce qu'il dit que par l'effort qui arrache ce «dit». Or, cet arrachement ne peut découler de la seule contrainte, mais engage un sujet à coïncider par lui-même avec sa propre parole. De l'Inquisition aux cures des psychiatres du XIX ${ }^{\mathrm{e}}$ siècle, il faut relever cet incroyable réquisit de l'aveu : il doit être libre - ce qui ne l'empêche pas de s'inscrire dans une relation de pouvoir qu' il relance et intensifie.

Cette performativité de l'aveu, au sens où son énonciation accomplit en elle-même, par sa seule mise en œuvre, un «quelque chose », nous

chaire Francqui, Université catholique de Louvain, 1981. - Tapuscrit, 159 pages ; $30 \mathrm{~cm}$ [Archives IMEC]. Nous en corrigeons cependant les erreurs d'orthographe et de ponctuation les plus évidentes.

$2 D E \ll$ Structuralisme et poststructuralisme », no 330 , II, p. 1264. Le terme allemand cité par Foucault semble croiser le Wahrsager d'Ainsi parlait Zarathoustra avec Aurore, I, 91.

3 Cours Mal faire, dire vrai. Fonctions de l'aveu.

$4 \ll$ «'essaierai d'abord de vous proposer une brève analyse de ce qu'on peut entendre par aveu et d'esquisser une très brève théorie de l'aveu comme un "speech act" » (Cours Mal faire, dire vrai. Fonctions de l'aveu). La notion de speech act (acte de parole) a été forgée par le philosophe anglais John L. Austin dans une conférence célèbre de 1955, pour indiquer que le langage ne sert pas d'abord à décrire la réalité mais à accomplir des actes, comme lorsqu'on déclare «la séance est ouverte » (J. L. Austin, How to do things with words, Oxford, Clarendon Press, 1962). Cette assimilation de l'aveu à un acte de langage est d'autant plus intéressante que L'archéologie du savoir avait exclu ce modèle pour définir l'énoncé, objet de l'archéologie (L'archéologie du savoir, p. 110-112). Quand l'expression revient en 1981, elle doit beaucoup également à la rencontre du philosophe américain John R. Searle (voir J. R. Searle, Speech Acts, Cambridge, Cambridge University Press, 1969), avec qui Foucault dialogue longuement en 1979. La discussion se poursuivra dans le cours de 1982-1983 : Foucault ne prononce nulle critique vis-à-vis de la philosophie du langage anglo-saxonne, mais montre simplement pourquoi les énoncés «parrèsiastiques » ne sont pas des énoncés performatifs : Le gouvernement de soi et des autres, p. 59 et suiv. 
la retrouvons intacte dans la pratique chrétienne de la confession. Mais, comme nous l'avons vu dans la première partie de notre recherche, Foucault sait qu'il ne va pas attraper avec ce grand filet de l'aveu des poissons de la même taille ni de la même espèce : le confesseur interrogeant n'est pas le psychanalyste analysant. Rappelons la place qu'occupe le dossier des confesseurs dans le cours Les anormaux.

En cette année 1975, Foucault propose à ses auditeurs du Collège d'étudier la naissance du thème de l'anormal, qui s'impose à la psychiatrie des années 1845-1850 en remplacement de la vieille figure du monstre. La notion d'anomalie a permis l'introduction du problème de la sexualité dans la psychiatrie, jusqu'à ce que ladite sexualité ne soit plus un champ parmi d'autres d'anomalies, mais la racine première, le « principe étiologique général $\gg^{5}$ de toutes les anomalies. Or, pour traiter efficacement l'anomalie nouvelle, le dispositif des sciences empiriques - c'est-à-dire l'examen de signes et de symptômes déchiffrables sur le corps et dans le comportement des malades - s'est accompagné d'une multiplication des procédures pour faire parler le sujet. Foucault relève qu'une telle injonction n'était au départ absolument pas évidente, son arrivée nécessitant un accommodement du fonctionnement traditionnel de la vérité médicale, afin que le discours du malade puisse légitimement s'introduire entre le médecin et la maladie. Pour ce faire, deux conditions ont été posées : 1) il ne fallait pas que le malade produise lui-même la vérité, mais que sa parole soit un signe à déchiffrer par le médecin, seul capable de l'interpréter pour produire un discours vrai ${ }^{6} ; 2$ ) il fallait justifier cette introduction de l'aveu dans la formation régulière du discours médical, non seulement par sa collaboration au diagnostic, mais également par son effet curatif. C'est le récit, trois fois narré par Foucault, d'une cure du psychiatre Leuret en 1840. La folie s'efface quand le fou la dit: « Je le reconnais. Je suis fou $\gg^{7}$.

De l'aveu religieux à l'aveu médical, il n'y a semble-t-il qu'un pas, mais que ne franchit pas Foucault. Certes, il y a bien réutilisation par la médecine du XIX ${ }^{\mathrm{e}}$ d'une procédure née du côté des confessionnaux de l'Église catholique. Mais l'analyse tient à respecter la spécificité de la forme religieuse de l'aveu, telle que le concile du Latran IV l'a généralisée

$5 \quad$ Les anormaux, p. 156.

6 La volonté de savoir, p. 89-9o.

$7 D E n^{\circ} 16$, « L'eau et la folie », I, p. 298-299; $D E$ n $^{\circ} 295$, « Sexualité et solitude », II, p. 988. Repris dans le cours Malfaire, dire vrai. Fonctions de l'aveu. 
en 1215 à la totalité du peuple chrétien. L'émergence du nouveau pouvoir médical n'aurait pas été possible si la chair chrétienne n'avait pas croisé d'autres technologies du corps. Foucault a soin de distinguer «l'anatomie politique du corps » que déploie l'État à partir de l'âge classique dans les écoles et les asiles, de « la physiologie morale de la chair $\gg^{8}$ que déploie conjointement l'Église dans les confessionnaux. La première vise à produire un corps utile, la seconde un désir purifié. L'analyse foucaldienne ne cesse d'enfoncer le coin des différences, en amont des transformations, pour mieux montrer les effets ultérieurs inattendus du croisement de dispositifs hétérogènes : ainsi de la médicalisation de la masturbation qui fera la jointure au XIX ${ }^{\mathrm{e}}$ siècle entre la chair chrétienne et le dressage éducatif; exemple parfait d'un croisement stratégique.

Mais la différence entre les dispositifs disciplinaires ne concerne pas seulement l'objet de leurs discours («chair-sujet » pour l'Église ou « corps-objet » pour l'État ${ }^{9}$ ) et leur référent (les plaisirs du corps ou ses aptitudes), elle est proprement technique. Foucault évoque en effet une étrange caractéristique de la confession chrétienne, qui est même sa règle d'or : le confesseur doit oublier ce qu'il a entendu, amnésie nécessaire qui signifie l'oubli suprême accompagnant le pardon divin ${ }^{10}$. Alors que cet oubli ouvre une béance entre l'aveu religieux et toutes les autres formes d'aveu - judiciaire, psychiatrique -, il n'est que furtivement mentionné dans Les anormaux. Le seul texte de Foucault qui s'attarde un tant soit peu sur cet aspect est «La vie des hommes infâmes » en 1977, même s'il se borne finalement à marquer la coupure : l'enregistrement administratif a aujourd' hui remplacé le pardon religieux, le premier conservant ce que le second effaçait ${ }^{11}$. Foucault souligne cependant le paradoxe :

$[\ldots]$ étonnante contrainte $[\ldots]$ de tout dire pour tout effacer, de formuler jusqu'aux moindres fautes dans un murmure ininterrompu, acharné, exhaustif,

8 Les anormaux, p. 180.

9 Pour reprendre un thème développé dans Surveiller et punir, p. 179-183.

10 Les anormaux, p. 172. Dans l'Ancien Testament, cet effacement complet est exprimé dans le Psaume 51 (50), verset 11 : « Détourne ta face de mes fautes, et tout mon mal efface-le » (Ps. 51, 11, traduction Bible de Jérusalem).

$11 D E \mathrm{n}^{\circ} 198$, « La vie des hommes infâmes », II, p. 245. Foucault signale cependant dans une conférence de 1980 que l'aveu dans la cure psychiatrique a également vertu d'effacement, mais cet effacement ne concerne pas celui qui reçoit l'aveu (le psychiatre), mais la matière avouée (la folie). Dire « je suis fou » marque la fin de la folie; de même que dire «je suis pécheur » efface aussitôt le péché par l'absolution reçue du prêtre. 
auquel rien ne devait échapper, mais qui ne devait pas un instant se survivre à luimême. Pour des centaines de millions d'hommes et pendant des siècles, le mal a dû s'avouer en première personne, dans un chuchotement obligatoire et fugitif..$^{2}$

Nous sommes effectivement loin du dossier médical ou du casier judiciaire. À bien y regarder, la distance est même considérable : la confession signe en fait l'impossibilité d'épingler le savoir d'une subjectivité sur un corps - si ce n'est un savoir général des fonctionnements de la chair, qui vaut dès lors pour tous sans distinction. S'il y a bien un assujettissement de l'individu à lui-même dans l'aveu chrétien, il se fait sans la médiation d'une connaissance singulière ou individualisante, puisque le péché est doublement effacé : de la mémoire du prêtre, par l'oubli, et de l'âme du pécheur, par le pardon. Que vaudrait en effet une telle connaissance sans son archivage, sa fixation temporelle, son inscription sur quelque support? Ne restent à l'issue de la confession que le repentir et les œuvres de pénitence. Ce qui lie, dans l'aveu religieux, n'est donc pas l'énoncé mais la seule énonciation; non pas la vérité dans son contenu, mais le seul acte de produire la vérité devant autrui. On peut même se demander si l'acte de vérité ne s'efface pas finalement au profit d'un simple acte énonciatif qui est d'abord reconnaissance d'une autorité, obéissance et soumission. Ceci serait corroboré par le fait que le confesseur se doit en temps normal de croire ce que le pénitent lui dit, sans chercher à juger outre mesure ses dispositions actuelles ${ }^{13}$. À la limite, même si Foucault se garde de tirer une conclusion aussi extrême, peu importe ce qui est dit, à partir du moment où le mouvement de conversion est affirmé par le sujet ${ }^{14}$.

$12 D E n^{\circ} 198$, « La vie des hommes infâmes », II, p. 245, nous soulignons.

13 Telle est la règle Credere tenetur (citée par J. Delumeau, L'aveu et le pardon. Les difficultés

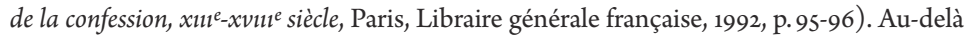
de la période prérévolutionnaire étudiée par Jean Delumeau, notons que cette maxime se retrouve dans un ouvrage majeur du XIX ${ }^{\mathrm{e}}$ siècle consacré à la confession, qui rappelle avec force : «Un simple soupçon sur la sincérité du pénitent, la crainte qu'il ne retombe dans le péché, ne sont pas des raisons suffisantes de lui refuser l'absolution. [...] Au tribunal sacré, il faut s'en rapporter au témoignage du pénitent pour ou contre lui » (T. Gousset, Théologie morale à l'usage des curés et des confesseurs, t. II, $9^{\mathrm{e}}$ édition, Paris, Jacques Lecoffre et Cie, 1853, p.353-354). Cet ouvrage est cependant trop tardif pour être mentionné par Foucault.

14 Selon le Catéchisme du concile de Trente, au moment de la confession, le mouvement sincère de conversion et la détestation globale de tous les péchés passés priment sur le souvenir de chaque péché en particulier et de leurs circonstances (J. Delumeau, L'aveu et le pardon, déjà cité, p.93). 
Revenons alors à ce qui intéresse Foucault dans ce vaste mouvement qui, du Moyen Âge à la Renaissance, va étendre et imposer la pratique de la confession à l'ensemble des laïcs. La leçon du 19 février 1975 fait halte à cette époque précise où l'extension du nombre des sujets avouant s'accompagne d'une triple obligation de régularité (une fois par an), d'exhaustivité (même les péchés véniels) et d'exclusivité (au curé uniquement). Bien entendu, ce qui se dit dans l'étroitesse d'un petit meuble grillagé s'inscrit dans un processus plus large de christianisation des couches populaires, charriant avec lui bien d'autres décisions doctrinales ou pastorales : définition conciliaire des sacrements, quadrillage géographique des paroisses, extension du pouvoir du curé, ouverture des séminaires pour la formation du clergé, etc. Mais en dépit de ce riche contexte historique, l'aveu confidentiel et chuchotant au prêtre concentre pour Foucault toute la nouveauté et l'efficace du pouvoir qui se déploie du XIII ${ }^{\mathrm{e}}$ au XVII ${ }^{\mathrm{e}}$ siècle.

Pourquoi réserver ainsi à l'aveu une importance que Foucault qualifie de $\ll$ presque exclusive $»^{15}$ ? Parce que dans cette obligation de tout avouer au prêtre, l'aspect proprement sacramentel de la confession, qui est transmission du pardon de Dieu par le truchement de son ministre ${ }^{16}$, va passer au second plan par rapport à l'opération générale de conduite des âmes. C'est-à-dire qu'avant l'absolution décisive, le prêtre va devoir exceller et déployer ses talents dans la manière d'accueillir le pécheur, manière de le questionner, manière de le corriger, manière de le conseiller. Par rapport aux théories médiévales du sacrement de confession, ceci est une nouveauté ${ }^{17}$, qui ouvre immédiatement le champ immense du goude l'Église. Dans les Écritures, le terme «sacrement » ne désigne pas un rite. Dans les Bibles latines, sacramentum traduit le terme grec $\mu v \sigma \tau \eta \dot{\rho} ı$ ov (le mystère). C'est dans le droit romain que le sacramentum prend le sens de serment sacré à l'empereur, d'engagement militaire. Ce sens juridique est repris par Tertullien, bon connaisseur du droit romain, pour désigner la foi et le baptême (C. Mohrmann, «Sacramentum dans les plus anciens textes chrétiens », The Harvard Theological Review, t. 47, vol.3, 1954, p.141152). Mais la définition de sept sacrements distincts et ritualisés apparaît seulement au $\mathrm{XIII}^{\mathrm{e}}$ siècle; elle sera confirmée par le concile de Trente.

17

Les anormaux, p. 165 .

La définition doctrinale de la confession comme «sacrement » est tardive dans l'histoire Selon le Docteur angélique, saint Thomas d'Aquin, le ministre reçoit un pouvoir spirituel pour dispenser les sacrements, ceux-ci désignant d'abord l'action salvifique de Dieu luimême. Le Christ est la cause principale du sacrement; le prêtre en est la cause instrumentale. Or, dans sa Somme contre les gentils (1258-1265), Thomas se borne à constater que l'Eucharistie, sacrement principal, nécessite une préparation qui est le sacrement de la confession. Mais il n'est pas encore question d'une préparation à cette préparation. Voir 
vernement des existences. Le $\mathrm{XVI}^{\mathrm{e}}$ siècle va ainsi connaître une prolifération de manuels des confesseurs, proposant un quadrillage toujours plus serré de la vie quotidienne du pénitent. Les questions à poser, qui commençaient au Moyen Âge par la liste des péchés (fornication, défloration d'une vierge, inceste, etc.), se déploient désormais en suivant les sens du corps (le toucher, la vue, la langue, l'oreille); chacun de ces sens se prêtant à de nouvelles subdivisions pour détailler, non pas les différents types d'actes, mais les différents types de pensées et les différents moments dans l'histoire de ces pensées : la première émotion ressentie, la première délectation que la pensée a eue de cette émotion, la première pensée d'exécuter le mal, la naissance de l'intention claire, etc. Ce n'est plus le simple registre de la faute, de l'infraction à la loi, mais celui de l'examen indéfini d'un corps de désir et de plaisir, sans cesse traversé, de façons multiples et souvent infinitésimales, par la concupiscence.

Nous avons vu que ce discours de la concupiscence n'était pas pour autant en continuité directe avec la somatisation de la masturbation qui surgit brusquement au XVIII ${ }^{\mathrm{e}}$ siècle, encore moins avec la psychopathologie sexuelle du XIX ${ }^{\mathrm{e}}$ siècle. Il ne l'est ni dans ses objets, ni dans ses référents, ni dans ses procédures. Mais si le petit meuble grillagé devenu la chambre d'écho de l'âme n'est ni le cabinet du médecin ni le bureau du juge, on peut alors s'étonner des conclusions des lectures foucaldiennes. La singularité historique affirmée est immédiatement temporisée par la monotonie des effets disciplinaires décrits : «contrôle $\gg^{18}$, $\ll$ correction $\gg($ ibid., p. 179), «quadrillages $\gg($ p. 179), «bouclage $\gg($ p. 170), « surveillance constante $\gg$ (p.188), etc. La spécificité du dispositif confessant est annulée par la monotonie de ses performances techniques, qui semblent finalement aligner la forme religieuse de l'aveu sur toutes ses autres formes (psychiatrique, judiciaire), dans la banalité commune du résultat escompté sur les corps.

Nous allons voir que c'est justement la manière de lire les textes qui rend possible cet alignement. Ce n'est pas une théorie générale du pouvoir, encore moins du pastorat chrétien, qui produit, du côté de dispositifs par ailleurs soigneusement distingués au plan historique, cette homogénéisation des effets; c'est une théorie des pratiques discursives, dont découlent des règles de lecture précises. Derrière cette

Thomas d'Aquin, Somme contre les gentils, IV, 74, R. Bernier, M. Corvez, M.-J. Gerlaud, F. Kerouanton, L.-J. Moreau trad., Paris, Cerf, 1993. 
théorie, s'entraperçoit à nouveau le projet déjà ancien d'établir « dans l'épaisseur du discours les conditions de son histoire $\gg^{19}$. La méthode archéologique des années 1960 est loin d'avoir été oubliée par les travaux de la décennie suivante.

\section{La confession est-elle une pratique coercitive?}

Reprenons la leçon du 19 février 1975, qui parcourt un nombre limité de manuels des XVII ${ }^{\mathrm{e}}$ et XVIII ${ }^{\mathrm{e}}$ siècles : Pierre Milhard (1617); Charles Borromée $\left(1648^{20}\right)$; diocèse de Strasbourg $(1722)$; Louis Habert $\left(1688^{21}\right)^{22}$. Parcours au premier abord décevant. Foucault s'attarde sur le type de questions posées au pénitent, la manière dont celles-ci découpent de manière toujours plus fine la totalité de la vie individuelle, mais l'analyse passe plus vite sur la relation de pouvoir entre le confesseur et le pénitent, c'est-à-dire sur la situation concrète qui engage la liberté de deux sujets. Ce que cette relation a véritablement été dans l'histoire chrétienne est d'autant plus absent de la leçon que Foucault prend sobrement acte de la distance incommensurable qui sépare la théorie enseignée de la pratique

\section{doublement à confusion. Cette édition de 1748 est une réédition posthume d'un manuel} édité sous ce titre pour la première fois en 1714. Or, ce manuel de 1714 est lui-même la version revue, corrigée et augmentée par Habert de son ouvrage de 1688 intitulé Pratique du sacrement de pénitence à destination du diocèse de Verdun. Ce n'est pas la version revue et corrigée qui est la référence de Foucault, mais bien la première édition de 1688. Foucault parle en effet clairement d'un manuel écrit «à la fin du XVII ${ }^{e} \gg($ Les anormaux, p. 165). Dans le répertoire bibliographique personnel de Foucault (Répertoire $A Z$ ), un ouvrage de Habert est d'ailleurs indiqué avec l'édition suivante : «1689 (1691)». Il ne peut s'agir que de la Pratique du sacrement de pénitence à destination du diocèse de Verdun, même si la première édition est en fait 1688. L'autre ouvrage noté sous le nom de Habert dans le répertoire, avec pour seule mention la date de 1708, est fort probablement sa Theologia dogmatica et moralis, non citée dans Les anormaux. Si ce répertoire est contemporain de la préparation du cours, cela vérifierait, s'il en est besoin, que Foucault lisait beaucoup plus d'ouvrages pour préparer ses enseignements qu'il n'en citait.

Alphonse de Liguori n'est que brièvement cité comme la reprise tardive et simplifiée de ce qui était déjà présent chez Habert ou dans le manuel du diocèse de Strasbourg (Les anormaux, p. 177). Foucault cite également deux ouvrages écrits à destination des séminaristes : Félix Vialart (1664, $2^{\mathrm{e}}$ édition) et Matthieu Beuvelet (1652). 
pastorale, populaire, forcément massive et rudimentaire. En fait, ce n'est pas l'événement de la confession qui intéresse en premier lieu le philosophe, mais la littérature qui le précède et la manière dont elle fait passer la vie du pénitent par un «filtre » discursif ${ }^{23}$. Pour bien saisir la spécificité de l'approche proposée par Les anormaux, il est intéressant de la comparer à celle ultérieurement proposée par l'historien Jean Delumeau, ainsi qu'au cours déjà croisé de 1977-1978 : Sécurité, territoire, population.

Publié en 1990, en complément d'une vaste étude sur le sentiment de sécurité en Occident, L'aveu et le pardon vise à reconstituer, entre les lignes des textes qui en décrivent les procédures formelles, le «vécu» de la confession du XIII ${ }^{\mathrm{e}}$ au XVIII ${ }^{\mathrm{e}}$ siècle, jusque dans les attitudes et la psychologie de ses acteurs ${ }^{24}$. Pour atteindre cet objectif, Jean Delumeau diversifie le plus possible ses sources documentaires, bien au-delà des seuls manuels normatifs écrits par et pour les confesseurs, là où Foucault se contente de citer quatre de ceux-ci. En particulier, l'historien du sentiment religieux prête toute son attention à un corpus volontairement occulté par la leçon du 19 février 1975 : l’abondante littérature rédigée par les clercs en direction du peuple chrétien, celle-ci étant composée de manuels pour se confesser mais également de sermons et conseils divers. Contrairement à ce qu'avance Foucault ${ }^{25}$, rien n'est moins sûr en effet que ce deuxième corpus ne soit que le prolongement tactique du premier, si l'on redonne au pénitent du $\mathrm{XVI}^{\mathrm{e}}$ siècle, à ses peurs et à ses attentes, une place dans l'ana$\mathrm{lys}^{26}$. Le prétendre serait entériner un fonctionnement homogène et univoque du pouvoir, au risque de déséquilibrer l'analyse en faveur du celui qui, seul, détient le « pouvoir des clés ${ }^{27}$ : le prêtre qui ouvre ou ferme la porte du salut. Rééquilibrant ce privilège redoutable, les manuels pour se confesser - à dominante bienveillante, issus le plus souvent des milieux

La difficulté et l'intérêt Decond degré pour y deviner, entre les lignes, et le comportement réel des confesseurs et les réactions des chrétiens ordinaires soumis à l'obligation de la confession. Comment cette contrainte a-t-elle été concrètement vécue ? 》 (J. Delumeau, L'aveu et le pardon, déjà cité, p. 7).

25 Les anormaux, p. 165.

26 La bienveillance qui s'exprime généralement dans cette littérature avait certes une fonction tactique : attirer un maximum de fidèles dans le confessionnal. Mais elle répondait également à l'inflation du sentiment de peur devant la question du salut, à l'aube des réformes protestantes et catholiques (J. Delumeau, L'aveu et le pardon, déjà cité, p. 39).

27

Les anormaux, p. 163 . 
jésuites ou casuistes ${ }^{28}$ - redonnent aux pénitents du savoir et du pouvoir : ils leur expliquent les règles de la confession, leur fournissent les moyens de surmonter leurs craintes et, surtout, tentent de combler la distance qui les sépare du confesseur, insistant sur l'égalité de tous devant le péché :

Pourquoi fuyez-vous des ministres qui sont pécheurs comme vous; des ministres qui manquent quelquefois à leurs devoirs les plus essentiels comme vous; des ministres qui, ayant vos mêmes faiblesses et vos mêmes imperfections, sont obligés de se confesser comme vous ?29

Face à ces textes écrits pour tranquilliser, les sévères manuels des confesseurs le sont d'abord vis-à-vis des clercs, dont la pratique du sacrement, au moins jusqu'aux conflits rigoristes du XVII ${ }^{\mathrm{e}}$ siècle, est jugée souvent distraite, expéditive, bâclée ${ }^{30}$.

Toute la littérature en direction du peuple chrétien insiste de son côté lourdement sur le fait que le prêtre est pécheur comme le laïc et se confesse de fautes semblables ${ }^{31}$. Par son universalité, la confession brouille en fait ce que Foucault appellera dans un article méthodologique de 1982 le «système des différenciations $\gg^{32}$ juridiques ou traditionnelles, de statut ou de privilège. Dans l'un de ses sermons, le jésuite Jacques Giroust, grand prédicateur du XVII ${ }^{\mathrm{e}}$ siècle, donne ainsi la parole au pénitent en des termes juridiques qui sont au bénéfice de ce dernier, là où Foucault ne mentionne que les droits infrangibles du confesseur ${ }^{33}$. Il est intéressant de noter que cette parole, adressée directement à Dieu, fait disparaître le prêtre de la scène du sacrement :

Je n'ai qu'à approcher de votre saint tribunal, et tout à coup vous répandez sur moi vos bénédictions ; je rentre auprès de vous dans tous mes droits. ${ }^{34}$

Dans le même sens, mentionnons également un autre sermon du même jésuite, rappelant que la fonction « principale et directe » du prêtre

Plus largement, ils sont issus de cette tradition bienveillante qui remonte à saint Thomas d'Aquin, et croise aussi bien saint François de Sales et saint Jean Eudes.

Joseph Chevassu, curé de Rouses, cité dans J. Delumeau, L'aveu et le pardon, déjà cité, p. 37.

30 J. Delumeau, L'aveu et le pardon, déjà cité, p.15-17.

31 Ibid., chap. III : «La confession pour tranquilliser ».

$32 D E \mathrm{n}^{\circ} 306$, « Le sujet et le pouvoir », II, p. 1059.

33 À deux reprises, Foucault parle du « droit d'examen » réservé au prêtre (Les anormaux, p. 165 et 198), qui semble un droit absolu, tout du moins «à la discrétion et à l'initiative du prêtre $\gg($ p.165).

Cité dans J. Delumeau, L'aveu et le pardon, déjà cité, p. 39, nous soulignons. 
se limite au pardon et à l'absolution ${ }^{35}$. Il n'a donc pas à trop discourir et creuser en deçà.

Si le prêtre n'est qu'un intermédiaire, cela implique que son pouvoir peut à son tour être interrogé et critiqué. Si nous étendons l'enquête aux lettres de direction spirituelle, nous découvrons que le quadrillage de la vie par l'examen s'accompagne de mises en garde incessantes, de limites posées au pouvoir du directeur spirituel. Certes, tout le monde doit être dirigé, sous le regard exclusif d'un autre qui cumule les fonctions de juge et de médecin, et n'est pas aisément destituable. Mais cet autre ne peut prétendre être absolument maître de la relation spirituelle, encore moins de sa finalité. Ainsi, saint François de Sales n’hésite pas à enjoindre madame de Chantal de se libérer en toute légitimité d'un directeur trop exigeant, dans une lettre du 14 juin 1604 :

Je loue infiniment le respect religieux que vous portez à votre directeur et vous exhorte de soigneusement y persévérer; mais il faut que je vous dise encore ce mot. Ce respect $[\ldots]$ ne vous doit pas gêner, ni étouffer la juste liberté que l'Esprit de Dieu donne à ceux qu'il possède..$^{36}$

Pour Monsieur de Genève, le directeur de Jeanne de Chantal avait outrepassé ses droits, et la mention de «l'Esprit de Dieu » exerce ici une fonction critique, donnant tout son poids à l'appréciation personnelle du dirigé ${ }^{37}$ : ce que le directeur impose doit s'accorder aux fruits et mouvements propres de l'Esprit, dont il n'est pas l'initiateur et dont seul le dirigé est le témoin direct. Celui qui détient le pouvoir est donc celui qui s'efface, et n'a en matière d'exigences et de prérogatives que ce qui peut mener autrui à son bien. En disant cela, nous ne qualifions pas de «bien » ou de «bon» un pouvoir qui s'occupe de ce bien. Il faut ici adopter la ligne nominaliste revendiquée par Foucault lui-même à la suite de Paul Veyne. Nous reprenons en fait simplement la définition du pouvoir pastoral donnée ultérieurement dans le cours Sécurité, territoire, population : l'originalité de ce pouvoir est de ne pas manifester d'abord sa force et sa supériorité mais d'être bienfaisant et médicinal. clée de Brouwer, 1980, p. 158.

Le directeur dont il est ici question avait fait prêter quatre vœux à Jeanne de Chantal : lui obéir, ne pas changer de directeur de conscience, garder le secret de ce qu' il lui dirait, ne confier à personne d'autres les tourments de son âme. 
Or, cette qualité intrigante du pastorat, Foucault la croise sans la voir en 1975 quand il recopie les manuels des confesseurs. Sans doute a-t-il peur d'y lire un desserrement du caractère disciplinaire de ces manuels, alors qu'il s'agit seulement d'une autre modalité du pouvoir. Ce n'est qu'en 1978 que Foucault inscrit, non seulement la confession, mais la totalité du pouvoir pastoral, dans une généalogie non plus des disciplines mais de ce qu'on pourrait appeler les pouvoirs de bienveillance. Un pont peut alors être jeté avec les analyses ultérieures de Jean Delumeau qui insiste tant sur la dimension tranquillisante et apaisante de la $\ll$ tactique $\gg^{38}$ pastorale. Ces pouvoirs se donnent une fin qui n'est pas subordonnée à la reconduction incessante de leur exercice - comme le pouvoir de souveraineté - mais au développement « naturel $»$, « convenable ${ }^{39}$ de leur objet, en fonction d'une certaine prédisposition qu'ils lui reconnaissent. Ces pouvoirs ne s'exercent pas à partir de leur intensification et de leur prolifération, mais, au contraire, à partir de leur autolimitation. Rappelons que le pouvoir pastoral est étudié dans le même cours où Foucault propose une histoire du libéralisme comme gouvernement «à l'économie ».

N'accentuons pas cependant le caractère unilatéralement coercitif des analyses de Foucault en 1975. La leçon mentionne en bonne rigueur les dispositions que doit expressément respecter le confesseur : celui-ci doit s'engager personnellement, avec certains affects, dans la confession; il doit y investir son « zèle $»$, son $\ll$ amour $»$, son $\ll$ désir $\gg^{40}$ de voir le pécheur pardonné. Cela requiert qu'il combatte son impatience, le souci pour ses propres activités et ses préférences personnelles; il doit résolument interrompre ses occupations pour toujours accueillir avec bienveillance et promptitude le pénitent, quel qu'il soit (ibid., p.167168). Mais dans ces dispositions bienveillantes, le professeur lit uniquement ce que L'archéologie du savoir avait nommé en 1969 des «modalités énonciatives $\gg^{41}$, c'est-à-dire ce qui permet au confesseur de tenir son discours, de se situer par rapport au pénitent et d'appliquer sa grille d'entretien. Fonctionnant en amont de la confession, comme ce qui en définit le sujet questionnant, ces dispositions ne sont là que pour « quapour tranquilliser $\gg$.

39 Guillaume de La Perrière, cité dans Sécurité, territoire, population, p. 99.

40 Les anormaux, p. 165.

41 L'archéologie du savoir, partie II, chap. IV : « La formation des modalités énonciatives », p. 68-74. 
lifier le prêtre comme personne intervenant en tant que telle $\gg^{42}-$ vocabulaire qui est ici clairement celui de L'ordre du discours ${ }^{43}$. Cette qualification n'ouvre pas la relation à la réversibilité ou à l'imprévisibilité, elle se borne à légitimer, à l'un de ses pôles, la prise de parole.

Concentré surl'étude des modalités énonciatives, le philosophe ne tire pas des textes qu'il cite la conséquence majeure qui se fera jour en 1978 dans Sécurité, territoire, population : le confesseur ou le directeur - comme l'évêque ou le supérieur de communauté monastique - doivent également vivre une forme d'obéissance ${ }^{44}$. L'amour du directeur spirituel autorise sa parole, mais, par un étrange effet de retour, il la fragilise et la menace en même temps. Les manuels des confesseurs vont même plus loin, comme le mentionne furtivement le cours de 1974-1975: c'est un amour qui « attache le confesseur aux intérêts des autres ${ }^{45}$, lit-on dans le manuel de Louis Habert en 1688. Cette phrase est importante car elle annonce l'une des caractéristiques du pouvoir pastoral décrites dans la leçon du 8 février 1978 : le berger doit se sacrifier pour ses brebis, il est moralement lié, attaché à leur salut ${ }^{46}$. Pour cette raison, si quelqu'un encourt un risque dans la confession, c'est d'abord le confesseur, qui s'expose lui-même à la tentation en entendant les péchés des autres. D'où l'appel à la vigilance, omniprésent dans les manuels en direction des clercs : ne demander que le nécessaire.

Seul le cours Sécurité, territoire, population effectue le croisement en apparence paradoxal. L'obéissance pastorale est bien une soumission d'individu à individu. En cela, elle échappe au modèle de la loi - au même titre que la discipline - car cette soumission ne peut se réfléchir dans aucun principe général. Mais ce pouvoir « ne conduit à aucune maîtrise, ni de soi ni des autres » (ibid., p. 182); ou encore, il s'exerce : «tel que même les points de maîtrise y sont encore des effets d'obéissance » (p.182). Phrase d'une audace confondante, qui signifie que nulle volonté propre du supérieur ne doit guider l'exercice de son autorité, mais seulement sa propre soumission à ce qui le dépasse : un supérieur plus élevé, mais surtout, ultimement, Dieu et le salut d'autrui. En 1975, Foucault ne dit mot de ces bornes qui révèlent, non pas un contre-pouvoir, mais

Les anormaux, p. 167.

Pour l'usage, en ce sens précis, du terme « qualification »: L'ordre du discours, p. 41.

Sécurité, territoire, population, p. 182.

L. Habert, Pratique du sacrement de pénitence..., cité dans Les anormaux, p. 166.

Sécurité, territoire, population, p. 132. 
un autre fonctionnement, plus subtil, du pouvoir : le pouvoir de bienveillance est un pouvoir qui généralise l'obéissance au point de s'y soumettre lui-même. Il l'exige pour lui-même, car il reconnaît à son objet une fin dont il ne peut se prétendre absolument le maitre. Le pouvoir pastoral peut en effet collaborer au salut mais ne peut l'assurer, puisqu'il n'appartient qu'à Dieu. Il opère ainsi sur fond d'incertitude.

Nous pourrions alors montrer, dans une tout autre direction que celle du cours Les anormaux, que la confession n'est pas caractérisée par l'asymétrie de la coercition, mais par un double investissement des partenaires en vue d'une fin qui leur échappe à tous deux, sur fond d'une égalité de condition (universalité du péché, généralisation de l'obéissance). La condition de cette relation n'est cependant pas la réversibilité du rapport de force, comme définie par Foucault dans un article de $1982^{47}$, mais elle est ce que nous pourrions appeler une « communication des affects $\gg$ : ce qui doit accompagner et guider l'investissement mutuel du pénitent et du confesseur est une réciprocité de leurs affects dans la relation pastorale. L'amour du confesseur suscite les bons sentiments du pénitent et le confesseur, en retour, est affecté par la situation du pénitent. De même, la peine que ressent ce dernier amène la peine et la consolation du confesseur qui suscite à son tour la consolation du pénitent. Ceci est corroboré par les exemples donnés par Jean Delumeau dans son étude : au sujet du confesseur, saint Antonin de Florence écrit dans un manuel de 1582 : «qu'il participe à la peine, s'il veut partager la joie $\gg{ }^{4}$. Là encore, cette réciprocité sera thématisée par le cours de 1977-1978 sous l'appellation « principe du transfert exhaustif et instantané $\gg^{49}$ : tout ce qui arrive de bien ou de mal à une brebis doit être considéré par le berger comme son propre bien et son propre mal.

Tel n'est pas l'horizon de lecture en 1975, où Foucault s'exprime exclusivement en termes de surveillance et de quadrillage. Il serait pourtant hâtif d'incriminer des hypothèses fallacieuses ou des a priori massifs de lecture concernant la pastorale chrétienne. Notre étude se voudrait plus affinée et, surtout, plus littérale, afin d'expliquer les limites de cette première histoire de l'aveu.

48 J. Delumeau, L'aveu et le pardon, déjà cité, p. 22. 
Revenons un peu en arrière et rappelons-nous que les dispositions bienveillantes du confesseur n'étaient à aucun moment replacées dans un jeu de pouvoir entre partenaires, mais réduites à des modalités énonciatives particulières. Notre hypothèse est alors la suivante : si Foucault ne fait pas droit à la subtilité d'une direction des âmes qui tend à s'effacer elle-même, s'il dénie au pénitent toute qualité de partenaire dans une relation pourtant irréductible à une coercition, c'est tout simplement que l'objet de sa lecture n'est pas le pouvoir mais uniquement le discours.

Comme nous allons tenter de le montrer, à quelques ajouts et corrections près, Foucault continue de lire les manuels exclusivement comme des pratiques discursives. Notre enquête ne prétend cependant pas aller au-delà des seules leçons concernant la confession, encore moins décalquer la totalité d'un cours foisonnant sur la seule Archéologie du savoir. Mais l'étude de la confession constitue assurément dans le cours de 1974-1975 un moment «archéologique », qui s'articule sans doute difficilement avec les analyses « généalogiques » qui l'entourent.

Les manuels des confesseurs comme pratique discursive

Si Foucault avance que les textes à usage des laïcs peuvent être considérés comme $\ll$ l'envers $\gg^{50}$ de ceux à usage des confesseurs, c'est la preuve qu' il ne situe pas son analyse au niveau de l'exercice effectif du pouvoir. Les deux corpus ne peuvent être considérés comme des figures semblables et inversées qu'à la condition expresse de ne pas s'intéresser à la relation entre le confesseur et le pénitent, à ce que le philosophe décrira plus tard très généralement comme le « pouvoir », c'est-à-dire un « ensemble d'actions qui s'induisent et se répondent les unes les autres $\gg^{51}$. La leçon du 19 février 1975 a une ambition beaucoup plus réduite, qui use sans la nommer d'une méthode éprouvée quelques années auparavant dans le contexte des sciences humaines : celle de L'archéologie du savoir. En lisant les manuels post-tridentins, il s'agit en fait de décrire les pratiques discursives qui rendent possible la production de l'ensemble de leurs énoncés, quelles que soient leur réception et leurs utilisations concrètes. Une certaine hésitation s'entend d'ailleurs dans la voix du professeur, entre le discours de la confession et sa pratique réelle. Foucault parle d'abord de la «pratique 
nouvelle de la pénitence », avant de se corriger : « ou plutôt cette extension nouvelle et formidable des mécanismes de discours $\gg^{52}$, soulignant le point nodal de sa recherche.

L'analyse des pratiques discursives a pour but de rendre compte de l'existence historique des discours, en deçà d'une étude de la langue (linguistique) et d'une analyse du sens des propositions (sémiotique). La méthode archéologique, définie en 1969 dans l'espoir de ressaisir dans une forme unitaire les travaux courant de Folie et déraison aux Mots et les choses, se tient à la lisière des mots, sans pour autant revenir aux « choses mêmes ${ }^{53}$, dans un domaine que Foucault nomme avec une ambiguité assumée «prédiscursif» (ibid., p. 100). L'analyse ne sort pas du domaine du discours, refusant de réduire son existence à des processus économiques ou sociaux, mais elle se tient à sa limite, là où un système reliant des éléments hétérogènes à la fois verbaux (grilles de spécifications, grilles d'interrogations) et non verbaux (des institutions, des groupes sociaux) permet l'apparition dans le discours de ce qui n'appartient qu'à lui, regroupé selon quatre domaines : 1) objets, 2) modes d'énonciation, 3) concepts, 4) thèmes et théories.

Les difficultés précédemment évoquées disparaissent à partir du moment où l'on considère la leçon de 1975 comme un effort pour rendre compte des régularités propres à un discours, ou encore du système qui permet aux manuels de parler de ces objets-là (le corps, l'âme), de circonscrire ces modalités d'énonciation (les vertus du confesseur, son statut, ses droits), de définir ces concepts (désir, délectation, plaisir) et d'élaborer ces stratégies (thèmes et théories de la concupiscence). C'est bien un changement d'objets et de thèmes à l'intérieur du discours de direction spirituelle qui est relevé par Foucault :

Il y a eu, à partir de ce moment-là, formation ou élaboration de toute une série d'objets nouveaux, qui sont à la fois de l'ordre de l'âme et du corps, formes de plaisir, modalités de plaisir. C'est ainsi qu'on passe du vieux thème que le corps était à l'origine de tous les péchés, à cette idée qu' il y a de la concupiscence dans toutes les fautes. ${ }^{54}$

Il faut donc rendre compte de ce passage, définir les règles discursives auxquelles sont soumis ces objets et thèmes nouveaux, dans la ligne 
de L'archéologie du savoir que l'on a cru à tort brisée alors qu'elle s'était seulement courbée. Courbée, elle l'est cependant, car Foucault s'efforce constamment de montrer comment ce discours de la confession est à l'origine d'un nouveau mode d'exercice du pouvoir. Ce nouveau mode n'est pas tant l'acte de confession en lui-même qu'une technique d'intervention plus large, non exclusivement discursive, incluant la construction d'institutions comme les collèges et les séminaires ${ }^{55}$. Or, dans les analyses de L'archéologie du savoir, les institutions n'apparaissaient que dans les « instances de délimitation » (p.57) qui précèdent la formation des objets du discours ou dans les «emplacements institutionnels » (p.69-70) qui en permettent les modalités énonciatives. Pour l'archéologie, les institutions et les décisions politiques devancent le savoir et le rendent possible, elles n'en découlent pas $^{56}$. À la différence de 1969, les mécanismes de discours étudiés en 1975 ont désormais des effets concrets, qui dépassent le seul champ du savoir pour lequel l'archéologie avait été initialement modélisée. Celle-ci ne s'intéressait qu'aux règles de formation de certains objets du discours, sans réfléchir à la manière dont ces objets permettent en retour une intervention sur les choses et les personnes. La leçon du 19 février 1975 complète en fait l'archéologie pour montrer comment une pratique discursive a également prise sur les corps.

Pour paraphraser le commentaire que faisait Foucault des incorporels stoïciens en 1970, le corps est à la fois une réalité biologique, accessible à l'expérience sensible, et ce qui se dit de ce corps dans un énoncés7.

Les institutions ne sont pas dans la leçon de 1975 la condition de possibilité où un discours se déploie, mais bien l'effet d'un quadrillage que Foucault a défini comme étant d'abord discursif : «Les quadrillages plus fins de la nouvelle christianisation, celle qui commence au XVI ${ }^{\mathrm{e}}$ siècle, ont amené des institutions de pouvoir et des spécialisations de savoir, qui ont pris forme dans les séminaires, dans les collèges » (Les anormaux, p. 179).

56 C'est tout l'enjeu des chapitres que l'on pourrait qualifier «d'histoire sociale » dans Naissance de la clinique (chapitres II à v). Le chapitre III montre comment les changements politiques et institutionnels autour de la Révolution ouvrent un espace où peut naître et se déployer le discours clinique.

$D E \mathrm{n}^{\circ} 80$, « Theatrum philosophicum », I, p.950. Nous avions déjà repéré l'importance de cette notion d'incorporel, empruntée aux stoïciens par l'intermédiaire de Deleuze. Elle avait permis à Foucault de définir la fonction de l'âme dans le dispositif punitif. Il est intéressant de noter à nouveau sa présence discrète dans le cours de 1974-1975, même si le vocable n'apparaît pas. Il est possible en effet de mettre en série l'article de 1970 sur Deleuze et les stoïciens, les pages de Surveiller et punir se réappropriant cette double référence, et la leçon du 19 février 1975. Les similitudes sont frappantes : 1) même métaphore $\mathrm{du} \ll$ flottement dans le cours de 1975 et dans l'article de 1970 pour décrire cette zone 
Et c'est au croisement des deux, c'est-à-dire d'un domaine discursif et d'un champ d'intervention sensible, qu'apparaît ce qui n'est ni un mot ni une chose mais un « domaine à la fois complexe et flottant $\gg^{58}$ qui ouvre conjointement une possibilité de discours et d'action. Ce lieu situé à la limite du discours, comme l'était le domaine des pratiques discursives, se révèle donc également limitrophe à l'action. Autrement dit, la pratique discursive a désormais une face tournée vers les mots et une face tournée vers les choses. Cette zone de flottaison, que Foucault nomme « corps sensible et complexe de la concupiscence », ou encore « corps de désir et de plaisir » (ibid., p.187), apparaît au XVI ${ }^{\mathrm{e}}$ siècle. Et si elle «apparaît » (p.187, nous soulignons), c'est bien qu'elle ne désigne pour Foucault ni une réalité biologique ni un mot - le terme « concupiscence » existait déjà au premier millénaire -, mais le point de contact entre un exercice du pouvoir et un discours de connaissance; ce point où, pour reprendre une remarque de L'archéologie du savoir, tout en lui donnant un sens différent, « parler, c'est faire quelque chose ${ }^{59}$.

Cette performativité du discours n'est cependant pas encore clairement expliquée ni même thématisée dans la leçon de 1975, le lien entre le « quadrillage discursif ${ }^{60}$ et le quadrillage des corps semblant aller de soi, alors qu'il demeure un point aveugle de l'analyse. Mais pour notre question présente portant sur la manière de lire les textes, il suffit de remarquer que nous avons bien une étude des manuels catholiques qui

entre les mots et les choses : «Plutôt que de resserrer le sens dans un noyau noématique qui forme comme le cœur de l'objet connaissable, laissons-le flotter à la limite des choses et des mots $\gg\left(D E \mathrm{n}^{\circ} 80\right.$, « Theatrum philosophicum », I, p. 950); «domaine à la fois complexe et flottant » (Les anormaux, p. 187) ; 2) même rôle assigné aux inventions discursives de «corrélatif [d'une] technique de pouvoir » dans le cours de 1975 (Les anormaux, p.187) et dans le livre sur la prison (Surveiller et punir, p. 38).

58 Les anormaux, p. 187.

59 L'archéologie du savoir, p. 272. Ce clin d'œil à John L. Austin (How to do things with words, déjà cité) ne signifie pas une réappropriation par l'archéologie de la philosophie analytique. En 1969, l'assertion selon laquelle on fait des choses en parlant se limite à noter le caractère «compliqué et coûteux » de la parole, conséquence de la lourdeur des règles et des conditions que le sujet parlant met en œuvre à son insu. Par contre, comme cité plus haut (voir note 4, ce chapitre), dans les conférences inédites de Louvain en 1981, Foucault se réapproprie pleinement l'expression speech act, au sens que lui donne Austin, pour décrire l'aveu : non pas ce que la parole met en œuvre comme contraintes internes, mais ce qu'elle opère en dehors d'elle, par le simple fait qu'elle a été prononcée dans certaines circonstances. C'est dans ce deuxième sens que nous l'employons ici.

60 Les anormaux, p. 188 
en contourne la lettre, pour retrouver en dessous de celle-ci le système qui leur permet de parler d'objets nouveaux. Système sous-jacent contre lexique et théorie apparents : cette opposition se retrouve clairement dans La chair et le corps, manuscrit inédit de 1978 portant également sur la confession tridentine ${ }^{61}$. Foucault $y$ invite le lecteur à ne pas se laisser obnubiler par les similitudes lexicales des traités sur la confession du Moyen Âge à l'âge classique pour prêter une attention nouvelle à leurs «modes d'intervention discursive », c'est-à-dire « de quoi il est question, par où passent les partages $\gg$ et comment se déploie la «mise en discours $\gg$. Cette analyse permet alors de dégager, dans l'histoire de l'aveu au deuxième millénaire, deux «systèmes » successifs (que nous appellerons a. et b.) de mise en relation d'éléments verbaux (a. « pollution $\gg / \ll$ acte $\gg ;$ b. « délectation $\gg / \ll$ affect $\gg)$ et non verbaux (a. relation à autrui / émission de la semence; b. relation à soi /moment du plaisir) qui ont chacun leurs règles spécifiques de partage (a. opposition binaire volontaire / involontaire; b. division à l'infini des degrés de consentement) :

La notion de concupiscence qui se retrouve tout au long de la conception chrétienne de la chair ne doit pas faire illusion; elle ne peut valoir comme un fil directeur continu. Si on porte attention moins aux élaborations théoriques qu'aux modes d'intervention discursive, si on essaye de repérer de quoi il est question, par où passent les partages, comment se déploie la nervure non seulement des interdits, mais de la mise en discours, on s'aperçoit que ce n'est pas le problème de la concupiscence qui est central, mais celui de l'émission de semence et de la délectation; et sous ce mot de concupiscence, constamment employé à travers tant de siècles, une transformation importante s'est effectuée, que l'usage de ce mot risque d'éluder : c'est celle qui permet de passer d'un système juridico-pénitentiel centré sur l'émission de semence et ses effets sur un ensemble de relations, à un autre système - plus correctif que juridique, plus directionnel que pénitentiel - qui est centré sur la délectation avec ses déterminations multiples dans le corps et l'âme. ${ }^{62}$

Dans ce texte inédit, les éléments verbaux étudiés ne sont pas tant les mots en eux-mêmes (puisque les champs lexicaux appartenant à des systèmes distincts peuvent se recouper) que les «notions $»$, terme que

61 La chair et le corps, Fragment de la $1^{\text {re }}$ version du tome II d'Histoire de la sexualité, 1978. - Manuscrit, 40 pages; $30 \mathrm{~cm}$ [Archives Daniel Defert]. Cette première version des Aveux de la chair a probablement été rédigée au début de l'année 1978. 
Foucault privilégie sur celui de $\ll$ concepts $»^{63}$. La « notion $\gg$ désigne en effet une connaissance élémentaire, un peu lâche, qui se limite à certains détails d'un objet, non à sa description complète. Si la «notion » se raccroche bien à un vocable relativement fixe, elle rayonne au-delà. En particulier, elle ne renvoie pas à une définition exhaustive de la chose, mais indique d'abord, dans le discours, un certain type d'analyse, une manière de faire jouer les mots entre eux.

La lecture des manuels des confesseurs ne vise donc pas à reconstituer, même indirectement, la réalité de la relation sacramentelle, encore moins son vécu subjectif (les émotions de crainte ou de soulagement qui l'accompagnent). Seule compte l'apparition d'une formation discursive nouvelle, dont la mise en œuvre ou non dans la pastorale de terrain importe peu, attendu que ses effets réels excèdent de toute façon celle-ci.

Cette évacuation de la réalité vécue de la confession - reconnue plus rustique que le raffinement des manuels - au profit d'une archéologie du discours sur la confession a trois conséquences majeures sur la lecture des textes.

1) L'analyse proposée suspend toute étude sémantique. Nous l'avons rappelé, la lettre du texte n'est pas le domaine où l'archéologie se déploie. Pour reprendre les termes précis de L'archéologie du savoir, sa matière première n'est pas « les textes (ou les paroles) tels qu' ils se donnent avec leur vocabulaire, leur syntaxe, leur structure logique ou leur organisation rhétorique $\gg^{64}$.

Cette distinction des niveaux dans la constitution d'un discours permet d'expliquer le caractère quelque peu flottant du commentaire de Foucault quant au vocabulaire utilisé dans les manuels : la leçon sur la confession ne parle dans un premier temps que du «corps » - là où Habert distingue ce qui relève du «corps » et ce qui relève de l'« âme ${ }^{65}$ - avant de rapatrier subrepticement l'âme, assimilée désormais à la conscience ${ }^{66}$, dans un champ où la notion de « corps » se bat

$63 \mathrm{La} \ll$ pollution $\gg$, la « délectation $\gg$, la « concupiscence $\gg$ sont des « notions $\gg($ ibid.). Par contre, le terme « concept » n'apparait à aucun endroit de la partie conservée du manuscrit.

64 L'archéologie du savoir, p. 100.

65 Les anormaux, p. 174.

66 Dans la suite de la leçon, Foucault assimile l'âme aux plaisirs et désirs qui habitent le corps (ibid., p.178), puis à la conscience que le sujet a de ce qui se passe dans le corps (p.179). 
désormais en duel avec celle de $\ll$ chair ${ }^{67}$, sans jamais que ces termes soient définis. Si l'analyse semble se structurer dans sa conclusion autour du binôme désir-plaisir (p.175 et 179), elle use aussi bien des partitions corps-plaisir (p.175) ou désir-plaisir-complaisance (p.177), au gré des textes commentés, supposés constituer un champ discursif homogène malgré leurs déplacements théoriques. Cette circulation sémantique déconcertante n'a pas d'autres buts que de désigner une transformation qui n'appartient en propre ni aux mots ni aux choses, mais court sous leurs usages. Cette transformation concerne ce que Foucault appelle «l'épinglage » (p. 175) ou «le point d'accrochage » du discours (p.173), qui n'est pas exactement son objet, mais plutôt ses plans de différenciation où les objets du discours peuvent apparaître : du manuel de Milhard (1617) à celui de Habert (1688), l'examen requis pour la confession ne porte plus d'abord sur les relations sexuelles mais sur le rapport du pénitent à son propre corps. La transformation affecte ensuite ce que L'archéologie du savoir nomme les « grilles de spécification ${ }^{68}$ appliquées par le discours : non plus le permis et le défendu, selon un code juridique portant sur les actes, mais les différents degrés de consentement, selon un code physiologique portant sur les parties du corps et leurs effets de désir et de plaisir.

Jamais dans sa lecture des manuels catholiques Foucault ne s'arrête sur un mot, n'en propose l'étymologie ou ne s'en sert comme point d'appui de son analyse. La disposition des mots sur la page constitue un domaine supérieur du discours, tantôt trompeur (une constance lexicale peut masquer une transformation sous-jacente), tantôt indifférent (la définition de l'âme ne joue aucun rôle dans la description des transformations).

2) L'archéologie ne reconnaît que des positions du sujet énonçant, définies par le discours lui-même (statut, emplacement institutionnel), et évacue toute référence à l'intériorité. Réduit à un « espace d'extériorité $\gg^{69}$, le sujet du discours ne coïncide donc avec aucun sujet psychologique. Tout rapport que le pénitent établit à lui-même dans la confession est donc absent de l'analyse des textes.

C'est ainsi que Foucault se refuse à entrer plus avant dans le grand

67 Foucault parle indifféremment de la «cartographie peccamineuse du corps » (ibid., p. 174) et de la « physiologie morale de la chair $\gg$ (p.18o).

$68 \ll[\ldots]$ il s'agit des systèmes selon lesquels on sépare, on oppose, on apparente, on regroupe, on classe, on dérive les unes des autres les différentes "folies" » (L'archéologie du savoir, p. 58). Ceci peut être appliqué aux différents « péchés ».

69 L'archéologie du savoir, p. 74. 
débat théologique qui agita la pratique de la confession à lâge classique : celui de la contrition et de l'attrition. Les brèves mentions dans le cours sont volontairement laconiques et lacunaires ${ }^{70}$, inversement proportionnelles à la profondeur des sillons que ce débat, en apparence théorique, a creusés dans la vie religieuse, politique et sociale du XvII ${ }^{\mathrm{e}}$ siècle. Contre les jésuites et autres casuistes, les théologiens rigoristes exigeaient que le pénitent éprouvât la pleine contrition, c'est-à-dire un regret libre et sincère de ses fautes, porté par l'amour de Dieu et non par la peur du châtiment divin - laquelle était encore entachée d'amour de soi. Les jansénistes, le Grand Arnauld en tête, insistaient sur le fait que la peur servile de l'enfer ne pouvait suffire à une juste confession. Boileau écrira de même en 1695 que « les froids remords d'un esclave craintif $\gg^{71}$ n'ont aucune valeur. Le concile de Trente avait pour sa part maintenu une position moyenne : une peur de l'enfer excluant la volonté de péché et unie à l'espérance du pardon suffisait ${ }^{72}$.

En deçà des querelles théologiques, il importe de noter que le pardon n'est accordé que sous certaines dispositions de la volonté du sujet (ses « volitions »), qu'il n'est donc pas automatiquement déclenché par le seul aveu. La notion de « régime de vérité », rencontrée dans le cours de 1979-1980, permettrait justement d'intégrer ces dispositions à l'étude du rapport entre pouvoir et vérité dans la pratique de confession, tout en écartant une analyse d'ordre strictement psychologique. Il ne s'agirait pas en effet d'évaluer l'état de conscience des pénitents d'après le degré de rigorisme de leur confesseur, mais de déterminer ce que le pénitent

70 La contrition est bien définie par Foucault comme un pré-requis (Les anormaux, p. 168), mais le débat avec les attritionnistes n'est pas même mentionné. Plus loin, Foucault fait une allusion rapide à la casuistique, mais ne juge pas ces controverses pertinentes pour l'analyse : la casuistique, «vieux juridisme de la pénitence », n'est pas nouvelle, alors que cette « technologie de l'âme et du corps » l'est (ibid., p.178). Le manuscrit inédit de 1978 évacue également le débat de surface, pour revenir au système discursif quil'a rendu possible : «Le "laxisme" de certains membres de la Société de Jésus et de plusieurs confesseurs mondains $\mathrm{du} \mathrm{XVII}^{\mathrm{e}}$ siècle [illisible] beaucoup pour cette prolifération de la casuistique; mais on peut dire en retour que ce "laxisme" n'aurait pas pu avoir de telles possibilités d'intervention s'il n'avait eu à sa disposition l'analyse subtile de la concupiscence. Peu importe d'ailleurs le laxisme : lui-même et son adversaire rigoriste se sont développés sur le fond de ce qu'on pourrait appeler la grande "pénitentialisation de l'âme", aux XVI ${ }^{\mathrm{e}}$ et XVII ${ }^{\mathrm{e}}$ siècles. De l'âme, je devrais dire cette couture de l'âme et du corps où naissent les passions, les images, les excitations sensibles, les délectations et les voluptés » (La chair et le corps).

71 Cité dans J. Delumeau, L'aveu et le pardon, déjà cité, p. 47.

72 J. Delumeau, L'aveu et le pardon, déjà cité, p. 50. 
doit dire ou faire pour être reconnu «attrit $\gg$ ou « contrit $\gg$, c'est-à-dire avoir accès au pardon et à la lumière divine. Dans la relation sacramentelle, l'attrition et la contrition sont d'abord des paroles et des gestes, avant d'être des dispositions mentales ${ }^{73}$.

Il semblerait d'autant plus important de faire droit ici aux « régimes de vérité » que c'est la ligne janséniste qui va dominer la seconde moitié du XVII ${ }^{\mathrm{e}}$ siècle. Or, cette ligne dure exigeait beaucoup du pénitent en tant que sujet de sa confession; c'est là d'ailleurs sa modernité paradoxale. Le manuel de Habert (1688), dont Foucault souligne à juste titre le rigorisme, imposait ainsi une pleine participation du confessant, c'est-à-dire un plein assentiment de sa volonté, qui allait jusqu'à la fixation de la peine par le pénitent lui-même ${ }^{74}$. Cette recommandation était ancienne, puisqu'on la trouve chez Jean de Gerson (1363-1429) - demandant que la pénitence choisie par le confesseur ait le « consentement » du pécheur 75 - mais elle fut remise à l'honneur par un courant rigoriste refusant les confessions serviles et automatiques. De cette subtile intrication entre rigorisme et convocation du pécheur en tant que sujet, Foucault ne tire en 1975 aucune conséquence historique particulière, n'y voyant qu'une tactique discursive supplémentaire accentuant un contrôle particulièrement retors sur le pénitent : en effet, si la peine fixée par le pénitent est trop faible, le confesseur reste libre de l'augmenter ${ }^{76}$.

3) Nous touchons là à une troisième conséquence importante de la réduction des manuels à des pratiques discursives : en ne distinguant pas contrition et attrition comme deux régimes distincts de vérité - dissous ici dans un débat théorique de surface - Foucault ne peut distinguer deux mouvements politiques contradictoires : l'un de développement

73 Pour connaître les dispositions du sujet, il y a des questions précises que le confesseur doit poser, comme l'indique un manuel de Gerson (cité par Jean Delumeau, ibid., p. 77-78), que l'on peut lire en se rappelant que le confesseur doit croire ce que lui dit le pénitent (principe du credere tenetur : voir note 13 , ce chapitre). Le régime de vérité de la contrition renverrait alors strictement à la manière dont le sujet doit parler, se comporter, pour être jugé « contrit». Par contre, Jean Delumeau en reste bien à la dimension psychologique du débat, en cohérence avec son projet d'une histoire des sentiments de peur et de sécurité en Occident : «Il n'était pas indifférent pour le catholique d'autrefois d'avoir en face de lui $[\ldots]$ un prêtre rigoriste ou indulgent. Son confort psychique, sa vie de relations, ses comportements quotidiens pouvaient [en] être modifiés » (p.12).

74 Les anormaux, p. 169.

75 J. Delumeau, L'aveu et le pardon, déjà cité, p. 96.

76 Les anormaux, p. 169. 
du pouvoir pastoral avec ses confessions populaires massives; l'autre d'obstruction à ce vaste mouvement de «christianisation » dans lequel la leçon de 1975 englobe tous les manuels sans distinction. Or, en réagissant au laxisme des attritionnistes, c'est-à-dire en refusant le pardon aux fidèles simplement craintifs, les contritionnistes empêchèrent de fait que la confession demeure un large instrument de pastorale des âmes, comme elle l'avait été au XVI ${ }^{\mathrm{e}}$ siècle ${ }^{77}$. À l'opposé, dans la première partie de l'âge tridentin, seul un régime de vérité faible et relâché avait permis d'amener les masses des campagnes au confessionnal.

La réduction de la confession à une pratique coercitive peut donc s'expliquer par une lecture des textes comme pratiques discursives, celle-ci mettant entre parenthèses le niveau de la langue et celui du rapport à soi, pour venir se loger entre les sujets et les mots. Ce choix de lecture permet de réduire un corpus de textes foisonnant - du fait de la diversité des destinataires (confesseurs/pénitents) et des écoles théologiques (rigoristes/casuistes) - à un unique système de formation. Ne reste alors, au terme de la leçon de 1975, qu'une opération discursive de découpe - « grille », « filtre », « quadrillage »-, appliquée à la vie d'une âme.

C'est un tout autre rapport au texte qui va s'entendre dans le cours $D u$ gouvernement des vivants. À cette division brutale que nous opérons entre 1975 et 1980, on pourrait objecter qu'une chose est de lire les manuels du $\mathrm{XVII}^{\mathrm{e}}$ siècle, une autre de lire les textes patristiques du IV ${ }^{\mathrm{e}}$ siècle - autre corpus, autre méthode. En fait, les textes patristiques font l'objet d'un premier commentaire dès le cours Sécurité, territoire, population (1977-1978), sans qu' ils soient encore reçus par Foucault dans leur lettre même. Lire un texte dans sa lettre, c'est en effet le lire à ce niveau terminal du discours que contournait l'archéologie, là où les mots se donnent dans une certaine organisation syntaxique, logique et rhétorique ${ }^{78}$. C'est de cette différence entre les pères lus en 1978 et les Pères lus en 1980 que nous allons maintenant tenter d'approcher. Restons donc dans la même salle du Collège de France, mais projetons-nous successivement trois ans, puis cinq ans plus tard.

77 J. Delumeau, L'aveu et le pardon, déjà cité, p. 67.

78 Ce que Foucault appelle précisément «l'étage terminal » du discours (L'archéologie $d u$ savoir, p. 99). Le paragraphe continue ainsi : «L'analyse reste en deçà de ce niveau manifeste, qui est celui de la construction achevée [... ] bref, elle laisse en pointillés la mise en place finale du texte » (L'archéologie du savoir, p. 100, l'auteur souligne). 


\section{Le retour au texte : le virage de 1980}

\section{Abandon du principe d'exemplarité}

À deux ans d'écart, le corpus patristique chez Foucault permet en effet une intéressante comparaison : si la vaste entreprise de commentaire des Pères au Collège de France commence bien le 6 février 1980, une leçon du cours de 1978 portait déjà intégralement sur le gouvernement des âmes dans les premiers siècles chrétiens. Or, de 1978 à 1980, le rapport aux textes anciens se modifie radicalement, alors même que la période ( $\mathrm{II}^{\mathrm{e}}-\mathrm{V}^{\mathrm{e}}$ siècle) et les auteurs de référence (saint Cyprien, saint Ambroise, Jean Cassien, etc.) sont quasiment les mêmes. Une simple étude quantitative nous indique déjà qu'un changement d'objet induit bien un changement de méthode. Décomptons par exemple, pour une même durée d'enseignement, les textes matériellement présents dans les analyses de Foucault. En une heure de cours, le 22 février 1978, sont proposées seize citations directes des Pères, souvent très brèves, extraites de sept auteurs et de huit ouvrages différents. Le 13 février 1980, Foucault utilise un nombre très légèrement supérieur de citations (vingt au total, couvrant dix-sept versets), mais elles sont extraites seulement de deux ouvrages d'un unique auteur (le De baptismo et le De paenitentia de Tertullien). Non seulement Foucault réduit considérablement la matière livresque utilisée, mais ses citations sont extrêmement resserrées : sur les dix-sept versets lus en cours, onze sont extraits de deux chapitres consécutifs du De paenitentia.

Dans la progression de l'argumentation ensuite, les citations jouent un rôle différent. En 1978, la leçon est organisée autour des trois domaines concernés par le pouvoir pastoral (le salut, la loi et la vérité). Le premier domaine est à son tour subdivisé en quatre principes qui régissent l'exercice du pouvoir en vue du salut (responsabilité analytique, transfert exhaustif et instantané, inversion du sacrifice, correspondance alternée). Mais ce découpage complexe ne se trouve tel quel chez aucun des Pères; il relève uniquement de l'analyse propre à Foucault, qui en avoue volontiers le caractère «schématique et caricatural $\gg^{79}$. En ce sens, la présentation du pouvoir pastoral rappelle ce que Foucault nommait très rudimentaire, très élémentaire $\gg)$. 
dans le cours Le pouvoir psychiatrique la «description fictive $\gg^{80}$ du rôle du médecin au XIX ${ }^{\mathrm{e}}$, description élaborée sur quatre séries différentes de texte. Dans ces deux cours, 1973-1974 et 1977-1978, la construction théorique et synchronique d'ensemble efface ouvertement la diversité historique des textes rassemblés (auteurs et contextes) et exonère de leur commentaire séparé. À une différence près : en 1978, une fois énoncé et expliqué dans son mécanisme interne, chaque principe de la pastorale du salut est illustré par une ou deux citations patristiques, réduites souvent à quelques mots. Introduite de la manière la plus directe qui soit par «Et saint Cyprien $[. .$.$] dit \gg^{81}$, « Jérôme le dit $\gg($ ibid., p. 174), «Comme le disait saint Grégoire » (p.175), la parole citée joue le rôle d'attestation conclusive de ce qui a été préalablement défini. Elle n'est ensuite ni reprise ni expliquée, comme si son sens était obvie et son adéquation au propos du professeur immédiate. Pour ce faire, l'énoncé citant est volontairement le plus neutre possible, se réduisant presque exclusivement au verbe dire ${ }^{82}$, qui devient alors subrepticement, par l'appui de la conjonction «comme » ou de l'adverbe « ainsi », synonyme de redire ou dire autrement : «Comme le disait saint Grégoire » (p. 175, nous soulignons), « et c'est ainsi que saint Benoît dit » (p. 175 et 181 , nous soulignons). En aucun cas, il ne s'agit de commenter le texte cité, de reprendre l'une de ses expressions ou de définir l'un de ses mots - comme le fera constamment Foucault en 1980. Il s'agit seulement de retrouver dans les relations entre les actants de la phrase (le pasteur, la brebis, l’abbé, la ville, le lait, la main), les rapports réels de pouvoir établis entre les sujets du pastorat.

Nous ne sommes plus dans une analyse des pratiques discursives, mais dans un rapport au texte beaucoup plus lâche et incertain. Tout se passe comme si, résolu à abandonner le modèle archéologique, Foucault ne lui avait pas encore trouvé de remplaçant. Sa lecture ne se situe plus en deçà des discours, pour en retrouver le système de formation, mais résolument au-delà, dans un fonctionnement concret du pastorat qui traverse le corps

«Oui, tout ceci est une description fictive, en ce sens, que je la bâtis à partir d'un certain nombre de textes qui ne sont pas d'un seul psychiatre; car s'ils étaient d'un seul psychiatre, la démonstration ne vaudrait pas » (Le pouvoir psychiatrique, p. 5, nous soulignons).

81 Sécurité, territoire, population, p. 173.

82 Sur les seize citations, treize sont introduites par le nom de l'auteur suivi du verbe $\ll$ dire $»$. 
social et auquel ces discours participent sur un mode obscur. Il reste en effet à définir comment cette analyse du pouvoir, qui prétend serrer de près son « usage quotidien $\gg^{8_{3}}$, peut s'articuler à une nouvelle analyse des textes. En prenant ses distances avec la première histoire de l'aveu, le cours Sécurité, territoire, population permet, comme nous l'avons vu, de réintégrer la relation entre le directeur et le dirigé à l'intérieur de l'étude du pouvoir, mettant ainsi en évidence l'autolimitation de la direction spirituelle. Mais cette avancée n'explique pas comment cette relation vécue est déduite de l'archive, avec quelles précautions. En l'absence de toute possibilité d'établir un lien direct entre un texte et une situation réelle, si l'historien ne veut pas, comme s'y risque Jean Delumeau, lire cette situation «entre les lignes $\gg^{84}$, encore moins revenir à une histoire des représentations, la marge de manœuvre est étroite. Nous pourrions alors tenter de définir ce rapport incertain de Foucault au texte, à mi-chemin entre le cours de 1974-1975 et celui de 1979-1980, par la notion d'« exemplarité ».

Ce que Foucault définit lui-même comme une $\ll$ esquisse $»^{85}$ du pouvoir pastoral, chevauchant les textes de manière cavalière, pourrait sans doute s'expliquer par les circonstances : il s'agit de présenter en un temps réduit un sujet vaste, à la fois connexe mais secondaire par rapport au thème général du cours (les dispositifs de sécurité). Cependant, des similitudes troublantes dans la manière d'approcher les textes se retrouvent tout au long du cours Sécurité, territoire, population, de la lecture des physiocrates du XVIII ${ }^{\mathrm{e}}$ siècle à celle des Pères de l'Église. Écoutons en particulier la manière dont Foucault introduit respectivement la Lettre d'un négociant sur la nature du commerce du grain (Louis-Paul Abeille, 1763), Le miroir politique (Guillaume de La Perrière, 1555), et La règle pastorale (Grégoire le Grand, 590). Nous allons voir que ces introductions n'engagent pas un quelconque manque de rigueur dans l'étude des documents, mais une approche critique de leur originalité et de leur pertinence :

83 Cette année 1978, peu de temps après son cours au Collège de France, Foucault insiste dans une conférence prononcée à Tokyo sur l'importance de répondre à la question «empirique » de «l'usage quotidien » du pouvoir : $D E \mathrm{n}^{\circ} 232$, « La philosophie analytique de la politique $\gg$, II, p. 541 .

84 J. Delumeau, L'aveu et le pardon, déjà cité, p. 7.

85 Sécurité, territoire, population, p. 139. 
Lettre d'un négociant sur la nature du commerce du grain :

Donc on a tout un paquet, là, de textes, de projets, de programmes, d'explications. Je me référerai simplement à [celui, parmi eux,] qui est à la fois le plus schématique, le plus clair [...]. Alors, [si l'on prend] ce texte comme référence - mais il est simplement exemplaire de toute une série d'autres $[\ldots]$. (p.37, nous soulignons)

\section{Le miroir politique :}

Je vais prendre, pour essayer de repérer les choses dans leur état encore fruste, un des premiers textes de cette grande littérature antimachiavélienne [...]. Dans ce texte, encore une fois très décevant $[\ldots]$ on voit cependant s'esquisser un certain nombre de choses qui sont, je crois, importantes. (p.96, nous soulignons)

La règle pastorale:

On le voit dans toute une série de textes comme celui de saint Grégoire, par exemple $[\ldots]$. (p. 178, nous soulignons)

La mise en parallèle de ces extraits amène deux séries de remarques. Tout d'abord, chaque texte n'est jamais lu dans sa singularité, mais comme «exemplaire » d'une série de documents dont l'analyse définit le thème commun et tente d'extraire les conséquences pour l'action politique. Cette exemplarité pose le caractère interchangeable d'un texte qui ne peut se réclamer d'aucune position particulière au sein de la série. Dans le cours Il faut défendre la société (1975-1976), Foucault hésitait déjà à donner une place originaire ou un rôle fondateur à une déclaration aussi importante que Qu'est-ce que le Tiers État? rédigée par Sieyès. Or, cette hésitation le faisait aussitôt retomber sur la notion prudente d'exemplarité : «Et je prendrais, sinon exactement comme point de départ, du moins comme exemple de cette transformation, évidemment, le fameux texte de Sieyès $\gg{ }^{86}$. D'où une deuxième remarque importante : si un texte est sélectionné parmi d'autres, ce n'est pas pour la richesse de son argumentation, mais au contraire pour son caractère « fruste », «schématique », non spéculatif ${ }^{87}$, qui va permettre d'illustrer le plus clairement possible des stratégies nouvelles, des objectifs d'intervention

87 Pour comprendre l'art effectif de gouverner, Foucault prend ses distances avec les textes de Giovanni Botero, Giovanni Antonio Palazzo et Bogislaw Philipp von Chemnitz, penseurs de la raison d'État, car ils sont jugés trop théoriques : « la plupart de ces textes sont tout de même des textes un petit peu théoriques et spéculatifs qui ont encore quelque chose comme un relent platonicien » (Sécurité, territoire, population, p. 296). Foucault leur préfère des textes «qui émanent de gens plus proches à coup sûr de la pratique politique, qui y ont été directement mêlés » (ibid., p. 297), comme Sully ou Richelieu. 
nouveaux, dont il n'est que l'annonce ou l'esquisse sur le papier. Ce n'est pas exactement une feuille de route, plutôt une manière inédite de réfléchir des actions possibles, sans pour autant faire un usage transcendantal des idées qui les organiserait en système. La maladresse formelle de l'esquisse, sa rudesse argumentative sont le signe pour Foucault qu'elle est tournée vers l'action et non vers la contemplation, qu'elle est œuvre d'un décisionnaire et non d'un théoricien. Le texte reste ainsi toujours en léger décalage par rapport à la transformation décrite - qui concerne d'abord les pratiques réelles ${ }^{88}$ - sans pour autant se perdre dans l'abstraction ou l'utopie prospective. Le commentaire y gagne par conséquent en liberté, étant donné que l'objet de l'analyse demeure toujours hors texte. La lecture peut alors être méticuleuse (ainsi des premières pages du Miroir politique de La Perrière, dont le vocabulaire est longuement discuté par Foucault) ou se contenter de résumer un argument général (ainsi du Politique de Platon, auquel une partie de la leçon du 15 février 1978 est consacrée), le texte ne vaut jamais pour lui-même, mais uniquement pour la rémanence de ses thèmes dans une série plus large, qui rend probables et intéressantes ses « corrélations dans le réel ${ }^{89}$.

Par rapport à cette pratique audible dans Sécurité, territoire, population, le cours de 1979-1980 va faire exactement l'inverse. Ce sont les textes eux-mêmes, lus dans leur irréductible singularité, qui définissent les problématiques. Ce sont leurs mots qui mènent l'argumentation et définissent le découpage précis de celle-ci, avec un minimum de remarques sur le contexte historique et les pratiques réelles. Un exemple va suffire à nous en convaincre : la lecture d'Édipe roi, proposée en ouverture du cours Du gouvernement des vivants. Cette lecture nous semble emblématique d'une nouvelle manière d'interroger les jeux de la vérité et du pouvoir à l'intérieur des textes anciens. La perception de cette nouveauté est facilitée par le contraste entre la lecture de 1980 et celle précédemment proposée du même Edipe en mai 1973, à Rio de Janeiro. La conférence brésilienne reprend en fait dans ses grandes

Revenant sur les textes théoriques de Botero et Palazzo qu'il avait commentés, Foucault précise : «Tout cela évidemment est tout à fait insuffisant pour arriver à repérer ce qu'a été réellement le fonctionnement de cette raison d'État 》 (ibid., p. 295, nous soulignons); «Et là on voit que cette théorie du maintien de l'État est tout à fait insuffisante pour recouvrir la pratique réelle de la politique et la mise en œuvre de la raison d'État » (p. 297, nous soulignons).

89 Sécurité, territoire, population, p. 104. 
lignes une conférence donnée en mars 1972 à l'université de Buffalo90, prolongeant elle-même une première étude proposée dans le cours de 1970-1971 au Collège de France : La volonté de savoir.

Cette série d'études consacrées à $O E$ dipe roi sur près de dix ans permet de nous assurer que ce n'est pas d'abord la spécificité du corpus patristique qui explique ces nouvelles pratiques de lecture, mais bien le virage théorique des «rituels » puis «régimes de vérité ». La lecture des Pères chrétiens n'aura plus ensuite qu'à s'inscrire dans la ligne tracée par le commentaire de Sophocle - à quelques torsions près, que nous évaluerons au terme de ce parcours.

\section{Quoi de neuf? CEdipe}

À l'écoute successive des conférences de 1973 (Rio de Janeiro) et de 1980 (Collège de France) consacrées à $O$ Edipe roi de Sophocle, la première impression est étrange, sinon dérangeante. À sept ans d'écart, en dépit du virage méthodologique que nous avons tenté de souligner, les deux lectures d' $O$ dipe semblent en tous points similaires : même mention de la «loi des moitiés ${ }^{91}$ pour définir le mécanisme de la vérité mis en intrigue par Sophocle; même relation établie entre cette loi et la technique juridico-politique du $\sigma v ́ \mu \beta o \lambda o v$ grec; même mise en parallèle finale du savoir d'OEdipe et de son pouvoir tyrannique. Foucault luimême ne cache pas la redite ${ }^{92}$.

Dès le début de la deuxième conférence de 1973, Foucault limite le statut du texte de Sophocle : la pièce est «représentative et d'une certaine façon instauratrice d'un type déterminé de relation entre pouvoir et savoir, entre pouvoir politique et connaissance ${ }^{93}$. Par cette affirma-

Cette conférence est reproduite dans Leçons sur la volonté de savoir. ĐEdipe fera à nouveau retour dans les conférences de Louvain en 1981, qui reprennent quant à elles le cours au Collège de France de 1979-1980, sans modification notable : Cours Mal faire, dire vrai. Fonctions de l'aveu.

$91 D E n^{\circ} 139$, « La vérité et les formes juridiques », I, p. 1425 ; la leçon du 16 janvier 1980 parle de «mécanique des moitiés » ou d'un «jeu de six moitiés ».

92 Redite qui lui permet, non sans malice, de vérifier que le public ne suit pas ou ne revient pas à ses cours - rappelons que les conférences de Rio reprennent en partie une leçon au Collège de 1970-1971 : «c'est un exemple dont j'ai un peu traité, dont j'ai un peu parlé, il $\mathrm{y}$ a au moins $[\ldots]$ neuf ans à peu près. Alors $\mathrm{j}$ 'ai fait quelques sondages, il semble que peu de gens s'en souviennent, ce qui prouve que grâce au ciel, ils ne restent pas là neuf ans » (leçon du 16 janvier 1980). 
tion, il s'agit d'écarter toute intrusion de la psychanalyse dans l'interprétation proposée du mythe. Il n'y a pas de vérité générale d'œEdipe à retrouver en deçà de Sophocle et pas d'identification possible d'GEdipe avec le lecteur; il n'y a qu'un texte ancien racontant une histoire, où sont lisibles un certain nombre de procédures de connaissance, apparues à un moment précis de l'histoire grecque. Mais en cherchant à définir, dans la phrase que nous venons de citer, la fonction du texte par rapport à ces procédures, Foucault utilise deux termes que l'on pourrait juger insatisfaisants : « représentative $\gg$ et $\ll$ instauratrice $\gg$.

Le substantif « instauratrice » est téméraire au regard des conclusions à venir : le reste du commentaire va en effet systématiquement relier les éléments de la tragédie à des pratiques religieuses, judiciaires ou politiques que Sophocle n'a en rien inventées, encore moins instaurées. Ainsi, la procédure de recherche de vérité que déploie la pièce, avec la place décisive occupée par le témoignage des esclaves, «obéit exactement aux pratiques judiciaires grecques de l'époque $\gg$ (ibid., p. 1423). Le verbe « obéir » est une deuxième fois employé pour décrire le rapport du texte à une technique bien réelle et largement répandue au même moment

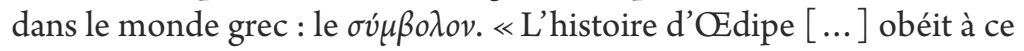
$\sigma v \mu \beta 0 \lambda o v$ : forme non pas rhétorique, mais religieuse, politique, quasi magique de l'exercice du pouvoir $\gg$ (p.1428). Rien de nouveau, donc, du côté de Sophocle. Ce que semblent pareillement reconnaître les leçons des 16 et 23 janvier 1980, laissant soupçonner que Foucault ne s'est pas beaucoup renouvelé entre-temps, reprenant sans doute une bonne partie de ses notes brésiliennes. Ainsi, en 1980, le «dire-vrai » des esclaves, distingué de celui des oracles et des devins, est pareillement rapporté aux «règles des procédures judiciaires nouvelles que les constitutions et les lois, à la fin $\mathrm{du} \mathrm{VI}^{\mathrm{e}}$ et surtout au début du $\mathrm{V}^{\mathrm{e}}$ siècle, avaient mises en place dans un certain nombre de cités grecques $\gg^{94}$. Sophocle n'a pas eu besoin de les nommer explicitement, seulement de les mettre en scène, étant donné qu'elles appartenaient déjà aux « réalités historiques » (ibid.).

Il faut se convaincre alors que le premier terme employé pour décrire le statut du texte par rapport aux pratiques réelles est le plus juste : la pièce est « représentative » d'un nouveau dispositif de savoir-pouvoir. L'adjectif « représentative » peut néanmoins surprendre au regard d'un 
travail historique qui considère les textes comme des faits, non seulement linguistiques, mais polémiques et stratégiques, ayant leur matérialité et leurs effets propres dans l'histoire, à distance des représentations mentales dont ils seraient la trace écrite. Pourtant, la conférence de Buffalo en 1972 en appelle bien à Edipe comme à une « figure ${ }^{95}$, vocabulaire par excellence de la représentation. Tout se passe comme si le statut que Foucault cherchait à accorder au texte, induisant une certaine méthode de lecture, se jouait finalement entre les deux termes - «instauratrice » et «représentative »-le premier en disant trop, le second trop peu. Si dans les postulats méthodologiques présentés à Rio les textes sont considérés comme des stratégies «qui font partie des pratiques sociales ${ }^{96}$, Foucault tend cependant à considérer la pièce de Sophocle comme simplement emblématique ou, plus précisément, exemplaire de ces stratégies. Nous réutilisons volontairement ce terme, mais en lui donnant le sens précis qu'il a en grammaire : est exemplaire un texte, phrase ou membre de phrase que l'on cite pour étayer ou illustrer une règle, une tournure, l'emploi particulier d'un mot. En ce sens, la pièce ne représente pas des faits mentaux (une certaine vision du pouvoir), mais elle réutilise des procédures discursives fonctionnant réellement dans la société comme stratégie de pouvoir, pour en exemplifier simplement l'usage. Sur ce point, les deux lectures d' $E$ dipe s'accordent parfaitement.

Cette même convergence de vue entre la conférence brésilienne de 1973 et la leçon parisienne de 1980 se retrouve dans leur reconnaissance finale de la dimension critique de la pièce : les péripéties de la tragédie œdipienne ont pour fonction principale de montrer l'échec d'une certaine forme de pouvoir politique. Or, cette forme, Foucault l'identifie immédiatement à une figure historique précise, apparue en Grèce aux $\mathrm{VII}^{\mathrm{e}}$ et $\mathrm{VI}^{\mathrm{e}}$ siècles avant notre ère : celle du tyran, c'est-à-dire l'homme arrivé au pouvoir non par une descendance légitime mais par ses exploits personnels ${ }^{97}$. À nouveau, la vérité politique du texte précède ce dernier - ce qui n'est bien entendu pas faux, mais annule du même coup la singularité de la tragédie au profit d'une réalité culturelle et sociale qui lui est contemporaine : l'avènement de la Grèce classique qui vient progres-

« Edipe - non pas blason de l'inconscient, [... ] mais figure du souverain porteur d'un savoir excessif » (Leçons sur la volonté de savoir, p. 250).

$96 D E \mathrm{n}^{\circ} 139$, «La vérité et les formes juridiques », I, p. 1408.

97 Ibid., p. 1432-1434; et leçon du 23 janvier 1980. 
sivement déchirer au $\mathrm{v}^{\mathrm{e}}$ siècle l'ancienne union du pouvoir et du savoir dans la figure tyrannique. Une fois reconnu tyran au même titre qu'un autre, Edipe peut en effet être rapproché de rois ou législateurs bien réels comme Kypsélos ou Solon ${ }^{98}$; tandis que la pièce elle-même, dans son réquisitoire contre les procédures tyranniques de gouvernement par le savoir, annonce à sa manière La République de Platon ${ }^{99}$. La pièce participe ainsi à ce vaste mouvement de dénonciation et de décomposition d'une Grèce archaïque, héritière des empires assyriens.

Un philologue comme Jean Bollack pourrait à juste titre reprocher à Foucault de ne pas faire suffisamment droit ici à l'autonomie des textes littéraires par rapport à la société dans laquelle ils s'inscrivent. À distance de l'anthropologie historique d'un Jean-Pierre Vernant, à laquelle Foucault portait un vif intérêt ${ }^{100}$, Jean Bollack refuse une étude globale de la société qui permettrait d'articuler dans une même vision les domaines de la vie politique, culturelle et intellectuelle ${ }^{101}$. Rendant au domaine des lettres et de la philosophie une autonomie relative, il cherche à établir la différence d'un texte par rapport à une tradition littéraire, signe d'un

$98 D E \mathrm{n}^{\circ} 139$, « La vérité et les formes juridiques », I, p. 1433.

$99 D E \mathrm{n}^{\circ} 139$, « La vérité et les formes juridiques », I, p. 1436

$100 \mathrm{DE} \mathrm{n}^{\circ}{ }_{139}$, «La vérité et les formes juridiques », I, p. 1503-1504. Les travaux de Jean-Pierre Vernant sont cités dans la leçon du 9 janvier 1980. La lecture que livre Foucault d'EEdipe roi est cependant peu dépendante de l'essai de l'anthropologue français «Ambiguité et renversement. Sur la structure énigmatique d'Edipe roi $\gg$ publié en 1970 (dans Échanges et communications. Mélanges offerts à Claude Lévi-Strauss, J. Pouillon et P. Maranda éd., t. II, La Hague-Paris, Mouton, 1970). Même si Vernant fait le lien entre Edipe et la figure tyrannique, il s'agit d'abord de relier la pièce de Sophocle à la pratique politique de « l'ostracisme $\gg$. Cet essai est cependant évoqué dans la leçon du 16 janvier 1980. L'influence commune à Foucault et Vernant est le philologue et sociologue Louis Gernet qui, le premier, établit le lien entre la tragédie et les pratiques juridiques à l'occasion de l'apparition en Grèce des tribunaux. Il le fit dans son séminaire de sociologie juridique à l'École pratique des hautes études (voir J.-P. Vernant et P. Vidal-Naquet, Mythe et tragédie en Grèce ancienne, t. I, Paris, La Découverte, 2001, p. 15). Foucault cite effectivement Gernet comme l'une de ses influences significatives aux côtés de Glotz, Dumézil et Vernant : DE $\mathrm{n}^{\circ} 139$, «La vérité et les formes juridiques », I, p. 1504.

101 J. Bollack, Sens contre sens. Comment lit-on? Entretiens avec Patrick Llored, Genouilleux, La passe du vent, 2000, p. 72. Replacée dans un certain «moment historique », la tragédie est effectivement analysée par Jean-Pierre Vernant et Pierre Vidal-Naquet « comme phénomène indissolublement social, esthétique, psychologique » (J.-P. Vernant et P. VidalNaquet, Mythe et tragédie en Grèce ancienne, t. I, déjà cité, p.9). Ces trois faces du phénomène font l'objet d'une analyse «structurale » qui montre comment la réalité sociale, la création esthétique et la mutation psychologique se rencontrent et se combinent. 
écart réflexif et critique par rapport à la société102. Mais cette différence n'a pas d'autre lieu que la lettre du texte, c'est-à-dire sa syntaxe et sa grammaire, qui constituent en propre le sens d'un énoncé. Aux dires du philologue formé à l'école allemande, les anthropologues français continuent de dessiner le «système des représentations collectives qu'ils voient naturellement et donc inconsciemment reproduit dans les œuvres $\gg^{103}$. Ce qui n'est pas inexact, Jean-Pierre Vernant et Pierre Vidal-Naquet n'hésitant pas à parler en $1972 \mathrm{du}$ «surgissement d'une conscience et d'un homme tragique $\gg^{104}$, contemporain des tragédies. Sans faire sien un vocabulaire issu de la psychologie historique d'Ignace Meyerson, maître de Vernant, Foucault épouse dans sa lecture d'OEdipe la même vision totale de la société grecque, croisant les domaines politiques, judiciaires et esthétiques.

Dans les développements rigoureusement parallèles des deux lectures foucaldiennes d'Edipe, en 1973 et en 1980, nous vérifions que la lettre du texte, ses choix lexicaux et ses formes rhétoriques n'intéressent le philosophe qu'en tant qu'ils illustrent des pratiques réelles d'exercice du pouvoir, que d'autres documents permettent par ailleurs d'attester et de faire fonctionner. À une différence près, à la fois discrète mais décisive.

La seule nouveauté introduite par le cours de 1980 par rapport aux conférences de Rio concerne justement ce qui appartient en propre à Edipe et n'apparaît nulle part ailleurs que dans le texte de Sophocle. La question posée est pourtant la même qu'en 1973 : «qu'est-ce que c'est que ce savoir œdipien ? $\gg{ }^{105}$. Mais la réponse brésilienne était sur ce point hâtive, tout du moins incomplète : à l'université de Rio, Foucault ne distingue pas tout d'abord la $\gamma v \omega ́ \mu \eta$ - la connaissance qui a permis à Edipe de résoudre l'énigme du Sphinx - de l'acte de trouver $\varepsilon \dot{v} \rho i ́ \sigma \mathcal{K} \varepsilon v$

102 J. Bollack, Sens contre sens, déjà cité, p.36. La recherche de Jean-Pierre Vernant et Pierre Vidal-Naquet fait cependant droit à la dimension critique des tragédies par rapport à la réalité que l'auteur tragique avait sous les yeux. La tragédie met en question ce que dit et croit la cité. Mais cette dimension critique rejoint finalement un moment historique d'incertitude et de tension entre les traditions mythiques qui s'effacent et les formes nouvelles de la pensée politique qui apparaissent. On retrouve donc à nouveau un système de représentations collectives.

103 J. Bollack, Sens contre sens, déjà cité, p. 72.

104 J.-P. Vernant et P. Vidal-Naquet, Mythe et tragédie en Grèce ancienne, t. I, déjà cité, p. 15.

105 Leçon du 23 janvier 1980 ; et $D E \mathrm{n}^{\circ} 139$, «La vérité et les formes juridiques », I, p. 1434 : «Qu'est-ce que ce savoir d'Edipe?» 
- qui mène Edipe à l'esclave ayant assisté au meurtre, à travers une certaine $\tau \dot{\varepsilon} \chi v \eta^{106}$. Au sujet du verbe $\varepsilon \dot{v} \rho i \sigma \kappa \varepsilon l v$, Foucault se contente de préciser qu'il s'agit d'une activité qui se fait seul; caractéristique qui lui permet aussitôt de relier l'usage du terme chez Sophocle à une certaine procédure de «savoir-pouvoir» apparue à un moment de l'histoire politique grecque. Quel savoir en effet se pratique seul, sans écouter les dieux ni personne, sinon le savoir autocratique du tyran, « qui par luimême, peut et est capable de gouverner la cité $\gg$ (ibid., p. 1435) ? Foucault compose alors, autour du personnage d'Edipe, la série suivante : «le tyran qui sait, l'homme de la $\tau \dot{\varepsilon} \chi v \eta$, de la $\gamma \nu \omega ́ \mu \eta \gg\left(\right.$ p. 1436) ${ }^{107}$. Sans s'arrêter aux choix lexicaux de Sophocle, le commentaire de Rio rassemble ainsi dans une même procédure de «savoir-pouvoir » : 1) la figure historique du tyran ; 2) la technique par laquelle Edipe mène l'enquête sur le meurtre de Laïos ; 3 ) la résolution par Edipe de l'énigme qui a guéri la cité. Trois éléments que le cours $D u$ gouvernement des vivants va au contraire soigneusement distinguer.

Au lieu de glisser trop vite de la vérité tragique à la vérité politique, Foucault insère dans le cours de 1979-1980, à l'exacte intersection entre le non-savoir initial d'Edipe et le savoir du tyran, un commentaire méticuleux du texte de Sophocle, s'attachant à ses mots et à leurs usages dans la tragédie. Ce moment bien particulier recouvre la première partie de la leçon du 23 janvier 1980. Il peut être localisé plus précisément entre une question portant sur le savoir («Qu'est-ce que c'est que ce savoir œdipien?») et l'annonce du virage vers le pouvoir («Alors quelques mots, maintenant, sur le pouvoir ») - le choix de ces bornes n'étant bien entendu pas indifférent. Comme nous allons tenter de le montrer, l'auditeur y respire un air renouvelé par rapport aux conférences brésiliennes.

Dans la leçon précédente du 16 janvier 1980, Foucault s'est attaché à définir les «alèthurgies » caractéristiques respectivement des dieux et

106 « Edipe dit à tout moment qu'il a vaincu les autres, qu'il a résolu l'énigme du Sphinx, qu'il a guéri la cité au moyen de ce qu'il appelle $\gamma v \omega ́ \mu \eta$, sa connaissance ou sa $\tau \dot{x} \chi v \eta \gg(D E$ $\mathrm{n}^{\circ} 139$, « La vérité et les formes juridiques », I, p. 1434).

107 Le séminaire de Buffalo est encore plus expéditif : à peine Foucault a-t-il posé la question du savoir d'Edipe, qu'il y répond par la tyrannie : « Ce savoir, à mi-chemin [de ceux] du dieu et de l'esclave, est celui du "tyran" » (Leçons sur la volonté de savoir, p. 234). Dans la suite du développement, comme à Rio en 1973, le savoir du tyran, la $\gamma v \omega ́ \mu \eta$ et la $\tau \dot{\chi} \chi v \eta$

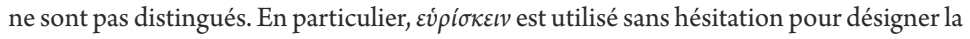
résolution de l'énigme du Sphinx. 
des esclaves, c'est-à-dire les « rituels » par lesquels ces derniers manifestent une vérité, après l'avoir arrachée au faux, au caché, à l'oubli. Toujours en excès par rapport aux besoins intrinsèques du pouvoir, même si ce dernier ne peut s'exercer sans eux, ces rituels ostentatoires délimitent le domaine à l'intérieur duquel Foucault découpera ensuite celui plus restreint des « régimes de vérité ». À l'intérieur des procédures réglées par lesquelles une vérité est manifestée, le «régime de vérité » décrira plus précisément la part qui revient à un sujet dans cette manifestation - part qui peut être celle de l'agent, du spectateur ou de l'objet de la manifestation. Notons pour le moment que la notion de « rituel $\gg$ nous laisse encore en deçà du rapport à soi.

Il reste alors à définir l'alèthurgie propre au personnage d'CEdipe $\mathrm{e}^{108}$ - procédure cette fois-ci individuelle, qu'il nous faut soigneusement distinguer du $\sigma v ́ \mu \beta o \lambda o v$ précédemment évoqué. En effet, le $\sigma v ́ \mu \beta o \lambda o v$ - pratique sociale courante dans la Grèce de ce temps ${ }^{109}$ - permet à Foucault de décrire l'alèthurgie générale de la pièce, le fil directeur de son intrigue, qui va de moitié de vérité en moitié de vérité, engageant à sa suite l'ensemble des personnages et des événements. Mais c'est seulement au sein de ce jeu du $\sigma u ́ \mu \beta o \lambda o v$, méticuleusement déplié par la leçon du 13 janvier 1980, que des procédures de vérité spécifiques sont engagées ${ }^{110}$.

En suivant la lettre du texte, Foucault distingue tout d'abord la $\tau \dot{\chi} \chi \nu \eta$ d'Edipe des manières de faire de Créon et de Tirésias, car celles-ci obéissent à d'autres champs sémantiques. Il rattache alors cette $\tau \dot{\chi} \chi v \eta$ au verbe $\varepsilon \dot{v} \rho i \sigma \kappa \varepsilon l v$, qui signifie «trouver, découvrir ». Le commentaire pose alors une deuxième distinction, à l'intérieur cette fois des propres

108 Foucault parle avec insistance d'un « savoir propre à OEdipe » (leçon du 23 janvier 1980). Ce savoir est « un savoir spécifique, qui a une forme particulière et que l'on peut parfaitement décrire par rapport aux autres types de savoir, que ce soit celui du devin, que ce soit celui du témoin, etc. » (ibid.). Il ne s'agit bien entendu pas ici du contenu de ce savoir, mais de la procédure en elle-même, de son rituel.

109 Foucault l'avait souligné à Rio et le rappelle à nouveau dans le cours de 1979-1980: le $\sigma v ́ \mu \beta o \lambda o v, \ll c$ 'est l'authentification d'une alliance privée entre deux familles, c'est la reconnaissance d'un individu par un autre, c'est la marque de validation d'un message, c'est tout cela, c'est cette forme-là qui est en jeu dans GEdipe » (leçon du 16 janvier 1980).

Le cours est clair sur ce point. Le jeu des moitiés permet ainsi de composer deux alèthurgies distinctes : «moitié divine, alèthurgie religieuse, prophétique, rituelle $[. .$.$] et puis ensuite$ moitié humaine qui est là, l'alèthurgie individuelle du souvenir et de l'enquête » (leçon du 16 janvier 1980). Le séminaire à Buffalo de 1972 insiste pareillement sur le fait que le $\sigma u ́ \mu \beta o \lambda o v$ permet de décrire la totalité de la tragédie de Sophocle : « OEdipe est une histoire "symbolique", une histoire de fragments qui circulent » (Leçons sur la volonté de savoir, p. 229). 
manières de faire d'Edipe. Il appuie cette distinction sur une nuance lexicale : la résolution de l'énigme du Sphinx est désignée par le substantif

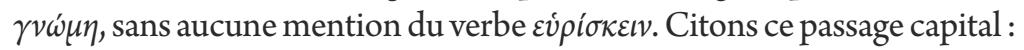

De sorte que cette activité si proprement œdipienne et à laquelle il va lier son destin, activité qui consiste à aller chercher, à partir à la découverte de la vérité, ce n'est pas du tout avec ça qu'il a résolu l'énigme du Sphinx. Il l'a résolu avec un tout autre type d'activité qui est $\gamma v \omega ́ \mu \eta$, qui est l'opinion."11

La distinction est une nouveauté décisive par rapport à $1973 \mathrm{car}$ elle permet d'isoler dans le texte une activité proprement œedipienne. Appartenant au vocabulaire juridico-politique de la Grèce du ve siècle, la $\gamma v \omega ́ \mu \eta$ désigne un élément précis dans la recherche d'une vérité : l'avis ou l'opinion donné par le citoyen au cours d'un procès. Ce n'est cependant pas pour cette raison que la $\gamma v \omega ́ \mu \eta$ est ici écartée : à ce stade de l'analyse, Foucault ne fait volontairement pas mention de la pratique historique qui consistait à demander aux jurés et magistrats de donner leur opinion. Il ne le fera qu'ultérieurement, dans la deuxième partie du cours traitant des implications politiques de la tragédie. Gardant ici une ligne de conduite ferme, il écarte la $\gamma v \omega ́ \mu \eta$ uniquement parce qu'elle n’appartient pas à la « série » (ibid.) des termes se rattachant dans la tragédie à $\varepsilon \dot{v} \rho i \sigma \kappa \varepsilon v v$, et n'est par ailleurs l'objet d'aucun usage dans le texte permettant de lui conférer une signification particulière pour Edipe ${ }^{112}$.

Foucault le redit tout au long de son commentaire, c'est le « vocabulaire $\gg^{113}$ - avec ses insistances, ses séries, ses choix caractéristiques et exclusifs - qui guide son enquête, semblable à des signaux qui clignotent à la surface du texte. Il s'agit de repérer les champs sémantiques permettant de discriminer les pratiques de vérité et d'isoler ainsi celle qui

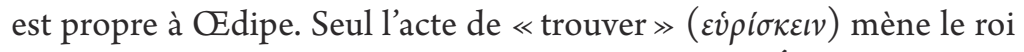
solitaire à se démasquer lui-même comme meurtrier. Écoutons alors la manière dont la leçon s'empare de ces quelques mots.

Définir ce que le texte entend par «trouver », c'est réveiller à sa surface un sens en apparence trivial, c'est redonner leur chance à des mots érodés

111 Leçon du 23 janvier 1980, nous soulignons.

112 De manière significative, ne voulant pas faire intervenir des éléments extérieurs au texte dans cette partie du commentaire, Foucault se contente de noter que $\gamma \nu \omega ́ \mu \eta$ est un « mot un petit peu pâle, un petit peu fade, un petit peu neutre $\gg($ ibid.).

113 《Tous les témoignages sont très caractéristiques au niveau du vocabulaire » (leçon du 16 janvier 1980); « Alors là, le vocabulaire est très clair, très net, très insistant 》 (ibid.). 
par un usage trop fréquent. Qu'est-ce que trouver? «Trouver, découvrir [commente Foucault], c'est un acte par lequel celui qui ne savait pas devient celui qui sait $\gg$ ( $i b i d$.). Et cet acte est rendu possible par la présence de «signes », de «repères », marqués du sceau d'un unique vocable sur

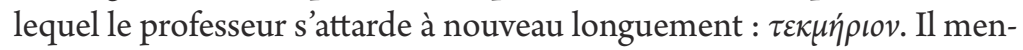
tionne ses usages divers chez les auteurs grecs (Alcméon, Aristote), précisant celui moins rigoureux et à connotation fortement médicale qu'en fait Sophocle. La priorité est cependant de décrire le mécanisme à la fois très simple et singulier suivant lequel ce $\tau \varepsilon \kappa \mu \eta ́ p ı v$ opère, non pas en général, mais dans l'intrigue d'Edipe roi; puis de regarder les objets qu'il y pro-

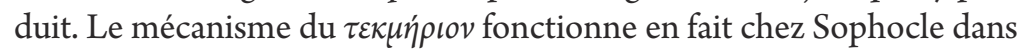
quatre directions différentes : 1) du présent au passé ; 2 ) du passé au présent ; 3) de la présence à l'absence ; 4) de l'absence à la présence. Foucault prend son temps et construit ses définitions par divisions successives, vers

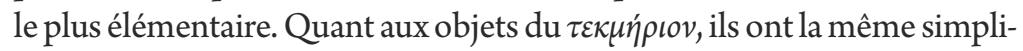
cité apparente : «ce qui s'est effectivement passé » (ibid.), c'est-à-dire à la fois l'événement en lui-même et l'écart de celui-ci par rapport au décret des dieux. Il reste alors à définir l'événement, comme le «croisement des choses, des séries, des gestes, des hommes $\gg$ (ibid.).

Si ces définitions peuvent sembler ingénues, il faut accorder à cette ingénuité le bénéfice d'une véritable posture méthodologique : ne pas être certain de savoir a priori ce que signifient les mots les plus évidents et laisser le texte reformuler lui-même cette signification, à partir des éléments mêmes de l'intrigue. Si l'événement est par exemple défini comme un croisement de choses et d'hommes, c'est parce qu'il porte dans $Q$ Edipe roi sur la croisée des chemins où mourut Laïos.

Dans ce commentaire qui ne redoute pas les inférences simples, voire minimales, la pensée se déploie à même le texte, déjouant les abstractions qui risqueraient de le surplomber, les significations qui prétendraient le précéder. Nul terme n'est trop usité pour se refuser à une définition neuve (ainsi de «trouver», «signe », «événement »). Pour reprendre une métaphore de Jean Bollack, il s'agit de lire le texte comme on apprend une langue étrangère, comme si les mots nous en étaient inconnus ${ }^{114}$. À la différence près que Foucault, contrairement à Bollack,

114 J. Bollack, Sens contre sens, déjà cité, p. 21 : «C’est comme si, dans une langue, le grec ou le français, on en apprenait une autre plus idiomatique, par un travail de décodage des éléments constitutifs. On découvre un nouvel emploi, qui repose sur d'autres éléments, 
ne s'intéresse pas tant à la syntaxe qu'aux mots eux-mêmes, leurs apparitions et leurs rémanences à chaque étape de l'intrigue, dans sa progression vers la reconnaissance finale.

Sous ces règles particulières de lecture, le texte n'a plus cette épaisseur que lui conférait le lourd outillage des pratiques discursives, il n'est plus que surface. C'est en ce sens qu'il faut entendre le qualificatif de « positiviste $\gg$ que Foucault applique en 1980 à sa propre méthode de lecture, sur un ton d'autodérision ${ }^{115}$. Bien entendu, à cette étape de la leçon, le professeur continue de relier les notions rencontrées à un contexte de débats et d'institutions qui les déterminent et les débordent ${ }^{116}$. Mais telles des notes en bas de page, ces remarques n'influent aucunement sur le mouvement de lecture du texte qui se poursuit sans nulle référence extérieure à celui-ci. Dans cette partie à la fois limpide et littérale du commentaire, la «tyrannie » est mentionnée comme vocable de la tragédie ( $\tau$ $\rho \alpha v \iota)$, nullement comme figure historique précise. Le fait qu'OEdipe ne daigne compter que sur ses propres capacités est bien indiqué, mais sans être encore raccroché à un quelconque régime politique. Dans ce moment précis de la lecture, la solitude d'OEdipe n'est que la condition de sa propre recherche d'une vérité dont il veut être le seul opérateur.

Ce n'est en fait que dans le deuxième temps de l'analyse que Foucault développe ce qui était déjà audible dans les conférences de Rio, non sans avoir préalablement indiqué la coupure : «Alors quelques mots, maintenant, sur le pouvoir $\gg$ (ibid.). Suit un long développement qui reprend la question du gouvernement mis en œuvre par OEdipe - sa prétention à piloter seul le navire - dans le contexte cette fois-ci de la Grèce $\mathrm{du} \mathrm{v}^{\mathrm{e}}$ siècle et sa critique du modèle tyrannique. La tragédie se retrouve alors insérée dans une lecture globale de la vie politique grecque, à la naissance de la démocratie. Mais entre la $\tau \dot{\varepsilon} \chi v \eta$ d’OEdipe et ses corrélations

empruntés ou modifiés, servant à la construction d'un sens dont on peut dire qu'il est toujours déjà une interprétation du sujet traité. »

115 «Je ne suis pas très sûr que l'interprétation ultra, agressivement et platement positiviste que je vous donne soit tout à fait vraie $\gg$ (leçon du 23 janvier 1980).

116 Citons deux de ces contextualisations, qui restent secondaires par rapport au mouvement de l'argumentation : «Cette transformation de celui qui ne savait pas en celui qui sait, vous savez bien que c'est le problème des sophistes, le problème de Socrate, ça sera encore le problème de Platon. C'est tout le problème de l'éducation, de la rhétorique, de l'art de persuader » (ibid.). Également, dans cette même partie de l'analyse : « Édipe est une affaire de médecine et de droit. C'est [... ] une des premières articulations d'une alèthurgie en forme judiciaire avec une alèthurgie en forme médicale. » 
avec des formes historiques de savoir-pouvoir, il y a le moment du texte, qui offre à la lecture la trace singulière d'un rituel de vérité.

Jusque dans la conclusion de la leçon, Foucault maintient cette singularité en distinguant la procédure elle-même qui mène OEdipe au vrai, de la manière dont le roi solitaire a voulu faire fonctionner cette procédure à l'intérieur du modèle tyrannique. La condamnation qu'entraîne avec lui le dénouement de la pièce ne porte donc pas sur la procédure en tant que telle - reconnue bonne et efficace en soi - mais sur son usage politique particulier ${ }^{117}$. La critique du pouvoir dans $E$ dipe roi, dont seule la référence à un certain contexte historique éclaire le sens, ne rencontre la $\tau \dot{\varepsilon} \chi v \eta$ d'Edipe qu'à partir du moment où la tragédie laisse cette dernière entre les mains d'un « maître unique » (ibid.), c'est-à-dire d'un oligarque.

\section{Du fils Edipe aux Pères chrétiens : radicalisation d'une lecture}

Si nous revenons à notre question première concernant le lien entre les présupposés méthodologiques et les pratiques de lecture, il importe de relever que les « rituels de vérité » ouvrent pour Foucault un nouveau rapport au texte, distinct des modèles qu'il avait utilisés dans le passé (pratiques discursives et exemplarité). Les leçons des 16 et 23 janvier 1980 proposent la lecture littérale, guidée par une lexicologie minutieuse, d'une œuvre considérée dans son autonomie et son originalité : Édipe roi. À l'opposé ou en contrepoint, les procédures de gouvernement politique continuent d'obéir à une lecture conjointement littérale et générale, passant aisément du texte au contexte, au risque de manquer la spécificité de l'espace propre à la tragédie. Ainsi du pouvoir tyrannique qui est conjointement ce que clame le chœur au milieu de la pièce et la 《matrice de la pensée politique ${ }^{118}$ grecque au v ${ }^{\mathrm{e}}$ siècle. À la fois le mot d'une intrigue et une figure historique précise.

Succédant à la lecture œdipienne, les lectures chrétiennes puis stoïciennes du cours $\mathrm{Du}$ gouvernement des vivants vont alors radicaliser le mouvement en ne conservant que le premier type de lecture. Il n'y a plus que le texte, car il n'y a plus que les régimes de vérité. Or, cette implication

117 «Le procédé est bon, la procédure est bonne, mais c'est le contexte du pouvoir tyrannique à l'intérieur duquel $\Xi$ dipe a voulu la faire fonctionner, $[\ldots]$ c'est cet usage-là qui condamne; qui condamne quoi ? Eh bien, celui-là même qui en fait usage » (ibid.). 
ne va pas de soi. En apparence, il n'y a nulle raison en effet de confiner ainsi les régimes de vérité au domaine textuel, étant entendu qu'ils n'ont pas moins de réalité dans la vie sociale des sujets que les régimes politiques. Le changement dans la manière de lire est pourtant patent à compter de la leçon du 6 février 1980 portant sur la Didachè et la Première Apologie de Justin, dont la lecture ne glisse jamais vers les marges du texte pour évoquer la situation politique ou la réalité sociale. À compter de ce moment, l'attention portée aux mots au détriment des choses va devenir exclusive de toute autre lecture, au point de suspendre les régimes de vérité au-dessus du bruit et de la fureur de l'histoire. Dans la première partie, nous avions relevé le caractère lapidaire des références historiques données par le cours, dessinant un simple horizon culturel ou politique ne déterminant en rien les régimes de vérité tels qu'ils sont concrètement mis en œuvre. Pour les premiers siècles chrétiens, la mention des hérésies ou des persécutions n'est l'occasion que de brèves notules; la conversion de l'empereur Constantin, qui demeure encore aujourd'hui un point de butée pour l'historien ${ }^{119}$, n'est pas même évoquée. Du fils Edipe aux Pères chrétiens, Foucault a considérablement réduit la voilure de sa recherche, alors que la notion de « régime de vérité » s'inscrit en apparence dans le prolongement direct de celle de « rituel de vérité » ou d'《alèthurgie », utilisée dans le commentaire d' Edipe.

Étrange modification des conditions de traversée des textes, donc. La délimitation d'un domaine distinct des régimes juridico-politiques - en ce sens qu'il est irréductible aux connaissances et aux sujétions que ces régimes réclament comme moyen et fin de leur exercice - était bien affirmée dès l'ouverture du cours, à l'occasion de la lecture d'un texte de Cassius concernant l'empereur Septime Sévère ${ }^{120}$. Mais avant de mettre le cap en direction du christianisme, le professeur ne semblait pas vouloir accorder à ce qu'il appelait encore « rituels de vérité » une place exclusive dans son analyse. Il avait choisi de respecter leur autonomie, mais dans l'unique fin de mieux les articuler aux arts de gouverner. Le projet initial était bien de décrire les relations entre exercice du pouvoir et manifestation de la vérité, ou encore entre « hégémonie » et « alèthurgie » (ibid.). L'analyse d' Édipe roi, dans les deux moments que nous venons d'isoler, en est un bon exemple. L'analyse des Pères, quant à elle, ne connaît plus 
qu'un unique moment : celui des régimes de vérité, dans leur inscription exclusivement textuelle. Il nous faut maintenant tenter de démêler les raisons de cet exclusivisme, en nous attardant tout d'abord sur un précurseur, dans l'œuvre de Foucault, de ce nouveau rapport au texte.

Ce précurseur, nous l'avons déjà rencontré dans notre recherche, et il convient d'autant plus d'y faire une nouvelle halte que s'y trouve l'annonce précoce des régimes de vérité : il s'agit de la réponse à Jacques Derrida au sujet des Méditations métaphysiques, où celui-ci se voit reprocher d'être allé «bien au-delà de ce que dit le texte cartésien : ou plutôt $[\ldots]$ bien en deçà de ses singularités $\gg{ }^{121}$. Cette remarque n'est pas aussi innocente qu' il y parait, replacée dans le contexte encore proche d'une Archéologie du savoir pour laquelle «ce que dit le texte cartésien » n'aurait précisément pas grand sens. C'est la volonté de contrecarrer Derrida sur son propre terrain, c'est-à-dire celui des $\ll$ traces textuelles $»^{122}$, qui mène Foucault à réinvestir le commentaire littéral, tout en cherchant à garder pour lui la fraîcheur de la méthode archéologique, qui s'accorde pourtant mal avec ce souci de la lettre. La gêne de Foucault est lisible dans le fait que l'adjectif « discursif» est tantôt employé en un sens banal, pour décrire le simple commentaire de texte, tantôt dans le sens précis qu'il avait en 1969, pour indiquer ce niveau de l'analyse sous-jacent aux mots et aux thèmes du discours $^{123}$. Arrêtons-nous sur la manière dont Foucault se ressaisit du texte de Descartes, une décennie après son commentaire dans Folie et déraison.

La troisième partie de la contre-démonstration de Foucault s'appuie sur le «vocabulaire employé $»^{124}$ par Descartes, en établissant des champs sémantiques qui permettent de soigneusement distinguer deux exercices différents dans le cheminement du doute : celui concernant la folie, celui concernant le rêve. Lisons attentivement les termes utilisés par Foucault : «Paragraphe de la folie : vocabulaire de la compa-

$121 D E \mathrm{n}^{\circ} 102$, « Mon corps, ce papier, ce feu $\gg$, I, p. 1116.

$122 D E \mathrm{n}^{\circ} 102$, « Mon corps, ce papier, ce feu », I, p. 1135 .

123 Le niveau inférieur de l'archéologie est en effet tantôt dénié, tantôt reconnu dans le texte de 1972. À certains endroits, Foucault appelle « analyse discursive » son simple commentaire littéral (étude du vocabulaire, des exemples et du récit de Descartes) : par exemple, page 1121. À un autre endroit, Foucault souligne pourtant bien que le discursif est un niveau plus profond de l'analyse : «si un lecteur, aussi remarquablement assidu que Derrida, a manqué tant de différences littéraires, thématiques ou textuelles, c'est pour avoir méconnu celles qui en forment le principe, à savoir les "différences discursives" » (ibid., p.1125).

124 Ibid., p. 1119, l'auteur souligne. 
raison $[\ldots]$. Paragraphe du rêve : vocabulaire de la mémoire $\gg$ (p.1119, nous soulignons). Dans sa progression, ce commentaire littéral consiste à dégager : 1) la «nature de l'exercice » (p.1119) cartésien indiquée par son vocabulaire; 2 ) les «thèmes » (p.1119) de l'exercice indiqués par les exemples choisis par Descartes; 3 ) l'« épreuve centrale » (p.1119) indiquée par le récit qu'en fait Descartes; 4) l'«effet de l'exercice » (p.11201121) indiqué par les phrases qui scandent les décisions de Descartes. Nous sommes bien dans une simple analyse de texte.

Au terme de sa plaidoirie méticuleuse, Foucault assimile cependant cette analyse à celle des «pratiques discursives » (p.1135), afin de souligner son incompatibilité avec la grammatologie de Derrida. Étrange rattachement, qui semble sinon trompeur, à tout le moins superflu. Il n'est pas sûr en effet que l'attention de Foucault dans son commentaire aux « différences littérales des mots » (p.1124) ait besoin du lourd outillage de l'archéologie pour s'opérer; surtout quand on se rappelle que les mots ne constituent pas le lieu d'investigation de l'archéologie : «pas plus de description d'un vocabulaire [écrivait Foucault en 1969] que de recours à la plénitude vivante de l'expérience $\gg^{125}$. Quant aux «événements $\gg^{126}$ qui se produisent dans le texte cartésien et aux «modes d'implication du sujet $\gg$ (ibid., p. 1135) dans celui-ci, ils ont un sens différent de ceux développés par L'archéologie du savoir. Dans son commentaire de Descartes, Foucault s'intéresse en effet à ce que dit le texte de la place occupée par le sujet méditant et des effets que cette méditation a sur lui. Pour l'archéologie, il s'agirait au contraire de se situer à un niveau inférieur, afin de définir le statut du sujet qui parle en ce lieu nommé «Descartes » : sa fonction institutionnelle et sa position par rapport à l'objet du discours, qui déterminent l'existence singulière des signes formant les Méditations métaphysiques. Bref, ce qui permet au sujet du discours de prendre ainsi la parole et de dire ces choses-là. Or, ces deux champs d'étude - le régime de vérité décrit par le texte et le régime de vérité $d u$ texte (génitif subjectif) - ne se recouvrent pas nécessairement, ils ne se recouvrent même que rarement. Les faire coïncider supposerait de faire appel sous le texte cartésien à une expérience « réelle » de la méditation qui serait homogène au discours qu'elle a produit. Force est de constater que Foucault glisse par moments vers cette supposition, comme si le sujet énonçant les Méditations et le sujet 
énoncé par elles $-\mathrm{le}$ 《Je $\gg$ du texte - étaient le même ${ }^{127}$. Mais pour une stricte analyse archéologique, ce serait trop accorder à Descartes d'entériner ainsi le rôle fondateur de la part mentale et subjective de l'exercice méditatif.

Pour le reste de la démonstration, l'exclusion de la folie qui permet à un sujet de se qualifier comme sujet doutant peut effectivement être mise au compte d'une analyse archéologique des Méditations, dégageant les conditions de production de leurs énoncés. La mise en lumière de cette exclusion n'est pas le résultat du commentaire littéral de l'exercice méditatif, puisque ce dernier n'aboutit qu'à montrer qu'il n'y a pas continuité entre l'expérience décrite de la folie et l'expérience décrite du rêve. Seul le rattachement des termes insanus, demens et amens à des pratiques médicales et judiciaires extérieures au texte - qualifiant respectivement le malade et l'incapable légal - permet de déduire leur rôle successivement « caractérisant » et «disqualifiant ${ }^{128}$. C'est bien le domaine juridique, la « question de droit $\gg^{129}$, qui vient déterminer de l'extérieur cette qualification du sujet pensant et méditant, tenant discours sur le rêve et la folie, et non ce que dit le texte cartésien à son égard. En faisant appel dans un second temps aux partages opérés par les institutions médicales et judiciaires, Foucault dégage ainsi ce que L'archéologie $d u$ savoir avait nommé des $\ll$ instances de délimitation $\gg^{130} \mathrm{du}$ discours.

En revanche, le « régime de vérité » $d u$ texte cartésien, si nous prenons le génitif en son sens maintenant objectif, c'est-à-dire ce qu'il propose au sujet de faire comme exercice de pensée, n'est rien d'autre que l'objet de son discours, dont le sens doit être suffisamment obvie pour guider les actes réflexifs du lecteur. L'interprétation littérale est alors

127 La volonté de recoller les morceaux entre le contenu du discours (ses mots, ses thèmes) et les pratiques discursives qui le sous-tendent amène cette déclaration pour le moins étrange de la part de Foucault : «Une "méditation" au contraire produit, comme autant d'événements discursifs, des énoncés nouveaux qui emportent avec eux une série de modifications du sujet énonçant » (ibid., p.1125). S'agit-il de l'exercice réel, subjectif, de la méditation ? Les guillemets indiquent-ils l'ambiguïté ? Dans l'article sur Blanchot en 1966, Foucault avait pourtant mis en doute l'assimilation entre le «sujet parlant » et « celui dont il est parlé » dans la proposition «Je parle $\gg\left(D E \mathrm{n}^{\circ} 38\right.$, « La pensée du dehors $\left.», \mathrm{I}, \mathrm{p} .547\right)$.

$128 D E \mathrm{n}^{\circ} 102$, « Mon corps, ce papier, ce feu », I, p. 1122.

129 Ibid., p.1122. Voir aussi p. 1128 : « Mais puis-je reprendre à mon compte cet exemple [des fous $]$ ? [... Impossible : isti sunt dementes, c'est-à-dire qu' ils sont juridiquement disqualifiés comme sujets raisonnables. »

130 L'archéologie du savoir, p. 57. 
justifiée si l'enjeu de l'analyse est de décrire cette fonction de guidage, caractéristique des écrits appartenant à la grande tradition des « conseils d'existence $\gg^{131}$. Les modifications dans la manière de lire les textes, apparues discrètement en 1972 et de manière encore plus nette en 1980, commencent ici à s'éclairer.

Lire certains régimes de vérité dans «l'économie visible du texte $\gg^{132}$, comme le propose à sa manière Foucault en 1972, c'est-à-dire dans le seul «jeu des oppositions sémantiques et grammaticales » (p.1131), n'est pas revenir à cette transparence du langage fustigée par L'archéologie $d u$ savoir. Il s'agit de faire droit à un type particulier d'écrits, qui doivent être étudiés par l'historien en fonction de ce qu'ils requièrent du côté du lecteur : une compréhension suffisamment immédiate pour qu'elle puisse être traduite en actes. Comme Foucault le rappellera à ses auditeurs en 1982, les Méditations cartésiennes plongent leurs racines dans l'usage grec de la $\mu \varepsilon \lambda \dot{\varepsilon} \tau \eta$ (meditatio) ${ }^{133}$. Or, parmi les supports de l'antique exercice de la $\mu \mathcal{\varepsilon} \lambda \dot{\varepsilon} \tau \eta$ se trouvent justement l'ensemble des recueils de conseils et résumés d'enseignements rédigés par le maître ou son disciple, dans le but d'être mémorisés et appliqués. Ainsi par exemple des Entretiens et du Manuel d'Épictète, régulièrement cités dans les derniers enseignements au Collège de France, de 1980 à 1984. Ces textes « avaient pour rôle d'être des opérateurs $\gg^{134}$, une « armature de la vie quotidienne $\gg($ p. 21), est-il précisé dans l'introduction à L'usage des plaisirs en 1984. Dans les leçons sur le monachisme chrétien, cela peut être éclairé par le choix préférentiel de Foucault pour l'œuvre de Cassien : si cette œuvre l'intéresse en priorité pour comprendre le fonctionnement réel du monachisme, c'est parce qu'elle n'est ni une règle générale de vie (comme la Règle de saint Pacôme) ni un témoignage sur la vie héroïque de quelques moines dessinant un idéal lointain (comme l'Histoire lausiaque). Elle est « un recueil de pratiques » par lequel Cassien «montre comment ça fonctionne, il montre comment on vit dans un monastère $\gg^{135}$.

131 Sous ce vocable, Foucault regroupe dans le cours Subjectivité et vérité (1980-1981) toute une littérature qui court de la période hellénistique et romaine jusqu'à l'âge classique (en particulier saint François de Sales) : leçon du 14 janvier 1981.

$132 D E \mathrm{n}^{\circ} 102$, « Mon corps, ce papier, ce feu », I, p. 1132.

133 L'herméneutique du sujet, p. 339-341.

134 L'usage des plaisirs, p. 21.

135 Leçon du 19 mars 1980. 
À distance de cette littérature, OEdipe roi n'est pas un écrit ascétique ; la $\tau \dot{\varepsilon} \chi v \eta$ individuelle mise en intrigue par Sophocle a d'abord pour but de manifester une vérité générale (vérité des faits ou vérité de soi), sans requérir une transformation préalable du sujet qui manie cette $\tau \dot{\varepsilon} \chi v \eta$. Les écrits ascétiques, qu'ils soient chrétiens ou gréco-romains, font au contraire de la vérité ce qui doit faire plier l'être même du sujet : régime de vérité, au sens de gouvernement de soi par la vérité. La finalité de leur lecture indéfiniment reprise n'est nullement de pénétrer la pensée de l'auteur, de découvrir le sens profond de ce que les mots signifient, mais, à force de les parcourir des yeux ou de les entendre, il s'agit de les graver dans son esprit afin de convertir un objet de méditation en principe d'action. La pensée d'une chose - par exemple la mort, le dénuement - doit nous préparer à l'expérimenter ${ }^{136}$.

Le nouvel usage des textes qui se fait jour en 1980 peut alors être éclairé par ce que dit la philosophie antique de la manière dont le disciple doit s'atteler à la lecture, au moins dans sa dimension proprement cognitive. En définissant le mode antique de lecture de ces manuels, Foucault définit par le même mouvement ses propres règles d'analyse. Le recoupement est pour le moins suggestif, si l'on se reporte au cours L’herméneutique du sujet (1981-1982):

$[\ldots]$ cette fonction méditative comme exercice du sujet se mettant par la pensée dans une situation fictive où il s'éprouve lui-même, c'est cela qui explique que la lecture philosophique soit - sinon totalement, du moins pour une bonne part - indifférente à l'auteur, indifférente au contexte de la phrase ou de la sentence. (p. 341, nous soulignons)

Cette double indifférence requise du côté du disciple, pour que soit respecté l'enjeu pratique et non théorique de ces textes, reflue sur la lecture de l'historien, si ce dernier veut les comprendre à l'horizon de la fin pour laquelle ils ont été écrits. Appliquer aux manuels spirituels une lecture « positiviste », mettant entre parenthèses à la fois leur contexte historique et leurs règles discursives de formation, c'est les considérer exclusivement comme des «actes de langage directs », pour reprendre une expression de la pragmatique. Sont ainsi contournées les questions posées par l'archéologie du savoir et la généalogie des formes de rationalités, aussi bien que celles posées par une analyse communicationnelle 
en termes de marquages linguistiques du locuteur et des destinataires. Sans se soucier des lieux où ils naissent, les textes sont lus du côté où ils sont supposés fonctionner. Or, cette performance discursive considérée comme acquise est bien la «transmission en quelque sorte nue de la vérité elle-même » (ibid., p.366), ou encore la concentration de l'auditeur sur la tâche à accomplir, sans que l'arrière-plan ou les conditions de possibilité de la parole dite viennent perturber celle-ci.

Analysant ainsi les régimes de vérité à même le texte, Foucault pratique ce que nous pourrions appeler une naïveté seconde. Cette posture méthodologique revient à étudier ces textes dans leur effort pour être transparents à l'action, immédiatement compréhensibles et applicables, comme le recommande par exemple Sénèque, commenté en 1982 dans le cours L'herméneutique du sujet. Notons que le passage suivant n'est pas exactement une citation du philosophe stoïcien, mais une libre reprise par Foucault d'un argument qui se trouve dans une lettre de Sénèque à Lucilius :

Et pour que ce discours fasse place à la vérité, il faut, dit [Sénèque], qu'il soit simplex, c'est-à-dire transparent : qu'il dise ce qu'il a à dire, qu' il n'essaie pas de le vêtir, de l'habiller, et par conséquent de le déguiser, soit par des ornements, soit par une dramaticité quelconque. Simple : il doit être simple comme de l'eau pure, la vérité doit y passer. (p. 383 )

L'historien devra ainsi recevoir le texte comme simplex, terme qu'il convient mieux de traduire par «sans artifice » et «sans détour », comme il peut se dire dans la littérature stoïcienne d'un timbre de voix ou d'un comportement moral ${ }^{137}$.

Justifiée, cette approche littérale des textes pose cependant immédiatement sa propre limite : pour les manuels grecs comme pour les manuels chrétiens, le revers de la lecture littérale est le déni de la rhétorique, que Foucault oppose trop vite au «franc-parler » $(\pi \alpha \rho \rho \eta \sigma i a)$ comme seule règle de la parole philosophique ${ }^{138}$. Dans le passage précédemment cité, c'est bien l'art rhétorique qui est fustigé, à travers les habits et ornements du discours mentionnés. Étudier ces ornements, c'est-à-dire les «manières de dire » et l'impact recherché sur l'auditoire, demanderait précisément de dépasser une compréhension littérale pour définir les

137 Comme l'atteste le Gaffiot (Le grand Gaffiot. Dictionnaire latin-français, édition revue et augmentée, Paris, Hachette, 2000), c'est l'usage qu'en fait par exemple Cicéron, respectivement dans De oratore et le De republica.

138 L'herméneutique du sujet, p. 350 et toute la leçon du 10 mars 1982. 
positions respectives de l'émetteur et du récepteur dans une situation concrète de transmission. Pourraient alors s'entendre dans le texte une ironie, une familiarité avec l'auditeur qui passent par l'évocation de notions communes (figure de la prolepse) et excèdent le seul franc-parler. Ainsi chez Épictète, même si l'organisation des éléments du discours ne doit pas viser des effets pathétiques chez le disciple (comme la persuasion ou la séduction), mais la seule appropriation des préceptes en vue de l'action, l'enseignement du philosophe doit néanmoins s'adapter aux différentes catégories d'interlocuteurs et à leur précompréhension de la philosophie $^{139}$. Il y a donc des modulations du discours philosophique qui méritent d'être étudiées, en fonction d'une certaine situation d'enseignement : la parole à destination d'une assemblée pouvant différer de la direction spirituelle individuelle. Seule une analyse rhétorique permet de saisir ces différences qui ne sont pas qu'ornements, mais touchent au contenu même du discours et à son interprétation.

À cette critique formulée par Laurent Jaffro ${ }^{140}$, à qui nous empruntons les références ci-dessus, nous pourrions joindre une semblable concernant le monde chrétien. En effet, l'oubli de la rhétorique vaut également pour les recommandations et histoires édifiantes à l'intention des moines dans le monachisme primitif: Foucault ne prend pas en considération les niveaux de langage utilisés par les Anciens en fonction de l'état d'avancement de leur interlocuteur (laïc, postulant, novice, moine éprouvé), prenant ainsi systématiquement au pied de la lettre les aphorismes extravagants des Pères du désert et leur reprise chez Cassien ${ }^{141}$. Ici encore, le style de l'apophtegme varie pourtant selon que la parole est destinée à un individu ou à une communauté; qu'elle est prononcée par un moine pourvu d'un charisme particulier ou par le commun des moines; en vue de répondre à une question anxieuse concernant le salut ou de donner un simple éclaircissement concernant l'Écriture ${ }^{142}$. À l'opposé, Foucault ne replace jamais l'apophtegme dans une situation concrète de communication.

139 Voir les Entretiens d'Épictète, II, 22, 1-3 ; IV, 1, 44-45. Ces prénotions (prolepse) sont toujours celles d'un public donné.

140 Cette lacune de L'herméneutique du sujet est longuement développée dans L. Jaffro, «Foucault et le stoïcisme. Sur l'historiographie de L'herméneutique du sujet », Foucault et la philosophie antique, F. Gros et C. Levy éd., Paris, Kimé, 2003, p. 51-83.

141 Sécurité, territoire, population, p. 209-210 et leçons du 19 et 26 mars 1980.

142 Spécialiste et éditeur de cette littérature des Pères du désert, Jean-Claude Guy distinguait ainsi trois types d'apophtegmes. Cette distinction est fonction respectivement de 
La modification dans la manière de lire les textes, initiée par l'étude de Sophocle et la notion d'《alèthurgie », s'accentue avec les Pères de l'Église, parce que la finalité de leurs écrits est différente : non seulement présenter un rite ou un exercice, mais inviter le lecteur à s'y appliquer. Par rapport à la lecture d'EEdipe roi proposée en ouverture du cours, nous avons relevé la radicalisation qu'opèrent les lectures chrétiennes immédiatement consécutives : les « rituels de vérité », comme corrélats singuliers de l'exercice du pouvoir, cèdent la place à des régimes autonomes de pratiques, dont l'articulation possible au domaine juridicopolitique est simplement annoncée, sans être mise en œuvre. Cela permet d'établir un lien entre la notion de régime de vérité et la méthode de lecture qui lui est immédiatement contemporaine.

Notre interprétation du changement méthodologique n'a cependant pas distingué la littérature ascétique chrétienne de la littérature ascétique gréco-romaine, alors que la chronologie du cours donne la priorité à la première. Nous pourrions alors risquer une hypothèse sur le rôle particulier joué ici par les textes chrétiens. Il ne serait pas incongru en effet de supposer que le virage a été accentué par la confrontation à la littérature patristique. Dans cette radicalisation des principes de lecture pourrait sourdre l'attention particulière que porte Foucault à la place occupée par le texte dans le christianisme - attention qui remonte ni plus ni moins à ses premiers écrits. Ce sera là notre dernier mot sur la question, à titre de simple présomption.

Dès les années 1960 en effet, le jeune philosophe avait ébauché une réflexion sur le livre chrétien par excellence - la Bible - et la tradition de lecture qu'elle avait suscitée d'Orient en Occident. Entre autres textes ${ }^{143}$, la postface de 1964 à La Tentation de saint Antoine de Flaubert prend acte

l'émetteur, du récepteur et du contexte. Par exemple, le premier type d'apophtegme requiert un charisme spécial, n'importe quel Ancien ne peut pas le donner (variation en fonction de l'émetteur); le deuxième type est fait pour être médité par une communauté, il perd tout caractère individuel (variation en fonction du récepteur); la troisième catégorie s'étend au-delà de l'anachorèse initiale à la cénobie (variation en fonction du contexte) : J.-C. Guy, «Remarques sur le texte des Apophtegmata Patrum », Recherches de science religieuse, t. 43,1955 , p. $252-258$.

143 Nous pourrions citer également l'article sur Klossowski, où Foucault oppose les « simulacres » étudiés par la religion grecque aux « signes » qui guident l'exégèse chrétienne du Livre ( $D E \mathrm{n}^{\circ}$ 21, «La prose d'Actéon », I, p. 358-359). Nous aurons l'occasion de revenir sur Klossowski dans la troisième partie. 
de la place singulière occupée par le Livre au milieu des autres livres. Mais elle le fait à un prix élevé par rapport aux propres hypothèses de Foucault : au lieu de réduire le texte à sa matérialité, c'est-à-dire à l'objet-livre, avec ses mots imprimés, la postface évoque le sens chatoyant et ineffable des Écritures, tournant le lecteur vers un « dehors » des mots à nouveau évoqué dans un article de 1966 consacré à Maurice Blanchot. Conscient de cette possibilité de chatoiement du sens au-delà ou en deçà des mots, Foucault, quinze ans plus tard, a la prévenance de lire les Pères dans la lettre, et seulement dans la lettre, pour que celle-ci signifie sans rien évoquer d'autre.

Relisons la postface de 1964. Mis en regard de Bouvard et Pécuchet qui le rejoint pour s'en écarter, le roman La Tentation de saint Antoine opère un mouvement singulier par rapport à lui-même : il cherche à se nier comme livre pour n'être plus qu'une scène de théâtre ${ }^{144}$. Sur cette scène, la Bible apparaît alors entre les mains d'Antoine comme «la présence souveraine de l'Écrit $\gg($ p. 337). La prolifération indéfinie des autres livres - en particulier ceux innombrables lus par Flaubert pour rédiger la Tentation : histoire des religions antiques, patristique, théologie, tradition tératologique, etc. - demeure invisible dans la lettre du roman et n'éclate par fragments que dans les fantasmes d'Antoine, suscités par le Livre seul. Les autres livres n'exercent leur fascination qu'à partir du texte saint, qui est le premier «lieu de la Tentation $»($ p.328), puisque c'est en ouvrant l'Ancien testament - «Écriture même de Dieu » (p.329) que l'anachorète voit se déployer devant lui une série d'images qui le bouleversent : scintillement des anciennes civilisations, nuits enivrantes d'Égypte, reine de Saba en son palais, etc. La différence entre les livres accumulés par Bouvard et Pécuchet et le Livre d'Antoine réside dans le motif de leur séduction : les premiers ne tiennent leur puissance que de leur matérialité - du papier imprimé rangé dans l'espace de la bibliothèque - tandis que le second s'impose par son pouvoir imaginant et synthétisant. Le Livre cèle en lui un trésor d'images ne demandant qu'à être libérées. La Bible n'est pas seulement ni d'abord un texte matériel, elle est un espace de vision qui synthétise l'Orient et l'Occident, le passé et l'avenir, les plus anciennes civilisations et celle, chrétienne, encore à venir. À un carrefour de l'histoire humaine, elle est formée des « der- 
niers reflets du monde antique sur l'ombre encore grise d'un univers en train de naître $\gg($ p. 335). Elle ne peut à ce titre être rangée sur les rayons d'une bibliothèque comme un parmi d'autres.

D'où la tradition d'interprétation que la Bible suscita chez les rabbins hellénisés et les premiers Pères d'Orient. Selon Foucault, cette tradition exégétique a déterminé notre rapport occidental aux textes. Dans sa préface à Naissance de la clinique, en parfait accord avec la postface au roman de Flaubert, il souligne que l'exégèse ne s'est pas tant exercée sur la lettre des textes que sur les «symboles » et les «images sensibles $\gg$ dont regorge le texte biblique ${ }^{145}$ - description par ailleurs exacte de l'exégèse allégorique, telle qu'elle était pratiquée aussi bien du côté du judaïsme alexandrin (Philon) que des Pères chrétiens (Clément d'Alexandrie, Origène $)^{146}$. Foucault s'attarde alors sur l'usage moderne $\mathrm{du} \ll$ commentaire $\gg$ qui présuppose toujours sous le texte la présence d'un reste inépuisable - le signifié - où une parole originelle reposerait silencieuse. Dans cette pratique du commentaire, survit et se réanime sans cesse le rapport au Livre saint :

Nous commentons depuis des années le langage de notre culture de ce point précisément où nous avions attendu en vain, pendant des siècles, la décision de la Parole. ${ }^{147}$

Cette attente postule que le signifié est toujours en excès par rapport au signifiant, offrant au commentateur une tâche herméneutique infinie que Naissance de la clinique barre d'un trait. En son lieu et place, Foucault se propose d'étudier le seul événement historique du discours : qu'est-ce qui fait que telle parole à tel moment de l'histoire a été dite ? Cependant, la double éviction du Livre et du commentaire infini n'est pas sans laisser

145 Naissance de la clinique, p. XIII.

146 Contrairement au judaïsme hellénisé, le judaïsme rabbinique de Palestine préférait commenter la lettre du texte saint, dans une visée d'abord juridique. De son côté, Philon d'Alexandrie, excellent connaisseur des commentaires mythologiques grecs, est l'un des grands maîtres d'œuvre de l'interprétation allégorique de l'Ancien Testament. Le recours à l'allégorie permet selon lui de résoudre les difficultés insolubles posées par le sens littéral du texte saint. Sous le sens obvie, il s'agit de retrouver une signification cachée, à l'aide de certaines règles précises d'interprétation. Voir J. Pépin, Mythe et allégorie. Les origines grecques et les contestations judéo-chrétiennes, édition revue et augmentée, Paris, Études augustiniennes, 1976. L'école chrétienne d'Alexandrie (Clément, mais surtout Origène qui développa une théorie complète des sens de l'Écriture) s'inscrit dans cette tradition conjointement juive et grecque.

147 Naissance de la clinique, p. xIII. 
quelques traces. Malgré les efforts déployés pour contourner ces usages exégétiques et les démettre de leur pouvoir révélant, Foucault ne ferme pas complètement la porte à un certain rapport religieux au monde de la parole et du texte : celui de la mystique.

La particularité du discours mystique est de nier sans cesse ce qu'il énonce concernant les attributs de Dieu : Dieu est non seulement au-delà du sensible, mais également au-delà des mots et des essences. Il faut donc à la fois le dire et se dédire aussitôt. Rappelons que l'adjectif «mystique »,

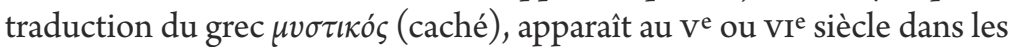
textes chrétiens, en particulier chez l'auteur de la Théologie mystique, le néoplatonicien Denys l'Aréopagite. À force de multiplier les chemins de traverse, l'archéologie proposée par Foucault semble rejoindre par certains aspects ce discours apophatique, jusque dans la progression de son argumentation qui ne cesse de définir sa position incertaine par la négation de toutes les positions connues ${ }^{148}$. En fait, l'analyse discursive des énoncés ne conjure pas totalement «l'excès » dénoncé par Naissance de la clinique comme condition et principe du commentaire. Bien entendu, cet excès ne renvoie pas ici à un signifié identique à lui-même, mais à des «systèmes de dispersion $\gg^{149}$ qui décrivent seulement des régularités de rapports entre objets, modes d'énonciation, concepts et stratégies. Mais l'objet de la recherche déborde néanmoins sans cesse la phrase prononcée ou le texte écrit, considérés dans leur matérialité. L'énoncé - qui n'est ni proprement linguistique ni proprement matériel - précède la parole ou le texte, étant leur « fonction d'existence $\gg($ ibid., p. 115); il reste en retrait par rapport à l'articulation déterminée de graphèmes ou de phonèmes, qui forme à chaque fois un système fermé. À la limite des discours prononcés, le geste archéologique ouvre alors un espace de pure extériorité où nul sujet, parlant ou écoutant, écrivant ou lisant, ne peut se retrouver. L'organisation de cet espace ne correspond en effet à aucune synthèse préalable, opérée dans et par le sujet pensant, mais il conditionne toutes les possibilités de synthèse.

De son côté, la mystique recherche ce qui excède à la fois les mots humains tout en étant leur envers silencieux, puisque l'Être au-delà du

148 C'est le style propre à L'archéologie du savoir : faire précéder toute proposition positive - qui n'en reste pas moins précaire - de longues séries de «il/ce n’est pas » : L'archéologie du savoir, p. 41, 65, 100 et 114-115; « on n'a pas voulu/on n'a pas essayé » (p. 73); «il ne faut pas/il n'est pas » (p.118-121 et 125).

149 L'archéologie du savoir, p. 53, l'auteur souligne. 
dicible et de l'intelligible est la Parole par excellence - « Parole ineffable $\gg^{150}$ dit Denys, qui suscite un jeu lexical jamais définitivement gagné. Les mystiques s'appuient sur le langage pour désigner par la négation ce qui, dépassant les capacités de nomination et d'intellection du sujet, échappe à la raison discursive (au sens platonicien de $\delta$ lávol $\alpha^{151}$ ) et s'apparente pour celle-ci à un néant. Seule la sortie du sujet hors de lui-même permet ainsi l'accès au Verbe divin. Là où l'archéologie refuse le thème de l'origine secrète - «si secrète et si originaire qu'on ne peut jamais la ressaisir tout à fait en elle-même $\gg^{152}-$, la quête mystique reste déterminée par un terme qui est en même temps le principe de tout : «Dieu ».

La différence est donc bien posée. Cependant, dans un article consacré à Maurice Blanchot en 1966, Foucault risque furtivement, sous la forme interrogative, un parallèle entre la pensée mystique et «l'expérience du dehors » décrite par l'auteur de L'espace littéraire. À la suite d'un certain nombre de romanciers modernes, Blanchot fait surgir en sa nudité «l'être du langage »153, rejoignant de plein droit l'effort philosophique objectivé par L'archéologie du savoir ${ }^{154}$. Foucault n'hésite pas alors à reconnaître que l'écriture de Blanchot et le discours mystique se partagent le même lieu : hors de la subjectivité, ils se tiennent sur le seuil de toute positivité (langage, conscience de soi, savoir) pour qu'apparaisse le vide où ces positivités peuvent se déployer.

Il faudra bien un jour essayer de définir les formes et les catégories fondamentales de cette «pensée du dehors ». Il faudra aussi s'efforcer de retrouver son cheminement, chercher d'où elle nous vient et dans quelle direction elle va. On peut bien supposer qu'elle est née de cette pensée mystique qui, depuis les textes du Pseudo-Denys, a rôdé aux confins du christianisme; peut-être s'estelle maintenue, pendant un millénaire ou presque, sous les formes d'une théologie négative. ${ }^{155}$

150 Denys l'Aréopagite (Pseudo-), Les noms divins, IV, \$11, dans E Euvres complètes, M. de Gandillac trad., Paris, Aubier-Montaigne, 1980, p. 105.

151 Pour Platon, la Siávola est le dialogue de l'âme avec elle-même, opposée à la simple opinion (Théétète, 189e-190a). Elle est un examen méthodique et exhaustif par l'âme des choses se présentant à elle. Cet examen atteint nécessairement un terme qui supprime toute dualité et aboutit à une certitude.

152 L'archéologie du savoir, p. 36, l'auteur souligne.

$153 D E n^{\circ} 38$, «La pensée du dehors », I, p. 549.

154 Qu'une certaine littérature fasse partie de la philosophie, Foucault l'affirme régulièrement. Par exemple : $D E n^{\circ} 39$, « L'homme est-il mort ? », I, p. 571-572. 
Foucault émet cependant aussitôt l'objection que nous venons de formuler un peu plus haut : la pensée mystique, même sortie d'ellemême, espère toujours se retrouver au terme de son errance aveugle dans l'Être qui est Parole (ibid., p. 549); tandis que l'expérience du dehors, au même titre que l'archéologie, n'ouvre que sur un « espace blanc, indifférent, sans intériorité $\gg^{156}$. La mystique demeure cependant dans le texte foucaldien comme « une possibilité encore incertaine ${ }^{157}$, une expérience à la fois lointaine et voisine de cette expérience du dehors qui précipite l'archéologie hors du sujet et de la trame visible des mots. Certains théologiens contemporains ne s'y sont d'ailleurs pas trompés dans leurs usages parfois surprenants de Foucault ${ }^{158}$.

Il n'est alors pas étonnant que Foucault, abordant une dizaine d'années plus tard des textes patristiques qui, rappelons-le, étaient encore lus au Moyen Âge comme faisant partie intégrante des Écritures, ait voulu écarter la proximité avec la mystique - proximité d'autant plus grande et dangereuse que certains régimes de vérité chrétiens, en particulier ceux de tradition néoplatonicienne, font de l'union obscure et indicible à Dieu leur fin ultime dès ici-bas ${ }^{159}$. Mais il fallait pour cela éviter l'am-

156 L'archéologie du savoir, p. 54.

$157 D E n^{\circ} 38$, «La pensée du dehors », I, p. 549.

158 H. Pinto, « The more which exceeds us : Foucault, roman catholicism and inter-faith dialogue $\gg$, Michel Foucault and Theology. The Politics of Religious Experience, J. Bernauer et J. Carrette éd., Aldershot, Ashgate, 2004, p. 191-216. Dans cet article, le théologien Henrique Pinto prend pour point de départ une phrase extraite de L'archéologie du savoir : «Certes, les discours sont faits de signes; mais ce qu' ils font, c'est plus que d'utiliser ces signes pour désigner des choses. C'est ce plus, qui les rend irréductibles à la langue et à la parole. C'est ce "plus" qu'il faut faire apparaître et qu'il faut décrire » (L'archéologie du savoir, p. 67, l'auteur souligne). Cette notion de «plus » est alors l'occasion de montrer non seulement que le discours théologique ne peut jamais être en adéquation avec la chose dite, mais que l'attention à ce «plus » doit devenir la règle de la théologie. L'article tend alors à conférer au «plus » un rôle quasi spirituel : le travail théologique n’est pas une obéissance au magistère, mais une «fidélité » à ce «plus » (H. Pinto, «The more wich exceeds us... », déjà cité, p. 199), qui peut être pensé comme «gratuité et néant» (ibid., p.192).

159 Chez Grégoire de Nysse, les trois degrés de l'union à Dieu sont les suivants : 1) l'éthique, où l'âme se purifie intérieurement par l'ascèse, pour se libérer de toute passion ; 2) la physique, où l'âme par son activité intellectuelle dépasse la connaissance des sens ; 3) la métaphysique, qui est l'union mystique de l'âme à Dieu, dans les ténèbres et l'inconnaissable (voir l'étude classique de Jean Daniélou : Platonisme et théologie mystique. Essai sur la doctrine spirituelle de saint Grégoire de Nysse, Paris, Aubier, 1944). Dans ses commentaires des pratiques baptismales et pénitentielles, Foucault cherche à couper le premier degré des deux autres, pour le considérer comme une fin en soi. Choix bien entendu discutable. 
biguiité entretenue quelques années auparavant d'un espace sous-jacent au discours, d'un excès de l'objet de la recherche sur les textes pris dans leur matérialité. La « chaîne manifeste, visible et colorée des mots »160, brisée en son temps par l'archéologie, devint dès lors le seul support possible de l'analyse, afin de lire les manuels uniquement comme des principes d'action. Au risque d'oublier ce que l'archéologie prenait pourtant en charge : les groupes sociaux, les espaces institutionnels et les types de gouvernement collectif qui précèdent le discours. Ceux-ci sont désormais relégués dans un domaine distinct, celui des « régimes juridico-politiques », qui finissent par disparaître complètement des leçons chrétiennes de 1980.

Après avoir défini ces principes de lecture, à la fois dans leur cohérence et leur limite intrinsèques, il nous faut maintenant descendre à ce niveau inférieur qui relève d'un art de la fabrication ou encore d'une poiétique, en ce lieu rarement visité où le philosophe-historien manipule les textes : comment les sélectionne-t-il, comment les traduit-il afin de les inclure dans une interprétation générale $\mathrm{du}$ 《christianisme » qu'un décès prématuré a malheureusement laissée en suspens? Dans la partie qui va suivre, nous nous concentrerons exclusivement sur les textes chrétiens des premiers siècles utilisés dans le cours $D u$ gouvernement des vivants. Par souci de méthode, nous privilégierons l'enregistrement sonore du cours, afin d'intégrer dans notre analyse la dramaturgie propre à l'énoncé oral. Le manuscrit préparatoire ne sera sollicité que pour vérifier occasionnellement certaines hypothèses ou mesurer l'écart entre la prise de notes et le moment singulier de la leçon publique ${ }^{161}$.

160 L'archéologie du savoir, p. 66

161 Ce choix nous a semblé d'autant plus justifié que le manuscrit du cours de 1980 est moins rédigé que certains des précédents manuscrits (Cours Du gouvernement des vivants, Notes préparatoires, 1980. - Manuscrit; $30 \mathrm{~cm}$ [Archives Daniel Defert]). Avec les années, le texte préparatoire était devenu un simple support pour l'énoncé oral, qui gagnait du même coup en liberté et en intensité. 



\section{CHAPITRE CINQ \\ Conséquences sur l'usage des textes: traduire les Pères}

Nous voudrions revenir ici à une question toute simple, d'ordre d'abord strictement matériel : comment Foucault utilise-t-il les textes patristiques dans ses leçons de 1980 ? Cette utilisation est nécessairement une pratique de traduction, dans le double sens du terme : traducere, c'està-dire passage à la fois d'un état du texte à l'autre (du latin ou du grec, vers la langue française) et d'un énoncé à l'autre (de la parole d'un Père à celle de Foucault, qui la coupe, la commente, l'inscrit dans sa propre argumentation).

Pour répondre à cette question autour de laquelle rôdent bien des suspicions et peu de réponses explicites, nous devons prendre le risque de l'érudition austère mais nécessaire. Sans mentionner la totalité des textes cités dans le cours de 1980, ni refaire intégralement un parcours de démonstration que d'aucuns jugeraient laborieux, il nous semble important de montrer quelques coutures du manteau. Il s'agit ce faisant de fonder, à partir de l'exemple précis de la patristique, la possibilité d'une étude des stratégies de travail de Foucault.

Pour pouvoir distinguer la parole personnelle de Foucault des textes des Pères, nous citerons les extraits du cours selon le code typographique suivant :

- les commentaires et apartés de Foucault sont indiqués en italiques. Quand une citation directe d'un Père intervient, elle est indiquée entre guillemets, précédée de sa numérotation la plus commune dans les collections de référence (souvent homogènes pour ce qui est du découpage en paragraphes); 
- entre crochets, nous indiquons deux types de précision, quand cela est nécessaire : 1) le texte original et sa traduction la plus littérale possible, 2) des variantes de traduction, chacune précédée des initiales de l'édition. La partie de la citation concernée par le complément entre crochets est indiquée en italique.

\section{Lire : des choix classiques ${ }^{1}$}

À quelques exceptions près, le cours de 1980 s'appuie sur les trois grandes éditions scientifiques françaises de textes patristiques. Toutes proposent une traduction en regard du texte original :

1) les «Textes et documents pour l'étude historique du christianisme » publiés sous la direction des abbés Hippolyte Hemmer et Paul Lejay, que nous abrégeons désormais : «HL, date d'édition $»$. Cette édition bilingue fut inaugurée en 1904, avec la volonté de s'adresser aux universitaires de l'École pratique des hautes études comme au simple lecteur chrétien. Elle prend acte des avancées de la recherche scientifique et corrige la grande patrologie de Migne (XIX ${ }^{e}$ siècle) pour l'établissement et l'attribution de certains textes;

2) la «Collection des universités de France» (dite collection Guillaume Budé) aux éditions des Belles Lettres, que nous abrégeons désormais «BL, date d'édition ». Avant son lancement, il n'existait en France aucune édition rigoureuse des classiques de la littérature grecque et latine. L'usage académique voulait que l'on se rapporte aux éditions allemandes. C'est en août 1920 que parurent conjointement le premier volume du De rerum natura de Lucrèce et le premier volume des Euvres complètes de Platon. La collection comprend aujourd'hui plus de huit cents volumes, parmi lesquels quelques œuvres de Pères latins (Augustin, Jérôme, etc.);

3) les «Sources chrétiennes » des éditions du Cerf, que nous abrégeons désormais : «SC, no du volume, date d'édition $»$. Cette édition

1 Exposer dans le détail les choix de Foucault et les chemins qui nous y ont mené risquerait d'alourdir cette partie méthodologique. Nous nous contentons de donner quelques éléments d'une recherche plus ample, que l'on trouvera exposée dans P. Chevallier, «Foucault et les sources patristiques », Foucault, P. Artières, J.-F. Bert, F. Gros et J. Revel éd., Paris, L'Herne (Cahiers de L’Herne ; 95), 2011, p. 137-142. 
bilingue, qui a aujourd'hui dépassé les cinq cents volumes, fut fondée en 1942 par les pères jésuites Jean Daniélou, Henri de Lubac et Claude Mondésert. Immédiatement devenue une référence internationale, elle a été reconnue par le CNRS comme unité de recherche associée en 1976, puis comme unité mixte de recherche ${ }^{2}$.

Si Foucault ne fait aucune mention explicite de cet usage, ni dans les leçons prononcées ${ }^{3}$ ni dans ses notes manuscrites, le style particulier de ces trois traductions s'entend distinctement dans ses propos. Leur objectif avoué est en effet de réinventer le texte original dans une nouvelle langue, contre la gageure d'un décalque systématique du lexique et de la syntaxe initiale ${ }^{4}$. Elles évitent ainsi soigneusement les lourdeurs du texte source et remplissent volontiers les blancs de son discours. Nous verrons au contraire que lorsque Foucault traduit lui-même un passage grec ou latin, il s'efforce d'être le plus près possible, lexicalement et syntaxiquement, du texte source.

À ces trois éditions classiques, maîtresses de rigueur quant à l'établissement des textes, s'ajoutent cependant trois exceptions. Le recours à la première s'explique par une simple raison de commodité matérielle : Foucault a également utilisé, sans doute à son domicile, la compilation de textes des premiers siècles éditée pour le grand public par François Louvel en $1963^{5}$. Cette édition de poche offre des traductions de qualité, mais sans texte original ni apparat critique. Elle est cependant plus malléable et surtout plus accessible que les éditions SC et surtout HL.

La deuxième entorse à la règle académique pourrait sembler plus

2 C. Mondésert, Pour lire les Pères de l'Église, dans la collection Sources chrétiennes, Paris, Cerf, 1979, p. 15 .

3 À une exception près : la leçon du 27 février 1980 mentionne l'édition du Pasteur d'Hermas par Robert Joly dans la collection des «Sources chrétiennes » (Cerf, 1958).

4 Cette tradition classique est depuis critiquée pour son excès d'élégance. Dans le champ des études grecques, le virage a été amorcé par Léon Robin. Même si sa traduction de Platon n'est pas allée jusqu'à un décalque systématique de la langue originale, elle marquait sa différence avec l'édition des Belles Lettres : «Mon principe a été de m’appliquer à ne laisser rien perdre du texte et, j'en conviens, de sacrifier pour cela l'élégance à la fidélité. $[\ldots]$ je me suis fait une règle de respecter les mots que, à peu de distance, Platon a cru devoir répéter : ces répétitions marquent avec plus de netteté l'enchaînement des idées et la continuité du raisonnement » (Platon, Euvres complètes, t. I, L. Robin, avec la collaboration de M.-J. Moreau trad., Paris, Gallimard, 1950, p. XVII).

5 Lesécrits des Pères apostoliques, F. Louvel éd., L. Bouyer préf., Paris, Cerf, 1963. Ce recueil, par ailleurs de grande qualité, reprend certaines traductions SC, auxquelles il ajoute d'autres traductions inédites. Il fut réédité par le Cerf en 2001. 
incongrue. Il s'agit d'une entreprise éditoriale aujourd'hui oubliée, dont les résonances à l'époque étaient clairement édifiantes et les prétentions littéraires : la traduction en trois volumes des œuvres complètes de Tertullien par l'abbé de Genoude (1792-1849). D'abord journaliste, ardent défenseur d'une monarchie qui l'avait anobli, Antoine-Eugène Genoud (dit aussi Genoude) avait épousé, une fois veuf, la carrière ecclésiastique. Mais c'est dès le début de sa vie publique de polémiste qu'il s'était lancé dans de vastes traductions de la Bible et des Pères qui sacrifiaient souvent à l'élégance, avec une forte tendance à l'abondance et à la pro-

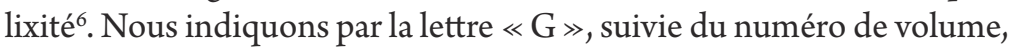
cette traduction de Tertullien.

Enfin, on trouve trace également, dans la leçon du 19 mars, de la traduction des Euvres complètes de Jean Chrysostome, sous la direction de Jean-Baptiste Jeannin et avec la collaboration de prêtres de l'Immaculée Conception de Saint-Dizier, publiée à Bar-le-Duc en 1864 .

Que le cours utilise ainsi majoritairement les traductions déjà disponibles, quelques apartés confirment par ailleurs ce résultat, tout en nous indiquant que Foucault ne se fie pas complètement à ce qui n'est pour lui qu'un appui. À deux reprises, le professeur rectifie en effet à l'oral une traduction qu'il est en train de lire : «Alors, je ne suis pas très fort en latin, mais je ne suis pas tout à fait d'accord avec la traduction $\gg^{7}$. Cette prudence audible se retrouve dans la leçon du 27 février 1980, encore plus instructive pour notre recherche : alors que la Didachè est citée dans la traduction de François Refoulé proposée par l'édition de François Louvel, Foucault la corrige en indiquant des termes grecs qui ne se trouvent ni dans son édition de référence ni dans ses notes préparatoires au cours. Il est donc évident qu'il a sous les yeux un livre qui lui permet le cas échéant de rectifier la traduction qu'il a recopiée dans ses notes. Sans doute pas HL, 1919 - par trop difficile d'accès ${ }^{8}$-, mais plus probablement l'édition SC de 1978, qui venait alors de paraitre. Celle-ci

6 Au sujet de sa traduction de la Bible, un proche contemporain note que celle-ci est «moins exacte que celle de Sacy, mais éloquente, et quelquefois emphatique (Article «Genoude (Antoine-Eugène Genoud, abbé de) 》, Dictionnaire universel des littératures, G. Vapereau, Paris, Hachette, 1876, p. 869).

7 Leçon du 5 mars 1980.

8 Contrairement aux éditions SC, les éditions HL ne se trouvaient plus qu'en bibliothèque. Foucault eût-il eu l'édition HL de 1919 à sa disposition, qu'il n'aurait pas eu besoin de 
n'aurait pas servi à la préparation du cours, puisque sa traduction n'est pas utilisée par Foucault, mais le professeur aurait pu se la procurer afin de disposer du texte grec pendant son enseignement ${ }^{9}$.

La lecture le 27 février est alors des plus significatives du laboratoire de recherche que constituait pour le philosophe une leçon au Collège. Le travail sur les sources permet de reconstituer la scène dans sa dimension quasi physique : le professeur lit à haute voix ses notes, tout en gardant un œil sur le volume SC qu'il a ouvert à côté de lui, pour y vérifier le texte grec. Il va donc de l'un à l'autre, discutant au passage la traduction proposée dans l'édition de 1963 par François Refoulé - indiquée ci-dessous en gras :

$(\mathrm{IV}, 14) \ll \grave{A}[\mathrm{dans}]$ l'assemblée, c'est-à-dire à cette réunion des croyants pour la prière quotidienne, la Didachè prescrit aussi : Tu confesseras tes manquements et tu n'iras pas à $l a[$ ta $]$ prière avec une conscience mauvaise $\gg-e n f i n, ~ « \sigma v v \varepsilon i \delta \eta ் \varepsilon i$ $\pi o v \eta \rho \tilde{a} \gg$, avec la conscience d'avoir mal fait, conscience mal à l'aise en quelque sorte. ${ }^{10}$

Ce même aller et retour entre les notes rédigées à l'avance et la lecture orale en direct se retrouve dans la leçon du 5 mars, où la même traduction de François Refoulé est à nouveau complétée par la mention du texte grec que l'édition Louvel ne fournissait pas ${ }^{11}$.

En rassemblant l'ensemble de ces informations, nous pouvons alors dresser le tableau (voir p. 193) des éditions utilisées par le cours $D u$ gouvernement des vivants, entre le 6 février et le $5 \mathrm{mars}^{12}$, avec un premier résultat étonnant : un même texte au cours d'une même leçon peut être cité dans deux éditions différentes. Des moments distincts de la recherche, avec pour chacun une prise de notes et des références

travailler chez lui sur l'édition de poche de Louvel. Bien entendu, nous choisissons les hypothèses les plus probables, qui ne tiennent pas lieu de certitude.

9 Foucault avait chez lui plusieurs éditions de la Didachè (entretien avec Daniel Defert, le 9 juillet 2008). Notre hypothèse est qu'il s'est d'abord procuré l'édition de poche de François Louvel (1963), pour travailler à son domicile, avant de se procurer l'édition SC, récemment parue (1978), pour disposer du texte original au Collège.

10 Leçon du 27 février 1980.

11 «Ainsi, au chapitre v [erratum : Iv], paragraphe 14 de la Didachè, vous voyez qu'il est dit:

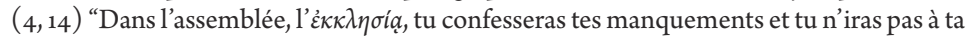
prière avec une conscience mauvaise". Donc, à l'assemblée où l'on prie collectivement, il faut

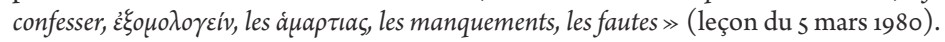

12 Nous avons volontairement laissé de côté les dernières leçons de 1980, consacrées essentiellement à Cassien. Le nombre réduit des éditions qui y sont manipulées les rendait moins riches pour cette étude. 
également distinctes, viennent ainsi se superposer dans le commentaire, comme si les traces d'expériences plus anciennes de lecture n'étaient pas effacées par le travail le plus récent, même dans le cas où celui-ci aurait été effectué sur une «meilleure » édition.

Pour compléter ce tableau, une fois repérées les citations les plus évidentes des éditions que nous venons de parcourir, il nous a fallu recourir à une autre source d'information.

Au regard de l'immensité du massif patristique, certains des lieux visités en 1980 ont bien entendu été indiqués par des sources secondaires, offrant au professeur une première cartographie du territoire qui n'exclut pas dans un second temps une exploration directe. La bibliographie «chrétienne » recopiée de la main même de Foucault dans un petit carnet de notes ${ }^{13}$ permet d'identifier trois études patristiques anciennes offrant les mêmes séries de citations que certains passages du cours, en particulier dans la première et la troisième leçon chrétienne : il s'agit de l'article d'Albert Dondeyne «La discipline des scrutins dans l'Église latine avant Charlemagne $\gg$, Revue d'Histoire ecclésiastique, t. 28, 1932, p. 5-33; du premier chapitre intitulé «Grundlagen zur Entstehung des Taufexorzismus » de la thèse d'habilitation de Franz Joseph Dölger, Der Exorzismus in altchristlichen Taufritual. Eine religionsgeschichtliche Studie, Paderborn, Schöningh, 1909 (réédité en 1967); et de l'article d'Henri Holstein «L'exhomologèse dans l'Adversus haereses de saint Irénée », Recherches de science religieuse, t. 35, 1948, p. 282-288. Nous insérons ces références dans le tableau dans la mesure où elles renvoient à des citations souvent extrêmement brèves - contrairement aux autres citations qui témoignent clairement d'une lecture de première main - extraites le plus souvent de textes rares ou peu visités (Les extraits de Théodote de Clément d'Alexandrie; un sermon de Quodvultdeus).

Le choix de ces sources secondaires permet au passage d'esquisser un portrait du philosophe en chercheur. Chercheur « classique » assurément dans ses choix de lecture, attiré par les études les plus érudites, et souvent les plus anciennes $(1909,1932)$, Foucault privilégie le format austère des thèses et articles scientifiques dont la rectitude des informations est souvent inversement proportionnelle à l'originalité de la pensée. Il les utilise essentiellement comme des recueils de citations, 
le regard d'abord attiré par les notes de bas de page. Pour cette raison, la littérature secondaire est volontairement choisie dans le champ historiographique le plus technique, excluant de fait tout ce qui ressemble déjà à une interprétation théologique ou à une synthèse historique trop large. Cela n'exclut bien entendu pas les lectures de fond plus contemporaines, comme les livres de Paul Veyne et de Peter Brown sur l'Antiquité tardive, mais les leçons chrétiennes de 1980 - qui se présentent d'abord comme de méticuleux commentaires de textes - n'en gardent nulle trace directe.

Enfin, la traduction personnelle que Foucault donne de Justin le 6 février 1980 est peut-être l'unique trace d'une lecture de la grande patristique de l'abbé Migne (abrégé ci-dessous $\ll M \gg$ ), attestée par Daniel Defert, celle-ci ne fournissant de traduction contrairement à HL et $\mathrm{SC}^{14}$.

Sources patristiques des premières leçons chrétiennes du cours de 1979-1980

\begin{tabular}{|c|c|c|c|}
\hline Leçon & Auteur & Texte & Édition ou source \\
\hline \multirow[t]{4}{*}{6 février } & Anonyme & Didachè & HL, 1919 \\
\hline & Justin & Première Apologie & M \\
\hline & Nouveau Testament & Épître de Paul à Tite & Livre de Dölger \\
\hline & Clément d'Alex. & Extraits de Théodote & Livre de Dölger \\
\hline \multirow[t]{3}{*}{13 février } & Tertullien & De paenitentia & G \\
\hline & Tertullien & De baptismo & $\mathrm{G}+\mathrm{SC}, 35,1952$ \\
\hline & Clément d'Alex. & Le pédagogue & SC, 70,1960 \\
\hline \multirow[t]{4}{*}{20 février } & Hippolyte & Tradition apostolique & SC, 11,1946 \\
\hline & Augustin & Sermon 216 & Article de Dondeyne \\
\hline & Quodvultdeus & Sermon $_{3}$ & Article de Dondeyne \\
\hline & Ambroise & Explication du symbole & Article de Dondeyne \\
\hline \multirow[t]{8}{*}{27 février } & Hermas & Le pasteur & SC, 53,1958 \\
\hline & Nouveau Testament & Épître aux Hébreux & Bible de Jérusalem \\
\hline & Ignace d'Antioche & Lettre aux Éphésiens & SC, 10,1951 \\
\hline & Anonyme & Épitre de Barnabé & HL, 1919 + Louvel 1963 \\
\hline & Clément de Rome & Épitre aux Corinthiens & HL, 1926 + Louvel 1963 \\
\hline & Anonyme & Didachè & HL, 1926 + Louvel 1963 \\
\hline & Polycarpe & Lettre aux Philippiens & SC, 10,1951 \\
\hline & Anonyme & Homélie du $u^{e}$ siècle & Louvel 1963 \\
\hline
\end{tabular}

14 Foucault était très fier d'avoir manié les vieux volumes poussiéreux de Migne (entretien avec Daniel Defert, 6 décembre 2006). Rappelons au passage que Foucault, dès 1963, avait

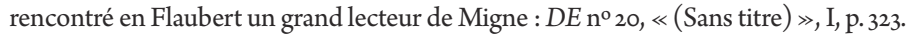




\begin{tabular}{llll}
\hline 5 mars & Cyprien & Correspondance & BL, 1925-1945 \\
Anonyme & Didachè & HL, 1919 + Louvel 1963 \\
Tertullien & De pudicitia & $\mathrm{G}$ \\
& De paenitentia & $\mathrm{HL}, 1906$ \\
& Paulin de Milan & Vie de saint Ambroise & $\mathrm{M}$ \\
Jérôme & Lettre 77 & $\mathrm{BL}, 1954$ \\
Irénée de Lyon & Adversus haereses & Article de Holstein \\
Ambroise & De paenitentia & SC, 179, 1971 \\
\hline
\end{tabular}

BL «Collection des universités de France » des éditions Les Belles Lettres

Louvel Les écrits des Pères apostoliques, édités par F. Louvel, Paris, Cerf, 1963

G cuvres de Tertullien traduites par l'abbé de Genoude

HL collection « Textes et documents pour l'étude historique du christianisme »

M patrologie de l'abbé Migne

SC collection « Sources chrétiennes »

\section{Traduire : Foucault, traducteur «sourcier»}

Comme nous avons eu l'occasion de le relever, dans ses leçons consacrées aux Pères chrétiens, Foucault se contente rarement des traductions fournies par les éditions à sa disposition et préfère repasser par le texte original. Le contraire eût étonné de la part d'un philosophe qui s'exerça très tôt au métier de traducteur : sa traduction précoce du Cycle de la structure de Viktor von Weizsäcker ${ }^{15}$ fut suivie de près par une traduction de l'Anthropologie de Kant pour sa thèse complémentaire ${ }^{16}$. À côté d'une grande vigilance concernant l'usage des mots dans des textes philosophiques dont la conceptualité ne correspond pas forcément à une lexie stable (distinguant ainsi chez Nietzsche un usage tantôt « neutre », tantôt «marqué » d'un même terme ${ }^{17}$ ), Foucault a jeté les bases d'une réflexion sur le lien entre philosophie et philologie, à l'ombre de la pra-

15 Traduction réalisée en collaboration avec Daniel Rocher et publiée en 1958 : DE I, p. 26.

16 E. Kant, Anthropologie du point de vue pragmatique, précédé de Michel Foucault, « Introduction à l'Anthropologie », M. Foucault trad., Paris, Vrin, 2008.

$17 D E n^{\circ} 84$, « Nietzsche, la généalogie, l'histoire », I, p. 1005 et 1006 : dans cet article, Foucault distingue deux emplois du mot Ursprung dans les textes de Nietzsche, l'un qui n'est pas «marqué », car interchangeable avec d'autres vocables (Entstehung, Herkunft, etc.); l'autre qui est au contraire spécifié, en particulier par son opposition à Herkunft. 
tique nietzschéenne de l'étymologie : la philosophie serait une philologie «toujours en suspens, une philologie sans terme $\gg^{18}$, attendu que les mots offerts à la réflexion du philosophe sont toujours déjà des interprétations de signes imposées par l'histoire, qu'il faut éclairer sans espérer atteindre un signifié originel. Cette attention ancienne aux ruses du vocabulaire pousse naturellement le philosophe à rencontrer le texte patristique original, grec ou latin.

En lisant les éditions Hemmer-Lejay, et, dans une moindre mesure, celle des Sources chrétiennes, Foucault s'affronte à certaines habitudes académiques concernant la traduction des textes classiques. Or, de ces habitudes, il fait souvent fi. L'usage hérité de la tradition philologique française était en effet de privilégier le style et le mouvement d'ensemble de l'œuvre sur telle ou telle maladresse de détail. Les éditions HemmerLejay n'y échappaient pas, partageant d'ailleurs avec les Belles Lettres certains traducteurs, comme le latiniste Pierre de Labriolle (1874-1940), traducteur de Tertullien et de Juvénal. Autant que possible, il fallait éviter de « faire traduction » et omettre si nécessaire les rudesses de la langue originale. La réinvention littéraire des textes prenait ainsi le pas sur le respect de la lettre. Soucieux d'exactitude et non d'appréciation littéraire des textes, Foucault conserve au contraire les répétitions que biffaient allégrement les éditions G, HL, et parfois encore SC. Ainsi, dans cet extrait du De paenitentia, Foucault souligne le caractère énigmatique mais décisif de la redondance :

D'où la phrase énigmatique, mais que, je crois, on peut comprendre maintenant: (VI, 16) « la foi, dit Tertullien, commence et se recommande par la paenitentiae fides $\gg$, la foi commence et se recommande par la foi de la pénitence. ${ }^{19}$

Voulant soigner la qualité littéraire de sa traduction, Labriolle efface quant à lui dans l'édition HL la répétition de fides et propose : « cette foi prend son point de départ et se recommande de la sincérité de la mologie nietzschéenne de ả $\gamma a \theta o ́ \varsigma$ dans La généalogie de la morale ( $1^{\mathrm{er}}$ traité, $\mathbb{S} 4$ et 5 ): «que peuvent bien signifier, au point de vue étymologique, les désignations du "bon" forgées dans les différentes langues ? : c'est là que j’ai découvert qu'elles renvoient toutes autant qu'elles sont à la même mutation conceptuelle » (F. Nietzsche, Éléments pour la généalogie de la morale. Écrit de combat ajouté à Par-delà le bien et le mal, P. Wotling trad., Paris, Librairie générale française, 2000, p. 70). 
pénitence $\gg^{20}$. En cela, le grand latiniste ne fait que suivre l'option stylistique de l'abbé de Genoude, dont Foucault avait consulté la traduction pour préparer sa leçon ${ }^{21}$. La redondance n'est pourtant nullement fortuite puisqu'il ne s'agit pas seulement pour Tertullien de faire dépendre la foi véritable de l'intégrité d'une pratique pénitentielle. Il s'agit d'abord et avant tout de faire de cette pratique l'expression d'une première forme de foi en Dieu qu'il définit par la «crainte» : (VI, 14) «Dès qu'on connaît le Seigneur, il faut le craindre [timeas $] \gg^{22}$. Dans cette crainte, qui est déjà une certaine relation à Dieu et un « premier baptême $\gg^{23}$, le pénitent désire être méritant, sans rien pouvoir exiger en retour. Il espère en un baptême qu'il ne reconnaît nullement comme un dû. Cette dimension votive de la pénitence est contenue dans le syntagme paenitentiae fides, qu'il convenait d'autant plus de garder intact qu'il n'est pas un hapax et apparaît déjà en VI, 13.

La Première Apologie de Justin va nous fournir un deuxième exemple de ce réajustement opéré par Foucault sur les traductions classiques. Nous sommes le 6 février 1980. La phrase citée de Justin est des plus complexes et les traducteurs successifs ont tous choisi la facilité, que ce soit Louis Pautigny (HL, 1904) ou plus récemment Charles Munier (SC, 507, 2006). Seule disponible en 1980, la vieille traduction de Pautigny va au plus court. Simplifiant le texte de Justin, elle remplace en fait par des substantifs commodes l'adjectif a $\lambda \eta \theta \tilde{\eta}$ et les participes passés $\delta i \delta a \sigma \kappa o ́ \mu \varepsilon v a$ et $\lambda \varepsilon \gamma o ́ \mu \varepsilon v a:$ Ceux qui croient à la vérité (ả $\lambda \eta \theta \tilde{\eta})$ de nos enseignements $(\delta i \delta a \sigma \kappa o ́ \mu \varepsilon v a)$ et de notre doctrine $(\lambda \varepsilon \gamma o ́ \mu \varepsilon v a) \gg^{24}$. La traduction proposée par Foucault est quant à elle sensiblement différente et tente de respecter la syntaxe originale :

Ony apprend que le baptême doitêtre donné, etne peutêtre donné, qu'à $(\mathrm{LXI}, 2)$ « ceux qui croient que sont vraies $[\dot{\alpha} \lambda \eta \theta \tilde{\eta}]$ les choses [cela même] que nous leur avons enseignées $[\delta i \delta \alpha \sigma \kappa o ́ \mu \varepsilon v \alpha]$ et dites $[\lambda \varepsilon \gamma o ́ \mu \varepsilon v \alpha] \gg$. (Ibid.)

Pour une juste compréhension de la phrase, le respect des participes

HL, 1906, p. 29, nous soulignons.

Rappelons que, pour cette leçon du 13 février, Foucault lit le De paenitentia dans la traduction de Genoude, qui propose en effet : « cette foi commence et se recommande par la sincérité de la pénitence » (G, II). Mais sur ce point précis, il a consulté le texte original pour vérifier et corriger une sentence qui lui semblait, à ses dires, « décisive ».

HL, 1906, p. 29.

HL, 1906, VI, 17, p. 29 : «Le premier baptême de l'écoutant, c'est une crainte parfaite ». HL, 1904, p. 127. Nous soulignons les difficultés susmentionnées. 


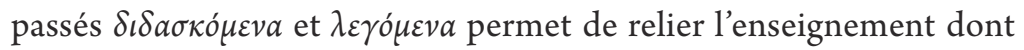
il est question au temps préparatoire du catéchuménat, comme le fait explicitement la Didach ${ }^{25}: \delta i \delta a \sigma \kappa o ́ \mu \varepsilon v a$ désigne un événement passé et non le simple acquiescement général à un corps de doctrine. Si la rigueur est à nouveau payante, regardons de près le tour que prend ici la traduction : l'équivalence recherchée est proche du mot à mot, au détriment de la dynamique de la phrase. Foucault est comme aimanté par la langue source, dont il veut garder intacte la syntaxe dans sa citation.

Le manuscrit du cours suffit à nous convaincre de cette vigilance constante qui amène Foucault à faire des mises au point au rythme de sa prise de notes : s'il lit en direct la version française sur la page de droite des éditions HL ou SC, son œil se reporte systématiquement à la page de gauche, où se trouve le grec ou le latin d'origine. Ça et là, entre les lignes de la traduction française qu'il recopie à la main, il ajoute un terme grec ou latin qui lui semble décisif ${ }^{26}$.

L'enregistrement sonore offre également maints exemples de ces mises au point. Ainsi du De paenitentia de Tertullien, déjà mentionné, que le professeur lit ici de toute évidence dans la traduction de Pierre de Labriolle chez Hemmer-Lejay (HL, 1906) ${ }^{27}$, tout en la corrigeant sur un point important concernant le champ d'application de l'exomologèse:

(IX, 3-4) « L'exomologèse, c'est la discipline qui prescrit à l'homme de se prosterner et de s'humilier, disciplina prosternendi et humilificandi, en s'imposant un

25 Peu avant, dans la même leçon, Foucault commente la Didachè en s'arrêtant sur l'expression qui ouvre le chapitre VII « tout ce qui précède » : « [...] et "tout ce qui précède" c'est ce qu'on trouve dans les premiers chapitres de la Didachè $\gg$. Cette remarque n'est pas anodine, car elle renvoie à un point débattu de l'établissement du texte. Dans leur édition (SC, 248, 1978), Willy Rordorf et André Tuilier mentionnent en effet, tout en l'écartant fermement, l'hypothèse de Jean-Paul Audet (dans une édition de 1958) selon laquelle « tout ce qui précède » serait une interpolation tardive. Foucault a donc raison d'insister sur cette expression qui lie la partie liturgique du texte à l'enseignement dit des « deux voies $\gg$, contenu dans les premiers chapitres (SC, 248, 1978, p.170, note 3 ).

Par exemple, dans les notes préparatoires à la leçon du ${ }_{13}$ février, au-dessus de sa citation du De paenitentia (VI, 9), entre « Mais pour y parvenir » et «il faut se mettre à la tâche », Foucault insère, par une accolade, le syntagme latin original sur lequel il va insister à l'oral : elaborandum est.

27 Contrairement à la leçon du 13 février, Foucault ne lit plus ici la traduction de l'abbé de Genoude. «En ce qui concerne la mise et la nourriture » est bien un choix caractéristique de Pierre de Labriolle, là où Genoude proposait : «L'exomologèse est donc un exercice qui a pour but d'humilier l'homme et de l'anéantir, en lui imposant une conduite qui attire la miséricorde, en réglant son extérieur et sa table » (G, II, nous soulignons). 
régime de nature à attirer sur lui la miséricorde. En ce qui concerne la mise et la nourriture... » Alors, je ne suis pas très fort en latin, mais je ne suis pas tout à fait d'accord avec la traduction. Il dit: « de ipso habitu atque victu ». «Habitu », c'est en fait, beaucoup plus vraisemblablement, le mode de vie général plus que l'habit. Ce que l'exomologèse concerne est une manière de vivre et pas simplement une manière de s'habiller à un moment donné. C'est une manière de vivre, c'est une manière d'être, c'est une manière de se nourrir. D'ailleurs, le texte le dit bien un peu plus haut, c'est une $(9,3)$ «conversatio », c'est une manière d'exister et d'avoir rapport aux autres, à soi, etc. ${ }^{28}$

Cette correction apportée par Foucault mérite un arrêt prolongé. Guidé par le respect de la lettre, Foucault est en effet amené à faire des choix, qui obéissent à deux motivations distinctes.

Dans l'exemple que nous venons de citer, le choix de traduction d'habitus par $\ll$ mode de vie $\gg$ au lieu de $\ll$ mise $\gg$ dénote en premier lieu le philosophe bon connaisseur de sa propre tradition. En fait, le sens propre et le sens figuré d'habitus sont ici tout autant légitimes. Labriolle peut étayer le recours au premier sur le fait qu'habitus est mis en série dans la phrase avec victus (nourriture) et précède la mention d'un corps que l'on doit sordibus obscurare (obscurcir de haillons). Foucault préfère quant à lui remonter un verset plus haut et rapprocher habitus de conversatio (régime, genre de vie). Dans cette manière d'interpréter Tertullien, il privilégie en fait implicitement la tradition philosophique sur l'acception matérielle du terme. L'habitus, traduction latine du grec $\mathcal{E}^{\prime \xi} \iota \varsigma$, est en effet longuement défini et discuté par Platon et surtout Aristote. Chez ce dernier, le terme désigne un engagement entier de l'être dans une disposition volontairement acquise, comme l'est par exemple la vertu ${ }^{29}$. La référence est d'autant plus plausible que Tertullien est régulièrement en débat avec la tradition aristotélicienne, à laquelle il emprunte certains $\operatorname{arguments}^{30}$. Il s'agit alors pour Foucault de donner à la phrase un sens le plus général possible, en respectant l'extension du concept philosophique dénoté par le terme habitus.

28 Leçon du 5 mars 1980.

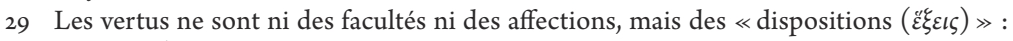
Aristote, Éthique à Nicomaque, II, 4, 1106a, 6-12.

30 Rappelons que Tertullien, s'il écrivait en latin, lisait et parlait le grec. Ses références explicites à Aristote sont cependant le plus souvent polémiques, surtout lorsqu'il fustige l'usage d'Aristote chez certains gnostiques : «Pitoyable Aristote qui leur a enseigné la dialectique » (Tertullien, Traité de la prescription contre les hérétiques, VII, 6, P. de Labriolle trad., Paris, Cerf, 1957, p.97). 
Dans un même souci d'opter pour le sens le plus riche, Foucault traduit la liberalitas de Dieu, sur laquelle insiste le De paenitentia, par « libéralité $\gg$, afin de conserver le double sens de liberté et de générosité, là où Labriolle, à la suite de Genoude, ne conserve que le deuxième sens ${ }^{31}$ :

Ce que Tertullien veut absolument maintenir, dans la conception qu'il se fait de la préparation au baptême, ce sont deux choses. Premièrement, ce qu'il appelle la $($ VI, 11$)$ «libéralité » de Dieu, « liberalitas » $[\ldots]$. Liberalitas dans les deux sens, générosité et liberté. ${ }^{22}$

Mais, étrangement, Foucault privilégie «libéralité » pour une autre raison, celle-ci nettement moins défendable : sa proximité phonique avec la liberalitas originale. D'autres exemples de traductions moins heureuses permettent de s'en rendre compte. Foucault traduit ainsi le verbe elaborare par «élaborer », ce qui n’a pas grand sens au regard du contexte :

Là, alors, je vous renvoie au chapitre vi du De paenitentia [...] (VI, 9) «Je ne conteste pas à ceux qui vont descendre dans l'eau l'efficacité [omnimodo salvum: l'intégrité complète] du bienfait de Dieu [qui est l'effacement des péchés], mais pour y parvenir il faut travailler, il faut se mettre à la tâche», enfin, « elaborandum est $\gg 33$, il faut élaborer, il faut se mettre à la peine. ${ }^{34}$

Notons au passage que la traduction par «il faut élaborer » est une improvisation orale ${ }^{35}$, qui fait partie d'une discrète théâtralité, propre à l'enseignement public de Foucault.

Quitte à rectifier ou affiner dans un second temps, le littéralisme, entendu ici comme simple décalque phonique, est toujours le premier réflexe. Au lieu de privilégier le contenu sémantique de la phrase, Foucault met en avant la langue source, choisissant la traduction la plus proche du signifiant comme forme acoustique. Trois leçons plus loin, il passe sans hésiter de publicatio sui à «se publier», avec une

HL, 1906, p. 27 : «Ils transforment sa générosité [liberalitatem] en servitude. » De son côté Genoude propose : «Ils font de la générosité de Dieu une servitude » (G, II). Leçon du 13 février 1980.

Foucault modifie l'ordre de la phrase qui aurait du être : « Je ne conteste pas que le bienfait de Dieu, qui est l'effacement des péchés, ne demeure tout à fait intact pour ceux qui vont descendre dans l'eau, mais pour y parvenir il faut travailler $\gg$. Mais il est plus littéral que Labriolle en 1906, qui substitue à «il faut travailler », «il faut souffrir », et ajoute le thème du bonheur, là où Tertullien ne parle que de «arriver à y parvenir » (sed ut eo pervenire contingat). 
homophonie également peu aidante pour le sens de la phrase originale ${ }^{36}$. La maladresse dans le premier cas est cependant compensée par la mention de plusieurs traductions possibles. Foucault est coutumier de ce tâtonnement, comme si la traduction idéale n'était approchée que par une série d'approximations : ainsi de probavimus traduit successivement par «nous avons éprouvé, nous avons prouvé, nous avons montré ${ }^{37}$.

Ceci nous amène à un deuxième constat, qui relève cette fois d'un choix théorique : si Foucault revient au texte original, c'est afin que la traduction proposée lui ressemble le plus possible, dans sa syntaxe comme dans sa phonie. Les exemples cités témoignent d'une résistance à quitter la langue source pour intégrer pleinement une langue cible qui altère forcément la chaîne des signifiants. À compter du cours Du gouvernement des vivants, l'usage des mots dans les textes anciens semble guider Foucault pour déterminer l'évolution des problèmes. La langue devient par conséquent le lieu de la rigueur, au point que le travail proposé n'est plus tant une interprétation du sens des textes, qu'une répétition des textes mêmes, simplement découpés d'une certaine manière afin d'être mis en série. Il nous reste alors à interpréter cette force d'attraction qui ne relève pas du seul goût pour les langues anciennes.

L'attachement de Foucault aux mots grecs et latins est exemplaire des citations proposées tout au long du cours de 1980 : autant que possible - au moins pour les syntagmes décisifs - les citations sont bilingues, le terme latin ou grec suivant ou précédant immédiatement le terme français, dans une redondance scrupuleuse. Dans les onze versets du De paenitentia de Tertullien cités au cours de la leçon du 13 février, pas moins de sept syntagmes voient ainsi leur traduction redoublée par le rappel du latin : liberalité /liberalitas; crainte /metus [erratum : timor]; danger /periculi; discipline de la pénitence / paenitentiae disciplina; baptême de la péni-

Leçon du 5 mars 1980 : « Tertullien a un mot qui est, je crois, fondamental : c'est la publicatio sui, il faut se publier ». Moins littérale, la traduction de Labriolle donne ici une idée plus juste du sens de cette expression, qui n'apparaît qu'une seule fois dans le De paenitentia : $(\mathrm{X}, 1)$ «Et pourtant, je présume que la plupart se dérobent à ce devoir [de l'exomologèse $][\ldots]$, parce qu'ils redoutent de s'afficher en public [ut publicationem sui $] \gg(\mathrm{HL}$, 1906 , p. 43). S'afficher en public n'est donc pas un devoir pour Tertullien, mais seulement la conséquence - dissuasive pour les hommes trop soucieux de leur image - d'une pénitence qui touche au comportement extérieur (nourriture, vêtement, pleurs, prosternations, etc.). Nous y reviendrons. 
tence / baptisma paenitentiae; épreuve de la pénitence / paenitentiae probatio; foi de la pénitence / paenitentiae fides. Dans certains cas, Foucault choisit de ne conserver que le terme grec ou latin, auquel il accole un article défini français : ainsi de «la paenitentiae disciplina ». Cette pratique du néologisme - au sens de l'importation directe d'un terme d'une langue dans une autre - se trouvait déjà dans la lecture inaugurale d' Edipe roi, le 9 janvier 1980, avec ses « alèthurgies » successives. Mais elle devient, dans les commentaires chrétiens, un style d'énonciation à part entière.

Par une sorte de dommage collatéral, cette pratique qui espère préserver dans leur intégrité des syntagmes de la langue source modifie en fait le texte original. Pour la simple raison tout d'abord que l'insertion du vocable grec ou latin dans une phrase française demande de modifier son mode ou son cas par souci de cohérence. À certains endroits, la phrase française, très librement traduite de l'original, n'est construite que pour mettre en valeur un terme grec ou latin, volontairement remis à l'infinitif s'il s'agit d'un verbe conjugué, ou au nominatif s'il s'agit d'un substantif. La conséquence de ces transpositions est de figer l'action initialement décrite par la phrase pour ne garder du texte original que son vocabulaire, réduit aux entrées lexicales d'un dictionnaire. C'est ainsi que, dans l'exemple suivant,

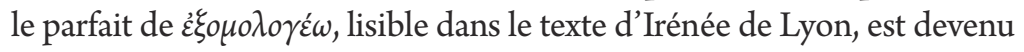
à l'intérieur de la phrase française un infinitif présent ( $\dot{\xi} \xi o \mu \nu \lambda o \gamma \varepsilon i v$ ), avant d'être traduit à l'aide d'un substantif («faire l'exomologèse») :

Livre I, chapitre xill, paragraphe 5, à propos d'une femme qui a été convertie, est revenue à l'Église, eh bien, à propos de cette fermme, il est dit : «Elle a passé toute sa

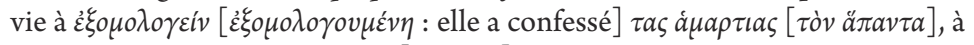
faire l'exomologèse de ses fautes [sa faute] $\gg .^{3^{8}}$

Dans ce passage d'Irénée, nous vérifions que le respect du texte original concerne d'abord et avant tout certains mots, véritables nœuds de l'argumentation, cités par Foucault dans la forme lexicale du dictionnaire (infinitif ou nominatif) et non dans celle du texte. Il s'agit de

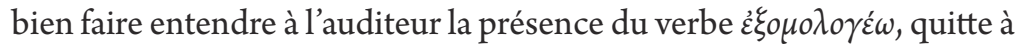
modifier sa forme verbale. Pour le reste, en dehors de ces mots-clés scrupuleusement isolés, un singulier peut devenir un pluriel et une phrase au passé peut se retrouver conjuguée au présent ${ }^{39}$. 
La raison première de cette hésitation à traduire, audible dans le recours à la citation bilingue, est la sédimentation de sens qui s'est opérée à travers les siècles sur le vocabulaire philosophique ou religieux : depuis le XIII ${ }^{\mathrm{e}}$ siècle, la confessio a ainsi pris un sens sacramentel à la fois trop restreint et codifié pour ne pas tromper l'auditeur moderne. La prudence requiert donc de translittérer le terme grec en parlant d'exomologèse, comme l'accepte d'ailleurs la langue latine et le Littré français ${ }^{40}$. Il en va de même pour la «pénitence » dont Foucault retrouve dans les textes $\mathrm{du} \mathrm{II}^{\mathrm{e}}$ siècle un sens spécifique, que le recours au terme grec

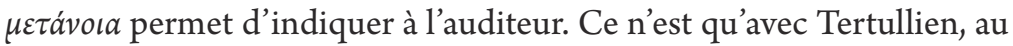
siècle suivant, que la $\mu \mathcal{\varepsilon} \tau \dot{v} v o$ เa, devenue en latin la paenitentia, vint à se détacher de l'illumination par la lumière divine pour devenir le seul acte

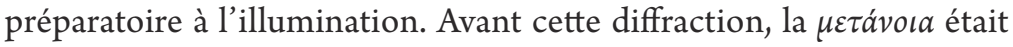
dans un seul mouvement ascèse et illumination ${ }^{41}$.

Préservés dans leur langue d'origine, les syntagmes en apparence triviaux comme paenitentiae disciplina laissent entendre à l'auditeur que leur traduction ultérieure s'est accompagnée subrepticement d'un glissement de sens - ou encore, si l'on veut avec Foucault passer du monde du texte au monde des signes, un changement de «signification » :

Donc, la traduction classique qu'on trouvait déjà de $\mu \varepsilon \tau \dot{q} v o l a$, c'est en latin paenitentia, la pénitence. Mais cette pénitence prend chez Tertullien une signification tout à fait différente..$^{42}$

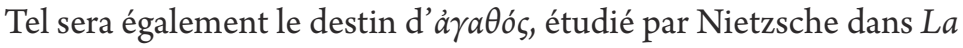

sur le commentaire : erreur de temps, de nombre, utilisation d'un mot pour un autre (tel « prêtre » pour « évêque » dans la citation de La vie de saint Ambroise, le 5 mars). L'écart le plus intéressant est l'invention d'une répétition pour accentuer un renversement dans l'argumentation. Ainsi de la citation du De paenitentia, le 13 février : (VI, 17 ) «Nous ne sommes pas plongés dans l'eau du baptême pour être purifiés [pour renoncer à nos péchés], mais nous sommes plongés dans l'eau du baptême [déjà moralement lavés] parce que nous sommes purifiés [nous avons renoncé]. » S'entend ici l'impact du caractère oral de l'enseignement. Comme le note Tertullien, au début du $\mathrm{III}^{\mathrm{e}}$ siècle, le terme grec avait déjà été transporté dans la langue latine : leçon du 5 mars 1980.

$41 \ll[\ldots]$ la $\mu \varepsilon \tau a ́ v o r a$, dans les textes grecs de la période hellénistique et du second siècle chrétien [...] c'est le changement de l'âme, c'est-à-dire c'est essentiellement ce mouvement par lequel l'âme pivote sur elle-même ou, plus précisément, le mouvement par lequel, d'une part, elle se détourne de ce qu'elle regardait jusque-là, les ombres, la matière, le monde, les apparences, etc., et à quoi elle était attachée. La $\mu \varepsilon \tau$ d́vola c'est aussi le mouvement $[\ldots]$ par lequel l'âme se tourne au contraire vers la lumière, vers le vrai, vers ce vrai qui l'illumine » (leçon du 13 février 1980). 
généalogie de la morale et évoqué par Foucault dans une conférence de $1964^{43}$. Ressaisissant la tâche de l'herméneutique moderne, telle qu'elle se déploie avec Nietzsche, Freud et Marx, cette conférence de jeunesse peut nous permettre de reformuler le problème rencontré en 1980 : si des mots ne renvoient jamais à un signifié originel mais toujours à une interprétation imposée de signes plus anciens ${ }^{44}$, la tâche de l'herméneute consiste à retrouver comment notre pénitence tridentine interprète la paenitentia de Tertullien qui interprète elle-même la $\mu \varepsilon \tau$ ćvoı a des Pères grecs et ainsi de suite - chaque interprétation devant être comprise comme une modification en profondeur de la précédente, non comme un simple additif ou prolongement ${ }^{45}$. Par rapport à sa conférence de 1964, Foucault abandonne seulement le caractère brusque et violent que revêt un tel changement chez Nietzsche.

Pour en revenir maintenant à une analyse strictement sémantique : ne pas traduire permet non seulement d'indiquer que la dénotation du terme a historiquement changé (la confession dénote depuis le XIII ${ }^{\mathrm{e}}$ la confession auriculaire privée à un membre du clergé); mais ce choix permet également et surtout de neutraliser toutes les connotations possibles liées à l'usage contemporain du terme (la confession renvoie au secret et à la peur, à l'assujettissement du fidèle et au pouvoir du clerc). A contrario, on peut considérer comme trompeuse la version du Pasteur d'Hermas par Robert Joly (SC, 53, 1958), qui traduit $\mu \varepsilon \tau$ d́vola par « pénitence » et $\ddot{\alpha} \varphi \varepsilon \sigma \iota \varsigma$ par «absolution $\gg{ }^{46}$, orientant de ce fait l'interprétation du lecteur contemporain vers le sacrement du deuxième millénaire.

Rassemblons maintenant nos deux constats pour tracer le portrait de Foucault en traducteur. N'hésitant pas à accoler le texte original à une traduction dénoncée par là même comme trompeuse, Foucault est un

Pour Nietzsche, relu par Foucault lors de ce colloque à Royaumont, les signes ne signifient que parce qu'ils sont déjà des interprétations, imposées de force par les classes supérieures : «Il n'y a rien d'absolument premier à interpréter, car au fond, tout est déjà interprétation, chaque signe est en lui-même non pas la chose qui s'offre à l'interprétation, mais interprétation d'autres signes » (ibid., p. 599).

45 «C'est un rapport tout autant de violence que d'élucidation qui s'établit dans l'interprétation. En effet, l'interprétation n'éclaire pas une matière à interpréter, qui s'offrirait à elle passivement ; elle ne peut que s'emparer, et violemment, d'une interprétation déjà là, qu'elle doit renverser, retourner » (ibid., p. 599). 
traducteur paradoxal qui voudrait ne pas avoir à traduire. Il recrée par conséquent un texte qui n'est à proprement parler ni une traduction ni une citation de l'original, mais un compromis où les syntagmes grecs ou latins, insérés dans le texte français, doivent curieusement lui être grammaticalement accordés. Cette caractéristique, qui pourrait sembler secondaire au regard de l'enjeu spécifique de l'interprétation, définit en fait une certaine catégorie de traducteurs que le linguiste Jean-René Ladmiral nomme les «sourciers $\gg 47$. Ces derniers ont tendance à se focaliser sur la structure lexico-syntaxique de la langue source, confortés par le principe avoué ou non d'une «intraduisibilité » ultime - c'est-à-dire de l'existence d'un reste incommunicable de sens, qu'une tournure inhabituelle dans la langue cible ou l'usage de néologismes doit rappeler au lecteur. Soulignant qu'il y a un « enjeu philosophique de toute traduction $\gg$ ( $\mathrm{p}$. XIII), Jean-René Ladmiral relève dans ce groupe - où sont convoqués Henri Meschonnic, Walter Benjamin et Antoine Berman - une conception religieuse de l'écrit, conférant au texte original une dimension quasi sacrée. Comme osait l'écrire Benjamin non sans provocation : «La version intralinéaire du texte sacré est le modèle ou l'idéal de toute traduction $\gg^{48}$. Pour cette école de traducteurs, l'exactitude sémantique doit être le produit de la conservation du signifiant, principe qui peut aller jusqu'à décalquer la syntaxe d'origine, la morphologie des mots (par exemple les préfixes), voire leur phonie. À l'opposé, ceux que Ladmiral nomme les « ciblistes » ne s'attardent ni au signifiant ni au signifié de la langue, mais au sens transmis par la parole, entendue avec Saussure comme l'exécution libre et individuelle de la langue. Il s'agit alors pour le traducteur d'interpréter cette parole, pour tenter de l'exprimer avec les ressources propres à la langue cible ${ }^{49}$.

S'il n'y a pas chez Foucault une philosophie de la traduction aussi consciente et volontariste que celle des vrais «sourciers », son effort pour respecter le signifiant du texte original n'est pas le simple reflet d'une prudence académique ou didactique. Cette posture, dont nous avons relevé à la fois les atouts et les dangers, indique à l'auditeur l'étran-

47 J.-R. Ladmiral, Traduire : théorèmes pour la traduction, Paris, Gallimard, 1994, p. XV. Disciple de Georges Mounin, Jean-René Ladmiral est l'un des rares auteurs d'un traité sur la question de la traduction en langue française.

48 W. Benjamin, «La tâche du traducteur », Mythe et violence, M. de Gandillac trad., Paris, Denoël, 1971, p. 275.

49 J.-R. Ladmiral, Traduire : théorèmes pour la traduction, déjà cité, p. XV. 
geté de textes que la traduction pourrait rendre faussement obvies ou contemporains, de la même manière que les auteurs latins avaient conservé dans sa translittération le terme grec exomologesis, « comme [s'il] désignait quelque chose que le mot latin, banal, de confessio n'arrive pas à saisir tout à fait $\gg^{50}$. Contrairement à ses premières traductions du Cycle de la structure et de l'Anthropologie du point de vue pragmatique, Foucault ne cherche pas tant ici un équivalent français au texte source, qu'un décalque le plus rigoureux possible de la phraséologie grecque ou latine d'origine, matière qui doit être travaillée par la découpe des phrases et l'extraction de certaines lexies, sans que la transposition dans un autre ordre des mots ne l'arrache à son site premier. Il suit ce faisant une leçon apprise du côté de Pierre Klossowski, dont la traduction de L'Énéide de Virgile en 1964 - proche à certains endroits du mot à mot l'avait grandement impressionné : dans cette traduction audacieuse mais rugueuse, il s'agissait de conserver le mince événement du «mot $\gg$ initial contre son étalement sur le «plan uniforme des langues $\gg^{51}$. À une traduction «latérale », expliquait Foucault en 1964, Klossowski avait substitué une traduction «verticale», faisant «tomber » une langue sur une autre, sans effacer le choc de la chute (ibid., p. 452).

Cette volonté de ne pas traduire, ou de traduire le moins possible, nous fait quitter la question du passage d'une langue à une autre pour aborder celle de la citation à proprement parler. Il nous faut montrer maintenant comment un mot grec comme $\dot{\varepsilon} \xi o \mu o \lambda o \gamma \varepsilon i v$ est extrait du texte original pour être placé, dans la dramaturgie propre au cours, au fronton d'une phrase, comme s'il tenait son sens de lui-même et non de son usage dans le discours. Objet à la fois prélevé et greffé, la citation réinvente le texte original, conférant une autonomie artificielle à une phrase qui devient ainsi une formule, un mot qui devient un emblème. Comme l'a montré Antoine Compagnon, la citation n'est pas seulement un énoncé répété, mais aussi et surtout une nouvelle énonciation singulière ${ }^{52}$.

Leçon du 5 mars 1980.

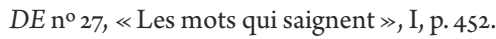

A. Compagnon, La seconde main ou le travail de la citation, Paris, Seuil, 1979, p.17-18 et $55-56$. 


\section{Citer : la citation comme pratique de la lexicalisation}

Foucault ne se contente pas de citer ni de traduire littéralement des versets entiers des Pères; il en détache des substantifs qu'il met en exergue par le recours à une tournure emphatique telle que : «c'est ce que Tertullien appelle $[\ldots] \gg$. Ce faisant, il fait porter sur l'auteur du texte la responsabilité d'un usage du langage que nous pourrions qualifier de «terminologique » : usage étroit qui consiste à lier de manière indissociable une expression linguistique à un concept. On peut noter six occurrences principales de cette tournure emphatique : elle est appliquée au «sceau de la vérité $\gg^{53}$, à la $\ll$ discipline de la pénitence $\gg^{54}$, à l'《épreuve de la pénitence $\gg^{55}$, à la $\ll$ pénitence seconde $\gg^{56}$, à $《$ l'exposition du cas $\gg^{57}$ et $\mathrm{au} \ll$ mode de vie $\gg^{58}$. Le groupe de mots ainsi isolé par la tournure emphatique est toujours donné également en latin ou en grec, non sans avoir préalablement été remis au nominatif. Or, une telle tournure n'est pas seulement ornementale, elle opère sur les textes bien plus qu'une simple césure.

Prenons un exemple en apparence banal, extrait du commentaire par Foucault du De paenitentia de Tertullien, le 13 février 1980 :

C'est ce que, dans le texte, Tertullien appelle la (VI, 5 ) paenitentiae probatio, l'épreuve de la pénitence ou encore la $(\mathrm{VI}, 6)$ « vérité de la pénitence $\gg .59$

Regardons d'abord la pratique du traducteur. Si une telle pratique peut être définie par les « unités sémantiques minimales traduisibles ${ }^{60}$

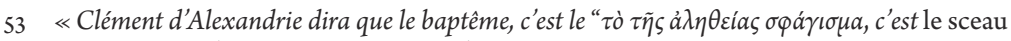
de la vérité" $\gg$ (leçon du 6 février 1980).

54 «C'est ce que Tertullien appelle, dans la phrase suivante "paenitentiae disciplina", la discipline de la pénitence $\gg$ (leçon du 13 février 1980).

55 «C'est ce que, dans le texte, Tertullien appelle la "paenitentiae probatio" » (leçon du 13 février 1980).

56 «ette "paenitentia secunda" [...] C'est comme dit Tertullien, "une autre planche de salut" » (leçon du 5 mars 1980).

57 «C'est ce que dans les textes de saint Cyprien, on appelle justement l'"expositio casus" » (leçon du 5 mars 1980).

58 «e que l'exomologèse concerne [...] c'est une manière de vivre, c'est une manière d'être, c'est une manière de se nourrir. D'ailleurs, le texte le dit bien un peu plus haut, c'est une "conversatio" $\gg$ (leçon du 5 mars 1980).

59 Leçon du 13 février 1980.

60 C'est-à-dire « le plus petit segment dont la cohésion des signes est telle qu'ils ne doivent pas être traduits séparément » ( J.P. Vinay et J. Darbelnet, Stylistique comparée du français 
qu'elle admet, il est intéressant de comparer la traduction d'un « sourcier » comme Foucault avec celle d'un « cibliste » comme Charles Munier, traducteur du même texte pour l'édition des Sources chrétiennes. Là où le premier conserve scrupuleusement certains mots ou groupes de mots, le second s'affranchit du signifiant pour privilégier des « unités de parole $\gg^{61}$ ou de $\ll$ message $»$ (Roman Jakobson), mettant l'accent non pas sur les mots, mais sur leur usage par des émetteurs dans un but de communication. D'un côté, le traducteur s'attarde sur des faits de langue, objets d'une analyse syntaxique; de l'autre, il interprète des actes de parole, objets d'une analyse pragmatique.

Difficile de ne pas donner raison à Munier, tant il est clair au plan sémantique qu'un mot déborde nécessairement sur ceux qui l'environnent, pour signifier au-delà des deux espaces blancs qui l'isolent au regard. Du même coup, la traduction de Munier dans l'édition SC fait disparaitre des unités lexicales élevées chez Foucault au rang d'unité de pensée : ainsi d'un mot simple (veritas) ou d'un groupe de mots (paenitentiae probatio). Là où une traduction littérale imposerait $(\mathrm{VI}, 5) \ll$ le Seigneur veut évaluer l'épreuve de la pénitence [paenitentiae probationem] », Munier propose «le Seigneur veut d'abord examiner de près notre pénitence $\gg^{62}$; et là où le sourcier se doit de traduire (VI, 6) « Mais retardons quelques temps encore la vérité de la pénitence [paenitentiae veritatem] », le cibliste s'autorise «Mais retardons quelques temps encore l'accomplissement de la véritable pénitence $\gg$ (p.167, nous soulignons). La transformation d'un substantif en verbe dans le premier cas, et d'un substantif en adjectif dans le second, entraîne à chaque fois une réorganisation plus large de la phrase, qui s'oppose à la tentation de diviser le signifiant en unités insécables.

À l'exact opposé, Foucault opère une lexicalisation d'un texte original qui est pourtant, rappelons-le, un texte à portée d'abord pratique et non théorique. En linguistique, sont dits «lexicalisés » un syntagme ou une périphrase qui viennent à être reconnus comme une unité fixe et autonome du lexique d'une langue (un lemme). Par extension, nous entendons ici par «lexicalisation $\gg$ la pratique de traduction consistant

et de l'anglais. Méthode de traduction, $4^{\mathrm{e}}$ édition, Paris, Didier, 1968, p. 16 et passim, cité dans J.-R. Ladmiral, Traduire : théorèmes pour la traduction, déjà cité, p. 203).

61 J.-R. Ladmiral, Traduire : théorèmes pour la traduction, déjà cité, p. 206.

62 SC, 316, 1984, p. 167, nous soulignons. 
à faire jouer des syntagmes comme des entrées lexicales. Cette pratique confère à un groupe de substantifs comme la «vérité de la pénitence » une unité terminologique. Une telle attribution est risquée, puisqu'il est loin d'être évident que la description d'un rituel ou son commentaire pastoral - comme celui du baptême proposé par la Didachè - puissent se lire comme un discours à finalité épistémologique. Roger Chartier fait remarquer à ce sujet qu'il n'est pas possible de lire des pratiques comme s'il s'agissait d'écritures - même si elles ont laissé des traces écrites ${ }^{63}$.

Certes, une pensée est bel et bien à l'œuvre dans ces conseils destinés aux pénitents ou aux évêques garants de la bonne volonté de ceux-ci. Comme Foucault le défendra dans un entretien l'année suivante : « La pensée, ça existe, bien au-delà, bien en deçà des systèmes et des édifices de discours. C'est quelque chose qui se cache souvent, mais anime toujours les comportements quotidiens $\gg{ }^{64}$. Mais justement, il n'est pas sûr que le découpage lexical, ou plus précisément les syntagmes isolés par le commentaire comme autant de sauts décisifs dans l'histoire des pratiques réfléchies de soi, soient substantiellement de l'ordre de ladite pensée. Si la tradition philosophique a toujours accordé au lexique une place décisive dans la détermination des concepts, cela ne veut pas dire que ces derniers ont nécessairement un ancrage verbal particulier, lié à une définition ou une glose précise. Ils peuvent tout aussi bien s'actualiser de manière labile et progressive dans des groupements d'unités sémantiques non lexicalisés, formant ce que Mathieu Valette appelle un «thème $\gg^{65}$. Concept et instabilité sémantique ne sont pas forcément exclusifs l'un de l'autre. A contrario, les lexiques et glossaires philosophiques tendent à figer le concept, oubliant sa naissance dans un environnement textuel qui est toujours un enchevêtrement complexe

63 «ire un rituel, qui est quelque chose comme une danse, comme s'il s'agissait d'un discours, comme quelque chose dont on peut donner une formulation algébrique, c'est me semble-t-il lui faire subir une altération essentielle » (Pratiques de la lecture, R. Chartier éd., Paris, Payot \& Rivages, 2003, p. 279).

$64 D E \mathrm{n}^{\circ} 296$, «Est-il donc important de penser? », II, p. 998. La même idée est exprimée dans un entretien de 1982 en compagnie d'Arlette Farge : « Nous ne croyons pas qu'ily ait, d'une part, l'analyse des comportements, d'autre part, l'histoire des idées ; pour nous, il y a de la pensée partout $\gg\left(D E n^{\circ}{ }^{322}\right.$, «L'âge d'or de la lettre de cachet », II, p. 1170).

$65 \mathrm{M}$. Valette, «La genèse textuelle des concepts scientifiques. Étude sémantique sur l'œuvre du linguiste Gustave Guillaume », Cahiers de lexicologie, t. 89, vol. 2, 2006, p. 125-142. 
de contraintes rhétoriques, d'expressions et de phraséologie ${ }^{66}$. Ainsi, le langage des Pères ne se réduit pas forcément à une terminologie stable qui donnerait à chaque occurrence d'un terme comme veritas le même contenu sémantique déterminé. Seul le langage scientifique peut prétendre réduire sa syntaxe à une combinatoire formelle d'unités terminologiques, permettant aux mots d'échapper à leurs connotations dans une phrase donnée. Un exemple d'apparence modeste va nous permettre d'approcher le point critique - et d'une certaine manière indécidable des traductions proposées par le cours de 1980.

Rappelons tout d'abord que l'étude de la pénitence en 1980 est guidée par la problématique de la vérité : il s'agit de montrer comment une certaine vérité du sujet doit venir à la lumière dans la pratique pénitentielle et comment cette vérité doit s'autoriser de preuves. Cette recherche $s^{\prime}$ inscrit dans le projet inaugural d'une histoire de la « force du vrai, une histoire du pouvoir de la vérité $\gg^{67}$. Une vérité, donc, dont l'effet n'est pas seulement d'illuminer l'organe de la connaissance (l'esprit), mais de modifier l'être de celui qui connaît, comme par exemple l'arracher à une vie d'erreurs ou à un état de ténèbres. Au sein de cette histoire, Tertullien est repéré dès $1977^{68}$ comme un relais important, sinon un point de départ pour la période chrétienne. Le cours de 1980 prolonge cette intuition avec des mots qu'il nous faut soigneusement peser : avec Tertullien, explique Foucault,

$[\ldots]$ on voit apparaître $[\ldots]$ l'idée que la pénitence, c'est ce qui doit manifester au regard de Dieu la vérité du pécheur lui-même, la sincérité de ses sentiments, l'authenticité de son remords, la réalité de son propos de ne plus recommencer. La pénitence, c'est donc l'affleurement à la surface de la vérité profonde de l'âme, et c'est en ce sens que l'on peut dire que la pénitence est une pièce de monnaie. Elle est ce qui permet la probatio. ${ }^{69}$

Le recours à l'informatique pour l'analyse linguistique a relancé depuis une trentaine d'années le vieux débat concernant les rapports entre pensée et lexique : voir M. Valette, «La genèse textuelle des concepts scientifiques... », déjà cité. Il est intéressant de noter que cet article remarquable, qui nous permet de questionner les pratiques de Foucault dans le cours de 1980, se réclame ouvertement d'un Foucault plus ancien : celui de L'archéologie du savoir et des stratégies discursives.

67 Leçon du 6 février 1980.

$68 D E n^{\circ} 206$, «Le jeu de Michel Foucault », II, p. 313. Foucault y fait de Tertullien le véritable inventeur de la « chair » chrétienne.

69 Leçon du 13 février 1980. 
Dans cette proposition extraite du cours de 1980, la vérité ne renvoie pas à l'établissement d'un système formel de connaissances, ni même à la simple opposition logique du vrai et du faux, mais à un mouvement volontaire de manifestation de soi, faisant figure d'événement dans la vie d'un sujet. Suscité par une pratique (la pénitence), ce mouvement obéit à une direction précise (il va de la profondeur à la surface) et porte sur un être qu'il saisit dans son unité ( «l'âme », «le pécheur »). Pour cette raison, il convient de distinguer le terme de « vérité » des trois autres qui le suivent de près dans la phrase : «sincérité », « authenticité », « réalité ». Alors qu'il serait tentant de les considérer comme des synonymes du premier, ces trois mots ne portent en fait que sur les « sentiments », sur les « remords » et les «propos » du sujet. À ce titre, ils ne désignent que des cas particuliers ou des composantes de ce qui semble irrésistiblement se manifester comme totalité : «la vérité du pécheur lui-même », ou, plus loin, «la vérité de l'âme », «la vérité de soi ».

Si le terme de «vérité » est fermement défini dans le commentaire proposé de Tertullien, faut-il pour autant s'attarder sur toutes les occurrences d'ả $\lambda \eta^{\prime} \theta \varepsilon \iota a$ ou de veritas dans les traités sur la pénitence du $\mathrm{II}^{\mathrm{e}}$ siècle et leur conférer le même poids conceptuel ? Là où Joly (Hermas, Le pasteur, SC, 53, 1958) et Labriolle (Tertullien, De paenitentia, HL, 1906) n'hésitent pas à recourir le cas échéant aux synonymes « authentique » ou «sincérité », Foucault choisit de toujours traduire à l'identique, comme si le terme de vérité, dans ces textes où la pénitence se constitue conjointement comme pratique et comme problème, était univoque. Regardons les deux exemples suivants :

[...] l'ange de la pénitence dit au chrétien, dit à Hermas : (XXVIII, 1) « Aime la vérité, qu'elle [seule] puisse sortir de ta bouche; de la sorte, l'esprit que Dieu a logé dans ta chair se trouvera vrai [SC : sera trouvé authentique] - à $\lambda \eta \theta \dot{\varepsilon} s .7^{\circ 0}$

C'est ce que, dans le texte, Tertullien appelle la $(\mathrm{VI}, 5)$ « paenitentiae probatio », l'épreuve de la pénitence ou encore la (VI, 6) « vérité [veritatem] de la pénitence $\left[\mathrm{HL}\right.$ : sincérité de la pénitence] $\gg .^{71}$

Dans la première phrase, le rétablissement par Foucault de l'adjectif «vrai » est tout à fait légitime par rapport au choix de son prédécesseur Joly - ce dernier ayant seulement cherché à éviter la redondance 
vérité/vrai pour honorer l'élégance littéraire. Le second exemple reste par contre ouvert à la discussion.

Tout d'abord, il convient de rappeler le contexte où apparaît le syntagme isolé par la citation : dans le De paenitentia, Tertullien ne s'attribue pas l'expression paenitentiae veritas mais la place dans la bouche d'un interlocuteur et objecteur fictif, qui lâche simplement, à titre de transition : (VI, 6) « Mais remettons à plus tard la vérité de la pénitence [sed differamus tantisper paenitentiae veritatem ] ». Par conséquent, il semble difficile d'en faire un solide nœud de l'argumentation. Cette difficulté permet au passage de relever un autre trait récurrent des citations proposées par Foucault, qui rejoint les règles d'interprétation des textes que nous avions préalablement évoquées : la manière de citer déjoue à l'avance toute possibilité d'une analyse rhétorique du discours qui ferait intervenir dans le commentaire les différents registres du langage. La forme dialoguée ou le mode subjonctif, qui inscrivent la lettre du texte dans une forme déterminée de communication, sont en effet systématiquement effacés des citations ${ }^{72}$.

Une fois précisé le contexte immédiat, il faut noter que le De paenitentia ne connait que deux autres occurrences de veritas. Or, celles-ci renvoient strictement à la vérité de Dieu, non du sujet humain ${ }^{73}$. En tant qu'elle concerne l'être divin, la vérité est définie comme rigoureusement opposée au mal et à l'erreur, « car, commente Tertullien, les contraires ne peuvent s'accorder $\gg{ }^{74}$. Qu'en est-il alors de la pénitence humaine et comment entendre son éventuelle veritas? Comme le rappelle Foucault, Tertullien la compare à de la monnaie dont les marchands vérifient qu'elle n'est pas élimée (scalptus), rognée (rasus) ou altérée (adulter). La métaphore monétaire doit ici être étudiée de près, car elle vient qualifier d'une manière singulière la veritas de la pénitence. Le vrai auquel elle

Ainsi dans le commentaire de la lettre 30 de saint Cyprien, le 5 mars 1980 : «Ceux qui font l'exomologèse, ce sont, dit saint Cyprien, $(\mathrm{XXX}, \mathrm{VI}, 3)$ "ceux qui frappent à la porte. Ils sont dans le vestibule, ils sollicitent l'entrée et avec leur bâton, ils cognent à la porte de l'église". » Cette libre citation est en fait, dans le texte original, un subjonctif exprimant un ordre : «Qu'ils frappent à la porte, mais qu'ils ne la brisent pas; qu'ils s'approchent du seuil de l'Église [sic], mais qu' ils ne sautent point dessus » (saint Cyprien, BL, 1945, p. 76). De paenitentia, III, 2 ; IV, 4.

74 HL, 1906, III, 2 : « quod inter contraria sibi nulla amicitia est ». Le Pasteur d'Hermas ne dit pas autre chose : «Le Seigneur est véridique en toutes ses paroles et il n'y a en lui aucun mensonge » (SC, 53, 1958, p. 151). Ce dernier verset est cité par Foucault dans la leçon du 27 février 1980. 
renvoie ne s'oppose pas tant au faux qu'à un vrai abîmé ou mélangé la fausse monnaie étant, dans le contexte du monnayage romain, une pièce dont le poids officiel a été modifié ou auquel un métal a été ajouté (cuivre et argent dans une pièce d'or). Comme l'atteste un texte d'Apulée ${ }^{75}$, cette vérification de la monnaie par le marchand - ou par le changeur de monnaie - était courante dans un contexte où la diffusion de pièces coulées (fondues dans un moule et non frappées au marteau), initiée par l'empereur Septime Sévère, avait produit des monnaies de piètre qualité et facilité le monnayage privé, imitant celui de l'État ${ }^{76}$.

Mais du même coup, ne faut-il pas voir dans cette vérification de la pénitence ce que Foucault commentera deux ans plus tard chez Sénèque en termes d'inspection administrative ${ }^{77}$, à l'image de l'inspection d'un navire ou d'un campement; inspection qui concerne le respect d'une procédure et non la remontée d'une vérité des profondeurs? Dans ce cas de figure, en nous référant simplement au texte de Tertullien qui abonde en ce sens, il s'agit simplement d'établir si la pénitence n'a pas connu des $\ll$ lenteurs $\gg$ (cunctationis) ou des « tergiversations $\gg(\text { tergiversationis })^{78}$. Pour reprendre les termes du cours de 1981-1982 consacré au stoïcisme, l'acte de montrer les preuves serait alors «instrumental » et non «opératoire »; c'est-à-dire que seule l'effectuation de la pénitence - et non sa monstration - aurait une valeur spirituelle en elle-même ${ }^{79}$. Telle serait l'interprétation immédiate du verset VI, $4 \ll[\mathrm{Dieu}]$ nous propose

$75 \ll$ De peur, lui dis-je, que l'une des pièces d'or que tu m'offres ne se trouve fausse [nequam] ou de mauvais aloi [adulter], nous allons les enfermer dans ce sac même; tu le marqueras de ton anneau, et demain, en présence d'un changeur, nous les éprouverons [comprobentur] » (Apulée, Les métamorphoses, Livres VII-XI, X, 9, 3, P. Vallette trad., Paris, Les Belles Lettres, 1945, p.110).

76 T. Mommsen, Histoire de la monnaie romaine, t. III, L. C. P. C. Blacas d'Aulps trad., Bologne, Forni editore, 1865-1875, p.14-15.

77 L'herméneutique du sujet, p. 462-463 : « quand [Sénèque] évoque les différentes opérations en quoi consiste l'examen qu'il pratique, il emploie des termes qui ne sont pas judiciaires, qui sont surtout administratifs. [... I Il emploie le verbe scrutari qui est le verbe technique pour vouloir dire : faire une inspection, l'inspection d'une armée, d'un campement, d'un navire, etc. ».

78 HL, 1906, VI, 3, p. 25.

79 L'herméneutique du sujet, p. 347 : « Ces obligations, pour celui qui est dirigé, de direvrai $[\ldots]$, ce sont des obligations en quelque sorte instrumentales. [...] Mais ces éléments de l'aveu sont instrumentaux, ils ne sont pas opérateurs. Ils n'ont pas en tant que tels une valeur spirituelle. » 
d'acheter l'impunité en donnant en échange la pénitence $\gg^{80}:$ il y a un achat; et l'achat requiert une légitime inspection, sans que celle-ci n'augmente la valeur de la chose achetée.

S'inscrivant en faux contre cette interprétation - pourtant cette fois littérale - Foucault lui objecte la disproportion incompréhensible et toute pascalienne qu'elle entérinerait entre un prix fini (la pénitence) et un gain infini (le pardon éternel). Il faut donc que le prix à payer ne soit pas la seule pénitence ni même sa seule intégrité, mais l'effet de sa manifestation ou encore sa force révélante ; pour la raison suffisante que celleci touche à la vérité même du sujet. En montrant notre pénitence, nous nous montrons nous-même, dans notre tréfonds. À la limite, du point de vue divin tel que l'interprète Foucault, il y a plus à attendre de cette monstration que du seul acte de pénitence honnêtement accompli :

C'est là la double fonction de la pénitence : préparer et assurer le cheminement qui va vers la vérité et montrer en quelque sorte, pour le regard orthogonal de Dieu qui voit tout et nous surveille sans cesse, manifester la vérité de ce que nous sommes. ${ }^{81}$

D'où l'importance, dans la traduction, d'insister sur la présence du terme veritas, accolé à la pénitence, et de ne pas en faire comme Labriolle une simple «sincérité ». Sauf que rien ne vient étayer cette deuxième interprétation, moins littérale. De la vérité du mouvement accompli par l'âme (la pénitence) à la vérité de l'âme elle-même, il y a un pas, que seul franchit Foucault ${ }^{82}$. Rien dans le texte de Tertullien ne permet de voir dans la vérité de la pénitence autre chose que le simple respect d'une procédure, la vérification tatillonne de l'honnêteté d'une conduite. Autour d'un simple écart de traduction entre Labriolle et Foucault, se joue en fait un conflit d'interprétation qui engage toute l'interprétation de l'œuvre de Tertullien.

80 HL, 1906, VI, 4, p. 25 .

81 Leçon du 13 février 1980 .

82 L'assimilation dans et par le commentaire de Foucault est progressive et se fait en trois étapes : 1) vérité du mouvement; 2 ) vérité de l'âme faisant le mouvement ; 3 ) vérité de l'âme («il y a une autre vérité, qui est la vérité du mouvement lui-même, qui est la vérité de l'âme elle-même faisant mouvement vers le bien [...] vérité de l'âme, vérité de soi sous le regard de Dieu », leçon du 13 février 1980). 
Un autre exemple de lexicalisation serait l'extraction du syntagme publicatio sui dans un passage du même De paenitentia, où Tertullien décrit les raisons poussant certains à ne pas faire pénitence. Le passage est ainsi traduit par Labriolle, qui préfère avoir recours à un verbe, contre la lettre du texte qui aurait imposé « publication de soi » :

$(\mathrm{X}, 1)$ «Je présume que la plupart se dérobent à ce devoir ou le diffèrent de jour en jour, parce qu' ils redoutent de s'afficher en public [publicationem sui] $\gg .{ }^{83}$

Pour sa part, Foucault insiste sur ce terme, en lui ajoutant l'idée d'obligation :

Tertullien a un mot qui est, je crois, fondamental : c'est la $(\mathrm{X}, 1)$ « publicatio sui, il faut se publier $\gg .^{84}$

S'il accepte également de recourir pour sa traduction à un verbe, qu'il choisit acoustiquement plus proche («se publier »), Foucault cite résolument le substantif latin, en forgeant au passage un néologisme : « la publicatio sui ». Or, ce néologisme va servir dans la suite de la leçon à désigner rétroactivement un épisode décisif de la procédure de pénitence déjà croisé par Foucault : celui de la manifestation publique et ritualisée, à la porte de l'église, du fait que l'on est pécheur, à grand renfort de cris et de larmes. Sommet théâtralisé de l'entière procédure, accomplissant l'ultime exigence de la pénitence, ce rituel avait été préalablement repéré dans la correspondance de saint Cyprien et dans le De pudicitia de Tertullien, qui ne lui donnent cependant aucun titre particulier ${ }^{85}$. C'est Foucault qui choisit de le baptiser publicatio sui, en empruntant cette expression à un traité qui ne se réfère pourtant pas explicitement à l'épisode des lamentations publiques. Pour le professeur, cette manifestation publique de soi sera appelée quelques siècles plus tard à croiser et à se confondre avec une autre procédure : l'aveu privé et juridique d'une faute particulière (l'expositio casus), engageant ainsi la mutation la plus importante qu'ait connue l'histoire des pratiques chrétiennes de vérité.

83 HL, 1906, p. 43.

84 Leçon du 5 mars 1980.

85 L'expression complète publicatio sui ne se trouve dans aucun de ces deux textes. Par contre, le De pudicitia fait mention à un endroit de la seule publicatio : les pécheurs coupables d'adultère et de fornication sont appelés à pleurer sur le seuil de l'église sans pouvoir y entrer et ne peuvent recevoir de l'Église «que la publication [publicationem] de leur déshonneur » (HL, 1906, p. 61). Cette unique occurrence, que Foucault n'évoque pas, aurait été plus convaincante que celle du De paenitentia. 
Pour autant, remis dans le contexte de la phrase de Tertullien, le substantif publicatio - qui prit le sens de «publication » seulement dans le latin tardif - ne décrit pas tant une obligation particulière qui viendrait s'ajouter à la pénitence pour la valider, que la simple conséquence d'actes pénitentiels ayant forcément leur composante visible. Jeûner, s'habiller pauvrement, se rouler aux pieds des prêtres : cela se voit et provoque nécessairement la gêne de celui qui se plie à ces obligations. À aucun moment Tertullien ne dit explicitement que la manifestation publique est essentielle à la pénitence. Pour corroborer cette minimisation de la portée du substantif dans l'argumentation, notons également que ce terme de publicatio n'apparaît dans le De paenitentia qu'à cet unique endroit.

Parfaitement traduits, les termes veritas et publicatio sui n'en sont pas moins partialement cités. L'un ne va pas sans l'autre : une certaine découpe et mise en exergue de mots peut faire du littéralisme une belle infidèle. En éclairant la raison d'être de ces pratiques de traduction et de citation, la notion de «lexicalisation » pointe à la fois l'apport et les limites des commentaires qu'elles induisent. D'un côté, elles permettent un repérage d'occurrences que les versions $\mathrm{HL}$ et SC empêchent. De l'autre, elles restent prisonnières d'une approche terminologique de la langue, qui peut surdéterminer les occurrences de certains termes clés et orienter l'interprétation.

À la lisière de la question de la citation, ou plutôt dans son redoublement même, une dernière caractéristique des pratiques textuelles de Foucault mérite d'être relevée : celles-ci restent indifférentes à l'intertextualité. Cette indifférence affichée est d'autant plus inattendue et provocante que, implicitement ou explicitement, les Pères chrétiens citent continuellement l'Écriture, considérée comme parole inspirée. Comme le fait remarquer Antoine Compagnon dans son étude sur la citation, « la citation demeure l'atome du discours théologal ${ }^{86}$; elle est la figure obligée des textes patristiques, qui deviendront à leur tour, pour la théologie médiévale, auctoritas, c'est-à-dire un recueil de propositions homologuées, pouvant être citées et interprétées mais non contestées ${ }^{87}$.

86 A. Compagnon, La seconde main ou le travail de la citation, déjà cité, p. 217

87 Ibid., p. 218-221 : à la différence de la citation biblique, la citation patristique dans la théologie médiévale met en avant le sujet de l'énonciation, l'auctor : un doctor authenticus qui 
Pour les premiers Pères chrétiens, les Écritures renvoient d'abord à l'Ancien Testament, corpus de textes reçus de la tradition juive et lus dans la traduction grecque de la Septante. La fixation du canon néotestamentaire est quant à elle le fruit d'une longue évolution, rythmée jusqu'aux $\mathrm{V}^{\mathrm{e}}$ et $\mathrm{VI}^{\mathrm{e}}$ siècles par les crises doctrinales, la répression des hérésies et l'institutionnalisation progressive de l'Église chrétienne. De par leur proximité avec l'événement christique, les premiers écrits chrétiens ont cependant très tôt été intégrés à l'argumentation théologique par un réseau complexe de citations et d'allusions qui dessine et isole déjà le futur « canon $»$. La réflexion théologique et le canon scripturaire se sont donc simultanément et réciproquement engendrés ${ }^{88}$.

Or, de ce réseau particulièrement dense de citations explicites et de simples allusions, Foucault ne fait nulle mention. À l'intérieur des textes patristiques, il neutralise au contraire la référence à des autorités extérieures, en premier lieu celle des Écritures, dont la mention dans une argumentation a pour fonction de «faire signe $»^{89}$ vers la sainteté du Livre, l'inspiration de la Parole. Autant Foucault ne cache nul emprunt à la philosophie païenne dans les chaînes de raisonnement de certains Pères $^{90}$, ni même les emprunts des Pères entre eux ${ }^{91}$, autant il passe sous silence la citation scripturaire, en tant que celle-ci appelle de la part du lecteur une reconnaissance ${ }^{92}$. En effet, à la différence du jeu souvent

détient les clés de la tradition. Dans un raisonnement, les auctorites doivent nécessairement renvoyer à un nom propre, un nom d'auteur. Par contre, la citation scripturaire chez les Pères peut très bien être implicite, complètement intégrée à la phrase, sans aucun nom d'auteur ni aucun marqueur particulier.

88 Y.-M. Blanchard, « Naissance du Nouveau Testament et canon biblique », L'autorité de l'Écriture, J.-M. Poffet éd., Paris, Cerf, 2002.

89 Antoine Compagnon a montré en quel sens la citation pouvait être dite « signe », alors même qu'une phrase, si elle est faite de signes, n'est pas elle-même un signe. Il faut pour cela se placer à un niveau d'analyse supérieur, interdiscursif : «La citation est un signe interdiscursif fait tel par la répétition, par la citation en tant qu'acte. L'énonciation de la répétition a le pouvoir de faire signe, elle fait de l'énoncé un signe dans le même temps qu'elle l'énonce » (A. Compagnon, La seconde main ... , déjà cité, p. 59, nous soulignons). Par exemple : l'emprunt par Tertullien aux stoïciens de l'idée d'une transmission par la semence d'une faute originelle (leçon du 13 février 1980).

91 Dans la leçon du 27 février 1980, Foucault note ainsi qu'un passage de l'Épitre de Barnabé reprend à l'identique un passage de la Didachè.

92 À l'exception, bien entendu, des textes patristiques se présentant explicitement et exclusivement comme des commentaires de l'Écriture : ainsi de l'interprétation du passage de la mer Rouge par Origène, qui se trouve dans le Commentaire sur saint Jean (cité dans la leçon du 20 février 1980). 
insaisissable des influences d'un corpus philosophique sur un autre, la citation n'est pas de l'ordre du seul emprunt. Elle suppose, à titre de réquisit supplémentaire, d'être repérée et interprétée comme telle. Elle n'avance pas masquée, attendu que sa découverte est au contraire sa raison d'être, même quand nul verbe introducteur n'est utilisé. Le texte patristique est ainsi tressé de citations scripturaires, complètement intégrées à son propre discours, comme autant d'expressions idiomatiques de sa propre langue. Citée par Foucault, la lettre suivante de Polycarpe, à propos d'un prêtre coupable d'avarice, en est un parfait exemple. Nous indiquons en gras les passages scripturaires :

(Lettre aux Philippiens, XI) «Je suis bien peiné pour lui et pour son épouse : veuille le Seigneur leur donner un vrai repentir [II Tim. 2, 25]. Soyez donc très modérés, vous aussi, sur ce point : ne les regardez pas comme des ennemis [II Thess. 3, 15], mais rappelez-les comme des membres souffrants et égarés $\gg .93$

Le professeur ne mentionne aucune de ces deux références. Alors même qu'il est non seulement dans les faits mais par essence citation, le texte patristique ne s'autorise plus que de lui-même : dans le cours de 1980, les citations des Écritures se fondent jusqu'à disparaître dans les citations patristiques. Par leur silence éloquent sur l'intertextualité, les citations de Foucault attribuent alors aux Pères ce qui, dans leur stratégie discursive propre, aurait dû au contraire leur échapper. Ainsi, par le truchement du verbe introducteur («il dit »), Tertullien en vient à être l'auteur d'énoncés extraits de la Genèse ou de l'évangile de Matthieu :

[...] mais, dit Tertullien, comment aurait-il pu façonner l'être humain, le corps de l'homme dans sa complexité et sa perfection, s'il n'avait eu que de la glaise, que de la terre [Gen. 2, 7], et qu'il n'avait pas d'eau? [...] un homme qui est précisément à l'image et à la ressemblance de Dieu [Gen. 1, 26], comme dit le texte.94

Tertullien ne dit que relativement peu de choses. Il donne des indications négatives [...]. On ne donne pas (De baptismo, XVIII, 1 ) « les choses saintes aux chiens, on ne donne pas aux pourceaux les perles $[\mathrm{Mt} .7,6] \gg .95$

À un seul endroit, Foucault évoque un « principe » extérieur au texte, tout en se gardant de préciser son origine évangélique ${ }^{96}$. Cette ignorance systématique des références bibliques lui fait d’ailleurs manquer l'origine 
paulinienne de la métaphore du tombeau pour désigner l'eau du baptême ${ }^{97}$. Mais, dans la majorité des cas, il n'y a pas seulement inattention, il y a ferme décision de ne pas faire jouer le texte patristique comme signe. Fait d'autant plus notable que le cours Sécurité, territoire, population ne voyait a contrario aucun empêchement à relever ces références ${ }^{98}$.

En mettant ainsi entre parenthèses ce qui, dans le texte patristique, fait autorité pour le lecteur - attendu que la citation biblique est définie par les Pères comme comprenant à elle seule l'Écriture, donc le Logos ${ }^{99}$ il s'agit en premier lieu d'empêcher le contexte littéraire de masquer, en les inscrivant dans une tradition, la nouveauté des pratiques pénitentielles. L'une des fonctions d'une telle inscription est en effet d'inventer une préhistoire à des décisions doctrinales par ailleurs strictement circonstancielles ou stratégiques. Une pratique récente vient ainsi à être justifiée depuis toujours, ou, plutôt, comme si elle avait déjà potentiellement existé au commencement. Mais il s'agit également pour Foucault de bien distinguer dans les actes de vérité chrétiens, ceux qui relèvent de l'adhésion à un contenu doctrinal et ceux qui relèvent de la manifestation de soi : actes de foi et actes d'aveu. Même si ces deux types d'actes ne sont jamais complètement indépendants l'un de l'autre, et peuvent se croiser dans un même rituel, ils ont des obligations et des effets différents sur le sujet. Or, la citation biblique a justement pour fonction de

principe évangélique « Ce que nous lions sur la terre sera lié au ciel » [Mt. 18, 18], qui guide l'argumentation de Cyprien, mais sans plus de précision (leçon du 5 mars 1980).

Foucault avance que l'usage du terme «tombeau » pour désigner «l'eau du baptême et la piscine du baptême » se trouve «explicitement, et pour la première fois d'une façon explicite chez Origène » (leçon du 20 février 1980). Le parallèle entre le baptême et l'ensevelissement est en fait d'abord paulinien (Col. 2, 12 ; Rom. 6, 4). Origène est bien le premier Père à y recourir dans ses Homélies sur l'Exode (SC, 16, 1947, V, 2, p.139), mais sans mention de la piscine. Foucault ne confondrait-il pas avec un passage plus connu de Cyrille de Jérusalem, dans ses Catéchèses mystagogiques : «Après cela vous avez été conduits par la main à la sainte piscine du divin baptême, comme le Christ de la croix au tombeau $\gg$ (SC, 126 bis, 1988, II, 4, 1) ?

98 Dans le cours de 1978, Foucault relève bien la référence de saint Grégoire à saint Paul (Sécurité, territoire, population, p. 184). Même si la référence aux Psaumes annoncée est incertaine, Foucault relève également la reprise constante dans les textes ascétiques chrétiens d'une sentence d'origine biblique : «Celui qui n'est pas dirigé tombe comme une feuille morte $\gg$ (Sécurité, territoire, population, p. 179; p. 191, note 29).

99 «Aussi le pouvoir de la phrase scripturale est-il sans limite : elle fait autorité, elle est argument $[\ldots]$ parce qu'elle porte en elle le Logos. [... ] Eil, talisman, fétiche, la citation biblique abrège le Verbe, comprend l'Écriture, concentre la puissance du Logos » (A. Compagnon, La seconde main ... , déjà cité, p. 202-203). 
susciter des «acceptations-engagements, adhésions-fidélités à certains contenus qui doivent être considérés comme vrais $\gg^{100}$. C'est donc en bonne rigueur de méthode que Foucault refuse de mêler l'adhésion au Logos à l'aveu de soi.

Pour Foucault, le sens d'un texte instituant ou décrivant un « régime d'aveu » ne se trouve ni en dessous (stratégies discursives) ni au-delà (intertextualité), mais dans sa lettre même, en particulier dans son lexique, qui concentre en lui les mutations des pratiques et la manière dont celles-ci sont réfléchies. Ce sens n'est pas équivoque, mais déterminé et indentifiable, par le biais d'une analyse lexicale qui relève les occurrences, l'importance nouvelle que prennent soudain certains mots, et détermine leurs usages. Pour retrouver ces usages, il convient de rendre les rituels baptismaux et pénitentiels à leur autonomie et leur littéralité : autonomie par rapport à un contexte littéraire et doctrinal qui n'engage pas la vérité du sujet (Bible et dogmes); littéralité que ne doit pas entacher des traductions induisant des connotations et des dénotations par trop contemporaines. Cette approche des textes, qui commande des pratiques rigides de citation et de traduction, rejoint le positivisme invoqué par Foucault dans son commentaire méticuleux d'Edipe roi. Cette position aurait toutes les faveurs d'un Jean Bollack, philologue farouchement opposé à la tradition herméneutique représentée par Hans Georg Gadamer ou Paul Ricœur, au nom d'un même positivisme affiché ${ }^{101}$. À l'hypothèse du sens introuvable ou inépuisable ouvrant sur un commentaire infini, Bollack oppose la clôture du texte et l'absence d'ambiguité de la syntaxe, qui vient fixer le «flux » de la langue en ses possibilités infinies : «le sens se constitue dans la lettre $\gg^{102}$.

Proche de Bollack quand il refuse l'hypothèse d'un sens précédant le texte et ne s'y déposant que pour soutenir et communiquer une oralité primordiale ${ }^{103}$, Foucault a cependant une autre pratique de la

100 Leçon du 6 février 1980.

101 J. Bollack, Sens contre sens, déjà cité, p. 49-5o.

$102 \mathrm{Ibid}$., p. 86; voir aussi p. 82-84. Cette proposition n'ignore pas mais inclut résolument l'écriture poétique : voir J. Bollack, Poésie contre poésie. Celan et la littérature, Paris, PUF, 2001.

103 Voir la critique, déjà rencontrée, de la préface à Naissance de la clinique : Foucault y dénonce le présupposé du «double fond de la parole», en dessous du discours, supposant toujours « un excès du signifié sur le signifiant, un reste nécessairement non formulé de la pensée que le langage a laissé dans l'ombre » (Naissance de la clinique, p.XII). 
lettre. Jean Bollack se méfie en effet de toute lecture ignorant les relations structurant la phrase. Il se concentre pour sa part sur la syntaxe et la grammaire, comme constituant le sens de manière univoque. À l'opposé, Foucault privilégie l'insistance et le rayonnement supposé de certains termes ( $\dot{\varepsilon} \xi o \mu o \lambda o \gamma \varepsilon i v$, veritas, probatio, etc.) qu'il coupe de leurs connexions à l'intérieur de la phrase pour les «lexicaliser ». Plus généralement, il s'agit d'isoler dans les textes des mots ou des constellations de mots désignant de manière relativement stable ${ }^{104}$ des pratiques où une manifestation de soi est engagée et commence à être l'objet d'une attention particulière de la part des écrivains chrétiens. Avant toute définition canonique explicite, Foucault distingue ainsi, dans les textes des $\mathrm{III}^{\mathrm{e}}$ et $\mathrm{IV}^{\mathrm{e}}$ siècles, l'épisode de l'aveu verbal et secret de la faute (désigné par le couple expositio casus / confessio) de la manifestation publique et spectaculaire du fait que l'on se reconnaît pécheur (désigné par le couple publicatio sui / exomologèse) ${ }^{105}$. L'argumentation se déploie ainsi à partir du repérage de syntagmes dont la particularité est de croiser les champs sémantiques du vrai et du soi. Même si le contexte direct semble accorder à des expressions comme paenitentiae veritas ou publicatio sui un sens trivial, voire insignifiant, Foucault en risque une autre interprétation. Il cite ces expressions de telle manière que le texte vient à être interrogé, et parfois violemment déchiré, par la question de la vérité.

Foucault succombe-t-il alors à l' «illusion terminologique $\gg^{106}$ fustigée par Jean-René Ladmiral chez certains traducteurs, réduisant les textes patristiques à une nomenclature de termes que le philosophe pourrait mettre en série, avec le présupposé que ceux-ci conserveraient, indépendamment de leur contexte, le même poids sémantique? Ne pourrait-on également lui faire le même reproche que Jean Bollack fait à l'encontre de Heidegger lisant Sophocle : «il lui importe de trouver le sens qu'il lui paraît philosophiquement s'imposer. [... I Il n'a pas besoin d'autres garants; d'autant moins qu'il s'en tient aux mots en les iso-

104 Dans la leçon du 5 mars 1980, Foucault reste cependant prudent sur cette relative stabilité des désignations : «Et là, les auteurs latins, tout comme pour l'épisode dont je vous parlais tout à l'heure, l'expositio casus, ils ont tendance à n'employer que confessio, ils ont tendance pour l'épisode dont je vais vous parler maintenant, à employer exclusivement le mot grec d'exomologèse. Je dis qu'ils ont tendance, ça veut dire que ce n'est pas absolument régulier. Mais, en général, exomologèse désigne cet épisode. »

105 Leçon du 5 mars 1980.

106 J.-R. ladmiral, Traduire : théorèmes pour la traduction, déjà cité, p. 222. 
lant $\gg^{107}$ ? Oui, si l'on suppose que ce sens reconstitué par la mise en série de termes lexicalisés est le dernier mot du philosophe sur les pratiques pénitentielles. Non, si l'on reconnaît dans ces choix drastiques un procédé d'abord heuristique. La pratique de traduction et de citation renverrait alors à ce que nous pourrions appeler un « anarchisme méthodologique $\gg$, qui prend le risque de partis pris inédits et de découpages nouveaux, voire provocants.

Il est alors intéressant de jeter un coup d'œil à l'unique référence méthodologique du cours, jetée apparemment en passant, sous la forme d'un simple conseil de lecture : il s'agit de l'ouvrage de Paul Feyerabend, Contre la méthode. Esquisse d'une théorie anarchiste de la connaissance (Londres, New Left Books, 1975 ; Paris, Seuil, 1979) qui venait à ce moment-là de paraître dans sa traduction française. 



\section{Vers une «anarchéologie» du christianisme}

Ceci dit, entre parenthèses, si vous voulez lire des livres intéressants de philosophie qui paraissent actuellement - il n'y en a pas tellement - je vous recommande, plutôt que d'autres plus bruyants, de lire le livre de Feyerabend qui vient de paraître au Seuil sur la science. Personne n'en parle, mais voilà, sur le problème anarchie et savoir, quelque chose qui est intéressant. ${ }^{1}$

Décidant d'appliquer l'anarchisme à l'épistémologie, Paul Feyerabend (1924-1994) ${ }^{3}$ montre que le pluralisme méthodologique - résumé par la formule lapidaire «tout est bon »- est non seulement un fait de l'histoire des sciences mais un principe raisonnable. Prenant Galilée comme archétype d'une révolution scientifique improbable et empiriquement peu convaincante pour la science de son temps, le philosophe d'origine autrichienne montre qu'aucune théorie ne s'est jamais accordée avec la totalité des faits connus dans le domaine où elle prétend

1 Leçon du 30 janvier 1980.

2 En employant ce terme, Feyerabend ne fait pas référence à l'anarchisme politique, qu'il n'apprécie guère (P. Feyerabend, Contre la méthode. Esquisse d'une théorie anarchiste de la connaissance, B. Jurdant et A. Schlumberger trad., Paris, Seuil, 1979, p.13). Il faut entendre ici par «anarchisme » une «pratique libérale $»($ p. 20$)$, que le philosophe réfère par exemple au mouvement esthétique du dadaïsme (p.18, note 2 ).

3 Ayant pérégriné dans de nombreux pays, Paul Feyerabend enseignait chaque année à Berkeley depuis 1958. D’abord influencé par Karl Popper, il bâtit finalement sa propre philosophie de la science contre celui-ci et son principe de «falsification ». On oublie souvent de préciser que l'influence philosophique majeure de Feyerabend fut le Wittgenstein des Investigations philosophiques, auquel il consacra plusieurs articles au début des années 1950 et qu'il n'a nul besoin de citer dans Contre la méthode (à une exception près : p. 144), tant la tournure d'esprit de l'auteur du Tractatus transparaît entre les lignes : voir la mention de la «structure des langages ordinaires $»($ p. 83 ; voir aussi p. 74-75). 
s'appliquer (ibid., p. 55). Par rapport à l'ancienne cosmologie aristotélicienne, celle de Galilée expliquait moins, à partir d'une théorie plus restreinte, et sans aucune évidence empirique nouvelle - les astres vus à la lunette astronomique requérant une nouvelle théorie optique, encore mal maîtrisée ou méconnue de Galilée lui-même ${ }^{4}$.

De tels constats empêchent d'attribuer la puissance des théories galiléennes à l'évidence d'une démarche nouvellement expérimentale. Ces théories dissimulaient en fait bien mal leurs écarts qualitatifs avec ladite expérience. Si la «discordance numérique » entre la théorie et les faits est bien connue des scientifiques, et mobilise comme témoin à charge la précision des instruments, les « échecs qualitatifs »le sont moins, les divergences constatées étant recouvertes par des hypothèses ad hocc'est-à-dire non testables, construites uniquement pour expliquer une incohérence. Ainsi de la théorie des couleurs chez Newton qui contredit ce que tout le monde peut voir dans un miroir et poussa le physicien à ajouter arbitrairement une hypothèse (ibid., p. 6o).

Par ces faits têtus, l'histoire des sciences nous enseigne qu'il n'y a pas de progrès de la science sans l'apparition de nouvelles théories heurtant les résultats empiriques les mieux établis. Pour la simple raison que les termes «faits 》 ou «observation » ne sont pas des objets précis, dégagés de toute composante subjective et distincts des théories reçues. Même l'impression sensorielle simple obéit à un certain « langage d'observation $\gg$ (p. 83) dont le noyau perceptif peut difficilement être isolé du concept qu'elle engage. L'écheveau d'interprétations constituant notre perception naturelle ne peut être débusqué que par un coup de force théorique qui vient introduire une «mesure de comparaison externe, incluant de nouvelles façons de relier concepts et percepts » (p.80). Seule une démarche « contre-inductive $»$ (p.26, 70 et passim), c'est-à-dire proposant des alternatives théoriques incompatibles avec les faits observés (p. 27), peut mettre à jour des faits nouveaux permettant de réfuter une précédente théorie.

Ce plaidoyer en faveur d'un anarchisme méthodologique peut éclairer le problème que les lectures foucaldiennes de 1980 nous posent au terme de cette deuxième partie. Ce n'est certainement pas un hasard si Foucault cite Feyerabend juste avant de se lancer dans une histoire des 
pratiques chrétiennes de vérité, alors même qu'il ne mentionnait que très rarement ses contemporains.

Devant les coupes franches opérées par le cours $D u$ gouvernement des vivants, non seulement dans la réalité historique du christianisme des premiers siècles (excluant toute considération sur la société romaine de l'époque, la sociologie des premières communautés chrétiennes, leur organisation politique), mais également dans le corpus des textes disponibles (excluant les traités dogmatiques et spirituels, la référence aux Écritures) et jusque dans ses argumentations (extrayant les mots par lexicalisation), il serait tentant de minorer l'objectif que se donne Foucault : il ne se serait en fait agi que d'étudier, non pas la totalité des pratiques chrétiennes, ni même la réalité de certaines pratiques baptisées « régime de vérité $\gg$, mais seulement leurs traces textuelles dans les débats pastoraux des premiers siècles. Et encore, le commentaire se serait-il borné à l'étude de syntagmes soigneusement choisis, renvoyant surtout aux préoccupations philosophiques du commentateur - plus précisément : les relations entre pouvoir et vérité. Exactement comme si la Généalogie de

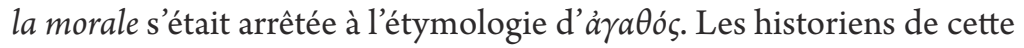
période, qui embrassent forcément un corpus et des perspectives plus larges, auront beau jeu de pointer les textes et les auteurs non cités, le détail oublié dans le tableau d'ensemble ou au contraire les dimensions essentielles occultées par le souci des petits faits (ainsi de la contemplation, de la foi)s. Foucault userait en 1980 d'une focale étonnamment courte. Peut-être. Mais encore faut-il se rappeler la duplicité optique des focales. Si elle déforme certains détails au premier plan, la focale courte ouvre également une profondeur de champ. La liberté et la radicalité des choix opérés par le cours de 1980 sont peut-être le prix à payer pour sortir d'une lecture commune du rapport entre l'Antiquité grécoromaine et l'Antiquité chrétienne - trop souvent interprété comme une

5 Les critiques érudites sont encore rares, les articles portant spécifiquement sur l'interprétation par Foucault du christianisme restant sporadiques. Dans un texte par ailleurs bienveillant, « Foucault, the Fathers and Sex », la patrologue Elizabeth A. Clark relativise les différences posées par Foucault entre l'Antiquité païenne et le christianisme, en montrant la complexité et la diversité des positions chrétiennes en présence : par exemple entre Clément d'Alexandrie et Cassien (Michel Foucault and Theology. The Politics of Religious Experience, J. Bernauer et J. Carrette éd., Aldershot, Ashgate, 2004, p. 50). Dans ces critiques érudites, il s'agit toujours de démultiplier les auteurs et les textes cités pour empêcher tout jugement tranché, toute véritable position. 
transition douce et sans doute jamais complètement achevée au plan des systèmes de pensée, l'originalité chrétienne ne se montrant que sur fond d'une reprise de la philosophie grecque ${ }^{6}$.

Car c'est bel et bien vers une interprétation globale du christianisme que se dirige Foucault dans la leçon du 27 février 1980, à partir d'une lecture en apparence limitée du Pasteur d'Hermas. Le ton est clairement affirmatif - rendant au christianisme son lieu propre : «c'est là [dans ce texte du II ${ }^{\mathrm{e}}$ siècle] que le christianisme a posé son problème ${ }^{7}$. Mais pour renverser les faits trop bien établis, interpréter autrement la spécificité historique du christianisme, il ne s'agit plus, comme dans le modèle stratégique des années 1970, de démultiplier au sein de vastes enquêtes historiques les connexions entre des pratiques hétérogènes. Le grain de l'analyse, désormais, se resserre.

Le premier pas en direction d'une nouvelle pensée du christianisme peut alors être légitimement interprété comme un pas en arrière, comme l'était la cosmologie de Galilée relue par Feyerabend : « des faits apparemment pertinents sont laissés de côté, de nouvelles données sont apportées par le recours à des liaisons ad hoc, le contenu empirique de la science est radicalement réduit $\gg^{8}$. Avec un clin d'œil à Feyerabend, Foucault présente ainsi sa méthode :

La position donc que je vous propose n'exclut pas l'anarchie, mais vous voyez qu'elle ne l'implique aucunement, qu'elle ne la recouvre pas et qu'elle ne s'identifie pas à elle [...]. Je vous dirais que ce que je vous propose serait plutôt une sorte d'(an)archéologie.9

Le jeu de mot n'est pas si innocent qu'il y paraît, car il a une signification double : le préfixe an- exclut l'archéologie, sans pour autant ouvrir à une simple anarchie, car l'anarchéologie conserve le statut de logos, de discours ordonné. Elle n'est pas anarchie, car elle accepte toujours un ả $\rho \chi \dot{\eta}-$ au sens le plus général de « principe »- qui est le texte

6 C'est l'idée d'un stoïcisme des premiers Pères : M. Spanneut, Le stoïcisme des Pères de l'Église. De Clément de Rome à Clément d'Alexandrie, Paris, Seuil, 1957 ; idée également d'un néoplatonisme chrétien qui lui succéda, par exemple chez Augustin : P. Courcelle, «Litiges sur la lecture du "Libri Platonicum" par saint Augustin », Augustiniana, t. IV, 1954, p. 225-239.

7 Leçon du 27 février 1980, nous soulignons.

8 P. feyerabend, Contre la méthode, déjà cité, p. 171.

9 Leçon du 30 janvier 1980. Nous reproduisons la manière dont Foucault écrit «(an) archéologie » dans le manuscrit du cours. 
en sa matérialité, étudié avec les outils habituels de la critique littéraire (établissement du texte, connaissance littéraire de la langue d'origine, recours à l'étymologie). Mais elle n'est plus archéologie, car elle ne suppose pas l'existence d'un ápxaíos, c'est-à-dire d'un niveau d'organisation originaire du discours, que celui-ci appartienne formellement à la langue (grammaire et syntaxe) ou à l'histoire (pratiques discursives). Si une part importante du cours peut ressembler à un commentaire traditionnel, qui se love sans difficulté autour des travaux de ses prédécesseurs historiens et philologues, à d'autres endroits, aux points nodaux de son argumentation, Foucault se donne une tout autre liberté : il s'empare du texte comme d'une succession de mots apposés plus que coordonnés par la syntaxe de la langue ou par les règles de formation sous-jacentes au discours. Telle la poésie homérique, le texte n'est plus qu'une succession d'éléments discrets, qui peuvent être extraits et mis en série en fonction du projet philosophique initial : une «histoire de la force du vrai ». Il nous reste alors à présenter ce que produit cette méthode comme lecture inédite du christianisme. 



\section{Une interprétation du christianisme} comme voie moyenne

Après ce long détour par la formation du concept et les méthodes de lecture afférentes, il nous faut revenir à la simplicité de ce que Foucault a dit et écrit au sujet de cet ensemble intriguant de faits nommé « christianisme », parfois déplié par le philosophe aux dimensions d'un Occident non moins problématique. De la préface à Folie et déraison (1961) au dernier cours au Collège de France Le courage de la vérité (1983-1984), nous n'assistons pas à l'affinement progressif d'une même approche conceptuelle, mais plutôt à une série d'essais et d'erreurs, qui ressemble à un brouillon sans cesse repris, sans cesse raturé.

Notre parcours désormais chronologique croisera bien entendu les partages que les deux premières parties de notre recherche ont mis en lumière, mais il tentera de rester au plus près de ce que nous dit Foucault, en mettant désormais de côté la question des méthodes et des sources. Le discours tantôt intrigué tantôt offusqué de l'historien de métier devant les recherches de Foucault peut souvent se révéler stérile : opposer à la subjectivité du philosophe l'objectivité de l'érudition méticuleuse, se contenter de pointer les oublis, les interprétations partielles, voire les anachronismes, risque d'effacer l'expérience de pensée que constitua pour lui la lecture des textes chrétiens - surtout quand aucune nouvelle intelligibilité de l'histoire n'est proposée à la place, sinon son inépuisable complexité.

Afin d'éviter les redites avec ce qui précède, nous avons fait le choix de nous concentrer sur les deux principales périodes où les références au 
christianisme sont à la fois nombreuses et resserrées autour des mêmes hypothèses : les études consacrées à la folie et à la littérature entre 1961 et 1966, et les cours au Collège de France de 1979-1980 à 1983-1984. Nous avions là deux ensembles à la fois plus cohérents et plus riches que les analyses finalement insatisfaisantes de la confession ou du pastorat. Hasards ou jeux de la mémoire, ces deux ensembles ont en commun de considérer le christianisme comme une totalité historique, dont l'apparition à un moment de l'histoire a fait rupture. Tout le travail d'interprétation de Michel Foucault sera précisément de tracer avec le plus de netteté possible cette ligne de partage, et d'abord, de lui assigner son lieu : dans quel domaine à la fois pratique et réflexifle christianisme a-t-il innové ? En quoi et dans quelle mesure s'est-il progressivement arraché à l'Antiquité gréco-romaine? 


\section{CHAPITRE SEPT \\ Le christianisme \\ comme Orient perdu}

\section{Tragédie de l'homme occidental}

Au commencement, il y a la tentative de dégager la «structure négative $\gg^{1}$ d'une expérience : celle de la folie à l'âge classique. Ni théorique ni pratique, cette expérience est qualifiée par Foucault de « fondamentale $\gg$, au sens où une culture $y$ engage et $y$ risque ses valeurs ${ }^{2}$. Elle n'est pas un fait massif se déployant progressivement dans l'histoire européenne, mais le produit du croisement entre des pratiques sociales, des savoirs et des productions artistiques initialement divergents. En même temps, cette expérience classique éclaire et unifie rétroactivement une multiplicité d'expériences hétérogènes, éloignées dans le temps et l'espace, entre lesquelles la folie auparavant se dispersait et se perdait :

L'homme européen depuis le fond du Moyen Âge a rapport à quelque chose qu'il appelle confusément : Folie, Démence, Déraison. ${ }^{3}$

1 Le terme «structure » apparait en $D E n^{\circ} 4$, « Préface », I, p. 192. C'est dans une conférence donnée au Japon en 1970 sur la folie que Foucault parle de «structure négative » à l'œuvre «dans toute société ou toute culture », à côté ou en deçà de ses phénomènes positifs ( $D E$ n $^{\circ} 83$, « La folie et la société », I, p. 996).

2 Histoire de la folie à l'âge classique, p. 228. Il s'agit de la réédition, sans sa préface d'origine, de l'ouvrage intitulé Folie et déraison. Histoire de la folie à l'âge classique, initialement paru chez Plon en 1961. Une version abrégée était entre-temps parue en collection «10/18 » en 1963. La préface d'origine a été reprise dans les Dits et écrits : $D E \mathrm{n}^{\circ} 4$, « Préface », I, p. 187-195.

$D E \mathrm{n}^{\circ} 4$, « Préface », I, p. 188. 
L'anthropologie, en particulier britannique (Alfred RadcliffeBrown ${ }^{4}$ ), utilisait depuis longtemps le terme de «structure » pour décrire les relations entre les individus en tant qu'elles sont institutionnellement contrôlées ou définies dans une société. Marqué par la phonologie de Roman Jakobson, Claude Lévi-Strauss en fit dès 1949 une propriété non consciente, intrinsèque à des ordres de faits bien délimités (parentés, alliances), à l'image des règles de formation des mots et des phrases dans la langue 5 . Se réclamant ouvertement en 1961 de cette analyse structurale, le projet de Foucault échappe pour cette raison à tout formalisme ou à la simple hypothèse heuristique, étant donné que la structure ainsi dégagée, reliant «notions, institutions, mesures juridiques et policières, concepts scientifiques $\gg^{6}$, ne prétend pas être la reprise du réel dans une forme abstraite qui lui serait étrangère, mais bien une propriété même du réel.

Une telle structure ne concerne que des $\ll$ ordres empiriques $\gg^{7}$ aux contours bien arrêtés, isolés par l'analyse à partir de leur unité fonctionnelle. La coexistence et l'articulation, dans un même espace géographique, de plusieurs ordres empiriques constituent alors un niveau supérieur dans la description que Foucault appelle une $\left\langle\right.$ culture $»^{8}$. Si l'auteur de Folie et déraison n'en donne à aucun moment une définition explicite, il est possible de déduire les réquisits de la notion : une culture a une « positivité $>^{9}$, c'est-à-dire qu'elle ne peut renvoyer à un inconscient collectif ou à une vérité cachée que l'analyste décrirait par tâtonnements ou analogies. Avant toute représentation mentale, elle désigne un ensemble d'ordres de

4 Foucault préfère pour sa part citer Franz Boas (1858-1942) comme « fondateur de la méthode structurale en ethnologie » $\left(D E \mathrm{n}^{\circ} 103\right.$, « Revenir à l'histoire », I, p. 1137). Radcliffe-Brown était anglais, Boas américain. Tous deux prirent leur distance avec l'ethnologie fonctionnaliste de Malinowski qui maintenait l'individu et ses besoins élémentaires au centre de ses analyses, dans une approche à la fois biologique et psychologique. À l'opposé, Radcliffe-Brown et Boas partent d'une société ou d'une culture comme totalité ou système de relations.

5 C. Lévi-Strauss, Les structures élémentaires de la parenté, $2^{\mathrm{e}}$ édition, Paris, Mouton, 1967. On se référera également au numéro spécial des Archives de philosophie : Dossier «Anthropologie structurale et philosophie : Lévi-Strauss », B. Karsenti éd., Archives de philosophie, t. 66, vol. 1, 2003, p. 5-88.

$6 D E \mathrm{n}^{\circ} 4$, « Préface $\gg$, I, p. 192.

7 Les mots et les choses, p. 11.

$8 D E n^{\circ} 4$, « Préface », I, p. 189 . Dans Les mots et les choses, Foucault évoque à nouveau «les codes fondamentaux d'une culture » (Les mots et les choses, p. 11). 
faits entre lesquels une continuité synchronique peut être établie, à la surface des institutions et des productions de l'esprit ${ }^{10}$.

Malgré cette synchronie qui permet de rassembler une diversité d'événements, nulle nécessité sous-jacente, nulle cause finale n'est jamais présupposée au niveau de la description historique. Relisons à ce sujet la phrase par laquelle est annoncé le grand renfermement à venir de l'âge classique. Dans le premier chapitre de Folie et déraison, contemplant les espaces désertés des léproseries à la fin du Moyen Âge, Foucault écrit :

La lèpre disparue, le lépreux effacé, ou presque, des mémoires, ces structures resteront. Dans les mêmes lieux souvent, les jeux de l'exclusion se retrouveront, étrangement semblables deux ou trois siècles plus tard. ${ }^{11}$

Pas d'unique moteur de l'histoire qui entraînerait dans un même mouvement la mémoire et les espaces, les subjectivités et les structures objectives. Ce que Foucault décrit dans la suite du chapitre comme « conscience » ou «expérience » de l'âge classique prend naissance dans un espace physique à la fois structuré et vide : vide de tout objet et vide de tout souvenir. Le lépreux n'est plus là, ni physiquement ni mentalement. Apparaît ici le principe, fondamental pour la suite des recherches archéologiques et généalogiques, selon lequel les ordres empiriques viennent s'articuler les uns aux autres de l'extérieur, par accident, et non à partir d'une nécessité plus ancienne ou plus profonde. L'éclatement affirmé du donné historique n'exclut pas cependant à d'autres endroits les grandes généralités et les secrètes continuités, comme nous allons le voir.

Comment le christianisme vient-il alors s'imprimer sur les pages de Folie et déraison? Nous nous limiterons à quelques remarques forcément incomplètes, compte tenu de l'étendue et de la densité de la thèse présentée par Foucault. La préface à l'édition de 1961 fera par contre l'objet d'une halte prolongée.

Au sein de la «structure » décrite, ce qui a trait à la religion ne constitue en apparence qu'une somme d'éléments parmi d'autres (institutionnels, discursifs, imaginaires), qui ne bénéficie dans la description d'aucune position privilégiée. Cette expérience de la folie, qui est « tout entière de 
l'histoire $\gg^{12}$, n'a ainsi que très épisodiquement à voir avec la théologie scolastique ou avec les pratiques chrétiennes de charité1 ${ }^{13}$. Tout au plus, la religion vaut-elle dans l'analyse de Foucault comme figure immémoriale d'un discours mystique qui a trouvé dans la société médiévale une certaine correspondance : le fou, rôdant aux confins des villes et des fêtes, envoyé de Dieu ou du Diable, détenteur à son insu d'un savoir inaccessible à la seule volonté humaine ${ }^{14}$. Cette errance tolérée, voire imposée, n'était pas sans lien en effet avec un enseignement traditionnel qui faisait du misérable le représentant secret du Christ et donnait à sa misère une «positivité mystique $\gg^{15}$. Mais cette proximité d'abord littéraire entre vérité de l'au-delà et folie ici-bas - rappelée en exergue du livre par les citations de Pascal et de Dostoïevski et, quelques pages plus loin, par la mention plus incertaine de l'épître aux Corinthiens ${ }^{16}$ - n'a eu que peu d'influence sur les événements en eux-mêmes : son inscription dans les pratiques sociales ne fut sans doute que fugace et, inversement, sa persistance nostalgique dans l'imaginaire européen n'entrava en rien l'institutionnalisation de l'enfermement des miséreux. Clé dramatique du livre de 1961, le fou naviguant ou errant aux abords des villes est d'abord une $\ll$ figure $\gg^{17}$ qui, tout en

$D E n^{\circ} 4, \ll$ Préface $»$, I, p. 192.

Par exemple, les maisons de charité fondées par saint Vincent de Paul apparaissent bien, mais seulement à titre de cheville dans la construction historique d'ensemble : elles participent de cette vaste assimilation du fou aux pauvres, miséreux et vagabonds qui ne travaillent pas et qu'il faut tenir à l'écart du vice (Histoire de la folie à l'âge classique, p. 106-107). Foucault se contente de relever «l'empreinte religieuse un peu plus marquée » de cette version catholique de l'enfermement par rapport aux workhouses anglaises : pour saint Vincent de Paul, il s'agit de « délivrer les pensionnaires d'un monde qui n'est pour leur faiblesse qu'une invitation au péché » (ibid., p. 107).

4 Le fou, le niais, le sot sont dépositaires de la vérité (p. 28-29). Le savoir du fou annonce le règne de Satan (p.38).

Ibid., p. 8o. Ce thème sera repris par Foucault dans son dernier cours au Collège de France, autour de la figure cynique, puis chrétienne, du « roi caché » (Le courage de la vérité, p. 263).

16 Histoire de la folie à l'âge classique, p. 51. «Je parle en fou l'étant plus que personne » n'est pas une citation littérale de l'épître aux Corinthiens, même dans la version latine de la Vulgate. Foucault a lu cette citation de l'apôtre chez Érasme (Ut insipiens, dico, plus ego, perinde quasi turpe sit uinci stultitia) qui la lui attribue. Plus précisément, Foucault a lu la traduction de l'Éloge de la folie par Pierre de Nolhac (Paris, Classiques Garnier, 1936), qu'il cite mot à mot. Dans le chapitre Lxiır de l'Éloge de la folie, Érasme donne en fait une très libre version de II Cor. 11, 21. La Bible de Jérusalem traduit de son côté : « Mais ce dont on se prévaut - c'est en insensé que je parle -, je puis m’en prévaloir, moi aussi. »

Au sujet de l'expérience de la folie à la Renaissance, annonçant le thème de la Nef des fous, Foucault écrit : «C'est cette présence, et quelques-unes de ses figures essentielles, 
conservant des liens avec le réel historique ${ }^{18}$, a pour fonction de rassembler les lambeaux d'une expérience révolue, dont seule subsiste encore la forme onirique. Le vocable latin figura a la même racine ( fig-) que les mots fingere (modeler), fictor (modeleur, sculpteur), figulus (potier). Le mot renvoie donc à une production artificielle, qui a cependant dans la matière une inscription physique. D'où les tensions internes à cette notion : la figure est à la fois une trace matérielle et une image ressemblante, une empreinte physique et une construction de l'esprit, un principe de vérité et un leurre. En témoigne la question que Foucault laisse en suspens du rapport de cause à effet entre le réel et l'imaginaire, entre l'apparition historique du rite d'embarquement et la rémanence à travers les siècles de figures similaires :

Est-ce ce rituel qui par ces valeurs est à l'origine de la longue parenté imaginaire qu'on peut suivre tout au long de la culture occidentale? Ou est-ce, inversement, cette parenté qui a, du fond des temps, appelé puis fixé le rite d'embarquement ?19

Ni cause d'un rite nouveau, ni effet d'un vieux rêve, la Nef des fous est en fait au croisement de deux histoires : une histoire immobile de l'imaginaire occidental, dont le commencement se perd dans le « fond des

qu'il faut maintenant rappeler d'une manière très hâtive » (Histoire de la folie à l'âge classique, p. 21, nous soulignons).

18 Foucault cite le tableau de Jérôme Bosch La Nef des fous, œuvre qui puise ses sources dans la littérature flamande du $\mathrm{Xv}^{\mathrm{e}}$ siècle (Histoire de la folie à l'âge classique, première partie, chap. I : «Stultifera navis », p. 15 et suiv.). Au-delà des représentations artistiques de cette « flotte de rêve », Foucault affirme bien : « ils ont existé, ces bateaux qui d'une ville à l'autre menaient leur cargaison insensée » (ibid., p. 22). Il étaye son propos sur les décrets d'expulsion des archives municipales (à partir d'une source secondaire : une histoire de la psychiatrie de 1912) et sur la pratique fort répandue des pèlerinages aux tombeaux des saints, en particulier pour guérir de la folie. Certains historiens ont cependant contesté la réalité historique de ces bateaux traversant les fleuves des Pays-Bas et de la Rhénanie : H. C. E. Midelfort, « Madness and civilization in early modern Europe : a reappraisal of Michel Foucault », After the Reformation. Essays in Honour of J. H. Hexter, B. C. Malament éd., Philadelphie, University of Pennsylvania Press, 1980, p. 247-265. Récemment, les travaux de Chris Philo, géographe et professeur à Glasgow, ont cependant corroboré la description foucaldienne du fou dans l'Europe médiévale, en particulier son statut de vagabond, son aura mystique et ses pèlerinages : C. Philo, A Geographical History of Institutional Provision for the Insane from Medieval Times to the 1860's in England and Wales. The Space Reserved for Insanity, Lewiston, Edwin Mellen Press, 2004. Nous empruntons ces références à l'article très complet et subtil de Colin Gordon : C. Gordon, «La réception de l'Histoire de la folie chez les historiens et les géographes : l'exemple anglo-saxon », Folie et justice : relire Foucault, P. Chevallier et T. Greacen éd., Toulouse, Érès, 2009, p. 23-35. Le débat reste donc ouvert. Histoire de la folie à l'âge classique, p. 26. 
temps » (ibid., p. 26) et se dénoue au XIX ${ }^{\mathrm{e}}$ siècle, et une histoire mouvementée des pratiques, où les ruptures sont au contraire parfaitement marquées. D'où la distinction qui peut se déduire du premier chapitre de Folie et déraison: en tant que symbole de la traversée d'un espace infini et incertain, la Nef des fous n'est qu' un motif imaginaire de plus dans une histoire à la fois longue et homogène, où Tristan et Iseut croise «l'âme nacelle » des mystiques et dont les derniers résidus se trouveraient du côté de l'hydrothérapie des psychiatres ${ }^{20}$. Cette histoire sans solution de continuité témoigne de l'alliance immémoriale, « dans le rêve de l'homme européen » (ibid., p. 26), des thèmes de l'eau et de la folie, qui permet à cette dernière de communiquer avec le reste du monde, à la faveur de son état fluide. En tant que bateau réel, la Nef cristallise au contraire, à la fin du Moyen Âge, une inquiétude nouvelle vis-à-vis de la folie, annonciatrice des grandes mutations et des enfermements à venir (p.28).

Dans cette configuration, l'appréhension positive de la folie par la mystique appartient clairement aux «motifs immémoriaux $\gg($ p. 28) du vieil imaginaire européen, cette longue histoire à l'origine et aux effets incertains. Cet imaginaire ne va cesser en fait de se décoller des pratiques réelles, qui vont venir progressivement s'appuyer sur un tout autre discours. Comme le fait remarquer Foucault, si la folie tient encore une place importante dans la littérature spirituelle du XVI ${ }^{\mathrm{e}}$ siècle, elle y perd déjà sa qualité de «puissance sourde » (p. 52), annonciatrice d'un «savoir interdit » (p.38). Folie est le monde comparé à la sagesse de Dieu. Folie est le mouvement de l'âme qui retourne à Lui, comparé aux réconforts sensibles. Mais à force d'être tout, la folie n'est plus rien : une simple question de mesure entre deux grandeurs - raison humaine / sagesse divine - qui s'appartiennent désormais dans un «cycle indéfini » (p.52). De son côté, l'institution ecclésiale scinde en deux son propre discours à l'âge classique, en distinguant désormais le

Pour ce survol de l'imaginaire européen, qui est ici d'abord un survol de l'histoire littéraire : ibid., p. 26-28. Au-delà des derniers feux du Moyen Âge, cette histoire se poursuit jusqu'au milieu du XIX ${ }^{\mathrm{e}}$ siècle, avant que le thème de la fumée (à travers l'opium) ne vienne remplacer le thème de l'eau $\left(D E \mathrm{n}^{\circ} 16\right.$, « L'eau et la folie », I, p. 299-300). En faisant des douches forcées du psychiatre Leuret la dernière manifestation de la représentation «aquatique » de la folie, l'article «L'eau et la folie » donne à cet imaginaire une fin plus précoce que dans la thèse de 1961. Pour Folie et déraison, cette «situation liminaire du fou », symbolisée par l'eau et la navigation, semble en effet demeurer « jusqu'à nos jours », au moins dans le «château de notre conscience » (Histoire de la folie à l'âge classique, p. 26). 
bon et le mauvais pauvre, ce dernier étant défini comme l'ennemi de l'ordre (ainsi du fainéant, de l'ivrogne, de l'impudique), là où le premier se montre en tout conforme et docile - la distinction n'empêchant pas l'Église de vouer les deux catégories de pauvres à un même enfermement (p. 86-89). C'est ce discours moral qui va accompagner le grand renfermement, lui tendant un miroir théologique approprié.

Il serait cependant réducteur de limiter l'importance historique du christianisme dans Folie et déraison à un simple relais discursif, finalement circonstanciel, du grand renfermement. Le rapport au christianisme dans la thèse inaugurale va en fait se jouer sur une scène beaucoup plus vaste, dans une tragédie où le personnage du fou n'apparaîtrait tout au plus qu'au deuxième ou au troisième acte. Cette scène élargie, c'est la préface de l'édition de 1961 qui la construit de toutes pièces.

L'expérience qui va se dessiner à l'âge classique comme rejet de la folie aux limites d'une raison désormais souveraine n'est en fait pour Foucault qu'un chapitre dans une «histoire des limites $»^{21}$ de notre culture - celle de l'homme européen. Et le jeune philosophe de citer dans sa préface deux autres exemples de limites, complétant le tracé de nos frontières occidentales : le rêve $\mathrm{e}^{22}$ et, surtout, les interdits sexuels. Or, chacune de ces limites imposées «signifie, en même temps, un partage originaire $\gg^{23}$. Ainsi, à la naissance de toute culture, sans exception, il y a un « déchirement qui est comme la naissance même de son histoire ${ }^{24}$; ou encore, pour reprendre les termes moins dramatiques des Mots et les choses : une manière de constituer des identités qui ordonnent les choses dans la figure du Même, renvoyant de ce fait tout autre ordonnancement à une altérité désormais incompréhensible.

$D E n^{\circ} 4, \ll$ Préface », I, p. 189, l'auteur souligne.

Ibid., p.190. L'importance lancinante du rêve dans notre culture avait fait l'objet en 1954 d'une longue étude historique de Foucault, à l'occasion de son introduction à la traduction française d'un ouvrage de Ludwig Binswanger : Traum und Existenz (DE $\mathrm{n}^{\circ} 1$, «Introduction $\left.\gg, I\right)$. Foucault est également marqué par l'œuvre inaugurale de Nietzsche, Naissance de la tragédie à partir de l'esprit de la musique (1872), qui accorde au rêve une place primordiale dans l'analyse de l'univers dionysiaque.

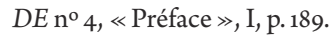

Ibid., p. 189. Foucault insistera sur ce point dans un article de 1964. Nul éden ne nous attend aux confins de l'Occident ou de l'histoire universelle : «Il n'y a pas une seule culture au monde où il soit permis de tout faire. Et on sait bien depuis longtemps que l'homme ne commence pas avec la liberté, mais avec la limite et la ligne de l'infranchissable $\gg\left(D E n^{\circ} 25, \ll\right.$ La folie, l'absence d'œuvre $\left.\gg, ~ I, ~ p .443\right)$. 
Malgré l'universalité de ces gestes de partage, celui que Foucault désigne par l'expression «l'homme européen $»^{25}$ garde un rapport singulier à la limite, à la fois acharné et inquiet, irrésolu en dépit de son acharnement. Dès 1961, ce rapport exacerbé à la limite est évoqué au sujet des interdits sexuels dans notre culture : pour les désigner, Foucault parle de ces « formes continuellement mouvantes et obstinées de la répression $\gg$ (ibid., p. 190, nous soulignons). Il ne s'agit pas seulement pour l'homme européen de partager mais aussi de rejeter. Et cette modalité particulière du rejet qu'est l'《exclusion sociale $\gg^{26}$ n'a pas attendu le grand renfermement ou le cogito de Descartes pour recouvrir en Occident le rapport à l'Autre. Dans Folie et déraison, Foucault fait remonter ce geste au Haut Moyen Âge, avec l'apparition des léproseries (ibid., p.15-19). Avec l'ouverture de l'Hôpital général, l'âge classique ne fera que conserver la forme de cette exclusion, tout en lui assignant un sens nouveau. Désormais, l'homme qui s'affirme au même moment citoyen du monde rejette loin de lui ce qui devient son contraire menaçant ou encore $\ll$ l'Extérieur ${ }^{27}$. Mais cette césure continue de le traverser de part en part dans la mesure même où il tente d'en faire une ligne claire et infranchissable qui passerait hors de lui. Le travail du négatif se révèle toujours dialectique, et le rapport à l'Autre devient spéculaire. Comme l'écrit Foucault dans un article de 1964 : «On dira non pas que nous avons été à distance de la folie, mais dans la distance de la folie $\gg^{28}$. Dès lors, cette proximité inquiétante ne peut que relancer sans cesse le geste d'exclusion qui l'avait paradoxalement produite.

L'homme européen, qui se constitue aux $\mathrm{XVI}^{\mathrm{e}}$ et $\mathrm{XVII}^{\mathrm{e}}$ siècles, ne serait donc pas seulement le produit d'une succession de partages, au même titre que n'importe quel indigena, mais également l'assomption du partage même, vécu sous l'unique mode du rejet, comme condition d'un certain règne de la vérité. Il faut donc relire la préface à Folie et déraison au-delà de la question particulière de la folie, comme une vaste épopée de l'histoire occidentale tout entière. La narration use en effet volontiers des procédés d'amplification et de dramatisation typiques de cette forme littéraire antique : 
Dans l'universalité de la ratio occidentale, il y a ce partage qu'est l'Orient : l'Orient, pensé comme l'origine, rêvé comme le point vertigineux d'où naissent les nostalgies et les promesses de retour $[\ldots]$ : nuit du commencement, en quoil'Occident s'est formé, mais dans laquelle il a tracé une ligne de partage, l'Orient est pour lui tout ce qu'il n'est pas, encore qu'il doive y chercher ce qu'est sa vérité primitive. ${ }^{29}$

Le partage historique originel est donc celui qui rejeta un monde loin d'un autre, alors même que l'un était né de l'autre. Reprenons le vocabulaire utilisé par la préface de Folie et déraison pour définir ces mondes. Les termes sont larges, volontiers surplombants : «la Raison occidentale $\gg($ p. 188), « la culture européenne $\gg($ p. 189), « la ratio occidentale $\gg(\text { p. 189 })^{30}$. À l'opposé de cette ratio définie comme essentiellement discriminante, apparaît un Orient relu par Foucault avec un unique maître et guide de lecture : Friedrich Nietzsche.

Rappelons quelques éléments de la leçon orientale du penseur prussien : union de la forme et de la force, de l'ordre et de la démesure, l'Orient est le nom propre qui désigne l'émergence dans l'histoire de la pensée d'une volonté d'accepter la totalité de l'être, défini comme pure apparence. Il s'agit, dans un même sursaut, de désirer et d'accepter les contradictions inhérentes au monde tel qu'il se donne à nous, sans chercher à les dépasser. À cette acceptation énergique s'oppose l'espoir ascétique d'accéder, par la négation de ce monde-ci, à un au-delà du sensible. Le premier mouvement est le mouvement tragique (Orient), le second est le mouvement dialectique (Occident). Dans la préface de 1961, Foucault se réfère explicitement à cette leçon nietzschéenne de Naissance de la tragédie à partir de l'esprit de la musique (1872), dont la puissance de monstration est affirmée :

Nietzsche ayant montré que la structure tragique à partir de laquelle se fait l'histoire du monde occidental n'est pas autre chose que le refus, l'oubli et la retombée silencieuse de la tragédie. [... ] L'étude qu'on va lire ne serait que la première, et la plus facile sans doute, de cette longue enquête, qui sous le soleil de la grande recherche nietzschéenne, voudrait confronter les dialectiques de l'histoire aux structures immobiles du tragique. ${ }^{31}$

$D E n^{\circ} 4$, « Préface $»$, I, p. 189-19o.

Il manquerait à cette série l'expression «Europe chrétienne »; elle se trouve dans un article de l'année suivante consacré à Hölderlin $\left(D E n^{\circ} 8\right.$, « Le "non" du père », I, p. 220). $D E n^{\circ} 4$, « Préface », I, p. 189-19o. On peut cependant relativiser la valeur historique de la grande thèse nietzschéenne. Le Dionysos de Nietzsche est en effet d'abord une reconstruction, même si celle-ci permit de libérer les études grecques d'une fixation exclusive 
La tragédie grecque exprime par son chant un vouloir vivre universel et primitif qui accepte le monde tel qu'il est, sans idéal, et se fond en lui dans une «union avec le cœur même du monde $\gg^{32}$, ou encore un «sentiment d'unité tout-puissant qui reconduit au sein même de la nature $\gg^{33}$. Cette unité n'est pas vécue sur le mode d'une synthèse heureuse et naïve, mais bien dans l'acception d'une contingence marquée par la douleur et la destruction. Même si Nietzsche met en avant la force positive et libératrice des pratiques orgiastiques, arrachant l'existence à un quotidien simplement subi, Dionysos reste un dieu de souffrance. Mais cette souffrance est justifiée par la vie elle-même et non par sa négation moralisatrice. À l'opposé, Platon se fait le critique de la réalité sensible, du miroitement trompeur de ses apparences, pour lui opposer une vérité au-delà des choses, que l'homme doit atteindre par l'ascèse et la pensée. À la suite de Platon, pour le jeune philologue de l'université de Bâle, l'Occident a refusé le chant tragique, ce chœur des satyres célébrant la nature et ses forces éternelles de génération, où l'individu pouvait s'oublier jusqu'à disparaître complètement.

Revenons maintenant au jeune Foucault. À ces termes généraux que sont l'Orient et l'Occident, il ne convient pas de donner des limites historiques trop précises, qu'elles soient géographiques ou temporelles. Il faut plutôt lire la préface à Folie et déraison en laissant jouer les oppositions qui en organisent la structure (Orient / Occident, tragique / dialectique), renvoyant à deux figures antithétiques primordiales. Au niveau le plus général de l'argumentation, ces deux figures assument le rôle de formes culturelles idéales ${ }^{34}$, tout à la fois expérience de vie et

sur l'harmonie apollinienne. La thèse de Nietzsche fut influencée par sa lecture de Plutarque, opposant déjà Dionysos à Apollon, mais également par celle d'Hérodote indiquant que le culte dionysiaque avait précédé le culte apollinien. Nietzsche, cependant, mélange beaucoup de sources diverses : des récits antiques ayant trait à cette divinité (les fragments qu'il nous reste des cultes à mystères de la Grèce antique : les mystères d'Éleusis ou l'orphisme) jusqu'aux références les plus contemporaines (la musique romantique du XIX ${ }^{e}$ siècle), en passant par ce que les philosophes et tragédiens grecs ont dit de Dionysos. Pour un exposé des sources nietzschéennes, voir A. Kremer-Marietti, «La démesure chez Nietzsche : Hybris ou Sublime? », Revue internationale de philosophie pénale et de criminologie de l'acte, $\mathrm{n}^{\mathrm{o}}$ 5-6, 1994, p. 69-84.

F. Nietzsche, La naissance de la tragédie suivi de Fragments posthumes (automne 1869-printemps 1872), M. Haar, P. Lacoue-Labarthe et J.-L. Nancy trad., Paris, Gallimard (Folio Essais), 1989, p. 44. Cette édition reprend celle de 1886.

Nous forgeons l'expression «formes idéales » en référence aux «idéaltypes » ou aux 
image du monde, qui tracent l'épure de deux millénaires d'histoire. Mais à ce niveau d'abstraction, qui n'est plus celui de l'analyse structurale initialement revendiquée, la pensée évite difficilement la tentation téléologique : à travers la référence constante à Nietzsche, non seulement dans la préface mais également dans le corps du texte ${ }^{35}$, les scansions de l'histoire européenne deviennent la répétition d'un grand partage originaire, plus ancien et plus essentiel que celui qui sépara un jour folie et déraison. Ce renvoi à l'origine s'effectue à travers un système de correspondances entre deux niveaux d'analyse, à la fois distincts et présentés en regard : un niveau idéal, indiqué dans la préface du livre, et un niveau concret, déplié ensuite par le corps du texte. Le premier décrit l'Occident comme totalité historique, le second décrit la folie comme péripétie de l’âge classique puis moderne. « Nietzsche » est le nom propre qui fait pivoter les descriptions l'une sur l'autre.

Mais la référence à Nietzsche joue ici un autre rôle, déterminant pour notre sujet : elle fait du christianisme une case vide, que les références extratextuelles invitent le lecteur à remplir. Tout l'art de la préface de 1961 consiste à indiquer les doublets Orient / Occident et tragique / dialectique sans jamais citer - mais non sans constamment faire signe vers lui - le doublet principal : Dionysos / Christ. En effet, si dans Naissance de la tragédie la rupture avec le chant du chœur antique s'accomplit avec Socrate et le platonisme, dans le reste de l'œuvre nietzschéenne, en particulier dans Le crépuscule des idoles (1888), le christianisme est rendu directement responsable de la pénétration de l'idéal platonicien jusque dans les couches populaires, infusant le refus du dionysiaque dans la culture tout entière ${ }^{36}$. Dès 1886 , un texte ajouté par Nietzsche à la nouvelle édition de Naissance la tragédie rompt bruyamment «le silence prudent et

«tableaux de pensée » chers à Max Weber, dont Foucault n'avait pas encore à cette époque fait la critique. Il s'agit bien en effet d'une construction de l'observateur à des fins de description et d'analyse.

$D E \mathrm{n}^{\circ} 4$, « Préface », I, p. 189 (deux références) et p. 190. Il réapparait ensuite dans le corps du texte : Histoire de la folie à l'âge classique, p. 47-48 et p. 661-663. Cette lecture téléologique de l'histoire transparaît chez Nietzsche dans des expressions comme « la grande fatalité du christianisme » (F. Nietzsche, Le crépuscule des idoles, suivi de Le cas Wagner, H. Albert trad., Paris, Flammarion, 1985, p. 173).

36 «Et combien il y a-t-il encore de traces de Platon dans l'idée de l'“Église", dans l'édification, le système, la pratique de l'Église ! 》 (F. Nietzsche, Le crépuscule des idoles, déjà cité, p. 173). 
hostile $\gg 37$ à l'égard du christianisme, pour désigner comme l'adversaire principal du livre la religion qui domina l'Occident. On nous objectera que l'invocation du penseur prussien au début de Folie et déraison tient plus de la figure emblématique, solaire mais aveuglante, que d'une thèse historique précise concernant le christianisme. À moins que l'opposition Dionysos / Christ ne soit finalement la plus à même de rendre compte de l'articulation entre les deux niveaux d'analyse que nous avons repérés ; comme la couture discrète qui fait tenir l'ensemble. Un indice permet de le laisser supposer : une brève mention du christianisme, à l'intérieur de l'argumentation proprement historique du livre, vient effectivement faire signe vers la préface et ses thèses nietzschéennes. Cette référence à l'horizon culturel chrétien est singulière, car elle n'a cette fois-ci rien à voir avec l'approche mystique de la folie, qui se perdait dans la nuit d'un commencement historique incertain, ni avec le rôle de justification morale offert par l'Église au grand renfermement. Il s'agit, plus profondément, du retour d'une pensée millénaire venue s'accomplir, à l'âge classique, dans la confiscation par la raison de l'expérience de la folie.

Dans le premier chapitre de Folie et déraison, Foucault évoque en effet l'influence platonicienne sur la spiritualité chrétienne du Xvi ${ }^{\mathrm{e}}$ siècle, dans cette conversion de l'âme qui se détourne du monde pour se tourner vers Dieu. Or, ce mouvement est justement celui qui conjure le péril d'une folie incontrôlable et infernale, en en faisant, comme nous l'avons $\mathrm{vu}$, une grandeur relative à la raison humaine et à la sagesse divine. Ainsi, pour Foucault, « sous l'influence majeure de la pensée chrétienne $3^{3}$, dans sa connivence discrètement évoquée avec le platonisme ${ }^{39}$, se trouve comblé l'abîme noir d'une folie annonciatrice chez Bosch ou Bruegel d'un réveil des forces primitives, ce « grand sabbat de la nature $\gg^{40}$ où le monde menaçait à chaque instant de s'effondrer. Il n'est pas étonnant

Dans ce texte («Essai d'autocritique »), Nietzsche parle du «silence prudent et hostile observé dans tout ce livre à l'égard du christianisme, - du christianisme considéré comme la variation sur le thème moral la plus extravagante qu'il ait été donné d'entendre jusqu'à ce jour à l'humanité » (F. Nietzsche, La naissance de la tragédie..., déjà cité, p.17). Histoire de la folie à l'âge classique, p. 52.

39 Foucault parle d'une « conversion, quasi platonicienne, vers le soleil de l'être » (ibid., p. 49). L'allusion peut sembler faible. Mais ce qu'il décrit dans ce passage est exactement le platonisme chrétien, celui par exemple d'un Grégoire de Nysse. Sur le platonisme chrétien, voir J. Daniélou, Platonisme et théologie mystique. Essai sur la doctrine spirituelle de saint Grégoire de Nysse, Paris, Aubier, 1944. Histoire de la folie à l'âge classique, p. 38. 
alors que Foucault, par-delà les siècles qui séparent le Moyen Âge de l'ère industrielle, établisse un lien entre l'œuvre de Nietzsche et ce qu'il nomme la «conscience tragique » de la folie, par opposition à «la conscience critique » qui apparaît comme constitutive de notre modernité (ibid., p. 45-47).

Dans ces jeunes années de recherche, Nietzsche est bien la figure en relief du texte foucaldien, comme le stéréotype de l'imprimeur. Il n'est donc pas étonnant qu'il hante à nouveau les articles que le philosophe consacre à la littérature au même moment : de l'hommage à Georges Bataille en 1963 à l'étude sur Maurice Blanchot en 1966. Dans ces textes que nous allons maintenant parcourir, ce qui a trait au christianisme s'inscrit pareillement dans le destin d'un Occident pensé avec Nietzsche comme perte et déni du tragique.

\section{Une expérience d'abord littéraire}

Pour Michel Foucault, plus frontalement encore que dans l'étude sur la folie, le choc avec ce que notre culture doit au christianisme se produit au contact de la littérature. Nous nous arrêterons principalement à cinq écrivains qui ont, chacun à leur manière, questionné cette dette de la littérature vis-à-vis de la religion chrétienne : Gustave Flaubert (18211880), Georges Bataille (1897-1962), Pierre Klossowski (1905-2001), Maurice Blanchot (1907-2003) et Roger Laporte (1925-2001).

Ce choc trouve sa forme réflexive dans une série d'études consacrées à ces auteurs, publiées dans les revues Critique et La Nouvelle Revue française entre 1963 et 1966. Commenter ces textes nous affronte à un certain nombre de difficultés : abordant la matière littéraire, ils prennent un plaisir évident à garder le secret et l'éclat de leur objet premier, sans en faire une analyse qui mettrait le texte à plat et lui appliquerait des critères ou des catégories étrangères. Participant à un débat sur le roman en 1963 à Cerisy-la-Salle, Foucault est le premier à rappeler que son titre de philosophe ne lui donne aucune compétence en la matière, si ce n'est une certaine « curiosité ». Derrière la fausse modestie et la politesse du moment, Foucault pose bien le problème et décrit l'effort qui sera le sien dans les articles que nous allons commenter. S’adressant à Philippe Sollers, Claude Ollier et quelques autres écrivains, il prévient : 
Je vais, toujours du fond de ma curiosité, essayer d'orienter le discours vers des choses beaucoup plus importantes, intérieures à vos œuvres, en laissant maintenant de côté la philosophie et les pourtours conceptuels. Ce sont les œuvres elles-mêmes qu'il faut interroger. ${ }^{41}$

Or, ces œuvres littéraires qui intéressent Foucault, seul le « problème religieux $\gg$ les rassemble, comme il le reconnaît dans un entretien de 1967. La reconnaissance de ce dénominateur commun à tous les auteurs qui l'ont passionné dans sa jeunesse lui permet de faire la suture entre deux de ses champs de prédilection : la littérature et l'ethnologie. Au-delà des indications biographiques qui pourraient étayer la proposition de Foucault - rappelons que Georges Bataille, Pierre Klossowski et Maurice Blanchot eurent tous trois une jeunesse catholique, et que les deux premiers se posèrent la question d'une vocation religieuse $\mathrm{e}^{42}-$ il convient de relever le lien établi entre le problème religieux et la « disparition du sujet » :

Pendant une longue période, il y a eu en moi une espèce de conflit mal résolu entre la passion pour Blanchot, Bataille et d'autre part, l'intérêt que je nourrissais pour certaines études positives, comme celles de Dumézil et de LéviStrauss, par exemple. Mais, au fond, ces deux orientations, dont l'unique dénominateur commun était peut-être constitué par le problème religieux, ont contribué dans une égale mesure à me conduire au thème de la disparition du sujet. ${ }^{43}$

Il faut ici préciser comment les deux thèmes s'articulent et, en particulier, dans quelle mesure la disparition du sujet serait concomitante en Occident de la disparition de Dieu. Les mots et les choses venaient en fait d'apporter la réponse, une année auparavant : à la fois Être infini et interlocuteur de notre finitude, le Dieu chrétien garantissait à l'être humain sa qualité de sujet libre et capable de vérité. Certes, l'homme du $\mathrm{XVII}^{\mathrm{e}}$ siècle était fragile, mais sa fragilité ne logeait pas tant dans les Chambéry, sur les recommandations d'une grande figure intellectuelle de la Compagnie de Jésus : le père Gaston Fessard (voir la chronologie détaillée de Pierre Klossowski dans A. Arnaud, Pierre Klossowski, Paris, Seuil, 1990, p.181-191). Il est intéressant de noter que le père Fessard (1897-1978), philosophe, spécialiste de Hegel et ami de Raymond Aron, avait suivi le séminaire d'Alexandre Kojève à l'École pratique des hautes études, comme Georges Bataille. Ce dernier, quant à lui, s'était converti au catholicisme à l'âge de vingt ans et avait même fait un séjour au Grand Séminaire de Saint-Flour (Cantal), avant de rompre définitivement avec la foi catholique et de proposer une a-théologie radicale. 
limites de son corps, de ses désirs ou de son langage, que dans son inadéquation première, métaphysique, à l'infini. Sa faiblesse essentielle n'était pas encore livrée à la seule empirie, mais toujours mesurée à plus grand que lui : une hauteur qui était à la fois interpellation et promesse. L'affrontement nouveau à la finitude, c'est la pensée des Lumières qui l'a rendu possible en vidant le ciel. Mais elle a ainsi précipité la chute de ce qu'elle croyait libérer et promouvoir : l'homme. De cette chute, les écrivains que nous allons lire sont les témoins.

\section{L'expérience de la transgression : Georges Bataille}

À travers sa fréquentation d'une certaine littérature - qu'il ressaisit sous la dénomination commune $\mathrm{d}^{\prime} \ll$ expérience limite ${ }^{44}$ - Foucault croise le christianisme au moment même où son évidence s'efface à la surface de notre culture. Lieu de prédilection où s'est affirmée et épanouie la subjectivité occidentale, la littérature a très vite pris la mesure de la mort qui se tramait, proclamée avec terreur par l'insensé du Gai savoir : «Dieu est mort ${ }^{45}$. Cette mort n'est ni un constat objectif ni l'opinion de quelques individus, mais, écrit Foucault, «l'espace désormais constant de notre expérience $\gg^{46}$. L'homonymie pourrait ici tromper. L'expérience à laquelle il est fait allusion n'a pas exactement le sens que revêtait l'expérience de la folie à l'âge classique. Elle ne doit pas non plus être entendue comme un vague sentiment existentiel de déréliction, même largement partagé par le siècle. Précisément, parce que la mort de Dieu n'est pas de l'ordre du constat. Le terme «expérience » doit être pris ici dans le sens précis et quasi scientifique des expériences que l'on fait : un protocole destiné à tester des hypothèses ${ }^{47}$. De la même manière, $l^{\prime}$ «expérience limite » est bien, chez Georges Bataille comme

Jusque dans le grand entretien de 1978 pour Il Contributo, Foucault conserve cette expression pour désigner les œuvres de Nietzsche, Blanchot et Bataille $\left(D E n^{\circ} 281\right.$, « Entretien avec Michel Foucault », II, p. 862).

F. Nietzsche, Le gai savoir, III, 125.

$46 D E n^{\circ} 13$, « Préface à la transgression », I, p. 263.

47 Ce sens du terme expérience s'accorde bien avec ce que Foucault dit de son travail en 1978 : «Je suis un expérimentateur en ce sens que j'écris pour me changer moi-même et ne plus penser la même chose qu'auparavant » $\left(D E \mathrm{n}^{\circ} 281\right.$, « Entretien avec Michel Foucault », II, p. 861). Dans le même entretien, Foucault distingue l'expérience du phénoménologue de l'expérience de Blanchot et de Bataille, alors qu'elles sont contemporaines - montrant bien qu'il ne s'agit pas ici de l'esprit d'une époque (p. 862). Voir aussi 
chez Maurice Blanchot, une décision personnelle ${ }^{48}$ qui consiste à expérimenter dans sa propre vie une certaine intensité la menant aux limites du vivable. Seule la fréquence et les similitudes de ces expériences personnelles, en particulier dans la littérature des années 1940-1960, permettent à Foucault d'y voir une modification en profondeur de notre culture, au sens que nous avons donné plus haut à ce terme. Dans ses jeunes années de recherche, Foucault évoque en effet à plusieurs reprises ces nœuds de notre histoire européenne qui commencent lentement à se dénouer : ceux de la folie, bien entendu, mais également, et sans doute d'abord, ceux d'un «monde chrétien des corps déchus et du péché $\gg 49$. Pour cette raison, la mort de Dieu est à la croisée de l'expérimentation personnelle et de l'événement culturel. Cette mort expérimentée est d'abord l'objet d'une interrogation inquiète sur notre actualité, sans jugement de valeur sur notre passé. Étudions alors la manière dont Foucault interprète cette mort dans l'œuvre de Georges Bataille.

Diplômé de l'École des chartes, romancier, essayiste, Georges Bataille a animé avec Roger Caillois le Collège de sociologie de 1937 à 1939. Bibliothécaire de métier, il est également fondateur de l'éphémère revue Acéphale, à laquelle ont collaboré Jean Wahl et Pierre Klossowski, ainsi que de la revue Critique en 1946, dont la reprise par les éditions de Minuit en 1950 assure finalement le succès. Proposant des comptes-rendus de publications dans tous les domaines de la création intellectuelle, ce mensuel retrouvait à sa manière l'intuition du Journal des savants du $\mathrm{XVII}^{\mathrm{e}}$ siècle, avec un idéal de partage des savoirs et de communications entre les disciplines.

Foucault découvre l'œuvre protéiforme de Bataille en 1953, conjointement à celles de Nietzsche et de Blanchot, après avoir vu au théâtre En attendant Godot de Samuel Beckett ${ }^{50}$. Dix ans plus tard, aux côtés de Roland Barthes, Maurice Blanchot, Jean Wahl et quelques autres, il rend hommage à l'auteur de L'expérience intérieure dans un numéro de Critique.

$D E n^{\circ} 23$, «Débat sur la poésie », I, p. $423:$ : l'expérience » y renvoie bien à des gestes volontaires, comme la contestation ou la transgression.

$D E n^{\circ} 281$, « Entretien avec Michel Foucault », II, p. 862.

$D E n^{\circ} 13$, «Préface à la transgression », I, p. 261.

$50 D E$ I, p. 21 et $D E n^{\circ} 343$, «Archéologie d'une passion », II, p. 1427. Dans un autre entretien, Foucault précise qu'il a découvert Nietzsche à travers la lecture de Bataille et de Blanchot $\left(D E \mathrm{n}^{\circ}\right.$ 281, «Entretien avec Michel Foucault », II, p. 867). 
Contre toute attente, l'article de Foucault déjoue la fascination facile, un rien esthète, que suscitent fréquemment les aspects les plus provocants de l'œuvre du chartiste : l'assomption par l'écriture de la cruauté et de l'érotisme - éros et thanatos. Pour Foucault, Bataille a tiré de la mort de Dieu la conséquence la plus immédiate et la plus extrême : non pas d'abord la permission absolue de tout faire, mais la reconnaissance que notre être est désormais sans extériorité. Par conséquent, il importe de ne pas réduire l'expérience de la transgression à une question morale ${ }^{51}$, mais de la placer résolument au cœur d'un questionnement ontologique. C'est sans doute là que se situe l'originalité de l'article de 1963, Foucault évitant habilement de faire de l'a-théologie de Bataille un simple christianisme inversé, comme s'y risquait son ami «le chrétien Pierre Klossowski $\gg^{52}$. La réduction de la transgression à une banale inversion des valeurs vient en effet lier le geste immoral à ce qu'il refuse, dans une inextricable dialectique ${ }^{53}$. Foucault délie au contraire les jeux

$D E n^{\circ} 13$, « Préface à la transgression », I, p. 265-266: « Cette existence si pure et si enchevêtrée, pour essayer de la penser [...], il faut la dégager de ses parentés louches avec l'éthique. La libérer de ce qui est le scandaleux et le subversif, c'est-à-dire de ce qui est animé par la puissance du négatif. »

52 L'expression est de Bataille, citée dans A. Pfersmann, « Klossowski avec Sade et Bataille », Traversées de Pierre Klossowski, L. Jenny et A. Pfersmann éd., Genève, Droz, 1999, p. 37. L'amitié entre Pierre Klossowski et Georges Bataille n'empêchait pas des prises de position contrastées, voire fortement opposées, en particulier sur la question religieuse. Klossowski rencontre Bataille en 1934 et les deux hommes commencent à se fréquenter autour du Collège de sociologie et de la revue Acéphale. En 1939, Klossowski donne au Collège une conférence sur « Sade et la Révolution ». Sa vision dialectique des rapports entre la mort de Dieu et la mort du Roi ne convainc pas Bataille, qui le dira dans La littérature et le mal (1957). Mais plus encore qu'une dialectique politique, c'est une dialectique religieuse que met en lumière Klossowski, traquant «l'antique système de la gnose manichéenne » dans «l'âme de ce grand seigneur libertin du siècle des lumières » (P. Klossowski, Sade mon prochain, précédé de Le philosophe scélérat, édition revue, complétée et corrigée, Paris, Seuil, 1967, p.140). Inversement, Klossowski n'accepte pas la fascination de Bataille pour le monde du péché et son potentiel d'expérimentations intellectuelles et existentielles.

Foucault ne pouvait ignorer cette interprétation, que son article s'ingénie à éviter. Cette interprétation trouve sa formulation la plus profonde dans un article de Pierre Klossowski publié en 1950 : «La messe de Georges Bataille. À propos de L'Abbé C. » (repris dans P. Klossowski, Un si funeste désir, Paris, Gallimard, 1963, p.121-132). Dans cet essai, Klossowski montre dans quelle mesure Bataille ne peut se passer de ce qu'il détruit : « [...] dans l'acte profanateur du nom le plus noble de l'existence, se révèle $s a$ présence. Ainsi Bataille, en dépit de son attitude athée, demeure solidaire de toute la structure cultuelle du christianisme $\gg($ p.127-128). Foucault avait certainement lu le recueil d'articles Un si funeste désir (déjà cité) où fut réédité cet essai l'année où il écrivait sur Bataille. C'est fort probablement ce livre que mentionne Daniel Defert dans sa 
dialectiques et tourne la transgression vers elle-même ou plutôt sur ellemême, pour en faire une expérience radicale de l'être qui en révèle la limite : «J'appelle expérience [écrit à ce sujet Bataille] un voyage au bout du possible de l'homme $\gg 54$.

Le sens de la transgression se voit dès lors renouvelé par rapport à l'acception commune du terme. Pour la religion chrétienne, rappelle en effet Foucault, l'infini n'est pas tant la négation ou la dénonciation de notre condition charnelle, que sa limite toujours mouvante, toujours poreuse, qui, dans un même mouvement, pose une distance et promet son abolition. Fine et fragile est la séparation entre Dieu et l'homme, entre l'infini et le fini, puisque ce qui borne notre être est aussi ce qui l'appelle au dépassement de ses bornes. À titre d'exemple, l'article mentionne en ouverture la continuité établie par la spiritualité chrétienne entre l'amour divin et l'amour sensible, entre éros et agapè :

Toute une mystique, toute une spiritualité le prouvent, qui ne savaient point diviser les formes continues du désir, de l'ivresse, de la pénétration, de l'extase et de l'épanchement qui défaille; tous ces mouvements, elles les sentaient se poursuivre, sans interruption ni limite, jusqu'au cœur d'un amour divin dont ils étaient le dernier évasement et la source en retour. ${ }^{55}$

S'il en va ainsi pour la mystique chrétienne, alors cette mort annoncée de Dieu, qui hante la littérature occidentale depuis la fin du XviII ${ }^{\mathrm{e}}$ siècle, ne marque pas tant la chute de toutes les limites - comme le laisserait entendre l'effroi moral d'un Mitia Karamazov - que leur relèvement. Parce que nulle contrainte ne nous est plus imposée du dehors par un Dieu ou un idéal, apparaissent dans toute leur dureté et leur naturalité les seules limites infranchissables : celles intrinsèques à notre finitude. Pour Foucault, dans l'article de 1963, ces limites sont principalement au nombre de trois : l'inconscient, la loi et le langage (p. 261). Sous ces trois mots, il est aisé de retrouver trois des sciences qui vont retenir l'attention du philosophe dans le dernier chapitre des Mots et les choses : psychanalyse, ethnologie et linguistique - même s'il donnera aux deux premières

chronologie, au mois d'août 1963 : «Lit Klossowski sur Nietzsche » (DE I, p. 32). Le recueil comprend en effet deux essais importants sur le penseur allemand. Foucault croise Klossowski un an plus tard au colloque de Royaumont consacré à Nietzsche et leurs relations deviennent régulières.

54 G. Bataille, L'expérience intérieure, Paris, Gallimard, 1978, p. 19.

$55 D E n^{\circ} 13$, « Préface à la transgression », I, p. 261, nous soulignons. 
un statut à part ${ }^{56}$. À elles trois, ces sciences défont la souveraineté du sujet conscient, agissant et parlant.

Que notre conscience soit déterminée par un inconscient dont elle ne pourra jamais faire le tour, que notre parole soit structurée par un langage toujours « déjà dit 》 dont le murmure originel nous est à jamais inaudible, cela va de soi. Il reste à cependant à expliquer la présence de la «loi » parmi les limites infranchissables de notre finitude. Même référée à un universel comme le tabou de l'inceste - évoqué à demi-mot par Foucault ${ }^{57}$ - il n'y a pas de raison qu'une telle loi ne puisse être transgressée dans les faits. Autant je ne puis espérer accéder à mon désir inconscient ni à l'être du langage, autant je peux coucher avec ma sœur. Certes. Mais la loi dégagée par l'ethnologue ou, pour employer le vocabulaire de Claude Lévi-Strauss, «l'exigence de la Règle comme Règle $\gg^{58}$, n'est pas forcément prescriptive, ni unilatéralement appliquée par tous les membres d'une société donnée. Pour l'anthropologie structurale, il y a «structure », déterminant un certain jeu social, à partir du moment où un fait est constaté avec suffisamment de régularité pour qu'il ne puisse être considéré comme le fruit du hasard ou des seuls choix individuels ${ }^{59}$. Qu'il le veuille ou non et quels que soient ses actes, l'individu vit dès lors dans une règle, qui ne prétend être rien d'autre, selon Claude LéviStrauss, qu'un fait objectif, sans aucune propriété qui lui soit extérieure.

Voici donc ce qui apparaît à l'homme moderne, une fois que la promesse d'un au-delà lui a été retirée : l'objectivité froide, impersonnelle et incontournable de la limite. Il y a de l'inconscient, il y a de la loi, il y a du langage. Pour Bataille, nulle autre expérience que celle de la sexualité, dans sa recherche constante d'un impossible dépassement, permet de révéler ces points de butée. D'où cette proposition paradoxale mais essentielle, formulée par Foucault : rien ne limite jamais le mouvement

Les mots et les choses, chap. $\mathrm{x}:$ « Les sciences humaines $\gg, \mathrm{p} \cdot 355-398$.

$D E n^{\circ} 13$, « Préface à la transgression », I, p. 261, où Foucault évoque «le seul contenu absolument universel de l'interdit ».

C. Lévi-Strauss, Les structures élémentaires de la parenté, déjà cité, p. 98.

Au sujet des mariages préférentiels, Claude Lévi-Strauss, dans la préface à la première édition de sa thèse, écrivait en 1949 : «Si j’avais le pouvoir de fixer la terminologie, j’appellerais préférentiel tout système où, à défaut d'une prescription clairement formulée, la proportion des mariages entre un certain type de parents réels ou classificatoires, que les membres du groupe le sachent ou l'ignorent, est plus élevée qu'il ne résulterait du hasard. Ce taux objectif reflète certaines propriétés structurales du système » (ibid., p. Ix). 
de la sexualité « parce qu'il est, depuis son origine et dans sa totalité, rencontre constante de la limite $\gg^{60}$. De la même manière que nous pouvons, à la limite, dire tout ce que nous voulons - nous ne le dirons jamais qu'à partir d'un langage qui nous précède et dont l'être nous échappe. C'est au cœur de ce paradoxe, que l'expérience de la transgression trouve son sens.

Mais ce sens doit être entièrement repensé hors de la sphère religieuse traditionnelle, puisqu'il s'opère désormais, comme le souligne Foucault, «dans un monde qui ne reconnaît plus de sens positif au sacré » (ibid., p. 262). Il ne s'agit donc plus de s'attaquer aux lois morales ou divines, mais aux limites même de notre être, sans promesse d'accéder à une quelconque positivité de l'autre côté, sans même croire que quelque chose nous attende sur l'autre rive. Si la spiritualité chrétienne risquait le franchissement, dans l'espoir d'embrasser définitivement l'Être divin, le geste de Bataille reste au contraire au bord de l'infranchissable, dans une contestation qui révèle simplement l'être conditionné que nous sommes. Il n'y a donc pas de troisième terme que délivrerait le travail du négatif; et la transgression, irréductible à la dialectique hégélienne, n'échappe pas à sa propre immanence. L'œuvre de Bataille mène ainsi à une « profanation sans objet, une profanation vide et repliée sur soi $\gg($ p. 262).

Mais pour quel résultat? La transgression serait un geste inutile si elle n'était, par une étrange propriété, une « expérience de l'impossible (l'impossible étant ce dont on fait l'expérience et ce qui la constitue) » (p. 263, l'auteur souligne). Arrêtons-nous un moment sur cette parenthèse sibylline de Foucault. Tenter de franchir la limite, c'est heurter cette zone de l'être qui ne peut à aucun moment devenir un objet pour ma conscience, pour mon action ou mon discours, car elle les constitue. C'est donc s'efforcer, dans un geste dérisoire car systématiquement voué à l'échec, de risquer une pensée, un acte ou une parole qui ne serait plus conditionné. Résultat vain en apparence. À moins qu'il ne s'agisse pas exactement d'accomplir l'impossible, mais seulement de se tenir au seuil de cet accomplissement, c'est-à-dire le plus loin possible des potentialités les plus communes de l'humain. La transgression ne cherche en fait pas tant à briser la ligne, qu'à s'enrouler autour d'elle, selon cette figure de la «vrille » convoquée à dessein par Foucault : 
La transgression n'est donc pas à la limite comme le noir est au blanc, le défendu au permis, l'extérieur à l'intérieur, l'exclu à l'espace protégé de la demeure. Elle lui est liée plutôt selon un rapport en vrille dont aucune effraction simple ne peut venir à bout. (p.265)

C'est ce lieu de coïncidence, dans un affrontement sans victoire, de l'individu à sa limite - donc à son être - que cherche à rejoindre l'auteur de L'expérience intérieure par l'écriture de l'innommable : la cruauté et la pornographie. Il ne s'agit pas tant ici de faire que de dire, puisque l'écriture a cette propriété de redoubler l'intensité de l'expérience, dans la conscience de l'événement passé : elle représente ce que Pierre Klossowski nomme, commentant Bataille, un «orgasme de l'esprit $\gg^{61}$.

Ce travail de contestation, Foucault le rapproche non sans audace de la philosophie critique de Kant, comme effort pour reconduire notre expérience sensible, et donc notre raison, à ses limites. Mais la reconduite kantienne à la frontière a sans doute manqué d'une audace que seul Bataille a eue : au lieu d'en rester à l'effort, propre à la pensée critique, de limitation des capacités de l'entendement, le chartiste s'est risqué à proposer une expérience de l'être même de la limite. Non pas seulement rester chez soi, et faire ce qui revient en propre au sujet humain, mais expérimenter quelque chose de ce qui fait que notre expérience est limitée. Or, cette possibilité, après quelques tergiversations, Kant l'avait refusée. Pouvait-il en être autrement ? En effet, faire une telle expérience, ce serait pour le sujet connaissant se donner la possibilité de contourner les conditions a priori incontournables qui nous permettent de saisir un objet. Pour reprendre les analyses ultérieures de Foucault dans Les mots et les choses, ce serait s'emparer, dans un même mouvement, de l'empirique et du transcendantal, alors que c'est leur distinction même qui permet la connaissance ${ }^{62}$. Mission impossible, au moins à l'horizon de la pensée critique. Une autre voie semble cependant praticable, si l'on

61 P. Klossowski, Un si funeste désir, déjà cité, p. 127, l'auteur souligne. Bataille évoque pourtant la faiblesse du dire sur le faire, la faillite du premier à exprimer l'intensité du second : «Absurdité de lire ce qui devrait déchirer à la limite de mourir » (G. Bataille, L'expérience intérieure, déjà cité, p.49); et la vie de Bataille renvoie à des expériences sensitives, bien réelles (drogues, yoga, débauches, etc.). Mais cette faiblesse initiale du dire va se renverser en une recherche d'autant plus intense de dire l'impossible. Dans Ma mère (1966, posthume et inachevé), le seul inceste s'accomplit finalement dans et par l'écrit : la lettre d'une mère à son fils.

62 Les mots et les choses, chap. IX, section IV : «L'empirique et le transcendantal », p. 329-333. 
accepte de délaisser la saisie objective pour risquer ce que Foucault nomme ailleurs une $\ll$ réflexion régressive ${ }^{6}{ }^{6}$. La régression en question consisterait à se diriger vers une réalité plus originelle que le soi pensant, parlant et agissant. C'est ici, dans l'analyse proposée par Foucault en 1963 du rapport entre Kant et Bataille, que l'activité littéraire est invitée à prendre le relais de l'activité proprement philosophique. Une telle réflexion pourrait en effet être accompagnée, voire suscitée, par un discours non exclusivement discursif ou démonstratif : méditation, journal, fragment, écriture romanesque ou parole poétique ${ }^{64}$. Au terme jamais atteint de cette réflexion, ce qui est donné dans l'expérience viendrait en sa limite coïncider avec ce qui rend l'expérience possible.

La possibilité d'une telle jointure sera longuement commentée par Foucault dans le chapitre IX des Mots et les choses «L'homme et ses doubles $\gg^{65}$, dans le prolongement direct de l'hommage à Bataille et dans une discussion serrée avec Kant et Heidegger. Nous aurons l'occasion d'y revenir. En attendant, l'article de 1963 évoque simplement ce lieu «de l'impossible» par la beauté de ses oxymores : «la transgression [écrit Foucault] porte la limite jusqu'à la limite de son être; elle la conduit à s'éveiller sur sa disparition imminente, à se retrouver dans ce qu'elle exclut $\gg{ }^{66}$. L'impossible ne signe plus alors l'échec ou la vacuité d'une expérience, mais l'effort pour se tenir au plus près de l'« "origine" $\gg^{67}$ à partir de laquelle un individu pense, parle et agit.

La proximité est alors évidente avec un discours mystique chrétien qui s'efforce depuis le $\mathrm{v}^{\mathrm{e}}$ siècle de repousser les frontières du langage pour dire le Mot au-dessus de tous les mots. Or, ce Mot n'est autre que le

63 Nous empruntons cette expression à la confrontation, dans la thèse complémentaire de Foucault, entre le Kant de l'Anthropologie et l'analyse existentiale de Heidegger : «Introduction à l'Anthropologie », dans E. Kant, Anthropologie du point de vue pragmatique, M. Foucault trad., Paris, Vrin, 2008, p. 77. Dans ce texte, l'expression est péjorative, dénonçant l'illusion heideggérienne d'un possible dépassement de Kant. Elle nous a semblé cependant tout à fait suggestive, d'autant plus que Heidegger demeure pour Foucault un interlocuteur irritant mais fortement présent.

64 Pour la multiplication des « niveaux différents de parole » chez Bataille, voir $D E \mathrm{n}^{\circ}{ }_{13}$, «Préface à la transgression », I, p. 271.

65 Voir en particulier Les mots et les choses, «Le recul et le retour de l'origine », chap. IX, section VI, p. 339-346.

$66 \mathrm{DE} \mathrm{n}^{\mathrm{o}} 13$, « Préface à la transgression », I, p. 265.

67 Ibid., p. 267. Les guillemets, insistants, sont de Foucault, en référence à Nietzsche. 
Verbe divin d'où viennent toute parole et toute vie, célébré par l'Évangile de Jean, comme Foucault le rappelle en citant la préface à Madame $E d w a r d a^{68}$. L'intense fréquentation des traités spirituels par Bataille reconduit alors la question que lui posait Pierre Klossowski par article interposé ${ }^{69}$ : si la transgression refait à sa manière le geste mystique, mais dans une expérience désormais intérieure et vide, pourquoi continuet-elle sans fin à héler le Dieu absent, à le désigner tout en le conjurant ? Ce besoin d'une transcendance, Klossowski l'avait lu pareillement chez Sade, avant même de le lire chez le chartiste athée ${ }^{70}$. Or, loin de passer cette question sous silence, l'article de Foucault insiste fortement sur l'horizon divin de la transgression. Chez Bataille, écrit-il, il s'agit moins d'une profanation sans Dieu, que d'une profanation «dans son absence rendue par là même scintillante $\gg^{71}$, dans cette nuit « où tous nos gestes s'adressent à cette absence $\gg$ (ibid., p. 262). Comme si la transgression ne pouvait survivre à la mort de Dieu et qu' il lui fallait sans cesse à nouveau «tuer Dieu qui n'existe pas» (p. 263, l'auteur souligne) - pour que la transgression vive de sa mort ou que le Dieu absent lui communique un peu de son énergie dissipée.

Telle est bien l'énigme la plus redoutable d'une œuvre qui ne l'est pas moins. Malgré la mise au point de Foucault, malgré son refus de faire de Bataille un nouveau prêtre de la profanation et de son œuvre une banale théologie inversée, un doute subsiste : le frisson de la transgression morale continuerait-il à parcourir l'échine d'une pensée dont la portée se voulait au départ strictement ontologique ? Le religieux serait-il invoqué pour réenchanter une expérience froide et sans esprit, dont Foucault décrit par ailleurs la «grande nervure squelettique » (ibid., p. 263) ?

Dans ses phrases à l'infinitif, qui déjouent la tentation de la prophétie ou de l'imprécation, l'hommage de Foucault à Bataille laisse entendre une vérité plus dérangeante, plus intrigante :

Tuer Dieu pour le ramener à ce néant qu'il est et pour manifester son existence au cour d'une lumière qui la fait flamboyer comme une présence (c'est l'extase). (p. 263, nous soulignons)

La mort de Dieu dit ainsi quelque chose de l'être même de Dieu;

68 Ibid., p. 264, où Foucault cite Bataille à propos du « mot qui dépasse les mots ».

69 P. Klossowski, «La messe de Georges Bataille. À propos de L’Abbé C. », déjà cité.

70 P. Klossowski, Sade mon prochain, déjà cité.

$71 D E \mathrm{n}^{\circ}{ }_{13}$, « Préface à la transgression », I, p. 262. 
elle le révèle sur la scène d'un déicide qui n'est blasphématoire qu'en apparence. Dans l'expérience limite, se manifeste l'existence de Dieu, négativement affirmée comme «néant qu'il est ». Cette phrase, proche de l'apophatisme, ne peut en fait se comprendre qu'avec la citation de Bataille qui la suit de près dans l'article. Il s'agit d'un extrait de la préface à Madame Edwarda, reprise dans L'érotisme (1957) :

Ce que le mysticisme n'a pu dire (au moment de le dire, il défaillait), l'érotisme le dit : Dieu n'est rien s'il n'est pas dépassement de Dieu dans tous les sens de l'être vulgaire, dans celui de l'horreur et de l'impureté; à la fin dans le sens de rien... (Cité par Foucault p. 264)

Analysons cette proposition : conscient des limites du langage humain et du caractère inconnaissable de son objet ultime, le mysticisme a tenté de parler de Dieu par paradoxe et négation; mais la peur du blasphème l'a empêché d'aller au bout de sa logique. Poussant à leur terme ces audaces seulement entrevues, car libre de toute inquiétude concernant l'issue fatale, Bataille assassine Dieu dans le langage en le mêlant au plus horrible et au plus vulgaire, pour finalement l'identifier à « rien ».

Mais en même temps, à travers la référence matricielle à la mystique, Bataille donne à ce rien un contenu bien précis : non pas une simple inexistence, mais le néant d'où émerge la finitude, la négativité à l'origine de toutes les positivités. À Dieu, il retire tout, sauf sa préséance dans l'ordre de l'être, comme le vérifient les expressions de Foucault pour désigner ce qui vient à briller dans la transgression : une « origine » (p. 267), une « ouverture $»($ p. 267). S'il reste dans la pensée moderne une trace de ce que Foucault nomme le «divin », elle est en ce lieu; non plus au-delà de nous, de l'autre côté de la limite, mais comme l'être même de la limite, au fondement de toutes les expériences accessibles à l'homme, comme au fondement de sa propre pensée : «En replaçant l'expérience du divin au cœur de la pensée, la philosophie depuis Nietzsche sait bien, ou devrait bien savoir, qu'elle interroge une origine sans positivité » (p.267). La vérité de l'au-delà est ainsi devenue, par un brusque mouvement de retour, une vérité de l'en deçà.

Mais ce mouvement de retour est-il pour autant un arrachement à la mystique? Rien n'est moins sûr. Il faut aussitôt ajouter qu'il appartient justement à la mystique de brouiller la distance qui sépare l'Être divin du néant, l'au-delà de l'homme de son en deçà. Au XIv'e siècle, Maître Eckhart n'hésite pas en effet à placer l'essence divine ou «déité » (Gottheit) audelà du Dieu trinitaire et révélé (Gott) et à identifier cette Déité à un néant 
(Niht), interdisant ainsi à toute autre qualification d'en faire un « quelque chose $\gg^{72}$. Le maître dominicain emmène son lecteur vers ce que des commentateurs récents n'ont pas hésité à nommer un «a-théisme mystique $\gg^{73}$. L'expérience de la transgression ne fait donc que porter à leur limite les contradictions qui structurent une certaine théologie, celle-là même qui a irrigué la pensée chrétienne, au moins jusqu'à l'âge classique et la condamnation du quiétisme par Bossuet. Un livre comme L'expérience intérieure en témoigne : Bataille n'écrit pas contre la mystique, il écrit souvent avec elle, parfois sans elle ; ne rejetant fermement que la théologie confessionnelle ${ }^{74}$. Mais rien ne peut sans doute ultimement distinguer l'en deçà de l'homme, dévoilé par la transgression, de l'au-delà mystique, dévoilé par le pur amour ${ }^{75}$, si ce n'est des considérations morales sur l'acte permettant le dévoilement. Dans les deux cas, ce qui est ainsi dévoilé est un être illimité, sans positivité et sans extériorité ${ }^{6}$. Pour s'en convaincre, il faut ici dire un mot, un seul, sur la mystique rhénane des essences.

72 L'audace des affirmations eckhartiennes se vérifie dans ce passage du sermon 71 : «Lorsque l'âme parvient dans l'Un et qu'elle entre là dans un limpide rejet d'elle-même, alors elle trouve Dieu comme dans un néant $\gg$ ( J. Eckhart, Du détachement et autres textes, G. Jarczyk et P.-J. Labarrière trad., Paris, Rivages poche, 1995, p. 96-97). Le sermon 52 décrit quant à lui l'état dans lequel l'âme se trouvait à l'origine, avant la création, c'est-àdire avant qu'elle soit jetée au milieu des choses : «Lorsque je me tenais dans ma cause première, je n'avais pas de Dieu, et j'étais alors cause de moi-même » (ibid., p. 76).

73 Titre de la «Reprise » finale de l'essai consacré à Eckhart par deux grands hégéliens : G. Jarczyk et P.-J. Labarrière, Maître Eckhart ou l'empreinte du désert, Paris, Albin Michel, 1995, p. 229-242.

74 Le premier chapitre de L'expérience intérieure s'intitule « Critique de la servitude dogmatique (et du mysticisme) $\gg$. Mais dès les premières lignes, Bataille établit un parallèle entre l'expérience intérieure et l'expérience mystique : «J'entends par expérience intérieure ce que d'habitude on nomme expérience mystique : les états d'extase, de ravissement, au moins d'émotion méditée » (G. Bataille, L'expérience intérieure, déjà cité, p. 15, l'auteur souligne). S'il émet une forte réserve vis-à-vis du mot «mystique », celle-ci porte uniquement sur la dimension confessionnelle de ce mouvement spirituel : contre cette dimension, il défend une «expérience nue, libre d'attaches, même d'origine, à quelque confession que ce soit. C'est pourquoi je n'aime pas le mot mystique » (ibid.). Dans la suite du livre, Bataille fait référence au Pseudo-Denys l'Aréopagite, à Jean de la Croix, à Angèle de Foligno et même, furtivement, à Maître Eckhart.

75 Pour une tradition mystique dont le représentant le plus éminent est Fénelon, il n'existe aucun rapport possible entre l'être imparfait qu'est l'homme et l'être infiniment parfait qu'est Dieu, si ce n'est un acte absolument désintéressé de « pur amour », sans calcul, sans motivation, sans récompense attendue : voir J. Le Brun, Le pur amour de Platon à Lacan, Paris, Seuil, 2002.

76 «a mort de Dieu ne nous restitue pas à un monde limité et positif $\gg\left(D E n^{\circ}{ }_{13}\right.$, « Préface à la transgression », I, p. 264). Au contraire, «la limite ouvre violemment sur l'illimité » (ibid., p. 265). Mais cet illimité n'est plus la propriété d'une Parole originaire 
Rappelons d'abord que les conditions historiques qui déterminent l'être de l'homme et le jettent contre les limites de l'inconscient, de la loi et du langage ne s'imposent pas à lui de l'extérieur, mais enveloppent de toute part sa vie empirique. Comme le diront Les mots et les choses : «le niveau de l'originaire est sans doute pour l'homme ce qui est le plus proche de lui $\gg{ }^{77}$, comme la trame silencieuse et secrète de ses jours. Mais vivre dans ces conditions, enveloppé par elles, c'est entretenir un rapport négatif à une origine qui se retire chaque fois que l'esprit tente de la saisir comme une chose. Cette origine qui affleure à la surface de l'homme, ce fond d'où lui viennent ses pensées, ses désirs et toutes ses capacités n'est pas sans rappeler le fond de l'âme dont parle Jean Tauler (1300-1361), disciple d'Eckhart et membre comme lui de l'Ordre des frères prêcheurs : ce Grund, qui est incréé, sans forme, sans distinction, sans modalité déterminée. 《Abîme » ou 《 gouffre $\gg^{78}$, le Grund est le lieu où l'âme peut s'unir à Dieu en essence. Par cette affirmation audacieuse, la mystique rhénane va ici beaucoup plus loin que la théologie d'un Thomas d'Aquin, pour qui l'union entre l'homme et Dieu ne peut se faire que par le biais de la mémoire, de l'intelligence ou de la volonté, c'est-à-dire toujours à distance. L'union des essences abolit au contraire toute extériorité entre Dieu et l'homme, puisque le Grund est toute simplicité, sans distinction possible. Il en va de même pour le monde empirique, qui n'est pas plus en dehors de Dieu que l'âme n'était à l'origine à l'extérieur du Grund. Comme l'écrit le théosophe Jakob Boehme (15751624), le monde visible

[... est une manifestation du monde intérieur et spirituel, provenant de la lumière éternelle et des ténèbres éternelles par une opération spirituelle. Il est objet de l'éternité, par lequel l'éternité s'est rendue visible. ${ }^{79}$

Pour Boehme, qui s'inscrit en cela dans la grande tradition des mystiques rhéno-flamands Eckhart ou Tauler, il n'y a pas de solution de continuité entre le créateur et la créature, mais un processus d'émanation ou

hors de notre langage, mais le langage lui-même dans son déploiement $\left(D E \mathrm{n}^{\circ}{ }_{14}\right.$, « Le langage à l'infini », I, p. 283).

77 Les mots et les choses, p. 341.

78 Sermon 44, J. Tauler, Sermons, E. Hugueny, G. Théry et M. A. L. Corin trad., J.-P. Jossua éd., Paris, Cerf, 1991.

79 J. Boehme, Confessions, A. Klimov préface, notes et commentaires, Paris, Fayard, 1973, p. 197 . 
de «flux » (signifié chez Maître Eckhart par le verbe fliezen), par lequel Dieu sort de lui-même tout en demeurant en lui-même. Créer ex-nihilo pour Boehme, ce n'est pas poser un quelque chose de différent, mais c'est créer Dieu à partir de lui-même, étant donné que Dieu est néant. Comme le résume d'ailleurs Maître Eckhart dans le sermon 28 : « Tout demeure l'Un qui jaillit en lui-même $\gg^{80}$. Nous voyons alors pourquoi la fermeté du postulat d'immanence, la mise en déroute systématique de l'extériorité divine, de tout ce qui dans la transgression évoquerait de près ou de loin un Dieu transcendant et créateur, n'évacuent pas pour Bataille la question théologique. Ce n'est pas parce qu'on ne sortira jamais du plan d'immanence que l'on en aura fini avec la question de Dieu.

Cette proximité, Foucault la connaît et la reconnaît en 1963. Mais par la suite, il va tenter de marquer plus fermement la césure entre l'expérience littéraire et l'expérience mystique, comme nous allons le voir dans les articles consacrés à Roger Laporte et à Maurice Blanchot. Au risque de taire des références théologiques évidentes chez l'un comme chez l'autre, le philosophe veillera à ne plus laisser affleurer de sous-entendus religieux dans ses commentaires. Dès l'hommage à Bataille, Foucault prend d'ailleurs soin de préciser que le retour du divin « au cœur de la pensée moderne $\gg^{{ }_{1}}$ est d'abord le retour nietzschéen à la Grèce, c'està-dire au dionysiaque - et non à la mystique chrétienne. À moins que...

\section{L'être du langage, l'être : Roger Laporte, Maurice Blanchot}

Pour Foucault, l'expérience fondamentale proposée par la littérature depuis la fin du XVIII ${ }^{\mathrm{e}}$ siècle est celle d'une disparition conjointe du sujet et de l'objet, à partir du moment où l'écrivain s'immerge dans le langage, au lieu de prétendre utiliser celui-ci comme un instrument. L'expérience souterraine vécue par la littérature est en quelque sorte la face sombre de l'avènement du sujet des Lumières, maître de lui-même et de son monde, par sa pensée et sa conscience.

À la place, ne resterait que le «sujet parlant $»^{82}$, expression que Foucault utilise par deux fois - en référence à Jacques $\operatorname{Lacan}^{83}$ - pour

80 J. Eckhart, Sermons, t. I : Sermons 1-3o, J. Ancelet-Hustache trad., Paris, Seuil, 1974, p. 234.

$81 D E n^{\circ} 13$, « Préface à la transgression », I, p. 267.

82 Ibid., p. 277 ; DE no 28 , «Le Mallarmé de J.-P. Richard », I, p. 460.

83 L'expression «sujet parlant » était devenue cependant courante en linguistique, à comp- 
désigner le mince espace de liberté et d'autonomie qui apparaît et disparaît à la surface d'un langage infini et impersonnel : sujet ni grammatical ni psychologique, contre lequel les sciences humaines et l'analyse littéraire viennent encore buter, faute d'une méthode appropriée pour le comprendre ${ }^{84}$. Quant au monde, il partage avec le langage ce point aveugle d'où nous arrivent les mots et les choses dans une distance insurmontable - affirmation audacieuse, puisqu'elle assimile l'être du langage à l'être, tout simplement : les mots et les choses se partageant la même origine silencieuse ${ }^{85}$. L'influence de Heidegger est notable dans ces réflexions sur ce que le penseur allemand, dans l'immédiat aprèsguerre, avait placé au cœur de sa pensée : die Sprache, événement par excellence de l'ouverture de l'être ${ }^{86}$.

Il nous faut ici faire une brève remarque lexicale : les traducteurs de Heidegger hésitent entre «langage » ou « parole » pour traduire le terme

ter de la distinction saussurienne entre langue et parole. Mentionnée furtivement dans les appendices aux $3^{\mathrm{e}}$ et $4^{\mathrm{e}}$ parties du Cours de linguistique général de Saussure, elle devint centrale chez l'un de ses élèves, le linguiste Charles Bally (C. Bally, Traité de stylistique

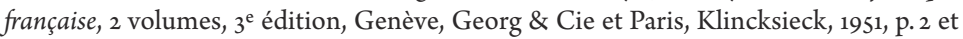
passim). Mais il s'agit alors seulement pour Bally de désigner une activité propre au sujet, qui exprime sa pensée dans une langue donnée. Jacques Lacan reprend cette expression en durcissant son sens : le sujet n'est que parole. Très tôt, Foucault lut et apprécia Lacan (voir $D E$ n $^{\circ}$ 299, « Lacan, le "libérateur" de la psychanalyse », II, p. 1024).

$84 D E n^{\circ} 28$, «Le Mallarmé de J.-P. Richard », I, p. 460-461.

$85 D E n^{\circ} 24$, «Le langage de l'espace », I, p. 436. Ce qui amène Foucault au colloque de Cerisy-la-Salle à prendre une position encore plus radicale : «Pleynet considère, et un certain nombre, je crois, d'entre nous, moi-même après tout, que la réalité n'existe pas, qu'il n'existe que le langage, et ce dont nous parlons, c'est du langage, nous parlons à l'intérieur du langage, etc. » $\left(D E \mathrm{n}^{\circ} 22\right.$, «Débat sur le roman $\left.\gg, \mathrm{I}, \mathrm{p} .408\right)$.

Selon la formule bien connue : «Le langage (Sprache) est la maison de l'Être (Sein)» (M. Heidegger, Lettre sur l'humanisme, R. Munier trad., Paris, Aubier-Montaigne, 1983, p. 27). Cette expression est commentée dans un texte rédigé par Heidegger en 1953-1954: M. Heidegger, «D'un entretien de la parole », Acheminement vers la parole, J. Beaufret, W. Brokmeier et F. Fédier trad., Paris, Gallimard, 1976, p. 87-140. Loin de clarifier le concept, cet entretien révèle combien la Sprache revêt chez Heidegger une signification délicate : elle est irréductible à l'aspect phénoménal de la parole (sons et syllabes) puisqu'elle est, dans la parole, ce qui lui permet de «donner à voir ». Mais «donner à voir » ne veut pas dire signifier quelque chose. Cette fonction de dévoilement, ou de « déploiement », est distincte de la stricte fonction du signe linguistique. Elle renvoie, de manière très obscure, à un dire originel : ce qui advient depuis le commencement dans la parole. Cependant, piégé par sa fascination pour la langue allemande, Heidegger attache à certains moments la parole à la structure de la langue : il lui arrive ainsi d'évoquer « la parole de la langue allemande » (ibid., p. 110), comme si cette langue avait une parole propre. 
allemand Sprache ${ }^{87}$. Il est intéressant de noter que l'on retrouve ce flottement chez Foucault ${ }^{88}$, même s'il ne cite pas directement Heidegger à l'appui de ses réflexions sur la littérature. Entre langage et parole, notons que c'est cependant le premier terme qui a la préférence du philosophe français, afin d'éviter sans doute la connotation saussurienne du deuxième.

Que faut-il alors entendre par langage, terme en apparence trop simple que Foucault se garde de définir ? Si une langue est un système particulier de signes prescrivant à ceux qui l'utilisent certaines règles contraignantes à des fins de communication, et si la parole, au sens que lui confère la linguistique de Saussure, est l'investissement personnel d'un sujet dans une langue, alors le langage, en deçà de la langue et de la parole, est le simple fait qu'il y a des énoncés, c'est-à-dire des assemblages de signes qui apparaissent dans l'histoire. La simplicité originelle de cette apparition, sa pure qualité de fait, seule la littérature permet de la capter, parce qu'elle peut interroger l'existence des signes avant leur réduction instrumentale aux besoins de communication d'une communauté. Pour Foucault, il ne s'agit donc pas d'étudier la faculté d'utiliser une langue pour transmettre des messages, mais d'approcher d'une réalité plus originelle, que seule une approche régressive peu appréhender. Ce que la sémiologie étudie depuis Ferdinand de Saussure n'est que la face sociale, extrinsèque, du langage. La science fondée par le linguiste suisse s'est en effet donnée pour but d'étudier « la vie des signes au sein de la vie sociale $\gg^{89}$; et c'est également dans cette direction que se sont orientés les travaux de Roland Barthes et d'Algirdas J. Greimas, afin de poser les bases d'une analyse scientifique de la signification ${ }^{90}$. Or, dans

87 La traduction par «langage » est le fait de Roger Munier : M. Heidegger, Lettre sur l'humanisme, déjà cité ; la traduction par «parole», aujourd'hui dominante, est le fait de François Fédier : M. Heidegger, Acheminement vers la parole, déjà cité. Cette dernière traduction ne résout pas tout à fait l'ambiguité du concept chez Heidegger, puisque l'expérience de l'essence de la Sprache reste liée à l'expérience de la langue maternelle ; surtout si elle est allemande ! Comme le dit Heidegger ( «D $\gg)$ à un Japonais ( $\ll \mathrm{J} »)$, au sujet des traductions par ce dernier de Hölderlin et Kleist, avec une emphase qui se passe de commentaires : «La parole de la langue allemande, dans tout son déploiement, a dû venir là sur vous comme un torrent » (M. Heidegger, «D'un entretien de la parole », déjà cité, p.110).

88 Par exemple dans l'article sur Blanchot qui entremêle constamment « parole » et «langage $\gg\left(D E n^{\circ} 38\right.$, «La pensée du dehors $»$, I, p. 553 et suiv. $)$.

89 F. de Saussure, Cours de linguistique générale, C. Bally, A. Sechehaye, A. Riedlinger éd., Paris, Payot, 1916, p. 33.

Avant leur éloignement temporaire à partir de 1964, Foucault fréquentait Roland Barthes, 
ses articles consacrés à Roger Laporte et Maurice Blanchot, comme dans ceux consacrés à Alain Robbe-Grillet, à aucun moment Foucault ne fait droit à la dimension conventionnelle, voire contractuelle du langage ${ }^{91}$. Un mot est lâché, qui pose la distance avec les approches développées au même moment par les sciences du langage : les œuvres littéraires sont mutiques $^{92}$. Elles n'ont rien de particulier à transmettre, nulle signification à dévoiler qui leur soit extérieure.

Dans le $\mathrm{xx}^{\mathrm{e}}$ siècle français, Michel Foucault identifie Georges Bataille et Maurice Blanchot comme figures tutélaires de ce nouveau rapport à la littérature. Si les deux hommes gardent leur farouche indépendance et n'adoubent nul disciple, Foucault les rapproche de quelques-uns de leurs cadets, en particulier le groupe un rien hétérogène d'écrivains rassemblés autour de la revue fondée par Philippe Sollers et Jean-Edern Hallier : Tel Quel. Le premier numéro sort en mars 1960, avec une épigraphe de Nietzsche en exergue : «Je veux le monde et le veux TEL QUEL, et le veux encore, le veux éternellement $[\ldots] \gg 93$. Ouvertement anti-sartrienne, la revue entend libérer la littérature des ornières de l'engagement politique pour la rendre à elle-même. Contre la littérature psychologique en premier lieu, contre la littérature de la représentation par la suite, il s'agit, comme l'explique Foucault, de promouvoir une « esthétique du langage, intérieure au langage ${ }^{94}$. À ses débuts et jusqu'en 1964, la revue apporte ainsi son soutien au Nouveau Roman et à Alain Robbe-Grillet, alors édité par les éditions de Minuit - maison

qui lui-même avait fait la connaissance d'Algirdas Greimas à Alexandrie quelques années auparavant. C'est sans doute autant par ses discussions avec Barthes, inaugurées en 1955, que par la lecture d'œuvres précises que Foucault croise la sémiologie, alors en pleine refondation dans le champ universitaire français. $\mathrm{Si}$, en France, le premier grand ouvrage théorique de Greimas ne paraît qu'en 1966 (A. J. Greimas, Sémantique structurale : recherche et méthode, Paris, Larousse, 1966), les premiers articles de Tzvetan Todorov ou encore les travaux de Vladimir Propp sont accessibles par l'intermédiaire de la revue Communications, fondée par Barthes en 1961.

91 À plusieurs reprises, Foucault prend ses distances avec la tentative de dégager les « structures » qui président à la «signification »: $D E \mathrm{n}^{\circ} 25$, «La folie, l'absence d'œuvre », I, p. $447 ; D E n^{\circ} 22$, «Débat sur le roman », I, p. 398-399. Lors du débat à Cerisy-la-Salle, c'est Jean-Pierre Faye qui relève l'écart entre Roland Barthes, théoricien du signe, et Alain RobbeGrillet, qui neutralise au contraire le signe dans son univers romanesque (ibid., p. 399).

92 Si les œuvres littéraires peuvent former un réseau de correspondances, celui-ci « est bien pour chacune d'elles son propre mutisme $\gg\left(D E \mathrm{n}^{\circ} 17\right.$, « Distance, aspect, origine $\left.\gg, \mathrm{I}, \mathrm{p} .306\right)$.

93 Cité dans P. Forest, Histoire de Tel Quel, 1960-1982, Paris, Seuil, 1995, p. 51.

$94 D E \mathrm{n}^{\circ} 22$, « Débat sur le roman », I, p. 415. 
hébergeant par ailleurs depuis 1950 la revue Critique. À l'occasion d'un colloque à Cerisy-la-Salle en 1963, qu'il préside à l'invitation de Philippe Sollers ${ }^{95}$, Foucault est le premier à ressaisir la cohérence du projet littéraire de Tel Quel et des écrivains qui gravitent autour (Philippe Sollers, Claude Ollier, Marcelin Pleynet, etc.). Mieux, il leur offre une tradition et une histoire : celle de la littérature occidentale depuis Sade.

Pour Foucault en effet, malgré les écarts et les tensions perceptibles entre des auteurs appartenant à des générations et des cultures différentes, l'expérience littéraire qui les traverse est une. De la même manière qu'il ne dit mot de l'écart idéologique entre Pierre Klossowski et Georges Bataille, il fait de Georges Bataille et de Maurice Blanchot une paire inséparable - presque gémellaire sous sa plume - et la met en regard d'une génération d'écrivains supposée homogène ${ }^{96}$. Certes, le philosophe repère discrètement les points de tensions et les écarts possibles, en particulier entre Sollers et Robbe-Grillet, un an avant leur divorce idéologique ${ }^{97}$. Mais ces décalages sont une condition pour décrire entre des œuvres qui conservent leur irréductible singularité, voire leur autisme, «l'étendue d'un réseau $\gg^{98}$. Ce réseau, dessiné en deçà des prises de position personnelles et des contextes historiques, est celui d'une littérature uniquement référée à elle-même : une littérature qui interroge «l'être de la littérature occidentale (ibid., p.306), contre ce que le philosophe appelle en 1963 la « littérature de la signification $\gg^{99}$, celle qui cherche à dégager le sens du monde et de l'homme.

À l'occasion de cette décade de Cerisy-la-Salle, une première radicalisation s'opère dans l'approche de la littérature par Foucault, qui touche de près le rapport aux textes spirituels. La question de «l'être de littérature » avait déjà été posée dans l’article consacré à Georges Bataille,

Ibid., p. 366-418; $D E \mathrm{n}^{\circ} 23$, « Débat sur la poésie », I, p. 418-434. Philippe Sollers avait lu avec enthousiasme, dès sa publication, Folie et déraison. Sur les relations entre Foucault et Tel Quel, voir P. Forest, Histoire de Tel Quel, 1960-1982, déjà cité, p.190-194. $D E n^{\circ} 22$, «Débat sur le roman », I, p. 367. L’article consacré à Robbe-Grillet est encore plus poreux : invoquant Klossowski, Don Quichotte, Le Château de Kafka, Sade, Mallarmé, Bataille ( $D E \mathrm{n}^{\circ}{ }_{17}$, « Distance, aspect, origine $»$, I, p. 300-313).

97 «On pourrait dire que si, chez Robbe-Grillet, les choses s'entêtent et s'obstinent, chez Sollers elles se simulent $\gg$ (ibid., p. 303). Par ailleurs, Sollers redonne un sens à la métaphore honnie par Robbe-Grillet ( $D E \mathrm{n}^{\circ} 22$, « Débat sur le roman $\left.», \mathrm{I}, \mathrm{p} .401\right)$.

$98 D E \mathrm{n}^{\circ} 17$, « Distance, aspect, origine », I, p. 306, l'auteur souligne.

$99 D E n^{\circ} 22$, «Débat sur le roman », I, p. 398. L'expression vise le manifeste sartrien pour une littérature engagée : J.-P. Sartre, Qu'est-ce que la littérature ? Paris, Gallimard, 1948. 
mais elle l'avait été sous la forme plus large et plus hospitalière de «l'être du langage $\gg^{100}$. Signe de cette hospitalité, Foucault posait cette question dans un apophatisme assumé, qui gardait une proximité avec le discours mystique jusque dans son style propre ${ }^{101}$. À cette époque, le philosophe n'avait pas encore formé ce réseau serré de l'expérience littéraire, intégrant comme nous l'avons vu des œuvres très variées, à condition qu'elles émargent à une même « esthétique du langage ${ }^{102}$. Or, dans les articles et interventions qui suivent de près l'hommage à Bataille jusqu'à la fin de l'année 1963, une nette inflexion apparaît : il s'agit d'écarter fermement toute autre manière d'écrire et de parler qui ne saurait éviter de personnaliser l'être du langage (écriture mystique) ou de lui reconnaître ne serait-ce qu'une simple positivité (sciences du langage). L'expérience littéraire ne s'ouvre qu'aux œuvres fermées sur elles-mêmes. C'est dans ce contexte, que Foucault va lire et étudier deux romanciers, dans leur proximité apparente et leur distance réelle avec le langage mystique.

La Veille (1963) est le premier roman de Roger Laporte, publié dans la collection exploratoire « Le chemin » de Gallimard, avec les encouragements de son ami Maurice Blanchot ${ }^{103}$. De taille modeste, le livre s'ouvre sur ces simples mots : «Il a disparu $\gg^{104}$. Pour Foucault, le sobre constat annonce à la fois ce que le roman raconte et ce qu' il montre de sa propre activité. Désormais, ce qui rend possible la littérature a perdu son évidence aussi bien subjective qu'objective : monde extérieur ou homme intérieur, l'origine a disparu.

Pour rejoindre cette origine, il ne sert à rien de mettre à jour les struc-

$100 \mathrm{DE} \mathrm{n}{ }^{\circ} 13$, « Préface à la transgression », I, p. 269.

101 Dans l'article consacré à Bataille, Foucault se plait lui-même à former des oxymores, caractéristiques du langage mystique, telle l'«absence rendue par là même scintillante $[\mathrm{du}$ sacré] » (p. 262 »). L'oxymore, écrit l'historien de la mystique Michel de Certeau, « ouvre le vide d'un innommable, il pointe une absence de correspondance entre les mots et les choses » (M. de Certeau, La fable mystique, $x v t^{e}-x v u^{e}$ siècle, Paris, Gallimard, 1987, p. 199).

$102 D E n^{\circ} 22$, « Débat sur le roman », I, p. 415.

103 Philosophe d'origine lyonnaise, Roger Laporte fréquentait Maurice Blanchot depuis le début des années 1950; il devint l'un de ses plus proches amis. Un numéro spécial de la revue Le Matricule des anges lui a été consacré, incluant un long entretien abordant son rapport à la fois intime et athée à la religion : T. Guichar, «Roger Laporte. L'épreuve par neuf », suivi d'un entretien avec Roger Laporte, Le Matricule des anges, $\mathrm{n}^{\circ} 32$, septembreoctobre 2000, p. 14-23.

104 Cité par Foucault dans $D E n^{\circ} 15$, «Guetter le jour qui vient», I, p. 293, c'est Roger Laporte qui souligne. 
tures qui rendent le langage signifiant, puisque l'accès à la signification ne suffit pas à rendre compte de l'existence du langage, à expliquer sa raison d'être. Ce que la littérature révèle - parfois dans sa proximité avec la folie - est un langage qui ne se définit plus par ce qu'il dit ${ }^{105}$. À rebours, le signe qui signifie sort le langage de lui-même pour le mettre en relation avec une vie sociale où se trouvent des significations partagées, liant des éléments phoniques à des éléments psychiques. Tel se présente bien le langage quotidien, mais également la littérature humaniste de la signification. Il revient au contraire à certaines œuvres littéraires singulières de dérouler le langage pour lui-même, afin de mettre à plat son « être brut $\gg^{106}$, cet $\ll$ espace vide et plein à la fois $\gg^{107}$ qui tout à la fois soutient sa possibilité et lui dérobe la maîtrise de ses moyens d'expression. Les métaphores spatiales («espace $\gg)$ et matérielles («brut ») sont choisies à dessein par Foucault : elles renvoient à l'extériorité du monde et des choses, désignant ainsi ce qui est à la fois discontinu et homogène. L'essence de l'espace est en effet d'être partes extra partes - contrairement à la conscience qui est continue. L'être révélé par l'expérience littéraire, celui à la surface duquel scintillent les objets de la perception et les paroles qui les nomment, vient d'un dehors qui n'a avec nos facultés - conscience, mémoire, volonté - aucune affinité. De telle manière que sa venue n'opère aucune réconciliation : que ce soit entre le monde et le signe dans la signification (objet de la sémiotique) ou entre le monde et le sens dans la conscience (objet de la phénoménologie) ${ }^{108}$.

S'il y a extériorité radicale de l'être, il y a donc un écart qui rend possible une attente, peut-être un espoir. Roger Laporte le laisse entendre à la toute fin du roman, en des termes qui évoquent l'eschatologie biblique $^{109}$; mais c'est justement cette dimension religieuse que tait le commentaire de Foucault. Pour le philosophe, l'écart indiqué par $\mathrm{La}$ Veille ne vient pas distinguer le sujet parlant (Je) de l'objet dont il parle (le mystérieux «Il »), puisque le sujet et l'objet émergent furtivement de

$105 D E \mathrm{n}^{\circ} 25$, «La folie, l'absence d'œuvre », I, p. 447.

$106 D E \mathrm{n}^{\circ} 38$, «La pensée du dehors », I, p. 547.

$107 D E n^{\circ} 22$, « Débat sur le roman $\gg$, I, p. 368 .

$108 D E n^{\circ} 24$, «L Le langage de l'espace », I, p. 435-436.

109 Avant de se refermer sur le thème d'une possible «offrande » à venir, le roman de Laporte pose en effet ces questions : «Va-t-il m'introduire dans mon propre matin? Un jour la Fête aura-t-elle lieu ? » (R. Laporte, La Veille, déjà cité, p. 126). 
la même origine ${ }^{110}$. Cet écart se loge au contraire dans le langage même, quand celui-ci cesse d'être un discours adressé à quelqu'un et parlant de quelque chose, et se prend pour son propre objet, se dédouble par cette prise et découvre en son sein un espace inédit qui n'est ni l'ancienne $p s y$ ché ni le monde avant toute nomination, mais un « vide qui circule entre ses mots $»^{111}$, un « murmure » (ibid., p. 553) ininterrompu. Mise à nu des possibilités et des limites du langage, la littérature vient alors coïncider avec la «pensée », dans une intuition à nouveau heideggérienne ${ }^{112}$. En effet, s'il n'y a plus de sujet souverain en retrait des mots et des choses, définissant leur juste relation, quel lieu reste-t-il en propre à la pensée si ce n'est ce lieu qui est la condition même de toute expérience?

Ce qui est attendu par la littérature, et qui peut-être ne viendra jamais, c'est bien la révélation de cet être devenu «il $\gg$ chez Roger Laporte, ou «le compagnon $»$ chez Maurice Blanchot ${ }^{113}$. Cet $\ll I l$ sans visage et sans regard $\gg($ ibid., p. 564) n'est pas une personne, n'est pas un principe. Avec ou sans majuscule, la force évocatrice du pronom demeure, volontairement ambiguë : «Sans doute, l'écriture a sans cesse affaire à lui; il la surplombe et la mine; $i l$ en est le don, mais aussi bien la force qui le dérobe ${ }^{114}$. Retour de l'élément mystique ? Non, car si le commentaire continue de dialoguer avec la spiritualité chrétienne, il affirme désormais la différence - même si la route n'est pas définitivement barrée :

Il faut lire le texte de Roger Laporte en laissant de côté, au moins pour un temps, ces guetteurs et ces veilles où la spiritualité occidentale a si souvent trouvé ses ressources métaphoriques. ${ }^{115}$

Dans l'article sur Blanchot, Foucault évoque cette ligne étroite qui « sépare le Je parlant du Il qu'il est en son être parlé » (DE no 38 , «La pensée du dehors », I, p. 564-565). $D E n^{\circ} 38$, «La pensée du dehors $»$, I, p. 553 .

Citons la question de Michel Foucault à Philippe Sollers en $1963: \ll[\ldots]$ est-ce qu'au fond, pour vous, l'œuvre, le livre, ce n'est pas cette trajectoire dans le volume du langage qui le dédouble et fait apparaitre à l'intérieur de ce langage un espace propre, un espace vide et plein à la fois, qui est celui de la pensée ? $\left(D E n^{\circ} 22\right.$, «Débat sur le roman », I, p. 368). À rapprocher ici directement des rapports entre das Denken, das Sein et die Sprache chez Heidegger : «La pensée (Denken) accomplit la relation de l'Être à l'essence de l'homme. Elle ne constitue ni ne produit elle-même cette relation. La pensée la présente seulement à l'Être, comme ce qui lui est remis à elle-même par l'Être. Cette offrande consiste en ceci, que dans la pensée l'Être vient au langage (Sprache). Le langage est la maison de l'Être (Sein) $\gg($ M. Heidegger, Lettre sur l'humanisme, déjà cité, p. 27).

$113 D E n^{\circ} 38$, « La pensée du dehors », I, p. 562-565.

$114 D E \mathrm{n}^{\circ} 15$, « Guetter le jour qui vient », I, p. 294.

115 Ibid., p. 291. Foucault fait ici nommément référence à Jean de la Croix et, plus discrète- 
Si l'attente chez Laporte se fait veille - thème évangélique évident $\ll$ nul ne veille en cette veille $[\ldots]$. Ce qui veille, c'est la veille ${ }^{116}$. Passant sous silence la dimension messianique, voire doxologique du roman de Roger Laporte ${ }^{117}$, Foucault n'en garde que la trame nue. Même revirement au colloque de Cerisy-la-Salle, où le philosophe avait pourtant été le premier à user de l'adjectif « spirituel » à l'ouverture du débat, non sans une précaution à la hauteur de son embarras ${ }^{118}$. Quelques minutes plus tard, au sujet des expériences «comme le rêve, comme la folie, comme la déraison, comme la répétition, le double, la déroute du temps, le retour, etc. », repérées dans l'œuvre de Philippe Sollers, Foucault se rattrape : «C'est pourquoi les catégories de la spiritualité, du mysticisme, etc. ne paraissent pas du tout coller $\gg($ p.368). Une dernière fois, dans l'article consacré à Maurice Blanchot, Foucault écarte tout en la reconnaissant la proximité entre la mystique et la « pensée du dehors » : «Malgré plusieurs consonances, on est ici fort loin de l'expérience où certains ont coutume de se perdre pour se retrouver $\gg^{119}$.

Pourquoi l'adjectif «spirituel », dont Foucault n'arrive ici ni à se satisfaire ni à se libérer complètement, est-il jugé impropre ? Son usage se heurte à une première objection simple, qui l'empêche d'éclairer d'une lumière trop blanche l'expérience littéraire contemporaine. Cette objection se trouve à la fois dans l'article sur Roger Laporte et dans celui sur Maurice Blanchot. Le premier la formule sous sa forme la plus

ment, à Luther par la mention de la feste Burg. Ein feste Burg ist unser Gott est en effet un cantique de Martin Luther.

$116 D E \mathrm{n}^{\circ} 15$, « Guetter le jour qui vient », I, p. 290.

117 Le mystérieux «il $\gg$ devient à la fin du roman une figure messianique, dont le possible retour en gloire est annoncé dans la dernière phrase : «Absolument inapparent et en secret de lui-même $i l$ s'élèvera dans la pureté de sa propre gloire : de l'œuvre tout à fait solitaire, car se suffisant à elle-même, je recevrai alors mon congé » (R. Laporte, $L a$ Veille, déjà cité, p. 126). Toute l'œuvre de Laporte est marquée par l'inspiration mystique et biblique; le titre de son deuxième roman suffit à le vérifier : Une voix de fin silence, Paris, Gallimard, 1966 ; allusion au Livre des Rois : I Rois 19,12. Emmanuel Levinas ne s'y trompa pas dans son compte-rendu pour la Nouvelle Revue française : «Roger Laporte et la voix de fin silence » (repris dans E. Levinas, Noms propres, Montpellier, Fata Morgana, 1976, p. 133-137), qui se termine par ces mots : «le langage, c'est le fait qu'un seul mot toujours se profère : Dieu $\gg$.

$118 \ll$ Sollers $[\ldots]$ fait sans cesse référence à un certain nombre d'expériences - si vous voulez, j'appellerai ça, avec beaucoup de guillemets, des expériences spirituelles (mais enfin le mot spirituel n'est pas bon $) \gg\left(D E n^{\circ} 22\right.$, «Débat sur le roman $\left.\gg, I, p .366\right)$.

$D E n^{\circ} 38$, «La pensée du dehors », I, p. 565. 
immédiate et la plus frustre : l'expérience mystique personnalise l'être qui rend possible sa parole, en lui donnant la silhouette de «l'Aimé $»^{120}$. La veille n'est donc pas vaine et le néant dont parle le mystique est toujours un néant habité : «Au milieu de cette ombre, une lumière est allumée » (ibid., p. 291). De l'autre côté de sa déréliction, le mystique espère à chaque instant trouver le repos en trouvant son Dieu. Le second article, sur Maurice Blanchot, témoigne quant à lui d'une connaissance affinée de cette tradition spirituelle, dont il s'agit cette fois de ne pas taire la radicalité : l'expérience décrite par la théologie négative, en particulier chez le Pseudo-Denys l'Aréopagite, se déroule bien « dans une nuit sans ombre $\gg^{121}$, c'est-à-dire une nuit sans nulle lueur ou éclair qui en dessinerait soudain les arêtes. La conclusion semble pourtant la même, le thème du repos apparaissant dans les deux articles pour pointer le déficit de l'écriture mystique par rapport à la littérature; comme si, au moment de sauter, le mystique s'arrêtait in extremis au bord du gouffre qu'il a longé des nuits durant. En effet, précise Foucault,

$[\ldots]$ la mystique cherche à rejoindre - dût-elle passer par la nuit - la positivité d'une existence en ouvrant vers elle une communication difficile. Et quand bien même cette existence se conteste elle-même, se creuse dans le travail de sa propre négativité pour se retirer indéfiniment dans un jour sans lumière, dans une nuit sans ombre, dans une pureté sans nom, dans une visibilité libre de toute figure, elle n'en est pas moins un abri où l'expérience peut trouver son repos. (Ibid.)

La quête d'une positivité assurant à l'expérience un havre de paix n'est pourtant pas l'objection la plus convaincante à l'encontre de la théologie négative. Trois cas de figure doivent en effet être distingués - que l'article consacré à Maurice Blanchot semble parfois confondre, assimilant trop vite pensée, être et langage. Soit cette positivité est effective et modifie par conséquent la forme même de l'expérience ; et c'est l'expérience littéraire qui se révèle alors incomplète ou définitivement aveugle à l'être véritable. Soit cette positivité ne relève que de la disposition mentale du mystique (sa croyance); et l'on ne voit pas en quoi elle serait déterminante pour juger d'une expérience interne au langage. Soit enfin cette quête d'une positivité est inscrite dans la lettre même des textes - comme elle l'est dans l'extrait de Jean de la Croix cité dans 
l'article sur Laporte ${ }^{122}$; et il convient alors d'établir la place qu'occupent les propositions affirmatives dans la rhétorique propre à la mystique, en les mettant en relation avec les autres formes d'expression qu'elle affectionne, comme la négation, l'analogie ou encore la métaphore. Pas de trace d'une telle analyse chez Foucault.

Une nouvelle proposition relative à la mystique apparait alors dans l'article sur Blanchot. Elle se présente d'abord comme une simple précision, qui infléchit en fait l'argument. Il s'agit cette fois de dégager la struc-

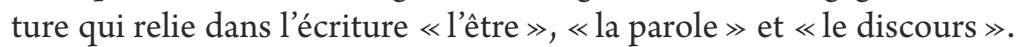
Ce faisant, Foucault ne nous convie pas à une analyse rhétorique des traités spirituels, qui en dégagerait les différentes figures ou tropes, mais à une étude de leurs conditions d'énonciation. Dans cette perspective, les textes sont considérés comme le lieu où s'inscrit un «sujet parlant $\gg$, comme le proposait déjà l'article de 1964 sur Mallarmé - à distance du sujet grammatical et du sujet psychologique ${ }^{123}$. Cette attention au sujet de l'énonciation renvoie en fait à la sémiotique littéraire du début des années 1960, croisant au passage la psychanalyse lacanienne.

Nous sommes ici quelques années avant le tournant linguistique que va connaître l'étude de la mystique dans l'université française, en particulier sous l'impulsion des historiens Michel de Certeau et Jacques Le Brun. La philologie et l'histoire littéraire, depuis l'abbé Brémond (1865-1933) jusqu'aux travaux de Jean Baruzi (1881-1953) et Jean Orcibal (1913-1991), avaient déjà ouvert la possibilité d'une approche horizontale de la spiritualité, hors des cadres confessionnels qui la dressaient à la verticale d'un Dieu supposé124. À la fin des années 1960, l'influence de la sémiotique va orienter les disciples d'Orcibal et de Baruzi vers une analyse linguistique du dire mystique, c'est-à-dire une définition des conditions discursives d'un tel acte, en deçà de ce qui est dit : comment un sujet prend la parole pour dire ce qu'il a à dire ? C'est exactement dans cette perspective que se glisse furtivement Foucault dans son article sur Maurice Blanchot. Le philosophe formule alors cette proposition étonnante : à travers son écriture, la mystique s'installe

122 La lumière dans le cœur du mystique « guide plus sûrement que la lumière de midi » $\left(D E n^{\circ} 15\right.$, « Guetter le jour qui vient », I, p. 291). Il s'agit d'une citation du poème La Nuit obscure.

$123 D E n^{\circ}$ 28, «Le Mallarmé de J.-P. Richard », I, p. 460, l'auteur souligne.

124 Pour une présentation du contexte, voir J. Le Brun, « Michel de Certeau historien de la spiritualité », Recherches de science religieuse, t. 91, vol. 4, 2003, p. 535-552. 
[... dans l'intériorité éblouissante d'une pensée qui est de plein droit Être et Parole. Discours donc, même si elle est, au-delà de tout langage, silence, au-delà de tout être, néant. ${ }^{125}$

L'enchevêtrement de notions, emportées par la brillance du style, demande une halte prolongée.

Dansl'expérience littéraire, nous avons vu que la parole, en tant qu'acte individuel, se résorbait dans le langage. Au contraire, dans l'effort pour communiquer à autrui un contenu par des signes appropriés, la parole devient discours, c'est-à-dire une activité langagière porteuse de sens, qui requiert nécessairement une pensée qui s'intériorise. Pas de discours, sans un retour à soi. Or, par cette intériorisation constamment manifestée par la grammaire énonciative du discours $(\ll$ Je pense que $[\ldots] \gg$, «J'ai bien réfléchi et je dirais que $[\ldots] \gg)$, le sujet qui dit «Je $\gg$ se présente comme responsable du discours prononcé ; il estl'instance qui répond de sa vérité. Pour cette raison, le terme de discours reçoit très tôt chez Foucault une connotation limitative, sinon péjorative, en particulier quand le philosophe compare ses limites intrinsèques aux possibilités vertigineuses du langage ${ }^{126}$. Alors que l'être du langage se donne à l'origine pour illimité, le discours, placé d'emblée dans l'intersubjectivité, s'affronte à trois limites que l'article consacré à Blanchot énumère de la sorte : 1) « celui auquel il s'adresse »; 2) 《la vérité de ce qu'il dit »; 3) 《les valeurs et les systèmes représentatifs qu'il utilise » (p.547). À l'opposé, délié de ces conventions, libre de se dire sans un sujet qui réponde de lui, «le langage échappe au mode d'être du discours » (p.548).

Que nous dit alors Foucault de l'énonciation mystique? La mystique ne se contente pas de postuler, en le nommant explicitement, un être aimant et tout-puissant qui l'attendrait au bout de la nuit. Elle opère, à l'intérieur même de ses énoncés, le mouvement inverse de l'expérience littéraire : au lieu de rendre son dire toujours plus impersonnel et inchoatif, la mystique dévoile comme condition de possibilité de son langage une « Parole » qui se révèle à son tour «Discours ». Précisons comment s'opère, dans les textes spirituels, une telle transmutation.

$125 D E \mathrm{n}^{\circ} 38$, « La pensée du dehors », I, p. 549, nous soulignons.

126 Le discours tente de conjurer la mort $\left(D E \mathrm{n}^{\circ}{ }_{14}\right.$, «Le langage à l'infini », I, p. 278), contrairement à l'image du poète chez Mallarmé qui en est «l'envers visible » $\left(D E \mathrm{n}^{\circ}{ }_{2} 8\right.$, «Le Mallarmé de J.-P. Richard », I, p. 462). Quant à Blanchot, il s'adresse au «non-discours de tout langage $»\left(D E \mathrm{n}^{\circ} 38\right.$, « La pensée du dehors $\left.», \mathrm{I}, \mathrm{p} .553\right)$. 
Le mystique ne parle que parce qu'une Parole lui a été adressée, non pas d'abord dans l'extériorité d'un livre ou de paroles articulées, mais, nous dit Foucault, dans «l'intériorité éblouissante d'une pensée » (ibid., p.549) - remarque encore un peu obscure qui pourrait être éclairée par un passage des Confessions de saint Augustin. L'évêque d'Hippone pose en effet que toute parole sur Dieu ne peut être reçue comme vraie quà la condition que la pensée de l'auditeur ait été au préalable illuminée par Dieu lui-même. Or, écrit Augustin, cette attestation est elle-même une parole «sans bruit de syllabe » qui, pour accréditer celui qui parle de Dieu, dit à la pensée de son auditeur : «Il dit vrai $\gg^{127}$. Voilà pourquoi Foucault peut légitimement assimiler la « Parole $\gg$ originelle à un «Discours », puisqu'il s'agit bien de la communication, d'un sujet à un autre, d'un contenu cognitif déterminé, qui vient juger en termes de vrai et de faux tout énoncé le concernant. C'est un discours qui décide de tous les autres discours. L'écriture mystique chrétienne est ainsi arrimée à un Logos, qui tient à la fois de la raison grecque et du Verbe créateur ouvrant les Écritures. La Parole divine, nous explique le Livre des Rois, peut très bien être la «voix d'un silence ténu ${ }^{128}$, elle reste une voix qui dit quelque chose à l'homme. Elle n'est pas le « murmure ${ }^{129}$ indiscernable qui circule entre les mots et défait tout ce qui se dit dans le langage. Elle est un principe discriminant dont la mystique doit attester la présence à l'intérieur de son propre discours. Les conditions d'énonciation de la mystique et celles d'une certaine expérience littéraire sont donc sur ce point incompatibles. Pour cette raison, après avoir fait remonter la «pensée du dehors » à la théologie négative du Pseudo-Denys l'Aréopagite ( $v^{\mathrm{e}}$ siècle), Foucault préfère finalement, par prudence, s'en tenir au Divin Marquis (de Sade) ${ }^{130}$.

127 Citons le passage : « Fais que j'entende et comprenne comment dans le principe tu as fait le ciel et la terre. Moïse l'a écrit. Il l'a écrit et il s'en est allé [... . S'il me parlait en hébreu, c'est en vain que sa voix frapperait mes sens : rien n'en toucherait mon intelligence. En revanche, s'il me parlait en latin, je saurais ce qu'il dirait. Mais d'où saurais-je s'il dit vrai ? Et quand bien même je le saurais, est-ce de lui que je le saurais ? C'est au-dedans de moi, oui, au-dedans, dans la demeure de la pensée, que la Vérité, qui n'est ni hébraïque, ni latine, ni grecque, ni barbare, sans se servir d'une bouche ni d'une langue, sans bruit de syllabes, me dirait : "Il dit vrai" » (Augustin, Les confessions, XI, III, 5, E. Tréhorel et G. Bouissou trad., Euvres de saint Augustin, t. XIV, Paris, Desclée de Brouwer, 1962).

128 I Rois 19, 12 (nous utilisons ici une traduction plus littérale de l'hébreu que celle, discutable, proposée par la Bible de Jérusalem : «le bruit d'une brise légère »).

$129 \mathrm{DE} \mathrm{n}^{\circ} 38$, « La pensée du dehors », I, p. 553 .

130 《Il est moins aventureux de supposer que la première déchirure par où la pensée du 
Cette mise à l'écart du langage mystique, pourtant constamment évoqué par Foucault et les écrivains qu'il commente, revêt à certains endroits une forme d'urgence, tant sa ressemblance avec une certaine littérature contemporaine est évidente et menaçante. Il ne suffit pas de distinguer radicalement l'être du langage du Logos divin, il faut repousser le mystérieux $i l$ le plus loin possible. Cet être anonyme, dont la saisie ultime par la littérature est toujours différée, doit perdre toute qualification, de peur qu' il se remplisse subrepticement d'un contenu, même incertain. C'est ici que Foucault prend implicitement ses distances avec Heidegger, voyant venir le danger d'une origine encore trop pleine et trop proche :

Mais n'est-ce pas encore capter cet $i$ absolument anonyme en une forme trop positive que de lui assigner comme être l'ouverture même de la pensée et de lui fixer comme lieu le langage d'une parole pensive ? ? $^{131}$

Sans limite et sans positivité, il n’a plus de figure.

L'Orient, tentation originelle du christianisme :

Pierre Klossowski, Gustave Flaubert

Cette cassure entre le Logos mystique et l'être du langage n'est pourtant pas aussi irrémédiable que celle qui sépara un jour l'Orient de l'Occident. Loin d'y voir un corps de doctrines et de pratiques immobiles, Foucault reconnait au christianisme une texture historique complexe, qui semble même dissimuler dans son étoffe des plis et des replis inattendus. La théologie négative et la mystique rhénane, mentionnées par Foucault dans ses lectures de Bataille et de Blanchot, en étaient une preuve précoce et certainement pas marginale, tant la postérité intellectuelle de cette tradition fut importante en Occident - pensons à l'idéalisme allemand, Hegel en tête, grand lecteur d'Eckhart et de Tauler. Cette ductilité du matériau religieux pourrait alors expliquer un certain flottement des références chrétiennes dans les écrits des années 1961-1966.

Non seulement l'adjectif «chrétien » flotte au-dessus des distinctions géographiques ou doctrinales ( « L’Europe chrétienne ${ }^{132}$

dehors s'est fait jour pour nous, c'est, d'une manière paradoxale, dans le monologue ressassant de Sade $\gg$ (ibid., p. 549).

$131 D E \mathrm{n}^{\circ} 15$, « Guetter le jour qui vient », I, p. 295.

$132 D E n^{\circ} 8$, «Le "non" du père », I, p. 220. 
embrasse d'un mot une réalité plurielle, voire hétérogène), mais il est miné de l'intérieur par le discret rappel de l'Orient païen qui l'a vu naître. C'est un mouvement à la fois distinct et complémentaire de celui que nous avons rencontré dans le cours Du gouvernement des vivants : malgré la vigueur avec laquelle le christianisme s'est doté dès les premiers siècles d'une forte identité - et l'étude des techniques de soi entend bien marquer la coupure avec l'Antiquité gréco-romaine sur ce point - le christianisme est resté en contact avec ce qu'il a prétendu rejeter de lui-même pour conquérir cette identité. Mais qu'aurait-il exactement rejeté ?

Encore moins défini que «l'Occident chrétien », «l'Orient » est une moitié de monde qui veut être le tout. Nous avons eu l'occasion de le souligner : c'est en référence constante au Dionysos de Nietzsche que Foucault fait se lever le soleil sur ses analyses. Or, dans Le crépuscule des idoles, le «dernier disciple du philosophe Dionysos » s'identifie au « maître de l'éternel retour ${ }^{133}$. L'affirmation de la vie dans le sentiment tragique unit en fait les deux leçons. Rappelons que l'éternel retour n'est pas chez Nietzsche une loi de l'être, une vérité individuelle ou cosmologique, elle est une valeur que l'homme doit littéralement s'incorpo$\operatorname{rer}^{134}$ : il nous revient, en chaque acte que nous posons, de vouloir le tout, c'est-à-dire d'agir dans le souci de la totalité de l'être, de son unité et de son homogénéité. Ce rapport neuf à la réalité - comme capacité de vivre chaque instant en voulant le revivre indéfiniment - n'est autre qu'un retour au tragique grec, à ce sentiment dionysien de ne faire qu' un avec le monde, jusque dans ses oppositions et ses contrastes, dans la jouissance des plaisirs de la terre et l'humble acceptation des maux qui l'accompagnent. Pour Nietzsche, l'éternel retour s'oppose en tout point aux idées chrétiennes de résurrection et d'immortalité, dont l'accès est conditionné par un partage mortifère : le jugement moral ${ }^{135}$.

Le divorce semble consommé entre les deux univers culturels. Puisque toute compréhension dialectique de l'histoire est exclue, Dionysos regarde le Christ comme son ennemi et son contraire, sans réconciliation

133 F. Nietzsche, Le crépuscule des idoles, déjà cité, p. 177.

134 Malgré la tentation, lisible à certains endroits de l'œuvre nietzschéenne, de trouver une confirmation de la doctrine de l'éternel retour dans la Physique de son temps : voir P. Wotling, $\mathrm{La}$ philosophie de l'esprit libre. Introduction à Nietzsche, Paris, Flammarion, 2008, p. 399-420.

135 F. Nietzsche, Le crépuscule des idoles, déjà cité, p. 97-102 : «La morale en tant que manifestation contre nature »; en particulier l'aphorisme $n^{\circ} 4: \ll$ Le saint qui plaît à Dieu, c'est le castrat idéal... La vie prend fin là où commence le "Royaume de Dieu”... » (p.100). 
possible. Sauf dans les pages de La Tentation de saint Antoine de Gustave Flaubert, dont Foucault rédige la postface en 1964. Ouvrant l'Ancien Testament, l'ermite Antoine, père des moines d'Orient, hume les parfums capiteux de l'ancienne Égypte, ceux de l'insouciance charnelle et du désir. En un raccourci fulgurant, Foucault propose alors une lecture nietzschéenne de Flaubert :

Antoine désire l'impossible retour à l'immobilité d'avant la vie : toute son existence, ainsi, rentrerait en sommeil, retrouverait son innocence, mais s'éveillerait à nouveau dans le bruissement des bêtes et des sources, dans l'éclat des étoiles. Être un autre, être tous les autres et que tout identiquement recommence, remonter au principe du temps pour que se noue le cercle des retours, c'est là le sommet de la Tentation. La vision de l'Engadine n'est pas loin. ${ }^{136}$

C'est bien là, en Engadine, région située au sud-est de la Suisse, que Nietzsche eut la révélation de l'éternel retour, devant le rocher pyramidal de Surlei qui surplombe le lac de Silvaplana. C'est également dans cette région, dans le village de Sils-Maria à 1800 mètres d'altitude, qu'il écrivit le deuxième tome d'Ainsi parlait Zarathoustra et ses derniers ouvrages importants.

Entre les rêveries de l'Orient soumises aux pouvoirs de l'imagination et le savoir occidental assujetti à l'obligation de vérité, le christianisme apparaît comme une très mince coupure, un point de basculement qui n'aurait quasiment aucune épaisseur :

L'Égypte chrétienne, et avec elle Alexandrie, et Antoine apparaissent au point zéro entre Asie et Europe, et comme au pli du temps : là où l'Antiquité, juchée au sommet de son passé, vacille et s'effondre sur elle-même, laissant revenir au jour ses monstres oubliés, et là où le monde moderne trouve son germe, avec les promesses d'un savoir indéfini. On est au creux de l'histoire. ${ }^{137}$

Le monde moderne, émergeant déjà à l'horizon du désert de HauteÉgypte, est caractérisé par la promotion et l'affinement de la connaissance objective : théologie au Moyen Âge, érudition à la Renaissance, science du monde et du vivant à l'âge moderne (ibid., p. 335). Mais en ce lieu de naissance de la culture européenne qu'est l'Orient, le partage n'est pas encore clairement opéré. Le basculement ne se voit qu'a posteriori; et pour Antoine, vivant au IV ${ }^{\mathrm{e}}$ siècle, le monde qui naît peut encore ressembler à celui qui meurt.

$136 D E \mathrm{n}^{\circ} 20$, «(Sans titre) $\gg, \mathrm{I}, \mathrm{p} .334$, nous soulignons.

$137 D E n^{\circ} 20$, «(Sans titre) $\gg$, I, p. 335 . 
C'est en fait un autre grand lecteur de Nietzsche qui oriente Foucault dans ces mêmes années vers la face orientale et cachée du christianisme : Pierre Klossowski. L'homme est secret, multi-instrumentiste. Tour à tour romancier, peintre, essayiste, excellent germaniste et latiniste, il passa également devant la caméra de Robert Bresson, le temps d'un film aux résonances christiques évidentes : Au hasard Balthazar (1966). C'est par sa vie autant que par sa pensée que le grand traducteur de Nietzsche et de Virgile se tient au point de rencontre entre paganisme et christianisme. Celui qui fut un proche ami de Georges Bataille - avec qui il partageait un même héritage catholique et une même vocation « suspendue »- publie entre 1953 et 1960 une trilogie romanesque (Les Lois de l'hospitalité) qui retint l'attention de Foucault, au milieu d'essais importants consacrés à Sade, Nietzsche, Bataille, mais aussi Kierkegaard. En 1964, le jeune Foucault lui consacre un article intitulé «La prose d'Actéon » dans La Nouvelle Revue française, organisé autour des figures du double et $\mathrm{du}$ «simulacre »- thème que Klossowski a repris de Lucrèce ${ }^{138}$. Dans cet article, Foucault ne salue rien moins qu' « un langage pour nous aussi essentiel que celui de Blanchot et de Bataille $\gg^{139}$.

À l'altérité divine, dont l'ombre portée découpe dans la vie du croyant des domaines inconciliables (bien/mal, lumière/ombre, vie/ mort), Klossowski oppose une pensée du Même que Foucault explicite sous la forme d'une question :

Mais si le Diable, au contraire, si l'Autre était le Même? Et si la Tentation n'était pas un des épisodes du grand antagonisme, mais la mince insinuation du Double? Si le duel se déroulait dans un espace de miroir? (Ibid., p.354)

À cette possible transmutation du religieux, qui supprimerait ici-bas les oppositions et les contradictions, il n'y a qu'une condition : l'abandon de toute idée d'un autre monde. S'il n'y a plus que ce monde-ci - tout simplement - alors plus rien ne vient faire radicalement ni réellement différence. Les signes qui nous environnent - qu'ils soient des figures,

138 Pour Lucrèce, les simulacres (simulacra, ou imagines, effigies, figurae, spectra) sont des «membranes légères » qui se détachent des corps existants pour en représenter la forme et l'aspect. Voltigeant en tous sens à l'air libre, ils pénètrent dans les sens et dans nos pensées, pour y être cause de nos sensations et de nos idées. Les simulacres sont donc des corps matériels, et, à ce titre, ils sont tous également vrais. Voir A. Gigandet, Fama deum. Lucrèce et les raisons du mythe, Paris, Vrin, 1998, p. 211 et suiv. 
des fantasmes ou de simples objets matériels - sont des simulacres, non pas en tant qu'ils nous cacheraient la vérité, mais parce qu'ils renvoient sans fin à autre chose qu'eux-mêmes; ils sont une perpétuelle monnaie d'échange. La Diane de la mythologie en serait un bel exemple : pure et sage, la Vierge chasseresse intéresse Klossowski en raison de l'étourdissante richesse de significations qu'elle recèle, du culte artémisien, dans la diversité de ses interprétations antiques, aux écrits néotestamentaires chrétiens. L'essai de Klossowski Le bain de Diane (1956) se termine d'ailleurs sur la citation des Actes des apôtres : « GRANDE EST LA DIANE DES ÉPHÉSIENS » (Act. 19, $28 ; 34)$, jetant ainsi un pont entre christianisme et paganisme, comme entre Christ et Dionysos ${ }^{140}$.

Dans la perte de tout principe discriminant, l'être et l'apparence, la vérité et le simulacre sont désormais une seule et même chose. Ne demeure qu'un jeu à l'intérieur de l'égalité : un écart qui sans cesse se creuse ou se résorbe entre deux éléments semblables. Foucault ne nomme pas la raison de cet écart. Mais si «les discours, les fables $\gg^{141}$ s'y engouffrent, il est aisé d'y voir, au moins en arrière-plan, l'écart qui habite le langage lui-même - comme l'article sur Blanchot le développera quelques années plus tard.

Dans l'univers infini des signes, le signe linguistique ne renvoie-t-il pas pourtant à un signifié, par un jeu rigoureux de différences qui interrompt le miroitement du monde et du langage ? Oui, reconnait Foucault, mais il existe au moins un domaine où le signe linguistique peut être un simulacre : c'est précisément le domaine religieux, malgré l'apparente appartenance de ses mots à une origine ferme et unique (le Dieu immuable et éternel). L'exemple d'un signe qui peut fonctionner comme signe sans déterminer aucun sens univoque, Foucault le trouve dans la figure religieuse de l'arbre de la Croix. Dans une analyse très subtile, il montre que tous les signes des Écritures renvoient à ce signe premier qui lui-même se dérobe à toute désignation ultime d'un sens. En effet, l'arbre de la Croix est lié «à l'histoire d'une manifestation qui n'est jamais achevée » (ibid., p. 358). Il est donc « tout entier suspendu à un avenir qu'il répète d'avance et qui le répétera à son tour $\gg$ (p.358). Dans cette structure temporelle complexe, que la théologie chrétienne a thématisée dans l'impossible conjonction du déjà là et du pas encore, le signe ne cesse de se dérober en avant et en arrière de lui-même. Il reviendra aux derniers philosophes 
païens (stoïciens, épicuriens) et aux premiers exégètes chrétiens (Origène) de dissocier le signe du simulacre, afin d'interrompre par des règles précises le mouvement décrit par Foucault : celui d'une interprétation infinie des textes qui ne sort jamais du jeu de renvoi. Quand il évoque la « patrie alexandrine » (p.359) de l'exégèse chrétienne, l'article sur Klossowski ne fait qu'effleurer cet immense problème de la lecture allégorique des Écritures, qui plonge ses racines dans l'œuvre de Philon d'Alexandrie $^{142}$. Mais ici encore, il s'agit d'une origine oubliée, recouverte par le triomphe d'une certaine volonté de vérité sur le monde des apparences.

De ce périple littéraire, qui va de Folie et déraison au grand article sur Maurice Blanchot, nous pouvons conclure que la peinture foucaldienne du christianisme occidental reste foncièrement nietzschéenne, guidée essentiellement par les leçons de Naissance de la tragédie : dénonciation d'un monde qui aurait perdu le sentiment de la totalité, l'union de tout ce qui est dans un infini jeu de reflets, au profit d'un autre monde, distinct du nôtre, posé comme un idéal invisible et lointain. D’où les métaphores lumineuses, en particulier solaires, qui forment la trame de l'article sur Klossowski - mais plus largement de tous les articles consacrés par Foucault à la littérature dans ces années de jeunesse : y brille à chaque page la «théophanie resplendissante des dieux grecs ${ }^{143}$, consacrant $\mathrm{l}^{\prime} \ll$ illumination de Midi et [le] retour éternel $\gg($ ibid., p. 358), jusqu'au jour maudit où «la divinité cesse de scintiller dans les clairières » (p.363). Cette Grèce rêvée n'est pas loin de la carte postale, avec son Ithaque personnelle (Alexandrie ${ }^{144}$ ), évoquée avec la nostalgie de ce qui revient plus sûrement dans les mémoires qu'il n’a jamais été.

Il ne faut donc pas voir dans ces pages une analyse des conditions historiques d'émergence du christianisme, mais une dramaturgie propre à l'écriture de Foucault, que les travaux des années 1970 effaceront complètement. Avec toute la puissance évocatrice recelée par ces noms propres, la «Grèce » ou l'« Orient » désignent la possibilité d'une autre manière de vivre et de penser. Non pour se défaire d'un rigorisme moral

142 Voir ce volume, p. 181 note 146.

$143 D E \mathrm{n}^{\circ} 21$, « La prose d'Actéon », I, p. 355 .

144 «Alexandrie, qui est notre lieu de naissance, avait prescrit ce cercle à tout le langage occidental : écrire, c'était faire retour, c'était revenir à l'origine, se ressaisir du premier moment ; c'était être de nouveau au matin $»\left(D E \mathrm{n}^{\circ} 24\right.$, «Le langage de l'espace $\left.», \mathrm{I}, \mathrm{p} .435\right)$. 
judéo-chrétien à peine évoqué par Foucault ${ }^{145}$, mais pour sortir de l'hégémonie $\mathrm{du}$ 《savoir occidental $\gg^{146}$ - thème déjà central dans le commentaire de Flaubert. Le jeune philosophe loue en effet le romancier d'avoir mis en scène la prolifération, moderne et dérisoire, des livres et de l'érudition : « La Tentation est la première œuvre littéraire qui tienne compte de ces institutions verdâtres où les livres s'accumulent et où croît doucement la lente, la certaine végétation de leur savoir » (ibid., p.326327). L'analyse de Bouvard et Pécuchet fait d'ailleurs suite et répond malicieusement à celle de La Tentation de saint Antoine. Les mésaventures des deux copistes retirés dans le Calvados rappellent la retraite d'Antoine, «comme son ombre grotesque $»($ p. 337). Le pacte occidental semble bien avoir été passé entre la science et la théologie, plus qu'entre la morale et la religion. Science et théologie sont en effet toutes deux citées par Foucault aux côtés de l'érudition, non seulement comme des moments singuliers de notre histoire intellectuelle, mais plus encore comme ses véritables maîtres d'œuvre ${ }^{147}$. Disciplines maîtresses du savoir occidental, science et théologie ont fait de l'Être un objet de connaissance, garanti par l'assurance de pouvoir en parler dans un langage transparent à ses procédures. Sur la base de cette prétention, le partage du vrai et du faux est venu discriminer le contenu même des énoncés, et non plus seulement les manières de prendre la parole, de s'engager dans le discours, comme c'était le cas dans la Grèce des poètes et des sophistes ${ }^{148}$.

Nous retrouvons ici, par le détour de la forme romanesque, le partage historique indiqué dans la préface à Folie et déraison. Mais ce qui se murmure dans les articles sur Gustave Flaubert et Pierre Klossowski, c'est la possibilité pour le christianisme de revenir à ce qu'il était avant le partage, dans cette zone où la religion nouvelle était encore orientale et bigarrée, où Christ et Dionysos étaient « simulacres l'un de l'autre ${ }^{149}$.

145 Nous l'avons indiqué, la « répression » et les «interdits sexuels » ne sont que rapidement mentionnés dans la préface à Folie et déraison: $D E \mathrm{n}^{\circ} 4$, « Préface », I, p. 190. L’article sur Klossowski indique en ouverture que le christianisme occidental « a maintenu en ses fantasmes les duels simplifiés de la Tentation $\gg\left(D E n^{\circ} 21\right.$, «La prose d'Actéon », I, p. 354). Mais c'est bien peu, comparé aux diatribes nietzschéennes incessantes contre la moralité chrétienne.

$146 D E \mathrm{n}^{\circ} 20$, «(Sans titre) $\gg$, I, p. 335 .

147 Foucault cite explicitement trois périodes historiques : Moyen Âge, Renaissance, âge moderne (ibid., p.335).

148 L'ordre du discours, p. 16-18.

$149 D E \mathrm{n}^{\circ} 21$, «La prose d'Actéon », I, p. 354 . 
Comme si, dans la complicité du divin avec le sacrilège, quelque chose de la lumière grecque sillonnait en éclair le fond de la nuit chrétienne. (Ibid., p. 355)

Dans la doctrine de l'éternel retour, telle que Pierre Klossowski l'interprète, il n'y a plus en effet de raison d'exclure certaines figures de dieux (Christ) au profit de certaines autres (Dionysos). Au sein d'un univers qui ne cesse de se retrouver et de se contredire, tous les dieux font éternellement retour en l'homme, quelle que soit la vigueur première de leurs oppositions ${ }^{150}$.

Ce regard tourné vers la lumière grecque est donc bien un regard tourné vers un certain divin, dont Foucault notait le récent retour dans la pensée contemporaine, sur fond de finitude ${ }^{151}$. Or, si l'Orient rode toujours aux confins du christianisme, la métaphysique rode pareillement aux limites de la pensée de Foucault. Les deux tentations sont d'ailleurs en bien des points semblables, à nouveau médiatisées par les œuvres de Nietzsche et de Heidegger, avec la même amitié-inimitié pour le second. Mais c'est surtout Kant, et les relations qu'il a successivement établies - et barrées - entre l'anthropologie et la métaphysique, qui va devenir dans ce contexte la référence majeure. Puisque nous sommes clairement, dans ces écrits des années 1960, du côté d'une histoire des idées et non du côté d'une histoire des pratiques, le rapport à la métaphysique éclaire ici et complète naturellement le rapport de Foucault au christianisme.

\section{Le retour de la métaphysique}

Le 20 mai 1961, Foucault présente devant Jean Hyppolite et Maurice de Gandillac sa thèse complémentaire intitulée « Kant : Anthropologie. Introduction, traduction et notes $\gg$. Si la traduction est rapidement publiée chez Vrin, la longue introduction reste dans les archives de la

150 Foucault se réfère ici à la conférence donnée par Pierre Klossowski au Collège de philosophie en 1957, qui développait longuement la question de la multiplicité des dieux présents dans l'œuvre de Nietzsche : «Nietzsche, le polythéisme et la parodie », repris dans P. Klossowski, Un si funeste désir, déjà cité, p. 187-228. Dans ces conditions, Christ et Dionysos, sans pour autant être réconciliés, doivent coexister : «L'existence en tant qu'éternel retour de toutes choses se produit dans les physionomies d'autant de multiples dieux qu'elle a de possibles explicitations dans l'âme des hommes » (ibid., p. 221).

$D E \mathrm{n}^{\circ} 13$, « Préface à la transgression », I, p. 267. 
Sorbonne. Bénéficiant aujourd'hui d'une édition intégrale ${ }^{152}$, cette thèse complémentaire est la preuve - dérangeante sans doute pour bien des contempteurs - que le lieu source de la pensée foucaldienne n'est pas d'abord la Forêt-Noire ou Sils-Maria, mais bien Königsberg. Si Foucault fut nietzschéen, il le fut par fidélité à ce qui, chez Nietzsche, et sans doute malgré lui, restait viscéralement kantien. Les éditeurs du texte intégral le disent d'entrée de jeu d'une même voix : c'est parce que la thèse complémentaire annonçait déjà la critique des sciences de l'homme et la mort de leur objet que Foucault n'avait pas souhaité la publier (p. 8-9). Sobre et rigoureux, l'avis mortuaire résonnerait cinq ans plus tard à la fin des Mots et les choses.

La parution en français quelques années plus tôt de l'étude de Heidegger Kant und das Problem der Metaphysik (1929) ${ }^{153}$ a certainement orienté le jeune étudiant vers l'Anthropologie, mais sans allégeance au maître de la Forêt-Noire, qui se trouve être le principal adversaire de la thèse complémentaire ${ }^{154}$. Il n'est d'ailleurs nul besoin d'aller chercher ailleurs que chez Foucault lui-même, dans les questions philosophiques qu'il porta trois décennies durant, son intérêt précoce pour l'Anthropologie. En cette zone

152 E. Kant, Anthropologie du point de vue pragmatique, précédé de M. Foucault, « Introduction à l'Anthropologie », M. Foucault trad., Paris, Vrin, 2008.

M. Heidegger, Kant et le problème de la métaphysique, A. De Waelhens et W. Biemel trad., Paris, Gallimard, 1953.

154 Foucault emprunte à Heidegger l'idée que l'anthropologie kantienne est la « répétition » de la philosophie critique (M. Heidegger, Kant et le problème de la métaphysique, A. De Walhens et W. Biemel trad., Paris, Gallimard (Tel), 1981, p. 261). D'autres passages de son introduction sont sous l'influence directe de l'interprétation heideggérienne, en particulier l'effort déployé pour jeter un pont entre l'usage régulateur des idées transcendantales et la vie concrète de l'homme, à travers la notion de Geist: « Introduction à l'Anthropologie », p. 40. Dans cette étude du Geist, se lit une familiarité évidente avec la partie A. de la quatrième section de l'essai de Heidegger intitulée «L'instauration du fondement de la métaphysique comme anthropologie » (Kant et le problème de la métaphysique, déjà cité, p. 261-275). Compte tenu de l'opposition finale à Heidegger, cet emprunt évident nous rappelle surtout que le texte de Foucault est d'abord une thèse universitaire, invitée à rendre compte des traditions d'interprétation du sujet traité. Il aurait en effet été difficile de ne pas évoquer, directement ou indirectement, l'essai de Heidegger, dont la traduction française était parue quelques années plus tôt. Dans ce passage que Foucault consacre au Geist, l'usage d'un vocabulaire heideggérien est tellement explicite qu'il prend une valeur, sinon ironique, au moins documentaire : le Geist, écrit Foucault, « est ce retrait, cette invisible et "visible réserve" dans l'inaccessible distance de laquelle le connaître prend place et positivité. Son être est de n'être pas là, dessinant, en ceci même le lieu de la vérité » («Introduction à l'Anthropologie », p. 41). Nous sommes à la limite du pastiche. 
intermédiaire et incertaine où l'homme manifeste non pas ce qu'il est, mais ce qu' il peut et doit faire dans l'espace et le temps, l'exercice quotidien de la liberté s'apparente à un « jeu » ou encore à un « art » (ibid., p. 33) par lequel l'homme acquiert le monde tout en étant formé par lui. Il ne s'agit donc pas d'une activité pérenne et fondatrice, mais d'un ensemble « de ruses, d'intentions louches et de dissimulations $\gg$ (p. 27), par lesquelles la loi morale cherche dans les us et coutumes des points d'équilibre précaires. Retour à la concrétude, donc, où l'homme n'est ni sujet de droit ni pur esprit, mais liberté donnée dans l'expérience. Pour Foucault, ce point de vue pragmatique offre à la connaissance de l'homme un champ «jamais offert en totalité, jamais en repos en soi-même» (p.32), où rien n'est donné en transparence et sans contrepartie. Ce champ n'est l'objet chez Kant ni d'une histoire de la culture ni d'une connaissance naturaliste, mais d'une exploration singulière qui répète la structure de la Critique dans l'exercice quotidien humain, où la vérité prend figure dans la dispersion temporelle et le langage. Quelques années à peine après ce commentaire affilé, le discours et l'histoire seront précisément, pour Foucault, les deux principaux lieux d'où émergera son interrogation sur la vérité.

Si Foucault insiste tellement sur l'appartenance de l'Anthropologie à l'entreprise critique, malgré le lien en apparence ténu dont le texte kantien semble se suffire, c'est pour refuser catégoriquement à toute anthropologie post-kantienne le droit de se faire connaissance empirique et autonome de l'homme. En effet, «l'empiricité de l'Anthropologie ne peut se fonder sur elle-même $\gg($ p. 75$)$ mais doit continuer à se référer aux conditions a priori de la connaissance, dans la relation à un objet, sans espoir de contourner ces conditions pour y découvrir la « vérité de la vérité » (p. 77). La phénoménologie husserlienne et les sciences humaines sont ici vertement tancées. Dans ce rappel vigoureux, où Foucault se fait presque plus kantien que Kant, sourd le grondement de la bataille qui se fera entendre dans Les mots et les choses. Car telle est bien la prétention de toutes les anthropologies philosophiques qui ont succédé à Kant : d'un côté, oublier la critique kantienne de la connaissance, pour fonder sans inquiétude une science de l'homme en sa positivité; de l'autre, faire de l'usage pur de la raison la nature profonde de notre finitude, le lieu où la vérité, sans cesse offerte et manquée, apparaît en sa retraite, comme « vérité de la vérité ». À quelques paragraphes de la fin de son introduction, Foucault décrit ce dernier dessein, en relevant les glissements successifs quil'ont rendu sinon possible, au moins pensable : 
Le caractère nécessaire de l'apparence transcendantale a été de plus en plus souvent interprété non pas comme une structure de la vérité, du phénomène de l'expérience, mais comme un des stigmates concrets de la finitude. Ce que Kant désignait en elle, d'une manière ambiguë, comme « naturel », a été oublié comme forme fondamentale du rapport à l'objet et récupéré comme «nature » de la nature humaine. L'illusion, par conséquent, au lieu d'être définie par le mouvement qui la critiquait dans une réflexion sur la connaissance, était référée à un niveau antérieur où elle apparaissait à la fois dédoublée et fondée : elle devenait vérité de la vérité. (p. 77)

Ce passage reprend très précisément l'introduction de la division «Dialectique transcendantale » de la Critique de la raison pure, en lui empruntant l'adjectif $\ll$ naturel (natürlich) $\gg^{155}$ - décisif pour le propos kantien - et en se permettant de tordre légèrement l'inévitable (unvermeidlich) vers le nécessaire ${ }^{156}$. Nécessaire ou simplement inévitable, l'apparence transcendantale est bien constitutive pour Kant de notre faculté de connaître. Elle n'est pas un mauvais ajustement de l'entendement à la sensibilité, elle ne correspond ni à une illusion d'optique (apparence empirique) ni à un défaut d'attention aux règles logiques (paralogisme), mais elle a son siège dans la raison pure, entendue comme faculté des principes. Elle est un défaut dans l'usage que nous faisons des idées, entendues comme synthèses subjectives, à partir du moment où celles-ci ne se rapportent plus à l'expérience : ainsi, par exemple, du principe selon lequel le monde doit avoir un commencement dans le temps. Si tout concept de l'entendement se rapporte aux objets de l'intuition et à leur synthèse dans l'imagination, l'idée à l'intérieur de la raison pure se rapporte quant à elle à l'entendement, mais uniquement dans le but de lui prescrire une certaine direction : une unité dont l'entendement n'a aucun concept et que Kant appelle « apparence transcendantale ». La découverte de cette apparence ne suffit pas à l'écarter comme un mirage, car elle répond à une nécessité interne à la raison. L'erreur consiste seulement à prendre la nécessité subjective pour une nécessité objective qui décrirait la chose en soi : le monde comme ayant effectivement un commencement.

155 E. Kant, Critique de la raison pure, III, 237, A. J. L. Delamarre, F. Marty, J. Barni trad., Paris, Gallimard, 1980, p. 321, l'auteur souligne : «Car nous avons à faire à une illusion naturelle et inévitable $[\ldots]$. Il y a donc une dialectique de la raison pure, naturelle et inévitable. »

156 Ibid., III, 237, p. 321 : c'est la liaison subjective opérée par la raison entre certains concepts qui répond à une « nécessité » (die subjective Nothwendigkeit), leur imputation à une réalité objective est quant à elle qualifiée par Kant d'«inévitable (unvermeidlich). 
La critique kantienne met le sujet au centre de la connaissance; mais c'est un sujet radicalement fini, aux capacités d'entendement limitées par des barrières infranchissables qui l'empêchent d'accéder à un inconditionné. Si la raison transcendantale, à travers ses idées, se projette le tout du monde à des fins d'unité, la constitution ontologique de la nature et la constitution téléologique de l'histoire lui sont interdites. C'est ici qu'intervient le coup de force de Heidegger, dénoncé par Foucault. On l'aura reconnu, même s'il n'est à aucun moment nommé, c'est l'auteur de Sein und Zeit qui est le premier visé par la finale du commentaire. En relevant comment l'illusion transcendantale kantienne a été ultérieurement réinterprétée comme le lieu où se dit une vérité de l'homme en sa finitude, sous la forme redoublée de la « vérité de la vérité », Foucault note en effet : «Cette illusion définie maintenant comme finitude devenait par excellence la retraite de la vérité : ce en quoi elle se cache et ce en quoi, toujours on peut la retrouver $\gg^{157}$. Le doctorant prend soin de souligner dans son texte le vocable « retraite », qui appartient au langage heideggérien de la manifestation de l'être ${ }^{158}$.

Pour Heidegger, le néokantisme de l'école de Marbourg - Hermann Cohen en particulier - a réduit la critique kantienne à une théorie de la connaissance ordonnée aux sciences de la nature; il a mis la philosophie au service de l'épistémologie ${ }^{159}$. En voulant extraire l'œuvre de Kant de ces mésinterprétations postérieures, Heidegger prétend dégager la spécificité de l'expérience que le sujet transcendantal fait de ses limites, aidé dans cette tâche par la phénoménologie husserlienne. Or, dans cette expérience des limites, il s'agit bien d'une expérience de l'être lui-même. Il faut donc renouer la question de l'a priori avec la question ontologique, ce qui ne peut se faire sans dépasser Kant. Le grand livre Sein und Zeit en 1927, l'enseignement lors du semestre d'hiver 1927-1928 consacré à la Critique de la raison pure $^{160}$ et l'étude sur Kant qui sortira de cet enseignement en

157 «ntroduction à l'Anthropologie », p. 77, l'auteur souligne.

158 M. Heidegger, Être et temps, F. Vezin trad., Paris, Gallimard, 1986, p. 62 : «l'être de l'étant » est « ce qui demeure en retrait (verborgen bleibt) ».

159 Les remarques contextuelles suivantes doivent beaucoup à C. Piché, « Heidegger et Cohen, lecteurs de Kant », Archives de philosophie, t. 61, vol. 4, 1998, p. 603-628 ; A. Schnell, De l'existence ouverte au monde fini. Heidegger 1925-1930, Paris, Vrin, 2005, p. 16-22.

160 M. Heidegger, Interprétation phénoménologique de la Critique de la raison pure, E. Martineau trad., Paris, Gallimard, 1982. 
$1929^{161}$ ne visent ni plus ni moins qu'à fonder ontologiquement l'idéalisme transcendantal. Si les conditions a priori de l'expérience ne peuvent être elles-mêmes objet d'une expérience comme le sont les choses sensibles, c'est bien que le transcendantal se retire toujours en nous donnant le monde. Il revient alors à l'analytique du Dasein d'ontologiser ce retrait du transcendantal en en faisant le retrait même de l'être. De l'expérience de ce retrait, une phénoménologie peut alors être envisagée. Cette phénoménologie nouvelle doit seulement veiller à ce que le domaine de l'a priori ne soit pas considéré comme une propriété du sujet connaissant, qui en revendiquerait dans sa solitude, avant même toute relation à l'étant, la maîtrise absolue, mais bien comme une modalité de la transcendance du Dasein. Car c'est par cette transcendance que le Dasein s'ouvre au monde, dans une relation qui n'est pas celle du sujet à l'objet.

D'une telle ontologisation de l'a priori, Foucault ne veut pas entendre parler. Il prévient surtout que cette manœuvre ne suffira pas à sortir la philosophie d'une pensée de la subjectivité, contrairement aux espoirs d'une ontologie qui voyait dans le retour au monde la possibilité d'échapper à la res cogitans cartésienne. Pour Heidegger en effet, le Dasein n'est ni une présence ni une substance, mais une existence toujours déjà engagée dans le monde, en relation avec lui, avant tout retour réflexif sur soi. Pour Foucault, l'être-au-monde ainsi décrit n'est qu'un écran de fumée entourant la vieille subjectivité :

Et le paradoxe est en ceci : en s'affranchissant d'une critique préalable de la connaissance et d'une question première sur le rapport à l'objet, la philosophie ne s'est pas libérée de la subjectivité comme thèse fondamentale et point de départ de sa réflexion. Elle s'y est au contraire enfermée en se la donnant épaissie, hypostasiée et close dans l'indépassable structure du «menschliches Wesen $\gg$, en quoi veille et se recueille silencieusement cette vérité exténuée qu'est la vérité de la vérité. ${ }^{162}$

Notons dans ce texte sévère, voire cinglant, que Das menschliches Wesen (l'essence de l'homme) est à nouveau une citation de Heidegger empruntée à l'essai sur $\mathrm{Kant}^{163}$. À la veille heideggérienne qui se voulait

161 M. Heidegger, Kant et le problème de la métaphysique, déjà cité.

162 «ntroduction à l'Anthropologie», p. 78 .

163 M. Heidegger, Kant et le problème de la métaphysique, quatrième section, A., $\mathbb{\$} 8: \ll \mathrm{La}$ question de l'essence de l'homme et le résultat véritable de l'instauration kantienne du fondement » (déjà cité, p. 270-275). Ce thème de «l'essence de l'homme » se retrouve également dans Lettre sur l'humanisme : «Tout humanisme se fonde sur une métaphy- 
« retour à l'origine, à l'authentique, à l'activité fondatrice $»^{164}$, Foucault préfère l'inconfort de l'anthropologie kantienne : une pensée qui est par le même mouvement connaissance objective de l'homme en son être naturel et interrogation sur les limites de cette connaissance. L'Anthropologie doit être lue comme «l'analogon empirique et extérieur » (p. 75) de la Critique, sans jamais pouvoir prétendre contourner ou envelopper cette dernière. C'est bien cette même impossibilité qui se trouvera à nouveau réaffirmée, cinq ans plus tard, dans la section «Le sommeil anthropologique » des Mots et les choses, palimpseste de la thèse complémentaire : «la vérité de toute vérité » et «l'homme en son essence » sont à nouveau dénoncés, cette fois comme le « Pli » où une philosophie de l'homme vient encore se cacher et espère se sauver ${ }^{165}$.

Entre la thèse de 1961 et le maître livre de 1966, si l'on retrouve le même hommage à Kant et la même défiance vis-à-vis de Heidegger, un troisième philosophe vient cependant modifier la donne et radicaliser le propos foucaldien. Le trublion s'appelle Nietzsche. Dans l'avant-dernier paragraphe de son introduction à l'Anthropologie, Foucault dessine déjà les linéaments d'une filiation inattendue : seul Nietzsche, en fin de compte, aurait retenu les leçons kantiennes, cherchant à être conséquent avec ces dernières jusque dans ses conclusions les plus radicales sur la mort de Dieu ${ }^{166}$. En effet, si la Critique de la raison pure a fait de l'Être suprême une simple idée régulatrice des concepts a priori, sans réalité objective décidable ${ }^{167}$, l'Anthropologie «montre du doigt l'absence de Dieu $\gg^{168}$ dans le domaine de l'activité naturelle de l'homme. Dieu n'est peut-être pas mort avec Kant, mais il s'est déjà retiré de ce domaine où l'homme s'appréhende comme phénomène dans la forme du temps. Plus d'infini, sinon celui insufflé par l'esprit de l'homme (Geist) à la

sique ou s'en fait lui-même le fondement. Toute détermination de l'essence de l'homme (menschliches Wesen) qui présuppose déjà, qu'elle le sache ou non, l'interprétation de l'étant sans poser la question portant sur la vérité de l'Être, est métaphysique » (M. Heidegger, Lettre sur l'humanisme, déjà cité, p. 51, nous soulignons).

164 «Introduction à l'Anthropologie », p. 78 .

165 Les mots et les choses, p. 352-353.

166 Foucault parle de « filiation et de fidélité » de Nietzsche à l'égard du « vieux "Chinois de Königsberg" » (《Introduction à l'Anthropologie », p. 68).

167 E. Kant, Critique de la raison pure, III, 426, déjà cité, p. 552.

168 «Introduction à l'Anthropologie », p. 75 . 
raison empirique (Gemüt), pour l'aider à ne pas se disperser ou s'assoupir dans le donné de l'expérience (ibid., p. 39). L’homme et l'infini vont encore de pair ; mais nulle ontologie ne peut plus fonder cette relation.

Malgré cette révolution, Foucault laisse entendre qu'il aurait manqué au grand Chinois de Königsberg l'audace nietzschéenne de critiquer radicalement, non seulement les anthropologies positivistes, mais toute anthropologie. En lieu et place de ce geste radical, Kant a préféré laisser à la connaissance phénoménale de l'homme une place marginale et incertaine dans sa pensée (p. 76). C'est de cette place que prétendront repartir les anthropologies philosophiques ultérieures, dans la quête illusoire d'un « originaire $»(\mathrm{p} .77)$ où la finitude humaine trouverait son fondement. Or, seule une critique radicale de l'homme, allant jusqu'à mettre en question son existence même, peut mener au sombre constat nietzschéen : «Dieu est mort ». Sans ce gommage définitif, aussi longtemps que l'homme demeurera objet d'une connaissance empirique, l'anthropologie continuera d'y recueillir la vérité de la finitude, cette « vérité de la vérité $\gg$ religieusement attendue. Au contraire, «c'est dans la mort de l'homme que s'accomplit la mort de Dieu $\gg($ p. 78$)$, c'est-à-dire de tout originaire. Sans autre explication, Foucault fait alors du «surhomme » nietzschéen le signe contemporain de cette mort, ou plutôt la nouvelle naissance qu'elle rend possible sur ses cendres.

En apparence, le chapitre IX des Mots et les choses ne dit pas autre chose. La proximité avec la finale de l'introduction de l'Anthropologie est patente, même si elle est précédée d'une réflexion neuve sur l'arrachement du langage, à compter du XIX ${ }^{\mathrm{e}}$ siècle, au domaine de la représentation. C'est d'abord «à l'intérieur de son langage ${ }^{169}$ que Nietzsche a tué l'homme et Dieu. Mais ce double assassinat a la même conséquence qu'en 1961 : la sortie de l'Anthropologie et de toute anthropologie, en tant que celles-ci continuent obstinément de faire de l'homme le lieu natif où une vérité attendue se dévoile, toujours légèrement en retrait de l'expérience. Foucault décrit ainsi l'expérience nietzschéenne :

Peut-être faudrait-il voir le premier effort de ce déracinement de l'Anthropologie [... ] dans l'expérience de Nietzsche : à travers une critique philologique, à travers une certaine forme de biologisme, Nietzsche a retrouvé le point où l'homme et Dieu s'appartiennent l'un l'autre, où la mort du second est synonyme de la dispa- 
rition du premier, et où la promesse du surhomme signifie d'abord et avant tout l'imminence de la mort de l'homme. (Ibid., p.353, nous soulignons)

L'usage du verbe pronominal «s'appartenir » indique pourtant une perspective nouvelle par rapport à 1961. En effet, le thème d'une appartenance entre l'homme et Dieu que Nietzsche aurait « retrouvé » n'apparait pas dans la thèse complémentaire ${ }^{170}$. Il faut donc se demander de quel ordre est cette appartenance tardivement formulée par l'archéologie des sciences humaines.

D'un point de vue strictement kantien, que ne dépasse jamais l'introduction à l'Anthropologie, Dieu n'est qu'une idée à la fois nécessaire mais sans contenu, dont la raison doit faire un usage hypothétique et régulateur ${ }^{171}$. Si une telle idée a également un rôle de «vivification $\gg$ de l'esprit, entendu dans sa diversité empirique comme Gemüt, cet usage ne peut prétendre envelopper ou totaliser l'existence concrète ${ }^{172}$. Confinée dans l'a priori, l'idée de Dieu n'a donc rien dans l'expérience sensible qui lui corresponde. Si l'on s'en tient à cette position de la thèse complémentaire, il n'y a pas d'appartenance entre Dieu et l'homme qui puisse être directement référée à l'expérience que nous faisons de l'être. Dans ces conditions, s'il faut parler d'un dénouement des relations entre Dieu et l'homme, il ne peut signifier autre chose qu'une simple déduction logique, non une mort tragique : si l'homme disparaît comme sujetobjet de connaissance, il emporte avec lui le Dieu qu'il a inévitablement pensé. Dans cette perspective, Nietzsche ne fait que mener à son terme la critique kantienne, à laquelle il reste fidèle jusque dans sa volonté d'aller plus loin que Königsberg.

170 Notons cependant que l'éternel retour est bien mentionné dans le corps de l'introduction. Mais il n'est pas question alors des rapports entre l'homme et le divin. Il s'agit d'indiquer la présence précoce, «pour une culture déjà éloignée», d'une réflexion sur «l'a priori, l'originaire et la finitude », partage posé par l'œuvre de Kant («Introduction à l'Anthropologie », p. 68). La pensée matinale retrouvée par Nietzsche, cette « aurore» évoquée par Foucault, reste donc de bout en bout kantienne, car elle reprend strictement la distinction entre les trois domaines, dont nulle fusion ultime n'est envisageable.

171 E. Kant, Critique de la raison pure, III, 426-442, déjà cité, p. 552-572.

172 Foucault est très clair sur ce point : le Gemüt ne chemine pas vers une «totalisation » de lui-même sous l'influence des idées régulatrices («Introduction à l'Anthropologie », p. 38). Foucault déploie ici une fine analyse de la deuxième sous-partie de l'Appendice à la dialectique transcendantale, «Du but final de la dialectique naturelle de la raison humaine » (E. Kant, Critique de la raison pure, III, 442-461, déjà cité, p. 572-597), à travers ce prisme du rapport entre le Geist et le Gemüt. 
En 1966, la mention d'un lieu «retrouvé » par Nietzsche nous emmène au contraire en deçà de Kant, dans une région reculée dont Foucault ose faire le « commencement de la philosophie ${ }^{173}$. L'appartenance entre Dieu et l'homme qui se révèle au terme de ce retour ${ }^{174}$, en ce lieu où la courbure du temps semble s'enrouler sur elle-même plutôt qu'atteindre une origine historique précise, ne peut plus désigner une simple liaison entre des concepts. Le verbe « appartenir » est par ailleurs trop fort. Il suggère un rapport concret, dont le contenu ne nous est pas donné par Foucault, mais que la figure de Dionysos pourrait nous indiquer silencieusement. En cette aurore de la pensée, le vocable «Dieu » semble en effet renvoyer à une détermination de l'être lui-même, et non plus à une simple idée régulatrice. Si l'essai de 1966 ne cite à aucun moment le dieu grec de la vigne, il laisse entrevoir la possibilité de penser cette appartenance mutuelle de Dieu et de l'homme autrement que sur le mode transcendantal. Si l'on suit cette voie dionysiaque entre les lignes du texte foucaldien, une telle appartenance ne serait autre que la disparition du sujet par immersion dans un tout qui n'est qu'apparences; une fusion complète entre l'homme et la totalité de ce qui advient (douleur, plaisir, création, destruction) dans « l'éternelle joie du devenir $\gg^{175}$ où tout est à la fois subi et indéfiniment voulu, où la volonté imprime aux choses sa puissance et revêt en retour toutes les enveloppes et émotions possibles. « Dieu est mort » ne fait pas perdre aux dieux leurs scintillements infinis, mais fait perdre au moi son unité. Dans un texte que Foucault connaissait, Pierre Klossowski ne disait pas autre chose :

Dieu est mort ne signifie pas que la divinité cesse en tant qu'une explicitation de l'existence, mais bien que le garant absolu de l'identité du moi responsable disparaît à l'horizon de la conscience de Nietzsche lequel, à son tour, se confond avec cette disparition. ${ }^{176}$

Mais pour que cette disparition ait un fondement au-delà de la seule expérimentation individuelle, encore faudrait-il transformer en une ontologie rigoureuse ce qui, chez le promeneur de Sils-Maria, n'est d'abord qu'une valeur à vouloir pour sa propre vie. Ce que Foucault nomme

173 Les mots et les choses, p. 353 .

174 Foucault se plaît d'ailleurs à mettre une majuscule à ce « Retour » (ibid., p. 353).

175 F. Nietzsche, Le crépuscule des idoles, suivi de Le cas Wagner, déjà cité, p. 177.

176 P. Klossowski, «Nietzsche, le polythéisme et la parodie », Un si funeste désir, déjà cité, p. 220. 
$l^{\prime} \ll$ expérience de Nietzsche ${ }^{177}$ nous dit-elle plus que cela ? Nous dit-elle en particulier quelque chose de l'être et de l'origine? Peu d'éléments dans le texte de 1966 nous permettent de répondre franchement par l'affirmative. Le passage à une compréhension ontologique des thèses nietzschéennes pourrait être indiqué par la curieuse mention d'une «certaine forme de biologisme $\gg(\mathrm{p} .353)$ chez le penseur prussien : selon Foucault, celle-ci est, avec la critique philologique, la source principale de sa pensée du retour. Mais le lyrisme dont use le philosophe à cet endroit ne permet pas de trancher définitivement. $L^{\prime} \ll$ ontologie purifiée $»(p .353)$ et la «pensée radicale de l'être $\gg(\mathrm{p} .353)$, indiquées quelques lignes plus haut, semblent quant à elles désigner Heidegger comme une étape intermédiaire qui n'aurait pas encore pris toute l'ampleur de la proposition nietzschéenne ${ }^{178}$. Dans Les mots et les choses, le passage à une vérité de l'être reste de l'ordre de l'interrogation ${ }^{179}$, et l'appartenance mutuelle de l'homme et de Dieu, un oracle sibyllin - à l'image du «scintillement multiple et recommencé des dieux $\gg$ (p.317-318) promis par Nietzsche.

Pourtant, un texte plus ancien vérifie ce glissement possible de l'interprétation foucaldienne hors de la filiation kantienne. Déjà longuement commenté, l'article consacré à Georges Bataille en 1961 noue ensemble, par l'intermédiaire de Nietzsche, l'expérience du divin et l'accès pour l'homme à une « origine sans positivité ${ }^{180}$. Situé chronologiquement entre la thèse complémentaire de 1961 et l'archéologie des sciences humaines de 1966, cet article évoque sans ambages la promesse d'une «pensée qui serait, absolument et dans le même mouvement,

177 Les mots et les choses, p. 353 .

178 Cette « ontologie purifiée », sans doute heideggérienne, ne désigne pour Foucault que l'un des nombreux efforts pour dépasser l'anthropologique. Si Nietzsche représente historiquement le premier de ces efforts, Foucault lui accorde une primauté durable, encore insurpassée : il «marque le seuil à partir duquel la philosophie contemporaine peut recommencer à penser; il continuera sans doute longtemps à surplomber son cheminement $\gg$ (ibid., p. 353).

179 C'était déjà sous forme de question que Foucault avait amené cette question de l'être : dans le détachement du langage du domaine de la représentation, « faut-il [...] pressentir la naissance, moins encore, la première lueur au bas du ciel d'un jour qui s'annonce à peine, mais où nous devinons déjà que la pensée $[\ldots]$ va se ressaisir en son entier et s'illuminer à nouveau dans l'éclair de l'être? N'est-ce pas ce que Nietzsche préparait, lorsqu'à l'intérieur de son langage, il tuait l'homme et Dieu à la fois, et promettait par là avec le Retour le scintillement multiple et recommencé des dieux? » (p. 317-318). Ici encore, la première question semble se référer à Heidegger plutôt qu'à Nietzsche.

$180 \mathrm{DE} \mathrm{n}^{\circ} 13$, « Préface à la transgression », I, p. 267. 
une Critique et une Ontologie » (p. 267); une pensée qui ferait de notre expérience des limites une expérience de l'être. Cette promesse, poursuit l'article, Nietzsche l'a formulée à sa manière « en replaçant l'expérience du divin au cœur de la pensée $\gg($ p. 267). Proposition théologique audacieuse, qui transgresse évidemment et volontairement l'interdit kantien - la Critique de la raison pure ayant rejeté Dieu et l'unité absolue des phénomènes hors de toute expérience possible.

Miroitement ironique des influences : cette interprétation du surhomme et de l'éternel retour en termes ontologiques, qui contredit à sa manière la thèse complémentaire de 1961, doit tout ou partie à l'essai de Heidegger sur Nietzsche, que Pierre Klossowski traduira cinq ans plus tard ${ }^{181}$.

Si nous rassemblons les résultats de cette partie, ouverte et refermée par l'article consacré à Georges Bataille, le divin désigne chez Foucault ce qu'expérimente l'homme quand il disparait comme sujet de son langage et de sa pensée : quand il va au bout de ses possibles et rencontre l'être de la limite, comme chez Bataille; quand il se perd dans l'ivresse d'une volonté perpétuelle, comme chez Nietzsche. Ces gestes sont d'abord des expériences langagières, dans lesquelles les mots cessent d'être transparents aux choses pour révéler «leur énigmatique épaisseur $\gg^{182}$ où disparaît le sujet parlant. Selon qu' il commente Blanchot ou Bataille, le langage est en effet présenté par Foucault à la fois comme le lieu originaire d'où naissent toutes les expériences ${ }^{183}$, et le lieu final où elles viennent perdre leur naturalité et se résorber ${ }^{184}$. La pensée du divin serait alors

181 M. Heidegger, Nietzsche, P. Klossowski trad., Paris, Gallimard (Bibliothèque de philosophie), 1971, 2 volumes. Dans son dernier entretien, Foucault souligne l'importance que l'interprétation heideggérienne de Nietzsche a eue pour sa propre lecture de Nietzsche : «Il est probable que si je n'avais pas lu Heidegger, je n'aurais pas lu Nietzsche. J'avais essayé de lire Nietzsche dans les années cinquante, mais Nietzsche tout seul ne me disait rien! Tandis que Nietzsche et Heidegger, ça a été le choc philosophique ! » (DE n ${ }^{\circ} 354$, «Le retour de la morale », II, p. 1522).

182 Les mots et les choses, p. 315 .

$183 \ll[\ldots]$ il n'existe que le langage, et ce dont nous parlons, c'est du langage, nous parlons à l'intérieur du langage, etc. $\gg\left(D E n^{\circ} 22\right.$, « Débat sur le roman $\gg, I$, p. 408$)$.

184 « Ce n'est pas notre langage qui a été, depuis bientôt deux siècles, érotisé : c'est notre sexualité qui depuis Sade et la mort de Dieu a été absorbée dans l'univers du langage, dénaturalisée par lui, placée par lui dans ce vide où il établit sa souveraineté 》 $\left(D E \mathrm{n}^{\circ}{ }^{13}\right.$, « Préface à la transgression », I, p. 276). 
non pas la saisie objective ou méditative du dieu, mais une expérience vécue aux limites mêmes du langage, sur lesquelles les textes que nous venons d'étudier se gardent bien de nous éclairer plus avant.

Dans ces conditions, inspiré ici par Heidegger, le philosophe français ne ferme pas la porte à une refonte de la métaphysique, subrepticement entrouverte par la Critique de la raison pure ${ }^{185}$. Ce que l'œuvre de Kant a laissé en suspens, c'est la possibilité de poser à nouveaux frais la question métaphysique à partir de notre finitude, au lieu d'en rester à un partage strict et mortifère : à la Critique, l'ordre de la connaissance, à l'Anthropologie, l'ordre de l'existence. Une autre métaphysique serait donc possible, qui n'exalterait plus l'homme comme lieu d'ouverture à un absolu pensé hors de ce monde-ci, ni même comme lieu originel d'interrogation de la finitude par elle-même. Car en faisant de l'homme la condition d'accès à la vérité - que cet accès se loge dans ses capacités cognitives ou, plus originellement, dans son être-au-monde - la philosophie occidentale a entériné une série de partages (sujet/objet, réalité/apparence, apparition/retrait) qui volent à l'homme une part de ses possibles.

Mais quelle autre métaphysique pourrait bien désigner Foucault dans son article sur Bataille, sinon la «métaphysique d'artiste » revendiquée par Nietzsche dans son Essai d'autocritique ${ }^{186}$ ? Celle qui ne croit pas en un autre monde et ne considère pas les oppositions dictées par la raison et le langage comme des oppositions réelles. Qu'elles soient d'origine scientifique, philosophique ou religieuse, les métaphysiques depuis Platon ont divisé l'être en deux, à l'image de l'ancienne gnose dont Foucault soupçonne le christianisme de ne s'être jamais complètement libéréé ${ }^{187}$. Nous avons eu l'occasion de le vérifier en relisant la préface de Folie et

185 Que cette porte ait été entrouverte par la première Critique, l'article sur Bataille l'indique clairement, en rappelant «le jour où [Kant] a articulé, sur un mode encore bien énigmatique, le discours métaphysique et la réflexion sur les limites de notre raison » (ibid., p. 267). Mais l'Anthropologie a refermé cette porte aussi sec. Cette position est ici directement inspirée par l'interprétation heideggérienne de Kant (M. Heidegger, Kant et le problème de la métaphysique, déjà cité).

186 «Artisten-Metaphysik» apparaît aux $\ 2$, 5 et 7 de l'Essai d'autocritique ajouté par Nietzsche à la nouvelle édition de La naissance de la tragédie. On trouve également chez Nietzsche les expressions synonymes : «métaphysique de l’art», «métaphysique du génie », «métaphysique dionysiaque », «métaphysique tragique ». Sur les multiples sens du terme «métaphysique » chez Nietzsche, voir P. Wotling, La philosophie de l'esprit libre. Introduction à Nietzsche, déjà cité, p. 53-85.

$187 D E n^{\circ} 21, \ll$ La prose d'Actéon », I, p. 354 . 
déraison, le grand partage de l'Occident, c'est le partage du partage, le partage de son objectivation. Chez quelques grands aînés, philosophes chresmologues ou simples expérimentateurs, Foucault guette une autre pensée de l'être - même s'il préfère en être le souffleur discret ou le coryphée occasionnel, sans la faire absolument sienne.

Si l'Orient a été la première tentation du christianisme, la métaphysique de «l'Un-originaire » (das Ur-Eine) ${ }^{188}$, célébrée par Nietzsche, est sans doute la dernière tentation de Foucault : la nostalgie d'un rapport au monde où la dénonciation de toute profondeur originaire de l'être, dans l'assomption des simulacres et des apparences, devient la révélation d'une réalité annoncée par le penseur allemand comme « plus profonde, plus primitive, plus métaphysique $\gg^{189}$; espace musical où tout est lié et revient sans cesse, sauf la subjectivité.

188 F. Nietzsche, La naissance de la tragédie..., déjà cité, p. 43-44.

189 «La volonté du paraître [...] y est donnée pour plus profonde (tiefer), plus primitive (ursprünglicher), plus métaphysique (metaphysicher), que la volonté de vérité, de réalité, d'être » (F. Nietzsche, Fragments posthumes, début 1888-début janvier 1889 [Euvres philosophiques complètes XIV], J.-C. Hémery trad., Paris, Gallimard, 1977, p. 269; il s'agit de l'extrait 17[3] du Nachlaß, datant de mai-juin 1888, portant sur les thèses de La naissance de la tragédie). 


\section{CHAPITRE HUIT \\ La relève d'un \\ temps précaire}

Faisons maintenant un saut d'une décennie : 1966-1976. Il nous faut pour cela accepter le résultat établi plus haut que Foucault cesse, jusqu’à $\mathrm{La}$ volonté de savoir inclus, de s'intéresser au christianisme comme totalité historique pour se concentrer sur certaines de ses péripéties tardives et circonstancielles, comme le christianisme tridentin. Après La volonté de savoir, le christianisme fait brusquement retour dans l'œuvre foucaldienne comme problème général. Après un détour par quelques hypothèses importantes formulées en 1977 et 1978, qui dénotent la volonté de penser à nouveaux frais la césure originelle entre le christianisme et l'Antiquité païenne, nous en viendrons à l'interprétation des grands cours au Collège, du Gouvernement des vivants (1979-1980) au Courage de la vérité (1983-1984). Loin de l'image facile d'un christianisme ascétique et intransigeant, Foucault y définit l'originalité chrétienne comme la reconnaissance et l'institution paradoxale d'un rapport précaire à la vérité.

Contrairement à ce qui précède, ce parcours s'assumera désormais comme essentiellement naiff. Il ne s'agira plus ici de questionner les sources de Foucault ni de vérifier la rectitude de ses informations, seulement d'écouter ce qu'il dit, qu'il le dise à partir d'un concept déterminé ou d'une constellation d'impressions subjectives, qu' il le lise dans la lettre des traités spirituels ou du fond d'une mémoire littéraire marquée par une intense fréquentation de Nietzsche et de Bataille. Aussi tenteronsnous, dans la dernière ligne droite de ce travail, quand il s'agira de commenter les leçons consacrées par Foucault au christianisme entre 1980 et 1984, de refermer les autres sources documentaires, pour ne pas freiner le galop de la pensée, tout en essayant d'en reproduire le tracé d'ensemble. 


\section{Premières hypothèses sur la différence chrétienne}

De l'œuvre nietzschéenne, plurielle et facétieuse, Foucault semble n'avoir conservé dans les années 1960 que l'idée d'une césure entre l'Orient et l'Occident ; césure mortifère dénoncée par Naissance de la tragédie, prolongée et approfondie dans Le gai savoir et Ainsi parlait Zarathoustra. C'est ce retour nietzschéen à l'Orient perdu, comme accès, par l'intermédiaire de la mythologie, à l'Ur-Eine, qui inspire le philosophe français dans ces années-là, même s'il ne se réfère pas directement au terme allemand Ursprung et parle prudemment d'une « origine sans positivité $\gg^{1}$. L'Ur-Eine est pour Nietzsche un magma originel où toutes les forces et émotions viennent se confondre sans principe d'individuation, en deçà de toute représentation. Seule la Grèce préhellénistique a véritablement compris et honoré cette fusion primordiale des forces de la nature.

L'adieu à cette conception peut être marqué symboliquement par l'article hommage à Jean Hyppolite publié en 1971 : « Nietzsche, la généalogie, l'histoire ». Dans ce texte qui prête une grande attention aux champs lexicaux, Foucault relève que le Nietzsche « généalogiste » récuse « au moins en certaines occasions, la recherche de l'origine (Ursprung) $\gg^{2}$. À la belle origine de l'Ursprung, qui suppose une identité première des choses sous leurs péripéties historiques, Foucault oppose alors l'origine basse de l'Herkunft, qu'il traduit par « souche » ou «provenance $\gg$ (ibid., p. 1008). Celle-ci ne renvoie qu'à un espace éclaté de luttes, sans harmoniques, où se reflètent déjà les analyses stratégiques à venir du philosophe français : celles consacrées à la prison et à la psychiatrie, croisant subrepticement la confession tridentine, l'Inquisition, ou même la sorcellerie, sans jamais prétendre y retrouver «ce qui était déjà $\gg^{3}$. En coupant en deux l'œuvre nietzschéenne, divisée désormais entre les œuvres qui opposent l'Ursprung à l'Herkunft (comme La généalogie de la morale) et celles qui les confondent encore (comme Humain, trop humain), Foucault barre la route à toute quête d'un commencement, même celui qui se donnerait comme pure négativité, ultérieurement recouverte par le déploiement dans l'histoire humaine d'une

$D E \mathrm{n}^{\circ}{ }_{13}$, « Préface à la transgression », I, p. 267.

$D E n^{\circ} 84$, « Nietzsche, la généalogie, l’histoire », I, p. 1006.

$D E n^{\circ} 84$, « Nietzsche, la généalogie, l’histoire », I, p. 1006. 
esthétique et d'un savoir conscient. Il abandonne ainsi pour dix ans l'opposition massive Orient/Occident qui sous-tendait aussi bien son étude sur la folie que ses exercices de critique littéraire. Dans un débat publié en 1972, Foucault réitère la coupure entre deux Nietzsche :

G. Preti : [...] Quel est le Nietzsche qui vous plait ?

M. Foucault : Évidemment, ce n'est pas celui de Zarathoustra ; c'est celui de L'origine de la tragédie, de La généalogie de la morale. ${ }^{4}$

Le découpage n'est pas exactement celui de l'article de 1971, puisque le préfixe $U r$ - («originaire ») appartient bien, comme nous l'avons vu, à Naissance de la tragédie - œuvre dont l'hommage à Jean Hyppolite ne dit étrangement mot, non plus d'ailleurs du Zarathoustra. Mais La généalogie de la morale, premier texte à opposer l'Ursprung et l'Herkunft, marque bien à nouveau la différence avec un autre Nietzsche, plus visionnaire qu'historiciste.

Dans ce contexte, le cours de 1979-1980 Du gouvernement des vivants peut être considéré comme un retour au questionnement initial, celui de la différence introduite par le christianisme dans l'histoire de la vérité. Reformulée à l'horizon neuf du sujet et des régimes de vérité, la question devient : qu'est-ce qui s'est joué dans les premiers siècles de notre ère pour que notre rapport à nous-mêmes et à la vérité se soit aussi radicalement modifié ? Retour à une périodisation longue, donc; retour surtout à l'équation culture occidentale = culture chrétienne ${ }^{5}$.

Revenir aux Pères, c'est en effet approcher de la déchirure entre deux mondes. Préalablement au cours de 1979-1980, l'entretien publié en 1977 dans la revue Ornicar ? et le cours Sécurité, territoire, population avaient déjà été glaner dans les sources patristiques, mais sans disposer de l'outil conceptuel des « régimes de vérité », ni d'un dispositif précis de lecture des textes tel que nous l'avons défini dans la deuxième partie. L'entretien et le cours proposaient pourtant tous deux une ébauche de réponse à la question de la différence chrétienne, que le cours de 1979-1980 va à la fois affiner et déplacer, par souci de méthode.

En 1977, dans une joute oratoire amicale avec Jacques-Alain Miller, la «chair » est brièvement indiquée par Foucault comme l'invention fon-

$4 D E \mathrm{n}^{\circ}$ 109, « Les problèmes de la culture. Un débat Foucault-Preti », I, p. 1240.

5 Leçon du 19 mars $1980:$ « [... ] ce qui constitue la subjectivité chrétienne et, par conséquent, la subjectivité occidentale ». 
damentale d'un dénommé Tertullien, écrivain chrétien décédé vers $220^{6}$. Cette proposition se situe clairement dans le champ d'une analyse des pratiques discursives, déjà visité par les leçons de 1975 sur les manuels des confesseurs, et retrouvé en 1978 dans le manuscrit de La chair et le corps. Il s'agit en effet, dans cet ensemble homogène de recherches, de préciser ce à quoi s'est appliqué à travers les siècles le «discours de sexualité $\gg^{7}$, non comme un objet préalable que les mots auraient désigné de l'extérieur, mais comme le produit d'un dispositif à la fois discursif et institutionnel, qui visait à répondre à un problème de gouvernement. Cette notion de chair est à nouveau évoquée à Tokyo dans la conférence «Sexualité et pouvoir » en avril 1978, comme innovation décisive qui marqua l'apparition d'un type inédit de pouvoir. La chair est un discours qui dépose sous la surface de la peau un entrelacs de sensations et de volitions dont le sujet doit désormais rendre compte :

La chair, c'est la subjectivité même du corps, la chair chrétienne, c'est la sexualité prise à l'intérieur de cette subjectivité, de cet assujettissement de l'individu à lui-même qui est l'effet premier de l'introduction dans la société romaine du pouvoir pastoral. ${ }^{8}$

La «chair » est un opérateur du discours qui permet d'exercer un pouvoir constant de surveillance en faisant du corps un objet inépuisable d'introspection et de verbalisation. Elle fait du corps le lieu principal de manifestation d'une volonté qui sans cesse cède ou se refuse au plaisir, et ne se découvre agissant de la sorte que par le truchement de ce qui deviendra ultérieurement l'aveu au directeur ou au confesseur.

Une telle définition apparaissait déjà dans le cours Les anormaux, au début de la leçon du 26 février 1975. Mais l'entretien publié dans Ornicar?, et à sa suite la conférence de Tokyo, prolongent la réflexion dans une direction nouvelle : reprenant en les modifiant les leçons prononcées sur le christianisme tridentin, Foucault raccroche l'invention discursive de la chair à un problème de gouvernement pastoral beaucoup plus ancien, dans la ligne du cours qui venait alors de s'achever, Sécurité, territoire, population. Si la chair a fleuri de manière remarquable sous le soleil de l'âge classique, le bourgeon était déjà perceptible aux $\mathrm{III}^{\mathrm{e}}$ et IV ${ }^{\mathrm{e}}$ siècles de notre ère : dans l'œuvre des Pères latins tout d'abord, aux

$6 D E \mathrm{n}^{\circ} 206$, «Le jeu de Michel Foucault », II, p. 313.

$7 D E \mathrm{n}^{\circ} 206$, « Le jeu de Michel Foucault », II, p. 313, nous soulignons.

$8 D E \mathrm{n}^{\circ} 233$, «Sexualité et pouvoir », II, p. 566. 
prises avec les hérésies gnostiques, dans une société chrétienne laïque ensuite, mise en question par la radicalité ascétique du premier monachisme. L'entretien d'Ornicar? indique brièvement le premier moment, la conférence à Tokyo développe plus longuement le second. Ensemble, les deux interventions dessinent à l'intérieur du christianisme des premiers siècles la même ligne de fracture : entre une société romaine à la morale monogame orientée vers la reproduction d'une part, et l'ascétisme extrême des gnostiques et des premiers moines, dénonçant la sexualité et la vie mondaine d'autre part. Or, cette possibilité d'éclatement, c'est l'invention de la chair qui la conjure :

Entre une société civile qui avait accepté un certain nombre d'impératifs moraux et cet idéal de l'ascétisme intégral, le christianisme a toujours hésité [...]. Je crois que c'est la conception très difficile, d'ailleurs très obscure, de la chair qui a servi, qui a permis d'établir cette sorte d'équilibre entre un ascétisme qui refusait le monde et une société civile qui était une société laïque. ${ }^{9}$

L'originalité chrétienne est cette définition neuve de la chair, qui permet de faire se rejoindre deux tendances opposées de la société de l'Antiquité tardive. Tel est bien l' «impératif stratégique $\gg^{10}$ des premiers siècles. La chair introduit l'inquiétude perpétuelle chez les laïcs en règle avec la morale d'alors enjoignant de se marier et d'avoir des rapports sexuels en vue de procréer. Mais en même temps, elle empêche que le corps soit méprisé dans une mortification excessive. Elle permet de trouver ce point d'équilibre entre deux extrêmes, réunissant l'homme marié et le moine dans un même dispositif pastoral, où tous deux sont aux prises avec un plaisir charnel qui risque à chaque instant de les conduire à la faute, sans pour autant être intrinsèquement condamnable.

Mais à y regarder de près, dans ces recherches de 1977 et 1978, la coupure ne passe pas exactement à l'intérieur du christianisme, mais entre le christianisme et des éléments extérieurs dont celui-ci doit s'accommoder : dans l'entretien publié dans Ornicar ?, il s'agit du dualisme des gnostiques (courant ésotérique venu principalement de Grèce ou d'Iran) ${ }^{11}$; dans la conférence de Tokyo, il s'agit à la fois de la morale essentiellement romaine, d'obédience stoïcienne, et du monachisme

$9 D E \mathrm{n}^{\circ} 233$, « Sexualité et pouvoir », II, p. 565 .

$10 D E n^{\circ} 206$, « Le jeu de Michel Foucault », II, p. 299.

11 Ibid., p. 313, nous soulignons. 
hindou et bouddhiste ${ }^{12}$. Cette extériorité permet au cours Sécurité, territoire, population de formuler la proposition discutée dans notre première partie selon laquelle, dans sa forme pastorale, le christianisme est «fondamentalement anti-ascétique $»^{13}$; et ce, malgré l'effort ultérieur du pouvoir ecclésial pour réglementer, et par là s'approprier, les contreconduites qui s'opposaient à son exercice.

De l'entretien pour Ornicar? à Sécurité, territoire, population, la réponse donnée à la question de l'originalité historique du christianisme est donc sensiblement la même : le christianisme a développé une forme inédite de pouvoir par souci d'intégration de ce qui ne lui appartenait pas en propre. Seule différence notable, l'entretien de 1977 répond par la solution, là où le cours de 1977-1978 répond par le problème : c'est d'abord parce que le christianisme a eu la prétention démesurée et inédite de gouverner toutes les âmes qu'il a été contraint ensuite de trouver un moyen permettant de concilier des stratégies morales différentes. La véritable innovation chrétienne est donc le pastorat, avant d'être la chair.

Revenons sur la définition du premier terme. « Pastoral $\gg$ est le pouvoir qui prend en charge la totalité des existences, de leur naissance à leur mort, pour les mener à une fin située au-delà de ce monde-ci, mais à laquelle toute chose, dès ici-bas, doit être ordonnée ${ }^{14}$. Dédiant son action à cette unique tâche, la pastorale chrétienne assure à chacun un salut spirituel par des techniques spécifiques de gouvernement qui ne peuvent être subordonnées à la réalisation ou au maintien d'une quelconque structure politique. Il ne s'agit donc pas pour Foucault de faire du salut une idéologie religieuse qui justifierait l'exercice caché d'un pouvoir séculier, mais au contraire de prendre au sérieux - sinon au pied de la lettre - cette volonté salvifique, dans sa singularité.

Si le théologique n'est pas ici au service du politique, le salut escompté n'est pourtant pas affaire de choix individuel, comme l'indique - tel un double nœud - la redondance des termes coercitifs dans un passage d'une autre conférence donnée à Tokyo, en juin 1978 : l'effet du pouvoir pastoral est un « rapport obligé de soi-même à soi-même en

$D E n^{\circ} 233$, « Sexualité et pouvoir », II, p. 565 .

Sécurité, territoire, population, p. 211. Voir notre discussion : Première partie, «Évaluation critique de l'histoire pastorale $\gg$, p. 78-91.

Sécurité, territoire, population, p. 240-241. 
terme de vérité et de discours obligé $\gg^{15}$. C'est donc un rapport modelé de l'extérieur par les techniques de direction, d'examen et de confession, sur fond d'hétéronomie du sujet.

Foucault va alors déplier les modalités de ce rapport dans trois directions : le rapport au salut, le rapport à la loi, le rapport à la vérité. Le cours Sécurité, territoire, population aboutit ainsi à une présentation méthodique, extrêmement organisée, des premiers siècles chrétiens. Nous la résumons dans le schéma suivant:

\begin{tabular}{|c|c|c|c|}
\hline Thème & $\begin{array}{l}\text { Type de } \\
\text { pouvoir }\end{array}$ & Caractéristiques & $\begin{array}{c}\text { Vocabulaire } \\
\text { de la direction }\end{array}$ \\
\hline SALUT & Distributif & $\begin{array}{l}\text { - Responsabilité analytique } \\
\text { - Transfert exhaustif instantané } \\
\text { - Inversion du sacrifice } \\
\text { - Correspondance alternée }\end{array}$ & $\ll$ Opération de direction $»^{16}$ \\
\hline LOI & $\begin{array}{l}\text { Dépendance } \\
\text { intégrale }\end{array}$ & $\begin{array}{l}\text { - Rapport à un individu } \\
\text { - Rapport non finalisé } \\
\text { - Rapport généralisé }\end{array}$ & $\ll$ Individu dirigé ${ }^{17}$ \\
\hline VÉRITÉ & Surveillance & & $\begin{array}{l}\text { «Direction de la conduite } \\
\text { quotidienne } » \\
\ll \text { Direction de conscience }{ }^{18}\end{array}$ \\
\hline
\end{tabular}

Comme nous pouvons le vérifier dans ce tableau, la «direction », entendue à la fois comme thème général de la recherche et comme technique spécifique de pouvoir, traverse l'ensemble de l'analyse. Au titre de notion générale, en partie forgée par l'historien, la direction apparait dès la partie consacrée au «salut » comme description large des relations entre le pasteur et ses brebis. Au titre de technique particulière, visant à établir un « rapport de soumission d'un individu à un autre individu » (ibid., p. 178), la notion apparaît ensuite dans la partie consacrée à la « loi », avec la vie monastique comme principal exemple. Cette direction, comme pratique codifiée de gouvernement, est alors directement lue dans les textes spirituels : les Institutions cénobitiques de Cassien et la Règle de saint Benoît. Enfin, à nouveau thème général, la

$D E \mathrm{n}^{\circ} 232$, « La philosophie analytique de la politique », II, p. 549, nous soulignons.

Sécurité, territoire, population, p. 172

Ibid., p. 178

Ibid., p. 184 
direction revient dans la partie consacrée à la «vérité », avec «la vie quotidienne $\gg$ et la « conscience $\gg$ comme objets principaux d'intervention. La référence est alors saint Grégoire et ses réflexions sur la charge du pasteur (la Regula pastoralis).

La transversalité de cette notion vient de l'homogénéité supposée, dans leur relation au pouvoir, des différents types de vie chrétienne : vie laïque et vie monastique. Dans le cours Sécurité, territoire, population, le mouvement monastique est partie prenante du développement global du pouvoir pastoral, comme le vérifie le parallélisme établi entre l'abbé et l'évêque, c'est-à-dire entre le chef du monastère et le chef de l'Église locale ${ }^{19}$, sans que le niveau où les deux domaines se rencontrent concrètement ne soit explicité ${ }^{20}$, et alors même que le droit de l'Église empêche les deux pouvoirs d'interférer directement ${ }^{21}$. Dans cette perspective, rien d'étonnant à ce que la Règle de saint Benoît, que ce dernier rédigea à l'intention de ses frères moines, soit citée après les conseils aux laïcs de l'évêque de Constantinople saint Jean Chrysostome ou du pape Grégoire le $\mathrm{Grand}^{22}$, sans distinction particulière. $\mathrm{Si}$, dans le monachisme qui se développe entre les $\mathrm{IV}^{\mathrm{e}}$ et $\mathrm{VI}^{\mathrm{e}}$ siècles, ce rapport de direction tend à l'obéissance parfaite, à l'«irréflexion » (ibid., p. 179), au « renoncement définitifà toute volonté propre $\gg($ p.181), cette description peut être appli-

bid., p. 182 : «le pasteur, qu'il soit abbé ou qu'il soit évêque ».

Ce n'est qu'en 1981, dans les leçons de Louvain, que Foucault repère explicitement cette rencontre dans certains textes patristiques : «C'est ainsi qu'on trouve cette définition qui date du $\mathrm{IV}^{\mathrm{e}}$ siècle dans l'Orient chrétien, le texte de Grégoire de Naziance, où il montre bien comment la direction spirituelle, pratique ascétique, pratique essentiellement destinée aux communautés monastiques, comment cette pratique est en train de se diffuser dans les communautés chrétiennes en général 》 (Cours Mal faire, dire vrai. Fonctions de l'aveu). Le texte en question est un discours, déjà cité dans le cours Sécurité, territoire, population, où Grégoire parle de la direction comme de l'« art des arts », la « science des sciences » (Sécurité, territoire, population, p. 154). Mais en même temps, comme nous allons le voir, malgré la mention de cette diffusion des pratiques ascétique au-delà de la clôture des monastères, le cours de Louvain insiste fortement sur la différence entre deux types de gouvernement au sein du christianisme. Plus précisément : c'est justement parce que Foucault perçoit en 1981 la césure, qu'il a besoin de montrer les interactions historiques entre deux pratiques hétérogènes. En 1978, la question ne se pose pas : le gouvernement pastoral est considéré comme une structure homogène s'appliquant à tous; et le philosophe cite Grégoire de Naziance sans distinguer les publics auxquels la direction est destinée.

Voir par exemple le concile d'Arles en 453-454 (455?) qui régla, en respectant strictement cette distinction des deux pouvoirs, le litige juridictionnel entre l'abbé de Lérins Fauste et l'évêque Théodose de Fréjus.

Sécurité, territoire, population, p. 172. 
quée d'une manière beaucoup plus large au «pastorat chrétien » (p.177). Foucault en arrive ainsi à la formule générale d'un dispositif qui connaît des degrés d'intensité mais pas de saut qualitatif : dans tous les cas, il s'agit de faire de chaque acte un acte ordonné par un autre, sans place laissée à la volonté propre. Du laïc au moine, il n'y a tout au plus qu'une gradation : « C'est vrai pour les laïcs, mais c'est vrai bien sûr, d'une façon beaucoup plus intense, pour les moines $\gg$ (p.179, nous soulignons).

L'entrelacement des références permet de faire de la direction la principale caractéristique du pouvoir pastoral, voire du christianisme tout court. Comme nous l'avons vu, Foucault hésite cependant à employer le terme générique de «christianisme »-qui recouvre une trop grande hétérogénéité de faits - et précise sa pensée :

$[\ldots]$ le terme «christianisme » n'est pas exact, il recouvre en vérité toute une série de réalités différentes. Il faudrait sans doute dire avec, sinon plus de précision, du moins un peu plus d'exactitude, que le pastorat commence avec un certain processus qui, lui, est absolument unique dans l' histoire et dont on ne trouve aucun exemple sans doute dans aucune autre civilisation : processus par lequel une religion, une communauté religieuse s'est constituée comme Église, c'està-dire comme une institution qui prétend au gouvernement des hommes dans leur vie quotidienne $[\ldots]$, et ceci à l'échelle non seulement d'un groupe défini, non seulement d'une cité ou d'un État, mais de l'humanité tout entière. (p. 151)

Le christianisme n'a pas d'unité historique évidente; son histoire complexe ne se déploie pas de manière homogène à partir d'une communauté primitive qui aurait vécu selon une certaine règle, en se rapportant à un certain modèle. Le pastorat « commence », nous dit Foucault, sans donner encore de date précise, avec la volonté d'universel, contemporaine du développement d'une institution nommée «Église », postérieure à la simple « communauté religieuse ».

Avec le pouvoir ecclésial, nous sommes au-delà d'une souveraineté qui s'exercerait sur un territoire et sur les sujets qui y résident. Une terminologie juridique et constitutionnelle serait ici impropre pour décrire un gouvernement qui se déploie d'abord comme art d'administrer n'importe quel individu, où qu'il se trouve, quelle que soit la constitution politique qui le protège et le contraint. Nous sommes dans un gouvernement qui ne correspond ni à une nation (requérant une communauté limitée d'hommes) ni à un État (requérant un territoire). La possession de territoires et le pouvoir administratif exercé par certains évêques sur leurs diocèses ne suffisent pas à définir le pouvoir ecclésial dans 
toute son étendue. Pour cette raison, Foucault ne se préoccupe pas de la conversion de l'empereur Constantin au IV ${ }^{\mathrm{e}}$ siècle, ni du passage de l'Empire tout entier au christianisme. Pour décrire l'essor du pastorat chrétien, la périodisation qu'il propose est plus large : «disons depuis le $\mathrm{II}^{\mathrm{e}}, \mathrm{III}^{\mathrm{e}}$ siècle après Jésus-Christ jusqu'au XVIII ${ }^{\mathrm{e}}$ siècle de notre ère » (p.152). Si l'origine christique est écartée par la mention du $\mathrm{II}^{\mathrm{e}}$ siècle, ce sont bien les premières grandes communautés chrétiennes organisées (Carthage au III siècle, avec la mention de l'évêque Cyprien page 173, Milan au IV e siècle, avec la mention de l'évêque Ambroise page 183) qui sont convoquées comme lieu de naissance d'une forme inédite de gouvernement des hommes, indépendante des évolutions théologicopolitiques de l'Empire : la direction de tous et de chacun.

Or, cette notion centrale de direction, le cours $D u$ gouvernement des vivants va à la fois en resserrer le champ d'application et en modifier la méthode d'analyse.

1) Resserrement tout d'abord de la direction autour de la technique particulière par laquelle un individu surveille continûment un autre et peut en permanence intervenir sur lui, par l'ordre ou le conseil. Plus question d'y voir un thème général par le biais d'une analyse qui engloberait, au-delà des différences de degrés et d'intensité dans les obligations requises, la charge de l'évêque et celle de l'abbé (ou du maître des novices). Plus de description synchronique d'un régime général de pouvoir, construit par l'historien au-dessus des particularités historiques, simplement exemplifié par les textes cités. Dans son sens étroit et technique, une telle relation codifiée n'existe pas dans le christianisme avant le IV ${ }^{\mathrm{e}}$ siècle et l'apparition de l'institution monastique. Il faut donc dissocier le gouvernement pastoral en son sens le plus général de la technique de direction :

Ce thème pastoral, donc, est important, il est évident, mais il ne coïncide pas avec l'idée ou avec la technique d'une direction. Il ne coïncide pas avec l'idée d'une intervention permanente $[\ldots]$. Le thème du pouvoir pastoral n'implique pas une technique de direction, même si, plus tard, lorsque cette technique de direction sera développée à l'intérieur du christianisme, c'est sous le signe du pastorat qu'elle sera placée. ${ }^{23}$ 
2) Modification ensuite des présupposés permettant d'étudier cette technique. Contrairement à ce que laissait entendre le cours Sécurité, territoire, population, la direction requiert de bout en bout la liberté du dirigé. Du point de vue du droit de l’Église, explique Foucault en 1980, la relation de direction n'est pas obligatoire, même dans les séminaires de la Contre-réforme, où seule l'est canoniquement la confession. La coupure entre les deux cours est si nette sur ce point, qu'il est intéressant de mettre en regard les deux propositions :

1978 :

Dans la pratique chrétienne, on va avoir une direction de conscience et des formes d'examen qui vont être tout à fait différentes [de l'Antiquité]. Premièrement, parce que la direction de conscience n'est pas exactement volontaire. Elle ne l'est pas en tout cas toujours, et dans le cas des moines par exemple la direction de conscience est absolument obligatoire, on ne peut pas ne pas avoir un directeur de conscience. ${ }^{24}$

1980 :

Dans la direction - et c'est bien ce qui fait la différence entre la direction de conscience $[\ldots]$ et toute structure de type politique ou juridique -, il n'y a ni sanction ni coercition. Le dirigé veut toujours être dirigé, et la direction ne tiendra, la direction ne fonctionnera, elle ne se déroulera que dans la mesure où le dirigé veut toujours être dirigé. [...] Le jeu de l'entière liberté, dans l'acceptation du lien de direction, est, je crois, fondamental. ${ }^{25}$

La liberté n'est donc pas une concession ou une forme de résistance qui limiterait de l'extérieur la direction, mais elle est au contraire la condition de possibilité de cet exercice du pouvoir. Le cours inédit de Louvain Mal faire, dire vrai insiste d'ailleurs sur le fait que la direction ininterrompue, supposant l'ouverture constante du cœur à un autre qui a charge d'autorité, afin que chaque acte et chaque volonté du sujet deviennent un effet de l'obéissance, est un «programme idéal et parfaitement irréalisable $\gg$, même dans les monastères. La direction est donc moins une sorte de panoptique spirituel qui mettrait constamment le sujet sous observation extérieure, que l'objet d'un effort intérieur, demandant au sujet de s'ajuster ${ }^{26}$ autant qu'il le peut, mais aussi

Sécurité, territoire, population, p. 185.

Leçon du 12 mars 1980.

Foucault ne dit pas littéralement que le sujet «s'ajuste » lui-même, mais qu'il y a une « nécessité d'ajuster cette règle impossible et intenable de l'exagoresis complet » (Cours Mal faire, dire vrai. Fonctions de l'aveu). 
autant qu'il le veut - puisque la liberté en est le principe premier. Cet ajustement va s'opérer en déclinant l'idéal recherché dans une série de pratiques toujours ponctuelles et incomplètes (examen de conscience le soir et le matin, confession des péchés à son directeur, entretiens réguliers avec le directeur, etc), dont la somme approche tangentiellement du résultat escompté.

Cette deuxième modification est décisive; elle répond aux exigences de la notion nouvelle de «régime de vérité », comme reconnaissance de la part qui revient en propre au sujet dans les techniques de soi. Elle ne nous permet pas cependant de pousser plus avant la compréhension de la différence chrétienne étant donné que ce nouveau présupposé de «l'entière liberté » s'applique aussi bien aux techniques païennes qu'aux techniques chrétiennes de direction.

Si la première modification ne répond pas non plus à la question, elle la rend cependant plus complexe en soulignant que l'expression « régime de vérité », pour ce qui est du christianisme, doit s'entendre au pluriel. Il nous faut donc corriger le titre donné dans notre première partie, qui se contentait d'un singulier : «Le christianisme comme régimes de vérité ». La notion de «pouvoir pastoral » rassemblait sous un même chef différentes techniques chrétiennes en mettant entre parenthèses le fait qu'elles étaient nées dans des lieux différents (diocèses/ monastères) et à des moments différents de l'histoire du christianisme (avant ou après le $\mathrm{IV}^{\mathrm{e}}$ siècle). Foucault affine désormais le découpage en distinguant plusieurs forces en présence et plusieurs vitesses au sein de l'histoire chrétienne.

Or, si l'on entend par subjectivation l'activité qui permet de donner au rapport à soi une forme définie, une pastorale sans technique de direction ne produit pas le même type de subjectivation qu'une pastorale reposant essentiellement sur ce mode bien particulier de liaison entre deux volontés qu'est la recherche d'un guidage permanent de l'une par l'autre. Il nous faut donc étudier jusqu'où, dans l'histoire désormais plurielle des techniques chrétiennes de soi, vaut cette différence entre le moine et le laïc. Si cette différence venait à être vérifiée au-delà du IV $^{\text {e }}$ siècle - date fatidique qui semble indiquer pour Foucault un véritable pivot de l'histoire chrétienne - elle ferait alors éclater la plupart des hypothèses de Sécurité, territoire, population. 


\section{La différence entre le moine et le pénitent}

Les techniques de soi chrétiennes dans le monachisme

Définitions : 1980

Dans les leçons données en 1980, tout se passe de prime abord comme si Foucault déplaçait la question de l'originalité du christianisme en général vers la question de l'originalité du monachisme en particulier, première véritable révolution dans les techniques de soi. L’opposition entre le monde gréco-romain et le monde chrétien se reporte en effet sur l'opposition entre la direction philosophique païenne, avec le stoïcisme comme forme exemplaire (Sénèque, Épictète), et la direction monastique chrétienne, illustrée par les écrits de Cassien. Dans la leçon du 12 mars 1980, Foucault propose de bien distinguer les techniques en fonction de la « nature » de leur objet, des « instruments » utilisés et des « objectifs » cherchés. Cette tripartition permet aux leçons suivantes, les 19 et 26 mars 1980, de fermement distinguer l'examen de conscience gréco-romain de l'examen chrétien. Foucault le fait par un strict jeu d'opposition, que nous résumons dans le tableau ci-dessous, en nous aidant également des leçons inédites Mal faire, dire vrai, données l'année suivante à Louvain, qui proposent la même antinomie entre les deux examens.

\begin{tabular}{|c|c|c|}
\hline & $\begin{array}{c}\text { Examen gréco-romain } \\
\text { (Pythagoriciens, Sénèque, Épictète) }\end{array}$ & $\begin{array}{l}\text { Examen chrétien } \\
\quad \text { (Cassien) }\end{array}$ \\
\hline $\begin{array}{l}\text { Nature } \\
\text { de leur objet }\end{array}$ & $\begin{array}{l}\text { - Les actions } \\
\text { en fonction d'un code de conduite } \\
\text { - Comptabilité de sa liberté } \\
\text { - Inspection des } \ll \text { erreurs de gestion }{ }^{27}\end{array}$ & $\begin{array}{l}\text { - Les pensées } \\
\text { en fonction de leur origine } \\
\text { - Vérité cachée de soi } \\
\text { - } \ll \text { Étiologie }{ }^{28} \text { de la faute }\end{array}$ \\
\hline Instruments & $\begin{array}{l}\text { - Exercice de la mémoire } \\
\text { sur les événements passés et à venir }\end{array}$ & $\begin{array}{l}\text { - Contrôle continu } \\
\text { de l'actualité de la pensée }\end{array}$ \\
\hline Objectifs & $\begin{array}{l}\text { - Trouver des principes rationnels de } \\
\text { conduite } \\
\text { - } \ll \text { Autonomie } »^{29}\end{array}$ & $\begin{array}{l}\text { - Mourir à soi-même } \\
\text { en tant que soi } \\
\text { - } \quad \text { «État } \gg \text { d'obéissance }{ }^{30}\end{array}$ \\
\hline
\end{tabular}


Pour Foucault, l'examen gréco-romain est une évaluation quasi administrative $^{31}$ de soi, qui ne confère à l'acte nulle autre qualité que celle d'avoir été réussi ou raté en fonction d'un code préétabli.

À l'opposé, l'examen chrétien creuse la pensée pour y retrouver une vérité cachée de son désir qui doit aussitôt être verbalisée au directeur. Le monde païen connaissait de multiples formes d'aveu, dans les pratiques religieuses comme dans les pratiques judiciaires, certaines déjà étrangement proches de l'aveu chrétien, comme en témoigne ce passage du Gorgias cité dans le cours Le gouvernement de soi et des autres (19821983) : «s'il arrive qu'on commette une faute [...], il faut aller en toute hâte, de son plein gré, là où l'on obtiendra la plus rapide punition, chez le juge, comme on irait chez le médecin $\gg^{32}$. Pour désigner la véridiction

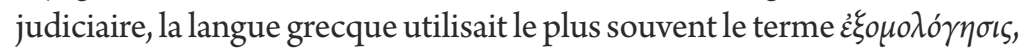
qui apparaît sous sa forme verbale dans $C_{\text {dipe }}$ roij3, où il exprime l'acte de témoigner, c'est-à-dire l'engagement personnel du sujet dans la reconnaissance d'un fait. Mais si ces formes précoces désignent bien une procédure d'aveu, elles ne sont pas en elles-mêmes productrices d'une vérité supplémentaire. C'est bien ce surcroît de vérité apporté par l'aveu, audible dans l'effort qui arrache les mots au silence, que Foucault relève comme étant l'originalité chrétienne. L'aveu monastique, auquel le cours Du gouvernement des vivants accroche le terme d' en discours perpétuel de soi $^{34}$, n'a pas pour simple fonction d'informer son directeur du résultat de ses cogitations. Il est en lui-même principe révélant et discriminant : la difficulté à avouer indique la présence de pensées mauvaises, là où l'absence de honte est le signe au contraire des bonnes pensées. Dans le monde chrétien, l'aveu gagne ainsi son efficace propre : c'est l'extériorisation de soi dans et par le discours qui devient opérateur de tri, de sélection, et donc de vérité.

Au sujet de l'examen pythagoricien du sommeil : «c'est beaucoup plus un vocabulaire administratif qu'un vocabulaire judiciaire. C'est le vocabulaire de la gestion d'un bien, d'un territoire, du gouvernement de l'administration » (Cours Mal faire, dire vrai).

Le gouvernement de soi et des autres, p. 33o. Foucault relève cette proximité, tout en restant prudent sur l'interprétation possible. En effet, aucun auteur chrétien à sa connaissance ne s'est référé à ce texte, « comme si effectivement ils savaient bien que ce n'était pas tout à fait ce dont [il] s'agit » (ibid., p. 331). Pour un exposé détaillé des formes païennes de «véridiction de soi » et d'aveu de la faute : cours Mal faire, dire vrai. Fonctions de l'aveu.

33 Foucault cite la forme ỏ $\mu o \lambda o \gamma \varepsilon ́ \omega$ qu'il traduit par «je reconnais, j'avoue, oui, c'est bien ce qui s'est passé » (leçon du 16 janvier 1980).

Leçon du 26 mars 1980. 
Mais surtout, l'aveu pénètre plus profondément le domaine de la subjectivité que ne le faisait la communication des états d'âme du sage stoïcien à son directeur ou à son ami quand il gagnait de l'argent, affrontait ses passions, participait aux affaires de la cité, etc. Ce ne sont pas tant les actes ni les états d'âme subséquents qui sont objets conjoints d'examen et d'aveu, mais les pensées qui viennent à l'esprit (le logismos ou la cogitatio ${ }^{35}$ ), au moment subreptice où celles-ci apparaissent. Et dans ce flux incessant et anarchique des pensées, il ne s'agit pas tant d'éprouver celles qui se rapportent à quelque chose de réel ou non, il ne s'agit pas d'étudier leur « contenu objectif» (ibid.) pour mettre de côté celles qui ne seraient pas vraies en soi, mais d'examiner leur « réalité matérielle $\gg($ ibid.), c'est-à-dire leur origine : de quoi elles sont faites, c'est-à-dire si elles viennent de Dieu ou de Satan.

Loin d'être une obligation parmi d'autres, l'aveu exhaustif rend possible et authentifie la soumission totale au supérieur, qui est la fin des techniques de soi monastiques : tout dire pour obéir en tout; et tout dire comme signe que l'on obéit en tout. Mais pour le moine suffisamment avancé, ce rapport asymétrique à autrui devient peu à peu un rapport asymétrique à soi, où autrui est tellement intériorisé qu'il n'a même plus besoin d'être physiquement présent ${ }^{36}$. Le cours de 1979-1980 insiste sur la dimension temporelle de ce que Foucault décrit comme un « devenir obéissant $\gg^{37}$, qui pénètre la totalité du comportement et indique un au-delà du modèle politique de la $\operatorname{loi}^{38}$ et même un au-delà de la simple regula vitae du moine. Librement consentie, l'obéissance tend à devenir une pure relation, indépendante des qualités de la personne à qui elle est due et d'une quelconque norme objective. Cette obéissance est moins l'observance d'un règlement extérieur ou la réponse ponctuelle à l'ordre d'un supérieur, qu'une «manière d'être, antérieure à tout ordre, plus fondamentale que toute situation de commandement $\gg^{39}$. sance, comme si autrui était en permanence présent au moi : «N'être jamais maître de soi et faire en sorte qu'il y ait toujours en soi, en vous-même, quelqu'un qui soit le maître et le maître de tout. $\gg$

37 Leçon du 19 mars 1980.

38 « Ici, il ne s'agit pas d'obéir à la loi comme code d'obligation ou d'interdit, il s'agit en fait de laisser le principe d'obéissance pénétrer tout le comportement » (cours Mal faire, dire vrai). 
Paradoxalement, à la fine pointe de l'obéissance, il n'y a plus ni supérieur ni ordre extérieur, puisque « tout autre [moine], tout événement doit fonctionner comme un ordre qui est donné » (ibid.). Le sujet se trouve dans un état de pure obéissance qui se manifeste par trois vertus : la subditio (la soumission : «vouloir ce que veut l'autre »), la patientia (la passivité : « vouloir ne pas vouloir ») et enfin l'humilitas (l'humilité : « ne pas vouloir vouloir $\gg$ ). Il serait d'ailleurs intéressant de se demander si la dimension quasi cosmique que Foucault donne à l'obéissance monastique - «le moine vit dans un monde qui est peuplé d'ordres » (ibid.) ne vient pas temporiser l'importance fondatrice donnée par ailleurs au rapport à autrui dans la direction chrétienne.

Avec l'examen permanent et l'aveu exhaustif, l'obéissance sans fin forme ainsi le triangle de la direction monastique. En apparence, nous ne sommes pas très loin des résultats du cours Sécurité, territoire, population, qui opposait de la même manière les derniers Grecs aux premiers chrétiens, en faisant jouer le couple autonomie/obéissance comme deux fins possibles mais inconciliables de la direction spirituelle. Dans la direction chrétienne, «Je veux que l'autre me dise ce que je dois vouloir $»^{\circ}$ : le but recherché est bien la libre renonciation à soi-même comme sujet voulant.

\section{Reprise de ces défnitions et généralisation}

à tout le christianisme : 1981 et 1982

Comme nous l'avons indiqué, cette jonction de l'exploration et de la verbalisation de soi, dans un rapport d'obéissance permanent à un autre, seul le monachisme la réalise «à partir de ce IV e siècle [insiste Foucault] et de ce IV siècle seulement $\gg^{41}$. Cependant, malgré l'attention scrupuleuse à cet écart initial, le cours laisse entendre à certains endroits que le christianisme postérieur aurait fortement convergé vers une forme unifiée de subjectivation. Il semble en effet que la distinction nouvelle, posée au Collège de France en 1980, entre «le christianisme en général $\gg$ et «le monachisme, précisément $\gg(i b i d$.$) , ne vaille que tempo-$ rairement, comme l'attesterait l'assimilation ultérieure des techniques 
monastiques par le gouvernement pastoral ${ }^{42}$. Les chemins de l'histoire sont peut-être plus longs et plus compliqués que ceux décrits dans Sécurité, territoire, population, la technique de direction peut ne s'insérer que très progressivement dans la pastorale globale du peuple chrétien, les routes mènent finalement au même lieu. La confession annuelle imposée à tous les fidèles par le canon 21 du concile du Latran en 1215 en serait le témoignage le plus probant ${ }^{43}$.

À travers l'exemple de l'examen monastique, si l'on en croit le cours Du gouvernement des vivants, il s'agirait en fait toujours de décrire « un processus finalement très long où s'élabore la subjectivité de l'homme occidental $\gg 44$. En effet, «la subjectivation de l'homme occidental, elle est chrétienne, elle n'est pas gréco-romaine $\gg$ (ibid.). Et cette subjectivation chrétienne consiste essentiellement à devenir obéissant, dans la forme de l'examen et de l'aveu. La différence entre le monde grécoromain et le monde chrétien passerait donc bien entre un sujet dirigé afin qu'il puisse se diriger lui-même parfaitement et un sujet dirigé afin qu'il le soit continuellement : être dirigé pour ne plus l'être ou être dirigé pour l'être ad vitam aeternam.

Cette thèse est d'autant plus plausible qu'elle est répétée tout au long des cours Subjectivité et vérité (1981-1982) et L'herméneutique $d u$ sujet (1981-1982). Le premier cours pose les données du problème et la méthode pour le traiter : un passage de l'Introduction à la vie dévote (1609) de saint François de Sales concernant la sexualité des éléphants montre la difficulté de saisir la différence exacte entre la morale païenne et la morale chrétienne, les deux ayant célébré pour les mêmes raisons apparentes la vertu conjugale du mammifère ${ }^{45}$. Pour saisir cette différence, l'analyse historique doit en fait se déporter des lois et des codes moraux vers le « genre mineur des conseils d'existence $\gg{ }^{46}$ prescrivant le type d'expériences que le sujet doit faire de lui-même. D'une manière encore rudimentaire, Foucault oppose alors la subjectivité grecque, qu'il assimile à la notion de ßios, comme fin que l'on se donne à soi, à ce qu'il appelle la « matrice générale de la subjectivité occidentale

Ibid : «plus tard, [...] cette technique de direction sera développée à l'intérieur du christianisme, $[\ldots]$ sous le signe du pastorat $\gg$.

43 Cité par Foucault dans le cours Mal faire, dire vrai. Fonctions de l'aveu.

44 Leçon du 12 mars 1980.

45 Leçon du 7 janvier 1981.

46 Leçon du 14 janvier 1981. 
et chrétienne $\gg 47$, définie comme rapport à la fois à un au-delà qu'il faut attendre et à une vérité de soi qu'il faut découvrir ${ }^{48}$.

L'herméneutique du sujet poursuit ce souci de construction d'une altérité irréductible entre Grecs et chrétiens, en mettant volontairement de côté toute distinction interne au christianisme. Même si Foucault semble par moments resserrer son interprétation autour du seul « modèle "ascétique-monastique" $\gg^{49}$, le reste du cours en reste à un niveau élevé de généralité. Il est ainsi indistinctement question de la « conversion chrétienne $\gg\left(\right.$ p. 202), de l'« ascèse chrétienne $\gg^{50}$, alors que les références les plus explicites viennent à nouveau de Cassien ${ }^{51}$, comme en témoigne d'ailleurs l'extrait livré la même année du dernier volume en préparation de l'Histoire de la sexualité : «Le combat de la chasteté $\gg^{52}$. Sans doute ne faut-il pas prêter une trop grande attention à ces généralisations, étant donné que le christianisme n'est pas le véritable sujet du cours et qu'il y joue d'abord un rôle évident de modèle repoussoir permettant à Foucault de définir ce qu'il nomme l'《âge d'or ${ }^{53}$ du souci de soi : les I $^{\text {er }}$ et $\mathrm{II}^{\mathrm{e}}$ siècles de notre ère, avant l'essor du christianisme. Mais nous y trouvons une fois de plus l'opposition de fond que le cours Du gouvernement des vivants et les leçons de Louvain avaient préalablement développée. Pour en saisir les tenants et les aboutissants, résumons les grandes lignes de l'argumentation proposée en 1982.

La notion de « souci de soi » permet à Foucault de distinguer trois grands modèles de rapport à soi : le modèle platonicien, le modèle hellénistique et le modèle chrétien. C'est sur le second modèle que s'at-

Leçon du 18 mars 1981.

Ibid. Foucault use en fait d'un triple jeu d'oppositions entre les subjectivités grecque et chrétienne : 1) fin immanente à soi / rapport à un au-delà ; 2) travail continu de soi sur soi / conversion comme arrachement à soi ; 3 ) recherche jamais achevée d'une certaine fin / accès à une vérité profonde de soi.

L'hésitation de Foucault sur ce point est intéressante : «En face [du modèle platonicien] s'est formé, à partir des $\mathrm{III}^{\mathrm{e}}$ et $\mathrm{IV}^{\mathrm{e}}$ siècles, le modèle chrétien. Il faudrait dire "modèle ascétique-monastique" plutôt que chrétien au sens général du terme. Mais appelons-le "chrétien" pour commencer » (L'herméneutique du sujet, p. 244).

o Ibid., p. 317. Les autres passages les plus significatifs dans ce cours sur la différence entre les philosophes païens et les Pères chrétiens sont ceux concernant la conversion (p. 202207) ; la connaissance de soi (p. 242-248); l'ascèse (p.315-317); la règle (p. 404-406).

1 Ibid., p. 286-287. Un peu plus tard, Foucault laisse entendre que le face-à-face entre le stoïcisme et le christianisme est d'abord un face-à-face entre Sénèque et Cassien (p. 404).

$D E \mathrm{n}^{\circ} 312$, « Le combat de la chasteté », II, p. 1114-1127.

L'herméneutique du sujet, p.79. 
tarde le cours de 1981-1982, moment singulier du stoïcisme romain ( $\mathrm{I}^{\mathrm{er}}$ et $\mathrm{II}^{\mathrm{e}}$ siècle), où le souci de soi s'étend à la vie entière du sujet et devient une fin pour lui-même. Si nous sommes ici du côté des philosophes stoïciens, et occasionnellement épicuriens ou cyniques, le cours est hanté par la question chrétienne. Entre les premiers chrétiens et les derniers Hellènes, les notions sont identiques (ascèse, conversion, examen), mais les problématiques sont radicalement hétérogènes. La question qui court silencieusement tout au long de cet enseignement pourrait être résumée de la sorte : que s'est-il donc passé dans cet écart de quelques siècles pour que notre rapport à nous-mêmes se soit aussi radicalement modifié, rendant possible une objectivation de notre être intime, faisant descendre la vérité au fond de l'âme avant de la faire remonter sous le regard d'une autorité qui, précisément, a charge d'âmes ?

Pour les stoïciens, le dire-vrai est d'abord du côté du maître qui enseigne ${ }^{54}$. Sa franchise doit avant tout opérer une certaine modification de l'être du disciple, pour lui permettre de constituer un rapport « plein et indépendant $\gg$ (p.312) à lui-même. Il ne s'agit pas ici d'une vérité des profondeurs que le dirigé atteindrait par introspection et déchiffrement de ses pensées, mais d'une vérité essentiellement ordonnée à l'action, celle qui pourra lui fournir une préparation digne d'un athlète, un bon «équipement » (p.312) lui permettant d'affronter les aléas de l'existence. Ce qu'enseigne le maître, le disciple doit non seulement le comprendre, le mémoriser, mais également en devenir sujet. Pour Foucault, cette incorporation de la vérité au sujet est l'exact opposé de l'objectivation du sujet dans un discours de vérité, telle que l'ère chrétienne la développera :

Il me semble que dans l'ascèse chrétienne on va trouver, donc, un mouvement de renonciation à soi qui passera, comme moment essentiel, par l'objectivation de soi dans un discours vrai. Il me semble que dans l'ascèse païenne, dans l'ascèse philosophique, dans l'ascèse de la pratique de soi à l'époque dont je vous parle, il s'agit de se rejoindre soi-même comme fin et objet d'une technique de vie, d'un art de vivre. [... C'est là cette espèce de différence fondamentale que j'essaie, au fond, depuis le début de ce cours, de faire apparaître. (p.317)

L'opposition est redoublée par l'impossibilité de réfléchir ce lien singulier entre sujet et vérité dans le modèle de la règle ; là où les techniques pastorales chrétiennes s'efforceront de définir l'exact ordonnancement des exercices entre eux, leurs modalités précises d'application et leur 
stricte régularité. Pour le philosophe stoïcien, ce lien est au contraire un art de faire, un exercice pratiqué avec assiduité et vigilance, mais dont la régularité n'est pas gage de réussite puisqu'il s'agit d'abord de donner à sa vie « une forma, une forme qui est belle $»($ p. 406).

Avec le monachisme chrétien, ces techniques de la vie philosophique vont être transposées à l'intérieur d'un rapport d'obéissance inédit « qui en altère et en inverse tous les effets $\gg^{55}$. Cette inversion radicale est la ligne de basse qui se fait entendre tout au long du cours L'herméneutique du sujet, où le christianisme est réduit à la fixation d'une subjectivité dans un discours de vérité, lui faisant perdre la plasticité éthique qu'elle avait dans l'Antiquité. La définition de l'âme stoïcienne selon Foucault pourrait tenir dans le verbe $\chi \rho \eta ́ \sigma \theta a l$ (se servir de) $)^{56}$ : l'âme est avec son corps, ses pensées, sa relation à autrui, dans le rapport de l'artisan à ses outils, sans secret ni double fond. Le but de l'ascèse est que l'âme trouve sa pleine souveraineté sur elle-même, en maîtrisant et en maniant convenablement à la fois ce qu'elle a à sa disposition et ce qui lui advient : «âme-sujet » (ibid., p. 56). À l'opposé, l'âme chrétienne tiendrait dans le substantif exploratio ${ }^{57}$ (exploration) : avant son commerce avec autrui et avant même toute relation avec le corps qu'elle anime, l'âme doit être connue et déchiffrée comme le siège de désirs, suscités par des représentations issues de sa nature peccamineuse. L'objet de l'ascèse est donc de se libérer des esprits mauvais en renonçant à soi par une relation d'obéissance : «âme-substance $\gg^{58}$. Mais cette lecture ne recouvre-t-elle pas la complexité du tableau historique peint précédemment en 1980 ? Foucault n'a-t-il pas, volontairement cette fois, joué des contrastes, avec de grands aplats noirs et blancs?

Revenons à ce lieu source qu'est pour nous le cours Du gouvernement des vivants, dans la mesure où il nous semble plus riche dans sa narration, plus subtile dans son approche du christianisme. En particulier, Foucault y présente longuement les techniques de soi qui s'appliquaient à tout chrétien avant le virage du monachisme. Il faut alors nous interroger sur le destin de cette ascèse primitive au-delà du $\mathrm{IV}^{\mathrm{e}}$ siècle.

56 L'herméneutique du sujet, p. 55 : «verbe très important en grec, aux significations très nombreuses », souligne Foucault. Les notions d'《utilisation » et d'《instrument » forment le porche d'entrée dans la conception platonicienne du souci de soi, dès la deuxième leçon du cours (p.54-57).

57 Exploratio conscientiae, cité par Foucault dans la leçon du 26 mars 1980.

58 L'herméneutique du sujet, p. 56. 
Les techniques de soi chrétiennes avant le monachisme

Ces techniques de soi, engageant un certain rapport du sujet à la vérité, se sont développées dans les premiers siècles chrétiens autour de deux rites, tous deux non répétables dans la vie du chrétien et marqués par une forte composante dramatique : le baptême et la seconde pénitence.

\section{Le baptême}

Le baptême permet au sujet d'accéder à la vérité dans un « rapport de connaissance immédiat et total avec Dieu $\gg^{59}$. Il est reçu après un temps de préparation qui est essentiellement un temps d'enseignement et de jeûne, sanctionné par un acte de foi. Illuminée par Dieu dans le baptême, l'âme est en même temps éclairée sur elle-même et reconnaît dans cette lumière son appartenance originelle à l'essence divine. Cependant, une nouveauté va être apportée par Tertullien au tournant du $\mathrm{II}^{\mathrm{e}}$ et du $\mathrm{III}^{\mathrm{e}}$ siècle. Le rapport de l'âme à la vérité va se dédoubler, de telle manière que le baptême va être désormais divisé en deux temps distincts : celui de la pénitence et celui de l'illumination; celui d'une épreuve à traverser en faisant la lumière sur soi et celui de la lumière divine inondant l'âme au terme du parcours pour la libérer définitivement. Mais c'est en fait la vie chrétienne dans son ensemble qui va être désormais tendue entre ces deux pôles, l'épreuve pré-baptismale ne faisant que préfigurer et équiper le fidèle pour un combat appelé à durer toute une vie. Expliquons-nous.

Sujet de connaissance dans la lumière baptismale, l'âme devient avec Tertullien objet de connaissance dans le temps de la préparation. Contrairement aux premiers siècles, ce temps de la préparation n'est plus seulement acquisition des connaissances nécessaires à l'illumination, mais également travail sur soi par l'ascèse, en vue d'une purification. La conversion ne signifie plus seulement mouvement de l'âme vers la lumière, mais d'abord et avant tout pénitence (paenitentia) dans la crainte et le danger des attaques continuelles de Satan. En posant non seulement la perversion originelle de la nature humaine, mais également l'installation d'un Autre - Satan - à l'intérieur de l'âme, Tertullien transforme le temps de 
purification extérieur en un combat intime : «l'autre [explique Foucault] s'est introduit en nous. [... ] il a établi son empire à l'intérieur même de l'âme des hommes $\gg^{60}$, il faut donc le vaincre.

Certes, comme nous l'avons indiqué, les pratiques de jeûne existaient déjà dans les premières préparations au baptême, mais elles ne requéraient aucune manifestation de soi particulière. Désormais, la purification doit pouvoir être authentifiée en montrant ses preuves; tel est le but de ce que Tertullien nomme la paenitentiae probatio. Le mouvement de l'âme vers la vérité divine doit être précédé d'un mouvement de l'âme vers sa propre vérité. C'est ce que Foucault repère dans l'institution du catéchuménat : l'apparition de rituels de vérité qui ne sont pas encore exactement manifestation de l'âme dans le détail de sa vérité singulière et profonde, mais manifestation de l'âme dans sa situation générale. Ces rituels sont principalement au nombre de quatre :

1) le «questionnaire-enquête » qui vérifie auprès de témoins et de l'intéressé que celui-ci mène une vie conforme à ce qui est attendu de lui;

2) l'exorcisme - appelé également «scrutin » ou « examen »- qui certifie que l'esprit mauvais a bien quitté le postulant;

3) la profession de foi par laquelle le postulant manifeste son adhésion nouvelle;

4) l'« exomologèse » ou la reconnaissance par le postulant de son état de pécheur, rituel plus proche d'un acte d'oraison que de l'aveu de péchés particuliers ${ }^{61}$.

Dans une pratique baptismale encore marquée par le schéma grec de l'illumination totale et définitive, Tertullien enfonce le coin de la faute originelle et de l'inquiétude salutaire. La relation entre la vérité et la subjectivité n'a plus la forme d'une unité à retrouver entre l'âme et son Dieu, comme le permettait le travail platonicien de la mémoire, mais elle se diffracte entre, d'une part, la «probation de la vérité de l'âme », qui oblige celle-ci à dire ce qu'elle est, et, d'autre part, «l'initiation à la vérité [divine] $\gg^{62}$ qui devient adhésion à une tradition instituée, c'està-dire au dogme. Le cours de Louvain Mal faire, dire vrai. Fonctions de l'aveu parlera dans le même sens du «lien profond » qui unit en les distinguant la «vérité de soi » et la «vérité du texte » : deux pôles tantôt 
complémentaires, tantôt conflictuels dans l'histoire du christianisme. Ces deux pôles, la Réforme protestante tentera tardivement de les réunir, pour « faire en sorte que ce que l'on découvre au fond de soi-même ce soit la vérité même de la foi, c'est-à-dire la vérité même qui est donnée dans le texte $\gg^{63}$. De son côté, le catholicisme maintiendra au contraire la distinction, et donc la tension, entre les deux vérités.

\section{La seconde pénitence}

La seconde pénitence (paenitentia secunda) concerne les fidèles qui, en dépit du baptême reçu, sont gravement retombés dans le péché. Son institution date du début du $\mathrm{III}^{\mathrm{e}}$ siècle, mais elle se développe surtout à la faveur des persécutions, occasion malheureuse pour certains fidèles de reniements forcés. Seconde planche de salut offerte aux relaps, cette pénitence ouvre la possibilité d'une pleine réintégration au sein de l'Église. Elle n'est cependant pas répétable. À l'image du baptême, dont elle est sinon la régénération au moins le rappel, la paenitentia secunda est un événement unique qui donne au fidèle fautif un nouveau statut pour une durée déterminée. Cette procédure est à son tour scandée par une série $\mathrm{d}^{\text {' }}$ 《actes de vérité ${ }^{64}$, sensiblement proches de la préparation baptismale. Foucault en cite trois principaux :

1) deux examens « extérieurs » du postulant, à la fois individuels et publics, au début et au terme de son entrée en pénitence ;

2) une nouvelle « exomologèse », qui est la manifestation publique par le pénitent du fait qu'il se reconnaît pécheur.

Cette dernière manifestation, fortement dramatisée, également désignée dans le cours par le syntagme publicatio sui, s'effectue à la porte de l'église, avant l'imposition des mains. Elle peut s'accompagner de pleurs, de gémissements, d'auto-humiliations. Mais à nouveau, insiste Foucault, nulle trace ici d'une verbalisation détaillée de soi, entendue comme « description analytique de la faute avec ses caractères et ses circonstances $\gg^{6}$. Si parole il y a à la porte de l'église, elle a une fonction expressive et non descriptive; elle ramasse et concentre en un cri la situation présente du sujet. 
Pourquoi le laïc ne peut être dirigé comme le moine

Ces deux rituels de vérité, baptême et seconde pénitence, marquent d'abord des événements, qui sont autant de passages nets d'un état à un autre, arrachant le sujet à ce qu'il était pour le rendre autre que luimême : passages de la mort à la vie, du péché au salut, de l'erreur à la vérité. Quand elle deviendra plus tard exploration et verbalisation de soi, par l'examen et la confession, la pratique pénitentielle s'étendra au contraire à la durée d'une vie, pour y devenir injonction permanente :

$[\ldots]$ il s'agira pour le sujet de cheminer de jour en jour, de moment en moment, d'instant en instant, vers une perfection sans cesse plus grande, et à ce momentlà le rapport de vérité du sujet à lui-même devra nécessairement être structuré sur un tout autre mode que celui de la rupture. ${ }^{66}$

Pour le moment, la seconde pénitence et le baptême coupent en deux la vie du sujet et ne lui demandent aucune confession particulière de ses péchés comme prix à payer.

Cependant, dès le $\mathrm{III}^{\mathrm{e}}$ siècle, Foucault relève avant l'entrée en seconde pénitence la présence d'une expositio casus, également exprimée par le verbe confessio, par laquelle le pécheur est invité à exposer son cas à l'évêque, très probablement en privé. Il faut bien en effet que l'évêque sache exactement de quoi il retourne pour évaluer si l'entrée en pénitence est justifiée ou non. Cette exposition, qui appelle un récit circonstancié des faits, annonce la forme secrète et détaillée de la confession, telle que le moine la pratiquera, et telle que le Moyen Âge l'imposera ensuite à tous les fidèles. Pour Foucault, l'histoire de l'aveu chrétien est finalement l'histoire de l'intégration progressive de cette expositio casus à l'intérieur de la pénitence, où elle va être appelée à remplacer peu à peu la publicatio sui qui en constituait auparavant le sommet et l'aboutissement.

La leçon du 5 mars 1980 insiste fortement sur la distinction, au $\mathrm{IV}^{\mathrm{e}}$ siècle, entre ces deux pratiques : l'expositio qui précède la pénitence et la publicatio qui la referme. D'un côté une pratique privée, verbale, analytique et subjective, et de l'autre, une pratique publique, non verbale, synthétique et objective. À compter du moment où la première se 
substituera à la deuxième dans la pénitence, les rapports entre la subjectivité et la vérité deviendront essentiellement juridiques et discursifs :

[... ] l'aveu, c'est-à-dire la publicatio sui par le chemin de l'expositio casus, l'exomologèse transformée en pratique juridico-verbale, cela est une chose tardive, lente, qui a demandé presque un millénaire ou, en tout cas, sept ou huit cents ans pour être accomplie. ${ }^{67}$

À cet accomplissement, les techniques monastiques préalablement exposées, exigeant la mise en discours de la vie entière du sujet ( $\dot{\xi} \xi$ a ó $\rho \varepsilon v \sigma ı \zeta)$, auraient bien entendu largement contribué. À écouter ce résumé, où Foucault coupe au plus court par souci de clarté, on pourrait se représenter un christianisme progressivement colonisé par un ensemble de techniques nées à la fois tardivement et dans ses marges. Dans le cours Mal faire, dire vrai, le philosophe semble encore plus catégorique sur ce point :

Mais si on s'en tient à nos sociétés - aux sociétés chrétiennes occidentales - il me semble qu'on pourrait sans trop de spéculation parler de croissance massive de l'aveu, non pas croissance continue, mais par paliers et par plages, par à-coups, avec arrêts et brusques accélérations.

Ainsi, l'ancienne exomologèse de la seconde pénitence aurait été remplacée par l'exagorèse du confessionnal ou de la cellule; et tout laïc serait peu à peu devenu un moine à sa manière, entérinant l'hégémonie de la direction et de l'examen sur tout autre rapport à soi. Cette thèse n'est cependant pas recevable pour deux raisons principales, l'une historique et l'autre méthodologique.

\section{Raison historique}

Si la seconde pénitence a peu à peu disparu du christianisme en tant que rituel, cédant la place à une pénitence réitérable, puis à une pénitence tarifée inspirée du droit germanique, elle est demeurée dans l'histoire chrétienne comme manifestation dramatique du combat et de la victoire sur le péché, distincte de l'examen intime et continuel de soi. Prendre conscience de la distance insurmontable entre ces deux rapports à la vérité est fondamental pour comprendre ce que Foucault dit du christianisme non seulement en 1980, mais également dans son dernier cours au Collège de France : Le courage de la vérité. 
Loin de la réduire à une péripétie historique passagère, Foucault fait de la tension entre la nouvelle pénitence juridico-discursive de

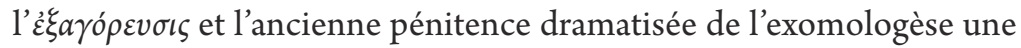
tension constitutive du christianisme. Cette dialectique non résolue entre les deux termes est exposée brièvement dans la leçon du 19 mars 1980 comme opposition entre « pénitence » et «monachisme ». Elle est reprise d'une manière beaucoup plus détaillée dans le cours de 1981 à Louvain comme opposition entre «exomologèse 》 et « $\dot{\varepsilon} \xi \alpha \gamma o ́ \rho \varepsilon v \sigma l \zeta 》$, avant d'être reformulée en 1984 comme opposition entre un «pôle parrèsiastique $\gg$ ou «mystique $» d u$ christianisme et un «pôle antiparrèsiastique $\gg$ ou $\ll$ ascétique ${ }^{68}$. Il nous faut être ici extrêmement précis, car les termes « pénitence » et «ascèse » peuvent prendre dans les écrits de Foucault des sens différents, voire contradictoires suivant les contextes. Si le moine, bien entendu, est lui aussi un pénitent et un ascète, il ne l'est pas au sens conféré à ces termes par la seconde pénitence. Réécoutons pour s'en convaincre la leçon du 5 mars 1980, en prêtant une attention particulière aux termes choisis par le professeur.

La pénitence seconde est un «statut qui concerne l'individu tout entier ${ }^{69}$, marqué par un temps d'ascèse pouvant durer plusieurs années et sanctionné par un événement final : l'imposition des mains. Avant cette imposition, le pénitent doit manifester par des actes l'épreuve qu'il est en train de traverser :

La $\mu \varepsilon \tau$ d́vola caractéristique de la pénitence ne doit pas être simplement une conversion de la pensée $[\ldots]$ il faut qu'elle soit un acte. Cet acte, qu'est-ce que c'est ? C'est l'extériorisation de [...] la conversion de pensée, c'est sa transcription en comportement, et cette pénitence en tant qu'acte, c'est cela qui est l'exomologèse. (Ibid.)

Pour Foucault, l'exomologèse est donc à la fois le nom d'un rituel précis qui se déroule sur le parvis de l'église avant l'imposition des mains et le terme générique qui englobe la totalité de la pénitence : ce temps où le pénitent jeûne, s'habille pauvrement, gémit, pleure, s'agenouille aux pieds du prêtre, etc. C'est même le terme générique qui prime dans la description, attendu que le rituel précis n'est en fait que

[... ] l'intensification, le resserrement, la dramatisation en un épisode public, à 
la porte de l'église et au moment de la réconciliation, de ce qui était au fond le drame permanent de la pénitence, cette espèce de théâtralisation - je dis ça sans aucune connotation péjorative - de la $\mu \mathcal{\varepsilon} \tau \dot{v} v o \iota \alpha$. (Ibid.)

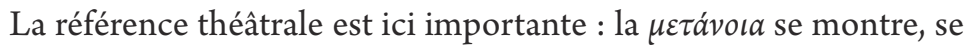
donne à voir en public. Il est alors possible, en reprenant l'argumentation de la leçon du 5 mars 1980, de parler d'《événementialisation » du travail ascétique par la pénitence, et ceci en deux sens complémentaires : ce travail sur soi s'inscrit dans une durée déterminée et il fait rupture dans la vie du sujet.

Dans le temps de la pénitence et jusqu'à l'imposition des mains,

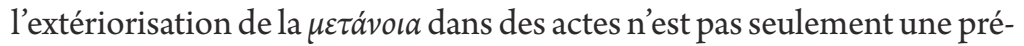
paration ou un équipement pour un combat à venir (le combat contre Satan), elle est l'expression d'un combat réel dont elle garantit en partie l'issue victorieuse. De ce combat en effet, la pénitence est directement contemporaine : non seulement elle l'atteste, en le représentant comme sur une scène de théâtre par les pleurs et les gémissements, mais cette attestation précipite la victoire, au point qu'il n'y a plus de distance entre la manifestation et la chose manifestée. Ce qui est publiquement exprimé dans l'exomologèse est bien l'être du pénitent : il est un pécheur, vivant une vie semblable à la mort, comme l'exprime la cendre sur la tête et les haillons. Mais par la manifestation publique de sa mort, il manifeste en même temps qu'il renonce à ce qu'il est pour vivre une autre vie : «Il s'agit, dans cette supplication de manifester ce qu'on est et, en même temps, de l'effacer $\gg$ (ibid.). Réécoutons bien cette phrase : Foucault ne dit pas « et, en même temps, de manifester qu'on l'efface », mais il rend la manifestation directement responsable de l'effacement; il lui confère, à la lecture de saint Jérôme ou de Tertullien, une efficacité décisive ${ }^{70}$.

L'ascèse monastique est tout autre, dans son fonctionnement et dans sa finalité. Elle ne manifeste nul événement particulier, mais elle est un effort continu. Loin des expressions exacerbées ou ostentatoires, elle est une pratique sévèrement limitée par l'usage de la discretio, entendue comme

70 Foucault dit cependant bien, quelques minutes plus tard que par l'exomologèse, on «manifeste qu'on meurt à cet état de pécheur », mais aussitôt il souligne que la manifestation a bien un effet réel : «cette énonciation, elle a pour effet quoi ? Précisément de montrer, non seulement de montrer, mais elle opère même cette espèce de détachement à l'égard de l'état de pécheur, puisque c'est à travers l'état de pécheur que l'on effectue, in $a c t u$, réellement la $\mu \varepsilon \tau a ́ v o l a$, le détachement à l'égard du péché ». 
capacité à distinguer les choses dans le but de déterminer la bonne mesure, le $\ll$ juste milieu ${{ }^{71}}^{7}$. Gardienne de toutes les vertus, la discretio se tient à égale distance des excès contraires, du zèle comme du relâchement. Non seulement les moines ne sont pas les champions de l'exploit ascétique, mais ils ont appris à s'en méfier, en particulier dans ses manifestations les plus spectaculaires : méfiance à l'égard de tout pouvoir personnel permettant d'agir sur soi ou autrui, méfiance vis-à-vis de tout état qui serait présomptueusement considéré comme d'origine divine. Encadrer l'ascèse par la discretio, comme le montre la leçon du 26 mars 1980, c'est la soumettre à une obéissance qui met sans cesse en question l'édification personnelle et le jugement propre, surtout s'ils se parent des meilleures intentions. Entre Dieu et le moine, vient toujours s'intercaler la relation à autrui.

Ce principe de modération va alors transmuer l'ascèse en une activité régulière, mesurée, n'ayant d'autre but que de favoriser l'humilité et la maîtrise des pensées. Le cours Mal faire, dire vrai mentionne en ce sens le triomphe de l'augustinisme dans les monastères occidentaux, en particulier bénédictins, contre les tendances contemplatives ou perfectionnistes des institutions cénobitiques issues de Cassien :

L'augustinisme veut dire : [...] que l'on ne peut par soi-même et par des actions spirituelles s'assurer son propre salut et purifier son propre cœur. [...] il est bien évident que tout ce qui peut être exercice spirituel va perdre un peu ou une grande part de son efficace quant à l'assurance de son salut. [...] ceci va disparaître au profit d'un travail qui va être imposé, organisé, réglementé.

Assomption du travail comme activité fondamentale du moine, méfiance vis-à-vis de tout événement salvifique qui déroge à la règle :

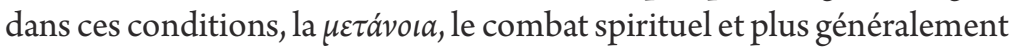
l'action directe de Dieu à l'intérieur de l'âme ne sont plus visibles « in $a c t u »$, et encore moins dans une ascèse désormais vidée de ses soubresauts dramatiques. De drame, ne reste que celui feutré et murmurant de l'obéissance au supérieur. Refuser la dramatisation de l'ascèse, c'est bien entendu refuser Tartuffe - comme le signale non sans humour Foucault $^{72}$ - mais c'est aussi et surtout refuser de faire de la vie du moine le théâtre d'événements décisifs, où la relation de l'âme à Dieu serait directement engagée et transformée. 
On comprend alors que Foucault rebaptise en 1984 le pôle monastique «pôle anti-parrèsiastique », la parrêsia désignant chez Philon d'Alexandrie une «modalité pleine et positive ${ }^{73}$ du rapport à Dieu, radicalement indépendante du rapport aux autres hommes. En un sens légèrement différent, le Nouveau Testament et les premiers Pères chrétiens vont faire de la parrêsia un « mode d'être ${ }^{74}$, témoignant à la fois du courage de dire la vérité aux hommes et de la confiance inébranlable en Dieu, avec une dimension nouvelle d'obéissance à sa volonté. Mais dans les deux cas, l'accent est mis sur l'intimité entre l'âme et Dieu - ce qui permet à Foucault de voir dans la parrêsia chrétienne l'origine de la grande tradition mystique (ibid., p. 307). Cette grande tradition rejointelle pour autant le pôle précédemment décrit de la pénitence ? Oui, si l'on se permet de tirer un fil beaucoup plus long, mais non moins solide, entre la parrêsia des cyniques et la seconde pénitence des premiers siècles. Même si Foucault ne fait pas lui-même le rapprochement, il n'en est pas moins suggestif. Longuement étudiée en 1984, la parrêsia cynique est cette idée que la vie peut être en elle-même l'expression directe, sans détour, de la vérité ; que nos gestes, paroles, manières de s'habiller et de se nourrir peuvent constituer le « corps même de la vérité »(p.160), sans solution de continuité. Or, il n'est pas question d'autre chose dans l'exomologèse du pénitent : vérité manifeste du processus de conversion dans l'habitus, c'est-à-dire la «manière de vivre », la «manière de se nourrir $\gg 75$, etc., mais aussi et surtout vérité manifeste de sa victoire sur le péché et sur la mort. La réduction de la vie à son dénuement atteste la transformation en cours et clame l'issue d'un combat universel. Il est d'ailleurs intéressant de noter que Foucault, pour décrire la parrêsia cynique en 1984, reprend exactement la même métaphore que celle utilisée en 1980 pour décrire la seconde pénitence : celle du théâtre ${ }^{76}$.

Pour contrer cette intimité de l'être avec la vérité, manifestée dans une ascèse qui prétend avoir une efficace propre, le monachisme va introduire le directeur ou le supérieur entre Dieu et le pénitent, jetant l'opprobre

Le courage de la vérité, p. 297.

Le courage de la vérité, p. 300.

Leçon du 5 mars 1980.

« Le choix de vie comme scandale de la vérité, le dépouillement de la vie comme manière de constituer, dans le corps même, le théâtre visible de la vérité $\gg$ (Le courage de la vérité, p.168169). Voir aussi page 234 : la «dramatisation » et la «théâtralisation $\gg$ des principes de la vie cynique. Le 5 mars 1980, Foucault parlait également de la «théâtralisation » de la $\mu \varepsilon \tau$ ávoıa. 
sur la notion de parrêsia, accusée de nourrir l'orgueil et la présomption. «Là où il y a obéissance [explique Foucault en 1984], il ne peut pas y avoir parrêsia $\gg^{77}$. La parrêsia est alors réduite à n'être plus que la parole outrecuidante, signe d'une trop grande confiance en soi. Mais il faut aussitôt remarquer que ce nouveau rapport entre subjectivité et vérité, tel qu'il a été défini dans le monachisme ancien, n’a pas eu complètement raison de la parrêsia. C'est du côté des ordres mendiants, franciscains et dominicains, que Foucault voit le retour au Moyen Âge d'une vérité vécue comme une provocation, dans ce dépouillement complet qui manifeste ce qu'est véritablement le monde et ce qu'est véritablement la vie (ibid., p.168-169). Ces exemples donnés en 1984 attestent à nouveau de la survivance d'une dramatisation non discursive des rapports du sujet à la vérité, telle que la pénitence seconde l'avait lointainement ritualisée.

Nous tenons ici une première raison importante de ne pas signer trop vite le permis d'inhumer de la vieille pénitence. «Pénitence » et «monachisme », indique Foucault en 1980, renvoient à « deux institutions qui sont à la fois voisines, qui sont en un sens parallèles, mais qui vont dans deux directions inverses et opposées $\gg^{78}$. La pénitence s'efforce de maintenir et de manifester la possibilité du salut dans une vie toujours menacée par le péché malgré le baptême, tandis que le monachisme maintient l'exigence d'un perfectionnement intime et secret dans une vie déjà sauvée par le sacrifice du Christ. Ces deux pratiques vont sans cesse interférer sans jamais complètement se recouvrir, même si «le monachisme sera, jusqu'à un certain point, une vie de pénitence [tandis que] la pratique de pénitence elle-même empruntera, tout au long de son histoire, beaucoup d'éléments au monachisme ${ }^{79}$. Même écho à Louvain en 1981: «On a donc, dans ces deux formes une compénétration, contamination réciproque, une tendance à s'homogénéiser jusqu'à un certain point sans bien sûr que le dimorphisme entre ces deux formes de vie soit jamais supprimé $\gg^{80}$.

78 Leçon du 19 mars 1980. Dans le cours Mal faire, dire vrai, Foucault corrige le terme « institution $\gg$ qu'il trouve trop fort, mais maintient la différence : « Ces deux pratiques, exomologèse pénitentielle, exagorèse spirituelle, en réalité, représentent beaucoup plus deux pôles entre lesquels il y a une série de formes graduées que deux institutions ou pratiques parfaitement distinctes. »

79 Leçon du 19 mars 1980.

80 Cours Mal faire, dire vrai, nous soulignons. 
Fidèle ici aux principes de l'analyse stratégique, Foucault établit une connexion entre des domaines de pratique qui n'en viennent jamais pour autant à se fondre en une nouvelle unité. Pour cette raison, « pénitence » et «monachisme » sont eux-mêmes parcourus par une tension interne qui n'est que l'effet de leurs croisements incessants : le paradoxe de la pénitence, rencontré chez Tertullien, entre une vie qui est perpétuelle mort à soi et une vie définitivement sauvée par l'eau du baptême, se retrouve dans le monachisme entre une expérience spirituelle tendue vers l'union définitive à Dieu et un risque de chute d'autant plus élevé que le moine se croit arrivé au but.

\section{Raisons méthodologiques}

Mais c'est tout autant le nouveau mode d'analyse des techniques de soi proposé par Foucault qui empêche de confondre la pénitence commune et la vie monastique, alors même que les deux domaines n'ont cessé historiquement de se croiser et d'influer l'un sur l'autre. En effet, la différence entre le moine et le laïc ne permet pas seulement de distinguer les sujets qui vont être soumis à telle ou telle obligation, mais également de définir les conditions sous lesquelles ces sujets vont pouvoir se constituer comme objets de cette obligation. La notion de «régimes de vérité » pénètre en effet beaucoup plus profondément le champ des pratiques que les seules codifications juridiques. Le moine se soumet à la direction spirituelle parce qu'il est entré dans un monastère, de la même manière que le logicien - dans l'exemple étudié dans notre première partie - s'incline devant la vérité logique « parce qu'il fait de la logique, c'est-à-dire parce qu'il s'est constitué lui-même, ou qu'il a été invité à se constituer comme opérateur dans un certain nombre de pratiques ou comme partenaire dans un certain type de jeu $\gg^{81}$. Il ne s'agit pas seulement de recevoir de l'extérieur un statut canonique avec des obligations afférentes, mais de se laisser librement transformer par un certain nombre d'expériences probatoires successives. Le terme «expérience » ne renvoie pas ici à un sentiment existentiel immédiat, mais bien à une épreuve codifiée dont le but est de modifier durablement le rapport qu'un sujet entretient à lui-même ${ }^{82}$.

81 Leçon du 6 février 1980, nous soulignons.

82 Dans la leçon du 13 février 1980, Foucault insiste pour donner à ce terme un sens non trivial et non spontané : «expérience comme ce qui, à la fois, qualifie le sujet, l'illumine 
Cette importance décisive de «l'expérience » se vérifie dans la place accordée par Foucault, dans le cours de 1979-1980, à la manière dont les jeunes gens sont acceptés et progressivement intégrés à la communauté monastique, à travers une série précise d'épreuves qui ont chacune leur durée propre : dix jours à la porte du couvent, un an comme postulant, plusieurs années comme novice ${ }^{83}$.

Si Foucault indique clairement en 1980 le caractère déterminant de ces probations, il n'en fait pas encore explicitement un axe particulier de sa recherche. Réécoutons à ce sujet le découpage proposé par le cours pour discriminer les pratiques de soi. Foucault distingue trois champs d'étude : «nature », «instruments $»$ et $\ll$ objectifs $»^{84}$. Sous des dénominations plus complexes, nous retrouvons ce même découpage dans l'introduction générale des trois volumes à venir de L'histoire de la sexualité, publiée dans Le Débat en novembre 1983 : «substance éthique», «formes de l'“élaboration" du travail éthique » et «téléologie du sujet moral ${ }^{85}$. Entre la « substance éthique $\gg$ et les « formes d'élaboration », l'article de 1983 insère cependant le «mode d'assujettissement » (p.1375), c'est-à-dire la « façon dont l'individu établit son rapport à cette règle et se reconnaît comme lié à l'obligation de la mettre en œuvre $\gg$ (p.1375). En donnant immédiatement après les exemples de l'appartenance à un groupe, de l'adhésion à une tradition spirituelle et de la réponse à un appel personnel, Foucault explicite sa proposition : le mode d'assujettissement désigne le type de lien qui lie le sujet à son devoir, du fait par exemple de son histoire personnelle, de sa situation sociale ou de ses choix de vie. Autrement dit : la raison que le sujet se donne à luimême d'obéir à une règle, étant entendu qu'une même règle peut obliger de bien des manières différentes ${ }^{86}$. Loin d'être la seule conséquence

sur soi et sur le monde et, en même temps, le transforme ». Dans la leçon du 19 mars 1980, Foucault cite de manière significative le mot experimentum, qui désigne chez Cassien les dix jours passés par le postulant à la porte du monastère où il demande à entrer. Foucault met à cette occasion experimentum en série avec « probation » : il s'agit dans les deux cas de désigner une épreuve transformatrice du sujet. C'est dans ces mêmes années 1978-1980 que le terme «expérience » devient décisif dans la manière dont Foucault présente son propre travail : $D E \mathrm{n}^{\circ}$ 281, «Entretien avec Michel Foucault », II, p. 863-864. Il restera thématiquement important jusque dans ses derniers travaux : L'usage des plaisirs, p. 10.

83 Leçon du 19 mars 1980.

84 Leçon du 12 mars 1980.

$85 D E \mathrm{n}^{\circ} 338$, « Usage des plaisirs et techniques de soi », II, p. 1375-1376.

86 Dans un entretien à Berkeley en avril 1983, Foucault mentionne en ce sens la raison 
d'un statut social ou juridique, le mode d'assujettissement peut aussi renvoyer à une certaine $\ll$ habitude $\gg^{87}$, terme faussement simple dont Foucault avait souligné l'importance chez Tertullien en le traduisant par «manière de vivre ${ }^{88}$. Dans les exemples donnés en 1983 , cette manière de vivre ne renvoie pas tant à un choix ponctuel et strictement individuel qu'à une pratique inscrite dans une durée et une mémoire collective.

Ces réflexions de l'année 1983 nous permettent de distinguer sous une même règle des manières différentes de s'y soumettre. Or, c'est bien la prise en considération $\mathrm{du}$ 《mode d'assujettissement » qui force à distinguer l'examen monastique des techniques appliquées aux simples laïcs, quand bien même ces deux groupes devraient en apparence se plier aux mêmes examens et lâcher les mêmes aveux : l'homme marié et le moine ne se reconnaissent pas liés par les mêmes obligations, parce qu'ils n'ont pas traversé les mêmes expériences, ne se réfèrent pas à la même histoire collective, n'ont pas répondu au même appel personnel. Dans ces conditions, étendre la technique de direction aux laïcs ne peut pas consister simplement à leur ajouter d'autorité une obligation de plus. Pour l'historien, il s'agit de se demander par quels jeux d'obéissance et de vérité les laïcs vont être préparés à choisir d'être dirigés de cette manière-là. Cette simple interrogation permet de ne pas considérer l'extension des techniques monastiques à l'ensemble du peuple chrétien comme une simple translation d'autorité. Il y a bel et bien une coupure initiale à surmonter, qui n'est pas seulement juridique, mais engage des modes de subjectivation différents.

Cette coupure et ses conséquences sur les techniques de gouvernement, Foucault les avait très indirectement mentionnées dans ses

donnée par Nicoclès de sa fidélité conjugale : «Parce que je suis roi et quelqu'un qui gouverne les autres, je dois montrer que je suis capable de me gouverner moi-même » $\left(D E \mathrm{n}^{\circ} 326\right.$, «À propos de la généalogie de l'éthique : un aperçu du travail en cours », II, p. 1214). Le «mode d'assujettissement » renvoie donc au type d'autorité que le sujet choisit de reconnaître pour régler sa conduite : loi naturelle, loi rationnelle, ordre cosmologique, révélation, etc. La soumission à une révélation se vit effectivement sur un mode différent que la soumission à un ordre rationnel. «Et l'on voit donc que la manière dont la même loi est acceptée par Nicoclès et par un stoïcien est très différente » (ibid.).

$87 D E n^{\circ} 338$, « Usage des plaisirs et techniques de soi », II, p. 1375-1376, nous soulignons : «On peut, par exemple, pratiquer la fidélité conjugale, et se soumettre au précepte qui l'impose, parce qu'on se reconnait comme faisant partie du groupe social qui l'accepte, qui s'en réclame à haute voix et qui en conserve silencieusement l'habitude. »

88 Voir la note 192 du chapitre «Le christianisme au risque de l'analyse stratégique ». 
travaux des années 1977 et 1978. Dans l'entretien pour la revue Ornicar ?, dans le cours Sécurité, territoire et population et enfin dans la conférence du 20 avril 1978 à Tokyo, il s'agissait bien déjà de reconnaître l'écart entre le moine et le chrétien marié, plus précisément entre une doctrine de l'ascétisme intégral qui refusait le monde et une morale stoïcienne modérée qui l'acceptait. Entre les deux, notait alors Foucault, « le christianisme a toujours hésité ${ }^{89}$, comme le vérifie un monachisme chrétien à la fois continuateur de pratiques ascétiques plus anciennes et contrôleur vigilant de leurs excès, ces derniers étant considérés comme autant de foyers possibles d'insoumission. Mais en disant cela, comme nous l'avons indiqué plus haut, Foucault faisait finalement passer la coupure à l'extérieur du christianisme - religion supposée être « fondamentalement anti-ascétique $\gg^{90}$. Ou tout du moins, il la faisait passer à proximité immédiate de ses marges, contaminées par les hérésies gnostiques ou un certain monachisme sauvage (l'anachorèse syrienne et égyptienne).

Le cours de 1980 remet au contraire cette question, non seulement au cœur de son analyse, mais aussi et surtout au cœur du christianisme. Il est d'autant plus étrange que le « mode d'assujettissement » n'apparaisse pas en toutes lettres comme critère de différenciation des techniques de soi, aux côtés des questions de «nature », d'《instruments » et $d^{\prime}$ « objectif ». Mais au moins le critère est-il implicitement pris en compte dans l'argumentation.

\section{La différence entre le salut et la perfection}

La division entre pénitence et monachisme semble malheureusement nous empêcher de ressaisir l'originalité chrétienne dans une forme unique - à moins de considérer que l'une des deux voies aurait des origines anté-chrétiennes, baptisant de fait l'autre. La fin du cours Le courage de la vérité (1983-1984) laisse effectivement entendre que la voie «parrèsiastique » des martyrs et des mystiques serait un simple prolongement de l'ancienne voie cynique. Si l'on accepte cette thèse, l'autre voie chrétienne - celle que Foucault nomme «anti-parrèsiastique » ou «ascétique » - ne serait pas tant un second rameau du même arbre 
qu'un greffon ultérieur qui constituerait finalement, peut-être, la seule et unique différence chrétienne : avoir remplacé la parole franche née de la confiance en Dieu par la défiance vis-à-vis de soi, impliquant direction et examen perpétuels. Péripétie du $\mathrm{IV}^{\mathrm{e}}$ siècle, donc, comme en retard sur l'origine, mais aux conséquences durables.

Ce greffon rendrait alors nulle et non avenue la question posée en introduction de notre recherche de savoir ce qui pourrait unifier, du point de vue de l'histoire de la pensée, l'ascétisme des Pères du désert et la pastorale augustinienne, la doctrine de la confession à Port-Royal et la doctrine de la confession chez Medina ou Suárez. Absolument rien, semble nous dire la dernière leçon de 1984 : les régimes de vérité chrétiens seraient seulement renvoyés à deux ensembles distincts de pratiques, sans origine commune, que seules les secousses de l'histoire auraient forcés à s'entrechoquer et à interagir. Ainsi disparaîtrait le christianisme, « comme à la limite de la mer un visage de sable ».

À ceci près que «pénitence » et «monachisme » ne se rencontrent pas dans l'hétérogénéité d'une histoire vouée à l'éclatement, mais ils naissent de la scission, dans l'histoire de la spiritualité, entre deux états du sujet : le salut et la perfection. Voilà en effet ce que Foucault retrouve en 1980 à la racine des deux voies : non pas un débat avec le vieux thème philosophique de la parrêsia, mais une réponse inédite à la question du jeune homme riche « Que dois-je faire pour avoir la vie éternelle ? ${ }^{91}$; ou plus précisément : «Dois-je être parfait pour être sauvé ? » Rappelons que la définition du salut et de la perfection a beau être d'ordre théologique, elle induit aussitôt des manières d'être, des pratiques reconnaissables et des institutions précises. En ce sens, le salut et la perfection appartiennent aussi au réel de l'histoire. Foucault use à ce titre d'une gradation intéressante pour désigner les états moraux ou spirituels dont parlent les textes anciens : en les désignant, il va méthodiquement de l'expression la plus abstraite à l'image la plus concrète. En étudiant les rapports entre le salut et la perfection, il s'agit d'étudier la «forme de lien », la « forme d'insertion $\gg, l^{\prime} \ll$ ancrage $\gg$ du sujet dans la vérité et de la vérité dans le sujet ${ }^{92}$.

Or, une telle scission entre salut et perfection ne va pas de soi. L'audace chrétienne qui a consisté à les distinguer, dans un arrachement qui rend chaque terme incertain aux yeux du commun et arbitraire aux yeux 
du sage, est justement ce qui permet à Foucault de définir l'événement de pensée ${ }^{93}$ qui, dans les premiers siècles de notre ère, a bouleversé les rapports du sujet à la vérité. Le cours $D u$ gouvernement des vivants insiste en effet sur la grande difficulté qu'il y avait à séparer ce que le contexte hellénistique et romain avait si fermement et universellement uni. Une telle séparation était incompréhensible pour un pythagoricien, un philosophe de l'Académie ou encore un stoïcien :

Le christianisme est une religion du salut dans la non-perfection. Tầche extraordinairement difficile à réaliser à une époque où, justement, la plupart des mouvements religieux du monde antique, du monde hellénistique et romain associaient d'une façon profonde et fondamentale la promesse du salut à l'accès à la perfection. Je crois que le grand effort et la grande singularité historique du christianisme, qui explique sans doute un grand nombre des traits de son développement et de sa permanence, c'est d'être arrivé à dissocier salut et perfection. ${ }^{94}$

Dans cet extrait du cours particulièrement assertif, Foucault propose une définition à la fois simple et aiguë de la singularité chrétienne, dans laquelle le professeur s'engage personnellement à la première personne. Étrangement, cette définition disparaîtra de ses réflexions ultérieures, alors qu'elle est la seule véritablement opérationnelle, la seule qui permette d'interpréter sans les dissocier les deux formes principales de techniques de soi chrétiennes :

Pénitence et monachisme $[\ldots]$ viennent se loger dans cet écart instauré, dans l'histoire du christianisme, par l'histoire du christianisme, entre perfection et salut. (Ibid.)

Qu'elle soit ou non un hapax dans l'œuvre, une hypothèse oubliée ou révisée, une telle proposition mérite d'être étudiée dans toutes ses conséquences. Il nous faut pour cela rappeler un point de méthode : nous avons choisi dans cette partie d'écouter Foucault pour lui-même, c'est-àdire dans la cohérence de sa pensée, ressaisie ici au niveau le plus général des «formes de rationalités » qu'il décrit. Nous avons donc choisi d'estomper les distinctions parfois subtiles entre les écoles philosophiques, qu'elles soient hellénistiques ou chrétiennes - même si les cours au Collège de France ne les ignorent pas -, une fois reconnue et acceptée l'hypothèse d'une césure essentielle survenue au début de notre ère dans

93 Les problématisations nouvelles seront définies quelques années après comme des « événements de pensée »: $D E$ n $^{\circ}$ 340, « Préface à l'Histoire de la sexualité », II, p. 1399. 
les manières de se rapporter à soi. En parlant $\mathrm{du}$ «christianisme $\gg$ ou du «stoïcisme », il ne s'agit donc pas tant de décrire un espace complet que de saisir les éléments de cet espace qui s'écartent absolument des espaces avoisinants ; il s'agit moins de nommer des identités que des différences.

Revenons à l'événement de la césure. Rien de moins évident en apparence que de dissocier le salut de la perfection : qu'est-ce en effet qu'une perfection qui n'apporterait pas le salut? Qu'est-ce qu'un salut qui ne récompenserait pas une perfection? Pour parvenir à un tel résultat, il a fallu au christianisme repenser de fond en comble le « problème » 95 du salut individuel, ou plutôt la manière de l'élaborer. Dans la leçon du 27 février 1980, Foucault décrit en effet la singularité chrétienne en des termes qui annoncent clairement ce qu'il définira quelques années plus tard comme un travail de «problématisation ». Contre la connotation encore trop négative et sommaire du terme « problème », le néologisme utilisé à plusieurs reprises par Foucault dans ses dernières années a pour fonction de souligner le caractère positif du mouvement qui permet d'élaborer, à partir d'une constellation de difficultés rencontrées autour d'un même domaine de pratiques, une trame nouvelle de réflexion ${ }^{96}$. Un «problème » ne doit pas être perçu par l'historien comme un obstacle qui apparaîtrait naturellement à la surface de l'histoire, bloquant temporairement la pensée et l'action; il est déjà en lui-même un riche matériau mental - un jugement synthétique a priori pourrait-on dire ${ }^{97}$ -

96 Voir les notes 6 et 7 (ce volume, p. 12-13) pour les occurrences principales du terme $\ll$ problématisation $\gg$.

97 Pour Kant, un tel jugement n'est ni un jugement d'expérience ni un jugement analytique a priori (par lequel j'extrais d'un concept A le prédicat B qui le contient). Il est l'acte par lequel la pensée, avant toute expérience, pose un lien entre deux concepts qui n'ont entre eux aucune relation d'inclusion : E. Kant, Critique de la raison pure, III, 35, A. J. L. Delamarre, F. Marty, J. Barni trad., Paris, Gallimard, 1980, p. 73. La métaphysique est précisément l'un des domaines indiqués par Kant où règnent de tels jugements synthétiques a priori (ibid., III, 39, p. 77). En définissant en 1984 ce qu'est pour lui une «problématisation », Foucault retrouve bien le vocabulaire de l'a priori kantien, même s'il s'agit toujours d'un a priori historique, s'imposant à la pensée uniquement pour une période donnée (et non de toute éternité comme le jugement cité en exemple par Kant : «Tout ce qui arrive à sa cause »). Sans invoquer nommément les « conditions de possibilité », Foucault parle des «conditions dans lesquelles des réponses possibles peuvent être données », mises en lumière par une « analyse critique » $\left(D E n^{\circ} 342, \ll\right.$ Polémique, politique et problématisations $\gg$, II, p. 1417). 
définissant les éléments et les conditions qui permettront ultérieurement la formulation de solutions. Sa généralité, qui permet de rassembler le divers contrariant de l'expérience, se retrouve bien dans l'expression proposée par Foucault de « forme générale de problématisation $\gg^{98}$. Appliquons cette définition au christianisme des premiers siècles.

Les difficultés rencontrées autour des pratiques de salut par les premières communautés chrétiennes sont principalement :

1) la réintégration des relaps : peut-on abandonner ceux qui ont renié leur profession de foi dans un contexte de persécution?

2) les dérives ascétiques et antinomiques des mouvements gnostiques, quand ceux-ci poussent à l'extrême le principe de la délivrance définitive par la connaissance : peut-on accepter, au nom de cette pureté absolue, le rejet complet du monde et des lois humaines ${ }^{99}$ ?

Mais plus généralement, au-delà de ces deux exemples mentionnés par Foucault, c'est le caractère élitiste de la philosophie antique - celle en particulier du premier stoïcisme ${ }^{100}$ - comme union définitive du salut et de la perfection, qui ne peut s'accorder avec les vues populaires du christianisme naissant. L'exigence démesurée du «souci de soi »stoïcien s'accompagnait en particulier de l'exclusion de fait d'une bonne partie de l'humanité : femmes, enfants et esclaves, là où Clément d'Alexandrie fera au contraire de l'enfant le modèle de la vie chrétienne ${ }^{101}$. « S'occuper de soi-même, c'est évidemment un privilège élitaire » remarque Foucault dans L'herméneutique du sujet (p.109). Refusant de prendre l'Antiquité pour modèle, le philosophe n'aura de cesse, en particulier dans l'entretien avec Hubert Dreyfus et Paul Rabinow, de rappeler combien la morale grecque était liée « à une société virile, à l'idée de dissymétrie, à l'exclusion de l'autre $\gg^{102}$.

Pour Foucault, le succès historique du christianisme, sa «perma-

$98 D E \mathrm{n}^{\circ} 342$, « Polémique, politique et problématisations », II, p. 1417, nous soulignons.

99 Leçon du 27 février 1980.

100 Ibid. C'est-à-dire avant «l'âge d'or » des $\mathrm{I}^{\mathrm{er}}$ et II $\mathrm{I}^{\mathrm{e}}$ siècles, étudié par L'herméneutique du sujet. Dans le dernier stoïcisme, le souci de soi devient un «principe universel » s'appliquant le plus largement possible, au-delà des différences sociales. Cependant, malgrél'élargissement du souci de soi, Foucault insiste sur le fait que «pareille prescription $[\ldots]$ ne peut être mise en œuvre que par un nombre évidemment très limité d'individus » (L'herméneutique $d u$ sujet, p.109). Le sage montre la porte à tout le monde, mais la porte reste étroite.

101 Leçon du 13 février 1980.

$102 D E n^{\circ} 326$, «À propos de la généalogie de l'éthique : un aperçu du travail en cours », II, p. 1207. 
nence $\gg^{103}$ ou sa longévité, s'expliquent au contraire par sa promotion d'une voie moyenne, abandonnant la vieille conception binaire de l'illumination. Auparavant, la voie haute, dictée par une philosophie de l'identité de l'âme et du divin, pouvait s'énoncer comme suit : «Ou on a l'illumination, et à ce moment-là on y reste, ou on ne reste pas dans l'illumination et c'est-à-dire que l'on n'avait pas été réellement illuminé $\gg^{104}$. Malgré la prégnance de cette philosophie sur les premiers penseurs chrétiens, malgré l'irréversibilité de la purification baptismale affirmée par quelques écrits anciens comme l'Épitre aux Hébreux, l'Épittre de Barnabé et même Le pasteur d'Hermas, Foucault insiste sur le fait qu'un grand nombre de textes chrétiens, entre la fin du I ${ }^{\text {er }}$ siècle et la première moitié du $\mathrm{II}^{\mathrm{e}}$ siècle, empruntent déjà une autre voie. La Didachè, la Première épitre de Clément, la Lettre aux Philippiens de Polycarpe, la Seconde épittre attribuée à Timon « empêchent cependant de considérer que le christianisme, sous ses formes primitives, se considérait comme une communauté d'individus parfaits, purs et ne pouvant retomber $\gg^{105}$. En cas de faute morale importante, comme l'adultère, des exclusions temporaires de la table eucharistique pouvaient être prononcées au sein de la communauté, mais sans empêcher une pleine réintégration du baptisé après une période de jeûnes et de prières. Par la seconde pénitence, en dépit des débats qu'elle a suscités autour des $\mathrm{II}^{\mathrm{e}}$ et $\mathrm{III}^{\mathrm{e}}$ siècles, cette possibilité sera étendue aux fautes d'une gravité extrême, comme l'abjuration de la foi. Le problème nouveau, comme fruit d'une lente et difficile «problématisation », va donc être de maintenir les effets de l'illumination dans une vie d'imperfection, ou encore d'affirmer le salut d'une existence reconnue comme perpétuellement et universellement faillible - puisque l'homme parfait n'existe pas ici-bas, pas même dans les rangs des saints apôtres, encore moins dans ceux des monastères ${ }^{106}$.

Sil'on relit l'entretien de 1984 avec Paul Rabinow, on comprend alors pourquoi «pénitence » et «monachisme », en dépit de leur apparente

103 Leçon du 19 mars 198 o.

104 Leçon du 27 février 1980.

105 Leçon du 27 février 1980.

106 C'est le récit par Cassien d'une réunion des apôtres où ceux-ci sont incapables par eux-mêmes de décider du principe de leur discretio : «Même chez les personnages les plus saints, même chez ceux qui étaient les plus proches de la vérité, il y avait une tâche aveugle, il y avait un point qui leur échappait, ils ne pouvaient être à eux-mêmes leur propre mesure » (leçon du 26 mars 1980). 
opposition, ont pu naître sur ce «sol » commun, ancienne agora philosophique désormais vidée de ses sages, ouverte à tout le monde :

À un même ensemble de difficultés plusieurs réponses peuvent être données. Et la plupart du temps, des réponses diverses sont effectivement proposées. Or ce qu'il faut comprendre, c'est ce qui les rend simultanément possibles; c'est le point où s'enracine leur simultanéité; c'est le sol qui peut les nourrir les unes et les autres, dans leur diversité et en dépit parfois de leurs contradictions. ${ }^{107}$

Malgré leurs différences longuement détaillées par Foucault, voire leur incompatibilité foncière, la théâtralité de la seconde pénitence et la discretio du moine disent une même vie précaire, à l'intégrité constamment menacée, dont l'individu le plus exercé et le mieux équipé ne saurait s'affirmer le maître ultime. Parce qu'il reste toujours une seconde planche de salut pour le plus grand des pécheurs et parce qu'il n'y a jamais de perfection certaine pour le cénobite, se sont développées deux économies de crise, assurant un gain maximal pour une mise de départ médiocre - étant entendu que l'homme chrétien ne peut rien par lui-même, ne peut se sauver par ses propres forces. La possibilité de répéter dramatiquement la première pénitence et l'obligation d'être perpétuellement dirigé modifient en profondeur les relations de l'être à la vérité : le christianisme affirme l'irréversibilité du rapport à Dieu dans la répétition de la faute. Ce salut est garanti à la seule condition que le sujet accepte, dans un même mouvement de déprise, sa nature pécheresse et l'assistance d'autrui : celle du supérieur du monastère ou celle de la communauté ecclésiale représentée par l'évêque.

De ces deux économies de crise, c'est la seconde pénitence qui a la faveur de Foucault, non seulement parce qu'elle est première chronologiquement, mais également parce qu'elle est la manifestation éclatante d'une lumière qui ne l'est plus : même si le sujet a véritablement et pleinement eu accès à la vérité divine par l'illumination baptismale, il peut rompre ce lien en toute connaissance de cause. En ne faisant pas de la chute une simple méconnaissance, un faux pas au cours de l'ascension de l'âme vers le $\lambda o ́ \gamma o \varsigma$ divin, la seconde pénitence défait le prestige que la contemplation de l'être avait dans le souci de soi antique et même dans les premières théologies baptismales. Il ne suffit pas de connaitre véritablement ce qui est pour s'y accorder harmonieusement. Conséquence 
vertigineuse, si l'on pousse à son terme les réflexions de Foucault : la vérité n'est plus ce qui sauve l'homme, n'est plus ce qui le rend libre, n'est plus ce qui le protège définitivement de l'imperfection et de la faute. Ce qui sauve le moine, précise en effet Foucault, dans une accentuation volontairement paulinienne de la théologie chrétienne, ce sont uniquement $\ll$ les effets du sacrifice salvateur du Christ $»^{108}$.

Réminiscence possible d'une lecture ancienne de Kierkegaard - enseigné en khâgne par Jean Hyppolite et à la Sorbonne par Jean Wahl ${ }^{109}$ - c'est la notion de « répétition ${ }^{110}$ ou encore de $\ll$ recommencement » qui est au cœur de la leçon du 27 février 1980. Même s'il n'est pas nommément cité, la référence indirecte au penseur danois n'aurait rien pour nous surprendre : Foucault avait en effet conservé dans sa bibliothèque le petit volume de Kierkegaard intitulé La répétition, dans l'édition de 1933 annotée de sa main ${ }^{111}$. Par le détour d'une fiction mettant en scène un amant pris de remords après avoir traitreusement rompu avec sa fiancée, cet « essai de psychologie expérimentale » interroge la signification existentielle du pardon divin, qui n'est autre qu'un nouveau don ou une « répétition $»(\text { Gjentagelsen })^{112}$. Comme l'indique

108 Leçon du 19 mars 1980.

109 On n'en saura pas beaucoup plus sur le rapport de Foucault à Kierkegaard. La chronologie de Daniel Defert note à l'année 1951 : « Pense au Danemark. Lit Kafka et Kierkegaard, expliqué à la Sorbonne par Jean Wahl » (DE I, p. 19) et en décembre 1955: « Foucault accueille en Suède Jean Hyppolite, qui donne deux conférences sur "Histoire et existence" et "Hegel et Kierkegaard" dans la pensée française contemporaine » (DE I, p. 25). Foucault ne parlera plus de Kierkegaard par la suite, à de très brèves exceptions près, peu significatives. Notons cependant les thématiques fortement kierkegaardiennes de l'hommage à Maurice Clavel en 1979, en particulier la référence à «l'instant », comme ce qui échappe radicalement à l'histoire : $D E \mathrm{n}^{\circ} 268$, «Vivre autrement le temps », II, p. 790. Nous sommes bien alors du côté des Miettes philosophiques (1844).

110 Quatre occurrences dans la leçon du 27 février 1980.

111 S. Kierkegaard, La répétition, P.-H. Tisseau trad., Paris, Alcan, 1933. Ce volume nous a été montré par Daniel Defert. Les annotations manuscrites sont surtout celles d'un étudiant surlignant un mot, un passage.

112 Le mot «pardon » n'apparaît pas dans le texte de Kierkegaard, mais c'est bien de cela dont il est question dans la «vraie » répétition, une fois écartées toutes les «fausses » répétitions (répétition comme ressouvenir, répétition comme effacement, répétition comme imitation, etc.). C'est bien l'idée de réconciliation entre Dieu et l'homme que l'on retrouve dans l'évocation par le jeune homme de la figure de Job : « le Seigneur et Job se sont compris, ils se sont réconciliés [...] - Job est béni et a tout reçu en double. - On appelle cela : une répétition » (S. Kierkegaard, La répétition, J. Privat trad., Paris, Payot \& Rivages, 2003, p.160). 
le pseudonyme Constantin Constantius, la possibilité de se réconcilier avec soi-même et avec Dieu ne repose ni sur les forces de la volonté ni sur les puissances de l'esprit. Stricte transcendance, elle n'est offerte ni par la philosophie grecque, dans le ressouvenir platonicien, ni par la philosophie moderne, dans l'Aufhebung hégélien :

[... en effet, les Grecs font le mouvement inverse, et un Grec choisirait à ce propos le ressouvenir, sans que sa conscience ne le torture; quant à la philosophie moderne, elle ne fait aucun mouvement, $[\ldots]$ et si elle se risque à en faire un, c'est toujours dans l'immanence; en revanche, la répétition est et demeure une transcendance. (Ibid., p. 121)

Lestée ou non de cette référence kierkegaardienne, la notion de répétition fait pivoter le cours sur lui-même : la réflexion sur le vrai que le sujet doit dire à propos de lui-même devient réflexion sur le vrai que le sujet ne peut être à lui-même. Sans cesse, il lui faut refonder son rapport à une vérité divine avec laquelle il ne peut coïncider. La scansion nouvelle du temps par la «répétition » amène alors Foucault à cette proposition forte : ce que le christianisme a introduit dans le monde romain et hellénistique n'est pas le péché ou la chute, mais la possibilité de la rechute. En effet, «l'idée même de rechute était une idée qui était étrangère aussi bien à la culture grecque, hellénistique et romaine qu'à la religion hébraïque $\gg^{113}$. Le christianisme a introduit le rapport à la vérité dans une temporalité incertaine, où les liens se distendent et les fidélités se rompent, transformant l'unique fois de l'adhésion du sujet à la vérité en simple première fois, permettant ainsi une seconde fois (seconde pénitence), qui ne sera bientôt plus qu'une deuxième fois (pénitence indéfiniment répétable de la confession).

Avant l'ère chrétienne, Foucault dégage deux grands types de pensée morale : le «système de la loi », dans lequel l'action bonne est définie uniquement en fonction de sa forme, de ses composantes et de ses effets, réduisant ainsi le sujet à n'être qu'un «élément modificateur de l'action » (ibid.); et le «schéma du salut», dans lequel le statut du sujet vient au contraire directement qualifier l'action, quelle que soit la forme de celle-ci. Ainsi du philosophe dans le premier stoïcisme : «son action est forcément bonne parce qu' il est sage $\gg$ ( $i b i d$.). Dans le premier cas, la faute est indéfiniment répétable, dans le second, le statut qui lie le sujet à la vérité est irréversible. Le tableau ci-dessous permet de résumer l'opposition : 


\begin{tabular}{llllll}
\hline & $\begin{array}{c}\text { Déterminant du } \\
\text { bien et du mal }\end{array}$ & \multicolumn{1}{c}{$\begin{array}{c}\text { Objet du } \\
\text { partage }\end{array}$} & $\begin{array}{c}\text { Type de } \\
\text { partage }\end{array}$ & $\begin{array}{c}\text { Type de } \\
\text { sujet }\end{array}$ & $\begin{array}{c}\text { Rapport } \\
\text { au temps }\end{array}$ \\
\hline $\begin{array}{l}\text { Système } \\
\text { de la loi }\end{array}$ & $\begin{array}{l}\text { Qualité de } \\
\text { l'action }\end{array}$ & $\begin{array}{l}\text { Actions bonnes / } \\
\text { actions mauvaises }\end{array}$ & Formel & $\begin{array}{l}\text { Sujet de } \\
\text { volonté }\end{array}$ & Répétable \\
\hline $\begin{array}{l}\text { Schéma } \\
\text { du salut }\end{array}$ & $\begin{array}{l}\text { Qualité du } \\
\text { sujet }\end{array}$ & $\begin{array}{l}\text { Individus sauvés / } \\
\text { individus non } \\
\text { sauvés }\end{array}$ & Temporel & $\begin{array}{l}\text { Sujet de } \\
\text { connaissance }\end{array}$ & Irréversible \\
\hline
\end{tabular}

Comme à son habitude, Foucault décrit ce faisant deux ensembles à la fois disjoints et strictement complémentaires - réflexe mathématique qui permet de s'assurer que tous les exemples historiques entreront bien dans la réunion des deux ensembles. Malgré ce découpage formel, Foucault précise aussitôt que les deux ensembles n'ont cessé de se croiser, de se contaminer mutuellement - transformant par exemple le vrai sage en un simple «idéal régulateur de comportement » (ibid.) ou encadrant, comme chez les pythagoriciens, la vie de sagesse par un ensemble méticuleux de prescriptions. Mais ils n'ont jamais reçu une commune intelligibilité, car ils n’ont jamais croisé un problème général qui permette de les penser ensemble, jusqu'au christianisme.

Le christianisme ne synthétise pas le système de la loi et le schéma du salut, mais il les maintient paradoxalement face à face, dans la distance posée par le libre arbitre. Perfection et salut sont à ce point décollés que leur union dans le temps ne saurait être qu'illusoire, fruit vénéneux de

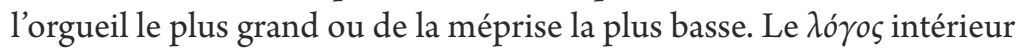
guidait le sage antique, lui assurant la parfaite maîtrise de lui-même, de ses affects, de ses passions. L'homme chrétien n'a pas d'empire, que des obscurités, faiblement éclairées par une discretio qui ne lui appartient pas en propre, puisque - comme le précise Foucault en lisant Cassien - «Il n'y a pas de discretio naturelle, immanente à l'homme $\gg^{114}$. Depuis Tertullien, Satan habite dans l'âme humaine, altérant celle-ci, la divisant avec elle-même, sans que jamais ne cesse le combat ni ne s'éloigne le danger ${ }^{115}$. Comment alors offrir un salut à une subjectivité aussi irrésolue dans ses actions et aussi opaque dans ses pensées ? La réponse chrétienne à cette question n'a pas été de resserrer la loi pour qu'elle mène plus sûrement à 
la perfection, encore moins d'abaisser l'idéal pour relativiser les chutes successives. Pour reprendre le titre du bel hommage de Michel Foucault au philosophe catholique Maurice Clavel, elle a été de « vivre autrement le temps $»^{116}$ : le vivre sur un autre mode que celui de «l'avant $\gg$ et de «l'après » qui caractérisait l'ancien schéma du salut et scindait l'existence en deux parties incomparables. L'ancien schéma ne permettait pas le retour en arrière, « puisque le temps ne retourne pas sur lui-même en tout cas le temps des individus $\gg{ }^{117}$. Foucault montre au contraire que la seconde pénitence répète, non pas le baptême, mais la pénitence prébaptismale : elle est le « recommencement même de l'acte tout entier par lequel on est purifié des péchés que l'on a commis $\gg^{118}$.

Cette nouvelle économie du salut intègre la rechute, qu'elle finira dans la pratique pastorale par considérer comme inéluctable. En ce sens, la sotériologie chrétienne trace une voie moyenne, non élitiste, méfiante vis-à-vis de tout rapport à Dieu qui n'intégrerait pas mais prétendrait dépasser les infirmités de l'homme dans son rapport à la vérité. Le christianisme ne rapproche pas pour autant le ciel de la terre, maintenant la radicalité et la violence des ruptures, qu'elles soient le fait du péché ou de la $\mu \varepsilon \tau$ ávola (ibid.). Comme l'explique Foucault dans l'hommage à Clavel, la rencontre du temps et de l'éternité prend désormais la forme de $\ll$ la fracture, $[\mathrm{du}]$ déchirement, $[\mathrm{de}] \mathrm{l}^{\prime}$ interruption $\gg^{119}:$ nulle vérité ne s'installe à demeure dans l'histoire sinon celle, objective, du Dogme révélé, et celle, subjective, du labeur patient de la pénitence.

La patience requise n'est pas seulement à entendre comme l'attente soutenue et docile du jour dernier. En commentant la patientia du moine, Foucault lui donne un sens beaucoup plus riche que celui découlant de sa seule inscription dans une liste des caractéristiques de la vie conventuelle. Notre hypothèse est que la patientia résume à elle seule l'attitude fondamentale de l'homme obéissant, en révélant un nouveau rapport du sujet au monde extérieur.

DE n 268, «Vivre autrement le temps », II, p. 788. Sur les rapports de Michel Foucault à Maurice Clavel, Daniel Defert fait remarquer : «Clavel croyait en Dieu mais n'aimait pas l'Église ; Foucault ne croyait pas en Dieu mais s'intéressait beaucoup à l'Église ! » (entretien avec Daniel Defert, 6 décembre 2006).

117 Leçon du 19 mars 1980.

118 Leçon du 27 février 1980.

$119 D E \mathrm{n}^{\circ} 268$, «Vivre autrement le temps », II, p. 790. 
À première vue, nulle raison d'accorder à la patientia une place particulière dans l'analyse : comme nous l'avons vu, la leçon du 19 mars 1980 en fait simplement l'une des caractéristiques de l'obéissance monastique, à côté de la subditio et de l'humilitas, formant ainsi le triangle de l'assujettissement par la passivité. Mais la radicalité avec laquelle Foucault interprète cette obéissance, lue dans la lettre de textes auxquels il n'accorde le bénéfice d'aucune ironie pédagogique ni d'aucun effet rhétorique, nous invite à ne pas redouter de mener ce raisonnement à son terme. Si l'obéissance mène aussi loin, prenons le risque de l'y accompagner.

Considérons tout d'abord l'humilitas. Comme nous l'explique Foucault, cette vertu concerne le « rapport à soi $\gg^{120}$ : il s'agit de se disqualifier soi-même comme volonté ou encore «ne pas vouloir vouloir » (ibid.). Mais que devient donc le soi quand il n'est plus volonté voulant vouloir? Foucault le dit à demi-mot : il s'agit de «se mettre aussi bas que possible $\gg(i b i d$.), et encore «plus bas que tout $\gg(i b i d$.), c'est-à-dire ne différer en rien « d'un corps inanimé ou de la matière première employée par un artiste » (ibid.). Sans retour sur soi, le sujet n'est plus qu'extériorité, exposé aux aléas de la nature comme le grain de sable ou au bon vouloir du potier comme l'argile. Peu importe dès lors qui le manipule.

Regardons maintenant la subditio, qui concerne cette fois le « rapport aux autres » (ibid.). À sa fine pointe, comme nous l'avons vu, la subditio n'est plus soumission à une loi, ni même à quelqu'un en particulier : elle devient une attitude permanente qui n'a plus d'objet pour lui assigner une limite ou une mesure. La subditio transmue tout ce qui advient - «tout autre, tout événement » (ibid.) - en ordre, de telle manière que «le moine vit dans un monde qui est peuplé d'ordres » (ibid.). Foucault insiste : «le monde tout entier est ordre » (ibid.). C'est-à-dire que la subditio véritable précède le rapport à des individus pour devenir une disposition première du sujet, une attitude fondamentale vis-à-vis du dehors ${ }^{121}$.

Dans les deux cas, l'humilitas et la subditio annulent leur objet (le soi, les autres) pour se transformer en autre chose que ce pour quoi ils avaient initialement été définis : le rapport d'assujettissement d'individu

120 Leçon du 19 mars 1980.

121 Notons qu'à Louvain en 1981, Foucault fait de la subditio la caractéristique « la plus fondamentale de l'obéissance $\gg$, mais ne dit mot de cette intériorisation radicale de la subditio, pourtant requise du moine et soulignée en 1980. Foucault l'inscrit strictement dans la relation à autrui. Il y a là un appauvrissement notable du sens (cours Mal faire, dire vrai). 
à individu. Étrangement, ces deux notions viennent alors converger vers un point qui n'est autre que le rapport au monde extérieur, objet précisément de la troisième vertu caractéristique de l'obéissance monastique : la patientia. « Notion difficile ${ }^{122}$ nous prévient aussitôt Foucault qui se garde bien pour cette raison de la traduire. La patientia dit d'abord une passivité absolue par rapport aux ordres donnés : pas d'hésitation, pas de résistance, pas de réflexion. Mais elle dit autre chose, qui a rapport aux événements, à la contingence, à tout ce qui advient : en ce sens, elle est bien « une attitude à l'égard du monde extérieur » (ibid.). Sous les coups du sort, les violents assauts du dehors, elle est « une certaine endurance, une capacité à résister ou, en tout cas, à supporter » (ibid.).

À l'idée que la patientia chrétienne serait une résistance héroïque à l'événement douloureux, une objection vient aussitôt : le sage stoïcien ne faisait-il pas de même? La distinction posée par Foucault est en fait extrêmement subtile. L'exemple choisi pour illustrer la notion est un extrait des Institutions cénobitiques de Cassien, déjà cité - mais pour d'autres raisons - dans le cours Sécurité, territoire, population ${ }^{123}$ : Patermutus, arrivant au monastère avec son fils, supporte de voir celui-ci frappé et humilié par les autres moines, avant d'accepter de le noyer à la demande de l'ancien; jusqu'au moment où ce dernier, ayant ainsi éprouvé l'obéissance de Patermutus, interrompt l'exercice. Un rien scandaleuse, cette histoire n'est pourtant pas évoquée pour mettre une nouvelle fois en scène l'absurdité de l'obéissance monastique, mais uniquement pour opposer l'ả $\pi \dot{\theta} \theta \varepsilon$ la grecque à la patientia chrétienne : inflexible, Patermutus a tout supporté, mais il ne l'a pas fait en philosophe. À cela, les définitions de l'ả $\pi \dot{a} \theta \varepsilon ı a$ fournies par le cours Sécurité, territoire, population offrent un premier élément d'explication :

$\dot{\alpha} \pi \dot{\theta} \theta \varepsilon \iota a$ grecque :

N'avoir pas de passions, c'est n'avoir plus de passivité. Je veux dire, c'est éliminer de soi-même tous ces mouvements, toutes ces forces, tous ces orages dont on n'est pas maître et qui vous exposent ainsi à être l'esclave soit de ce qui se passe en vous, soit de ce qui se passe dans votre corps, soit éventuellement de ce qui se passe dans le monde. L'apatheia grecque garantit la maîtrise de soi. (p.181)

123 Sécurité, territoire, population, p.180, avec une erreur de Foucault quant à la référence, mentionnée par l'éditeur (voir p.191, note 33). En 1978, contrairement à la leçon de 1980, il s'agit uniquement de donner un exemple de l'absurdité de ce qui est demandé aux moines pour les éprouver. Chéri par Foucault, cet exemple reviendra dans le cours de Louvain en 1981. 


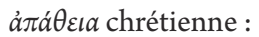

Cela veut dire essentiellement renoncer à cet égoïsme, à cette volonté singulière qui est la mienne. Et ce qu'on va reprocher aux plaisirs de la chair, ce n'est pas qu'ils rendent passif - c'était le thème stö̈cien et même épicurien -, ce qu'on reproche aux plaisirs de la chair, c'est que s'y déploie au contraire une activité qui est une activité individuelle, personnelle, égoïste. C'est que le moi, c'est que moi-même, j'y suis directement intéressé. (p. 182)

De part et d'autre de l'histoire de la pensée, la condamnation des

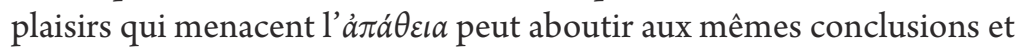
aux mêmes interdits, les raisons de cette condamnation diffèrent cependant grandement. Le stoïcien discrimine simplement la passivité et l'activité du sujet. Pour le chrétien, il s'agit plus finement de mesurer le degré d'engagement et d'intérêt de la volonté propre, jusque dans ses apparentes passivités. L'ả đá $\theta \varepsilon \varepsilon a$ grecque s'attaque essentiellement aux passivités, la patientia à la volonté.

Le cours Du gouvernement des vivants fait alors un pas de plus en ajoutant une seconde différence, déterminante pour notre propos. Celle-ci ne concerne plus le rapport du sujet à lui-même, mais le rapport du sujet

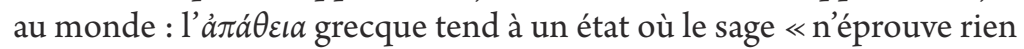
qui puisse, d'une manière ou d'une autre, l'agiter ou l'affecter $\gg^{124}$; la patientia chrétienne accepte au contraire « dans ce qu'elle peut avoir de plus vif et de plus mordant, la souffrance, l'épreuve qui vous vient des autres ou du monde $\gg$ (ibid.). Patermutus résiste donc à la souffrance tout en étant affecté. À l'opposé, le sage antique n'est affecté par rien qui puisse devenir pour lui souffrance.

Cette mise entre parenthèses du monde par le sage, nous la trouvons clairement formulée dans le cours L’herméneutique du sujet, autour de l'exercice de la praemeditatio malorum : écrivant à un ami qui a perdu son fils - deuil paternel qui fait écho pour nous à la mésaventure de Patermutus - Sénèque ne le console pas vraiment, mais l'encourage pour l'avenir à «montrer une âme haute face à la fortune, à prévoir les offensives de cette fortune, je ne dis pas comme événement possible, mais comme devant se produire à coup sûr $\gg^{125}$. Dans cet exercice de la praemeditatio malorum, il ne s'agit pas de rendre l'avenir plus présent, mais au contraire de l'annuler; il ne s'agit pas de rendre le malheur à venir 
plus réel, mais au contraire de réduire par avance sa réalitée ${ }^{126}$. Il s'agit, nous dit Foucault, de parvenir à comprendre qu'un tel événement est «sans importance et sans durée » (ibid., p. 453). Rien de tel chez Patermutus qui, pour ne pas réagir, n’en éprouve pas moins dans son cœur la morsure de ce qu'il voit se dérouler devant ses yeux : les humiliations puis la noyade de son fils.

S'écartant imperceptiblement des problèmes initiaux de la subjectivité et du rapport à soi, Foucault nous emmène ici du côté de deux épreuves du monde, fondamentalement différentes. Le terme «épreuve » est utilisé par ses soins pour décrire à la fois l'ả đá $\theta \varepsilon \imath a$ grecque et la patientia chrétienne, mais en deux sens bien distincts.

Si l'on reprend la définition proposée par L'herméneutique du sujet, l'épreuve antique est à la fois une expérience - «ce à travers quoi nous faisons l'expérience de nous-mêmes $\gg$ (p. 466) - et un exercice - «ce à partir de quoi $[\ldots]$ nous allons nous former $\gg$ (p. 466) par une $\tau \varepsilon \chi v \eta ́$ appropriée. Cette définition a des conséquences importantes, puisqu'elle permet à Foucault de tracer à la toute fin du cours de 19811982 un raccourci historique inattendu : à travers les péripéties complexes de la $\tau \varepsilon \chi v \eta ́$ - outil de la maîtrise de soi devenue à l'âge moderne outil de la maîtrise du monde - cette double acception de l'épreuve va entraîner avec elle toute la pensée occidentale jusqu’à la Phénoménologie de l'esprit de Hegel. Au « sommet de cette philosophie ${ }^{127}$, l'épreuve de soi et la connaissance du monde se rejoignent et s'éclairent mutuellement : après le dernier tour de roue de l'Histoire, le monde connu comme objet et le monde occasion de se connaître comme sujet reconnaissent le mouvement commun qui les habite comme étant le mouvement même de l'Esprit. Retenons pour le moment de ce raccourci que l'épreuve est bien un mouvement dialectique qui va du soi vers le monde et du monde vers le soi.

Cette double acception de l'épreuve, nous la retrouvons tout d'abord inchangée dans les leçons chrétiennes de 1980. Faisons un rapide relevé de ses occurrences :

- le terme «épreuve » entendu comme exercice a deux occurrences ${ }^{128}$. L'exercice est alors synonyme de purification (purification baptismale); 
- le terme « épreuve » entendu comme expérience a quinze occurrences $^{129}$. L'expérience est alors synonyme de vérification (par exemple : l'exorcisme).

En revanche, au moment précis où elle advient, l'épreuve dont parle Foucault quand il commente la patientia du moine en 1980 n'est ni une expérience ni un exercice. Elle est rapport à un dehors qui n'est pas interprété comme rapport aux autres (objet de la subditio) et ne permet pas non plus le retour à soi (objet de l'épreuve antique).

À travers l'exemple de Patermutus, il s'agit de saisir la douleur dans son seul effet sur le sujet : ce qui est insupportable à voir pour un père, quelle qu'en soit la cause. Sans doute l'humiliation est-elle un « exercice » commandé par l'ancien pour fortifier Patermutus ; sans doute estelle intégralement « expérience » pour vérifier les justes dispositions de ce dernier. Mais à cet endroit du cours, Foucault a soin de ne pas focaliser l'attention sur l'ordonnateur à l'origine de l'épreuve endurée. Il étend au contraire son champ d'émergence à la totalité du réel : l'épreuve lui vient «des autres ou du monde $\gg^{130}$. «Éprouver $\gg$ n'a pas ici le sens étymologique que lui confère le verbe latin probare, qui a donné esprover en ancien français («fournir des preuves », comme dans «l'épreuve de vérité $\gg$ de l'exorcisme ${ }^{131}$ ), mais il a le sens moderne de ce que l'on subit et de ce que l'on ressent. Pour le moine, l'«épreuve du monde » est toujours un génitif objectif.

Comme la praemeditatio malorum nous l'a rappelé, le sage stoïcien n'éprouve pas le monde comme un dehors irréductible, essentiellement résistant : le monde extérieur n'est que l'occasion qui le déprend de lui-

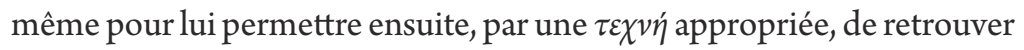
son empire originel, dans un parfait accord avec la totalité de l'être qui est $\lambda o ́ \gamma o s$. Que ce soit dans le premier stoïcisme, de Zénon à Chrysippe, ou dans le dernier stoïcisme des $\mathrm{I}^{\mathrm{er}}$ et $\mathrm{II}^{\mathrm{e}}$ siècles, les maux extérieurs,

129 Les occurrences se trouvent majoritairement dans la leçon du 20 février 1980 : «épreuve d'exorcisme » (deux occurrences); «épreuve du feu » (une occurrence); «épreuve de vérité 》 (cinq occurrences); «épreuve de vérification 》 (une occurrence); «épreuve de l'authenticité » (une occurrence); «épreuve » sans épithète (deux occurrences). «Épreuve » est également employé comme synonyme de probatio/preuve (une occurrence le 20 février et une le 5 mars). Enfin, citons l'épreuve pour intégrer le monastère (une occurrence le 19 mars).

130 Leçon du 19 mars 1980, nous soulignons.

131 Leçon du 20 février 1980. 
et avec eux toutes nos passivités, ne font que passer. Rien de tel dans la description de la patientia chrétienne. Il ne s'agit pas d'abord de la nécessité pour l'ascète de se donner des épreuves à traverser - même si celles-ci jalonnent constamment la vie monastique - mais de la possibilité initiale de faire l'épreuve du monde, c'est-à-dire d'être affecté par lui, «dans ce qu'il peut avoir de plus vif et de plus mordant $\gg^{132}$. Or, ce rapport - et c'est là une rupture avec le monde grec - n'est pas dialectique : le chemin entre l'âme et «l'ordre du monde ${ }^{133}$ dans lequel le philosophe reconnaissait à la fois le $\lambda o ́ \gamma o \varsigma$ divin et son propre $\lambda o ́ \gamma o \varsigma$ est désormais barré. L'épreuve ne peut être surmontée dans l'harmonie retrouvée avec la rationalité de l'être, elle ne peut être qu'endurée. La patientia comme tâche éthique, devançant tout rapport à autrui et toute probation de soi, fait apparaître une irréductible altérité : le réel résiste et nulle $\tau \varepsilon \chi v \eta ́$ philosophique ne peut le réduire.

Par effet de retour, le moi chrétien se reconnaît blessé - car il y a bien morsure et perte de pouvoir sur soi. Le postulat du monachisme selon lequel «l'homme divin $»^{134}$ n'existe pas n'est pas seulement une affirmation théologique sur le diable habitant de l'âme, il est également une conception nouvelle de l'homme habitant du monde : l'homme a perdu l'accès à la totalité.

Dans L'herméneutique du sujet, Foucault définit l'expérience philosophique antique comme «ce à travers quoi nous nous connaissons, ce à travers quoi nous nous découvrons, ce à travers quoi nous nous révélons à nous-mêmes $\gg(p .466)$. Si elle ne venait refermer un enseignement sur le souci de soi stoïcien, on prêterait volontiers à cette définition des accents chrétiens. Foucault n'invoque-t-il pas sans cesse l'obsession, apparue dans les premiers siècles de notre ère, de la « découverte de soi » (ibid., p.116), de l'« exégèse de soi » (p.228) ? Mais la vérité qui semble obséder le christianisme, et dont Foucault parle en des termes qui prennent parfois une consistance trompeuse à force d'être répétés, n'est qu'une vérité discontinue, sans identité fixe à découvrir au terme de

132 Leçon du 19 mars 1980.

133 Ibid. Sur ce thème, voir L'herméneutique du sujet, p. 246, au sujet des mouvements gnostiques reprenant le schéma platonicien : «connaissance de l'être et reconnaissance de soi ne constituent qu'une seule et même chose ».

134 Leçon du 19 mars 1980. 
la recherche. Le moi individuel n'est que le récit commun de ses brisures et de ses recollements. Quant aux miettes peccamineuses rapportées au confesseur par le pénitent, elles n'ont d'autre but que d'aider à renoncer à soi, c'est-à-dire à perdre définitivement ce que l'on connaît subrepticement $^{135}$. Rien d'étonnant à ce que cette vérité inessentielle soit vouée à l'oubli de celui qui l'a une fois entendue, comme nous l'avons déjà évoqué en citant ce passage important de «La vie des hommes infâmes » :

$[\ldots]$ étonnante contrainte $[\ldots]$ de tout dire pour tout effacer, de formuler jusqu'aux moindres fautes dans un murmure ininterrompu, acharné, exhaustif, auquel rien ne devait échapper, mais qui ne devait pas un instant se survivre à lui-même. ${ }^{136}$

Comme l'explique le séminaire à l'université du Vermont en 1982, c'est là toute la différence entre le soi chrétien et le soi défini au $\mathrm{XVIII}^{\mathrm{e}}$ siècle par les sciences humaines ${ }^{137}$. Contrairement à la subjectivité chrétienne, la subjectivité moderne, constituée au croisement du droit, de la science et de l'administration, enferme l'homme dans une « identité » déterminée (biologique ou psychologique), naturalisée par le savoir, enregistrée par le pouvoir ${ }^{138}$.

Étrange destin du lexique dans l'œuvre de Foucault : le terme «identité » n'est jamais utilisé par le philosophe pour désigner le sujet chrétien $^{139}$. Par contre, le terme a bien un sens pour la subjectivité grecque,

135 Foucault le dit bien à propos du christianisme en général : «Paradoxalement, se connaître soi-même a constitué un moyen de renoncer à soi $\gg\left(D E \mathrm{n}^{\circ} 363\right.$, « Les techniques de soi », II, p.1607). Ce que l'exomologèse théâtralise encore plus radicalement : «La révélation de soi est dans le même temps la destruction de soi » (ibid., p. 1627).

$136 D E n^{\circ} 198$, « La vie des hommes infâmes », II, p. 245 .

$137 D E n^{\circ}{ }_{36} 6_{3}$, «Les techniques de soi », II, p. 1632 : «À partir du XVIII ${ }^{\mathrm{e}}$ siècle et jusqu’à l'époque présente, les "sciences humaines" ont réinséré les techniques de verbalisation dans un contexte différent, faisant d'elles non pas l'instrument du renoncement du sujet à lui-même, mais l'instrument positif de la constitution d'un nouveau sujet. »

138 Citons quelques-unes des occurrences les plus significatives, en notant au passage qu'elles ont toutes une connotation négative : l'identité est un «épinglage à soi-même » $\left(D E n^{\circ} 169\right.$, «Questions à Michel Foucault sur la géographie », II, p. 36); «Il est dangereux, selon moi, de considérer l'identité et la subjectivité comme des composantes profondes et naturelles $\gg\left(D E \mathrm{n}^{\circ} 272\right.$, « Foucault étudie la raison d'État $\gg$, II, p. 801); par le détour du savoir médical et de l'enregistrement administratif, l'identité sexuelle est fixée : «À chacun son identité sexuelle première, profonde, déterminée et déterminante » ( $D E$ $\mathrm{n}^{\circ} 287$, « Le vrai sexe $\gg$, II, p. 936 ).

139 À l'exception de l'expression «identité chrétienne » qui ne décrit pas une identité du 
comme le vérifie le lexique du cours de $1981-1982^{140}$ : revenant toujours à lui-même, le soi se rassemble et se recueille dans la contemplation de l'essence de l'âme ou dans la subordination de ses facultés à la raison. La tâche éthique du souci de soi est d'échapper en son être propre à la dispersion et à la discontinuité, en accomplissant par la mémoire une «totalisation de la vie sociale, de la vie active, de la vie de plaisir, de la vie de loisir $\gg^{141}$. On objectera que l'identité grecque ne désigne ici rien d'autre que la réflexivité pronominale du soi, librement dépliée et repliée à partir d'elle-même. Mais ce serait oublier la distinction que pose Foucault entre la simple ipséité et la formation d'un soi que le sujet grec doit trouver ou retrouver en lui, et qui devient ainsi la norme de son développement :

«Soi » est un pronom réfléchi, dont la signification est double. Auto veut dire «le même », mais il renvoie aussi à la notion d'identité. Ce deuxième sens permet de passer de la question « quel est ce soi ? » à la question «à partir de quel fondement trouverai-je mon identité ? ».142

Il y a donc bien un fondement qui donne au soi sa juste forme : dans l'Alcibiade, l'âme s'élève ainsi par un mouvement dialectique jusqu'à l'élément divin où elle se reconnaît elle-même, identique à elle-même, comme dans un miroir ${ }^{143}$; chez Épictète, le regard sur soi épouse par analogie le regard divin qui se gouverne lui-même dans une parfaite indépendance ${ }^{144}$; chez Marc Aurèle, le soi trouve son identité comme « partie de la raison qui préside au monde » (ibid., p. 294).

Au contraire, dans le cours consacré au christianisme, l'identité à soi ne peut survivre à la « répétition ». Elle n'est plus ce que construit patiemment l'esthétique de l'existence, ni ce que l'âme retrouve en se remémorant son origine divine, elle est ce qui se défait au long d'une vie,

sujet, mais bien une identité collective ( $D E \mathrm{n}^{\circ}$ 291, « "Omnes et singulatim" : vers une critique de la raison politique », II, p. 966).

140 Dix occurrences dans L'herméneutique du sujet.

141 L'herméneutique du sujet, p. 448. Sans le souci de soi, « on ne peut pas se constituer soimême comme une identité » (ibid.). Voilà pourquoi le délire amoureux décrit par Ovide est «une expérience tardive qui ne correspond absolument pas à celle de Platon ou d'Aristote $»$. Le délire amoureux est ignorance de son «identité », « oubli de soi » $(D E$ $\mathrm{n}^{\mathrm{o}} 354$, « Le retour de la morale $»$, II, p. 1520).

$142 D E n^{\circ} 363$, « Les techniques de soi », II, p. 1610.

143 L'herméneutique du sujet, p. 436 ; $D E \mathrm{n}^{\circ}{ }_{363}$, «Les techniques de soi », II, p.1610-1611.

144 L'herméneutique du sujet, p. 441. 
dans une succession inexorable de ruptures : rupture du sujet avec luimême dans la pénitence pré-baptismale, rupture du sujet avec la vérité dans la rechute, et répétition à perpétuité de la première pénitence. Emporté par cette découverte, avec toute la liberté que lui procure la situation d'enseignement, Foucault en tire une conséquence provocante, faisant du christianisme la pensée par excellence de la rupture :

Ce n'est pas l'appartenance du sujet à la vérité ou l'appartenance de la vérité au sujet, c'est leur distance qui fait problème. Et ce n'est pas la question de l'identité du sujet, c'est la question de la rupture qui fait problème. ${ }^{145}$

Il y a bien une première ligne de démarcation qui passe entre le soi grec d'un côté et le soi chrétien et moderne de l'autre. Celle-ci est une ligne éthique, engageant la liberté des sujets, vers la maîtrise de soi ou la soumission à autrui. Mais il y a également une deuxième ligne qui passe entre le soi chrétien d'un côté et le soi grec et moderne de l'autre. Celleci est une ligne que l'on pourrait appeler ontologique ${ }^{146}$, déterminant les possibles de l'homme : non pas ce qu'il doit faire, mais ce qu'il peut ou non être. La première ligne sépare l'autonomie de l'hétéronomie, comme le cours Sécurité, territoire, population l'avait précocement indiqué. La seconde, tracée par le cours Du gouvernement des vivants, sépare un sujet capable de se ressaisir comme totalité d'un sujet qui ne peut jamais coïncider avec lui-même, encore moins avec sa destinée. Cette deuxième ligne fait et défait, de part et d'autre d'elle-même, l'identité à soi. Où situer Foucault dès lors, sinon derrière cette curiosité qui le pousse constamment à franchir chacune de ces lignes?

145 Leçon du 27 février 1980.

146 Bien entendu, l'adjectif « ontologique » ne désigne pas autre chose ici qu'un objet de l'analyse historique. L'ontologie dont il est question ne dit pas la vérité définitive de l'être, mais elle est une pensée qui a des effets historiques importants. 



\section{CONCLUSION}

Le christianisme est un objet historique à la fois extrêmement présent dans les travaux de Michel Foucault - peut-être même est-il l'objet le plus présent - et pourtant largement disséminé, éclaté en plusieurs endroits de l'œuvre, rarement étudié de front.

Références suggestives à l'Occident opposé à un Orient nietzschéen dès la préface à Folie et déraison en 1961, dialogue avec Bataille, Blanchot ou Klossowski tout au long des années 1960, dossiers historiques de l'aveu, de la chair, de la pastorale chrétienne ouverts par les cours au Collège de France et, surtout, ébauche plusieurs fois remise sur le métier et toujours inédite d'un grand livre sur les Aveux de la chair : Foucault n'a cessé de s'intéresser au christianisme; au point qu'en 1978 il manqua une interview pour suivre à la télévision l'élection du pape Jean Paul $\mathrm{II}^{1}$. Mais l'intérêt avéré ne crée pas de lui-même un objet historique bien défini comme le système pénal ou la psychiatrie; ce qui explique en partie le relatif silence des commentateurs habituels sur ce sujet, mais aussi celui des utilisateurs potentiels des travaux de Foucault, tels les historiens et les théologiens du christianisme qui n'ont été, encore à ce jour, que très peu à recourir à ses travaux. Tenter de ressaisir la cohérence de la pensée de Foucault sur le christianisme, chercher tout d'abord si cette cohérence existe dans une œuvre multiforme, qui n’a cessé de varier ses méthodes et ses objets, telle fut notre motivation première.

À première vue, cette cohérence se joue autour de l'aveu, comme dispositif central et décisif de l'histoire chrétienne : pas de salut possible 
pour le chrétien sans verbalisation constante de sa vérité intime, vérité de son désir, de sa sexualité. L'aveu se déploie dans cette triple injonction : il faut avouer, il faut tout avouer, il faut tout avouer au prêtre. L'aveu n'a de sens que s'il est effectué sous le regard d'un autre qui a autorité et à qui l'on doit une obéissance totale. Le sujet chrétien viendrait alors simplement se glisser entre un certain pouvoir et un certain savoir dont il dépendrait sa vie durant. Face à ce sujet, se dresserait en contraste le sujet grec, pour qui autrui n'est que l'occasion amicale d'affermir et de perfectionner la maîtrise de son corps et de ses pensées. Autonomie de la Rome antique, hétéronomie radicale de la Rome chrétienne.

Assurément, l'aveu est une pièce maîtresse de l'interprétation foucaldienne du christianisme, tout comme son corollaire dans l'ordre du discours qui est la chair, désignant un corps habité par une infinité de désirs et de plaisirs ; il suffit pour s'en convaincre de relire le beau titre que Foucault donne à ses conférences de 1981 à l'Université catholique de Louvain : Mal faire, dire vrai. Mais la lecture proposée par Foucault se limite-t-elle à la description monotone des confessionnaux, de ces visages contrits qui se tiennent dans la pénombre du petit meuble grillagé ? Foucault n'a-t-il rien fait d'autre que de dénombrer les liens qui nous enserrent depuis que le Verbe s'est fait chair? A priori oui : «le christianisme est la religion de l'aveu » répète à plusieurs reprises le philosophe. Pourtant, le cours au Collège de France Du gouvernement des vivants (1979-1980), qui a joué pour nous ici le rôle du $\sigma \dot{u} \mu \beta o \lambda o v$ d'Edipe, laisse entendre une interprétation différente, qui donne soudain aux hypothèses précédentes une profondeur de champ nouvelle : le christianisme n'est pas d'abord la religion de l'aveu, mais la religion $\mathrm{du} \ll$ salut dans l'imperfection $\gg$. Restait à comprendre cette étrange expression utilisée en 1980.

Les leçons de 1980 sur le christianisme sont l'occasion pour Michel Foucault de renouveler à la fois ses concepts et sa méthode. Définition d'un nouveau domaine d'étude qui vient se glisser entre le savoir et le pouvoir : les « régimes de vérité »; et mise en œuvre d'une nouvelle manière d'aborder les textes : une lecture faussement littérale qui se révèle travail radical de «lexicalisation ». Établir une cartographie de ces nouveaux espaces a été l'enjeu principal des deux premières parties de cette recherche. Dans la troisième partie, nous nous sommes efforcé de dégager la nouveauté de cet enseignement, au cours duquel Foucault 
étudie le christianisme non seulement comme une nouvelle éthique, mais également comme une nouvelle ontologie du soi. L'insistance indéniable sur la forclusion de la liberté individuelle dans l'obéissance, sur l'hétéronomie nouvelle induite par la Parole révélée, sur la pastorale à la fois intime et universelle d'une institution ecclésiale briguant l'hyperprésidence des âmes, nous a semblé devoir être relue à la lumière des changements ayant affecté, au cours des premiers siècles, le champ des expériences possibles offertes à l'individu. La description proposée par Foucault insiste en effet sur l'opacité impénétrable que le sujet est devenu à lui-même à partir de l'ère chrétienne : il me faut vivre, croire, prier, faire pénitence «dans l'incertitude de ce qui se passe au fond de moi $»^{2}$.

Bien entendu, cette incertitude ne fait que rendre plus urgente la recherche des profondeurs, plus insistant l'examen tatillon de soi - les manuels des confesseurs ne feront pas autre chose. Mais Foucault décrit en 1980 un autre mouvement, qui ne contredit pas mais infléchit le premier : l'effort inédit, non pas pour faire passer la lumière là où elle n'est pas encore, mais pour faire cohabiter, dans une même existence, le jour avec la nuit, l'imperfection avec le salut, sans heureuse synthèse à espérer ici-bas, sans béatitude à attendre, au moins à l'horizon de ce monde-ci. La répétition de la pénitence est l'expression rituelle d'un moi exilé de lui-même et de son Dieu, déchu de ses prérogatives et attributs antiques.

Si l'éthique chrétienne décrite par Foucault semble entourée par les murs du catholicisme, du fait de l'insistance du philosophe sur le gouvernement pastoral et les médiations ecclésiales garantissant le salut individuel, l'ontologie qui l'intéresse, et rend compte selon lui du renouvellement complet des techniques de soi dans les premiers siècles de notre ère, serait à chercher du côté d'une vision plus radicale et certainement plus paradoxale de l'âme humaine. Celle défendue par le luthérien Kierkegaard, contre le savoir absolu hégélien ? Peut-être, même si l'intriguant Danois n'est pas nommé. Celle inventée entre le $\mathrm{II}^{\mathrm{e}}$ et le $\mathrm{III}^{\mathrm{e}}$ siècle par Tertullien, faisant de l'Autre (Satan) l'indélogeable habitant de l'âme ? Certainement. Mais celle surtout initiée par saint Paul, avec un sens de l'aporie qui ne lui venait ni de son héritage juif ni de son héritage grec. À travers les thèmes de la seconde pénitence, de l'obscurité à soi ou encore de la mesure introuvable que nul moine ne peut prétendre 
être à lui-même, les leçons de 1980 ne sont-elles pas finalement un long commentaire du verset de l'Épître de Paul aux Romains : «Je ne fais pas le bien que je veux et commets le mal que je ne veux pas $\gg^{3}$ ?

La question n'est plus alors, comme pour le philosophe stoïcien, « Par quelles techniques je vais pouvoir accéder définitivement à la vérité, me l'incorporer au point que je ne ferai plus qu'un avec le $\lambda o ́ \gamma o \varsigma$ ? », mais «Par quelles techniques je vais pouvoir continuer à vivre alors même que la vérité n'est pas ce que je découvre sans cesse un peu plus tous les jours mais ce que je manque constamment ? » - non plus une inlassable quête de savoir et de pouvoir, mais une indépassable fragilité de l'être. Tout ce qui a été préalablement décrit dans Les anormaux ou La volonté de savoir en termes d'obéissance absolue, d'examen perpétuel de soi et d'aveu continu de ses fautes est bien entendu vrai, mais ne se comprend qu'à l'aune de ce rapport paradoxal à la vérité; puisque l'éternité n'est jamais perdue, mais n'est jamais non plus définitivement gagnée.

À sa manière, le cours de 1980 reprend et approfondit ce que Foucault écrivait en 1954 sur la liberté chrétienne. Dans son introduction à la traduction française de l'ouvrage de Ludwig Binswanger, Traum und Existenz (1930), le jeune philosophe parlait déjà de «cette liberté humaine toujours précaire qui est inclinée sans se laisser déterminer, qui est éclairée sans pouvoir être contrainte, et qui est avertie sans être réduite à l'évidence $\gg^{4}$.

3 Rom. 7, 19 (traduction de la Bible de Jérusalem).

$4 D E \mathrm{n}^{\circ} 1$, « Introduction $\gg$, I, p. 111. 


\section{APPENDICE \\ Possibilité de compléter I'histoire stratégique du christianisme}

Si le cours de 1979-1980 se présente d'abord comme l'ébauche d'une histoire du christianisme comme «régime de vérité », cela ne signifie pas qu'une histoire du christianisme comme savoir et comme pouvoir, c'est-àdire comme économie dogmatique et comme régime juridico-politique, n'ait pas également sa consistance et sa régularité propre au sein de l'analyse stratégique engagée. Il ne serait pas juste d'incriminer chez Foucault le dédain ou l'ignorance des autres manifestations historiques du christianisme - que ce soient ses doctrines ou ses institutions - comme conséquences des franches réductions méthodologiques mentionnées. Contre le reproche récurrent de morcellement et d'oublis qu'essuient bien souvent les travaux historiques du philosophe, nous voudrions montrer la possibilité d'une description complète du christianisme dans ses trois « régimes » d'existence, institutions et dogmes inclus.

\section{*}

Dans une certaine mesure, le cahier des charges d'une histoire juridicopolitique du christianisme est en partie rempli par le cours de 19771978, Sécurité, territoire, population, si nous entendons par «politique », comme nous l'avons établi précédemment, une stratégie globale qui essaie de coordonner et de finaliser les rapports de force présents dans une société donnée. Le pastorat est bien la traduction, en termes de 
relations concrètes de pouvoir, de ce processus spécifique au christianisme par lequel une religion s'est constituée comme Église, c'est-à-dire comme une institution prenant en charge la vie quotidienne de chacun de ses membres pour les mener au salut ${ }^{1}$. Le jugement critique que nous avons porté sur les modèles de contre-conduites construits par Foucault à cette occasion ne doit pas masquer l'intérêt des pistes proposées par le cours pour décrire le gouvernement pastoral : en particulier l'organisation des paroisses et leur quadrillage territorial à partir $\mathrm{XIV}^{\mathrm{e}}$ siècle, thème encore peu visité à l'époque du cours par les historiens, au moins dans sa forme médiévale ${ }^{2}$. À l'intersection des laïcs et des clercs, la paroisse est précisément un lieu qui permet de prendre l'institution ecclésiale dans son fonctionnement mobile et adaptable, en combinant une analyse microscopique et macroscopique du pouvoir - la fonction pastorale s'appliquant en effet à tous les degrés de la hiérarchie ecclésiale et de ses enseignements, du plus local (le curé) au plus universel (l'évêque).

Le plus grand silence dans les dits et écrits de Foucault semble en revanche entourer ce qui constitue pourtant le principal objet des recherches historiques traditionnelles sur les premiers siècles chrétiens : l'économie dogmatique du christianisme, avec ses controverses théologiques, la constitution progressive de sa normativité en lien étroit avec le combat contre les hérésies - autant d'événements discursifs à peine évoqués par les cours Sécurité, territoire, population et Du gouvernement des vivants. Mais si Foucault n'a effectivement jamais écrit cette histoire critique de l'économie dogmatique chrétienne, certaines remarques méthodologiques des années 1968 et 1969 dessinent en filigrane ce qu'elle pourrait être. Il nous a semblé pertinent sur ce point de ne pas nous contenter de l'apparent silence de Foucault, mais d'utiliser librement les outils qu'il a laissés dans ses analyses plus anciennes afin de

1 Sécurité, territoire, population, p. 151.

2 Ibid., p. 156. L'organisation et la vie des paroisses étaient peu étudiées par les historiens du Moyen Âge, compte tenu en partie de la faiblesse des archives. La première étude magistrale fut celle de Jacques Toussaert : Le sentiment religieux en Flandre à la fin du Moyen Âge, Paris, Plon, 1963. Marquée par l'histoire des mentalités, cette recherche s'attardait longuement sur les comptes paroissiaux, les listes de pèlerins, les registres judiciaires et même les décomptes d'hosties, pour décrire la pratique réelle des fidèles. Vinrent ensuite l'étude de Paul Adam, La vie paroissiale en France au $x v^{e}$ siècle, Paris, Sirey, 1964, dans la lignée sociologique de Gabriel Le Bras, et surtout Pierre Chaunu : Le temps des réformes. Histoire religieuse et système de civilisation. La crise de la chrétienté. L'éclatement 1250-1550, chap. III : «La vie religieuse des humbles », Paris, Fayard, 1975, p. 147-209. 
compléter ses propres travaux. Ces analyses des années 1968 et 1969 concernent l'unité de pratiques discursives d'un type bien particulier : celles qui engagent un rapport problématique à une origine.

Dans la réponse à la revue Esprit de 1968, Foucault met radicalement en question les deux critères traditionnels d'individualisation des discours, quand le renvoi à un unique sujet de l'énonciation fait défaut : le « recours historico-transcendantal » à un projet initial toujours en recul sur ses manifestations historiques; et le « recours empirique ou psychologique » à un sujet fondateur ${ }^{3}$. Ce découpage semble tout à fait pertinent pour rendre compte des approches classiques de l'histoire de la dogmatique chrétienne, dans la manière dont elles constituent l'unité de leur objet. En effet, la doctrine chrétienne n'est pas le fait d'une unique source scripturaire encore moins d'un unique auteur ; seule une méprise sur le statut et l'histoire des Écritures saintes peut laisser espérer à certains fondamentalistes une origine simple et univoque. Les efforts incessants à travers l'histoire de la théologie pour approcher d'un noyau initial «chrétien » illustrent au contraire avec beaucoup de justesse les réflexions de Foucault. Un bref rappel historique est ici nécessaire.

Pour comprendre le destin de la Bible en monde chrétien, il faut oublier ce qui constitue pour nous aujourd'hui l'objet « livre ». Il faudrait imaginer un livre sans auteur, sans éditeur et, à la limite, sans existence matérielle bien définie. Aux premiers siècles de notre ère, pour les communautés chrétiennes naissantes, «Les Écritures » désignent seulement les Écritures juives, et il n'est pas encore question de leur adjoindre un deuxième livre, suite de la saga initiale. Bien entendu, des écrits sur Jésus apparaissent très tôt, dans un buissonnement rédactionnel dont nos quatre évangiles actuels ne sont sans doute que des rameaux. Mais ces écrits de circonstance, accompagnant les enseignements itinérants des apôtres et des prophètes, ne sont que l'ombre portée de l'événement fondateur : la mort et la résurrection d'un homme qui n'a rien écrit, sinon quelques signes sur le sable ${ }^{4}$. Rouleaux encore transportés dans des tubes fermés, des sacs de cuir ou des jarres, les premiers écrits chrétiens n'ont ni la cohérence de nos livres d'histoire ni l'autorité des anciens livres sacrés. Ce sont des livres nomades, qui n’ont 
pas besoin de décliner leur identité pour circuler. Pour les jeunes Églises chrétiennes, la question de rassembler et sélectionner les témoignages les plus fiables sur Jésus émerge seulement dans le courant du $\mathrm{II}^{\mathrm{e}}$ siècle, quand l'événement fondateur commence à s'éloigner, les mémoires à devenir incertaines, et l'éclatement doctrinal à se profiler. Ainsi naît l'idée d'un «canon » des Écritures. L'idée, mais pas la chose. Car cette règle définitive, nul ne l'a dictée au premier millénaire. Si l'on veut être rigoureux, la clôture définitive de la Bible chrétienne ne sera prononcée qu'au concile de Florence en 1442, issu du dialogue avec les jacobites, avant d'être réaffirmée au concile de Trente (session de 1546). Avant 1442, les décisions autoritaires concernant le canon sont rares et surtout locales. C'est en fait l'usage qui va progressivement faire loi : il ne s'agit pas tant, comme le ferait aujourd'hui un historien, de vérifier l'adéquation des choses dites à une certaine vérité des faits, que de reconnaître les textes qui ont été le plus largement reçus et priés dans les communautés ${ }^{5}$. Ce type de jugement reste cependant sujet à caution, et le débat se poursuivra jusqu'à la Réforme, où Luther remettra en question ce qui avait fait jusque-là l'unité de la doctrine chrétienne.

À la période moderne, les réponses contrastées apportées à cette question de l'unité doctrinale peuvent être reformulées dans la problématique de Foucault : «recours historico-transcendantal » d'un côté, «recours empirique ou psychologique » de l'autre. Le premier recours tente de ressaisir derrière un ensemble désormais clos de discours (les Écritures) une idée directrice ou un message originel. Mais il ne peut le faire que sous une forme évolutive qui intègre les interprétations successives apparues dans l'histoire pour s'affiner sans cesse dans un développement conceptuel jamais achevé. «Chrétien» décrit alors la continuité historique de ce processus de clarification et d'explicita-

5 C'est l'idée de «tradition » qui apparaît chez Irénée de Lyon. Dès le II ${ }^{\mathrm{e}}$ siècle, ce penseur chrétien a recours aux Écritures juives et aux quatre évangiles pour réfuter les mouvements gnostiques qui déferlent alors sur le pourtour méditerranéen. Après une réfutation par la raison (Livre II), le Livre III de l'Adversus haereses annonce une réfutation à partir des «Écritures ». Si le terme connaît des glissements de sens chez Irénée, il joint cependant dans la preuve les Écritures juives et chrétiennes qui font déjà système pour lui (Livre III). On peut en effet légitimement supposer qu'Irénée avait bien les quatre évangiles devant les yeux sous une forme textuelle et ne se référait donc pas uniquement à des traditions orales. Le travail très précis auquel se livre Irénée de comparaison des péricopes de Luc avec celles de Matthieu et de Marc rend en effet douteux le recours exclusif à des traditions orales. 
tion, toujours à reprendre. L'image de Foucault est ici très juste : il s'agit d'une « esquisse toujours dénouée d'une unité qui ne s'achève pas $\gg^{6}$. Le second critère met au contraire en avant le rapport existentiel à la personnalité d'un fondateur - rapport sans cesse confessé par un individu ou une communauté. « Chrétien » décrit alors une affirmation quant à un être historique (Jésus-Christ), sur ce qu'il a été et ce qu'il doit être pour tous ceux qui parlent en son nom. Si l'on voulait inscrire ces deux modèles dans l'histoire de la théologie chrétienne, en choisissant volontairement deux figures à la fois emblématiques et opposées, la première école serait celle d'Alfred Loisy (1857-1940), la seconde celle d'Adolf von Harnack (1851-1930). Pour les deux penseurs, il y a bien à l'origine de l'histoire une « essence » de la vérité chrétienne, qui s'accomplit dans des milieux culturels divers ou au contraire s'y pervertit.

Dans sa désormais classique Histoire des dogmes (Lehrbuch der Dogmengeschichte, 1886-1889), Harnack réduit ainsi le christianisme dogmatique à une contamination précoce de «l'esprit grec » sur l'Évangile. Aux dogmes impurs, Harnack oppose l'authenticité de la personne du Christ, dans une ligne qu'il veut à la fois paulinienne et augustinienne. «L'Évangile » n'est pas le corpus scripturaire clôturé par l'institution ecclésiale à un moment donné de son histoire, mais il est, en son essence, ce qu'une personne historique a vécu et ce qu'elle nous appelle à vivre à sa suite : «L'Évangile est indissolublement lié à Jésus-Christ. [...] L'Évangile est en lui parole et acte, l'Évangile est devenu sa nourriture, donc aussi la vie de son être, et il entraîne avec lui tous les autres hommes à entrer dans cette vie qui est la sienne $\gg^{7}$. Le travail historique doit donc avancer dans le sens d'une purification : la recherche des paroles les plus authentiques de Jésus, leur resserrement autour du kérygme originel.

Si L'Évangile et l'Église (1902) d'Alfred Loisy fut condamné par les autorités ecclésiales catholiques, ce petit livre n'en était pas moins au départ une verte réponse à Harnack et à son refus tout luthérien de prendre en considération la tradition de l'Église. Pour Loisy, nul kérygme originel ne peut être isolé du mouvement global initié par Jésus, c'està-dire de la réception de son message par les premiers disciples et les premières communautés croyantes, formant à leur manière l'Église en

$6 D E \mathrm{n}^{\circ} 58$, « Réponse à une question », I, p. 703.

7 Nous citons la réédition française de l'ouvrage : A. von Harnack, Histoire des dogmes, E. Choisy trad., Paris, Cerf, 1993, p. XVIII. 
voie de constitution : «Autant dire que le Christ est inséparable de son œuvre, et que l'on tente une entreprise qui n'est qu'à moitié réalisable, quand on veut définir l'essence du christianisme d'après le pur Évangile de Jésus $\gg^{8}$. En 1922, bien après les multiples controverses suscitées par son livre, celui qui est devenu entre-temps professeur d'histoire des religions au Collège de France écrit encore : «Ainsi s'explique le développement de ce qu'on a coutume d'appeler la tradition évangélique. C'était une tradition effervescente, une évolution créatrice, qui emportait les vieux textes dans son mouvement grandissant $\gg^{9}$. À la recherche du pur rapport qui obsède Harnack et nombre d'exégètes protestants, Loisy oppose la nécessité du développement doctrinal, la cohérence de son hellénisation et la liberté de ses voies.

Sans beaucoup simplifier, on peut avancer que toutes les histoires dogmatiques du christianisme publiées au XIX et au XX ${ }^{\mathrm{e}}$ siècle se rattachent à l'une de ces deux écoles, même si les plus récentes études ont cherché une voie moyenne entre les deux ${ }^{10}$. Or, une analyse attentive révèle que Foucault n'ignore rien de ces deux voies, soit qu'il les mentionne directement dans le cours de 1980, soit qu'il les rencontre à l'occasion de ses réflexions sur le marxisme ou la psychanalyse. Ces deux dernières pratiques discursives ont effectivement en commun avecl'économie dogmatique chrétienne un rapport à la fois nécessaire et constamment remis en cause à une origine fondatrice : un texte ou une personne.

Prenons alors le premier critère proposé par l'approche classique de l'histoire des dogmes : celui d'une approche dialectique du texte source comme témoignage essentiel d'une réception encore inachevée, ou

8 A. Loisy, L'Évangile et l'Église, $2^{\mathrm{e}}$ édition augmentée, Bellevue, Chez l'auteur, 1903, p. XxI.

9 A. Loisy, Les Livres du Nouveau Testament traduits du grec en français, Paris, Émile Noury, 1922, p. 11.

10

C'est exactement le mouvement qu'opère Jean Daniélou dans son Histoire des doctrines chrétiennes avant Nicée. Dans l'« Avant-propos » du premier volume, il écrit : « L'essence de la foi chrétienne est d'affirmer que seul le Christ a pénétré au-delà du voile et que seul il a ouvert les sceaux du Livre scellé. Mais cette affirmation, la théologie judéo-chrétienne la développe au moyen des catégories qui étaient celles de l'apocalyptique [juive] » (J. Daniélou, Histoire des doctrines chrétiennes avant Nicée, t. I : Théologie du Judéo-christianisme, M.-O. Boulnois éd., $2^{\mathrm{e}}$ édition, Paris, Desclée/Cerf, 1991, p. 6). Dans cette proposition, Daniélou tient ensemble la pureté de l'affirmation chrétienne primordiale et la légitimité de sa traduction dans un contexte culturel particulier. Daniélou fait de même ensuite avec la philosophie hellénistique et le monde latin. 
«recours historico-transcendantal ». Que le développement dogmatique chrétien ne se fasse que dans le prolongement d'un texte originel, Foucault non seulement ne l'ignore pas ${ }^{11}$, mais il en souligne la force contraignante dans les débats théologiques : le cours Du gouvernement des vivants rappelle à ce sujet que Tertullien maintint contre ses propres tendances théologiques l'efficacité spirituelle de l'eau du baptême, car il la savait être un thème constant des Écritures ${ }^{12}$. Mais ce retour nécessaire à la source, dont la normativité ne sera instituée que tardivement avec la définition du canon des Écritures, n'empêche pas une série de décalages et d'inventions par rapport au texte initial. Dans une même leçon, le 28 février 1980, Foucault note à la fois la fixation ecclésiale d'une mémoire et d'une tradition - « dont le garant se trouve dans le texte, dans l'Écriture, et dans l'autorité même de l'institution ecclésiale » - et les libertés herméneutiques d'un Origène, opérant par rapport à l'exégèse typologique traditionnelle du Livre de l'Exode une série de « déplacements » et de «ré-interprétations $\gg^{13}$. À ces opérations audacieuses et peu normées, fait écho, en contexte juif, le commentaire « tout à fait curieux $»^{14}$ que donne Philon d'Alexandrie d'un passage du Livre des Nombres. Ni plus ni moins, relève alors Foucault, l'écrivain juif « réaménage complètement » le texte de la Bible, dans une visée allégorique (ibid.). Cette remarque pourrait sembler secondaire par rapport au propos du cours ; elle est pourtant révélatrice de la perception qu'a Foucault du discours théologique et de son fonctionnement singulier. Ce qu'il décrit ici n'est pas un simple rapport anarchique à une origine textuelle finalement peu contraignante; c'est en fait une pratique discursive bien précise, qu'il analyse à la fin d'une communication importante de 1969 intitulée « Qu'est-ce qu'un auteur? » ${ }^{15}$.

$\mathrm{Au}$ terme de cette communication, Foucault dégage la spécificité de ceux qu'il nomme les « fondateurs de discursivité » (p. 832), en les distinguant des fondateurs de sciences et des fondateurs de genres littéraires. Dans le contexte de l'archéologie du savoir, il faut préciser qu'il ne s'agit pas d'abord ici de personnalités historiques mais de corpus de textes portés par une même «fonction-auteur ». Les deux exemples

11 Plus que le cours de 1979-1980, c'est surtout le cours de 1981-1982 qui insiste sur l'extériorité normative des Écritures : L’herméneutique du sujet, p. 245 et 345.

12 Leçon du 13 février 1980.

13 Leçon du 20 février 1980.

14 Leçon du 23 janvier 1980.

$15 D E n^{\circ} 69$, « Qu'est-ce qu'un auteur? », I, p. 817-849. 
choisis par Foucault sont les textes de Marx et de Freud, en tant qu'ils ont ouvert une «possibilité indéfinie de discours » (p. 833) appelée «marxisme » ou «psychanalyse ». Il s'agit donc, à travers cette activité de fondation, d'un jeu subtil de proximité et d'écart avec un texte originel : « [Marx et Freud] ont ouvert l'espace pour autre chose qu'eux et qui pourtant appartient à ce qu'ils ont fondé $\gg$ (p. 833). Foucault avance alors l'idée qu'il existe un certain type de discours dont la spécificité serait d'instaurer des règles de différenciation par rapport à leurs propres concepts et hypothèses. Marx et Freud n'ont pas fondé une postérité intellectuelle qui répéterait ou imiterait indéfiniment leur propre discours, mais ils ont rendu possible une différence par rapport à euxmêmes : des transformations qui prennent appui sur leurs textes pour s'en écarter.

Bien entendu, un pur écart ne permettrait pas de penser l'unité de cette pratique discursive spécifique. Inspiré par les retours à Freud (Jacques Lacan) et à Marx (Louis Althusser) qui ont secoué dans les années 1950-1960 les orthodoxies psychanalytique et marxiste, Foucault montre alors habilement comment un certain mouvement de « retour à » accompagne toujours les transformations en apparence anarchiques d'un corpus primordial : à la fois, il les motive et permet de les identifier. L'appréciation de ce retour n'est donc pas ici uniquement négative, bien au contraire. Certes, Foucault souligne l'ambivalence d'un geste par lequel on prétend revenir au texte même du fondateur, dans sa simplicité première, alors qu'en même temps «il s'agit plutôt de ce qui est dit à travers les mots, dans leur espacement $\gg$ (p. 836). Il note également l'arbitraire des choix opérés quand il s'agit de discriminer dans un corpus ce qui est essentiel et ce qui est second ou dérivé. Mais il montre en même temps comment ces ambiguités permettent justement, non pas une réplique à l'identique, mais une série de décrochages et de renouvellements parfois complets de la discursivité initiale.

Or, cette fonction productrice du « retour à » s'inscrit dans cela même que Freud et Marx ont instauré. Dès lors, «l'oubli », comme postulat du « retour à », n'est plus accidentel, et donc fautif, mais « essentiel et constitutif » (p. 836). Sont ainsi fondateurs de discursivité ceux qui fondent une possibilité de discours revenant sans cesse à eux comme référence première, mais sur le mode toujours d'une découverte (un texte oublié de Freud, un passage négligé de Marx) et donc d'un écart : 
Il s'ensuit naturellement que ce retour, qui fait partie du discours lui-même, ne cesse de le modifier, que le retour au texte n'est pas un supplément historique qui viendrait s'ajouter à la discursivité elle-même et la redoublerait d'un ornement qui, après tout, n'est pas essentiel; il est un travail effectif et nécessaire de transformation de la discursivité elle-même. (p. 836)

La critique initiale chez Foucault du rapport normatif à un texte comme horizon de sens sans cesse reculé se renverse ici en marquage rigoureux d'une invention permanente. Or, une telle description pourrait très bien s'appliquer à l'économie des discours dogmatiques chrétiens : un discours n'est pas «chrétien » parce qu'il cite l'Écriture, mais parce qu'il s'efforce sans cesse de revenir à elle, en toute liberté d'invention et dans l'exacte mesure où ce geste appartient aux règles mêmes de sa formation discursive. Il resterait seulement à préciser en quoi l'Écriture rend possible, dans sa constitution singulière, ce jeu incessant de décalages et de retours, au même titre que le corpus freudien ou marxien.

Arrêtons-nous alors plus brièvement sur le second critère : celui du rapport à une personne historique ou «recours empirique ou psychologique ». Ce n'est plus ici la matérialité d'un texte qui est décisive, mais l'existence d'un être, comme volonté et parole singulières. Un tel rapport s'efforce précisément de contourner toute trace textuelle pour retrouver ce qu'un individu historique a véritablement voulu dire ou faire. Ce n'est pas une surprise : à aucun moment du cours de 1980 la personne historique de Jésus de Nazareth ou le rapport confessant du croyant à celle-ci ne sont mentionnés. Dans ce silence éloquent, on peut entendre l'écho du double travail critique effectué par Foucault sur une autre personnalité historique dont tout un ensemble de discours, d'institutions et de pratiques s'est réclamé : à nouveau, Karl Marx. Il s'agit à la fois pour Foucault de mettre en question le nom propre - en ce qu'il renverrait à la personnalité d'un fondateur dans l'unité historique indubitable de sa vie, de ses intentions, de sa pensée - et le rapport « confessant » et normatif à ce nom.

Recouverte par le nom propre, la cohérence supposée de la vie et de la pensée d'un homme ne survit pas à la discontinuité des pratiques discursives : suivant le niveau des événements historiques étudiés, la périodisation variera et zébrera les unités traditionnelles, sans souci de l'intégrité apparente des personnes et des doctrines. Comme Foucault le précise dans un entretien de 1967, Les mots et les choses dénient toute originalité à la pensée de Karl Marx, non pas globalement, mais «dans le domaine 
épistémologique précis de l'économie politique $\gg^{16}$. Marx l'économiste est donc intégré, voire dissous, dans une épistémè qui le précède et le détermine. Mais si l'on passe de l'économie politique à la conscience historique d'une société, c'est un autre découpage qui prévaudra, faisant alors du nom propre « Karl Marx » une rupture et un commencement $t^{17}$. Dans cette possibilité indéfinie de découpage offerte par la description formelle des énoncés, le nom finalement s'efface, devient « vide et inutile $\gg$ (ibid., p. 624), ou simple indicateur d'une certaine transformation historique. Ainsi, de même que Foucault disait «Marx, pour moi, ça n'existe pas $\gg^{18}$, il faudrait sans doute dire $\ll$ Jésus-Christ, pour Foucault, ça n'existe pas $\gg$. Néanmoins, Foucault reconnaît que cet effacement du nom opéré par l'archéologie n'empêche pas un ensemble de discours et de pratiques de se réclamer d'un unique «Marx $\gg$, dans la vérité de son existence historique. C'est l'entretien déjà cité de 1978 avec le philosophe Yoshimoto qui aborde la question précise du rapport entre le marxisme et l'être historique « Karl Marx ».

Au cours de l'entretien, Foucault propose une définition minimaliste $\mathrm{du} \ll$ marxisme $\gg$ en termes exclusivement de pouvoir : le marxisme est «l'ensemble des modes de manifestation du pouvoir liés, d'une ou façon ou d'une autre, à la parole de Marx $\gg^{19}$. Dans l'analyse critique que le philosophe français se propose alors d'opérer, il s'agit d' « examiner systématiquement chacun de ces modes de manifestation $\gg$ (p.611), mais sans chercher à établir un quelconque lien de causalité avec l'homme Karl Marx, même si les manifestations de pouvoir qui composent l'ensemble «marxisme » se réfèrent toutes à ce dernier en son existence historique. Car si Marx est un événement majeur de la vie politique et intellectuelle du XIX ${ }^{\mathrm{e}}$ siècle, ce qu'il a un jour pensé, voulu, ou même tout simplement été, n'a pas de pertinence ni d'effet déterminant au-delà de son époque, sinon à reconstituer sa personne de manière arbitraire comme le font précisément les pouvoirs marxistes. En dehors de cette reprise autoritaire et artificielle, «Karl Marx » appartient à jamais au passé et une meilleure connaissance de ce qu'il a été ne sera pas source

$D E \mathrm{n}^{\circ} 48$, « Sur les façons d'écrire l'histoire », I, p. 615 ; cet exemple sera repris dans la réponse à la revue Esprit en $1968: D E \mathrm{n}^{\circ} 58$, « Réponse à une question », I, p. 704.

$17 D E n^{\circ} 48$, « Sur les façons d'écrire l'histoire », I, p. 615.

$18 D E \mathrm{n}^{\circ} 169$, «Questions à Michel Foucault sur la géographie », II, p. 38 .

$19 D E n^{\circ} 235$, « Méthodologie pour la connaissance du monde : comment se débarrasser du marxisme », II, p. 611 . 
de meilleurs effets politiques. « $[\ldots]$ remonter jusqu'à la source pour savoir ce que Marx a effectivement dit, saisir sa parole à l'état pur et la considérer comme l'unique loi » (ibid., p. 611) demeure sans intérêt pour une analyse contemporaine du marxisme. Cela signifie que l'opération de vérification des mécanismes de pouvoir que se propose de faire Foucault n'a pas son principe du côté de Marx lui-même. L'écart entre Marx et le marxisme n'est pas le fruit d'une erreur ou d'une mauvaise interprétation qui serait rectifiée par un quelconque retour à l'origine.

Il n'est donc pas possible en toute rigueur de définir d'inéluctables « effets de vérité » (ibid., p. 602) de la parole (vivante) de Marx qui, audelà de circonstances historiques précises, auraient produit une regrettable philosophie étatique ou au contraire permettraient de la corriger. Cette dernière remarque nous semble importante pour le sujet qui nous occupe : elle peut en effet être appliquée aux péripéties historiques du pastorat chrétien. Des analyses de Foucault, on peut déduire que les diverses modalités du pouvoir ecclésial ont un rapport historiquement indécidable avec les «vraies » paroles de Jésus. Pour cette raison, aucune des analyses du philosophe concernant les régimes disciplinaires ou ascétiques du christianisme ne se préoccupe de l'existence historique d'un homme nommé Jésus, le lien de cause à effet ne pouvant être objet d'étude. Des premiers siècles à aujourd' hui, n'a fonctionné continûment qu'un certain retour au texte fondateur : les apôtres ou évangélistes comme « fondateurs de discursivité », au même titre que Karl Marx et Sigmund Freud.

\section{*}

Concluons alors notre étude sur la possibilité d'une histoire dogmatique du christianisme. Si l'on utilise les outils laissés par Foucault, une histoire de l'économie dogmatique chrétienne ne peut recourir au rapport normatif à une personne historique, dont il est vain d'établir les vraies paroles et de rechercher quels vrais « effets $»$ elles ont eus ou auraient dû avoir à travers les siècles ; mais une telle histoire peut et doit intégrer le rapport à un texte fondateur dans la forme définie de «l'instauration d'une discursivité », qui permet de définir un jeu subtil d'écarts et de retours, d'inventions et de redécouvertes. Si l'on osait un parallèle confessionnel, on pourrait dire que la conception foucaldienne de l'unité doctrinale du christianisme se révèle plus catholique que protestante. 
L'opposition entre les deux critères n'est cependant pas aussi nette qu'il y paraît. Une discursivité caractérisée par un mouvement perpétuel de « retour à » conserve toujours un lien intrinsèque à la personne historique qui se trouve derrière le texte ; c'est même la condition principale du mouvement de « retour », comme Foucault le note à la toute fin de l'article de 1969:

Or pour caractériser ces retours, il faut ajouter un dernier caractère : ils se font vers une sorte de couture énigmatique de l'œuvre et de l'auteur. En effet, c'est bien en tant qu'il est texte de cet auteur-ci que le texte a valeur instauratrice, et c'est pour cela, parce qu'il est texte de cet auteur, qu' il faut revenir vers lui. ${ }^{20}$

On notera cependant que ce passage sur la «couture énigmatique» n’a pas été lu par Foucault lorsqu'il a repris ce texte à l'université de Buffalo en $1970^{21}$.

21 Comme l'indiquent les éditeurs des Dits et écrits, en mettant ce passage entre crochets. Foucault autorisera cependant indifféremment la réédition des deux versions : $D E n^{\circ} 69$, «Qu'est-ce qu'un auteur? », II, p. 817 . 


\section{BIBLIOGRAPHIE SÉLECTIVE}

\section{Philosophie}

\section{Textes}

Aristote, De l'âme, A. Jannone éd., E. Barbotin trad. et note, Paris, Les Belles Lettres, 2002.

Deleuze Gilles, Logique du sens, Paris, Éditions de Minuit, 1969.

Heidegger Martin, Acheminement vers la parole, J. Beaufret, W. Brokmeier et F. Fédier trad., Paris, Gallimard (Classiques de la philosophie), 1976.

- Kant et le problème de la métaphysique, A. De Waelhens et W. Biemel trad., Paris, Gallimard (Tel), 1981.

- Lettre sur l'humanisme, R. Munier trad., édition bilingue, Paris, Aubier Montaigne (Philosophie de l'esprit), 1983.

Kant Emmanuel, Critique de la raison pure, A. J. L. Delamarre, F. Marty, J. Barni trad., Paris, Gallimard (Folio. Essais), 1980.

- Anthropologie du point de vue pragmatique, précédé de M. Foucault, «Introduction à l'Anthropologie », M. Foucault trad., Paris, Vrin (Bibliothèque des textes philosophiques), 2008.

Kierkegaard Søren, La répétition, J. Privat trad., Paris, Payot \& Rivages (Petite bibliothèque), 2003.

Marx Karl et Engels Friedrich, L'idéologie allemande, précédé de Karl Marx, Thèses sur Feuerbach, H. Auger, G. Badia et J. Baudrillard trad., Paris, Éditions sociales, 1982.

Montaigne Michel de, Les essais en français moderne, A. Lanly éd., Paris, Gallimard (Quarto), 2009.

Nietzsche Friedrich, Fragments posthumes, début 1888-début janvier 1889 (Ouvres philosophiques complètes XIV), G. Colli et M. Montinari éd., J.-C. Hémery trad., Paris, Gallimard, 1977. 
- La naissance de la tragédie; Fragments posthumes automne 1869-printemps 1872, G. Colli et M. Montinari éd., M. Haar, P. Lacoue-Labarthe et J.-L. Nancy trad., Paris, Gallimard (Folio Essais), 1989.

- Le crépuscule des idoles, suivi de Le cas Wagner, H. Albert trad., Paris, Flammarion (GF), 1985.

- Éléments pour la généalogie de la morale. Écrit de combat ajouté à Par-delà le bien et le mal, P. Wotling trad., Paris, Librairie générale française (Le livre de poche. Classiques de la philosophie), 2000.

Platon, Théétète, M. Narcy trad., 2 e édition corrigée, Paris, Flammarion (GF), 1995. Wittgenstein Ludwig, Tractatus logico-philosophicus, suivi de Investigations philosophiques, P. Klossowski trad., Paris, Gallimard (Bibliothèque des idées), 1961.

\section{Études}

BRÉHIER Émile, La théorie des incorporels dans l'ancien stoïcisme, $9^{\mathrm{e}}$ édition, Paris, Vrin, 1997 [1907].

KARSENTi Bruno éd., dossier «Anthropologie structurale et philosophie : LéviStrauss $\gg$, Archives de philosophie, t. 66, vol.1, 2003, p. 5-88.

PICHé Claude, «Heidegger et Cohen, lecteurs de Kant », Archives de philosophie, t. 61, vol. 4, 1998, p. 603-628.

SCHNell Alexander, De l'existence ouverte au monde fini. Heidegger 1925-1930, Paris, Vrin (Bibliothèque d'histoire de la philosophie), 2005.

VERnANt Jean-Pierre, Mythe et pensée chez les Grecs. Étude de psychologie historique, Paris, Maspero, 1965.

Vernant Jean-Pierre et Vidal-Naquet Pierre, Mythe et tragédie en Grèce ancienne, t. I, Paris, La Découverte, 2001 [1972].

Wotling Patrick, La philosophie de l'esprit libre. Introduction à Nietzsche, Flammarion (Champs. Essais), 2008

\section{Épistémologie}

Aron Raymond, Introduction à la philosophie de l'histoire. Essai sur les limites de l'objectivité historique, édition revue et augmentée, Paris, Gallimard (Tel), 1981 [1938]. Bollack Jean, Sens contre sens. Comment lit-on? Entretiens avec Patrick Llored, Genouilleux, La passe du vent, 2000.

Compagnon Antoine, La seconde main ou le travail de la citation, Paris, Seuil, 1979 FEYerabend Paul, Contre la méthode. Esquisse d'une théorie anarchiste de la connaissance, B. Jurdant et A. Schlumberger trad., Paris, Seuil (Points. Sciences), 1988 [1979]. 
LAdMIRAL Jean-René, Traduire : théorèmes pour la traduction, Paris, Gallimard (Tel), 1994.

Le Goff Jacques et Nora Pierre, Faire de l'histoire, t. III : Nouveaux objets, Paris, Gallimard (Folio. Histoire), 1974.

Levi Giovanni, Le pouvoir au village. Histoire d'un exorciste dans le Piémont du $x v u^{e}$ siècle, M. Aymard trad., précédé de L'histoire au ras du sol par Jacques Revel, Paris, Gallimard (Bibliothèque des histoires), 1989.

Marrou Henri Irénée, Carnets posthumes, F. Marrou-Flamant éd., Paris, Cerf (Intimité du christianisme), 2006.

Ricoeur Paul, La mémoire, l'histoire, l'oubli, Paris, Seuil, 2000.

VALETTE Mathieu, « La genèse textuelle des concepts scientifiques. Étude sémantique sur l'œuvre du linguiste Gustave Guillaume », Cahiers de lexicologie, t. 89, vol. 2, 2006, p. 125-142.

Veyne Paul, Comment on écrit l'histoire, Paris, Seuil (L'univers historique), 1978.

Weber Max, L'éthique protestante et l'esprit du capitalisme, J. Chavy trad., Paris, Plon, 1964.

— Essais sur la théorie de la science, J. Freund trad., Paris, Plon, 1965.

\section{Histoire du christianisme}

\section{Période patristique}

\section{Textes}

La doctrine des douze apôtres (Didachè), W. Rordorf et A. Tuilier trad., $2^{\mathrm{e}}$ édition revue et augmentée, Paris, Cerf (Sources chrétiennes ; 248 bis), 1998 [1978].

Les écrits des Pères apostoliques, F. Louvel éd., L. Bouyer préf., Paris, Cerf (Chrétiens de tous les temps), 1963.

Les Pères apostoliques, t. I : Doctrine des Apôtres, Épître de Barnabé, H. Hemmer, G. Oger et A. Laurent trad., Paris, Librairie Alphonse Picard (Textes et documents pour l'étude historique du christianisme ; 5), 1919.

Les Pères apostoliques, t. II : Clément de Rome. Épitre aux Corinthiens, Homélie du $u^{e}$ siècle, H. Hemmer trad., $2^{e}$ édition, Paris, Librairie Alphonse Picard (Textes et documents pour l'étude historique du christianisme ; 10), 1926.

Athanase d'Alexandrie, Vie d'Antoine, G. J. M. Bartelink éd. et trad., Paris, Cerf (Sources chrétiennes; 400), 1994.

Augustin, Les confessions, A. Solignac éd., E. Tréhorel et G. Bouissou trad., Euvres de saint Augustin, t. XIV, Paris, Desclée de Brouwer, 1962. 
Cyprien, Correspondance, t. I, L. Bayard éd. et trad., Paris, Les Belles Lettres (Collection des universités de France), 1945 [1925].

- Correspondance, t. II, L. Bayard éd. et trad., Paris, Les Belles Lettres (Collection des universités de France), 1925.

Denys l'Aréopagite (Pseudo-), Euvres complètes, M. de Gandillac éd. et trad., Paris, Aubier-Montaigne (Bibliothèque philosophique), 1980 [1943].

Hermas, Le Pasteur, R. Joly éd. et trad., Paris, Cerf (Sources chrétiennes ; 53), 1958. IRÉNÉE de Lyon, Contre les hérésies, Livre I, t. II, A. Rousseau et L. Doutreleau éd. et trad., Cerf (Sources chrétiennes ; 264), 1979.

Jean Cassien, Institutions cénobitiques, J.-C. Guy éd. et trad., Paris, Cerf (Sources chrétiennes; 109), 1965.

- Conférences, t. I, dom E. Pichery éd. et trad., Paris, Cerf (Sources chrétiennes ; 42), 1966.

- Conférences, t. II, dom E. Pichery éd. et trad., Paris, Cerf (Sources chrétiennes ; 54), 1967.

- Conférences, t. III, dom E. Pichery éd. et trad., Paris, Cerf (Sources chrétiennes ; 64), 1971.

JÉRÔME, Lettres, t. IV, J. Labourt éd. et trad., Paris, Les Belles Lettres (Collection des universités de France), 1954.

Justin, Apologies, L. Pautigny éd. et trad., Paris, Librairie Alphonse Picard et Fils (Textes et documents pour l'étude historique du christianisme), 1904.

Palladius, Histoire lausiaque (Vies d'ascètes et de Pères du désert), A. Lucot éd. et trad., Paris, Librairie Alphonse Picard et Fils, 1912.

Tertullien, Opera omnia cum selectis praecedentium editionum lectionibus, PetitMontrouge, J.-P. Migne éd., Paris, Migne (Patrologia Latina), 1844, 2 volumes.

- Euvres, E.-A. de Genoude trad., 2édition, Paris, Louis Vivès, 1852, 3 volumes.

- De Paenitentia. De Pudicitia, P. de Labriolle trad., Paris, Alphonse Picard et Fils (Textes et documents pour l'étude historique du christianisme ; 3), 1906.

- Traité du baptême, R. F. Refoulé éd. et trad., M. Drouzy trad., Paris, Cerf (Sources chrétiennes; 35), 1952.

\section{Études}

«Pénitence », Dictionnaire de spiritualité ascétique et mystique, t. XII, $1^{\mathrm{re}}$ partie, Paris, Beauchesne, 1984.

Brown Peter, Genèse de l'Antiquité tardive, A. Rousselle trad., Paris, Gallimard (Bibliothèque des histoires), 1983 [édition originale américaine 1978].

- La vie de saint Augustin, J.-H. Marrou trad., $2^{\mathrm{e}}$ édition augmentée, Paris, Seuil (Points histoire), 2001 [édition originale américaine 1967].

CAPELLE Bernard, «L'introduction du catéchuménat à Rome », Recherches de théologie ancienne et médiévale, t. V, 1933, p.129-154. 
DANiÉLOU Jean, Platonisme et théologie mystique. Essai sur la doctrine spirituelle de saint Grégoire de Nysse, Paris, Aubier (Théologie), 1944.

- Histoire des doctrines chrétiennes avant Nicée, t. I : Théologie du Judéo-christianisme, M.-O. Boulnois éd. (texte établi à partir de l'édition italienne de 1974), $2^{\mathrm{e}}$ édition, Paris, Desclée/Cerf (Bibliothèque de théologie), 1991 [1958].

- Histoire des doctrines chrétiennes avant Nicée, t. II : Message évangélique et culture hellénistique aux $\imath^{e}$ et $\imath 11^{e}$ siècles, $2^{\mathrm{e}}$ édition, Paris, Desclée/Cerf (Bibliothèque de théologie), 1990 [1961].

- Histoire des doctrines chrétiennes avant Nicée, t. III : Les origines du christianisme latin, $2^{\mathrm{e}}$ édition, Paris, Desclée/Cerf, 1991 [1978].

DodDs Eric R., Pagan and Christian in an Age of Anxiety. Some Aspects of Religious Experience from Marcus Aurelius to Constantine, Cambridge, Cambridge University Press, 1990 [1965].

Dondeyne Albert, « La discipline des scrutins dans l'Église latine avant Charlemagne $\gg$, Revue d'Histoire ecclésiastique, t. 28, 1932, p. 5-33.

Dölger Franz Joseph, Der Exorzismus in altchristichen Taufritual. Eine religiongeschichtliche Studie, Paderborn, Schöningh, 1909.

Grotz Joseph, Die Entwicklung des Bußstufenwesens in vornicänischen Kirche, Fribourg, Herder, 1955.

Guy Jean-Claude, « Remarques sur le texte des Apophtegmata Patrum », Recherches de science religieuse, t. 43, 1955, p. 252-258.

Holstein Henri, «L'exhomologèse dans l'Adversus haereses de saint Irénée », Recherches de science religieuse, t. 35, 1948, p. 282-288.

Loisy Alfred, L'Évangile et l'Église, $2^{\mathrm{e}}$ édition augmentée, Bellevue, Chez l'auteur, 1903.

- Les Livres du Nouveau Testament traduits du grec en français, Paris, Émile Nourry, 1922.

Spanneut Michel, Le stoïcisme des Pères de l'Église. De Clément de Rome à Clément d'Alexandrie, Paris, Seuil (Patristica Sorbonensia ; 1), 1957.

Autres périodes

Textes

Boенмe Jakob, Confessions, A. Klimov préface, notes et commentaires, Paris, Fayard (Documents spirituels), 1973.

Eckhart Johannes dit Maître, Sermons, t. I : Sermons 1-30, J. Ancelet-Hustache trad., Paris, Seuil, 1974.

- Du détachement et autres textes, G. Jarczyk et P.-J. Labarrière éd. et trad., Paris, Rivages poche (Petite bibliothèque), 1995. 
FrançoIs de Sales, Correspondance : les lettres d'amitié spirituelle, A. Ravier éd., Paris, Desclée de Brouwer (Bibliothèque européenne), 1980.

TAuler Jean, Sermons, E. Hugueny, J.-P. Jossua éd., G. Théry et M. A. L. Corin trad., Paris, Cerf (Sagesses chrétiennes), 1991.

Thomas d'Aquin, Somme contre les gentils, R. Bernier, M. Corvez, M.-J. Gerlaud, F. Kerouanton, L.-J. Moreau trad., Paris, Cerf, 1993.

\section{Études}

«Doelger (François-Joseph) », Dictionnaire d'histoire et de géographie ecclésiastiques, t. XIV, Paris, Letouzay et Ané, 1960.

Aubert Roger, David Knowles et Ludovicus J. Rogier éd., Nouvelle histoire de l'Église, Paris, Seuil, 1963-1975, 5 volumes.

Chaunu Pierre, Le temps des réformes. Histoire religieuse et système de civilisation. La crise de la chrétienté. L'éclatement 1250-1550, Paris, Fayard (Le Monde sans frontières), 1975 .

CoHn Norman, The Pursuit of the Millennium. Revolutionary Millenarians and Mystical Anarchists of the Middle Ages, $3^{\mathrm{e}}$ édition revue et augmentée, Londres, Maurice Temple Smith, 1970 [1957].

Delumeau Jean, La peur en Occident ( $x v^{e}-x v ı \imath^{e}$ siècles), Paris, Fayard, 1978.

- Le péché et la peur. La culpabilisation en Occident, $x u 1{ }^{e}-x v u 1^{e}$ siècles, Paris, Fayard, 1983.

- L'aveu et le pardon. Les difficultés de la confession, xun ${ }^{e}-x v u u^{e}$ siècle, Paris, Libraire générale française (Le Livre de poche), 1992 [1990].

- Une histoire du Paradis, t. II : Mille ans de bonheur, Paris, Fayard, 1995.

Goldmann Lucien, Le Dieu caché. Étude sur la vision tragique dans les Pensées de Pascal et dans le théâtre de Racine, Paris, Gallimard (Bibliothèque des idées), 1955.

Harnack Adolf von, Histoire des dogmes, E. Choisy trad., Paris, Cerf (Patrimoines du christianisme), 1993 [édition originale allemande 1886-1889].

KoџAкоwsкi Leszek, Chrétiens sans Église. La conscience religieuse et le lien confessionnel au $x v u^{e}$ siècle, A. Posner trad., Paris, Gallimard (Bibliothèque de philosophie), 1969 [édition originale polonaise 1965].

LÉCRIVAIN Philippe, «Les millénarismes du christianisme antique et médiéval », Histoire raisonnée de la philosophie morale et politique, A. Caillé, C. Lazzeri et M. Senellart éd., Paris, La Découverte, 2001, p.151-161.

PÉPIN Jean, Mythe et allégorie. Les origines grecques et les contestations judéo-chrétiennes, édition revue et augmentée, Paris, Études augustiniennes, 1976. 


\section{Euvres et essais littéraires}

Arnaud Alain, Pierre Klossowski, Paris, Seuil (Les contemporains), 1990.

BataILle Georges, L'expérience intérieure, Paris, Gallimard (Tel), 1978 [1943].

Forest Philippe, Histoire de Tel Quel, 1960-1982, Paris, Seuil (Fiction \& Cie), 1995.

Jenny Laurent et A. Pfersmann Andreas éd., Traversées de Pierre Klossowski, Genève, Droz, 1999.

KLossowski Pierre, La vocation suspendue, Paris, Gallimard, 1950.

- Le bain de Diane, Paris, Gallimard, 1980 [1956].

- Un si funeste désir, Paris, Gallimard, 1963.

- Sade mon prochain, précédé de Le philosophe scélérat, édition revue et corrigée, Paris, Seuil (Pierres vives), 1967 [1947].

GUICHAR Thierry, « Roger Laporte. L'épreuve par neuf», suivi d'un entretien avec Roger Laporte, Le Matricule des anges, $\mathrm{n}^{\circ} 32$, septembre-octobre 200o, p. 14-23. LAPORTE Roger, La Veille, Paris, Gallimard (Le chemin), 1963. 



\section{INDEX}

A

Althusser, Louis - 21, 22, 356

Ambroise de Milan (saint) — 121, 155,300

Antoine (saint) - 82, 84, 123, 179, 272

Aristote - 37, 106, 107, 198, 342

Arnauld (le Grand) - 152

Aron, Raymond - 37, 38, 39, 120, 244

Artémidore d'Ephèse - 124

Athanase d'Alexandrie

$$
\text { (saint) }-82,84
$$

Augustin (saint) - 82, 89, 114, 121,

$122,123,125,226,269$

Austin, John L. - 32, 132, 148

B

Bally, Charles - 258

Barthes, Roland - 75, 246, 259, 260

Baruzi, Jean - 267

Bataille, Georges - 243, 244, 245, $246,247,248,249,250,251,252$,
$253,254,255,257,260,261,262$,

$270,273,287,288,289,291,345$

Benjamin, Walter — 204

Benoît (saint) - 297, 298

Bentham, Jeremy - 13, 43, 44, 45, $48,49,50$

Bernauer, James - 11, 184, 225

Blanchot, Maurice - 56, 174, 180, $183,243,244,245,246,257,259$, $260,261,262,264,265,266,267$, $268,270,273,274,275,288,345$

Bloch, Ernst - 74, 85

Bloch, Marc - 21

Boehme, Jakob - 256

Bollack, Jean - 163, 164, 168, 219, 220, 221

Borromée, Charles - 138

Bossuet, Jacques-Bénigne - 255

Bouyer, Louis - 82, 83

Bréhier, Émile - 24

Brown, Peter - 11, 122, 123, 124,

125,193 
C

Cassien, Jean - 83, 84, 85, 113, 114, $116,125,155,175,178,191,225,297$, $303,308,318,322,329,333,336$

Certeau, Michel de - 74, 78, 262, 267

Chartier, Roger - 208

Chaunu, Pierre - 36, 77, 78, 350

Cicéron - 177

Clark, Elizabeth A. - 225

Clavel, Maurice - 331, 334

Clément d'Alexandrie - 109, 181, $192,225,328$

Clément de Rome - 226, 329

Cohn, Norman $-73,74$

Compagnon, Antoine - 205, 215, 216,218

Courcelle, Pierre - 121, 129, 226

Cyprien (saint) - 113, 155, 211, 214, 217,300

Cyrille de Jérusalem (saint) -218

D

Daniélou, Jean - 88, 114, 120, 122, $184,189,242,354$

Defert, Daniel - 62, 81, 191, 193, $247,331,334,345$

Deleuze, Gilles — 23, 24, 106, 147

Delumeau, Jean - 56,60, 86, 135, 136, 139, 140, 142, 144, 152, 153, 154, 157

Denys l'Aréopagite

(Pseudo-) - 182, 183, 255, 266, 269

Derrida, Jacques - 98, 100, 102, $107,172,173$
Descartes, René - 97, 98,100,101, 172, 173, 238

Dodds, Eric R. - 122

Dölger, Franz Joseph - 192

Dondeyne, Albert — 192

Dumézil, Georges - 47, 163, 244

E

Eckhart, Johannes dit Maître - 254, 255, 256, 257, 270

Engels, Friedrich - 21, 74

Épictète - 175, 178, 303, 342

F

Febvre, Lucien - 21

Fénelon, François (de La Mothe) $-78,255$

Festugière, André-Jean — 122

Feyerabend, Paul - 221, 223, 224, 226

Flaubert, Gustave - 56, 179, 180, $181,193,243,270,272,276$

François de Sales (saint) - 140, 141, 175, 307

Freud, Sigmund - 62, 203, 356, 359

G

Gadamer, Hans Georg — 219

Galilée - 223, 226 
Genoude, Antoine E. - 114, 190, 196, 197, 199

Gernet, Louis - 163

Gerson, Jean de - 153

Giroust, Jacques - 140

Goldmann, Lucien - 19, 75

Gordon, Colin - 235

Grégoire de Naziance - 82, 298

Grégoire de Nysse - 114, 184

Grégoire le Grand - 157, 158, 218, 242,298

Greimas, Algirdas J. — 259, 260

Guy, Jean-Claude - 178

Guyon (Madame de la Mothe) $-76,78$

\section{$\mathrm{H}$}

Habert, Louis - 138, 143, 150, 153

Hadot, Ilsetraut - 111

Hadot, Pierre - 121

Harnack, Adolf (von) - 353

Hegel, Georg Wilhelm Friedrich

- $33,85,244,270,338$

Heidegger, Martin - 220, 252, 258, 259, 264, 270, 277, 278, 281, 282, $283,287,288,289$

Hemmer, Hippolyte - 114, 188

Hermas - 189, 203, 210, 211, 226, 329

Hippolyte de Rome - 114

Holstein, Henri - 192

Hyppolite, Jean — 117, 277, 292,

293, 331
Irénée de Lyon (saint) - 89, 201, 352

J

Jaffro, Laurent - 178

Jean Chrysostome (saint) - 190, 298

Jean de la Croix - 76, 153, 255, 264, 266

Jérôme (saint) - 89, 317

Joachim de Flore $-85,86,87,89$

Justin -89

Justin Martyr — 193,196

K

Kant, Emmanuel - 194, 251, 252, $277,278,279,280,281,282,283$, $284,285,286,289,327$

Kierkegaard, Sören - 273, 331, 347 Klossowski, Pierre - 56, 179, 205, 243, 244, 246, 247, 251, 253, 261, $270,273,274,275,276,277,286$, 288,345

Kojève, Alexandre - 244

Kołakowski, Leszek — 20, 73, 74, $75,76,77,78,79$

$\mathrm{L}$

Labriolle, Pierre de - 195, 197, 198, 199, 200, 210, 213, 214 Lacan, Jacques - 257, 258, 356 
N

Ladmiral, Jean-René — 204, 207, 220

La Perrière, Guillaume (de) - 68, $69,142,157,159$

Laporte, Roger - 243, 257, 260, $262,263,264,265$

Le Brun, Jacques - 78, 255, 267

Lécrivain, Philippe - 86

Lejay, Paul - 114, 188

Léonard, Jacques - 35, 42, 43, 51

Levi, Giovanni - 30, 68

Levinas, Emmanuel - 265

Lévi-Strauss, Claude - 232, 244, 249

Liguori, Alphonse de - 138

Loisy, Alfred - 353, 354

Lubac, Henri de - 86, 114, 189

Lucrèce - 273

Luther, Martin - 74, 265, 352

M

Mallarmé, Stéphane - 261, 267, 268

Marc Aurèle - 342

Marrou, Henri Irénée - 120, 121, 129

Marx, Karl - 21, 33, 75, 203, 356,

$357,358,359$

Migne, Jacques-Paul - 80, 81, 188,

193

Milhard, Pierre - 138, 151

Montaigne, Michel de -118

Nietzsche, Friedrich - 31, 36, 37, $132,194,202,203,237,239,240$, 241, 242, 243, 245, 246, 248, 252, 254, 260, 271, 272, 273, 277, 278, $283,284,285,286,287,288,289$, 290, 291, 292, 293

$\mathrm{O}$

Orcibal, Jean - 267

Origène - 114, 181, 216, 218, 275, 355

P

Palladius - 81, 83, 84, 85

Pascal, Blaise - 234

Paul (saint) - 81, 114, 123, 188, 218, 345,347

Philon d'Alexandrie - 181, 275, 319, 355

Platon - 22, 70, 115, 159, 163, 169, $183,189,198,240,241,289,342$

Polycarpe (saint) - 217, 329

Q

Quodvultdeus - 192

$\mathrm{R}$

Refoulé, François - 191

Ricœur, Paul - 22, 30, 219

Robbe-Grillet, Alain — 260, 261 
Sade, François (marquis de) - 253, $261,269,270,273,288$

Sartre, Jean-Paul — 261

Saussure, Ferdinand de — 204, 258,259

Searle, John R. - 32, 132

Senellart, Michel - 12, 93, 111

Sénèque - 177, 212, 303, 308, 337

Sollers, Philippe - 243, 260, 261, 264, 265

Sophocle - 127, 160, 161, 162, 163, $164,165,166,168,176,179,220$

Spanneut, Michel - 120, 121, 226

Surin, Jean-Joseph $-76,78$

$\mathrm{T}$

Tauler, Jean - 256, 270

Tertullien - 57, 89, 112, 114, 118, 122, $136,155,190,195,196,197,198,199$, 200, 202, 203, 206, 209, 210, 211,
$212,213,214,215,216,217,294,311$, $312,317,321,323,333,347,355$

Thomas d'Aquin (saint) - 136, 140,256

V

Vernant, Jean-Pierre - 21, 163, 164 Veyne, Paul - 26, 64, 123, 141, 171, 193

Vincent de Paul (saint) - 234

W

Wahl, Jean - 246, 331

Weber, Max - 17, 20, 29, 36, 37, 41, $42,44,52,55,75,241$

Wittgenstein, Ludwig - 32, 223 

Michel Foucault, bibliographie $\quad 7$

Introduction 11

CHAPITRE Un — Définition du modèle stratégique 19

Usage des concepts dans l'historiographie de Michel Foucault 19

La stratégie comme connexion de l'hétérogène 31

Les «formes de rationalité » sont-elles des idéalités?

CHAPITRE DEUX — Le christianisme

au risque de l'analyse stratégique $\quad 56$

Le christianisme éclaté : 1973-1977 56

Le christianisme comme «gouvernementalité» : 1978

Un nouveau regard sur les institutions politiques : Église et État 64

Pastorat chrétien et marxisme : un miroir déformant? 72

Évaluation critique de l'histoire pastorale $\quad 78$ 
Origine et mutation de la notion de « régime de vérité»

Postérité de la notion de « régimes de vérité» : vers le domaine de l'éthique? 103

Une double réduction de l'objet « christianisme»

CHAPITRE TROIS — Foucault et

l'historiographie de l'Antiquité tardive

\section{DEUXIÈME PARTIE \\ Une lecture singulière des Pères}

CHAPITRE QUATRE — Le christianisme dans le texte

Limites de la première histoire de l'aveu (1974-1978)

Spécificité de la forme religieuse de l'aveu

La confession est-elle une pratique coercitive?

Les manuels des confesseurs comme pratique discursive

Le retour au texte : le virage de 1980

Abandon du principe d'exemplarité

Quoi de neuf? Edipe

Du fils $\mathcal{E}$ dipe aux Pères chrétiens : radicalisation d'une lecture

CHAPITRE CINQ - Conséquences

sur l'usage des textes: traduire les Pères

Lire : des choix classiques

Traduire : Foucault, traducteur «sourcier»

Citer : la citation comme pratique de la lexicalisation 
TROISIÈME PARTIE

Une interprétation du christianisme comme voie moyenne

CHAPITRE SEPT — Le christianisme comme Orient perdu

Tragédie de l'homme occidental 231

Une expérience d'abord littéraire 243

L'expérience de la transgression: Georges Bataille 245

L'être du langage, l'être: Roger Laporte, Maurice Blanchot 257

L'Orient, tentation originelle du christianisme:

Pierre Klossowski, Gustave Flaubert 270

Le retour de la métaphysique

CHAPITRE HUIT — La relève d'un temps précaire 292

Premières hypothèses sur la différence chrétienne 292

La différence entre le moine et le pénitent

Les techniques de soi chrétiennes dans le monachisme 303

Les techniques de soi chrétiennes avant le monachisme

Pourquoi le laïc ne peut être dirigé comme le moine

La différence entre le salut et la perfection

Conclusion

Appendice - Possibilité de compléter l'histoire stratégique du christianisme

Bibliographie sélective

Index 



\section{Autres titres \\ de la collection}

Rousseau, politique et esthétique.

Sur la Lettre à d'Alembert

Sous la direction de Blaise Bachofen

et Bruno Bernardi

2011

Études sur Spinoza et les philosophies

de l'âge classique

Alexandre Matheron

2011

Raison pratique et normativité chez Kant Sous la direction de Jean-François Kervégan 2010

Utopies des Lumières

Sous la direction d'Antoine Hatzenberger

2010

Les Lumières en mouvement.

La circulation des idées au XVIII ${ }^{\mathrm{e}}$ siècle

Sous la direction d'Isabelle Moreau 
Les sources de la morale laïque. Héritages croisés

Sous la direction d'Anne-Claire Husser,

Bruno Barthelmé et Nicolas Piqué

2009

Hobbes, Spinoza ou les politiques de la Parole.

Critique de la sécularisation et usages

de l'histoire sainte à l'âge classique

Sous la direction de Julie Saada

2009

Cesare Beccaria. Des délits et des peines

Texte italien établi par Gianni Francioni.

Introduction, traduction et notes

par Philippe Audegean

2009

Le libéralisme au miroir du droit.

L'État, la personne, la propriété

Sous la direction de Blaise Bachofen

2008

Bakounine jeune hégélien.

La philosophie et son dehors

Jean-Christophe Angaut

2007

Simon Stevin. De la vie civile 1590

Présentation et traduction de Catherine Secretan.

Études réunies par Catherine Secretan

et Pim den Boer

2005

Nicolas de Cues,

penseur et artisan de l'unité

Sous la direction de

David Larre 
L'empire des gènes.

Histoire de la sociobiologie

Jacques Ruelland

2004

Matérialisme et passions

Sous la direction de Pierre-François Moreau

et Ann Thomson

2004 

Cet ouvrage, composé avec les caractères Arno Pro et Seria, a été mis en page par les soins du service d'édition de l'École normale supérieure de Lyon. L'ouvrage a été reproduit sur du papier Munken Print cream certifié FSC.

Il a été achevé d'imprimer par l'imprimerie Laballery en décembre 2011.

Dépot légal

décembre 2011

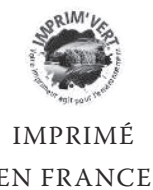


